Cochrane Database of Systematic Reviews

\title{
Immunosuppressive treatment for proliferative lupus nephritis
} (Review)

Tunnicliffe DJ, Palmer SC, Henderson L, Masson P, Craig JC, Tong A, Singh-Grewal D, Flanc RS, Roberts MA, Webster AC, Strippoli GFM

Tunnicliffe DJ, Palmer SC, Henderson L, Masson P, Craig JC, Tong A, Singh-Grewal D, Flanc RS, Roberts MA, Webster AC, Strippoli GFM.

Immunosuppressive treatment for proliferative lupus nephritis.

Cochrane Database of Systematic Reviews 2018, Issue 6. Art. No.: CD002922.

DOI: 10.1002/14651858.CD002922.pub4.

www.cochranelibrary.com 
TABLE OF CONTENTS

HEADER

ABSTRACT

PLAIN LANGUAGE SUMMARY

SUMMARY OF FINDINGS

BACKGROUND

OBJECTIVES

METHODS

RESULTS

Figure 1.

Figure 2.

DISCUSSION

AUTHORS' CONCLUSIONS

ACKNOWLEDGEMENTS

REFERENCES

CHARACTERISTICS OF STUDIES

DATA AND ANALYSES

Analysis 1.1. Comparison 1 Mycophenolate mofetil (MMF) versus IV cyclophosphamide (CPA), Outcome 1 Death.

Analysis 1.2. Comparison 1 Mycophenolate mofetil (MMF) versus IV cyclophosphamide (CPA), Outcome 2 Remission. .............

Analysis 1.3. Comparison 1 Mycophenolate mofetil (MMF) versus IV cyclophosphamide (CPA), Outcome 3 Adverse renal outcomes.

Analysis 1.4. Comparison 1 Mycophenolate mofetil (MMF) versus IV cyclophosphamide (CPA), Outcome 4 Stable kidney function.

Analysis 1.5. Comparison 1 Mycophenolate mofetil (MMF) versus IV cyclophosphamide (CPA), Outcome 5 Ovarian failure. ..... Analysis 1.6. Comparison 1 Mycophenolate mofetil (MMF) versus IV cyclophosphamide (CPA), Outcome 6 Menstrual irregularities.

Analysis 1.7. Comparison 1 Mycophenolate mofetil (MMF) versus IV cyclophosphamide (CPA), Outcome 7 Infection.

Analysis 1.8. Comparison 1 Mycophenolate mofetil (MMF) versus IV cyclophosphamide (CPA), Outcome 8 Malignancy.

Analysis 1.9. Comparison 1 Mycophenolate mofetil (MMF) versus IV cyclophosphamide (CPA), Outcome 9 Leucopenia.

Analysis 1.10. Comparison 1 Mycophenolate mofetil (MMF) versus IV cyclophosphamide (CPA), Outcome 10 Bladder toxicity. ..

Analysis 1.11. Comparison 1 Mycophenolate mofetil (MMF) versus IV cyclophosphamide (CPA), Outcome 11 Alopecia.

Analysis 1.12. Comparison 1 Mycophenolate mofetil (MMF) versus IV cyclophosphamide (CPA), Outcome 12 Gastrointestinal (GI) adverse events.

Analysis 1.13. Comparison 1 Mycophenolate mofetil (MMF) versus IV cyclophosphamide (CPA), Outcome 13 Daily proteinuria. Analysis 1.14. Comparison 1 Mycophenolate mofetil (MMF) versus IV cyclophosphamide (CPA), Outcome 14 Serum creatinine.

Analysis 2.1. Comparison 2 Mycophenolate mofetil (MMF) versus oral cyclophosphamide (CPA), Outcome 1 Death. ................ Analysis 2.2. Comparison 2 Mycophenolate mofetil (MMF) versus oral cyclophosphamide (CPA), Outcome 2 Remission. ......... Analysis 2.3. Comparison 2 Mycophenolate mofetil (MMF) versus oral cyclophosphamide (CPA), Outcome 3 Adverse renal outcomes.

Analysis 2.4. Comparison 2 Mycophenolate mofetil (MMF) versus oral cyclophosphamide (CPA), Outcome 4 Ovarian failure. ... Analysis 2.5. Comparison 2 Mycophenolate mofetil (MMF) versus oral cyclophosphamide (CPA), Outcome 5 Infection. ............ Analysis 2.6. Comparison 2 Mycophenolate mofetil (MMF) versus oral cyclophosphamide (CPA), Outcome 6 Leucopenia. ....... Analysis 2.7. Comparison 2 Mycophenolate mofetil (MMF) versus oral cyclophosphamide (CPA), Outcome 7 Bone toxicity. ..... Analysis 2.8. Comparison 2 Mycophenolate mofetil (MMF) versus oral cyclophosphamide (CPA), Outcome 8 Alopecia. ............ Analysis 2.9. Comparison 2 Mycophenolate mofetil (MMF) versus oral cyclophosphamide (CPA), Outcome 9 Gastrointestinal (GI) adverse events.

Analysis 2.10. Comparison 2 Mycophenolate mofetil (MMF) versus oral cyclophosphamide (CPA), Outcome 10 Daily proteinuria.

Analysis 3.1. Comparison 3 Mycophenolate mofetil (MMF) + tacrolimus (TAC) versus IV cyclophosphamide (CPA), Outcome 1 Death.

Analysis 3.2. Comparison 3 Mycophenolate mofetil (MMF) + tacrolimus (TAC) versus IV cyclophosphamide (CPA), Outcome 2 Remission.

Analysis 3.3. Comparison 3 Mycophenolate mofetil (MMF) + tacrolimus (TAC) versus IV cyclophosphamide (CPA), Outcome 3 Adverse renal outcomes. 
Analysis 3.4. Comparison 3 Mycophenolate mofetil (MMF) + tacrolimus (TAC) versus IV cyclophosphamide (CPA), Outcome 4 Stable kidney function.

Analysis 3.5. Comparison 3 Mycophenolate mofetil (MMF) + tacrolimus (TAC) versus IV cyclophosphamide (CPA), Outcome 5 Ovarian failure.

Analysis 3.6. Comparison 3 Mycophenolate mofetil (MMF) + tacrolimus (TAC) versus IV cyclophosphamide (CPA), Outcome 6 Menstrual irregularities.

Analysis 3.7. Comparison 3 Mycophenolate mofetil (MMF) + tacrolimus (TAC) versus IV cyclophosphamide (CPA), Outcome 7 Infection.

Analysis 3.8. Comparison 3 Mycophenolate mofetil (MMF) + tacrolimus (TAC) versus IV cyclophosphamide (CPA), Outcome 8 Leucopenia.

Analysis 3.9. Comparison 3 Mycophenolate mofetil (MMF) + tacrolimus (TAC) versus IV cyclophosphamide (CPA), Outcome 9 Bone toxicity.

Analysis 3.10. Comparison 3 Mycophenolate mofetil (MMF) + tacrolimus (TAC) versus IV cyclophosphamide (CPA), Outcome 10 Alopecia.

Analysis 3.11. Comparison 3 Mycophenolate mofetil (MMF) + tacrolimus (TAC) versus IV cyclophosphamide (CPA), Outcome 11 Gastrointestinal (GI) adverse events.

Analysis 3.12. Comparison 3 Mycophenolate mofetil (MMF) + tacrolimus (TAC) versus IV cyclophosphamide (CPA), Outcome 12 Daily proteinuria.

Analysis 4.1. Comparison 4 Mycophenolate mofetil (MMF) + IV cyclophosphamide (CPA) versus IV CPA, Outcome 1 Death. ...... Analysis 4.2. Comparison 4 Mycophenolate mofetil (MMF) + IV cyclophosphamide (CPA) versus IV CPA, Outcome 2 Remission. . Analysis 4.3. Comparison 4 Mycophenolate mofetil (MMF) + IV cyclophosphamide (CPA) versus IV CPA, Outcome 3 Menstrual irregularities.

Analysis 4.4. Comparison 4 Mycophenolate mofetil (MMF) + IV cyclophosphamide (CPA) versus IV CPA, Outcome 4 Infection. ... Analysis 4.5. Comparison 4 Mycophenolate mofetil (MMF) + IV cyclophosphamide (CPA) versus IV CPA, Outcome 5 Leucopenia. Analysis 4.6. Comparison 4 Mycophenolate mofetil (MMF) + IV cyclophosphamide (CPA) versus IV CPA, Outcome 6 Daily proteinuria.

Analysis 5.1. Comparison 5 Mycophenolate mofetil (MMF) versus tacrolimus (TAC), Outcome 1 Death.

Analysis 5.2. Comparison 5 Mycophenolate mofetil (MMF) versus tacrolimus (TAC), Outcome 2 Remission.

Analysis 5.3. Comparison 5 Mycophenolate mofetil (MMF) versus tacrolimus (TAC), Outcome 3 Adverse renal outcomes. ........ Analysis 5.4. Comparison 5 Mycophenolate mofetil (MMF) versus tacrolimus (TAC), Outcome 4 Stable kidney function. ........... Analysis 5.5. Comparison 5 Mycophenolate mofetil (MMF) versus tacrolimus (TAC), Outcome 5 Menstrual irregularities. ......... Analysis 5.6. Comparison 5 Mycophenolate mofetil (MMF) versus tacrolimus (TAC), Outcome 6 Infection.

Analysis 5.7. Comparison 5 Mycophenolate mofetil (MMF) versus tacrolimus (TAC), Outcome 7 Leucopenia.

Analysis 5.8. Comparison 5 Mycophenolate mofetil (MMF) versus tacrolimus (TAC), Outcome 8 Alopecia.

Analysis 5.9. Comparison 5 Mycophenolate mofetil (MMF) versus tacrolimus (TAC), Outcome 9 Daily proteinuria (at 24 weeks). Analysis 5.10. Comparison 5 Mycophenolate mofetil (MMF) versus tacrolimus (TAC), Outcome 10 Disease activity. ................. Analysis 5.11. Comparison 5 Mycophenolate mofetil (MMF) versus tacrolimus (TAC), Outcome 11 Serum creatinine. ............... Analysis 5.12. Comparison 5 Mycophenolate mofetil (MMF) versus tacrolimus (TAC), Outcome 12 Creatinine clearance. .......... Analysis 6.1. Comparison 6 Calcineurin inhibitors (CNI) versus IV cyclophosphamide (CPA), Outcome 1 Death. ........................ Analysis 6.2. Comparison 6 Calcineurin inhibitors (CNI) versus IV cyclophosphamide (CPA), Outcome 2 Remission. Analysis 6.3. Comparison 6 Calcineurin inhibitors (CNI) versus IV cyclophosphamide (CPA), Outcome 3 Adverse renal outcomes.

Analysis 6.4. Comparison 6 Calcineurin inhibitors (CNI) versus IV cyclophosphamide (CPA), Outcome 4 Stable kidney function. Analysis 6.5. Comparison 6 Calcineurin inhibitors (CNI) versus IV cyclophosphamide (CPA), Outcome 5 Ovarian failure. .......... Analysis 6.6. Comparison 6 Calcineurin inhibitors (CNI) versus IV cyclophosphamide (CPA), Outcome 6 Menstrual irregularities.

Analysis 6.7. Comparison 6 Calcineurin inhibitors (CNI) versus IV cyclophosphamide (CPA), Outcome 7 Infection.

Analysis 6.8. Comparison 6 Calcineurin inhibitors (CNI) versus IV cyclophosphamide (CPA), Outcome 8 Malignancy: extended follow-up.

Analysis 6.9. Comparison 6 Calcineurin inhibitors (CNI) versus IV cyclophosphamide (CPA), Outcome 9 Leucopenia.

Analysis 6.10. Comparison 6 Calcineurin inhibitors (CNI) versus IV cyclophosphamide (CPA), Outcome 10 Alopecia. ................ Analysis 6.11. Comparison 6 Calcineurin inhibitors (CNI) versus IV cyclophosphamide (CPA), Outcome 11 Gastrointestinal (GI) adverse events.

Analysis 6.12. Comparison 6 Calcineurin inhibitors (CNI) versus IV cyclophosphamide (CPA), Outcome 12 Daily proteinuria. ... 
Analysis 6.13. Comparison 6 Calcineurin inhibitors (CNI) versus IV cyclophosphamide (CPA), Outcome 13 Creatinine clearance.

Analysis 6.14. Comparison 6 Calcineurin inhibitors (CNI) versus IV cyclophosphamide (CPA), Outcome 14 Serum creatinine. ...

Analysis 7.1. Comparison 7 Cyclophosphamide (CPA) versus azathioprine (AZA), Outcome 1 Death.

Analysis 7.2. Comparison 7 Cyclophosphamide (CPA) versus azathioprine (AZA), Outcome 2 Remission in proteinuria. ...........

Analysis 7.3. Comparison 7 Cyclophosphamide (CPA) versus azathioprine (AZA), Outcome 3 Adverse renal outcomes. ............

Analysis 7.4. Comparison 7 Cyclophosphamide (CPA) versus azathioprine (AZA), Outcome 4 Stable kidney function. ...............

Analysis 7.5. Comparison 7 Cyclophosphamide (CPA) versus azathioprine (AZA), Outcome 5 Ovarian failure.

Analysis 7.6. Comparison 7 Cyclophosphamide (CPA) versus azathioprine (AZA), Outcome 6 Menstrual irregularities. .............

Analysis 7.7. Comparison 7 Cyclophosphamide (CPA) versus azathioprine (AZA), Outcome 7 Infection.

Analysis 7.8. Comparison 7 Cyclophosphamide (CPA) versus azathioprine (AZA), Outcome 8 Malignancy.

Analysis 7.9. Comparison 7 Cyclophosphamide (CPA) versus azathioprine (AZA), Outcome 9 Bone toxicity.

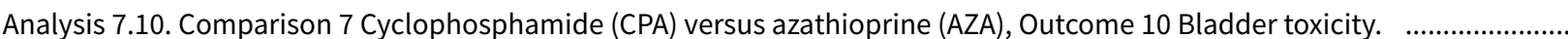

Analysis 8.1. Comparison 8 Rituximab (RTX) + mycophenolate mofetil (MMF) versus placebo + MMF, Outcome 1 Death. .......... Analysis 8.2. Comparison 8 Rituximab (RTX) + mycophenolate mofetil (MMF) versus placebo + MMF, Outcome 2 Remission. ....

Analysis 8.3. Comparison 8 Rituximab (RTX) + mycophenolate mofetil (MMF) versus placebo + MMF, Outcome 3 Stable kidney function.

Analysis 8.4. Comparison 8 Rituximab (RTX) + mycophenolate mofetil (MMF) versus placebo + MMF, Outcome 4 Infection. ..... Analysis 8.5. Comparison 8 Rituximab (RTX) + mycophenolate mofetil (MMF) versus placebo + MMF, Outcome 5 Leucopenia. ... Analysis 9.1. Comparison 9 Rituximab (RTX) + cyclophosphamide (CPA) versus RTX, Outcome 1 Remission.

Analysis 9.2. Comparison 9 Rituximab (RTX) + cyclophosphamide (CPA) versus RTX, Outcome 2 Infection.

Analysis 9.3. Comparison 9 Rituximab (RTX) + cyclophosphamide (CPA) versus RTX, Outcome 3 Daily proteinuria.

Analysis 9.4. Comparison 9 Rituximab (RTX) + cyclophosphamide (CPA) versus RTX Outcome 4 Creatinine clearance. Analysis 9.5. Comparison 9 Rituximab (RTX) + cyclophosphamide (CPA) versus RTX, Outcome 5 Serum creatinine. ................. Analysis 10.1. Comparison $10 \mathrm{Abatacept}+$ other immunosuppressive agent (IS) + versus placebo + other IS, Outcome 1 Death. Analysis 10.2. Comparison 10 Abatacept + other immunosuppressive agent (IS) + versus placebo + other IS, Outcome 2 Remission.

Analysis 10.3. Comparison $10 \mathrm{Abatacept}+$ other immunosuppressive agent (IS) + versus placebo + other IS, Outcome 3 Adverse renal outcomes.

Analysis 10.4. Comparison 10 Abatacept + other immunosuppressive agent (IS) + versus placebo + other IS, Outcome 4 Major Infection.

Analysis 10.5. Comparison 10 Abatacept + other immunosuppressive agent (IS) + versus placebo + other IS, Outcome 5 Herpes zoster virus.

Analysis 10.6. Comparison 10 Abatacept + other immunosuppressive agent (IS) + versus placebo + other IS, Outcome 6 Healthrelated quality of life.

Analysis 10.7. Comparison 10 Abatacept + other immunosuppressive agent (IS) + versus placebo + other IS, Outcome 7 Disease activity (BILAG).

Analysis 11.1. Comparison 11 Laquinimod + other immunosuppressive agent (IS) versus placebo + other IS, Outcome 1 Death.

Analysis 11.2. Comparison 11 Laquinimod + other immunosuppressive agent (IS) versus placebo + other IS, Outcome 2 Complete remission.

Analysis 12.1. Comparison 12 Ocrelizumab + other immunosuppressive agent (IS) versus placebo + other IS, Outcome 1 Death.

Analysis 12.2. Comparison 12 Ocrelizumab + other immunosuppressive agent (IS) versus placebo + other IS, Outcome 2 Remission.

Analysis 12.3. Comparison 12 Ocrelizumab + other immunosuppressive agent (IS) versus placebo + other IS, Outcome 3 Major Infection.

Analysis 13.1. Comparison 13 Sirukumab + other immunosuppressive agent (IS) versus placebo + other IS, Outcome 1 Death. Analysis 13.2. Comparison 13 Sirukumab + other immunosuppressive agent (IS) versus placebo + other IS, Outcome 2 Infection.

Analysis 13.3. Comparison 13 Sirukumab + other immunosuppressive agent (IS) versus placebo + other IS, Outcome 3 Malignancy.

Analysis 13.4. Comparison 13 Sirukumab + other immunosuppressive agent (IS) versus placebo + other IS, Outcome 4 Gastrointestinal (GI) adverse events.

Analysis 14.1. Comparison 14 IV versus oral cyclophosphamide (CPA), Outcome 1 Death.

Analysis 14.2. Comparison 14 IV versus oral cyclophosphamide (CPA), Outcome 2 Adverse renal outcomes.

Analysis 14.3. Comparison 14 IV versus oral cyclophosphamide (CPA), Outcome 3 Stable kidney function. 
Analysis 14.4. Comparison 14 IV versus oral cyclophosphamide (CPA), Outcome 4 Ovarian failure.

Analysis 14.5. Comparison 14 IV versus oral cyclophosphamide (CPA), Outcome 5 Infection.

Analysis 14.6. Comparison 14 IV versus oral cyclophosphamide (CPA), Outcome 6 Malignancy.

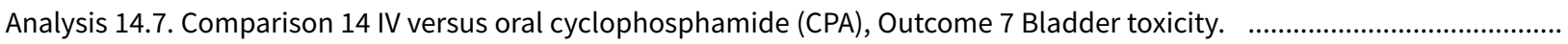

Analysis 14.8. Comparison 14 IV versus oral cyclophosphamide (CPA), Outcome 8 Gastrointestinal (GI) adverse events. ..........

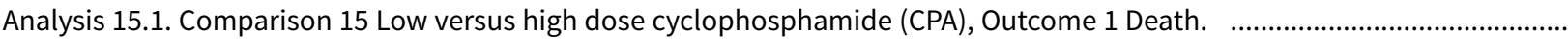

Analysis 15.2. Comparison 15 Low versus high dose cyclophosphamide (CPA), Outcome 2 Remission.

Analysis 15.3. Comparison 15 Low versus high dose cyclophosphamide (CPA), Outcome 3 Adverse renal outcomes.

Analysis 15.4. Comparison 15 Low versus high dose cyclophosphamide (CPA), Outcome 4 Stable kidney function. ..................

Analysis 15.5. Comparison 15 Low versus high dose cyclophosphamide (CPA), Outcome 5 Ovarian failure. ............................

Analysis 15.6. Comparison 15 Low versus high dose cyclophosphamide (CPA), Outcome 6 Infection.

Analysis 15.7. Comparison 15 Low versus high dose cyclophosphamide (CPA), Outcome 7 Malignancy.

Analysis 15.8. Comparison 15 Low versus high dose cyclophosphamide (CPA), Outcome 8 Leucopenia.

Analysis 15.9. Comparison 15 Low versus high dose cyclophosphamide (CPA), Outcome 9 Bone toxicity.

Analysis 15.10. Comparison 15 Low versus high dose cyclophosphamide (CPA), Outcome 10 Alopecia.

Analysis 15.11. Comparison 15 Low versus high dose cyclophosphamide (CPA), Outcome 11 Gastrointestinal (GI) adverse events.

Analysis 15.12. Comparison 15 Low versus high dose cyclophosphamide (CPA), Outcome 12 Daily proteinuria. ......................

Analysis 15.13. Comparison 15 Low versus high dose cyclophosphamide (CPA), Outcome 13 Creatinine clearance. .................

Analysis 15.14. Comparison 15 Low versus high dose cyclophosphamide (CPA), Outcome 14 Serum creatinine. ....................

Analysis 15.15. Comparison 15 Low versus high dose cyclophosphamide (CPA), Outcome 15 Disease activity (SLEDAI). ..........

Analysis 16.1. Comparison 16 Standard versus reduced dose oral corticosteroids, Outcome 1 Death.

Analysis 16.2. Comparison 16 Standard versus reduced dose oral corticosteroids, Outcome 2 Remission.

Analysis 16.3. Comparison 16 Standard versus reduced dose oral corticosteroids, Outcome 3 Relapse.

Analysis 16.4. Comparison 16 Standard versus reduced dose oral corticosteroids, Outcome 4 Infection.

Analysis 16.5. Comparison 16 Standard versus reduced dose oral corticosteroids, Outcome 5 Gastrointestinal (GI) adverse events.

Analysis 16.6. Comparison 16 Standard versus reduced dose oral corticosteroids, Outcome 6 Creatinine clearance.

Analysis 16.7. Comparison 16 Standard versus reduced dose oral corticosteroids, Outcome 7 Serum creatinine.

Analysis 17.1. Comparison 17 IV versus oral corticosteroids, Outcome 1 Death.

Analysis 17.2. Comparison 17 IV versus oral corticosteroids, Outcome 2 Adverse renal outcomes.

Analysis 18.1. Comparison 18 Cyclophosphamide (CPA) + corticosteroids versus corticosteroids, Outcome 1 Death. ................

Analysis 18.2. Comparison 18 Cyclophosphamide (CPA) + corticosteroids versus corticosteroids, Outcome 2 Complete remission of proteinuria.

Analysis 18.3. Comparison 18 Cyclophosphamide (CPA) + corticosteroids versus corticosteroids, Outcome 3 Adverse renal outcomes.

Analysis 18.4. Comparison 18 Cyclophosphamide (CPA) + corticosteroids versus corticosteroids, Outcome 4 Deterioration of kidney function.

Analysis 18.5. Comparison 18 Cyclophosphamide (CPA) + corticosteroids versus corticosteroids, Outcome 5 Stable kidney function.

Analysis 18.6. Comparison 18 Cyclophosphamide (CPA) + corticosteroids versus corticosteroids, Outcome 6 Ovarian failure. .... Analysis 18.7. Comparison 18 Cyclophosphamide (CPA) + corticosteroids versus corticosteroids, Outcome 7 Infection. ........... Analysis 18.8. Comparison 18 Cyclophosphamide (CPA) + corticosteroids versus corticosteroids, Outcome 8 Malignancy. ....... Analysis 18.9. Comparison 18 Cyclophosphamide (CPA) + corticosteroids versus corticosteroids, Outcome 9 Bone toxicity. .... Analysis 18.10. Comparison 18 Cyclophosphamide (CPA) + corticosteroids versus corticosteroids, Outcome 10 Bladder toxicity.

Analysis 18.11. Comparison 18 Cyclophosphamide (CPA) + corticosteroids versus corticosteroids, Outcome 11 Daily proteinuria.

Analysis 18.12. Comparison 18 Cyclophosphamide (CPA) + corticosteroids versus corticosteroids, Outcome 12 Serum creatinine.

Analysis 18.13. Comparison 18 Cyclophosphamide (CPA) + corticosteroids versus corticosteroids, Outcome 13 Creatinine clearance.

Analysis 19.1. Comparison 19 Cyclophosphamide (CPA) + azathioprine (AZA) + corticosteroids versus corticosteroids alone, Outcome 1 Death. 
Analysis 19.2. Comparison 19 Cyclophosphamide (CPA) + azathioprine (AZA) + corticosteroids versus corticosteroids alone, Outcome 2 Adverse renal outcomes.

Analysis 19.3. Comparison 19 Cyclophosphamide (CPA) + azathioprine (AZA) + corticosteroids versus corticosteroids alone, Outcome 3 Stable kidney function.

Analysis 19.4. Comparison 19 Cyclophosphamide (CPA) + azathioprine (AZA) + corticosteroids versus corticosteroids alone, Outcome 4 Ovarian failure.

Analysis 19.5. Comparison 19 Cyclophosphamide (CPA) + azathioprine (AZA) + corticosteroids versus corticosteroids alone, Outcome 5 Infection.

Analysis 19.6. Comparison 19 Cyclophosphamide (CPA) + azathioprine (AZA) + corticosteroids versus corticosteroids alone, Outcome 6 Bladder toxicity.

Analysis 20.1. Comparison 20 Azathioprine (AZA) + corticosteroids versus corticosteroids alone, Outcome 1 Death.

Analysis 20.2. Comparison 20 Azathioprine (AZA) + corticosteroids versus corticosteroids alone, Outcome 2 Complete remission of proteinuria.

Analysis 20.3. Comparison 20 Azathioprine (AZA) + corticosteroids versus corticosteroids alone, Outcome 3 Adverse renal outcomes.

Analysis 20.4. Comparison 20 Azathioprine (AZA) + corticosteroids versus corticosteroids alone, Outcome 4 Stable kidney function.

Analysis 20.5. Comparison 20 Azathioprine (AZA) + corticosteroids versus corticosteroids alone, Outcome 5 Ovarian failure. ... Analysis 20.6. Comparison 20 Azathioprine (AZA) + corticosteroids versus corticosteroids alone, Outcome 6 Infection. ............ Analysis 20.7. Comparison 20 Azathioprine (AZA) + corticosteroids versus corticosteroids alone, Outcome 7 Malignancy. ........ Analysis 20.8. Comparison 20 Azathioprine (AZA) + corticosteroids versus corticosteroids alone, Outcome 8 Bone toxicity. ...... Analysis 20.9. Comparison 20 Azathioprine $(A Z A)+$ corticosteroids versus corticosteroids alone, Outcome 9 Creatinine clearance.

Analysis 21.1. Comparison 21 Cyclosporin (CSA) + corticosteroids versus corticosteroids alone, Outcome 1 Daily proteinuria. ... Analysis 21.2. Comparison 21 Cyclosporin (CSA) + corticosteroids versus corticosteroids alone, Outcome 2 Serum creatinine. .. Analysis 21.3. Comparison 21 Cyclosporin (CSA) + corticosteroids versus corticosteroids alone, Outcome 3 Creatinine clearance.

Analysis 22.1. Comparison 22 Misoprostol + corticosteroids versus corticosteroids alone, Outcome 1 Adverse renal outcomes.

Analysis 23.1. Comparison 23 Plasma exchange (PE) + immunosuppression (IS) versus IS alone, Outcome 1 Death.

Analysis 23.2. Comparison 23 Plasma exchange (PE) + immunosuppression (IS) versus IS alone, Outcome 2 Adverse renal outcomes.

Analysis 23.3. Comparison 23 Plasma exchange (PE) + immunosuppression (IS) versus IS alone, Outcome 3 Stable kidney function.

Analysis 23.4. Comparison 23 Plasma exchange (PE) + immunosuppression (IS) versus IS alone, Outcome 4 Infection. ............ Analysis 23.5. Comparison 23 Plasma exchange (PE) + immunosuppression (IS) versus IS alone, Outcome 5 Leucopenia. ....... Analysis 23.6. Comparison 23 Plasma exchange (PE) + immunosuppression (IS) versus IS alone, Outcome 6 Daily proteinuria. . Analysis 23.7. Comparison 23 Plasma exchange (PE) + immunosuppression (IS) versus IS alone, Outcome 7 Serum creatinine. Analysis 23.8. Comparison 23 Plasma exchange (PE) + immunosuppression (IS) versus IS alone, Outcome 8 Creatinine clearance.

Analysis 23.9. Comparison 23 Plasma exchange (PE) + immunosuppression (IS) versus IS alone, Outcome 9 Disease activity (SLAM).

Analysis 24.1. Comparison 24 Plasma exchange (PE) versus immunosuppression (IS), Outcome 1 Adverse renal outcomes. .... Analysis 24.2. Comparison 24 Plasma exchange (PE) versus immunosuppression (IS), Outcome 2 Infection.

Analysis 24.3. Comparison 24 Plasma exchange (PE) versus immunosuppression (IS), Outcome 3 Leucopenia.

Analysis 24.4. Comparison 24 Plasma exchange (PE) versus immunosuppression (IS), Outcome 4 Alopecia.

Analysis 24.5. Comparison 24 Plasma exchange (PE) versus immunosuppression (IS), Outcome 5 Daily proteinuria. Analysis 24.6. Comparison 24 Plasma exchange (PE) versus immunosuppression (IS), Outcome 6 Creatinine clearance. ......... Analysis 25.1. Comparison 25 Long versus short duration cyclophosphamide (CPA), Outcome 1 Adverse renal outcomes. ....... Analysis 25.2. Comparison 25 Long versus short duration cyclophosphamide (CPA), Outcome 2 Stable kidney function. ......... Analysis 25.3. Comparison 25 Long versus short duration cyclophosphamide (CPA), Outcome 3 Ovarian failure. ..................... Analysis 25.4. Comparison 25 Long versus short duration cyclophosphamide (CPA), Outcome 4 Infection. 
Analysis 26.1. Comparison 26 Maintenance: azathioprine (AZA) versus mycophenolate mofetil (MMF), Outcome 1 Death. ....... Analysis 26.2. Comparison 26 Maintenance: azathioprine (AZA) versus mycophenolate mofetil (MMF), Outcome 2 Renal relapse.

Analysis 26.3. Comparison 26 Maintenance: azathioprine (AZA) versus mycophenolate mofetil (MMF), Outcome 3 End-stage kidney disease.

Analysis 26.4. Comparison 26 Maintenance: azathioprine (AZA) versus mycophenolate mofetil (MMF), Outcome 4 Doubling of serum creatinine.

Analysis 26.5. Comparison 26 Maintenance: azathioprine (AZA) versus mycophenolate mofetil (MMF), Outcome 5 Ovarian failure.

Analysis 26.6. Comparison 26 Maintenance: azathioprine (AZA) versus mycophenolate mofetil (MMF), Outcome 6 Infection. ... Analysis 26.7. Comparison 26 Maintenance: azathioprine (AZA) versus mycophenolate mofetil (MMF), Outcome 7 Malignancy. . Analysis 26.8. Comparison 26 Maintenance: azathioprine (AZA) versus mycophenolate mofetil (MMF), Outcome 8 Leucopenia. Analysis 26.9. Comparison 26 Maintenance: azathioprine (AZA) versus mycophenolate mofetil (MMF), Outcome 9 Bone toxicity.

Analysis 26.10. Comparison 26 Maintenance: azathioprine (AZA) versus mycophenolate mofetil (MMF), Outcome 10 Alopecia. . Analysis 26.11. Comparison 26 Maintenance: azathioprine (AZA) versus mycophenolate mofetil (MMF), Outcome 11 Gastrointestinal (GI) adverse events.

Analysis 26.12. Comparison 26 Maintenance: azathioprine (AZA) versus mycophenolate mofetil (MMF), Outcome 12 Daily proteinuria.

Analysis 27.1. Comparison 27 Maintenance: azathioprine (AZA) versus cyclosporin (CSA), Outcome 1 Death. ........................... Analysis 27.2. Comparison 27 Maintenance: azathioprine (AZA) versus cyclosporin (CSA), Outcome 2 Adverse renal outcomes. . Analysis 27.3. Comparison 27 Maintenance: azathioprine (AZA) versus cyclosporin (CSA), Outcome 3 Infection. ..................... Analysis 27.4. Comparison 27 Maintenance: azathioprine (AZA) versus cyclosporin (CSA), Outcome 4 Leucopenia. ................. Analysis 27.5. Comparison 27 Maintenance: azathioprine (AZA) versus cyclosporin (CSA), Outcome 5 Gastrointestinal (GI) adverse events.

Analysis 27.6. Comparison 27 Maintenance: azathioprine (AZA) versus cyclosporin (CSA), Outcome 6 Daily proteinuria. .......... Analysis 27.7. Comparison 27 Maintenance: azathioprine (AZA) versus cyclosporin (CSA), Outcome 7 Disease activity (SLEDAI). Analysis 28.1. Comparison 28 Maintenance: azathioprine (AZA) versus cyclophosphamide (CPA), Outcome 1 Death.

Analysis 28.2. Comparison 28 Maintenance: azathioprine (AZA) versus cyclophosphamide (CPA), Outcome 2 Adverse renal outcomes.

Analysis 28.3. Comparison 28 Maintenance: azathioprine (AZA) versus cyclophosphamide (CPA), Outcome 3 Bladder toxicity. . Analysis 28.4. Comparison 28 Maintenance: azathioprine (AZA) versus cyclophosphamide (CPA), Outcome 4 Creatinine clearance.

Analysis 29.1. Comparison 29 Maintenance: azathioprine (AZA) versus tacrolimus (TAC), Outcome 1 Adverse renal outcomes. .. Analysis 29.2. Comparison 29 Maintenance: azathioprine (AZA) versus tacrolimus (TAC), Outcome 2 Infection.

Analysis 29.3. Comparison 29 Maintenance: azathioprine (AZA) versus tacrolimus (TAC), Outcome 3 Gastrointestinal (GI) adverse events.

Analysis 30.1. Comparison 30 Maintenance: prednisone withdrawal versus prednisone continuation, Outcome 1 Relapse. ...... Analysis 30.2. Comparison 30 Maintenance: prednisone withdrawal versus prednisone continuation, Outcome 2 Major infection.

Analysis 31.1. Comparison 31 Maintenance: intravenous immunoglobulin (IVIG) versus intravenous cyclophosphamide (IV CPA), Outcome 1 Creatinine clearance.

Analysis 31.2. Comparison 31 Maintenance: intravenous immunoglobulin (IVIG) versus intravenous cyclophosphamide (IV CPA), Outcome 2 Daily proteinuria.

Analysis 31.3. Comparison 31 Maintenance: intravenous immunoglobulin (IVIG) versus intravenous cyclophosphamide (IV CPA), Outcome 3 Serum creatinine.

ADDITIONAL TABLES

APPENDICES 
[Intervention Review]

\section{Immunosuppressive treatment for proliferative lupus nephritis}

David J Tunnicliffe1,2, Suetonia C Palmer ${ }^{3}$, Lorna Henderson4, Philip Masson ${ }^{5}$, Jonathan C Craig1,6,7, Allison Tong1,2, Davinder SinghGrewal8, Robert S Flanc ${ }^{9}$, Matthew A Roberts 10 , Angela C Webster1,6,11, Giovanni FM Strippoli1,6,12,13,14

${ }^{1}$ Sydney School of Public Health, The University of Sydney, Sydney, Australia. ${ }^{2}$ Centre for Kidney Research, The Children's Hospital at Westmead, Westmead, Australia. ${ }^{3}$ Department of Medicine, University of Otago Christchurch, Christchurch, New Zealand. ${ }^{4}$ Renal Department, NHS Lothian, Edinburgh, UK. 5Department of Renal Medicine, Royal Free London NHS Foundation Trust, London, UK. ${ }^{6}$ Cochrane Kidney and Transplant, Centre for Kidney Research, The Children's Hospital at Westmead, Westmead, Australia. ${ }^{7}$ College of Medicine and Public Health, Flinders University, Adelaide, Australia. 8Department Paediatric Rheumatology, The Sydney Children's Hospitals Network, Westmead, Australia. ${ }^{9}$ Department of Nephrology, Monash Medical Centre, Clayton, Australia. ${ }^{10}$ Eastern Health Clinical School, Monash University, Box Hill, Australia. ${ }^{11}$ Centre for Transplant and Renal Research, Westmead Millennium Institute, The University of Sydney at Westmead, Westmead, Australia. 12Department of Emergency and Organ Transplantation, University of Bari, Bari, Italy. ${ }^{13}$ Medical Scientific Office, Diaverum, Lund, Sweden. ${ }^{14}$ Diaverum Academy, Bari, Italy

Contact address: David J Tunnicliffe, Sydney School of Public Health, The University of Sydney, c/- Centre for Kidney Research, The Children's Hospital at Westmead, Westmead, NSW, 2145, Australia. david.tunnicliffe@health.nsw.gov.au.

Editorial group: Cochrane Kidney and Transplant Group.

Publication status and date: New search for studies and content updated (conclusions changed), published in Issue 6, 2018.

Citation: Tunnicliffe DJ, Palmer SC, Henderson L, Masson P, Craig JC, Tong A, Singh-Grewal D, Flanc RS, Roberts MA, Webster AC, Strippoli GFM. Immunosuppressive treatment for proliferative lupus nephritis. Cochrane Database of Systematic Reviews 2018 , Issue 6. Art. No.: CD002922. DOI: 10.1002/14651858.CD002922.pub4.

Copyright @ 2018 The Cochrane Collaboration. Published by John Wiley \& Sons, Ltd.

\section{A B S T R A C T}

\section{Background}

Cyclophosphamide, in combination with corticosteroids, has been first-line treatment for inducing disease remission for proliferative lupus nephritis, reducing death at five years from over $50 \%$ in the 1950 s and 1960 s to less than $10 \%$ in recent years. Several treatment strategies designed to improve remission rates and minimise toxicity have become available. Treatments, including mycophenolate mofetil (MMF) and calcineurin inhibitors, alone and in combination, may have equivalent or improved rates of remission, lower toxicity (less alopecia and ovarian failure) and uncertain effects on death, end-stage kidney disease (ESKD) and infection. This is an update of a Cochrane review first published in 2004 and updated in 2012.

\section{Objectives}

Our objective was to assess the evidence and evaluate the benefits and harms of different immunosuppressive treatments in people with biopsy-proven lupus nephritis. The following questions relating to management of proliferative lupus nephritis were addressed: 1) Are new immunosuppressive agents superior to or as effective as cyclophosphamide plus corticosteroids? 2) Which agents, dosages, routes of administration and duration of therapy should be used? 3) Which toxicities occur with the different treatment regimens?

\section{Search methods}

We searched the Cochrane Kidney and Transplant Specialised Register up to 2 March 2018 with support from the Cochrane Information Specialist using search terms relevant to this review. Studies in the Specialised Register are identified through searches of CENTRAL, MEDLINE, and EMBASE, conference proceedings, the International Clinical Trials Register (ICTRP) Search Portal and ClinicalTrials.gov.

\section{Selection criteria}

Randomised controlled trials (RCTs) and quasi-RCTs comparing any immunosuppressive treatment for biopsy-proven class III, IV, V+III and $\mathrm{V}+\mathrm{VI}$ lupus nephritis in adult or paediatric patients were included. 


\section{Data collection and analysis}

Data were abstracted and the risks of bias were assessed independently by two authors. Dichotomous outcomes were calculated as risk ratio (RR) and measures on continuous scales calculated as mean differences (MD) with 95\% confidence intervals (Cl). The primary outcomes were death (all causes) and complete disease remission for induction therapy and disease relapse for maintenance therapy. Evidence certainty was determined using GRADE.

\section{Main results}

In this review update, 26 new studies were identified, to include 74 studies involving 5175 participants overall. Twenty-nine studies included children under the age of 18 years with lupus nephritis, however only two studies exclusively examined the treatment of lupus nephritis in patients less than 18 years of age.

\section{Induction therapy}

Sixty-seven studies (4791 participants; median 12 months duration (range 2.5 to 48 months)) reported induction therapy. The effects of all treatment strategies on death (all causes) and ESKD were uncertain (very low certainty evidence) as this outcome occurred very infrequently. Compared with intravenous (IV) cyclophosphamide, MMF may have increased complete disease remission (RR $1.17,95 \% \mathrm{Cl}$ 0.97 to 1.42; low certainty evidence), although the range of effects includes the possibility of little or no difference.

Compared to IV cyclophosphamide, MMF is probably associated with decreased alopecia (RR $0.29,95 \% \mathrm{Cl} 0.19$ to 0.46 ; 170 less (129 less to 194 less) per 1000 people) (moderate certainty evidence), increased diarrhoea (RR 2.42, 95\% Cl 1.64 to 3.58 ; 142 more (64 more to 257 more) per 1000 people) (moderate certainty evidence) and may have made little or no difference to major infection (RR $1.02,95 \% \mathrm{Cl} 0.67$ to 1.54; 2 less (38 less to 62 more) per 1000 people) (low certainty evidence). It is uncertain if MMF decreased ovarian failure compared to IV cyclophosphamide because the certainty of the evidence was very low (RR 0.36, 95\% CI 0.06 to 2.18; 26 less (39 less to 49 more) per 1000 people). Studies were not generally designed to measure ESKD.

MMF combined with tacrolimus may have increased complete disease remission (RR 2.38, 95\% Cl 1.07 to 5.30 ; 336 more (17 to 1048 more) per 1000 people (low certainty evidence) compared with IV cyclophosphamide, however the effects on alopecia, diarrhoea, ovarian failure, and major infection remain uncertain. Compared to standard of care, the effects of biologics on most outcomes were uncertain because of low to very low certainty of evidence.

\section{Maintenance therapy}

Nine studies (767 participants; median 30 months duration (range 6 to 63 months)) reported maintenance therapy. In maintenance therapy, disease relapse is probably increased with azathioprine compared with MMF (RR $1.75,95 \% \mathrm{Cl} 1.20$ to 2.55 ; 114 more ( 30 to 236 more) per 1000 people (moderate certainty evidence). Multiple other interventions were compared as maintenance therapy, but patient-outcome data were sparse leading to imprecise estimates.

\section{Authors' conclusions}

In this review update, studies assessing treatment for proliferative lupus nephritis were not designed to assess death (all causes) or ESKD. MMF may lead to increased complete disease remission compared with IV cyclophosphamide, with an acceptable adverse event profile, although evidence certainty was low and included the possibility of no difference. Calcineurin combined with lower dose MMF may improve induction of disease remission compared with IV cyclophosphamide, but the comparative safety profile of these therapies is uncertain. Azathioprine may increase disease relapse as maintenance therapy compared with MMF.

\section{PLAIN LANGUAGE SUMMARY}

\section{Immunosuppressive treatment for people with proliferative lupus nephritis}

\section{What is the issue?}

In lupus, the body's immune system for fighting infection attacks different parts of the body, including the kidneys. About half of all people with lupus have kidney problems. An estimated one in every 10 people who have lupus kidney disease (lupus nephritis) can develop kidney failure. The goal of treatment is to protect kidney function and avoid side-effects.

While the life expectancy of patients who have lupus has dramatically improved, available treatments can cause serious side effects such as hair loss, serious infection, and infertility. It is important to know about which treatments help to treat lupus while causing the fewest side-effects.

\section{What did we do?}

We searched the Cochrane Kidney and Transplant Specialised Register up to 2 March 2018 and we combined all studies testing treatments aimed to control the body's immune system for lupus nephritis. 


\section{What did we find?}

In this review update, 74 studies involving 5175 patients with lupus nephritis could be studied. Treatments included intravenous (given through a vein) cyclophosphamide, oral (tablets by mouth) mycophenolate mofetil (MMF), azathioprine, and tacrolimus (used alone or together with MMF). We also found studies of treatments called "biologic" therapies, that have been designed to change very specific parts of the body's immune system that cause it to attack itself. We looked particularly at key outcomes such as whether treatment prevented patients from needing dialysis and controlled the lupus damage to the kidney tissue (called remission). We also looked at serious sideeffects including death, infection, infertility, and hair loss.

After combining the available studies, compared with cyclophosphamide, MMF may be better at getting the lupus damage to the kidneys under control. However, the range where the actual effect may suggest that MMF may make little or no difference to disease remission compared to treatment with cyclophosphamide. MMF treatment given with tacrolimus may lead to more disease remission. MMF may result in less hair loss and worse diarrhoea, but we were not certain whether MMF reduces infertility or other serious side effects. MMF was better than azathioprine for preventing kidney disease in the longer term. None of the studies told us whether treatment had any effect on death or need for dialysis, and there was very low certainty of evidence for the use of biologics in patients with lupus nephritis.

\section{Conclusions}

Patients with lupus nephritis may have similar or slightly better outcomes when treated with MMF or MMF with tacrolimus compared to those patients who receive intravenous cyclophosphamide. We are still not certain which is the best treatment for lupus nephritis to protect against needing dialysis in the longer term. 
SUMMARY OF FINDINGS

\section{Summary of findings for the main comparison. Mycophenolate mofetil (MMF) versus IV cyclophosphamide (CPA) for induction therapy}

Patient or population: patients with induction therapy in lupus nephritis

Settings: all settings

Intervention: MMF

Comparison: IV CPA

\begin{tabular}{|c|c|c|c|c|c|c|}
\hline \multirow[t]{3}{*}{ Outcomes } & \multicolumn{2}{|c|}{$\begin{array}{l}\text { Illustrative comparative risks }{ }^{\star} \\
(95 \% \mathrm{CI})\end{array}$} & \multirow[t]{3}{*}{$\begin{array}{l}\text { Relative effect } \\
(95 \% \mathrm{CI})\end{array}$} & \multirow{3}{*}{$\begin{array}{l}\text { No. of partici- } \\
\text { pants } \\
\text { (studies) }\end{array}$} & \multirow{3}{*}{$\begin{array}{l}\text { Certainty of } \\
\text { evidence } \\
\text { (GRADE) }\end{array}$} & \multirow[t]{3}{*}{ Comments } \\
\hline & Assumed risk & $\begin{array}{l}\text { Corresponding } \\
\text { risk }\end{array}$ & & & & \\
\hline & IV CPA & MMF & & & & \\
\hline $\begin{array}{l}\text { Death } \\
\text { Follow-up: } \\
\text { mean } 24 \text { weeks }\end{array}$ & 40 per 1000 & $\begin{array}{l}\mathbf{5 3} \text { per } \mathbf{1 0 0 0} \\
(29 \text { to } 98)\end{array}$ & $\begin{array}{l}\text { RR } 1.12 \\
(0.61 \text { to } 2.06)\end{array}$ & $826(8)$ & $\begin{array}{l}\oplus \Theta \Theta \Theta \\
\text { very low1,2,3 }\end{array}$ & $\begin{array}{l}\text { Downgraded as follows: } \\
1 \text { Indirectness: time frame insufficient } \\
2 \text { Total number of events small } \\
3 \text { Severe imprecision ( } 2 \\
\text { grades): risk estimate includes null effect } \\
\text { and estimate consistent with both appreciable benefit } \\
\text { and harm }\end{array}$ \\
\hline $\begin{array}{l}\text { ESKD } \\
\text { Follow-up: } \\
\text { mean } 32 \text { weeks }\end{array}$ & 85 per 1000 & $\begin{array}{l}\mathbf{6 1} \text { per } 1000 \\
(23 \text { to } 157)\end{array}$ & $\begin{array}{l}\mathbf{R R} \mathbf{0 . 7 1}(0.27 \text { to } \\
1.84)\end{array}$ & $231(3)$ & $\begin{array}{l}\oplus \Theta \Theta \odot \\
\text { very low } 1,2,3\end{array}$ & $\begin{array}{l}\text { Downgraded as follows: } \\
1 \text { Indirectness: time frame insufficient } \\
2 \text { Total number of events small } \\
3 \text { Severe imprecision ( } 2 \\
\text { grades): risk estimate includes null effect } \\
\text { and estimate consistent with both appreciable benefit } \\
\text { and harm }\end{array}$ \\
\hline $\begin{array}{l}\text { Complete re- } \\
\text { nal remission } \\
\text { Follow-up: } \\
\text { mean } 24 \text { weeks }\end{array}$ & 222 per 1000 & $\begin{array}{l}\mathbf{2 6 0} \text { per } \mathbf{1 0 0 0} \\
\text { (216 to } 316)\end{array}$ & $\begin{array}{l}\text { RR } 1.17 \text { (0.97 to } \\
1.42)\end{array}$ & $828(8)$ & $\begin{array}{l}\oplus \oplus \ominus \ominus \\
\operatorname{low}^{1,2,3}\end{array}$ & $\begin{array}{l}\text { Downgraded as follows: } \\
1 \text { Study limitations } \\
2 \text { Total number of events small }\end{array}$ \\
\hline
\end{tabular}




\begin{tabular}{|c|c|c|c|c|c|c|}
\hline & & & & & & $\begin{array}{l}3 \text { Imprecision ( } 2 \text { grades): risk estimate includes null ef- } \\
\text { fect and estimate consistent with both appreciable } \\
\text { benefit and harm }\end{array}$ \\
\hline $\begin{array}{l}\text { Partial renal } \\
\text { remission } \\
\text { Follow-up: } \\
\text { mean } 24 \text { weeks }\end{array}$ & 415 per 1000 & $\begin{array}{l}\mathbf{4 2 3} \text { per } \mathbf{1 0 0 0} \\
\text { (369 to } 490)\end{array}$ & $\begin{array}{l}\text { RR } 1.02 \\
\text { (0.89 to } 1.18)\end{array}$ & $868(9)$ & $\begin{array}{l}\oplus \oplus \ominus \ominus \\
\operatorname{low} 1,2\end{array}$ & $\begin{array}{l}\text { Downgraded as follows: } \\
1 \text { Study limitations } \\
2 \text { Serious indirectness: differences in the outcome defin- } \\
\text { ition between studies. }\end{array}$ \\
\hline Ovarian failure & 41 per 1000 & $\begin{array}{l}15 \text { per } 1000 \\
(2 \text { to } 90)\end{array}$ & $\begin{array}{l}\text { RR } \mathbf{0 . 3 6} \\
(0.06 \text { to } 2.18)\end{array}$ & $539(3)$ & $\begin{array}{l}\oplus \ominus \odot \odot \\
\text { very low } 1,2,3\end{array}$ & $\begin{array}{l}\text { Downgraded as follows: } \\
1 \text { Study limitations } \\
2 \text { Severe heterogeneity: point estimates varied widely } \\
{ }^{3} \text { Total number of events small } \\
{ }^{4} \text { Severe imprecision ( } 2 \text { grades): risk estimate includes } \\
\text { null effect and estimate consistent } \\
\text { with both appreciable benefit and harm }\end{array}$ \\
\hline $\begin{array}{l}\text { Major infec- } \\
\text { tion } \\
\text { Follow-up: } \\
\text { mean } 24 \text { weeks }\end{array}$ & 114 per 1000 & $\begin{array}{l}\mathbf{1 1 6} \text { per } \mathbf{1 0 0 0} \\
\text { (76 to } 175)\end{array}$ & $\begin{array}{l}\text { RR 1.02 } \\
\text { (0.67 to } 1.54)\end{array}$ & $699(6)$ & $\begin{array}{l}\oplus \oplus \oplus \ominus \\
l o w^{1,2}\end{array}$ & $\begin{array}{l}\text { Downgraded as follows: } \\
1 \text { Study limitations } \\
2 \text { Total number of events small } \\
3 \text { Severe imprecision ( } 2 \text { grades): risk estimate includes } \\
\text { null effect and estimate consistent } \\
\text { with both appreciable benefit and harm }\end{array}$ \\
\hline $\begin{array}{l}\text { Alopecia } \\
\text { Follow-up: } \\
\text { mean } 24 \text { weeks }\end{array}$ & 239 per 1000 & $\begin{array}{l}69 \text { per } 1000 \\
(45 \text { to } 110)\end{array}$ & $\begin{array}{l}\text { RR } 0.29 \\
(0.19 \text { to } 0.46)\end{array}$ & $622(3)$ & $\begin{array}{l}\oplus \oplus \oplus \ominus \\
\text { moderate } e^{1,2,3}\end{array}$ & $\begin{array}{l}\text { Downgraded as follows: } \\
1 \text { Study limitations } \\
2 \text { Total number of events small } \\
\text { Upgraded as follows: } \\
3 \text { Large magnitude of effect }\end{array}$ \\
\hline $\begin{array}{l}\text { Diarrhoea } \\
\text { Follow-up: } \\
\text { mean } 24 \text { weeks }\end{array}$ & 100 per 1000 & $\begin{array}{l}\mathbf{2 4 1} \text { per } \mathbf{1 0 0 0} \\
\text { (163 to } 357)\end{array}$ & $\begin{array}{l}\text { RR 2.42 } \\
\text { (1.64 to } 3.58 \text { ) }\end{array}$ & $609(4)$ & $\begin{array}{l}\oplus \oplus \oplus \ominus \\
\text { moderate } 1,2,3\end{array}$ & $\begin{array}{l}\text { Downgraded as follows: } \\
1 \text { Study limitations } \\
2 \text { Total number of events small }\end{array}$ \\
\hline
\end{tabular}


*The basis for the assumed risk for partial renal remission was prognostic studies (Fernandes das Neves 2015; Moroni 2007; So 2011; Zakharova 2016); and the assumed risk for other outcomes was calculated using the median control group risk across studies in the meta-analysis. The corresponding risk (and its $95 \%$ confidence interval) is based on the assumed risk in the comparison group and the relative effect of the intervention (and its $95 \% \mathrm{Cl}$ )

Cl: Confidence interval; RR: risk ratio

GRADE Working Group certainty of evidence

High certainty: Further research is very unlikely to change our confidence in the estimate of effect

Moderate certainty: Further research is likely to have an important impact on our confidence in the estimate of effect and may change the estimate

Low certainty: Further research is very likely to have an important impact on our confidence in the estimate of effect and is likely to change the estimate

Very low certainty: We are very uncertain about the effect estimate

Summary of findings 2. Mycophenolate mofetil (MMF) + tacrolimus (TAC) versus IV cyclophosphamide (CPA) for induction therapy

MMF + TAC compared with IV CPA for lupus nephritis

Patient or population: Patients with proliferative lupus nephritis

Settings: all settings

Intervention: MMF + TAC

Comparison: IV CPA

\begin{tabular}{|c|c|c|c|c|c|c|}
\hline \multirow[t]{3}{*}{ Outcomes } & \multicolumn{2}{|c|}{$\begin{array}{l}\text { Illustrative comparative risks }{ }^{\star} \\
(95 \% \mathrm{Cl})\end{array}$} & \multirow[t]{3}{*}{$\begin{array}{l}\text { Relative effect } \\
(95 \% \mathrm{CI})\end{array}$} & \multirow{3}{*}{$\begin{array}{l}\text { No. of partici- } \\
\text { pants } \\
\text { (studies) }\end{array}$} & \multirow{3}{*}{$\begin{array}{l}\text { Certainty of } \\
\text { evidence } \\
\text { (GRADE) }\end{array}$} & \multirow[t]{3}{*}{ Comments } \\
\hline & Assumed risk & $\begin{array}{l}\text { Corresponding } \\
\text { risk }\end{array}$ & & & & \\
\hline & IV CPA & MMF + TAC & & & & \\
\hline $\begin{array}{l}\text { Complete re- } \\
\text { nal remission } \\
\text { follow-up: } \\
\text { mean } 24 \text { weeks }\end{array}$ & 244 per 1000 & $\begin{array}{l}\mathbf{5 8 0} \text { per } \mathbf{1 0 0 0} \\
(261 \text { to } 1000)\end{array}$ & $\begin{array}{l}\text { RR 2.38 (1.07 to } \\
5.30)\end{array}$ & $402(2)$ & $\begin{array}{l}\oplus \oplus \oplus \ominus \\
\operatorname{low}^{1,2,3,4}\end{array}$ & $\begin{array}{l}\text { Downgraded as follows: } \\
\text { 1Study limitation: concern regarding the incomplete re- } \\
\text { porting of IV CPA group } \\
2 \text { Heterogeneity: substantial heterogeneity indicated by } \\
{ }^{2} \text { statistic. Although } \mathrm{Chi}^{2} \text { test was satisfied, the small } \\
\text { number of studies may make this unreliable. }\end{array}$ \\
\hline
\end{tabular}




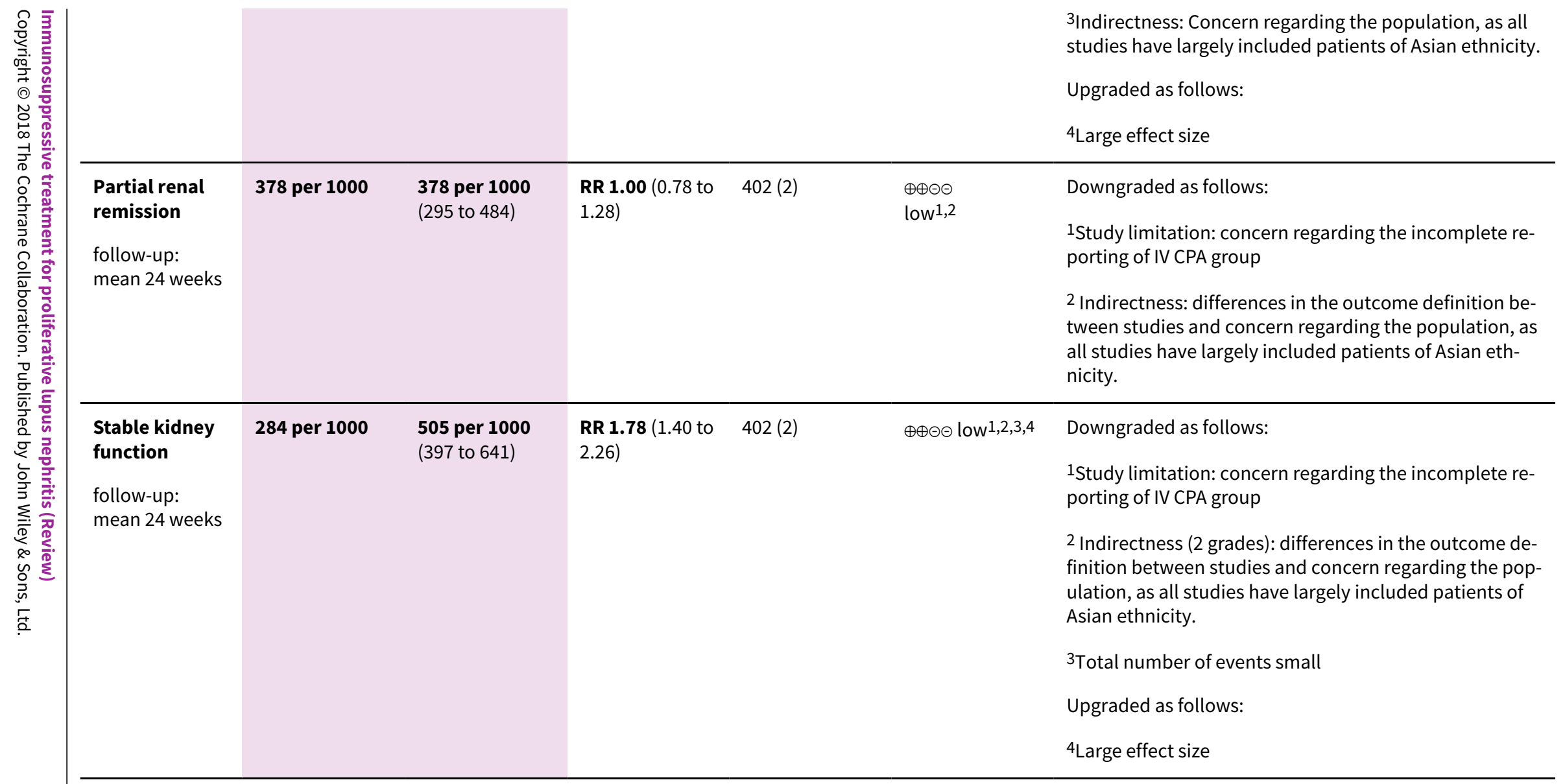

*The basis for the assumed risk was calculated using the median control group risk across studies in the meta-analyses. The corresponding risk (and its $95 \%$ confidence interval) is based on the assumed risk in the comparison group and the relative effect of the intervention (and its $95 \% \mathrm{Cl}$ ).

Cl: Confidence interval; RR: Risk Ratio

GRADE Working Group certainty of evidence

High certainty: Further research is very unlikely to change our confidence in the estimate of effect.

Moderate certainty: Further research is likely to have an important impact on our confidence in the estimate of effect and may change the estimate.

Low certainty: Further research is very likely to have an important impact on our confidence in the estimate of effect and is likely to change the estimate.

Very low certainty: We are very uncertain about the estimate. 


\begin{tabular}{|c|c|c|c|c|c|c|}
\hline \multicolumn{7}{|c|}{$\begin{array}{l}\text { Patient or population: patients with maintenance treatment in lupus nephritis } \\
\text { Settings: all settings } \\
\text { Intervention: AZA } \\
\text { Comparison: MMF }\end{array}$} \\
\hline \multirow[t]{3}{*}{ Outcomes } & \multicolumn{2}{|c|}{$\begin{array}{l}\text { Illustrative comparative risks* } \\
(95 \% \mathrm{Cl})\end{array}$} & \multirow[t]{3}{*}{$\begin{array}{l}\text { Relative effect } \\
(95 \% \mathrm{CI})\end{array}$} & \multirow{3}{*}{$\begin{array}{l}\text { No. of partici- } \\
\text { pants } \\
\text { (studies) }\end{array}$} & \multirow{3}{*}{$\begin{array}{l}\text { Certainty of } \\
\text { evidence } \\
\text { (GRADE) }\end{array}$} & \multirow[t]{3}{*}{ Comments } \\
\hline & Assumed risk & $\begin{array}{l}\text { Corresponding } \\
\text { risk }\end{array}$ & & & & \\
\hline & MMF & AZA & & & & \\
\hline $\begin{array}{l}\text { Death } \\
\text { Follow-up: } 36 \text { to } \\
72 \text { months }\end{array}$ & 22 per 1000 & $\begin{array}{l}\mathbf{2 5} \text { per } 1000 \\
\text { (7 to } 84)\end{array}$ & $\begin{array}{l}\text { RR } 1.15 \\
(0.34 \text { to } 3.87)\end{array}$ & $451(4)$ & $\begin{array}{l}\oplus \odot \odot \odot \\
\text { Very low } 1,2,3\end{array}$ & $\begin{array}{l}\text { Downgraded as follows: } \\
\text { 1Total number of events small } \\
\text { 2Severe imprecision ( } 2 \text { grades): risk estimate includes } \\
\text { null effect and estimate consistent with both appre- } \\
\text { ciable benefit and harm } \\
\text { 3Indirectness: time frame insufficient }\end{array}$ \\
\hline $\begin{array}{l}\text { ESKD } \\
\text { Follow-up: } 36 \text { to } \\
72 \text { months }\end{array}$ & 17 per 1000 & $\begin{array}{l}\mathbf{3 0} \text { per } 1000 \\
(9 \text { to } 96)\end{array}$ & $\begin{array}{l}\text { RR } 1.70 \\
(0.52 \text { to } 5.54)\end{array}$ & $452(4)$ & $\begin{array}{l}\oplus \ominus \ominus \ominus \\
\text { Very low } 1,2,3\end{array}$ & $\begin{array}{l}\text { Downgraded as follows: } \\
1 \text { Total number of events small } \\
\text { 2Severe imprecision ( } 2 \text { grades): risk estimate includes } \\
\text { null effect and estimate consistent with both appre- } \\
\text { ciable benefit and harm } \\
\text { 3Indirectness: time frame insufficient }\end{array}$ \\
\hline $\begin{array}{l}\text { Renal relapse } \\
\text { Follow-up: } 36 \text { to } \\
72 \text { months }\end{array}$ & 152 per 1000 & $\begin{array}{l}\mathbf{2 6 6} \text { per } \mathbf{1 0 0 0} \\
\text { (183 to } 388)\end{array}$ & $\begin{array}{l}\text { RR } 1.75 \\
\text { (1.20 to } 2.55)\end{array}$ & $452(4)$ & $\begin{array}{l}\oplus \oplus \oplus \ominus \\
\text { moderate } 1\end{array}$ & $\begin{array}{l}\text { Downgraded as follows: } \\
1 \text { Total number of events small }\end{array}$ \\
\hline $\begin{array}{l}\text { Doubling of } \\
\text { serum creati- } \\
\text { nine } \\
\text { Follow-up: } 36 \text { to } \\
72 \text { months }\end{array}$ & 39 per 1000 & $\begin{array}{l}86 \text { per } 1000 \\
(40 \text { to } 182)\end{array}$ & $\begin{array}{l}\text { RR } 2.19 \\
(1.03 \text { to } 4.66)\end{array}$ & $452(4)$ & $\begin{array}{l}\oplus \oplus \ominus \ominus \\
\operatorname{low}^{1,2}\end{array}$ & $\begin{array}{l}\text { Downgraded as follows: } \\
1 \text { Study limitations: (studies generally at unclear or } \\
\text { high risk of bias for many domains) } \\
2 \text { Total number of events small }\end{array}$ \\
\hline
\end{tabular}




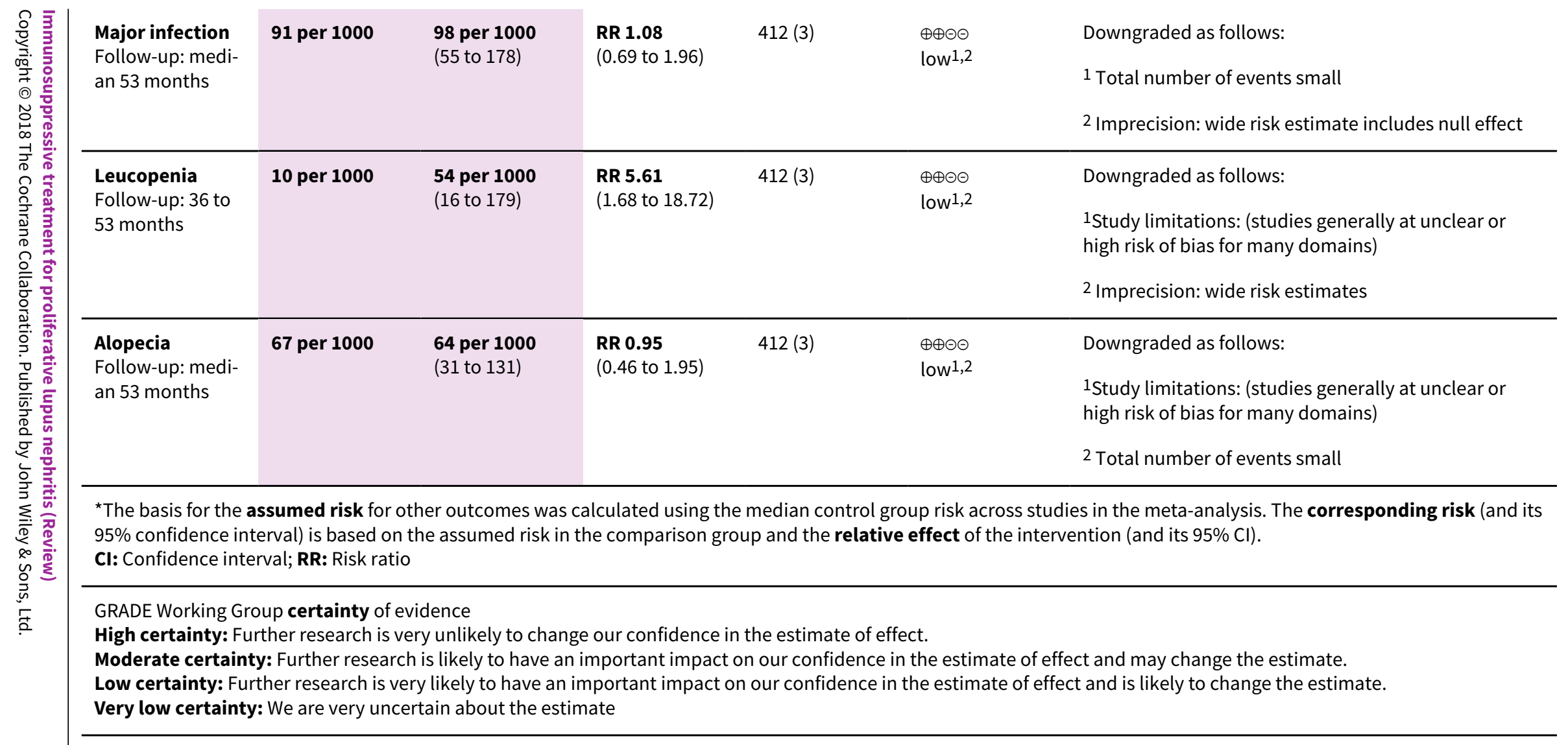




\section{B A C K G R O U N D}

\section{Description of the condition}

Lupus nephritis occurs in about $20 \%$ to $75 \%$ of all people with systemic lupus erythematosus (SLE) (Cervera 2009), leading to end-stage kidney disease (ESKD) in $10 \%$ to $17 \%$ of patients at 10 years (Houssiau 2010; Tektonidou 2016). Predominantly affecting young women, lupus nephritis is also more common in certain ethnic minority groups, particularly among African-Americans and Hispanics who may also have a more aggressive form of the disease that is less responsive to treatment (Hanly 2016; Korbet 2007; Sexton 2014).

Kidney involvement ranges from mild subclinical disease, which is associated with favourable outcomes and a low chance of progression to ESKD, to severe nephritic and/or nephrotic syndrome with kidney impairment and greater risk of progression to ESKD. In the United States of America, and Australia and New Zealand, approximately $1 \%$ of patients commencing dialysis had ESKD as a consequence of lupus nephritis (ANZDATA 2016; Costenbader 2011). Patients with SLE and active lupus nephritis have reduced health-related quality of life (Daleboudt 2011; McElhone 2006; Vu 1999). Fatigue is a frequent symptom and often identified as the most disrupting aspect of the disease in patients with lupus nephritis (Daleboudt 2011; Tench 2000), as it can limit their capacity to participate in the workforce, family, and social activities (Sutanto 2013).

Renal biopsy is required for the precise diagnosis and classification of lupus nephritis. Histological classification was introduced by the World Health Organization (WHO) in 1982 and revised in 2003 by the International Society of Nephrology (ISN) and the Renal Pathology Society (RPS). The ISN/RPS 2003 Class I and II lesions have a good prognosis and are generally not an indication for specific therapy, although some guidelines recommend therapy for people with WHO Class II lupus nephritis and proteinuria ( $>2$ g/d) (Tunnicliffe 2015). Proliferative disease (WHO Class III, IV, V +III and V+IV; ISN/RPS 2003 Class III (A) and (A/C), Class IV-G and IV-S, and Class III or IV in combination with Class V) is usually symptomatic, more fulminant, and requires treatment to induce remission and prevent significant kidney injury and premature death. Active proliferative (WHO Class IV) lupus nephritis is the most aggressive form of the condition, and has the worst prognosis without intensive immunosuppressive treatment.

\section{Description of the intervention}

Immunosuppressive therapy in the management of proliferative lupus nephritis aims to induce and maintain disease remission, in order to maximise patient and renal survival while minimising complications or treatment related adverse effects. The induction phase of therapy usually lasts six to 12 months. Common immunosuppressive agents in induction therapy include corticosteroid and an anti-proliferative agent such as cyclophosphamide, mycophenolate mofetil (MMF), or azathioprine. Less commonly used treatments that are added to corticosteroids include tacrolimus, cyclosporin, plasma exchange or plasmapheresis, or a biologic therapy such as rituximab. Intravenous (IV) cyclophosphamide in combination with corticosteroids became standard of care therapy for inducing remission based on a landmark National Institutes of Health (NIH) trial that showed cyclophosphamide was superior over corticosteroids alone in preventing renal flares and kidney failure (Decker 1975). A meta-analysis (Bansal 1997) and our earlier systematic review (Flanc 2004a) identified that the addition of an immunosuppressant to corticosteroids was superior to corticosteroids alone in managing proliferative lupus nephritis. Subsequently, low-dose cyclophosphamide (Euro-lupus regimen) has been reported to have equivalent efficacy to the NIH protocol (Houssiau 2002). The dose of corticosteroid is tapered as the disease activity is controlled and the anti-proliferative therapy is replaced with a less toxic alternative once remission is induced. Maintenance therapy aims to maintain remission and potential treatments include: azathioprine, MMF, tacrolimus and cyclosporin.

\section{How the intervention might work}

Active lupus nephritis is characterised by an inflammatory response to immune complexes in the kidneys. Mediators of inflammation, including complement, leukocytes, and cytokines injure the kidney and amplify inflammation. The release of kidney antigens in response to this inflammatory kidney injury may result in the production of kidney-specific autoantibodies. This organ-specific autoimmunity may perpetuate inflammation and result in kidney injury (Rovin 2014). The mechanisms of action of therapies used in the management of lupus nephritis are diverse, and aim to attenuate inflammation. Corticosteroids and IV cyclophosphamide and other conventional treatments have a broad spectrum immunosuppressive effect, while biologic therapies, which have been of increasing focus of trials in the last decade, target B-cells, T-cells, cytokines or growth factors to suppress the immune response (Murphy 2013).

First-line therapy has transformed lupus nephritis from an acute illness with five-year survival rates at less than $50 \%$ in the 1950 s to a chronic illness with five-year survival rates greater than $90 \%$ (Houssiau 2010; Mok 2002). Response to treatment is often slow, and although remission is induced in a significant proportion of patients, the risk of relapse has been reported between $18 \%$ and $46 \%$ (Ponticelli 1998), and treatment can cause considerable toxicity, and increase the risk of infertility (Henderson 2012).

\section{Why it is important to do this review}

We conducted a systematic review of immunosuppressive treatment of proliferative lupus nephritis in 2004 (Flanc 2004a), and updated this systematic review in 2012 (Henderson 2012). The 2012 review identified 50 randomised controlled trials (RCTs) that enrolled a total of 2846 participants. The conclusion was that compared with IV cyclophosphamide, MMF was as effective in inducing disease remission, but with a lower risk of ovarian failure. MMF was more effective than azathioprine in maintaining disease remission. A recent network meta-analysis identified that compared to IV cyclophosphamide either MMF or tacrolimus or their combination was more effective in inducing remission. Compared with IV cyclophosphamide, the combination of MMF and tacrolimus reduced ovarian failure, but either treatment alone conferred a similar risk of ovarian failure. The use of these newer therapies on outcomes such as: death, ESKD and doubling of serum creatinine (SCr) were inconclusive (Palmer 2017).

In the past five years, numerous studies have evaluated a number of regimens including MMF, tacrolimus or their combination and various biologic agents. Given the uncertainty that surrounds the safety and efficacy of these therapies, the aim of our 
updated review was to evaluate the relative effects of all available immunosuppressive therapies for the induction and maintenance treatment of lupus nephritis using IV cyclophosphamide as the main comparator in induction therapy and azathioprine as the main comparator in maintenance therapy.

\section{O B J E C T IVES}

Our objective was to assess the evidence and evaluate the benefits and harms of different immunosuppressive treatments in people with biopsy-proven lupus nephritis. The following questions relating to management of proliferative lupus nephritis were addressed:

1. Are new immunosuppressive agents superior to or as effective as cyclophosphamide plus corticosteroids?

2. Which agents, dosages, routes of administration and duration of therapy should be used?

3. Which toxicities occur with the different treatment regimens?

\section{METHODS}

\section{Criteria for considering studies for this review}

\section{Types of studies}

We included all RCTs and quasi-RCTs, whether published or available only in abstract form, which evaluated any of the treatment options in the focus of this review, singularly or in combination, determining the benefits and harms of different treatment options for lupus nephritis.

\section{Types of participants}

We included adults and children with biopsy-proven proliferative lupus nephritis.

\section{Types of interventions}

We considered studies that investigated the following treatment options for either induction or maintenance therapies for lupus nephritis.

- Corticosteroids including prednisone and methylprednisolone

- Other immunosuppressive agents including azathioprine, cyclophosphamide, MMF, tacrolimus and cyclosporin

- Plasma exchange or plasmapheresis

- Biologic therapy (for example, abatacept, atacicept, laquinimod, ocrelizumab, rituximab and sirukumab).

Non-specific treatment options (e.g. antihypertensive agents) were not included in the present analysis because these do not specifically aim to treat underlying lupus nephritis, but rather more generally, aim to prevent the progression of chronic kidney disease (CKD).

\section{Types of outcome measures}

\section{Primary outcomes}

- Death (all causes)

- ESKD, requirement for renal replacement therapy

- Complete renal remission: defined as return to normal SCr, urinary protein excretion $<0.5 \mathrm{~g} / 24 \mathrm{~h}$, and inactive urinary sediment) following induction therapy
- Relapse of lupus nephritis: maintenance therapy

\section{Secondary outcomes}

The following dichotomous outcome measures were considered:

- Partial renal remission: defined as a fall to $<3.0 \mathrm{~g} / \mathrm{d}$ protein if baseline $\geq 3.0 \mathrm{~g} / \mathrm{d}$ or $\geq 50 \%$ reduction if $<3.0 \mathrm{~g} / \mathrm{d}$ at baseline and stabilisation of $\mathrm{SCr} \pm 25 \%$ (ALMS 2007)

- Remission in proteinuria: complete and partial.

* Complete remission in proteinuria: defined as urinary protein excretion $\leq 0.3 \mathrm{~g} / 24 \mathrm{~h}$ (Chan 2000)

* Partial remission in proteinuria: defined as $<3.0 \mathrm{~g} / \mathrm{d}$ protein if baseline $\geq 3.0 \mathrm{~g} / \mathrm{d}$ or $\geq 50 \%$ reduction if $<3.0 \mathrm{~g} / \mathrm{d}$ at baseline (ALMS 2007)

- Relapse of lupus nephritis - induction therapy

- Doubling of SCr

- Deterioration of kidney function: defined as more than $20 \%$ worsening of $\mathrm{SCr}$

- Stable kidney function: defined as a less than $20 \%$ worsening of $\mathrm{SCr}$.

The following side effects (toxicity) of treatments were considered:

- Ovarian failure (sustained amenorrhoea)

- Menstrual irregularities

- Infection

* Major infection: all-cause infection excluding herpes zoster virus infection

* Herpes zoster virus infection

- Development of any malignancy

- Leucopenia (defined as $<4 \times 10^{9}$ cells/L)

- Bone toxicity (avascular necrosis or fracture)

- Bladder toxicity (haemorrhagic cystitis)

- Alopecia

- Gastrointestinal (GI) adverse effects including diarrhoea, vomiting and nausea.

The following continuous outcomes were analysed at the end of treatment.

- Daily proteinuria (24 hour urinary protein excretion) $(\mathrm{g} / 24 \mathrm{~h})$

- Creatinine clearance $(\mathrm{CrCl})(\mathrm{mL} / \mathrm{min})$

- $\mathrm{SCr}(\mu \mathrm{mol} / \mathrm{L})$

- Health-related quality of life

- Fatigue

- Disease activity (e.g. British Isles Lupus Assessment Group (BILAG), SLE Disease Activity Index (SLEDAI)

\section{Search methods for identification of studies}

\section{Electronic searches}

We searched the Cochrane Kidney and Transplant Specialised Register up to 2 March 2018 through contact with the Information Specialist using search terms relevant to this review. The Cochrane Kidney and Transplant Specialised Register contains studies identified from several sources.

1. Monthly searches of the Cochrane Central Register of Controlled Trials (CENTRAL) 
2. Weekly searches of MEDLINE OVID SP

3. Handsearching of kidney-related journals and the proceedings of major kidney conferences

4. Searching of the current year of EMBASE OVID SP

5. Weekly current awareness alerts for selected kidney and transplant journals

6. Searches of the International Clinical Trials Register (ICTRP) Search Portal and ClinicalTrials.gov.

Studies contained in the Specialised Register are identified through search strategies for CENTRAL, MEDLINE, and EMBASE based on the scope of Cochrane Kidney and Transplant. Details of these strategies, as well as a list of handsearched journals, conference proceedings and current awareness alerts, are available in the Specialised Register section of information about Cochrane Kidney and Transplant.

See Appendix 1 for search terms used in strategies for this review.

\section{Searching other resources}

1. Reference lists of review articles, relevant studies and clinical practice guidelines

2. Handsearching of proceedings of major rheumatology conferences

3. Letters seeking information about unpublished or incomplete studies to investigators known to be involved in previous studies.

\section{Data collection and analysis}

\section{Selection of studies}

The search strategy described was performed to identify eligible studies. The titles and abstracts resulting from the searches were screened by two authors who independently assessed retrieved abstracts, and if necessary the full text, to determine which studies satisfied the inclusion criteria. Disagreement about inclusion was resolved by discussion with a third author.

Where duplication reports of the same study were confirmed, the initial first complete publication was selected (the index publication) and was the primary data source, but any other additional prior or subsequent reports were also included. These additional prior or subsequent reports containing supplementary outcome data (such as longer-term follow up, or different outcomes) also contributed to the meta-analysis.

\section{Data extraction and management}

Data abstraction was performed independently by two authors using a standardised form. Unclear data were clarified by contacting the author of the study report and any relevant data obtained in this manner was included in the review (see Acknowledgements).

\section{Assessment of risk of bias in included studies}

The following items were independently assessed by two authors using the risk of bias assessment tool (Higgins 2011) (see Appendix 2).

- Was there adequate sequence generation (selection bias)?

- Was allocation adequately concealed (selection bias)?
- Was knowledge of the allocated interventions adequately prevented during the study (detection bias)?

* Participants and personnel (performance bias)

* Outcome assessors (detection bias)

- Were incomplete outcome data adequately addressed (attrition bias)?

- Are reports of the study free of suggestion of selective outcome reporting (reporting bias)?

- Was the study apparently free of other problems that could put it at a risk of bias?

Disagreements regarding the risk of bias adjudications were resolved by consultation with a third review author.

\section{Measures of treatment effect}

\section{Dichotomous data}

For dichotomous outcomes (death (all causes), complete or partial renal remission, complete or partial remission in proteinuria, ESKD, renal relapse, doubling of $\mathrm{SCr}$, stable kidney function, ovarian failure, menstrual irregularities, major infection, herpes zoster virus infection, malignancy, leucopenia, bone toxicity, bladder toxicity, alopecia and GI disorders) results were expressed as risk ratio (RR) with $95 \%$ confidence intervals $(\mathrm{Cl})$.

\section{Continuous data}

Where continuous scales of measurement were used to assess the effects of treatment (urinary protein excretion, $\mathrm{CrCl}$, SCr, healthrelated quality of life, fatigue, disease activity) the mean difference (MD) with $95 \% \mathrm{Cl}$ was used.

\section{Unit of analysis issues}

\section{Studies with multiple treatment groups}

In studies comparing the efficacy of more than two interventions we considered the following:

1. If different interventions were of different classes (for example, MMF or tacrolimus versus IV cyclophosphamide), we included each treatment group in separate meta-analyses, ensuring we did not include outcome data for the control group participants more than once in a single meta-analysis.

2. If interventions were of the same therapy (for example, high dose or low dose abatacept, laquinimod), we summarised into a single group that was compared with the control group for dichotomous outcomes (we summed the sample sizes and the number of people with events across the treatment groups). For continuous data, we entered the means and standard deviations of a single intervention group (usually the highest dosage) for comparison with the control group. Where appropriate, we considered sensitivity analyses, testing the impact of including the alternative intervention group in analyses.

\section{Dealing with missing data}

Where a study reported outcome data after excluding some randomised participants from the denominator, further information required from the original author was requested by electronic mail and any relevant information obtained in this manner was included in the review. Evaluation of important numerical data such as screened, randomised patients as well as intention-to-treat, as-treated and per-protocol population were 
carefully performed. Attrition rates, for example drop-outs, losses to follow-up and withdrawals were investigated. Issues of missing data and imputation methods (for example, last-observationcarried-forward) was critically appraised (Higgins 2011).

\section{Assessment of heterogeneity}

We first assessed for statistical heterogeneity visually by inspecting forest plots of standardised mean effect sizes and of risk ratios. Furthermore, we applied a $\mathrm{Chi}^{2}$ test to assess heterogeneity. With $\mathrm{P}$ $<0.05$ used to denote statistical significance, and with $1^{2}$ calculated to measure the proportion of total variation in the estimates of treatment effect that was due to heterogeneity beyond chance (Higgins 2011) and we used conventions of interpretation that were defined by Higgins 2003.

\section{Assessment of reporting biases}

Detection of potential for publication bias was planned for among the primary outcomes. We made every attempt to minimise publication bias by including unpublished studies (for example, by searching online trial registries). In order to assess publication bias we used funnel plots of the log odds ratio (OR) (effect versus standard error of the effect size) when sufficient number of studies were available (Higgins 2011). For the analysis and the interpretation of the funnel plots, other reasons for asymmetry besides publication bias were considered (for example, differences in methodological quality and true heterogeneity in intervention effects). However, the limited amount of study data published did not enable meaningful interpretation. We had also planned to conduct subgroup analysis and meta-regression to evaluate potential sources of heterogeneity but this was not possible because of the small number of studies of paired interventions.

\section{Data synthesis}

Data were abstracted from individual studies and then pooled for summary estimates using a random-effects model. The randomeffects model was chosen because it provides a more conservative estimate of effect in the presence of known or unknown potential heterogeneity (Deeks 2001).

\section{Subgroup analysis and investigation of heterogeneity}

Subgroup analyses are hypothesis-forming rather than hypothesistesting and should be treated with caution. We considered subgroup analyses on the ethnicity of participants, class of lupus nephritis, age of the patient (adults versus children) and the type of induction therapy patients were treated with before randomisation in maintenance therapy studies in order to explore whether clinical differences between the studies may have systematically influenced the differences that were observed in the treatment outcomes. However, insufficient data were available to conduct subgroup analyses for the primary outcomes.

\section{Sensitivity analysis}

The following sensitivity analyses were considered:

- Repeating the analysis excluding unpublished studies

- Repeating the analysis taking account of risk of bias, as specified
- Repeating the analysis excluding any very long or large studies to establish how much they dominate the results

- Repeating the analysis excluding studies using the following filters: language of publication, source of funding (industry versus other), and country the study was conducted in.

However insufficient data were available to determine these factors influence of the on effect size.

\section{'Summary of findings' tables}

We presented the main results of the review in 'Summary of findings' tables. These tables present key information concerning the quality of the evidence, the magnitude of the effects of the interventions examined, and the sum of the available data for the main outcomes (Schünemann 2011a). The 'Summary of findings' tables also include an overall grading of the evidence related to each of the main outcomes using the GRADE (Grades of Recommendation, Assessment, Development and Evaluation) approach (GRADE 2008; GRADE 2011). The GRADE approach defines the quality of a body of evidence as the extent to which one can be confident that an estimate of effect or association is close to the true quantity of specific interest. The quality of a body of evidence involves consideration of within-trial risk of bias (methodological quality), directness of evidence, heterogeneity, precision of effect estimates and risk of publication bias (Schünemann 2011b). We presented the following outcomes in the 'Summary of findings' tables.

- Death (all causes)

- ESKD, requirement for renal replacement therapy

- Complete renal remission

- Partial renal remission

- Renal relapse

- Doubling of SCr

- Stable kidney function

- Ovarian failure

- Major infection

- Leucopenia

- Alopecia

- Diarrhoea

\section{RES U L T S}

\section{Description of studies}

\section{Results of the search}

For this update, a search was conducted on 2 March 2018 (Figure 1). This new search identified 110 reports. After full-text review 71 new studies were identified. Twenty-six (43 reports) new studies were included and 17 (19 reports) were excluded. We identified 26 ongoing studies which will be assessed in a future update of this review. We also identified 20 new reports of 11 existing included studies. One study identified as a primary study in the 2012 review update was reallocated as a secondary report of ALMS 2007 (Sundel 2008). Four previously excluded studies have been included as they met our inclusion criteria (Abedi 2007; Florez-Suarez 2004; Loo 2010; Zhang 1995a). 


\section{Figure 1. Study flow diagram. *Non-RCTs have been deleted from this update}

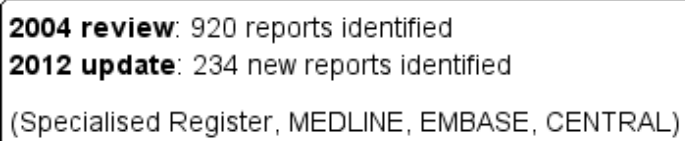

\begin{tabular}{|l|}
\hline Included studies \\
2004 review: 25 ( 36 reports, 915 participants) \\
2012 update: 48 (170 reports, 2846 participants) \\
Excluded studies \\
2004 review: 2 ( 2 reports) \\
2012 update: 55 (96 reports) \\
Ongoing studies \\
2004 review: 4 (4 reports) \\
2012 update: 11 (11 reports) \\
\hline
\end{tabular}

\section{8 review update}

Specialised register: 110 reports identified

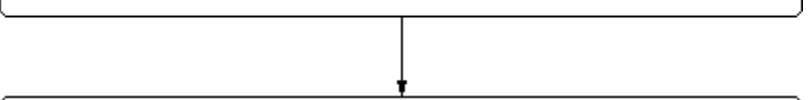

New included studies: 26 (43 reports)

New reports of existing studies: 20 (11 studies)

New ongoing studies: 28 (28 reports)

New excluded studies: 17 (19 reports) (wrong population or intervention)

New reports of existing excluded studies: 0

$$
\begin{aligned}
& 2018 \text { review update } \\
& \text { Included studies: } 74 \text { (236 reports; } 5175 \text { participants) } \\
& \text { Excluded studies" : } 48 \text { ( } 84 \text { reports) } \\
& \text { Ongoing studies: } 31 \text { ( } 31 \text { reports) }
\end{aligned}
$$

\section{Included studies}

\section{See Characteristics of included studies}

After including the studies identified from the 2018 update search, a total of 236 reports of 74 studies were included in this review (Figure 1) which included a total of 5175 randomised participants (Abedi 2007; ACCESS 2014; ALMS 2007; APRIL-LN 2012; AURA-LV 2016; Balletta 1992; Bao 2008; Barron 1982; Belmont 1995; BELONG 2013; Boedigheimer 2017; Boletis 1999; Boumpas 1992; Cade 1973; Chan 2000; Chen 2011; Clark 1981; Clark 1984; Contreras 2004; CYCLOFALUNE 2010; Decker 1975; Deng 2016; Derksen 1988; Donadio 1972; Donadio 1976; Doria 1994; Dyadyk 2001; El-Sehemy 2006; El-Shafey 2010; Florez-Suarez 2004; Fries 1973; Fu 1997; Furie 2014; Ginzler 1976; Ginzler 2005; Gourley 1996; Grootscholten 2006; Hahn 1975; Hong 2007; Houssiau 2002; Jayne 2013; Kaballo 2016; Kamanamool 2017; Lewis 1992; Li 2009c; Li 2012; Liou 2007; Liu 2015; Loo 2010; Lui 1997; LUNAR 2012; MAINTAIN Nephritis 2010; Mehra 2018; Mendonca 2017; Mitwalli 2011; Mok 2016; Moroni 2006; Mulic-Bacic 2008; MyLupus 2011; Nakamura 2002e; Ong 2005; Pal 2017; Rathi 2016; Rovin 2016; Sabry 2009; Sedhain 2016; Sesso 1994a; SIMPL 2014; Steinberg 1971; Sun 2015; Wallace 1998; Yap 2017; Yee 2004; Zhang 1995a).

Twenty-nine studies enrolled adults and children ( $<18$ years) (ACCESS 2014; ALMS 2007; Bao 2008; BELONG 2013; Boumpas 1992; Cade 1973; Chen 2011; Derksen 1988; Donadio 1972; Donadio 1976; Doria 1994; El-Shafey 2010; Houssiau 2002; Kaballo 2016; Lewis 1992; Li 2012; Loo 2010; LUNAR 2012; MAINTAIN Nephritis 2010; Mehra 2018; Mendonca 2017; Moroni 2006; Ong 2005; Rathi 2016; Sesso 1994a; Steinberg 1971; Sun 2015; Wallace 1998; Yee 2004), 29 only enrolled adults (APRIL-LN 2012; AURA-LV 2016; Balletta 1992; Belmont 1995; Boedigheimer 2017; Boletis 1999; Chan 2000; Clark 1984; Contreras 2004; CYCLOFA-LUNE 2010; Dyadyk 2001;
El-Sehemy 2006; Furie 2014; Ginzler 1976; Ginzler 2005; Gourley 1996; Grootscholten 2006; Hahn 1975; Hong 2007; Kamanamool 2017; Li 2009c; Lui 1997; Mitwalli 2011; Mok 2016; MyLupus 2011; Nakamura 2002e; Rovin 2016; Sabry 2009; SIMPL 2014), 2 only enrolled children (Barron 1982; Fu 1997), and 14 studies did not specify the age of the participants.

There were 67 studies of induction therapy (4791 participants), and nine studies of maintenance therapy (767 participants; 297 had already completed an induction phase study (ALMS 2007; Chen 2011)). Follow-up ranged from median 12 months duration (range 2.5 to 48 months) for induction therapy, and median 30 months duration (range 6 to 63 months) for maintenance therapy. The numbers of patients included in the studies ranged from 6 to 378 with a median number of 45 patients.

Of all authors contacted for further clarification for the 2012 review update, nine responded (Drs Belmont, Doria, Donadio, Fries, Gourley, Houssiau, Solomons, Wofsy and Florez-Suarez). For the 2018 update, one author provided supplementary data (Dr Rathi).

\section{Induction therapy}

Comparators for induction therapy included the following.

1. MMF plus corticosteroid versus IV cyclophosphamide plus corticosteroid (10 studies, 878 participants: Abedi 2007; ALMS 2007; El-Shafey 2010; Florez-Suarez 2004; Ginzler 2005; Li 2012; Mulic-Bacic 2008; Ong 2005; Rathi 2016; Sedhain 2016)

2. MMF plus corticosteroid versus oral cyclophosphamide plus corticosteroids (1 study, 62 participants: Chan 2000)

3. MMF plus tacrolimus plus corticosteroid versus IV cyclophosphamide plus corticosteroid (2 studies, 402 participants: Bao 2008; Liu 2015) 
4. MMF plus IV cyclophosphamide and corticosteroids versus cyclophosphamide plus corticosteroids (1 study, 82 participants: Sun 2015)

5. MMF plus corticosteroids versus tacrolimus plus corticosteroids (2 studies, 190 participants: Li 2012; Mok 2016)

6. Calcineurin inhibitors (tacrolimus or cyclosporin) plus corticosteroids versus IV cyclophosphamide plus corticosteroids (4 studies, 178 participants: Chen 2011; CYCLOFA-LUNE 2010; Hong 2007; Li 2012) or oral cyclophosphamide plus corticosteroids (1 study, 34 participants: Lui 1997)

7. Cyclophosphamide plus corticosteroid versus azathioprine plus corticosteroid (4 studies, 219 participants: El-Sehemy 2006; Decker 1975; Dyadyk 2001; Grootscholten 2006) or lefluomide plus corticosteroid (1 study, 30 participants: Deng 2016)

8. Rituximab plus MMF versus placebo plus MMF (both arms included corticosteroids) (1 study, 144 participants: LUNAR 2012)

9. Rituximab plus cyclophosphamide versus rituximab alone (both arms included corticosteroids) (1 study, 19 participants: Li 2009c)

10.Abatacept versus placebo (2 studies; 432 participants: ACCESS 2014, Furie 2014)

11.Low dose or high dose laquinimod versus placebo (1 study, 46 participants: Jayne 2013)

12.Low dose or high dose ocrelizumab versus placebo (1 study; 378 participants: BELONG 2013)

13.Sirukumab with or without corticosteroids plus MMF or azathioprine versus placebo with or without corticosteroids plus MMF or azathioprine (1 study, 25 participants: Rovin 2016)

14.IV versus oral cyclophosphamide (2 studies, 74 participants: Decker 1975; Yee 2004)

15.Low versus high dose IV cyclophosphamide (3 studies, 253 participants: Houssiau 2002; Mitwalli 2011; Sabry 2009)

16.Standard dose corticosteroid versus reduced dose corticosteroid with both arms receiving enteric-coated mycophenolate sodium (EC-MPS) (1 study, 81 participants: MyLupus 2011)

17.IV versus oral corticosteroid (1 study, 22 participants: Barron 1982).

18.IV cyclophosphamide with or without corticosteroid versus corticosteroid alone (5 studies, 261 participants: Decker 1975; Boumpas 1992; Gourley 1996; Sesso 1994a; Steinberg 1971)

19.Cyclophosphamide versus azathioprine with or without corticosteroids versus corticosteroid alone (4 studies, 94 participants: Decker 1975; Cade 1973; Donadio 1972; Hahn 1975)

20.Azathioprine plus corticosteroids versus corticosteroids alone (3 studies, 78 participants: Cade 1973; Decker 1975; Hahn 1975)

21.Cyclosporin plus corticosteroids versus corticosteroids alone (1 study, 10 participants: Balletta 1992)

22. Misoprostol plus corticosteroids versus corticosteroids alone (1 study, 14 participants: Belmont 1995)

23. Plasma exchange plus immunosuppression plus corticosteroids versus immunosuppression plus corticosteroids (5 studies, 174 participants; Clark 1981; Clark 1984; Doria 1994; Lewis 1992; Wallace 1998)

24.Plasma exchange versus immunosuppression alone ( 2 studies, 40 participants; Derksen 1988; Nakamura 2002e)
25.Long versus short duration IV cyclophosphamide (1 study, 40 participants: Boumpas 1992)

Other comparisons

- Plasma exchange versus immunoadsorption (1 study, 28 participants; Loo 2010)

- MMF versus cyclophosphamide (unclear if oral or IV) (1 study, 14 participants: Yap 2017)

- Tacrolimus + azathioprine versus IV cyclophosphamide (1 study, 58 participants: Pal 2017)

- Atacicept plus MMF and corticosteroid versus placebo plus MMF and corticosteroid (1 study, 6 participants: APRIL-LN 2012)

- Low dose or high dose voclosporin versus placebo (1 study; 256 participants: AURA-LV 2016)

- AMG811 (anti-IFN- $\gamma$ antibody) versus placebo (1 study; 28 participants: Boedigheimer 2017)

- Cyclophosphamide till remission versus cyclophosphamide for 1 year (1 study, 36 participants: Zhang 1995a).

\section{Maintenance therapy}

Six studies (541 participants) compared azathioprine plus corticosteroid to another immunosuppressive agent (MMF, cyclophosphamide, cyclosporin or tacrolimus) (ALMS 2007; Chen 2011; Contreras 2004; Kaballo 2016; MAINTAIN Nephritis 2010; Moroni 2006); two studies had already completed an induction phase (ALMS 2007; Chen 2011). One study (40 participants) compared cyclophosphamide with cyclosporin (Fu 1997), one study (14 participants) compared IV cyclophosphamide to IV immunoglobulin (IVIG) (Boletis 1999) and one study compared prednisone withdrawal versus prednisone continuation (SIMPL 2014).

The maintenance phase of one study (Chan 2000) underwent a significant post-randomisation protocol adjustment. The MMF induction arm originally switched to maintenance azathioprine at one year, but the protocol changed mid-trial to continue MMF for two years. This was prompted by an unexpectedly high rate of renal relapse in the azathioprine maintenance group. Data for those participants on the original protocol were not reported separately from the adjusted protocol, so accordingly, only the induction phase data of this study could be included in our synthesis.

\section{Excluded studies}

See Characteristics of excluded studies.

Forty-eight studies were excluded (Andrade-Ortega 2010; Antunes 2001; ASPEN 2008; ATLAS 2016; Austin 2009; Balow 1981; Balow 1984; Ble 2011; Chanchairujira 2009; Clark 1993; Clark 2001a; CONTROL 2016; Davis 1999; Daza 2005; Deng 2017a; Feng 2014; Frutos 1997; Hebert 1987; Khajehdehi 2012; Kuo 2001; Li 2005; Li 2014a; LJP 394-90-05 2003; LJP 394-90-09 2005; Lu 2002; Miyasaka 2009; NCT00001212; NCT00404157; NCT00429377; NCT00436438; NCT00539799; NCT00659217; NCT01299922; NCT01342016; NCT01930890; NCT02176486; Pierucci 1989; Schaumann 1992; Su 2007; Sztejnbok 1971; Wallace 2006; Wang 2007; Witte 1993; Yap 2012; Ye 2001; Yoshida 1996; Zhang 2015c; Zheng 2005a).

The major reasons for exclusion were: 
1. Diagnosis of lupus nephritis was not biopsy-proven or was not proliferative lupus nephritis

2. That the randomised treatment comparison was not immunosuppression.
For this update non-RCTs have been deleted.

\section{Risk of bias in included studies}

Reporting of details of study methodology was incomplete for the majority of studies, and are summarised in Figure 2.

Figure 2. Risk of bias graph: review authors' judgements about each risk of bias item presented as percentages across all included studies.

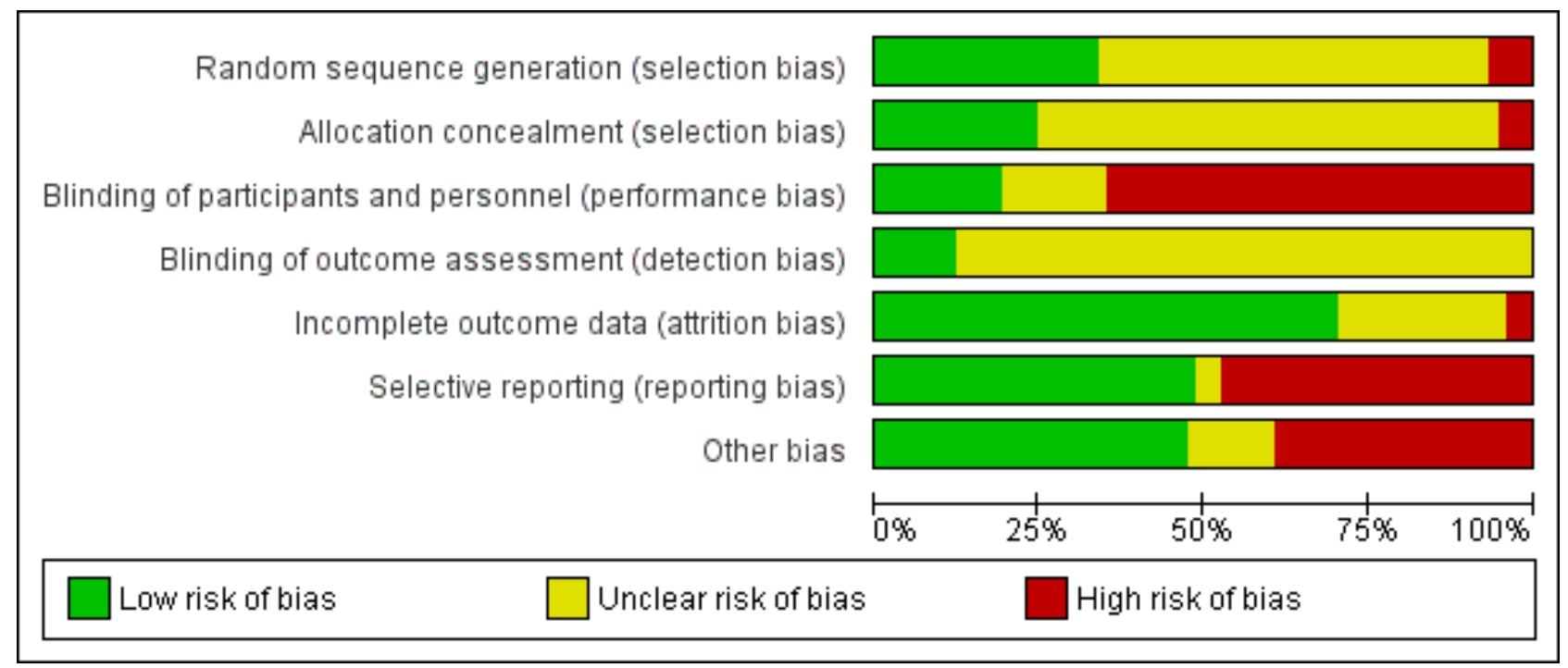

\section{Allocation}

\section{Random sequence generation}

Of the included studies, 25 reported adequate sequence generation (Bao 2008; Chan 2000; Chen 2011; Decker 1975; Derksen 1988; Donadio 1972; Donadio 1976; Fu 1997; Ginzler 2005; Gourley 1996; Grootscholten 2006; Hahn 1975; Houssiau 2002; Kaballo 2016; Kamanamool 2017; Lewis 1992; Li 2009c; Liu 2015; Mehra 2018; Mok 2016; Moroni 2006; Ong 2005; SIMPL 2014; Steinberg 1971; Yee 2004). Sequence generation was inadequate in five studies where alternation was used to allocate patients to treatment groups (Barron 1982; Cade 1973; Contreras 2004; Loo 2010; Sabry 2009). These studies were included in the review but deemed high risk for selection bias. Sequence generation was unclear in the remaining 44 studies.

\section{Allocation concealment}

Allocation concealment was adequate in 17 studies (ALMS 2007; Boletis 1999; Boumpas 1992; Chen 2011; Contreras 2004; CYCLOFALUNE 2010; Fu 1997; Ginzler 2005; Hahn 1975; Kamanamool 2017; Lewis 1992; Li 2009c; Liu 2015; Moroni 2006; Ong 2005; SIMPL 2014; Steinberg 1971), inadequate in four studies (Barron 1982; Cade 1973; MyLupus 2011; Sabry 2009), and unclear in the remaining 53 studies.

\section{Blinding}

\section{Performance bias}

Low risk of bias was assigned to 14 studies (ACCESS 2014; APRIL-LN 2012; AURA-LV 2016; Belmont 1995; BELONG 2013; Boedigheimer 2017; Furie 2014; Ginzler 1976; Jayne 2013; LUNAR 2012; Mitwalli 2011; Rovin 2016; SIMPL 2014; Steinberg 1971).
High risk was assigned to 47 studies, with 46 studies being openlabel (Abedi 2007; ALMS 2007; Bao 2008; Barron 1982; Boumpas 1992; Cade 1973; Chen 2011; Clark 1981; Clark 1984; Contreras 2004; CYCLOFA-LUNE 2010; Donadio 1972; Donadio 1976; Doria 1994; Dyadyk 2001; El-Shafey 2010; Florez-Suarez 2004; Fries 1973; Fu 1997; Ginzler 2005; Gourley 1996; Grootscholten 2006; Hahn 1975; Hong 2007; Kamanamool 2017; Lewis 1992; Li 2009c; Li 2012; Liou 2007; Liu 2015; Lui 1997; MAINTAIN Nephritis 2010; Mehra 2018; Mendonca 2017; Mok 2016; Moroni 2006; Mulic-Bacic 2008; MyLupus 2011; Nakamura 2002e; Ong 2005; Pal 2017; Rathi 2016; Sedhain 2016; Sun 2015; Wallace 1998; Yee 2004; Zhang 1995a), and one study was unlikely to have treatment allocation blinded (Loo 2010). The remaining 13 studies were unclear, as they did not report blinding.

\section{Detection bias}

Nine studies reported blinding of subjective outcomes adequately (ALMS 2007; AURA-LV 2016; Bao 2008; Chan 2000; Gourley 1996; Liu 2015; Mitwalli 2011; Moroni 2006; SIMPL 2014), the remaining studies were classified as unclear, as blinding of the outcome assessor was not reported.

\section{Incomplete outcome data}

Incomplete outcome data was addressed adequately in 54 studies (ACCESS 2014; ALMS 2007; APRIL-LN 2012; AURA-LV 2016; Balletta 1992; Bao 2008; Belmont 1995; Boedigheimer 2017; Boletis 1999; Boumpas 1992; Cade 1973; Chan 2000; Chen 2011; Clark 1981; Clark 1984; Contreras 2004; CYCLOFA-LUNE 2010; Decker 1975; Doria 1994; El-Sehemy 2006; Fu 1997; Furie 2014; Ginzler 1976; Ginzler 2005; Gourley 1996; Grootscholten 2006; Hahn 1975; Houssiau 2002; Jayne 2013; Kaballo 2016; Kamanamool 2017; Lewis 1992; Li 2009c; Li 2012; LUNAR 2012; MAINTAIN Nephritis 2010; Mehra 
2018; Mendonca 2017; Mitwalli 2011; Mok 2016; Moroni 2006; MulicBacic 2008; MyLupus 2011; Ong 2005; Rovin 2016; Sabry 2009; Sesso 1994a; SIMPL 2014; Steinberg 1971; Sun 2015; Wallace 1998; Yee 2004). Three studies were inadequate (Barron 1982; BELONG 2013; Liu 2015), and the remainder were unclear.

\section{Selective reporting}

We found that 36 studies were free of selective reporting bias (ACCESS 2014; ALMS 2007; Bao 2008; Belmont 1995; BELONG 2013; Boletis 1999; Boumpas 1992; Cade 1973; Chan 2000; Chen 2011; Clark 1981; Contreras 2004; CYCLOFA-LUNE 2010; Decker 1975; Donadio 1976; Doria 1994; El-Shafey 2010; Furie 2014; Ginzler 1976; Gourley 1996; Grootscholten 2006; Houssiau 2002; Kaballo 2016; Kamanamool 2017; Lewis 1992; Li 2012; LUNAR 2012; MAINTAIN Nephritis 2010; Mitwalli 2011; Mok 2016; Moroni 2006; Ong 2005; Rathi 2016; Sesso 1994a; Steinberg 1971; Sun 2015). Thirty-five studies were considered to be at high risk of reporting bias (Abedi 2007; APRIL-LN 2012; AURA-LV 2016; Balletta 1992; Barron 1982; Boedigheimer 2017; Clark 1984; Deng 2016; Derksen 1988; Donadio 1972; Dyadyk 2001; El-Sehemy 2006; Florez-Suarez 2004; Fries 1973; Fu 1997; Ginzler 2005; Hahn 1975; Hong 2007; Jayne 2013; Li 2009c; Liou 2007; Liu 2015; Loo 2010; Mehra 2018; Mendonca 2017; MulicBacic 2008; MyLupus 2011; Nakamura 2002e; Pal 2017; Rovin 2016; SIMPL 2014; Wallace 1998; Yap 2017; Yee 2004; Zhang 1995a), and the remaining three studies (Lui 1997; Sabry 2009; Sedhain 2016) were unclear.

\section{Other potential sources of bias}

Eighteen studies declared their funding sources to be independent or academic funding bodies and were judged to be free of other potential bias (Boumpas 1992; Clark 1981; Clark 1984; CYCLOFA-LUNE 2010; Donadio 1972; Donadio 1976; Gourley 1996; Grootscholten 2006; Houssiau 2002; Kamanamool 2017; Li 2012; Liou 2007; Liu 2015; MAINTAIN Nephritis 2010; Mendonca 2017; Mok 2016; Sun 2015; Yap 2017). Eight studies that declared independent funding sources were deemed high risk because of either early termination (Ginzler 2005; Lewis 1992; Yee 2004), heavy cross-over between treatment arms (Fries 1973; Ginzler 1976; Ginzler 2005; Steinberg 1971), pooling of studies (Decker 1975) or differences between treatment arms at baseline (Sesso 1994a). A further 20 studies declared sponsorship by a pharmaceutical industry company. Ten of the pharmaceutical sponsored studies included an author who declared pharmaceutical company affiliation; these were judged as carrying high risk of a potential source of bias (ACCESS 2014; ALMS 2007; APRIL-LN 2012; AURA-LV 2016; BELONG 2013; Contreras 2004; Furie 2014; LUNAR 2012; Moroni 2006; MyLupus 2011; Rovin 2016). Thirty-three studies did not disclose study funding sources. Eleven studies exhibited potential biases, which included inadequate reporting of results (Deng 2016; Sedhain 2016), pooling of interventions into study arms (Derksen 1988), low statistical power (Boedigheimer 2017; SIMPL 2014), and differences between treatment arms at baseline (El-Sehemy 2006; Mehra 2018; Mitwalli 2011; Loo 2010; Rathi 2016; Sabry 2009).

\section{Effects of interventions}

See: Summary of findings for the main comparison Mycophenolate mofetil (MMF) versus IV cyclophosphamide (CPA) for induction therapy; Summary of findings 2 Mycophenolate mofetil (MMF) + tacrolimus (TAC) versus IV cyclophosphamide (CPA) for induction therapy; Summary of findings 3 Azathioprine (AZA) versus mycophenolate mofetil (MMF) for maintenance therapy

\section{Induction therapy}

Main comparisons and outcomes for induction therapy, graded by certainty of the evidence, are presented in Summary of findings for the main comparison and Summary of findings 2.

\section{1 \& 2. MMF plus corticosteroids versus cyclophosphamide plus corticosteroid}

\section{Intravenous cyclophosphamide}

\section{Primary outcomes}

Compared to IV cyclophosphamide, treatment with MMF may have led to increased complete disease remission (Analysis 1.2.2 (8 studies, 828 participants): RR $1.17,95 \% \mathrm{Cl} 0.97$ to $1.42 ; \mathrm{I}^{2}=0 \%$ ) (low certainty evidence), although the range of effects includes the possibility of little or no difference. It is uncertain if MMF compared to IV cyclophosphamide reduced death and ESKD because the certainty of the evidence is very low (Analysis 1.1; Analysis 1.3.1).

\section{Secondary outcomes}

The studies reported that MMF may be as effective as IV cyclophosphamide in the stabilisation of kidney function (Analysis 1.4 ( 6 studies, 641 participants): RR $1.05,95 \% \mathrm{Cl} 0.94$ to 1.17 ; $12=0 \%$ ) (low certainty evidence), and may be as effective in inducing partial renal remission (Analysis 1.2.2 (9 studies, 868 participants): RR 1.02, $95 \% \mathrm{Cl} 0.89$ to $\left.1.18 ; \mathrm{I}^{2}=0 \%\right)$. It is uncertain if MMF compared to IV cyclophosphamide increased complete remission in proteinuria (Analysis 1.2.1) and partial renal remission in proteinuria (Analysis 1.2.4) because the certainty of the evidence was very low. In terms of adverse kidney outcomes, it is uncertain if MMF compared to IV cyclophosphamide reduced renal relapse (Analysis 1.3.2) and doubling of SCr (Analysis 1.3.3) because the certainty of the evidence was very low, as few studies reported these outcomes.

Compared to IV cyclophosphamide, treatment with MMF may have made little to no difference to $\mathrm{SCr}$ at the end of the study (Analysis 1.14 (6 studies, 759 participants): MD $2.14 \mu \mathrm{mol} / \mathrm{L}, 95 \%$ $\mathrm{Cl}-3.09$ to $7.37 ; \mathrm{I}^{2}=0 \%$ ) (low certainty evidence), although we cannot be certain of its effect on daily proteinuria (Analysis 1.13) because the certainty of evidence was very low. A considerable level of heterogeneity was observed among studies examining daily proteinuria $(12=63 \%)$. One study (Ong 2005) recruited patients with significantly greater proteinuria among cyclophosphamide treated patients at baseline, an observation which persisted to follow-up. Exclusion of this study reduced the level of heterogeneity slightly $(12=26 \%)$

MMF probably reduced alopecia (Analysis 1.11 (3 studies, 622 participants): RR $0.29,95 \% \mathrm{Cl} 0.19$ to $0.46 ; \mathrm{I}^{2}=0 \%$ ), but probably increased diarrhoea (Analysis 1.12.1 (4 studies, 609 participants): RR 2.42, $95 \% \mathrm{Cl} 1.64$ to 3.58 ) (moderate certainty evidence). Compared to IV cyclophosphamide, MMF may have made little or no difference to major infection (Analysis 1.7 .1 (6 studies, 699 participants): RR $1.02,95 \% \mathrm{Cl} 0.67$ to $1.54 ; \mathrm{I}^{2}=0 \%$ ) (low certainty evidence). We were unable to determine if MMF reduced ovarian failure (Analysis 1.5), herpes zoster virus infection (Analysis 1.7.2), malignancy (Analysis 1.8), leucopenia (Analysis 1.9), vomiting (Analysis 1.12.2), nausea (Analysis 1.12.3), or GI upset (Analysis 1.12.4) compared to IV cyclophosphamide because the certainty of evidence was very low, as few studies reported these outcomes and events. In this review update, the introduction of a new study increased heterogeneity and imprecision of the effect estimates, 
to include both appreciable benefit and harm for the outcomes ovarian failure ( $\mathrm{RR} 0.36,95 \% \mathrm{Cl} 0.06$ to $2.18 ; \mathrm{I}^{2}=39 \%$ ) and leucopenia (RR $0.59,95 \% \mathrm{Cl} 0.33$ to $1.08 ; \mathrm{I}^{2}=59 \%$ ). As a result, the certainty of the evidence for these outcomes was downgraded to very low. For the ovarian failure outcome, the inclusion Rathi 2016 which compared a low dose IV cyclophosphamide ("Euro-lupus") to MMF, introduced three events and the benefit of MMF demonstrated in the 2012 Cochrane review update was no longer apparent.

\section{Oral cyclophosphamide}

Only one study examined the use of MMF plus corticosteroids versus oral cyclophosphamide and corticosteroids in induction therapy of proliferative lupus nephritis (Chan 2000).

\section{Primary outcome}

We were unable to determine if MMF compared to oral cyclophosphamide reduced death because the certainty of the evidence was very low (Analysis 2.1). However, MMF may have made little or no difference to ESKD (Analysis 2.3.1 (62 participants): RR $0.19,95 \% \mathrm{Cl} 0.01$ to 3.76 )

\section{Secondary outcomes}

Chan 2000 reported MMF compared to oral cyclophosphamide may make little or no difference in the inducing complete remission in proteinuria (Analysis 2.2.1 (62 participants): RR 0.98, 95\% Cl 0.74 to 1.30 ) and partial remission in proteinuria (Analysis 2.2.2 (62 participants): RR $1.07,95 \% \mathrm{Cl} 0.44$ to 2.59 ) (low certainty evidence). Similarly, MMF may have made little or no difference to renal relapse (Analysis 2.3.2 (62 participants): RR 1.15, 95\% Cl 0.55 to 2.37 ), doubling of $\mathrm{SCr}$ (Analysis 2.3 .3 (62 participants): RR $0.63,95 \% \mathrm{Cl} 0.11$ to 3.48 ), and daily proteinuria (Analysis 2.10 (42 participants): MD $0.30 \mathrm{~g} / 24 \mathrm{~h}, 95 \% \mathrm{Cl}-0.19$ to 0.79 ) (low certainty evidence).

Chan 2000 reported the use of MMF may have reduced ovarian failure (Analysis 2.4 (53 participants): RR $0.10,95 \% \mathrm{Cl} 0.01$ to 0.73 ), major infection (Analysis 2.5.1 (62 participants): RR $0.21,95 \% \mathrm{Cl}$ 0.05 to 0.89 ), leucopenia (Analysis 2.6 (62 participants): RR 0.06, $95 \% \mathrm{Cl} 0.00$ to 0.92 ), and alopecia (Analysis 2.8 (62 participants): RR $0.05,95 \% \mathrm{Cl} 0.00$ to 0.81 ) compared to oral cyclophosphamide (low certainty evidence). MMF compared to oral cyclophosphamide may have made little or no difference to: herpes zoster virus infection (Analysis 2.5.2 (62 participants): RR $0.38,95 \% \mathrm{Cl} 0.08$ to 1.79 ) and GI upset (Analysis 2.9 (62 participants): RR $2.81,95 \% \mathrm{Cl} 0.31$ to 25.58) (low certainty evidence). We were unable to determine if MMF compared to oral cyclophosphamide reduced bone toxicity (Analysis 2.7) because the certainty of the evidence was very low.

\section{MMF plus tacrolimus and corticosteroid versus IV cyclophosphamide plus corticosteroid}

\section{Primary outcomes}

MMF in combination with tacrolimus may improve the induction of complete renal remission (Analysis 3.2.1 (2 studies, 402 participants): RR $2.38,95 \% \mathrm{Cl} 1.07$ to $5.30 ; \mathrm{I}^{2}=57 \%$ ) (low certainty evidence), while it is uncertain whether combination therapy reduces death (Analysis 3.1) because the certainty of the evidence was very low.

\section{Secondary outcomes}

MMF in combination with tacrolimus may have increased induction of complete remission in proteinuria (Analysis 3.2.3 (2 studies, 402 participants): $\mathrm{RR} 2.38,95 \% \mathrm{Cl} 1.07$ to $5.30 ; \mathrm{I}^{2}=57 \%$ ), and achievement of stable kidney function stable kidney function (Analysis 3.4 (2 studies, 402 participants): RR $1.78,95 \% \mathrm{Cl} 1.40$ to 2.26; $12=0 \%$ ) (low certainty evidence). Combination therapy may have made little or no difference in inducing partial renal remission (Analysis 3.2.2 (2 studies, 402 participants): RR 1.00, 95\% Cl 0.78 to $1.28 ; 1^{2}=0 \%$ ) and partial remission in proteinuria (Analysis 3.2.4 (2 studies, 402 participants): RR $0.98,95 \% \mathrm{Cl} 0.76$ to 1.26 ; 12 $=0 \%$ ) when compared with IV cyclophosphamide (low certainty evidence). It is uncertain if combination therapy compared to IV cyclophosphamide reduced daily proteinuria (Analysis 3.12 (1 study, 40 participants): MD $-1.69 \mathrm{~g} / 24 \mathrm{~h}, 95 \% \mathrm{Cl}-2.8$ to -0.57 ) because the certainty of the evidence was very low.

MMF plus tacrolimus compared to IV cyclophosphamide may have made little or no difference to menstrual irregularities (Analysis 3.6 ( 1 study, 323 participants): RR $0.28,95 \%$ Cl 0.06 to 1.35 ) (low certainty of evidence). It is uncertain the effects that MMF plus tacrolimus may have had on the following outcomes: doubling of $\mathrm{SCr}$ (Analysis 3.3.1), ovarian failure (Analysis 3.5), major infection (Analysis 3.7.1), herpes zoster virus infection (Analysis 3.7.2), leucopenia (Analysis 3.8), bone toxicity (Analysis 3.9), alopecia (Analysis 3.10), diarrhoea (Analysis 3.11.1) and GI upset (Analysis 3.11.2), because the certainty of the evidence was very low, due to risk of bias concerns, indirectness of the population and imprecision of the point estimates because of a small sample size and few event numbers.

\section{MMF plus IV cyclophosphamide versus IV cyclophosphamide alone}

One study compared MMF plus Iv cyclophosphamide versus IV cyclophosphamide alone (Sun 2015).

\section{Primary outcomes}

Compared to IV cyclophosphamide alone, It is uncertain if MMF in combination with cyclophosphamide improves the induction of complete renal remission (Analysis 4.2.1) and reduces death (Analysis 4.1) because the certainty of the evidence was very low.

\section{Secondary outcomes}

MMF in combination with IV cyclophosphamide may reduce major infection compared to treatment with IV cyclophosphamide alone (Analysis 4.4 .1 ( 82 participants): RR $0.37,95 \% \mathrm{Cl} 0.14$ to 0.93 ) and may make little or no difference to daily proteinuria (Analysis 4.6 (77 participants): MD $-0.54 \mathrm{~g} / 24 \mathrm{~h}, 95 \% \mathrm{Cl}-1.12$ to 0.04 ).

Compared to IV cyclophosphamide alone, It is uncertain if the combination of MMF and IV cyclophosphamide reduces menstrual irregularities (Analysis 4.3) or leucopenia (Analysis 4.5).

\section{MMF plus corticosteroid versus tacrolimus plus corticosteroid}

\section{Primary outcomes}

MMF compared to tacrolimus may have made little or no difference in inducing complete renal remission (Analysis 5.2.1 (3 studies, 273 participants): RR $1.02,95 \% \mathrm{Cl} 0.83$ to $1.26 ; \mathrm{I}^{2}=0 \%$ ) (low certainty evidence). It is uncertain if MMF compared to tacrolimus reduced 
death (Analysis 5.1) or ESKD (Analysis 5.3.1) because the certainty of the evidence was very low.

\section{Secondary outcomes}

For secondary efficacy outcomes, MMF compared to tacrolimus may have made little or no difference in achieving partial renal remission (Analysis 5.2.2 (2 studies, 190 participants): RR 0.83, 95\% $\mathrm{Cl} 0.51$ to $1.36 ; \mathrm{I}^{2}=0 \%$ ), complete remission in proteinuria (Analysis 5.2.3 (1 study, 40 participants): RR $1.00,95 \% \mathrm{Cl} 0.50$ to 1.98 ), partial remission in proteinuria (Analysis 5.2.4 (2 studies, 190 participants): RR $0.90,95 \% \mathrm{Cl} 0.79$ to $1.03 ; \mathrm{I}=0 \%$ ), deterioration in kidney function (Analysis 5.3.5 (1 study, 150 participants): RR 0.54, 95\% Cl 0.27 to 1.09 ), and stable kidney function (Analysis 5.4 ( 1 study, 40 participants): RR $1.00,95 \% \mathrm{Cl} 0.50$ to 1.98 ) (low certainty evidence). The use of MMF may have reduced renal relapse (Analysis 5.3.2 (1 study, 150 participants): RR $0.67,95 \% \mathrm{Cl} 0.48$ to 0.98 ) compared to tacrolimus (low certainty evidence). It is uncertain whether MMF improves daily proteinuria (Analysis 5.9), $\mathrm{SCr}$ (Analysis 5.11), and $\mathrm{CrCl}$ (Analysis 5.12), because the certainty of the evidence was very low. MMF compared to tacrolimus may have made little or no difference to renal disease activity (SLEDAI) (Analysis 5.10.1 ( 2 studies, 233 participants): MD $-0.21,95 \% \mathrm{Cl}-2.05$ to $1.63 ; \mathrm{I}^{2}=$ $71 \%$ ) and extrarenal disease activity (SLEDAI) (Analysis 5.10.2 (2 studies, 233 participants): MD $-0.26,95 \% \mathrm{Cl}-0.74,0.22 ; \mathrm{I}^{2}=0 \%$ ) (low evidence certainty).

For outcomes, menstrual irregularities (Analysis 5.5: 1 study, 40 participants), major infection (Analysis 5.6.1: 2 studies, 190 participants), herpes zoster virus infection (Analysis 5.6.2: 1 study, 150 participants), leucopenia (Analysis 5.7: 1 study, 40 participants), and alopecia (Analysis 5.8: 1 study, 150 participants), we were unable to be certain of the effect of the MMF compared to tacrolimus because the certainty of the evidence was very low.

\section{Calcineurin inhibitors plus corticosteroids versus cyclophosphamide plus corticosteroid}

\section{Primary outcomes}

Compared to IV cyclophosphamide, calcineurin inhibitors (tacrolimus and cyclosporin) may have made little or no difference to the induction of complete renal remission (Analysis 6.2.1 (4 studies, 178 participants): RR $1.35,95 \% \mathrm{Cl} 0.94$ to $1.93 ; \mathrm{I}^{2}=0 \%$ ) (low certainty evidence). It is uncertain if calcineurin inhibitors decreased death (Analysis 6.1) or ESKD (Analysis 6.3.1) compared to IV cyclophosphamide because the certainty of the evidence was very low.

\section{Secondary outcomes}

Compared to IV cyclophosphamide, calcineurin inhibitors may have improved the induction of complete remission in proteinuria (Analysis 6.2 .3 (3 studies, 105 participants): RR 1.71, 95\% Cl 1.08 to $2.70 ; 1^{2}=0 \%$ ) and may have made little or no difference to the induction of partial renal remission (Analysis 6.2.2 (4 studies, 178 participants): RR $0.88,95 \% \mathrm{Cl} 0.61$ to 1.26 ) (low certainty evidence). The effect of calcineurin inhibitors compared to IV cyclophosphamide on doubling of $\mathrm{SCr}$ (Analysis 6.3.2), stable kidney function (Analysis 6.4), ovarian failure (Analysis 6.5), menstrual irregularities (Analysis 6.6), major infection (Analysis 6.7.1), herpes zoster virus infection (Analysis 6.7.2), leucopenia (Analysis 6.9), alopecia (Analysis 6.10), and GI symptoms (Analysis 6.11 ) is unclear because the certainty of the evidence was very low. It is unclear the effect that calcineurin inhibitors have on continuous outcomes daily proteinuria (Analysis 6.12), $\mathrm{CrCl}$ (Analysis 6.13), and SCr (Analysis 6.14) at 9, 12 and 18 months compared to IV cyclophosphamide because the certainty of the evidence was very low.

An extended follow-up study of 38 participants from CYCLOFALUNE 2010 examined long-term safety and efficacy outcomes, but it was uncertain if cyclosporin reduced doubling of $\mathrm{SCr}$ (Analysis 6.3.3), premature ovarian failure (Analysis 6.5.3), and malignancy (Analysis 6.8), or improved daily proteinuria (Analysis 6.12) and $\mathrm{SCr}$ (Analysis 6.14) because the certainty of the evidence was very low.

\section{Cyclophosphamide plus corticosteroid versus azathioprine plus corticosteroids}

\section{Primary outcome}

The risk of death at five years (Analysis 7.1.1: 2 studies, 146 participants) and at 10 years (Analysis 7.1.2: 1 study, 59 participants) is uncertain because the certainty of the evidence was very low. Additionally, it is uncertain if azathioprine compared to cyclophosphamide reduced ESKD (Analysis 7.3.1: 2 studies, 144 participants).

\section{Secondary outcomes}

For efficacy outcomes it is uncertain if azathioprine compared to cyclophosphamide improved the rates of complete remission in proteinuria (Analysis 7.2.1: 1 study, 59 participants), partial remission in proteinuria (Analysis 7.2.2: 1 study, 59 participants), and stable kidney function (Analysis 7.4: 1 study, 57 participants) because the certainty of the evidence was very low. Similarly, for adverse renal outcomes it is not certain if azathioprine compared to cyclophosphamide reduced renal relapse (Analysis 7.3.3: 1 study, 87 participants) and deterioration of kidney function (Analysis 7.3.6: 1 study, 30 participants) because the certainty of evidence was very low; although, it may have reduced doubling of $\mathrm{SCr}$ (Analysis 7.3.5 (2 studies, 144 participants): RR $0.48,95 \% \mathrm{Cl} 0.24$ to $0.95 ; 1^{2}=0 \%$ ) (low certainty evidence).

For safety outcomes, azathioprine may have made little or no difference to ovarian failure (Analysis 7.5 (2 studies, 126 participants): RR $2.11,95 \% \mathrm{Cl} 0.59$ to $7.53 ; 12=34 \%$ ) (low certainty evidence). However, it is uncertain if it reduced menstrual irregularities (Analysis 7.6: 1 study, 15 participants), major infection (Analysis 7.7.1: 1 study 57 participants), herpes zoster virus infection (Analysis 7.7.2: 1 study, 57 participants), malignancy (Analysis 7.8: 2 studies, 144 participants), bone toxicity (Analysis 7.9: 1 study, 87 participants), and bladder toxicity (Analysis 7.10: 2 studies, 144 participants) because the certainty of the evidence was very low.

\section{Rituximab $+M M F$ versus placebo $+M M F$ (both arms included corticosteroids)}

\section{Primary outcomes}

It is uncertain if rituximab plus MMF versus placebo plus MMF improved the induction of complete renal remission (Analysis 8.2.1) or reduced death (Analysis 8.1.1), because the certainty of the evidence was very low. 


\section{Secondary outcomes}

Rituximab plus MMF compared to placebo plus MMF may have made little or no difference in the stabilisation of kidney function (Analysis 8.3 ( 1 study, 144 participants): RR $1.24,95 \% \mathrm{Cl} 0.90$ to 1.7 ) (low certainty evidence). It is uncertain if it improved the induction of complete remission in proteinuria (Analysis 8.2.3), partial renal remission (Analysis 8.2.2), or reduced major infection (Analysis 8.4.1), herpes zoster virus infection (Analysis 8.4.2), and leucopenia (Analysis 8.5 ) because the certainty of the evidence was very low.

\section{Rituximab plus cyclophosphamide versus rituximab alone}

One study compared rituximab plus cyclophosphamide versus rituximab alone (Li 2009c).

\section{Primary outcomes}

It is uncertain if rituximab plus cyclophosphamide compared to rituximab alone improved the induction of complete renal remission (Analysis 9.1.1) because the certainty of the evidence was very low.

\section{Secondary outcomes}

Similarly, it is uncertain if rituximab plus cyclophosphamide improved the induction of partial renal remission (Analysis 9.1.2), reduced major infection (Analysis 9.2.1) and herpes zoster virus infection (Analysis 9.2.2), or improved daily proteinuria (Analysis 9.3), $\mathrm{CrCl}$ (Analysis 9.4), and $\mathrm{SCr}$ (Analysis 9.5) compared to rituximab alone because the certainty of the evidence was very low.

\section{$10,11,12, \& 13$. Other biologics versus placebo (both arms included standard of care therapy (MMF or CPA))}

\section{Primary outcomes}

It is uncertain if biologics: abatacept, atacicpet, laquinimod, ocrelizumab and sirukumab improved the induction of complete renal remission (Analysis 10.2.(1,2,3); Analysis 11.2.(1,2,3); Analysis 12.2.(1,2,3)), reduced death (Analysis 10.1.(1,2,3); Analysis 11.1. (1.2.3); Analysis 12.1.(1,2,3); Analysis 13.1), and reduced ESKD (Analysis 10.3.(1,2,3)) compared to standard of care therapy because the certainty of the evidence was very low.

\section{Secondary outcomes}

It was uncertain if the abatacept or ocrelizumab improved the induction of partial renal remission (Analysis 10.2.(4.5.6); Analysis 12.2. $(4,5,6)$ ) because the certainty of the evidence was very low. Likewise, it was uncertain if the biologics compared to placebo plus standard of care therapy reduced renal relapse (Analysis 10.3.4); major infection (Analysis 10.4.(1,2,3); Analysis 12.3.(1,2,3); Analysis 13.2), herpes zoster virus infection (Analysis 10.5), malignancy (Analysis 13.3), and diarrhoea (Analysis 13.4) because the certainty of the evidence was very low.

It is uncertain if abatacept with standard of care therapy compared to placebo with standard of care therapy improved the physical and mental component of the health-related quality of life (SF-36) (Analysis 10.6) and disease activity (BILAG) (Analysis 10.7) because the certainty of the evidence was very low.

\section{Intravenous versus oral cyclophosphamide}

\section{Primary outcomes}

We were unable to determine if IV cyclophosphamide compared to oral cyclophosphamide reduced death (Analysis 14.1) because the certainty of the evidence was very low. IV cyclophosphamide compared to oral cyclophosphamide may have made little or no difference to ESKD (Analysis 14.2.1 (2 studies, 67 participants): RR $0.23,95 \% \mathrm{Cl} 0.04$ to $1.28 ; \mathrm{I}^{2}=0 \%$ ) (low certainty evidence).

\section{Secondary outcomes}

For adverse renal outcomes, IV cyclophosphamide may have made little or no difference to doubling of $\mathrm{SCr}$ (Analysis 14.2.2 (2 studies, 67 participants): RR $0.67,95 \% \mathrm{Cl} 0.23$ to $1.98 ; \mid 2=0 \%$ ) (low certainty evidence). It is uncertain if IV compared to oral cyclophosphamide reduced the deterioration of kidney function (Analysis 14.2.3) and improved the achievement of stable kidney function (Analysis 14.3) because the certainty of the evidence was very low. For safety outcomes, IV compared to oral cyclophosphamide may have made little or no difference to ovarian failure (Analysis 14.4 (2 studies, 56 participants): RR $0.70,95 \% \mathrm{Cl} 0.37$ to $1.30 ; 1^{2}=0 \%$ ) and major infection (Analysis 14.5.1 (2 studies, 67 participants): $\mathrm{RR} 1.16,95 \% \mathrm{Cl} 0.47$ to $2.90 ; \mathrm{I}^{2}=0 \%$ ) (low certainty evidence), and it is uncertain if IV cyclophosphamide reduced herpes zoster virus infection (Analysis 14.5.2), malignancy (Analysis 14.6), bladder toxicity (Analysis 14.7), and GI upset (Analysis 14.8.1) because the certainty of the evidence was very low.

\section{High versus low dose cyclophosphamide}

\section{Primary outcomes}

Compared to high dose cyclophosphamide, the use of low dose cyclophosphamide may have been as effective in inducing complete renal remission (Analysis 15.2.1 (3 studies, 267 participants): RR $1.09,95 \% \mathrm{Cl} 0.63$ to $1.86 ; \mathrm{I}^{2}=67 \%$ ) and may have made little or no difference to ESKD (Analysis 15.3.1 (2 studies, 135 participants): RR $0.49,95 \% \mathrm{Cl} 0.05$ to 5.20 ) (low certainty evidence). However, it is uncertain if compared to high dose cyclophosphamide, low dose cyclophosphamide reduced ESKD at 5 years (Analysis 15.3.2) and 10 years (Analysis 15.3.3), and reduced death at 6 months (Analysis 15.1.1), 12 months (Analysis 15.1.2), 5 years (Analysis 15.1.3), and 10 years (Analysis 15.1.4) because the certainty of the evidence was very low.

\section{Secondary outcomes}

Low dose cyclophosphamide may have made little or no difference to efficacy outcomes of partial renal remission (Analysis 15.2.2 (3 studies, 267 participants): RR $0.88,95 \% \mathrm{Cl} 0.69$ to $1.14 ; \mathrm{I}^{2}=0 \%$ ) and stabilisation of kidney function at 3 years (Analysis 15.4.1 (1 study, 89 participants): RR $0.72,95 \% \mathrm{Cl} 0.50$ to 1.03 ), and at 5 years (Analysis 15.4.2 ( 1 study, 85 participants): RR 0.96, 95\% 0.77 to 1.20 ) compared to high dose cyclophosphamide (low certainty evidence). It is uncertain if low dose cyclophosphamide improved daily proteinuria (Analysis 15.12: 3 studies, 242 participants), $\mathrm{CrCl}$ (Analysis 15.13: 1 study, 177 participants), and $\mathrm{SCr}$ (Analysis 15.14 (3 studies, 247 participants) compared to high dose cyclophosphamide because the certainty of the evidence was very low.

Compared to high dose cyclophosphamide, low dose cyclophosphamide may have made little or no difference to renal 
relapse (Analysis 15.3.4 (3 studies, 211 participants): RR 2.75, $95 \% \mathrm{Cl} 0.47$ to $15.98 ; 1^{2}=66 \%$ ) (low certainty evidence). The risk of ovarian failure may be two times higher in patients on high dose cyclophosphamide compared to those on low dose cyclophosphamide (Analysis 15.5 (4 studies, 299 participants): RR $1.73,95 \% \mathrm{Cl} 0.70$ to $4.31 ; \mathrm{I}^{2}=19 \%$ ) (low certainty evidence). Compared to high dose cyclophosphamide, low dose cyclophosphamide may make little or no difference to major infection (Analysis 15.6.1 (4 studies, 327 participants): RR 1.44, 95\% $\mathrm{Cl} 0.83$ to $2.49 ; \mathrm{I}^{2}=25 \%$ ), herpes zoster virus infection (Analysis 15.6.2 (3 studies, 281 participants): RR $1.58,95 \% \mathrm{Cl} 0.41$ to 6.05 ), malignancy (Analysis 15.7 (2 studies, 206 participants): RR 1.44, $95 \% \mathrm{Cl} 0.09$ to $23.31 ;\left.\right|^{2}=41 \%$ ), and leucopenia (Analysis 15.8 (3 studies, 281 participants): RR $0.82,95 \% \mathrm{Cl} 0.13$ to 5.15 ; $12=51 \%$ ) (low certainty evidence). It is uncertain if low dose cyclophosphamide use reduced bone toxicity (Analysis 15.9: 2 studies, 164 participants) compared to high dose cyclophosphamide because the certainty of the evidence was very low.

\section{Standard versus reduced dose oral corticosteroid}

One study compared standard versus reduced dose oral corticosteroid (MyLupus 2011).

\section{Primary outcomes}

It was uncertain if reduced dose oral corticosteroid compared to standard dose oral corticosteroid improved the induction of complete renal remission (Analysis 16.2.1: 81 participants) and reduced death (Analysis 16.1: 81 participants) because the certainty of the evidence was very low.

\section{Secondary outcomes}

It is uncertain of the effect of reduced dose oral corticosteroid compared to standard dose oral corticosteroid improved the induction of partial renal remission (Analysis 16.2.2: 81 participants), $\mathrm{CrCl}$ (Analysis 16.6: 74 participants), and $\mathrm{SCr}$ (Analysis 16.7: 81 participants), or reduced renal relapse (Analysis 16.3: 50 participants) because the certainty of the evidence was very low. For safety outcomes, compared to standard dose corticosteroids it was uncertain if reduced dose oral corticosteroids reduced major infection (Analysis 16.4.1: 81 participants), herpes zoster virus infection (Analysis 16.4.2: 81 participants), diarrhoea (Analysis 16.5.1: 81 participants), vomiting (Analysis 16.5.2: 81 participants), and nausea (Analysis 16.5.3: 81 participants) because the certainty of the evidence was very low

\section{Intravenous versus oral corticosteroids}

One study compared IV versus oral corticosteroids (Barron 1982).

It was uncertain if the use of pulsed methylprednisolone compared to oral corticosteroids alone reduced death (Analysis 17.1) or renal relapse (Analysis 17.2) because the certainty of the evidence was very low. The certainty of the evidence was downgraded because of the potential risk of bias, small sample size and small event numbers.

\section{Other comparisons (18 to 25)}

Older comparisons - immunosuppressive agent plus corticosteroids versus corticosteroids alone (18 to 22), plasma exchange plus immunosuppression versus immunosuppression alone (23), plasma exchange (no immunosuppression) versus immunosuppression (24) and long versus short-duration cyclophosphamide (25) - have been reported in the original Cochrane review (Flanc 2004a) and can also be found in the Data and analyses section of this review.

\section{Maintenance therapy}

Main outcomes for maintenance therapy, graded by certainty of the evidence, are presented in Summary of findings 3.

\section{Azathioprine plus corticosteroid versus mycophenolate mofetil plus corticosteroid}

\section{Primary outcomes}

Compared to MMF, azathioprine probably increased renal relapse (Analysis 26.2 (4 studies, 452 participants): RR 1.75, 95\% Cl 1.20 to $2.55 ; 1^{2}=0 \%$ ) (moderate certainty evidence). However, it is uncertain if azathioprine compared to MMF reduced death Analysis 26.1) or ESKD because the certainty of the evidence was very low (Analysis 26.3).

\section{Secondary outcomes}

It is uncertain if azathioprine compared to MMF improved daily proteinuria (Analysis 26.12) because the certainty of the evidence was very low; while it may have increased doubling of $\mathrm{SCr}$ (Analysis 26.4 (4 studies, 452 participants): RR 2.19, 95\% Cl 1.03, 4.66; 12 = 0\%) (low certainty evidence).

For safety outcomes, the use of azathioprine compared to MMF may have increased leucopenia (Analysis 26.8 (3 studies, 412 participants): RR $5.61,95 \% \mathrm{Cl} 1.68$ to $18.72 ; \mathrm{I}^{2}=0 \%$ ) and may have made little or no difference to major infection (Analysis 26.6 (3 studies, 412 participants): RR $1.08,95 \% \mathrm{Cl} 0.60$ to 1.96 ; $\mathrm{I}^{2}=0 \%$ ), alopecia (Analysis 26.10 (3 studies, 412 participants): RR 0.95, 95\% $\mathrm{Cl} 0.46$ to $1.95 ; \mathrm{I}^{2}=0 \%$ ), nausea (Analysis 26.11 .2 (2 studies, 307 participants): RR $1.08,95 \% \mathrm{Cl} 0.65$ to $1.80 ; \mathrm{I}^{2}=0 \%$ ), and diarrhoea (Analysis 26.11.3 (2 studies, 307 participants): RR $0.74,95 \% \mathrm{Cl}$ 0.31 to $1.73 ; 1^{2}=33 \%$ ) (low certainty evidence). It is unclear if azathioprine compared to MMF reduced ovarian failure (Analysis 26.5), herpes zoster virus infection (Analysis 26.6.2), malignancy (Analysis 26.7), bone toxicity (Analysis 26.9), and vomiting (Analysis 26.11.4) because the certainty of the evidence was very low.

\section{7, 28 \& 29. Azathioprine plus corticosteroid versus} cyclophosphamide, cyclosporin or tacrolimus plus corticosteroid

\section{Primary outcomes}

It is uncertain if azathioprine compared to cyclosporin, cyclophosphamide and tacrolimus made little or no difference to death (Analysis 27.1; Analysis 28.1; Analysis 29.1), ESKD (Analysis 27.2.1; Analysis 28.2.1), and renal relapse (Analysis 27.2.2; Analysis 28.2.2; Analysis 29.1.1) because the certainty of the evidence was very low.

\section{Secondary outcomes}

It is uncertain if azathioprine compared to cyclosporin, cyclophosphamide and tacrolimus made little or no difference to daily proteinuria (Analysis 27.6), $\mathrm{CrCl}$ (Analysis 28.4), disease activity (SLEDAI) (Analysis 27.7), doubling of SCr (Analysis 28.2.3), major infection (Analysis 27.3.1; Analysis 29.2.1), leucopenia (Analysis 27.4), bladder toxicity (Analysis 28.3), and GI disturbance 
(Analysis 27.5.1; Analysis 29.3.1) because the certainty of the evidence was very low.

\section{Prednisone withdrawal versus prednisone continuation}

\section{Primary outcomes}

It is uncertain if prednisone withdrawal compared to prednisone continuation made little or no difference to renal and non-renal relapse (Analysis 30.1) because the certainty of the evidence was very low.

\section{Secondary outcomes}

It is uncertain if prednisone withdrawal compared to prednisone continuation made little or no difference to major infection (Analysis 30.2) because the certainty of the evidence was very low.

\section{Intravenous immunoglobulin versus intravenous cyclophosphamide}

\section{Secondary outcomes}

It is uncertain if IV immunoglobulin compared to IV cyclophosphamide improved $\mathrm{SCr}, \mathrm{CrCl}$ or proteinuria (Analysis 31.1; Analysis 31.2; Analysis 31.3) because the certainty of the evidence was very low.

Three studies reported health-related quality of life, one study reported fatigue and 21 studies reported disease activity. Given the heterogeneity of reporting of these outcomes, the results have been presented in tables (Table 1; Table 2; Table 3).

\section{DISCUSSION}

The management of lupus nephritis has become complex and difficult to navigate because of the recent proliferation of new interventions and studies, which have been compared in numerous combination regimens. In the 1970s, it was demonstrated that compared with corticosteroids alone, the combined use of cyclophosphamide and corticosteroids induced remission, reduced ESKD and death, resulting in the use of this regimen as firstline therapy for over 30 years.

Our earlier systematic review (Flanc 2004a) of immunosuppressive treatment of proliferative lupus nephritis found that adding cyclophosphamide or azathioprine to steroids improved or preserved kidney function when compared to steroids alone, and that plasma exchange conferred no additional benefit. In the subsequent update of the review (Henderson 2012), we found that MMF compared to cyclophosphamide had similar effects on death and inducing complete renal remission at six months, with a better safety profile as indicated by a reduced risk of ovarian failure, alopecia and leucopenia but with an increased risk of diarrhoea. Additionally, for maintenance therapy, MMF was more effective than azathioprine at preventing renal relapse with less leucopenia and no difference in other safety outcomes. Data regarding newer agents such as tacrolimus, cyclosporin and rituximab were insufficient to permit any meaningful conclusions at the time of publication. Numerous recent studies have examined the combination of MMF and tacrolimus and the use of biologics in induction therapy.

\section{Summary of main results}

As shown by eight studies involving over 800 participants with proliferative lupus nephritis in the analysis of this updated review, MMF dosed at $2 \mathrm{~g}$ to $3 \mathrm{~g}$ daily may have increased the induction of complete disease remission and stable kidney function at six months compared to cyclophosphamide, although the certainty of the evidence was low, because of study limitations and imprecision concerns, with the risk estimate including the possibility of no effect. Treatment with MMF compared to cyclophosphamide reduced the risk of alopecia but increased the risk of diarrhoea. These data justify the current use of MMF as the first-line agent in proliferative lupus nephritis. MMF provided no benefit for other adverse events compared with cyclophosphamide, although its effect on ovarian failure is unclear. As the inclusion of one new study (Rathi 2016) has introduced greater imprecision in the ovarian failure treatment estimate, a total of three events has altered the summary estimate to suggest no benefit. This finding cannot be definitively stated as the treatment estimate is susceptible to change with addition of a few events; as a result, the certainty of the evidence has been downgraded to very low.

Compared to IV cyclophosphamide, the use of calcineurin inhibitors (tacrolimus and cyclosporin) may be as effective in inducing complete renal remission, while the combination of MMF and tacrolimus may improve the induction of complete renal remission, and achieving stable kidney function at six months. The generalisability of these findings may be limited as the two studies of combination therapy have largely included patients of Asian ethnicity, and have had serious concerns regarding selection bias and reporting bias. The safety of these therapies is unclear as the certainty of evidence is generally low to very low due to substantial imprecision in treatment effects and a small sample size and event numbers, limiting the applicability of the findings.

For maintenance therapy, MMF is probably more effective than azathioprine at preventing renal relapse with less leucopenia but there may be no difference in other outcomes (major infection, alopecia, and GI adverse events). The effectiveness and safety of many other interventions, including biologics (for example, rituximab and abatacept) and cyclosporin, is unclear because of very low certainty of the evidence, as they have only been trialled in a small number of studies with low numbers of events and inconsistent outcome reporting. The clinical role of these therapies therefore remains unclear and warrants caution.

\section{Overall completeness and applicability of evidence}

Our review was based on a highly sensitive electronic search of the Cochrane Kidney and Transplant's Specialised Register, which includes journal alerts and handsearching of all relevant conference proceedings, the reporting of existing studies evaluating induction and maintenance therapy of lupus nephritis means there are considerable gaps in the evidence. While some studies had moderate periods of follow-up over one to two years, others were much shorter and inadequately powered to detect events in the clinically important outcomes. The average time to remission with cyclophosphamide is about 10 months (Ioannidis 2000); however, the follow-up in the majority of induction therapy studies was six months. Furthermore, the risk of adverse events such as ovarian failure and the development of ESKD increases after six months, so there is considerable uncertainty in treatment effects across interventions, which results in an inability of patients and 
clinicians to evaluate the benefits and harms of therapy. Healthrelated quality of life and fatigue are included in a core set of outcomes for SLE developed by OMERACT (Strand 2000). Yet, very few lupus nephritis studies have reported these patient-reported outcomes. No standardised set of outcomes have been developed specifically for lupus nephritis studies. The development of a core set of outcomes by all stakeholders, including patients, with defined measures and definitions of renal remission (Liang 2006; van Vollenhoven 2017) would ease comparisons across studies and assist with building evidence for the induction and maintenance therapy of lupus nephritis. There were limited studies examining biologics, with sparse outcome data and confidence intervals were frequently very wide, indicating substantial uncertainty. Studies may not reflect usual clinical practice due to selection bias, with rituximab increasingly being used and showing benefit in patients who have not achieved remission with standard therapies (Weidenbusch 2013).

The disease spectrum and the proportion of patients within each class of lupus nephritis differed among studies. Furthermore, patient demographics varied among studies where environmental, socioeconomic, as well as clinical and genetic factors have been thought to play an important role explaining differences in the outcome of lupus nephritis by ethnicity. Comparing MMF with cyclophosphamide in induction therapy, six studies included primarily Asian patients (Bao 2008; Chan 2000; Li 2012; Liu 2015; Ong 2005; Rathi 2016; Sedhain 2016) and two of the largest studies comparing MMF with cyclophosphamide included higher proportions of African-American and Hispanic patients (ALMS 2007; Ginzler 2005). Non-Caucasian populations have a higher risk of relapse, death and CKD compared with Caucasian populations (Adler 2006; Contreras 2006) and often fail to respond to cyclophosphamide (Adler 2006; Contreras 2006; Dooley 1997). Ginzler 2005 included the largest percentage (56\%) of patients of African-American origin. This was the only study that showed a clear benefit in favour of MMF over IV cyclophosphamide for induction of remission. The Aspreva Lupus Management Study (ALMS) data which included 12\% African-American and $35 \%$ Hispanic patients, suggested interactions between group interventions and race that were not explained by differences in disease characteristics (ALMS 2007). ALMS 2007 was the only study to provide stratified results according to ethnicity and class of lupus in the update, and no studies provided stratified results according to severity of kidney impairment reducing the power to examine potential differences between these groups. Despite the lack of stratification of results, variation among studies could be considered a strength as despite clinical differences in population and histological classification, uniformity of effect demonstrated in the meta-analysis suggest that the results were valid across race and class of lupus nephritis.

\section{Quality of the evidence}

We graded our confidence in the certainty of the evidence using the Grading of Recommendations Assessment, Development and Evaluation (GRADE) approach (GRADE 2011), which considers study limitations, imprecision, indirectness, inconsistency and publication bias. Overall, most studies had high or unclear risks of bias for most domains of study reporting assessed (Figure 2). The internal validity of the design, conduct and analysis of the included RCTs was difficult to assess in some studies because of the omission of important methodological details. No study adequately reported all domains of the risk of bias assessment so that elements of internal bias may be present in the meta-analysis (Begg 1996; Moher 1999).

Estimated effects on efficacy and safety outcomes were frequently imprecise with confidence intervals that exhibited both considerable benefit and harm. The generalisability (directness) of the evidence was limited by the number of available studies on many treatment comparisons. Additionally, considerable clinical heterogeneity in interventions, definitions of remission and renal relapse and outcome reporting among studies hampered interpretation and presentation of important outcomes in this review. For example, comparing MMF with cyclophosphamide, there was variability among studies in therapeutic dosing, route of administration, definition of outcomes and co-interventions. The small number of studies for some treatment comparisons limited the power of statistical testing and important inconsistencies between studies could not excluded. Publication bias (the effects of small studies on treatment effects) could not be assessed, new reports from hand-searching conference proceedings in addition to those already searched by Cochrane Kidney and Transplant were included in the meta-analysis to minimise publication bias. Overall, based on important limitations, we have generally moderate to very low confidence in the certainty of the evidence for the benefits and harms of induction and maintenance therapy in people with proliferative lupus nephritis.

\section{Potential biases in the review process}

Although this systematic review is reported using Cochrane methods and includes a comprehensive evidence summary for this topic, the review has limitations that might be considered. Firstly, the analysis was limited by the reporting of outcomes in the primary studies. For example, the definitions of renal remission were variable across studies. While for the analysis of these outcomes, there was evidence of low heterogeneity, indicating the meta-analysis was appropriate, the small number of studies for treatment comparisons in this review may limit the statistical power to detect heterogeneity, and as a result it may still be present. Second, incomplete reporting of outcomes also limits the power of this review to detect differences among interventions. For example, although eight studies with 828 participants compared MMF with IV cyclophosphamide in induction therapy, only three reported on ovarian failure and one on doubling of SCr. Finally, different treatment effects for patients of different ethnic backgrounds has been hypothesised and observed (Isenberg 2010), although it could not be explored in this systematic review because of insufficient data for ethnicity in the original study reports to perform meta-regression analyses.

\section{Agreements and disagreements with other studies or reviews}

In contrast to previous meta-analyses (Mak 2009; Moore 2006), we re-organised interventions according to treatments for induction of disease remission or maintenance therapy, which better reflects clinical practice. Broad inclusion criteria also helped explore the totality of evidence available, rather than limiting meta-analysis by specific immunosuppression regimens as have previously published systematic reviews (Cao 2015; Deng 2012; Feng 2013; Hannah 2016; Kamanamool 2010; Lee 2010; Lee 2011; Liu 2012; Mak 2009; Maneiro 2014; Moore 2006; Radhakrishnan 2010; Touma 2011; Walsh 2007; Zhang 2016; Zhou 2011; Zhu 2007). A review 
of systematic reviews of meta-analyses of RCTs and observational studies (Chen 2017) also showed that induction therapy with MMF compared to IV cyclophosphamide had a higher response rate and decreased alopecia. However, in contrast, the review found that MMF decreased ovarian failure and leucopenia, and calcineurin inhibitors (tacrolimus) increased complete remission and decreased ovarian failure and GI adverse events. These differences may be because the other overview included systematic reviews of observational studies and did not assess the certainty of the available evidence, and we included more recent RCTs in our review. For example, our review included Rathi 2016, which introduced further uncertainty regarding the outcomes of ovarian failure and leucopenia for MMF versus cyclophosphamide induction therapy.

Similar findings between this review and recent network metaanalysis strengthen the conclusion that there is inconclusive evidence for therapy based on treatment effects on important safety outcomes and that MMF is the most effective therapy in maintaining disease remission (Palmer 2017; Tian 2015). While, some network meta-analyses found similar findings in that there may be no difference between MMF, calcineurin inhibitors or their combination in inducing renal remission compared to cyclophosphamide (Tian 2014; Singh 2016), other network metaanalyses have found that these therapies may be more effective than cyclophosphamide in inducing renal remission (Lee 2015; Palmer 2017). As there are vast options available for treatment, of which some have not been directly compared, a network metaanalysis may allow for greater certainty about all treatment options through the use of indirect evidence. Although, given the small number of studies, an imbalance of evidence in the network, particularly tacrolimus alone or its combination with MMF may affect the power and reliability for the overall analysis, and also the network meta-analysis may be underpowered to check for statistical heterogeneity, leading to incoherence between direct and indirect results. Considering the apparent lack of evidence and possible incoherency, the results from the network meta-analysis should be interpreted with a degree of caution (Mills 2013).

\section{AUTHORS' CONCLUSIONS}

\section{Implications for practice}

In this review, we found that MMF may lead to increased complete disease remission compared to IV cyclophosphamide, although the certainty of the evidence was low and included the possibility of no effect, however there was some evidence of better tolerability. The equivalent remission rates combined with a more favourable sideeffect profile compared to cyclophosphamide support the current practice of MMF along with corticosteroids as first-line induction therapy for proliferative lupus nephritis. Numerous published guidelines concur with our findings, recommending MMF or IV cyclophosphamide with corticosteroids for induction therapy in patients with ISN class III/IV lupus nephritis (Tunnicliffe 2015). The combination of MMF and tacrolimus may be more effective in inducing renal remission and achieving stable kidney function but this needs to be interpreted with a degree of caution, as it has largely been informed by one large study with participants of mainly Chinese ethnicity.

Although there are few study data on maintenance therapy, meta-analyses from two recent large RCTs (ALMS 2007; MAINTAIN Nephritis 2010) showed that MMF is superior to azathioprine in preventing renal relapse with no difference between the therapies in death, doubling of $\mathrm{SCr}$, major infection, leucopenia and GI disturbance. The data for newer biologic agents, including rituximab was very limited, so no conclusions about the relative benefit and harms of these agents could be made. Until further research becomes available, the lack of data on other agents and heterogeneity of dosing schedules make it difficult to offer recommendations about other agents and to be more specific about optimal dosing schedules.

\section{Implications for research}

There are four main implications for future research. In no particular order, firstly for the design of future studies, given the short duration of studies and imprecision for treatment estimates for death and ESKD, registry-based RCTs may clarify the risks and eventual harms of specific treatment regimens, as outcomes, are captured automatically during routine follow-up with registry databases. Efficient data linkage between hospital records, national and state-wide mortality databases and cancer registries may also help clarify the efficacy and safety of specific therapies. Secondly, standardisation of the reporting of safety and efficacy outcomes in studies evaluating therapies for lupus nephritis might facilitate better comparison and improve our understanding of the benefits and harms of treatment. Thirdly, future studies should further examine the long-term safety and efficacy of MMF as maintenance therapy to provide guidance around tapering or when to stop treatment; further studies should also examine the safety and efficacy of MMF plus tacrolimus as induction therapy in the management of lupus nephritis across all ethnic groups. Further studies are needed in patient populations that carry greater disease burden, such as children, AfricanAmericans, Hispanics and Asians, different histopathological classes of lupus nephritis and patients presenting with advanced renal impairment.

\section{ACKNOWLEDGEMENTS}

The 2018 version of this systematic review was funded by the Cochrane Review Support Programme 2017.

We are grateful to Cochrane Kidney and Transplant for their assistance, in particular, Gail Higgins who carried out the search, and Fiona Russell and Narelle Willis for editorial support and copy editing, and Steve Chadban, Peter Kerr and Robert Atkins who were involved in the original design of the review.

We are indebted to Drs Belmont, Doria, Donadio, Fries, Gourley, Houssiau, Rathi, Solomons and Wofsy who supplied data relating to their studies upon request.

We are grateful to Yu-Tian Xiao, School of Clinical Medicine, Fudan University for translation of articles from Chinese to English. 


\section{REFERE N C E S}

\section{References to studies included in this review}

\section{Abedi 2007 \{published data only\}}

Abedi A, Nakhjavani MR, Gafari N, Argani H. Comparison between corticosteroid and mycophenolate mofetil and corticosteroid and cyclophosphamide in the treatment of lupus nephritis [abstract no: FO031]. Nephrology Dialysis Transplantation 2007;22(Suppl 6):vi14.

\section{ACCESS 2014 \{published data only\}}

* ACCESS Trial Group. Treatment of lupus nephritis with abatacept: the Abatacept and Cyclophosphamide Combination Efficacy and Safety Study.[Erratum appears in Arthritis Rheumatol. 2015 Feb;67(2):487 Note: Aranow, Cynthia [added]]. Arthritis \& Rheumatology 2014;66(11):3096-104. [MEDLINE: 25403681]

Shidham GB, Birmingham DJ, Rovin B, Hebert LA. 24 hour protein: creatinine ration (24 PCR), not spot PCR (Spot $P(R)$, should be used to monitor the treatment of severe lupus nephritis (LN): the experience of ACCESS [abstract no: FR-P0604]. Journal of the American Society of Nephrology 2016;27(Abstract Suppl):501A.

Shidham GB, Birmingham DJ, Rovin B, Hebert LA. Ability of spot urine protein/creatinine ratio (spot PCR) to correctly identify the proteinuria endpoints of complete remission (CR), partial remission (PR), and treatment failure (TF) as determined by 24 hour urine PCR (24 PCR): experience of abatacept and cyclophosphamide combination efficacy and safety study (ACCESS) [abstract no: TH-PO764]. Journal of the American Society of Nephrology 2015;26(Abstract Suppl):265A.

Wofsy D, Askanase A, Cagnoli PC, Chatham WW, Contreras G, Dall'Era M, et al. Treatment of lupus nephritis with abatacept plus low-dose pulse cyclophosphamide followed by azathioprine (the Euro-Lupus regimen): twenty-four week data from a double-blind controlled trial [abstract no: 884]. Arthritis \& Rheumatology 2013;66(10 Suppl):S379-80. [EMBASE: 71318364]

Wofsy D, Hillson JL, Diamond B. Abatacept for lupus nephritis: alternative definitions of complete response support conflicting conclusions. Arthritis \& Rheumatology 2012;64(11):3660-5. [MEDLINE: 22806274]

Wofsy D, Hillson JL, Diamond B. Comparison of alternative primary outcome measures for use in lupus nephritis clinical trials. Arthritis \& Rheumatology 2013;65(6):1586-91. [MEDLINE: 23529285]

Wofsy D, Shropshire SM, Hillson JL, Diamond B. Abatacept for lupus nephritis: alternative outcome measures support opposing interpretations of data from a multicenter, randomized, double-blind, placebo-controlled phase II/III study [abstract]. Arthritis \& Rheumatology 2011;63(10 Suppl 1):S964-5. [EMBASE: 70784721]

\section{ALMS 2007 \{published and unpublished data\}}

* Appel GB, Contreras G, Dooley MA, Ginzler EM, Isenberg D, Jayne $D$, et al. Mycophenolate mofetil versus cyclophosphamide for induction treatment of lupus nephritis. Journal of the American Society of Nephrology 2009;20(5):1103-12. [MEDLINE: 19369404]

Appel GB, Dooley MA, Ginzler EM, Isenberg D, Jayne D, Solomons N, et al. Mycophenolate mofetil compared with intravenous cyclophosphamide as induction therapy for lupus nephritis: Aspreva Lupus Management Study (ALMS) results [abstract no: SA-FC057]. Journal of the American Society of Nephrology 2007;18(Abstracts):47A.

Clancy RM, Ginzler EM, Kim M. Association of adiponectin and soluble endothelial protein $C$ receptor (SEPCR) with longitudinal assessments in the induction phase of a randomized multicenter trial comparing mycophenolate mofetil and intravenous cyclophosphamide [abstract no: 263]. ACR/ ARHP Annual Scientific Meeting; 2009 Oct 17-21; Philadelphia (PA). 2009.

Dall'Era M, Stone D, Levesque V, Cisternas M, Wofsy D. Identification of biomarkers that predict response to treatment of lupus nephritis with mycophenolate mofetil or pulse cyclophosphamide. Arthritis Care \& Research 2011;63(3):351-7. [MEDLINE: 21080348]

Dooley MA, Appel GB, Ginzler EM, Isenberg D, Jayne D, Wofsy D, et al. Aspreva lupus management study (ALMS): maintenance results analysis by racial subgroup [abstract no: OP0168]. Annals of the Rheumatic Diseases 2011;70(Suppl 3):125.

Dooley MA, Jayne D, Ginzler EM, Isenberg D, Olsen NJ, Wofsy D, et al. Mycophenolate versus azathioprine as maintenance therapy for lupus nephritis. New England Journal of Medicine 2011;365(20):1886-95. [MEDLINE: 22087680]

Ginzler E. Contribution of vascular well being to the therapeutic response in the induction phase of a randomized multicenter trial comparing MMF to IVC [abstract no: 2063]. ACR/ARHP Annual Scientific Meeting; 2008 Oct 24-29; San Francisco (CA). 2008.

Ginzler EM, Appel GB, Dooley MA, Isenberg D, Jayne D, Solomons N, et al. Mycophenolate mofetil and intravenous cyclophosphamide in the Aspreva Lupus Management Study (ALMS): efficacy by racial group [abstract no: L13]. ACR/ARHP Annual Scientific Meeting; 2007 Nov 6-11; Boston (MA). 2007.

Ginzler EM, Wofsy D, Isenberg D, Gordon C, Lisk L, Dooley MA, et al. Nonrenal disease activity following mycophenolate mofetil or intravenous cyclophosphamide as induction treatment for lupus nephritis: findings in a multicenter, prospective, randomized, open-label, parallel-group clinical trial. Arthritis \& Rheumatism 2010;62(1):211-21. [MEDLINE: 20039429]

Isenberg D. Lessons from the Aspreva Lupus Management Study [abstract no: SP0073]. Annals of the Rheumatic Diseases 2009;68(Suppl 3):23.

Isenberg D, Appel GB, Contreras G, Dooley MA, Ginzler EM, Jayne $D$, et al. Influence of race/ethnicity on response to lupus nephritis treatment: the ALMS study. Rheumatology 2010;49(1):128-40. [MEDLINE: 19933596] 
Isenberg D, Appel GB, Dooley MA, Ginzler EM, Jayne D, Lisk L, et al. Mycophenolate mofetil compared with intravenous cyclophosphamide as induction for lupus nephritis: ALMS results and BILAG responses [abstract no: OP-0018]. Annals of the Rheumatic Diseases 2008;67(Supp II):53.

Isenberg D, Appel GB, Dooley MA, Ginzler EM, Jayne D, Solomons N, et al. Mycophenolate mofetil (MMF, Cellcept) for induction and maintenance treatment of lupus nephritis (LN): baseline demographics of patients from the phase III randomized, controlled ASPREVA Lupus Management Study (ALMS) [abstract no: AB0424]. Annals of the Rheumatic Diseases 2007;66(Suppl II):606.

Jayne DR, Appel GB, Dooley MA, Ginzler E, Isenberg D, Wofsy D, et al. Results of the Aspreva Lupus Management Study (ALMS) maintenance phase [abstract no: TH-FC111]. Journal of the American Society of Nephrology 2010;21:25A.

Nadig RS. Efficacy, toxicity and tolerability of mycophenolate mofetil in patients with lupus nephritis, based on dose/weight ratio [abstract no: 431]. ACR/ARHP Annual Scientific Meeting; 2007 Nov 6-11; Boston (MA). 2007.

Schwartz N, Patel T, Ginzler EM, Solomons N, Buyon JP, Clancy RM. Response to MMF therapy for lupus nephritis is independent of genetic variation of inosine monophosphate dehydrogenase [abstract no: 837]. Arthritis \& Rheumatology 2012;64(10 Suppl):S365. [EMBASE: 70998834]

Schwartz N, Patel T, Ginzler EM, Solomons N, Buyon JP, Clancy RM. Response to MMF therapy for lupus nephritis is independent of genetic variation of inosine monophosphate dehydrogenase [abstract no: 837]. Arthritis \& Rheumatology 2012;64(10 Suppl):S365. [EMBASE: 70998834]

Silva-Fernandez L, Nadig RS, von Gizycki H, Ginzler EM. Efficacy, toxicity and tolerability of mycophenolate mofetil in patients with lupus nephritis, based on dose/weight ratio [abstract no: 431]. ACR/ARHP Annual Scientific Meeting; 2007 Nov 6-11; Boston (MA). 2007.

Sinclair A, Appel G, Dooley MA, Ginzler E, Isenberg D, Jayne D, et al. Mycophenolate mofetil as induction and maintenance therapy for lupus nephritis: rationale and protocol for the randomized, controlled Aspreva Lupus Management Study (ALMS). Lupus 2007;16(12):972-80. [MEDLINE: 18042591]

Sinclair A, Appel G, Dooley MA, Ginzler E, Isenberg D, Jayne D, et al. Protocol for the Aspreva Lupus Management Study (ALMS) [abstract no: F-PO882]. Journal of the American Society of Nephrology 2005;16:528A.

Stone DH, Dall'Era M, Levesque V, Cisternas MG, Wofsy D. Identification of biomarkers that predict response to treatment of lupus nephritis with mycophenolate mofetil (MMF) or pulse cyclophosphamide (IV) [abstract no: 2073]. ACR/ARHP Annual Scientific Meeting; 2009 Oct 17-21; Philadelphia (PA). 2009.

Sundel R, Solomons N, Lisk L, Aspreva Lupus Management Study (ALMS) Group. Efficacy of mycophenolate mofetil in adolescent patients with lupus nephritis: evidence from a two-phase, prospective randomized trial. Lupus 2012;21(13):1433-43. [MEDLINE: 22922564]
Sundel RP, Lisk L. Mycophenolate mofetil compared with intravenous cyclophosphamide as induction treatment for pediatric lupus nephritis: a randomized trial [abstract no: 1274]. ACR/ARHP Annual Scientific Meeting; 2008 Oct 24-29; San Francisco, CL. 2008.

Walsh M, Solomons N, Lisk L, Jayne DR. Mycophenolate mofetil or intravenous cyclophosphamide for lupus nephritis with poor kidney function: a subgroup analysis of the Aspreva Lupus Management Study. American Journal of Kidney Diseases 2013;61(5):710-5. [MEDLINE: 23375819]

Wofsy D, Appel GB, Dooley MA, Ginzler EM, Isenberg D, Jayne D, et al. Aspreva lupus management study maintenance results [abstract no: CS12.6]. Lupus 2010;19(1 Suppl):27.

Wofsy D, Appel GB, Dooley MA, Ginzler EM, Isenberg D, Jayne D, et al. Mycophenolate mofetil compared with intravenous cyclophosphamide in the treatment of lupus nephritis: predictors of response [abstract no: SAT0188]. Annals of the Rheumatic Diseases 2008;67(Suppl II):493.

\section{APRIL-LN 2012 \{published data only\}}

* Ginzler EM, Wax S, Rajeswaran A, Copt S, Hillson J, Ramos E, et al. Atacicept in combination with MMF and corticosteroids in lupus nephritis: results of a prematurely terminated trial. Arthritis Research \& Therapy 2012;14(1):R33. [MEDLINE: 22325903]

\section{AURA-LV 2016 \{published data only\}}

Dooley MA, Pendergraft WF, Ginzler EM, Olsen NJ, Tumlin J, Rovin B, et al. Speed of remission with the use of voclosporin, MMF and low dose steroids: results of a global lupus nephritis study [abstract no: 5L]. Arthritis \& Rheumatology 2016;68(Suppl 10):4362-3. [EMBASE: 613889173]

Parikh SV, Pendergraft WF, Tumlin JA, Saxena R, Solomons N, Huizinga RB. Treatment of active lupus nephritis with voclosporin: 48 week data from the AURA-LV study [abstract no: 4]. American Journal of Kidney Diseases 2017;69(5):A2.

* Pendergraft WF, Tumlin JA, Rovin B, Dooley MA, Jayne DR, Wofsy D, et al. AURA-LV: successful treatment of active lupus nephritis with voclosporin [abstract no: HI-OR05]. Journal of the American Society of Nephrology 2016;27(Abstract Suppl):2B.

\section{Balletta 1992 \{published data only\}}

Balletta M, Sabella D, Magri P, Sepe V, Stanziale P, Di Luccio R, et al. Cyclosporin plus steroids versus steroids alone in the treatment of lupus nephritis. Contributions to Nephrology 1992;99:129-30. [MEDLINE: 1458919]

Bao 2008 \{published and unpublished data\}

* Bao H, Liu ZH, Xie HL, Hu WX, Zhang HT, Li LS. Successful treatment of class $\mathrm{V}+\mathrm{IV}$ lupus nephritis with multi-target therapy. Journal of the American Society of Nephrology 2008;19(10):2001-10. [MEDLINE: 18596121]

Bao H, Xie HL, Zhang HT, Zhang X, Hu WX, Liu ZH, et al. Successful treatment of class V+IV lupus nephritis with multi-target immunosuppressive therapy [abstract no: SA-FC060]. Journal of the American Society of Nephrology 2007;18(Abstracts):48a. 


\section{Barron 1982 \{published data only\}}

Barron KS, Person DA, Brewer EJ Jr, Beale MG, Robson AM. Pulse methylprednisolone therapy in diffuse proliferative lupus nephritis. Journal of Pediatrics 1982;101(1):137-41. [MEDLINE: 7045314]

\section{Belmont 1995 \{published data only\}}

Belmont HM, Kitsis E, Skovron ML, McCullagh E, Abramson S. Misoprostol and prednisone treatment of lupus nephritis. American Journal of Therapeutics 1995;2(12):928-32. [PUBMED: 11854810]

\section{BELONG 2013 \{published data only\}}

Mysler E, Spindler A, Guzman R, Renato B. Study design and baseline patient characteristics of BELONG, the randomized double-blind, placebo-controlled phase III trial of ocrelizumab, a humanized anti-CD20 antibody, in lupus nephritis [abstract no: PO2.E20]. Lupus 2010;19(1 Suppl):156.

* Mysler EF, Spindler AJ, Guzman R, Bijl M, Jayne D, Furie RA, et al. Efficacy and safety of ocrelizumab in active proliferative lupus nephritis: results from a randomized, double-blind, phase III study. Arthritis \& Rheumatism 2013;65(9):2368-79. [MEDLINE: 23740801]

Mysler EF, Spindler AJ, Guzman R, Bijl M, Jayne D, Furie RA, et al. Efficacy and safety of ocrelizumab, a humanized antiCD20 antibody, in patients with active proliferative lupus nephritis (LN): results from the randomized, double-blind phase III BELONG Study [abstract]. Arthritis \& Rheumatism 2010;62(Suppl 10):1455. [EMBASE: 70380848]

\section{Boedigheimer 2017 \{published data only\}}

Boedigheimer MJ, Martin DA, Amoura Z, Sanchez-Guerrero J, Romero-Diaz J, Kivitz A, et al. Safety, pharmacokinetics and pharmacodynamics of AMG 811, an anti-interferon-gamma monoclonal antibody, in SLE subjects without or with lupus nephritis. Lupus Science \& Medicine 2017;4(1):e000226. [MEDLINE: 29018537]

\section{Boletis 1999 \{published data only\}}

Boletis JN, Boki C, Michael S, Stamatiadis DN, Nakopoulou L, Moutsopoulos C. Comparative study of pulse treatment with cyclophosphamide or immunoglobulin to sustain the remission of lupus nephritis [abstract]. Journal of the American Society of Nephrology 1998;9(Program \& Abstracts):140A-1A.

* Boletis JN, loannidis JP, Boki KA, Moutsopoulos HM. Intravenous immunoglobulin compared with cyclophosphamide for proliferative lupus nephritis. Lancet 1999;354(9178):569-70. [MEDLINE: 10470708]

\section{Boumpas 1992 \{published data only\}}

Austin HA, Fessler BJ, Boumpas DT, Vaughan EM, Klippel JH, Balow JE. Prognostic indicators supporting use of short courses of pulse immunosuppression for severe lupus nephritis (LN) [abstract no: 130]. Journal of the American Society of Nephrology 1995;6(3):411.

* Boumpas DT, Austin HA, Vaughn EM, Klippel JH, Steinberg AD, Yarboro $\mathrm{CH}$, et al. Controlled trial of pulse methylprednisolone versus two regimens of pulse cyclophosphamide in severe lupus nephritis. Lancet 1992;340(8822):741-5. [MEDLINE: 1356175]

Illei GG, Takada K, Parkin D, Austin HA, Crane M, Yarboro CH, et al. Renal flares are common in patients with severe proliferative lupus nephritis treated with pulse immunosuppressive therapy: long-term followup of a cohort of 145 patients participating in randomized controlled studies. Arthritis \& Rheumatism 2002;46(4):995-1002. [MEDLINE: 11953977]

\section{Cade 1973 \{published data only\}}

Cade R, Spooner G, Schlein E, Pickering M, DeQuesada A, Holcomb A, et al. Comparison of azathioprine, prednisone, and heparin alone or combined in treating lupus nephritis. Nephron 1973;10(1):37-56. [MEDLINE: 4695164]

Chan 2000 \{published data only\}

Chan T, Wong W, Lau C, Tsang EW, Ji Y, Mok M, et al. Prolonged follow-up of patients with diffuse proliferative lupus nephritis (DPLN) treated with prednisolone and mycophenolate mofetil (MMF) [abstract no: A1010]. Journal of the American Society of Nephrology 2001;12(Program \& Abstracts):195A.

Chan TM. Mycophenolate mofetil in the treatment of lupus nephritis: 7 years on. Lupus 2008;17(7):617-21. [MEDLINE: 18625633]

* Chan TM, Li FK, Tang CS, Wong RW, Fang GX, Ji YL, et al. Efficacy of mycophenolate mofetil in patients with diffuse proliferative lupus nephritis. Hong Kong-Guangzhou Nephrology Study Group. New England Journal of Medicine 2000;343(16):1156-62. [MEDLINE: 11036121]

Chan TM, Tse KC, Tang CS, Mok MY, Li FK, Hong Kong Nephrology Study Group. Long-term study of mycophenolate mofetil as continuous induction and maintenance treatment for diffuse proliferative lupus nephritis. Journal of the American Society of Nephrology 2005;16(4):1076-84. [MEDLINE: 15728784]

Karassa FB, Isenberg DA. Efficacy of mycophenolate mofetil in patients with diffuse proliferative lupus nephritis. New England Journal of Medicine 2001;344(5):382-3. [MEDLINE: 11195799]

\section{Chen 2011 \{published data only\}}

Chen W, Liu Q, Chen W, Fu P, Liao Y, Zhang J, et al. A prospective multicenter randomized trial of treatment of active lupus nephritis with tacrolimus versus cyclophosphamide/ azathioprine in Chinese adults [abstract no: SU248]. World Congress of Nephrology; 2009 May 22-26; Milan (Italy). 2009.

Chen W, Liu Q, Chen W, Tang X, Fu P, Liu F, et al. Outcomes of maintenance therapy with tacrolimus versus azathioprine for active lupus nephritis: a multicenter randomized clinical trial. Lupus 2012;21(9):944-52. [MEDLINE: 22438027]

* Chen W, Tang X, Liu Q, Chen W, Fu P, Liu F, et al. Shortterm outcomes of induction therapy with tacrolimus versus cyclophosphamide for active lupus nephritis: a multicenter randomized clinical trial. American Journal of Kidney Diseases 2011;57(2):235-44. [MEDLINE: 21177013] 
Clark 1981 \{published data only\}

* Clark WF, Lindsay RM, Cattran DC, Chodirker WB, Barnes CC, Linton AL. Monthly plasmapheresis for systemic lupus erythematosus with diffuse proliferative glomerulonephritis: a pilot study. Canadian Medical Association Journal 1981;125(2):171-4. [MEDLINE: 7272867]

Clark WF, Lindsay RM, Chodirker WB, Cattran D, Linton AL. Elective plasmapheresis in S.L.E. nephritis: pilot for a controlled prospective study [abstract]. Kidney International 1979;16(6):928.

\section{Clark 1984 \{published data only\}}

* Clark WF, Balfe JW, Cattran DC. Long-term plasma exchange in patients with systemic lupus erythematosus and diffuse proliferative glomerulonephritis. Plasma Therapy \& Transfusion Technology 1984;5(3):353-60. [EMBASE: 14000064]

Clark WF, Cattran D, Balfe JW, Williams W, Lindsay RM, Linton AL. Chronic plasma exchange in SLE nephritis [abstract]. Kidney International 1983;24(3):424.

* Clark WF, Cattran DC, Balfe JW, Williams W, Lindsay RM, Linton AL. Chronic plasma exchange in systemic lupus erythematosus nephritis. Proceedings of the European Dialysis \& Transplant Association 1983;20:629-35. [MEDLINE: 6361759]

Clark WF, Williams W, Cattran DC, Balfe JW, Chodirker WB, Koval JJ, et al. A controlled trial of chronic plasma exchange therapy in S.L.E. nephritis [abstract]. Kidney International 1984;25(1):161.

Clark WF, Williams W, Cattran DC, Chodirker WB, Koval JJ, Lindsay RM, et al. Controlled trial of chronic plasma exchange therapy in SLE nephritis [abstract]. Kidney International 1982;21(1):147.

Linton AL, Williams W, Cattran D, Lindsay R, Clark W. Controlled trial of chronic plasma exchange in SLE nephritis [abstract]. Australasian Society of Nephrology.19th Annual Scientific Meeting. 2nd Asian Congress of Nephrology; 1983 Feb 14-18; Melbourne, Australia. 1983:48.

\section{Contreras 2004 \{published and unpublished data\}}

Contreras G, Pardo V, Leclercq B, Gomez E, Reich J, O'Nan P, et al. Maintenance therapy for proliferative forms of lupus nephritis: a randomized clinical trial comparing quarterly intravenous cyclophosphamide (IVCY) versus oral mycophenolate mofetil (MMF) or azathioprine (AZA) [abstract no: F-FC069]. Journal of the American Society of Nephrology 2002;13(Sept Program \& Abstracts):15A.

Contreras G, Pardo V, Leclercq B, Lenz O, ONan P, Tozman E, et al. Lupus nephritis: sequential therapy with short-term intravenous cyclophosphamide followed by maintenance oral mycophenolate mofetil or oral azathioprine is more efficacious and safer than long-term intravenous cyclophosphamide [abstract no: SA-FC173]. Journal of the American Society of Nephrology 2003;14(Nov):38A.

* Contreras G, Pardo V, Leclercq B, Lenz O, Tozman E, O'Nan P, et al. Sequential therapies for proliferative lupus nephritis. New
England Journal of Medicine 2004;350(10):971-80. [MEDLINE: 14999109]

Contreras G, Roth D, Berho M, Perez G, Gomez E, Acosta M, et al. Immunosupressive therapy for proliferative lupus nephritis: preliminary report of a prospective, randomized clinical trial with mycophenolate mofetil (MMF) [abstract]. Journal of the American Society of Nephrology 1999;10(Program \& Abstracts):99A.

Contreras G, Tozman E, Nahar N, Metz D. Maintenance therapies for proliferative lupus nephritis: mycophenolate mofetil, azathioprine and intravenous cyclophosphamide. Lupus 2005;14(Suppl 1):S33-8. [MEDLINE: 15803929]

\section{CYCLOFA-LUNE 2010 \{published and unpublished data\}}

Dostal C, Tesar V, Rysava R, Rovensky J, Rychlik I, Hrneio Z, et al. Cyclofa-Lune (2002) lupus nephritis - randomized controlled multicentric therapeutic comparative study with cyclosporin $A$ versus cyclophosphamide: running evaluation [abstract no: THU0390]. Annual European Congress of Rheumatology EULAR 2004; 2004 Jun 9-12, Berlin (Germany). 2004.

Zavada AJ, Pesickova S, Rysava R, Horak P, Hrncir Z, Rovensky J, et al. Extended follow-up of a investigator-initiated trial comparing two sequential induction and maintenance treatment regimens for proliferative lupus nephritis based either on cyclophosphamide or cyclosporine [abstract no: 596]. Arthritis and Rheumatism 2013;65(10 Suppl):S256-S7. [EMBASE: 71318082]

* Zavada J, Pesickova S, Rysava R, Olejarova M, Horak P, Hmcir Z, et al. Cyclosporine A or intravenous cyclophosphamide for lupus nephritis: the Cyclofa-Lune study. Lupus 2010;19(11):1281-9. [MEDLINE: 20605876]

Zavada J, Pesickova SS, Rysava R, Horak P, Hrncir Z, Lukac J, et al. Extended follow-up of the CYCLOFA-LUNE trial comparing two sequential induction and maintenance treatment regimens for proliferative lupus nephritis based either on cyclophosphamide or on cyclosporine A. Lupus 2014;23(1):69-74. [MEDLINE: 24213308]

\section{Decker 1975 \{published data only\}}

* Austin HA 3rd, Klippel JH, Balow JE, le Riche NG, Steinberg AD, Plotz PH, et al. Therapy of lupus nephritis. Controlled trial of prednisone and cytotoxic drugs. New England Journal of Medicine 1986;314(10):614-9. [MEDLINE: 3511372]

Austin HA, Klippel J, le Riche N, Decker JL, Balow JE. Immunosuppressive therapy of lupus nephritis [abstract]. Kidney International 1985;27(1):204. [CENTRAL: CN-00550415]

Austin, HA 3rd, Muenz LR, Joyce KM, Antonovych TA, Kullick ME, Klippel JH, et al. Prognostic factors in lupus nephritis. Contribution of renal histologic data. American Journal of Medicine 1983;75(3):382-91. [MEDLINE: 6351607]

Carette S, Klippel JH, Decker JL, Austin HA, Plotz PH, Steinberg AD, et al. Controlled studies of oral immunosuppressive drugs in lupus nephritis. A long-term follow-up. Annals of Internal Medicine 1983;99(1):1-8. [MEDLINE: 6344715] 
Decker JL, Klippel JH, Plotz PH, Steinberg AD.

Cyclophosphamide or azathioprine in lupus

glomerulonephritis. A controlled trial: results at 28 months. Annals of Internal Medicine 1975;83(5):606-15. [MEDLINE: 1106278]

Decker JL, Steinberg AD, Reinertsen JL, Plotz PH, Balow JE, Klippel JH. NIH conference. Systemic lupus erythematosus: evolving concepts. Annals of Internal Medicine 1979;91(4):587-604. [MEDLINE: 158324]

Dinant HJ, Decker JL, Klippel JH, Balow JE, Plotz PH, Steinberg AD. Alternative modes of cyclophosphamide and azathioprine therapy in lupus nephritis. Annals of Internal Medicine 1982;96(6 Pt 1):728-36. [MEDLINE: 7046543]

Steinberg AD, Steinberg SC. Long-term preservation of renal function in patients with lupus nephritis receiving treatment that includes cyclophosphamide versus those treated with prednisone only. Arthritis \& Rheumatology 1991;34(8):945-50. [MEDLINE: 1859488]

\section{Deng 2016 \{published data only\}}

Deng F, Hong D, Wang L. A clinical research of the effect of leflunomide as an induction treatment for proliferative lupus nephritis [abstract no: FR-P0593]. Journal of the American Society of Nephrology 2016;27(Abstract Suppl):499A.

\section{Derksen 1988 \{published data only\}}

* Derksen RH, Hene RJ, Kallenberg CG, Valentijn RM, Kater L. Prospective multicentre trial on the short- term effects of plasma exchange versus cytotoxic drugs in corticosteroidresistant lupus nephritis. Netherlands Journal of Medicine 1988;33(3-4):168-77. [MEDLINE: 3067098]

\section{Donadio 1972 \{published data only\}}

* Donadio JVJ, Holley KE, Wagoner RD, Ferguson RH, McDuffie FC. Further observations on the treatment of lupus nephritis with prednisone and combined prednisone and azathioprine. Arthritis \& Rheumatism 1974;17(5):573-81. [MEDLINE: 4278104]

Donadio JVJ, Holley KE, Wagoner RD, Ferguson RH, McDuffie FC. Treatment of lupus nephritis with prednisone and combined prednisone and azathioprine. Annals of Internal Medicine 1972;77(6):829-35. [MEDLINE: 4566283]

\section{Donadio 1976 \{published data only\}}

Donadio JV, Holley KE, Ferguson RH, Ilstrup D. Long-term treatment of diffuse proliferative lupus nephritis (DPLN) with prednisone and combined prednisone and cyclophosphamide [abstract]. Kidney International 1977;12(6):465.

Donadio JV, Holley KE, Ferguson RH, Ilstrup DM. Progressive lupus glomerulonephritis. Treatment with prednisone and combined prednisone and cyclophosphamide. Mayo Clinic Proceedings 1976;51(8):484-94. [MEDLINE: 950801]

* Donadio JV, Holley KE, Ferguson RH, Ilstrup DM. Treatment of diffuse proliferative lupus nephritis with prednisone and combined prednisone and cyclophosphamide. New England Journal of Medicine 1978;299(21):1151-5. [MEDLINE: 309095]

\section{Doria 1994 \{published data only\}}

* Doria A, Piccoli A, Vesco P, Vaccaro E, Marson P, De Silvestro G, et al. Therapy of lupus nephritis. A two-year prospective study. Annales de Medecine Interne 1994;145(5):307-11. [MEDLINE: 7985937]

\section{Dyadyk 2001 \{published and unpublished data\}}

* Dyadyk A, Vasilenko I, Bagriy A, Dyadyk O, Yarovaya N, Roschin Y, et al. Azathioprine and cyclophosphamide in treatment of patients with diffuse proliferative lupus nephritis - a randomized controlled study [abstract]. Nephrology Dialysis Transplantation 2001;16(6):A57.

Dyadyk OI, Vasilenko IV, Bagriy AE, Kholopov LS, Dyadyk OO, Yarovaya NF, et al. Azathioprine and cyclophosphamide in diffuse proliferative lupus nephritis treatment - a randomized controlled study [abstract no: SAT0125]. Annals of the Rheumatic Diseases 2007;66(Suppl II):467.

\section{El-Sehemy 2006 \{published data only\}}

Abd-ELHady El-Sehemy M, Al-Saaran AM, Al-Saeed Baddour NM, El-Sayed Moez P, Adam AG. Comparative clinical prospective therapeutic study between cyclophosphamide, cyclosporine and azathioprine in the treatment of lupus nephritis [abstract]. 41st Congress. European Renal Association. European Dialysis and Transplantation Association; 2004 May 15-18; Lisbon (Portugal). 2004:48.

Abd-ELHady El-Sehemy M, Al-Saaran AM, Al-Saeed Baddour NM, El-Sayed Moez P, Gaber Adam A. Cyclosporine but not cyclophosphamide is a rescue therapy in resistant lupus nephritis even in class iv proliferative GN [abstract]. 41st Congress. European Renal Association. European Dialysis and Transplantation Association; 2004 May 15-18; Lisbon (Portugal). 2004:48.

Adam AG, El-Sehemy MS, Baddour NM, Moez PE, Al Saaran AM. Prospective comparative study between azathioprine "AZA", cyclophosphamide "CYP", cyclosporine "CsA" and mycophenolate mofetil "MMF" in the treatment of lupus nephritis: is CYP still the preferred therapy against the newcomer MMF. AZA: an old drug with still a great potential? [abstract no: F-PO258]. Journal of the American Society of Nephrology 2004;15(Oct):122A.

* El-Sehemy MS, Al-Saaran AM, Baddour NM, Adam AG, Moez PE. Comparative clinical prospective therapeutic study between cyclophosphamide, cyclosporine and azathioprine in the treatment of lupus nephritis. Egyptian Journal of Immunology/Egyptian Association of Immunologists 2006;13(1):39-52. [MEDLINE: 17974149]

\section{El-Shafey 2010 \{published and unpublished data\}}

* El Shafey EM, Abdou SH, Shareef MM. Is mofetil superior to pulse intravenous cyclophosphamide for induction therapy of proliferative lupus nephritis in Egyptian patients?. Clinical \& Experimental Nephrology 2010;14(3):214-21. [MEDLINE: 20169461]

\section{Florez-Suarez 2004 \{published and unpublished data\}}

Flores-Suarez LF, Villa AR. Open randomized trial comparing mycophenolate mofetil (MMF) vs intravenous 
cyclophosphamide (IV-CYC) as induction therapy for severe lupus nephritis (LN) [abstract no: F-PO257]. Journal of the American Society of Nephrology 2004;15(Oct):122A.

Flores-Suarez LF, Villa AR. Preliminary results of an open randomised clinical trial comparing mycophenolate mofetil (MMF) vs intravenous cyclophosphamide (IV-CYC) as induction therapy for severe lupus nephritis [abstract no: THU0413]. Annual European Congress of Rheumatology EULAR 2004; 2004 Jun 9-12, Berlin, Germany. 2004.

\section{Fries 1973 \{published data only\}}

* Fries JF, Sharp GC, McDevitt HO, Holman HR. Cyclophosphamide therapy in systemic lupus erythematosus and polymyositis. Arthritis \& Rheumatism 1973;16(2):154-62. [MEDLINE: 4716431]

\section{Fu 1997 \{published data only\}}

* Fu LW, Yang LY, Chen WP, Lin CY. Clinical efficacy of cyclosporin A neoral in the treatment of paediatric lupus nephritis with heavy proteinuria. British Journal of Rheumatology 1998;37(2):217-21. [MEDLINE: 9569080]

Fu LW, Yang LY, Chen WP, Lin CY. Cyclosporin pharmacokinetics following administration of capsules and Neoral in paediatric patients with lupus nephritis. British Journal of Clinical Pharmacology 1997;44(2):125-7. [MEDLINE: 9278195]

\section{Furie 2014 \{published data only\}}

Furie R, Nicholls K, Cheng TT, Houssiau F, Burgos-Vargas R, Chen SL. Efficacy and safety of abatacept over 12 months in patients with lupus nephritis: results from a multicenter, randomized, double-blind, placebo-controlled phase II/III study [abstract]. Arthritis \& Rheumatism 2014;63(10 Suppl 1):S962-3. [EMBASE: 70785447]

* Furie R, Nicholls K, Cheng TT, Houssiau F, Burgos-Vargas R, Chen SL, et al. Efficacy and safety of abatacept in lupus nephritis: a twelve-month, randomized, double-blind study. Arthritis \& Rheumatology 2014;66(2):379-89. [MEDLINE: 24504810]

Wofsy D, Hillson JL, Diamond B. Abatacept for lupus nephritis: alternative definitions of complete response support conflicting conclusions. Arthritis \& Rheumatism 2012;64(11):3660-5. [MEDLINE: 22806274]

Wofsy D, Hillson JL, Diamond B. Comparison of alternative primary outcome measures for use in lupus nephritis clinical trials. Arthritis \& Rheumatism 2013;65(6):1586-91. [MEDLINE: 23529285]

\section{Ginzler 1976 \{published data only\}}

* Ginzler E, Diamond H, Guttadauria M, Kaplan D. Prednisone and azathioprine compared to prednisone plus low-dose azathioprine and cyclophosphamide in the treatment of diffuse lupus nephritis. Arthritis \& Rheumatism 1976;19(4):693-9. [MEDLINE: 782464]

\section{Ginzler 2005 \{published and unpublished data\}}

Appel G, Ginzler E, Radhakrishnan J, Aranow C, Buyon J, Dooley M, et al. Multicenter controlled trial of mycophenolate mofetil (MMF) vs intravenous cyclophosphamide (IVC) as induction therapy for severe lupus nephritis (LN) [abstract no: SA-FC171]. Journal of the American Society of Nephrology 2003;14(Nov):38A.

Burchardi C, Schlondorff D. Induction therapy for active lupus nephritis: mycophenolate mofetil versus cyclophosphamide. Nature Clinical Practice Nephrology 2006;2(6):314-5. [MEDLINE: 16932452]

Elliott JR, Manzi S. Induction therapy for active lupus nephritis: mycophenolate mofetil is superior to cyclophosphamide. Nature Clinical Practice Rheumatology 2006;2(7):354-5. [MEDLINE: 16932717]

* Ginzler EM, Dooley MA, Aranow C, Kim MY, Buyon J, Merrill JT, et al. Mycophenolate mofetil or intravenous cyclophosphamide for lupus nephritis. New England Journal of Medicine 2005;353(21):2219-28. [MEDLINE: 16306519]

Karassa FB. Mycophenolate mofetil or intravenous cyclophosphamide in lupus nephritis. New England Journal of Medicine 2006;354(7):764-5. [MEDLINE: 16481648]

Radhakrishnan J, Ginzler E, Appel G. Mycophenolate mofetil (MMF) vs. intravenous cyclophosphamide (IVC) for severe lupus nephritis (LN): subgroup analysis of patients with membranous nephropathy (SLE-V) [abstract no: TH-FC036]. Journal of the American Society of Nephrology 2005;16:8A.

Radhakrishnan J, Moutzouris D, Ginzler E, Appel G. Lupus membranous nephropathy IV cyclophosphamide (IVC) vs. mycophenolate mofetil (MMF) [abstract no: SA-PO2951]. Journal of the American Society of Nephrology 2008;19(Abstracts Issue): $779 \mathrm{~A}$.

Robert C, Mo S, Kim M, Ginzler EM. Should therapy go beyond the control of immediate injury? Biomarkers of the vasculature and their association with longitudinal assessments in the induction phase of a randomized multicenter trial comparing mycophenolate mofetil and intravenous cyclophosphamide [abstract no: PO1.B.31]. Lupus 2010;19(1 Suppl):55.

\section{Gourley 1996 \{published data only\}}

* Gourley MF, Austin HA, Scott D, Yarboro CH, Vaughan EM, Muir J, et al. Methylprednisolone and cyclophosphamide, alone or in combination, in patients with lupus nephritis. A randomized, controlled trial. Annals of Internal Medicine 1996;125(7):549-57. [MEDLINE: 8815753]

Illei GG, Austin HA, Crane M, Collins L, Gourley MF, Yarboro CH, et al. Combination therapy with pulse cyclophosphamide plus pulse methylprednisolone improves long-term renal outcome without adding toxicity in patients with lupus nephritis. Annals of Internal Medicine 2001;135(4):248-57. [MEDLINE: 11511139]

Illei GG, Takada K, Parkin D, Austin HA, Crane M, Yarboro CH, et al. Renal flares are common in patients with severe proliferative lupus nephritis treated with pulse immunosuppressive therapy: long-term followup of a cohort of 145 patients participating in randomized controlled studies. Arthritis \& Rheumatism 2002;46(4):995-1002. [MEDLINE: 11953977] 


\section{Grootscholten 2006 \{published and unpublished data\}}

Arends S, Grootscholten C, Bijl M, Berger SP, De Sevaux R, Voskuyl AE, et al. Cyclophosphamide versus azathioprine/ methylprednisolone: long-term follow-up of the first Dutch lupus nephritis study [abstract]. Lupus 2011;20(4):350. [EMBASE: 70414154]

Arends S, Grootscholten C, Derksen RH, Berger SP, de Sevaux RG, Voskuyl AE, et al. Long-term followup of a randomised controlled trial of azathioprine/ methylprednisolone versus cyclophosphamide in patients with proliferative lupus nephritis. Annals of the Rheumatic Diseases 2012;71(6):966-73. [MEDLINE: 22128082]

Grootscholten C, Bajema IM, Florquin S, Steenbergen EJ, Peutz-Kootstra CJ, Goldschmeding R, et al. Interobserver agreement of scoring of histopathological characteristics and classification of lupus nephritis. Nephrology Dialysis Transplantation 2008;23(1):223-30. [MEDLINE: 17981886]

Grootscholten C, Bajema IM, Florquin S, Steenbergen EJ, Peutz-Kootstra CJ, Goldschmeding R, et al. Treatment with cyclophosphamide delays the progression of chronic lesions more effectively than does treatment with azathioprine plus methylprednisolone in patients with proliferative lupus nephritis. Arthritis \& Rheumatism 2007;56(3):924-37. [MEDLINE: 17328070]

Grootscholten C, Bijl M, Ligtenberg G, Hagen EC, van den Wall Bake AW, de Glas-Vos JW, et al. Is azathioprine an alternative for cyclophosphamide in the treatment of patients with proliferative lupus nephritis? Two year results of the Dutch Lupus Nephritis Study [abstract no: OP0176]. Annual European Congress of Rheumatology EULAR; 2004 Jun 9-12; Berlin (Germany). 2004.

Grootscholten C, Dieker JW, McGrath FD, Roos A, Derksen RH, van der Vlag J, et al. A prospective study of anti-chromatin and anti-C1q autoantibodies in patients with proliferative lupus nephritis treated with cyclophosphamide pulses or azathioprine/methylprednisolone. Annals of the Rheumatic Diseases 2007;66(5):693-6. [MEDLINE: 17135217]

* Grootscholten C, Ligtenberg G, Hagen EC, van den Wall Bake AW, de Glas-Vos JW, Bijl M, et al. Azathioprine/ methylprednisolone versus cyclophosphamide in proliferative lupus nephritis. A randomized controlled trial. Kidney International 2006;70(4):732-42. [MEDLINE: 16820790]

Grootscholten C, Ligtenberg G, van Houwelingen HC, Derksen RHW, Berden JH. Randomized controlled trial comparing cyclophosphamide pulse therapy with azathioprine/ methylprednisolone in proliferative lupus nephritis [abstract no: SU-FC047]. Journal of the American Society of Nephrology 2004;15(Oct):54A.

Grootscholten C, Snoek FJ, Bijl M, van Houwelingen HC, Derksen $\mathrm{RH}$, Berden JH, et al. Health-related quality of life and treatment burden in patients with proliferative lupus nephritis treated with cyclophosphamide or azathioprine/ methylprednisolone in a randomized controlled trial. Journal of Rheumatology 2007;34(8):1699-707. [MEDLINE: 17659757]
Ligtenberg G, Grootscholten CM, Derksen RH, Berden JH. Cyclophosphamide pulse therapy versus azathioprine and methylprednisolone pulses in proliferative lupus nephritis: first results of a randomized, prospective multicenter study [abstract no: F-FC067]. Journal of the American Society of Nephrology 2002;13(Program \& Abstracts):14A.

\section{Hahn 1975 \{published data only\}}

Hahn BH, Kantor OS, Osterland CK. Azathioprine plus prednisone compared with prednisone alone in the treatment of systemic lupus erythematosus. Report of a prospective controlled trial in 24 patients. Annals of Internal Medicine 1975;83(5):597-605. [MEDLINE: 1106277]

\section{Hong 2007 \{published and unpublished data\}}

Hong R, Haijin Y, Xianglin W, Cuilan H, Nan C. A preliminary study of tacrolimus versus cyclophosphamide in patients with diffuse proliferative lupus nephritis [abstract no: SaP131]. Nephrology Dialysis Transplantation 2007;22(Suppl 6):vi276.

\section{Houssiau 2002 \{published data only\}}

Dall'Era M, Cisternas MG, Smilek DE, Straub L, Houssiau FA, Cervera $\mathrm{R}$, et al. Predictors of long-term renal outcome in lupus nephritis trials: lessons learned from the Euro-Lupus Nephritis cohort. Arthritis \& Rheumatology 2015;67(5):1305-13. [MEDLINE: 25605554]

Houssiau FA. The 10-year followup of the Euro-Lupus Nephritis Trial (ELNT), a randomized prospective trial comparing low-dose (LD) versus high-dose (HD) intravenous (IV) cyclophosphamide (CY) followed by azathioprine (AZA) as treatment of proliferative lupus nephritis [abstract no: 2060]. ACR/ARHP Annual Scientific Meeting; 2008 Oct 24-29; San Francisco (CA). 2008.

Houssiau FA, Cosyns J, Cervera R. The Euro-Lupus Nephritis Trial: 5-year follow-up [abstract no: OP0054]. Annual European Congress of Rheumatology EULAR; 2003 June 18-21; Lisbon (Portugal). 2003.

Houssiau FA, Vasconcelos C, D'Cruz D, Sebastiani G, de Ramon Garrido E, Danieli M, et al. Long term outcome of patients randomized in the Euro-Lupus Nephritis Trial: further evidence that a low-dose IV cyclophosphamide induction regimen achieves good results [abstract no: OP0034]. Annals of the Rheumatic Diseases 2006;65(Suppl II):64.

* Houssiau FA, Vasconcelos C, D'Cruz D, Sebastiani GD, Garrido Ed Ede R, Danieli MG, et al. Immunosuppressive therapy in lupus nephritis: the Euro-Lupus Nephritis Trial, a randomized trial of low-dose versus high-dose intravenous cyclophosphamide. Arthritis \& Rheumatism 2002;46(8):2121-31. [MEDLINE: 12209517]

Houssiau FA, Vasconcelos C, D'Cruz D, Sebastiani GD, de Ramon Garrido E, Danieli MG, et al. Early response to immunosuppressive therapy predicts good long-term renal outcome in lupus nephritis: lessons from the Euro-Lupus Nephritis trial (ELNT) [abstract no: 67]. Lupus 2004; 7th International Congress on SLE and Related Conditions; 2004 May 9-13; New York (NY). 2004. 
Houssiau FA, Vasconcelos C, D'Cruz D, Sebastiani GD, de Ramon Garrido E, Danieli MG, et al. Early response to immunosuppressive therapy predicts good long-term renal outcome in proliferative lupus glomerulonephritis: lessons from the Euro-Lupus Nephritis Trial [abstract no: OP0174]. Annual European Congress of Rheumatology EULAR; 2004 Jun 9-12; Berlin (Germany). 2004

Houssiau FA, Vasconcelos C, D'Cruz D, Sebastiani GD, de Ramon Garrido E, Danieli MG, et al. Early response to immunosuppressive therapy predicts good renal outcome in lupus nephritis: lessons from long-term followup of patients in the Euro-Lupus Nephritis Trial. Arthritis \& Rheumatism 2004;50(12):3934-40. [MEDLINE: 15593207]

Houssiau FA, Vasconcelos C, D'Cruz D, Sebastiani GD, de Ramon Garrido E, Danieli MG, et al. The 10-year follow-up data of the Euro-Lupus Nephritis Trial comparing low-dose and highdose intravenous cyclophosphamide. Annals of the Rheumatic Diseases 2010;69(1):61-4. [MEDLINE: 19155235]

\section{Jayne 2013 \{published data only\}}

Jayne D, Appel G, Chan TM, Barkay H, Weiss R, Wofsy D. A randomized controlled study of laquinimod in active lupus nephritis patients in combination with standard of care [abstract no: LB0003]. Annals of the Rheumatic Diseases 2013;72(Suppl 3):164.

\section{Kaballo 2016 \{published data only\}}

* Kaballo BG, Ahmed AE, Nur MM, Khalid IO, Abu-Aisha H. Mycophenolate mofetil versus azathioprine for maintenance treatment of lupus nephritis. Saudi Journal of Kidney Diseases \& Transplantation 2016;27(4):717-25. [MEDLINE: 27424688]

\section{Kamanamool 2017 \{published data only\}}

* Kamanamool N, Ingsathit A, Rattanasiri S, Ngamjanyaporn P, Kasitanont N, Chawanasuntorapoj R, et al. Comparison of disease activity between tacrolimus and mycophenolate mofetil in lupus nephritis: a randomized controlled trial. Lupus 2017;27(4):647-56. [MEDLINE: 29105558]

Sumethkul V. Comparison between tacrolimus (TAC) and mycophenolate mofetil (MMF) for induction of remission in lupus nephritis. clinicaltrials.gov/ct2/show/NCT01580865 (first received 17 April 2012).

\section{Lewis 1992 \{published data only\}}

Chen YE, Korbet SM, Katz RS, Schwartz MM, Lewis EJ. The value of a partial remission in severe lupus nephritis [abstract no: SA-P0743]. Journal of the American Society of Nephrology 2006;17(Abstracts):731A.

Chen YE, Korbet SM, Katz RS, Schwartz MM, Lewis EJ, Collaborative Study Group. Value of a complete or partial remission in severe lupus nephritis. Clinical Journal of the American Society of Nephrology: CJASN 2008;3(1):46-53. [MEDLINE: 18003764]

Clough JD, Lewis EJ, Lachin JM. Treatment protocols of the lupus nephritis collaborative study of plasmapheresis in severe lupus nephritis. The Lupus Nephritis Collaborative Study Group. Progress in Clinical \& Biological Research 1990;337:301-7. [MEDLINE: 2352989]
Korbet SM, Schwartz MM, Evans J, Lewis EJ, Collaborative Study Group. Severe lupus nephritis: racial differences in presentation and outcome. Journal of the American Society of Nephrology 2007;18(1):244-54. [MEDLINE: 17167111]

Korbet SM, Whittier WL, Lewis EJ. Complete remission rate and outcome in severe lupus nephritis: the impact of baseline serum creatinine [abstract no: TH-PO769]. Journal of the American Society of Nephrology 2015;26(Abstract Suppl):266A.

Lachin JM, Lan SP. Termination of a clinical trial with no treatment group difference: the Lupus Nephritis Collaborative Study. Controlled Clinical Trials 1992;13(1):62-79. [MEDLINE: 1315665]

Levey AS, Lan SP, Corwin HL, Kasinath BS, Lachin J, Neilson EG, et al. Progression and remission of renal disease in the Lupus Nephritis Collaborative Study. Results of treatment with prednisone and short-term oral cyclophosphamide. Annals of Internal Medicine 1992;116(2):114-23. [MEDLINE: 1727614]

Lewis E, Lachin J, Lupus Nephritis Collaborative Study Group (LNCSG). Primary outcomes in the controlled trial of plasmapheresis therapy (PPT) in severe lupus nephritis [abstract]. Kidney International 1987;31(1):208.

Lewis EJ. Plasmapheresis therapy is ineffective in SLE. Lupus Nephritis Collaborative Study Group. Journal of Clinical Apheresis 1992;7(3):153. [MEDLINE: 1286995]

* Lewis EJ, Hunsicker LG, Lan SP, Rohde RD, Lachin JM, Lupus Nephritis Collaborative Study Group. A controlled trial of plasmapheresis therapy in severe lupus nephritis. The Lupus Nephritis Collaborative Study Group. New England Journal of Medicine 1992;326(21):1373-9. [MEDLINE: 1569973]

Patel SB, Korbet SM, Lewis EJ. The prognosis of severe lupus nephritis based on the Modification of Diet in Renal Disease (MDRD) study estimated glomerular filtration rate. Lupus 2011;20(3):256-64. [MEDLINE: 21138981]

Pohl M, Berl T, Lan S, Lupus Nephritis Collaborative Study Group (LNCS). Plasmapheresis (PP) does not enhance the susceptibility to infections in immunosuppressed patients with lupus nephritis [abstract]. Kidney International 1989;35(1):230.

Pohl MA, Lan SP, Berl T. Plasmapheresis does not increase the risk for infection in immunosuppressed patients with severe lupus nephritis. The Lupus Nephritis Collaborative Study Group. Annals of Internal Medicine 1991;114(11):924-9. [MEDLINE: 2024858]

\section{Li 2009c \{published and unpublished data\}}

Li EK, Tam LS, Zhu TY, Kwok CL, Leung YY, Szeto CC. Rituximab monotherapy is an effective induction therapy in proliferative lupus nephritis: a pilot study [abstract no: THU0223]. Annals of the Rheumatic Diseases 2009;68(Suppl 3):247.

* Li EK, Tam LS, Zhu TY, Li M, Kwok CL, Li TK, et al. Is combination rituximab with cyclophosphamide better than rituximab alone in the treatment of lupus nephritis?. Rheumatology 2009;48(8):892-8. [MEDLINE: 19478041] 


\section{Li 2012 \{published and unpublished data\}}

* Li X, Ren H, Zhang Q, Zhang W, Wu X, Xu Y, et al. Mycophenolate mofetil or tacrolimus compared with intravenous cyclophosphamide in the induction treatment for active lupus nephritis. Nephrology Dialysis Transplantation 2012;27(4):1467-72. [MEDLINE: 21917733]

Li X, Ren H, Zhang W, Xu Y, Shen P, Zhang Q, et al. Induction therapies for proliferative lupus nephritis: mycophenolate mofetil, tacrolimus and intravenous cyclophosphamide [abstract]. Journal of the American Society of Nephrology 2009;20:391A.

\section{Liou 2007 \{published data only\}}

Liou C, Ni Z, Qian J, Lin A, Zhang W, Fang W, et al. Treatment of lupus nephritis type_and_with leflunomide: A two year prospective sequential therapies [abstract no: M-PO-1081]. 4th World Congress of Nephrology.19th International Congress of the International Society of Nephrology (ISN); 2007 Apr 21-25; Rio de Janeiro, Brazil. 2007:349.

\section{Liu 2015 \{published data only\}}

Liu Z, Zhang H, Liu Z, Xing C, Fu P, Ni Z, et al. Multitarget therapy for induction treatment of lupus nephritis: a randomized trial. [Summary for patients in Ann Intern Med. 2015 Jan 6;162(1):I24; PMID: 25383607]. Annals of Internal Medicine 2015;162(1):18-26. [MEDLINE: 25383558]

\section{Loo 2010 \{published data only\}}

Loo CY, Kong NCT, Shahrir M, Suriani A, Halim AG, Rozita M, et al. Plasmapheresis versus immunoadsorption in severe lupus nephritis (LN) [abstract no: M-PO-0967]. 4th World Congress of Nephrology.19th International Congress of the International Society of Nephrology (ISN); 2007 Apr 21-25; Rio de Janeiro, Brazil. 2007:319.

* Loo CY, Mohamed Said MS, Mohd R, Abdul Gafor AH, Saidin R, Halim NA, et al. Immunoadsorption and plasmapheresis are equally efficacious as adjunctive therapies for severe lupus nephritis. Transfusion \& Apheresis Science 2010;43(3):335-40. [MEDLINE: 21051293]

\section{Lui 1997 \{published and unpublished data\}}

Lui SF, Cheng IKP, Tong KL, Li CS, Wong KC, Chan TM, et al. Treatment of type iv lupus nephritis (LN) - comparison of 2 triple therapy regimens: cyclosporin a (CSA), prednisolone (PRED), azathioprine (AZA) vs. oral cyclophosphamide (POCP), prednisolone, azathioprine [abstract no: P1613]. Nephrology 1997;3(Suppl 1):S476.

\section{LUNAR 2012 \{published and unpublished data\}}

Appel GB, Looney RJ, Eisenberg RA, Rovin BH, Ginzler EM, Adler SG, et al. Protocol for the Lupus Nephritis Assessment with rituximab (LUNAR) Study [abstract no: F-PO1121]. Journal of the American Society of Nephrology 2006;17(Abstracts):573A.

Furie R, Looney J, Rovin B, Latinis K, Appel G, SanchezGuerrero J, et al. Efficacy and safety of rituximab (RTX) in patients (Pts) with proliferative lupus nephritis (LN): results from randomized, double-blind phase III LUNAR study at week 52 [abstract no: CS6.6]. Lupus 2010;19(1 Suppl):15.
Furie R, Looney J, Rovin B, Latinis K, Appel G, SanchezGuerrero J, et al. Efficacy and safety of rituximab in patients with proliferative lupus nephritis: results from the randomized, double-blind phase III LUNAR study [abstract no: SAT0185]. Annals of the Rheumatic Diseases 2010;69(Suppl 3):549.

Furie R, Looney RJ, Rovin B, Latinis KM, Appel G, SanchezGuerrero J, et al. Efficacy and safety of rituximab in subjects with active proliferative lupus nephritis (LN): results from the randomized, double-blind phase III LUNAR study [abstract no: 1149]. ACR/ARHP Annual Scientific Meeting; 2009 Oct 17-21; Philadelphia (PA). 2009.

Furie R, Rovin B, Appel G, Kamen D, Fervenza F, Spindler A, et al. Effect of rituximab (RTX) on anti-dsDNA and C3 levels and relationship to response: results from the LUNAR trial [abstract no: PO2.E.22]. Lupus 2010;19(1 Suppl):157.

Furie R, Rovin B, Appel G, Kamen D, Fervenza FC, Spindler A, et al. Effect of rituximab (RTX) on anti -double-stranded DNA antibody and $c 3$ levels and relationship to response: results from the LUNAR trial [abstract no: SAT0186]. Annals of the Rheumatic Diseases 2010;69(Suppl 3):550.

Furie R, Rovin B, Appel G, Kamen DL, Fervenza FC, Spindler A, et al. Effect of rituximab (RTX) on anti-dsDNA and C3 levels and relationship to response: results from the LUNAR trial [abstract no: 271]. ACR/ARHP Annual Scientific Meeting; 2009 Oct 17-21; Philadelphia (PA). 2009.

Furie R, Rovin BH, Kamen DL, Latinis KL, Appel GB, SanchezGuerrero J, et al. Trial design and baseline characteristics of patients in the randomized double-blind, placebo-controlled phase III lupus nephritis assessment with rituximab study (LUNAR) [abstract no: THU-0242]. Annals of the Rheumatic Diseases 2009;68(Suppl 3):253.

Rovin B, Appel G, Furie R, Fervenza F, Guerrero JS, Lenz O, et al. Trial design and baseline characteristics of patients in the randomized double-blind, placebo-controlled phase III LUpus Nephritis Assessment with Rituximab study (LUNAR) [abstract no: M353]. World Congress of Nephrology; 2009 May 22-26; Milan, Italy. 2009.

Rovin B, Appel G, Furie R, Kamen DL, Fervenza FC, Spindler A, et al. Effect of Rituximab (RTX) on anti-dsDNA and C3 levels and relationship to response: Results from the LUNAR Trial [abstract no: F-P01281]. Journal of the American Society of Nephrology 2009;20:406A.

Rovin BH, Appel G, Furie R, Looney J, Latinis K, Fervenza FC, et al. Efficacy and safety of rituximab (RTX) in subjects with proliferative lupus nephritis (LN): results from the randomized, double-blind phase III LUNAR study [abstract no: SA-FC332]. Journal of the American Society of Nephrology 2009;20:77A.

* Rovin BH, Furie R, Latinis K, Looney RJ, Fervenza FC, SanchezGuerrero J, et al. Efficacy and safety of rituximab in patients with active proliferative lupus nephritis: the Lupus Nephritis Assessment with Rituximab study. Arthritis \& Rheumatism 2012;64(4):1215-26. [MEDLINE: 22231479] 
MAINTAIN Nephritis 2010 \{published and unpublished data\}

* Houssiau FA, D'Cruz D, Sangle S, Remy P, Vasconcelos C, Petrovic R, et al. Azathioprine versus mycophenolate mofetil for long-term immunosuppression in lupus nephritis: results from the MAINTAIN Nephritis Trial. Annals of the Rheumatic Diseases 2010;69(12):2083-9. [MEDLINE: 20833738]

Houssiau FA, D'Cruz D, Sangle S, Remy P, Vasconcelos C, Petrovic $R$, et al. Longterm renal outcome in lupus nephritis patients can be predicted by the kinetics of proteinuria drop in response to therapy: data from the MAINTAIN NEPHRITIS trial [abstract no: SAT0193]. Annals of the Rheumatic Diseases 2010;69(Suppl 3):552.

Houssiau FA, D'Cruz DP, Sangle SR, Remy P, Vasconcelos C, De Ramon Garrido E, et al. Azathioprine versus mycophenolate mofetil for maintenance immunosuppression of proliferative lupus nephritis: results of a randomized trial (MAINTAIN) [abstract no: 1150]. ACR/ARHP Annual Scientific Meeting; 2009 Oct 17-21; Philadelphia (PA). 2009.

Stoenoiu MS, Aydin S, Tektonidou M, Ravelingien I, le Guern V, Fiehn C, et al. Repeat kidney biopsies fail to detect differences between azathioprine and mycophenolate mofetil maintenance therapy for lupus nephritis: data from the MAINTAIN Nephritis Trial. Nephrology Dialysis Transplantation 2012;27(5):1924-30. [MEDLINE: 22110048]

Tamirou F, D'Cruz D, Sangle S, Remy P, Vasconcelos C, Fiehn C, et al. Long-term follow-up of the MAINTAIN Nephritis Trial, comparing azathioprine and mycophenolate mofetil as maintenance therapy of lupus nephritis. Annals of the Rheumatic Diseases 2016;75(3):526-31. [MEDLINE: 25757867]

Tamirou F, Lauwerys BR, Dall'Era M, MacKay M, Rovin B, Cervera R, et al. A proteinuria cut-off level of $0.7 \mathrm{~g} /$ day after 12 months of treatment best predicts long-term renal outcome in lupus nephritis: data from the MAINTAIN Nephritis Trial. Lupus Science \& Medicine 2015;2(1):e000123. [MEDLINE: 26629352]

\section{Mehra 2018 \{published data only\}}

Mehra S, Usdadiya JB, Jain VK, Misra DP, Negi VS. Comparing the efficacy of low-dose vs high-dose cyclophosphamide regimen as induction therapy in the treatment of proliferative lupus nephritis: a single center study. Rheumatology International 2018;38(4):577-68. [MEDLINE: 29450636]

\section{Mendonca 2017 \{published data only\}}

Mendonca S, Gupta D, Ali S, Gupta P. Mycophenolate mofetil or cyclophosphamide in Indian patients with lupus nephritis: Which is better? A single-center experience. Saudi Journal of Kidney Diseases \& Transplantation 2017;28(5):1069-77. [MEDLINE: 28937065]

\section{Mitwalli 2011 \{published data only\}}

Mitwalli AH, Al Wakeel JS, Hurraib S, Aisha A, Al Suwaida A, Alam A, et al. Comparison of high and low dose of cyclophosphamide in lupus nephritis patients: a long-term randomized controlled trial. Saudi Journal of Kidney Diseases \& Transplantation 2011;22(5):935-40. [MEDLINE: 21912022]

\section{Mok 2016 \{published and unpublished data}

Mok C, Ying K, Tong K, Siu Y, To C, Yim C, et al. Mycophenolate mofetil versus tacrolimus for active lupus nephritis: an extended observation of a randomized controlled trial [abstract no: 1073]. ACR/ARHP Annual Scientific Meeting; 2008 Oct 24-29; San Francisco (CA). 2008.

Mok CC. Factors determining response in patients with acute lupus nephritis treated with glucocorticoids and mycophenolate mofetil (MMF) [abstract]. Arthritis and Rheumatism 2009;60(Suppl 10):923.

Mok CC, Ho LY, To CH, Chan KL. Factors associated with renal remission, relapse and long-term renal function decline in lupusnephritis treated with combined prednisolone and mycophenolate mofetil (MMF) or tacrolimus(TAC) [abstract no: 988]. Arthritis \& Rheumatology 2015;67(10 Suppl):988. [EMBASE: 72094876]

Mok CC, To CH, Ying KY, Yim C, Ng WL. Factors associated with long-term renal function deterioration in lupus nephritis treated initially with combined prednisolone and mycophenolate mofetil (MMF) or tacrolimus (TAC) [abstract no: 600]. Arthritis \& Rheumatism 2013;65(Suppl 10):S258-9. [EMBASE: 71318086]

Mok CC, Ying KY, Ng CW, Ng WL. Risk of renal flares and decline in renal function in patients with active lupus nephritis treated with mycophenolate mofetil (MMF) [abstract no: SAT0202]. Annals of the Rheumatic Diseases 2010;69(3):555.

Mok CC, Ying KY, Yim CW, Ng WL. Risk of renal flares and decline in renal function in patients with active lupus nephritis treated with mycophenolate mofetil (MMF) [abstract]. Arthritis and Rheumatism 2010;60(10 Suppl):477. [EMBASE: 70381867]

* Mok CC, Ying KY, Yim CW, Siu YP, Tong KH, To CH, et al. Tacrolimus versus mycophenolate mofetil for induction therapy of lupus nephritis: a randomised controlled trial and long-term follow-up. Annals of the Rheumatic Diseases 2016;75(1):30-6. [MEDLINE: 25550339]

Mok CC, Ying S, Yim CW, Ng WL. Factors determining response in patients with active lupus nephritis treated with glucocorticoids and mycophenolate mofetil (MMF) [abstract no: PO2.E.17]. Lupus 2010;19(1 Suppl):155.

Mok CC, Ying S, Yim CW, Ng WL. Tacrolimus (TAC) versus mycophenolate mofetil (MMF) for the treatment of membranous lupus nephritis: a randomized controlled trial [abstract no: OP0069]. Annals of the Rheumatic Diseases 2010;69(3):75.

Mok CC, Ying S, Yim CW, Ng WL. Tacrolimus (Tac) versus mycophenolate mofetil (MMF) for the treatment of membranous lupus nephritis: a randomized controlled trial [abstract no: CS6.8]. Lupus 2010;19(1 Suppl):16.

Mok CC, Ying SK, Tong KH, Siu YP, To CH, Yim CW, et al. Mycophenolate mofetil versus tacrolimus for active lupus nephritis: an extended observation of a randomized controlled trial [abstract no: THU0220]. Annals of the Rheumatic Diseases 2009;68(Suppl 3):246. 
Moroni 2006 \{published and unpublished data\}

Doria A, Ponticelli C, Mosca M, Ferraccioli GF, Moroni G, Todesco S, et al. A randomized trial comparing cyclosporine versus azathioprine for maintenance therapy in diffuse lupus nephritis [abstract]. Lupus 2004; 7th International Congress on SLE and Related Conditions; 2004 May 9-13; New York (NY). 2004.

* Moroni G, Doria A, Mosca M, Alberighi O, Ferraccioli G, Todesco S, et al. A randomized pilot trial comparing cyclosporine and azathioprine for maintenance therapy in diffuse lupus nephritis over four years. Clinical Journal of the American Society of Nephrology: CJASN 2006;1(5):925-32. [MEDLINE: 17699309]

Moroni G, Doria A, Mosca M, Ferraccioli G, Todesco S, Manno C, et al. A randomized trial comparing cyclosporine versus azathioprine for maintenance therapy in diffuse lupus nephritis [abstract no: F-PO255]. Journal of the American Society of Nephrology 2004;15(Oct):121A.

Moroni G, Doria A, Mosca M, Ferraccioli G, Todesco S, Schena P, et al. A randomized trial comparing cyclosporine versus azathioprine for maintenance therapy in diffuse lupus nephritis [abstract no: M030]. 41st Congress. European Renal Association. European Dialysis and Transplantation Association; 2004 May 15-18; Lisbon, Portugal. 2004:224.

Mosca M, Doria A, Moroni G, Ferrara R, Todesco S, Ponticelli C, et al. Induction therapy with oral cyclophosphamide in lupus nephritis [abstract no: THU0166]. Annual European Congress of Rheumatology EULAR; 2002 June 12-15; Stockholm, Sweden. 2002.

\section{Mulic-Bacic 2008 \{published and unpublished data\}}

Mulic-Bacic S, Antic D, Krizic M, Hajdarovic A, Mulic E. Mycophenolate mofetil or intravenous cyclophosphamide in treatment of lupus nephritis [abstract no: FRI0201]. Annals of the Rheumatic Diseases 2008;67(Suppl II):349.

\section{MyLupus 2011 \{published and unpublished data\}}

Jayne D, Amoura Z, Zeher M, Boletis J, Hiepe F. Enteric-coated mycophenolate sodium in patients with lupus nephritis: rationale and design of a multicenter randomized steroidsparing trial [abstract no: M-PO-1114]. 4th World Congress of Nephrology. 19th International Congress of the International Society of Nephrology (ISN); 2007 Apr 21-25; Rio de Janeiro, Brazil. 2007:357.

Jayne DR, Zeher M. Enteric-coated mycophenolate sodium (ED-MPS) for the treatment of lupus nephritis - MyLupus study [abstract no: SA-PO2253]. Journal of the American Society of Nephrology 2010;21:626A.

* Zeher M, Doria A, Lan J, Aroca G, Jayne D, Boletis I, et al. Efficacy and safety of enteric-coated mycophenolate sodium in combination with two glucocorticoid regimens for the treatment of active lupus nephritis. Lupus 2011;20(14):1484-93. [MEDLINE: 21976398]

Zeher M, Doria A, Lan JL, Aroca G, Amoura Z, Jayne D, et al. Efficacy and safety of enteric-coated mycophenolate sodium (EC-MPS) in combination with two corticosteroid regimens for the treatment of lupus nephritis flare - results of the MYLUPUS study [abstract no: SAT0213]. Annals of the Rheumatic Diseases 2010;69(3):559.

Zeher M, Lan J, Doria A, Amoura Z, Jayne D, Hiepe F. Enteric coated mycophenolate sodium (EC-MPS) treatment of lupus nephritis flare - interim results from a multicenter, randomized trial [abstract no: THU0234]. Annals of the Rheumatic Diseases 2009;68(Suppl 3):250.

\section{Nakamura 2002e \{published data only\}}

Nakamura T, Ushiyama C, Hara M, Osada S, Ugai K, Shimada N, et al. Comparative effects of plasmapheresis and intravenous cyclophosphamide on urinary podocyte excretion in patients with proliferative lupus nephritis. Clinical Nephrology 2002;57(2):108-13. [MEDLINE: 11863119]

\section{Ong 2005 \{published data only\}}

* Ong LM, Hooi LS, Lim TO, Goh BL, Ahmad G, Ghazalli R, et al. Randomized controlled trial of pulse intravenous cyclophosphamide versus mycophenolate mofetil in the induction therapy of proliferative lupus nephritis. Nephrology 2005;10(5):504-10. [MEDLINE: 16221103]

Ong LM, Hooi LS, Lim TO, Goh BL, Ahmad G, Ghazalli R, et al. Randomized controlled trial of pulse intravenous cyclophosphamide versus mycophenolate mofetil in the induction therapy of proliferative lupus nephritis [abstract no: FC30041]. Nephrology 2005;10(Suppl):A121.

\section{Pal 2017 \{published data only\}}

Pal A, Roychowdhury A, Ghosh P. Comparison of tacrolimusazathioprine combination versus cyclophosphamide for induction treatment of proliferative lupus nephritis [abstract no: OC014]. Indian Journal of Rheumatology 2017;12(5 Suppl 1):S9. [EMBASE: 619738235]

\section{Rathi 2016 \{published data only\}}

Goyal A, Rathi M, Jha V, Sharma A, Joshi K, Nada R, et al. Randomized controlled trial of low dose intravenous cyclophosphamide versus oral mycophenolate mofetil in treatment of lupus nephritis [abstract no: FR-OR053]. Journal of the American Society of Nephrology 2013;24(Abstracts):48A-9A.

Rathi M, Goyal A, Gupta PK, Jaryal A, Sharma A, Jha V, et al. Randomized controlled trial of low-dose intravenous cyclophosphamide versus oral mycophenolate mofetil in treatment of lupus nephritis [abstract]. Nephrology Dialysis Transplantation 2014;29(Suppl 3):iii28-iii2. [EMBASE: 71491534]

* Rathi M, Goyal A, Jaryal A, Sharma A, Gupta PK, Ramachandran $\mathrm{R}$, et al. Comparison of low-dose intravenous cyclophosphamide with oral mycophenolate mofetil in the treatment of lupus nephritis. Kidney International 2016;89(1):235-42. [MEDLINE: 26489028]

Rathi M, Jaryal A, Goyal A, Sharma A, Gupta P, Gupta K. Outcomes in lupus nephritis patients previously randomized to receive either low dose cyclophosphamide versus oral mycophenolate mofetil on azathioprine maintenance [abstract no: 2941]. Arthritis \& Rheumatology 2015;67(Suppl 10):2941. [EMBASE: 72096818] 
Rovin 2016 \{published data only\}

Aranow C, van Vollenhoven R, Rovin B, Wagner C, Zhou B, Gordon $\mathrm{R}$, et al. A phase 2, multicenter, randomized, doubleblind, placebo-controlled, proof-of-concept study to evaluate the efficacy and safety of sirukumab in patients with active lupus nephritis [abstract no: 2835]. Arthritis \& Rheumatology 2014;66(Suppl 10):S1239. [EMBASE: 71738820]

* Rovin B, van Vollenhoven R, Aranow C, Wagner C, Gordon R, Zhuang $\mathrm{Y}$, et al. A multicenter, randomized, double-blind, placebo-controlled study to evaluate the efficacy and safety of treatment with sirukumab (CNTO 136) in patients with active lupus nephritis. Arthritis \& Rheumatology 2016;68(9):2174-83. [MEDLINE: 27110697]

Rovin, BH, van Vollenhoven R, Aranow C, Wagner C, Gordon R, Hsu B, et al. Efficacy and safety of sirukumab in patients with active lupus nephritis: results from a phase 2 , multicenter, randomized, double-blind, placebo-controlled, proof-ofconcept study [abstract no: TH-PO426]. Journal of the American Society of Nephrology 2014;25:204A.

\section{Sabry 2009 \{published and unpublished data\}}

* Sabry A, Abo-Zenah H, Medhat T, Sheashaa H, Mahmoud K, El-Huseini A. A comparative study of two intensified pulse cyclophosphamide remission-inducing regimens for diffuse proliferative lupus nephritis: an Egyptian experience. International Urology \& Nephrology 2009;41(1):153-61. [MEDLINE: 18214709]

Sabry A, Sheashaa H, Mahmoud K, El-Huseini A, El Dahshan K. A comparative study of two intensified pulse cyclophosphamide remission-inducing regimen for diffuse proliferative lupus nephritis: an Egyptian experience [abstract no: FP036]. Nephrology Dialysis Transplantation 2007;22(Suppl 6):vi28.

\section{Sedhain 2016 \{published data only\}}

Sedhain A, Hada R, Agrawal RK, Baral A, Bhattarai GR. Effect of cyclophosphamide versus mycophenolate mofetil in induction therapy of lupus nephritis in Nepalese population [abstract no: FR-PO598]. Journal of the American Society of Nephrology 2016;27(Abstract Suppl):500A.

\section{Sesso 1994a \{published data only\}}

* Sesso R, Monteiro M, Sato E, Kirsztajn G, Silva L, Ajzen H. A controlled trial of pulse cyclophosphamide versus pulse methylprednisolone in severe lupus nephritis. Lupus 1994;3(2):107-12. [MEDLINE: 7920609]

Sesso R, Monteiro M, Silva L, Sato E, Ajzen H. Pulse cyclophosphamide $(\mathrm{CY})$ versus pulse methylprednisolone (MP) in severe lupus nephritis [abstract no: 97P]. Journal of the American Society of Nephrology 1993;4(Program \& Abstracts):286.

\section{SIMPL 2014 \{published data only\}}

Galbraith L, Manns B, Hemmelgarn B, Walsh M. The Steroids In the Maintenance of remission of Proliferative Lupus nephritis (SIMPL) pilot trial. Canadian Journal of Kidney Health \& Disease 2014;1:30. [MEDLINE: 25780619]

\section{Steinberg 1971 \{published data only\}}

Steinberg AD, Kaltreider HB, Staples PJ, Goetzl EJ, Talal N, Decker JL. Cyclophosphamide in lupus nephritis: a controlled trial. Annals of Internal Medicine 1971;75(2):165-71. [MEDLINE: 4104337]

\section{Sun 2015 \{published data only\}}

Sun J, Zhang H, Ji Y, Gui M, Yi B, Wang J, et al. Efficacy and safety of cyclophosphamide combined with mycophenolate mofetil for induction treatment of class IV lupus nephritis. International Journal of Clinical \& Experimental Medicine 2015;8(11):21572-8. [MEDLINE: 26885107]

Wallace 1998 \{published data only\}

Wallace DJ, Goldfinger D, Pepkowitz SH, Fichman M, Metzger Al, Schroeder JO, et al. Randomized controlled trial of pulse/ synchronization cyclophosphamide/apheresis for proliferative lupus nephritis. Journal of Clinical Apheresis 1998;13(4):163-6. [MEDLINE: 9886795]

\section{Yap 2017 \{published data only\}}

Yap DY, Lee P, Yam I, Tam CH, Wong S, Yung S, et al. Effect of cyclophosphamide and mycophenolate mofetil on lymphocyte subsets in patients with active lupus nephritis effect of cyclophosphamide and mycophenolate mofetil on lymphocyte subsets in patients with active lupus nephritis [abstract no: MP230]. Nephrology Dialysis Transplantation 2017;32(Suppl 3):iii510. [EMBASE: 617289724]

\section{Yee 2004 \{published data only\}}

Yee CS, Gordon C, Dostal C, Petera P, Dadoniene J, Griffiths B, et al. EULAR randomised controlled trial of pulse cyclophosphamide and methylprednisolone versus continuous cyclophosphamide and prednisolone followed by azathioprine and prednisolone in lupus nephritis. Annals of the Rheumatic Diseases 2004;63(5):525-9. [MEDLINE: 15082482]

\section{Zhang 1995a \{published data only\}}

Zhang J, Yang H. Cyclophosphamide (CTX) pulse therapy in lupus nephritis (LN): short term is better [abstract no: T235]. Nephrology Dialysis Transplantation 2003;18(Suppl 4):356.

Zhang JH, Yang H. Cyclophosphamide (CYX) pulse therapy in lupus nephritis (In): short term is better [abstract]. ISN XIII International Congress of Nephrology; 1995 Jul 2-6; Madrid (Spain). 1995:290.

\section{References to studies excluded from this review \\ Andrade-Ortega 2010 \{published data only\}}

Andrade-Ortega L, Irazoque-Palazuelos F, Lopez-Villanueva R, Barragan-Navarro Y, Bourget-Pietrasanta F, Diaz-Ceballos ML, et al. Efficacy of rituximab versus cyclophosphamide in lupus patients with severe manifestations. A randomized and multicenter study [Eficacia de rituximab comparado con ciclofosfamida en pacientes con manifestaciones graves de lupus eritematoso generalizado. Estudio aleatorizado y multicentrico]. Reumatologia Clinica 2010;6(5):250-5. [EMBASE: 2010499791] 
Antunes 2001 \{published data only\}

Antunes I, Woronik V, Sabagga E, Machado MM, Barros RT. ACE inhibition reduces proteinuria, hematuria and renal expression of inflammatory mediators in human lupus nephritis [abstract]. Journal of the American Society of Nephrology 2001;12(Program \& Abstracts):90A. [CENTRAL: CN-00444198]

\section{ASPEN 2008 \{published data only\}}

Linnik MD, Tansey MJ, Joh T. Dose dependent reduction in antidsDNA antibody levels observed with abetimus sodium through 52 weeks in the phase 3 ASPEN study (abetimus sodium in patients with a history of lupus nephritis) [abstract no: 1077]. ACR/ARHP Annual Scientific Meeting; 2008 Oct 24-29; San Francisco (CA). 2008.

Tansey MJ, Joh T, Linnik MD. Interim analysis reveals dose dependent reduction in anti-dsDNA antibody levels with Abetimus sodium through 52 weeks in the phase 3 ASPEN study (Abetimus Sodium in Patients with a history of lupus Nephritis) [abstract no: SA-PO2958]. Journal of the American Society of Nephrology 2008;19(Abstracts issue):781A.

Tansey MJ, Joh T, Linnik MD. Interim analysis reveals dose dependent reduction in anti-dsDNA antibody levels with Abetimus sodium through 52 weeks in the phase 3 ASPEN study (Abetimus Sodium in patients with a History of lupus Nephritis) [abstract no: THU0237]. Annals of the Rheumatic Diseases 2009;68(Suppl 3):251.

\section{ATLAS 2016 \{published data only\}}

Furie R, Malyar V. Efficacy, safety, and tolerability of BIIB023 as an adjunct to standard of care in subjects with lupus nephritis [abstract no: 749]. Arthritis \& Rheumatology 2017;68(Suppl 10):749.

Rovin B, Wofsy D, Jayne DR, Mysler E, Smirnakis K, Duffield JS, et al. Results from the ATLAS Trial: a phase 2 study to evaluate efficacy and safety of BIIB023 in subjects with lupus nephritis [abstract no:TH-OR051]. Journal of the American Society of Nephrology 2016;27(Abstract Suppl):13A.

\section{Austin 2009 \{published and unpublished data\}}

Austin HA III, Illei GG, Braun MJ, Balow JE. Randomized, controlled trial of prednisone, cyclophosphamide, and cyclosporine in lupus membranous nephropathy. Journal of the American Society of Nephrology 2009;20(4):901-11. [MEDLINE: 19297556]

Austin HA, Balow JE. Long-term observations in a prospective clinical trial of prednisone, cyclosporine and cyclophosphamide for lupus membranous nephropathy (LMN) [abstract no: SU-FO048]. Journal of the American Society of Nephrology 2004;15(Oct):54A.

Austin HA, Vaughan EM, Balow JE. Lupus membranous nephropathy: randomized controlled trial of prednisolone, cyclosporine and cyclophosphamide [abstract no: A0439]. Journal of the American Society of Nephrology 2000;11:81A.

Austin HA, Vaughan EM, Boumpas DT, Klippel JH, Balow JE. Lupus membranous nephropathy: controlled trial of prednisolone, pulse cyclophosphamide, and cyclosporine
A [abstract no: A0411]. Journal of the American Society of Nephrology 1996;7(9):1328.

Balow 1981 \{published data only\}

Balow JE, Dinant HJ, Decker JL, Klippel JH, Plotz PH, Steinberg AD. Clinical trial of cyclophosphamide plus azathioprine and pulse cyclophosphamide in lupus nephritis [abstract]. Kidney International 1981;19:119.

Balow 1984 \{published data only\}

Balow J, Austin H, Muenz L, Joyce K, Klippel J, Antonovych T. Influence of treatment on the evolution of renal pathology in lupus nephritis [abstract]. Kidney International 1984;25(1):158.

Ble 2011 \{published data only\}

Ble A, Mosca M, Di Loreto G, Guglielmotti A, Biondi G, Bombardieri S, et al. Antiproteinuric effect of chemokine $\mathrm{C}-\mathrm{C}$ motif ligand 2 inhibition in subjects with acute proliferative lupus nephritis. American Journal of Nephrology 2011;34(4):367-72. [MEDLINE: 21876349]

\section{Chanchairujira 2009 \{published data only\}}

Chanchairujira T, Mahasukont J, Shayakul C, Ong-Aj-Yooth L, Cheunsuchon B, Parichaitiranond P. Histopathological outcome in proliferative lupus nephritis patients who did not achieve good response after randomized to eurolupus or high-dose IV cyclophosphamide [abstract no: TH-PO861]. Journal of the American Society of Nephrology 2009;20:309A.

Clark 1993 \{published and unpublished data\}

Clark WF, Parbtani A, Naylor CD, Levinton CM, Muirhead N, Spanner $\mathrm{E}$, et al. Fish oil in lupus nephritis: clinical findings and methodological implications. Kidney International 1993:44(1):75-86. [MEDLINE: 8355469]

Clark WF, Parbtani A, Naylor CD, Muirhead N, Huff MW, Philbrick DJ, et al. Fish oil in lupus nephritis a double-blind randomized crossover study [abstract no: 37P]. Journal of the American Society of Nephrology 1992;3(3):309.

\section{Clark 2001a \{published and unpublished data\}}

Clark WF, Kortas C, Heidenheim AP, Garland J, Spanner E, Parbtani A. Flaxseed in lupus nephritis: a two-year nonplacebocontrolled crossover study. Journal of the American College of Nutrition 2001;20(2 Suppl):143-8. [MEDLINE: 11349937]

Clark WF, Parbtani A, Macdonald G, Heidenheim P, Holub B, Kortas $C$. Flaxseed in lupus nephritis: a two year randomized cross-over study [abstract no: T141]. Journal of the American Society of Nephrology 1998;9(Program \& Abstracts):143A.

\section{CONTROL 2016 \{published data only\}}

Anutrakulchai S, Panaput T, Wongchinsri J, Chaishayanon S, Satirapoj B, Traitanon O, et al. A multicentre, randomised controlled study of enteric-coated mycophenolate sodium for the treatment of relapsed or resistant proliferative lupus nephritis: an Asian experience. Lupus Science \& Medicine 2016;3(1):e000120. [MEDLINE: 26835147] 
Davis 1999 \{published data only\}

Davis JC, Manzi S, Yarboro C, Rairie J, Mcinnes I, Averthelyi D, et al. Recombinant human Dnase I (rhDNase) in patients with lupus nephritis. Lupus 1999;8(1):68-76.

\section{Daza 2005 \{published data only\}}

Daza L, Kornhauser C, Zamora L, Flores J. Captopril effect on prostaglandin E2, thromboxane B2 and proteinuria in lupus nephritis patients. Prostaglandins \& Other Lipid Mediators 2005;78(1-4):194-201.

\section{Deng 2017a \{published and unpublished data\}}

Deng D, Zhang P, Guo Y, Lim TO. A randomised double-blind, placebo-controlled trial of allogeneic umbilical cord-derived mesenchymal stem cell for lupus nephritis. Annals of the Rheumatic Diseases 2017;76(8):1436-9. [MEDLINE: 28478399]

\section{Feng 2014 \{published data only\}}

Feng X, Gu F, Chen W, Liu Y, Wei H, Liu L, et al. Mizoribine versus mycophenolate mofetil or intravenous cyclophosphamide for induction treatment of active lupus nephritis. Chinese Medical Journal 2014;127(21):3718-23. [MEDLINE: 25382325]

\section{Frutos 1997 \{published data only\}}

Frutos MA, Valera A, Martinez JM, Martin-Reyes G, Lopez de Novales E. Intravenous cyclophosphamide pulse therapy for severe lupus nephritis: finding an optimal dose and duration [abstract no: P1614]. Nephrology 1997;3(Suppl 1):S476.

\section{Hebert 1987 \{published data only\}}

Hebert I, Nielsen E, Pohl M, Lachin J, Hunsicker L, Lewis E. Clinical course of severe lupus nephritis during the controlled clinical trial of plasmapheresis [abstract]. Kidney International 1987;31(1):201.

\section{Khajehdehi 2012 \{published data only\}}

Khajehdehi P, Zanjaninejad B, Aflaki E, Nazarinia M, Azad F, Malekmakan L, et al. Oral supplementation of turmeric decreases proteinuria, hematuria, and systolic blood pressure in patients suffering from relapsing or refractory lupus nephritis: a randomized and placebo-controlled study. Journal of Renal Nutrition 2012;22(1):50-7. [MEDLINE: 21742514]

\section{Kuo 2001 \{published data only\}}

Kuo GM, Boumpas DT, Illei GG, Yarboro C, Pucino F, Burstein AH. Fludarabine pharmacokinetics after subcutaneous and intravenous administration in patients with lupus nephritis. Pharmacotherapy 2001;21(5):528-33. [MEDLINE: 11349741]

\section{Li 2005 \{published data only\}}

Li L, Zhang H, Shen S, Hu W, Liu Z. Controlled trial of tacrolimus (FK506) vs intravenous cyclophosphamide (IVC) as induction therapy for severe lupus nephritis [abstract no: F-PO1006]. Journal of the American Society of Nephrology 2005;16:556A.

\section{Li 2014a \{published data only\}}

Li M, Ma JJ, Zhao XL, Zhu Y. Treating lupus nephritis by a drug pair of radix astragali and rehmanniae radix combined with glucocorticoid: a preliminary clinical study. Zhongguo Zhongxiyi Jiehe Zazhi [Chinese Journal of Integrated Traditional and Western Medicine] 2014;34(8):956-9. [MEDLINE: 25223180]

\section{LJP 394-90-05 2003 \{published data only\}}

Alarcon-Segovia D, Tumlin J, Furie R, McKay J, Cariel M, Linnik M, et al. SLE trial shows fewer renal flares in LJP 394treated patients with high affinity antibodies to LJP 394: 90-05 trial results [abstract]. Arthritis \& Rheumatism 2000;43:S272.

Alarcon-Segovia D, Tumlin JA, Furie RA, McKay JD, Cardiel MH, Strand V, et al. LJP 394 for the prevention of renal flare in patients with systemic lupus erythematosus: results from a randomized, double-blind, placebo-controlled study. Arthritis \& Rheumatism 2003;48(2):442-54. [MEDLINE: 12571854]

Hura C, Tumlin JA, Heilbrunn KR. Integrated safety results from studies of LJP394 in SLE patients [abstract no: FP0218]. Journal of the American Society of Nephrology 2004;15(Oct):113A.

Linnik MD, Hu JZ, Heilbrunn KR, Strand V, Hurley FL, Joh T. AntidsDNA antibodies and exacerbation of renal disease in patients with systemic lupus erythematosus: results from 2 randomized controlled trials with LJP394 [abstract]. Lupus 2004; 7th International Congress on SLE and Related Conditions; 2004 May 9-13; New York (NY). 2004.

Linnik MD, Hu JZ, Heilbrunn KR, Strand V, Hurley FL, Joh T, et al. Relationship between anti-double-stranded DNA antibodies and exacerbation of renal disease in patients with systemic lupus erythematosus. Arthritis \& Rheumatism 2005;52(4):1129-37. [MEDLINE: 15818711]

Linnik MD, Joh T, Tumlin JA. Reductions in anti-dsDNA antibodies and reduced risk of SLE renal flare and major SLE flare [abstract no: SA-P0695]. Journal of the American Society of Nephrology 2003;14(Nov):450A.

Tumlin JA, Cardiel MH, Furie RA, Wallace DJ, Hura C. Renal flare in SLE patients with impaired renal function in a RCT of LJP 394 [abstract no: SA-P0693]. Journal of the American Society of Nephrology 2003;14(Nov):449A.

Tumlin JA, Cardiel MH, Furie RA, Wallace DJ, Hura C, Foster T, et al. Reductions in 24 hour urine protein levels associated with treatment of lupus patients withLJP 394 in two randomized, placebo controlled, double-blind clinical trials [abstract]. Lupus 2004; 7th International Congress on SLE and Related Conditions; 2004 May 9-13; New York (NY). 2004.

Tumlin JA, Hura C, Joh T, Heilbrunn KR. Reductions in 24-hour urine protein levels associated with treatment of SLE patients with LJP394 in two randomized, placebo-controlled clinical trials [abstract no: F-PO256]. Journal of the American Society of Nephrology 2004;15(Oct):121A.

Tumlin JA, Linnik MD, Appel GB. The B-cell tolerogen LJP-394 reduces renal flares in patients with lupus nephritis: a prospective, double-blinded, placebo controlled trial [abstract]. Journal of the American Society of Nephrology 2001;12(Program \& Abstracts):253A.

\section{LJP 394-90-09 2005 \{published and unpublished data\}}

Cardiel MH, LJP 394-90-09 Study Investigators. Randomized, placebo controlled, double blind phase III trial for the evaluation of LJP 394 (Abetimus sodium) in the treatment of 
patients with SLE who are at risk of renal flare [abstract no: OP0053]. Annual European Congress of Rheumatology EULAR; 2003 June 18-21; Lisbon (Portugal). 2003

Cardiel MH, Tumlin JA, Furie RA, Wallace DJ, Joh T, Linnik MD, et al. Abetimus sodium for renal flare in systemic lupus erythematosus: results of a randomized, controlled phase III trial. Arthritis \& Rheumatism 2008;58(8):2470-80. [MEDLINE: 18668592]

Hura C, Tumlin JA, Heilbrunn KR. Integrated safety results from studies of LJP394 in SLE patients [abstract no: FP0218]. Journal of the American Society of Nephrology 2004;15(Oct):113A.

Linnik MD, Hu JZ, Heilbrunn KR, Strand V, Hurley FL, Joh T. AntidsDNA antibodies and exacerbation of renal disease in patients with systemic lupus erythematosus: results from 2 randomized controlled trials with LJP394 [abstract]. Lupus 2004; 7th International Congress on SLE and Related Conditions; 2004 May 9-13; New York (NY). 2004.

Linnik MD, Hu JZ, Heilbrunn KR, Strand V, Hurley FL, Joh T, et al. Relationship between anti-double-stranded DNA antibodies and exacerbation of renal disease in patients with systemic lupus erythematosus. Arthritis \& Rheumatism 2005;52(4):1129-37. [MEDLINE: 15818711]

Linnik MD, Joh T, Tumlin JA. Reductions in anti-dsDNA antibodies and reduced risk of SLE renal flare and major SLE flare [abstract no: SA-P0695]. Journal of the American Society of Nephrology 2003;14(Nov):450A.

Tumlin JA, Cardiel MH, Furie RA, Wallace DJ, Hura C. Renal flare in SLE patients with impaired renal function in a RCT of LJP 394 [abstract no: SA-P0693]. Journal of the American Society of Nephrology 2003;14(Nov):449A.

Tumlin JA, Cardiel MH, Furie RA, Wallace DJ, Hura C, Foster T, et al. Reductions in 24 hour urine protein levels associated with treatment of lupus patients with LJP 394 in two randomized, placebo controlled, double-blind clinical trials [abstract]. Lupus 2004; 7th International Congress on SLE and Related Conditions; 2004 May 9-13; New York (NY). 2004.

Tumlin JA, Hura C, Appel G. Efficacy results from a RCT of LJP 394 in SLE patients with history of renal disease [abstract no: SA-P0694]. Journal of the American Society of Nephrology 2003;14(Nov):449A-50A.

Tumlin JA, Hura C, Heilbrunn KR. The effect of LJP394 and concomitant immunosuppressive agents on levels of anti-dsDNA antibodies in SLE patients [abstract no: PUB137]. Journal of the American Society of Nephrology 2004;15(Oct):791A.

Tumlin JA, Hura C, Joh T, Heilbrunn KR. Reductions in 24-hour urine protein levels associated with treatment of SLE patients with LJP394 in two randomized, placebo-controlled clinical trials [abstract no: F-PO256]. Journal of the American Society of Nephrology 2004;15(Oct):121A.

Wallace DJ, Cardiel MH, Tumlin JA, Furie R, Hura C, Strand V, et al. Safety results from a RCT of LJP 394 in SLE patients with a history of renal disease [abstract no: SA-P0692]. Journal of the American Society of Nephrology 2003;14(Nov):449A.

Lu 2002 \{published data only\}

Lu L. Study on effect of Cordyceps sinensis and artemisinin in preventing recurrence of lupus nephritis. Zhongguo Zhongxiyi Jiehe Zazhi [Chinese Journal of Integrated Traditional and Western Medicine] 2002;22(3):169-71. [MEDLINE: 12585097]

Miyasaka 2009 \{published data only\}

Miyasaka N, Kawai S, Hashimoto H. Efficacy and safety of tacrolimus for lupus nephritis: a placebo-controlled double-blind multicenter study. Modern Rheumatology 2009;19(6):606-15. [MEDLINE: 19688181]

\section{NCT00001212 \{published data only\}}

NCT00001212. Immunosuppressive drug therapy in membranous lupus nephropathy. www.clinicaltrials.gov/ct2/ show/NCT00001212 (first received 3 November 1999).

\section{NCT00404157 \{published data only\}}

Garg J. A study to evaluate the safety of rituximab retreatment in subjects with lupus nephritis previously enrolled in study U2970g. www.clinicalTrials.gov/show/NCT00404157 (first received 27 November 2006)

\section{NCT00429377 \{published data only\}}

NCT00429377. Phase 3 study of tacrolimus (FK506) for lupus nephritis: a placebo controlled, double-blind multicenter, comparative study. www.clinicaltrials.gov/ct2/show/ NCT00429377 (first received 29 January 2007).

\section{NCT00436438 \{published data only\}}

NCT00425438. A randomized, open-label study to compare the effect of cellcept plus corticosteroids, and cyclophosphamide plus corticosteroids followed by azathioprine, on remission rate in patients with lupus nephritis. www.clinicaltrials.gov/ct2/ show/NCT00425438 (first received 23 January 2007).

\section{NCT00539799 \{unpublished data only\}}

Jayne D, Walsh M. Corticosteroids in the maintenance therapy of proliferative lupus nephritis: a randomized pilot study (SIMPL). www.clinicaltrials.gov/ct2/show/NCT00539799 (first received 4 October 2007).

\section{NCT00659217 \{published and unpublished data\}}

Tan JM. Effect of mesenchymal stem cell transplantation for lupus nephritis. www.clinicaltrials.gov/ct2/show/NCT00659217 (first received 14 April 2008).

\section{NCT01299922 \{published data only\}}

Praga M, Gema FJ. Phase III, open, randomized, parallel-group clinical trial, to evaluate the efficacy and safety of treatment with prednisone, cyclosporine, mycophenolic acid versus prednisone and mycophenolic acid in lupus nephritis type III-IVV. www.clinicaltrials.gov/ct2/show/NCT01299922 (first received 18 February 2011).

\section{NCT01342016 \{published data only\}}

NCT01342016. A randomized, double-blind double dummy, parallel control and multi-center clinical trial to compare the 
efficacy and safety of tacrolimus capsules in treatment of lupus nephritis with leflunomide tablets. www.clinicaltrials.gov/ct2/ show/NCT01342016 (first received 25 April 2011).

\section{NCT01930890 \{published data only\}}

NCT01930890. BIIB023 long-term extension study in subjects with lupus nephritis. www.clinicaltrials.gov/ct2/show/ NCT01930890 (first received 29 August 2013).

\section{NCT02176486 \{published data only\}}

NCT02176486. A phase 1b, randomized, double-blind, placebo-controlled, safety, tolerability and pharmacokinetic study of multiple rising doses of MLN9708 for the treatment of subjects with ISN/RPS class III or IV lupus nephritis. www.clinicaltrials.gov/ct2/show/NCT02176486 (first received 25 June 2014).

\section{Pierucci 1989 \{published data only\}}

Pierucci A, Simonetti BM, Pecci G, Feriozzi S, Mavrikakis G, Cinotti GA, et al. Low does aspirin in patients with lupus nephritis [abstract]. Kidney International 1988;33(1):281.

Pierucci A, Simonetti BM, Pecci G, Mavrikakis G, Feriozzi S, Cinotti GA, et al. Acute effects of a thromboxane receptor antagonist on renal function in patients with lupus nephritis [abstract]. Kidney International 1987;31(1):283.

Pierucci A, Simonetti BM, Pecci G, Mavrikakis G, Feriozzi S, Cinotti GA, et al. Improvement of renal function with selective thromboxane antagonism in lupus nephritis. New England Journal of Medicine 1989;320(7):421-5. [MEDLINE: 2643773]

\section{Schaumann 1992 \{published data only\}}

Schaumann D, Hein R, Neumann KH, Brunkhorst R, Schmidt RE, Koch KM. Short term cyclophosphamide (CP) bolus therapy for lupus nephritis [abstract]. Journal of the American Society of Nephrology 1994;5(3):360A.

Schaumann D, Hein R, Schmidt RE, Brunkhorst R, Neumann KH, Deicher $\mathrm{H}$, et al. Intravenous cyclophosphamide (CP) pulse therapy for lupus nephritis - duration of induction therapy [abstract no: 028]. International Symposium on Pathogenesis, Pathophysiology \& Therapy of Glomerulonephritis; 1992 Dec 3-5; Bruges, Belgium. 1992:60.

\section{Su 2007 \{published data only\}}

Su L, Mao JC, Gu JH. Effect of intravenous drip infusion of cyclophosphamide with high-dose Astragalus injection in treating lupus nephritis. Zhong Xi Yi Jie He Xue Bao [Journal of Chinese Integrative Medicine] 2007;5(3):272-5. [MEDLINE: 17498486]

\section{Sztejnbok 1971 \{published data only\}}

Sztejnbok M, Stewart A, Diamond H, Kaplan D. Azathioprine in the treatment of systemic lupus erythematosus. A controlled study. Arthritis \& Rheumatism 1971;14(5):639-45.

\section{Wallace 2006 \{published data only\}}

Wallace DJ, Lisse J, Stohl W, McKay J, Boling E, Merrill JT, et al. Belimumab (BmAb) reduces SLE disease activity and demonstrates durable bioactivity at 76 weeks [abstract no:
2012]. ACR/ARHP Annual Scientific Meeting; 2006 Nov 11-15; Washington (DC). 2006.

\section{Wang 2007 \{published data only\}}

Wang J, Hu W, Xie H, Zhang H, Chen H, Zeng C, et al. Induction therapies for class IV lupus nephritis with non-inflammatory necrotizing vasculopathy: mycophenolate mofetil or intravenous cyclophosphamide. Lupus 2007;16(9):707-12. [MEDLINE: 17728363]

\section{Witte 1993 \{published data only\}}

Hein R, Neumann KH, Koch KM, Deicher H, Schmidt RE. Cyclophosphamide pulse therapy of systemic lupus erythematosus with renal involvement [CyclophosphamidStosstherapie des systemischen Lupus erythematodes mit Nierenbeteiligung]. Immunitat und Infektion 1991;19(2):57-8. [MEDLINE: 1855811]

Witte T, Schaumann D, Hein R, Helmchen U, Neumann KH, Koch KM, et al. Cyclophosphamide bolus therapy in lupus nephritis [Cyclophosphamid-Bolustherapie bei Lupusnephritis]. Deutsche Medizinische Wochenschrift 1993;118(27-28):1005-10. [MEDLINE: 8334946]

Witte T, Schaumann D, Hein R, Neumann KH, Koch KM, Deicher $\mathrm{H}$, et al. Cyclophosphamide bolus therapy in lupus nephritis - status of the clinical study [CyclophosphamidbolusTherapie bei Lupusnephritis--Stand der Therapiestudie]. Immunitat und Infektion 1993;21(Suppl 1):24-6. [MEDLINE: 8344680]

\section{Yap 2012 \{published data only\}}

Yap DY, Yu X, Chen XM, Lu F, Chen N, Li XW, et al. Pilot 24 month study to compare mycophenolate mofetil and tacrolimus in the treatment of membranous lupus nephritis with nephrotic syndrome. Nephrology 2012;17(4):352-7. [MEDLINE: 22295934]

\section{Ye 2001 \{published data only\}}

Ye Z, Tan YH, Hong XP, Feng XX, Xiao XL. MMF vs CTX in the treatment of severe SLE patients [abstract no: P1610]. Lupus 2001;10(Suppl):S99.

\section{Yoshida 1996 \{published data only\}}

Yoshida T, Kameda H, Ichikawa Y, Tojo T, Homma M. Improvement of renal function with a selective thromboxane A2 synthetase inhibitor, DP-1904, in lupus nephritis. Journal of Rheumatology 1996;23(10):1719-24. [MEDLINE: 8895147]

Yoshida T, Kameda H, Masashi A, Homma M, Ikeda Y. Improvement of renal function with selective thromboxane A2 synthetase inhibitor, DP-1904 in lupus nephritis. Advances in Experimental Medicine \& Biology 1997;433:113-7. [MEDLINE: 9561116]

\section{Zhang 2015c \{published and unpublished data\}}

Zhang J, Zhao Z, Hu X. Effect of Rituximab on serum levels of anti-C1q and antineutrophil cytoplasmic autoantibodies in refractory severe lupus nephritis. Cell Biochemistry \& Biophysics 2015;72(1):197-201. [MEDLINE: 25490907] 
Zheng 2005a \{published data only\}

Zheng WC, Hu SJ, Fang Q. Intervention of liuwei dihuang pill on lupus nephropathy treated with cyclophosphamide and glucocorticoids. Zhongguo Zhongxiyi Jiehe Zazhi [Chinese Journal of Integrated Traditional and Western Medicine] 2005;25(11):983-5.

\section{References to ongoing studies}

\section{2nd Dutch Lupus Trial \{published data only\}}

Bijl M. Comparison of short course cyclophosphamide followed by mycophenolate mofetil versus long course cyclophosphamide in the treatment of proliferative lupus nephritis. www.controlled-trials.com/ISRCTN34634478 (first received 27 January 2006).

\section{ChiCTR-TRC-09000587 \{published data only\}}

Li Z. The intensive therapy of severe lupus nephritis: a multicenter, randomized, controlled prospective clinical trial. www.chictr.org.cn/showprojen.aspx?proj=8948 (first received 1 October 2009).

\section{ChiCTR-TRC-10000931 \{published data only\}}

Mei C. Treatment of severe lupus nephritis with tacrolimus (FK 506) based immunosuppression. www.chictr.org.cn/ showprojen.aspx?proj=8607 (first received 9 July 2010).

\section{CTRI/2016/01/006488 \{published data only\}}

Gupta KL. Comparison of two steroid dose regimen in lupus nephritis. www.ctri.nic.in/Clinicaltrials/pdf_generate.php? trialid $=13735 \&$ EncHid $=\&$ modid $=\&$ compid $=\% 27, \% 2713735 \mathrm{det}$ $\% 27$ (first received 7 January 2016).

\section{CTRI/2017/05/008697 \{published data only\}}

Gupta KL. A study to compare the efficacy of cyclophosphamide versus multi-drug therapy in the treatment of lupus nephritis. www.ctri.nic.in/Clinicaltrials/showallp.php? mid1 $=19083 \&$ EncHid $=\&$ userName $=$ CTRI/2017/05/008697 (first received 30 May 2017).

\section{ISRCTN66475575 \{published data only\}}

Chartapisak W. Enteric coat mycophenolate sodium versus intravenous cyclophosphamide for severe paediatric lupus nephritis. www.isrctn.com/ISRCTN66475575 (first received 29 July 2009).

\section{NCT00302549 \{published data only\}}

Li LS. To compare the efficacy and safety of FK506 vs IVC in the treatment of class III-IV LN. www.clinicaltrials.gov/ct2/show/ NCT00302549 (first received 14 March 2006).

\section{NCT00705367 \{published data only\}}

NCT00705367. Phase I study in China - tolerability of a single dose of abatacept $30 \mathrm{mg} / \mathrm{kg}$. www.clinicaltrials.gov/show/ NCT00705367 (first received 26 June 2008).

NCT00881309 \{published data only\}

Hu W. To compare the efficacy and safety of tripterygium (TW) vs AZA in the maintenance therapy for lupus nephritis.
www.clinicaltrials.gov/ct2/show/NCT00881309 (first received 14 April 2009).

NCT01056237 \{published data only\}

Liu ZH. Long-term study of multi-target therapy as maintenance treatment for lupus nephritis. www.clinicaltrials.gov/ct2/show/ NCT01056237 (first received 26 January 2010).

NCT01172002 \{published data only\}

NCT01172002. A randomized multicenter trial comparing leflunomide and azathioprine as remission-maintaining treatment for proliferative lupus glomerulonephritis. www.clinicaltrials.gov/ct2/show/NCT01172002 (first received 27 July 2010).

NCT01284725 \{published data only\}

Belaigues, B. Weaning of Immunosuppression in Nephritis of Lupus (WIN-Lupus). www.clinicaltrials.gov/ct2/show/ NCT01284725 (first received 21 January 2011).

NCT01639339 \{published data only\} NCT01639339. A phase 3, randomized, double-blind, placebocontrolled study to evaluate the efficacy and safety of belimumab plus standard of care versus placebo plus standard of care in adult subjects with active lupus nephritis (BLISS-LN). www.clinicaltrials.gov/ct2/show/NCT01639339 (first received 10 July 2012).

\section{NCT01714817 \{published data only\}}

NCT01714817. Efficacy and safety study of abatacept to treat lupus nephritis. www.clinicaltrials.gov/ct2/show/NCT01714817 (first received 26 October 2012).

\section{NCT01845740 \{published data only\}}

Wegener W. Phase Ib study of SC milatuzumab in SLE. www.clinicaltrials.gov/ct2/show/NCT01845740 (first received 3 May 2013).

NCT01861561 \{published data only\}

Piyaphanee N, Pattaragarn A. Efficacy and infectious complications of induction therapy with low-dose versus high-dose intravenous cyclophosphamide for proliferative lupus nephritis in children. www.clinicaltrials.gov/ct2/show/ NCT01861561 (first received 19 May 2013).

\section{NCT02226341 \{published data only\}}

NCT02226341. Open-label prospective randomized study to determine the efficacy and safety of two dosing regimens of ACTHar in the treatment of proliferative lupus nephritis. www.clinicaltrials.gov/ct2/show/NCT02226341 (first received 25 August 2014)

\section{NCT02256150 \{published data only\}}

NCT02256150. A multi-center, randomized, controlled, openlabel clinical study to evaluate the efficacy and safety of mizoribine in comparison with cyclophosphamide in the treatment of lupus nephritis. www.clinicaltrials.gov/ct2/show/ NCT02256150 (first received 1 October 2014). 


\section{NCT02260934 \{published data only\}}

Diamond B, Wofsy D, Dall'Era M, Aranow C. Rituximab plus cyclophosphamide followed by belimumab for the treatment of lupus nephritis (ITN055AI) (CALIBRATE). www.clinicaltrials.gov/ ct2/show/NCT02260934 (first received 6 October 2014).

\section{NCT02457221 \{published data only\}}

NCT02457221. A phase III, randomized, open, parallelcontrolled, multi-center study to compare the efficacy and safety of tacrolimus capsules and cyclophosphamide injection in treatment of lupus nephritis. www.clinicaltrials.gov/ct2/ show/NCT02457221 (first received 22 May 2015).

\section{NCT02547922 \{published data only\}}

NCT02547922. A multicentre, randomised, double-blind, placebo-controlled, phase 2 study evaluating the efficacy and safety of anifrolumab in adult subjects with active proliferative lupus nephritis (TULIP-LN1). www.clinicaltrials.gov/ct2/show/ NCT02547922 (first received 31 August 2015).

\section{NCT02550652 \{published data only\}}

NCT02550652. A randomized, double-blind, placebo-controlled, multi-center study to evaluate the safety and efficacy of obinutuzumab in subjects with ISN/RPS 2003 class III or IV lupus nephritis. www.clinicaltrials.gov/ct2/show/NCT02550652 (first received 14 September 2015).

\section{NCT02630628 \{published data only\}}

Chan DT. A randomized open-label study to evaluate the efficacy and safety of tacrolimus and corticosteroids in comparison with mycophenolate mofetil and corticosteroids in subjects with class III/IV \pm V lupus nephritis. www.clinicaltrials.gov/ct2/show/NCT02630628 (first received 5 December 2015).

\section{NCT02770170 \{published data only\}}

NCT02770170. A double-blind, randomised, placebocontrolled trial evaluating the effect of BI 655064 administered as sub-cutaneous injections, on renal response after one year treatment in patients with lupus nephritis. www.clinicaltrials.gov/ct2/show/NCT02770170 (first received 11 May 2016).

\section{NCT02936375 \{published data only\}}

Bao C. Iguratimod as treatment for active lupus nephritis. www.clinicaltrials.gov/ct2/show/NCT02936375 (first received12 October 2016).

\section{NCT02954939 \{published data only\}}

Yap D. A randomized, controlled double-blind study comparing the efficacy and safety of orelvo (voclosporin) (23.7 mg twice daily) with placebo in achieving renal response in subjects with active lupus nephritis. www.clinicaltrials.gov/ct2/show/ NCT02954939 (first received 13 September 2016).

\section{NCT03021499 \{published data only\}}

Dooley MA. Aurinia renal response in active lupus with voclosporin. www.clinicaltrials.gov/ct2/show/NCT03021499 (first received 16 January 2017).

\section{NCT03214731 \{published data only\}}

Yu XQ. Efficacy and safety of artesunate plus standard of care in active lupus nephritis. www.clinicaltrials.gov/ct2/show/ NCT03214731 (first received 12 July 2017).

\section{PER-062-15 \{published data only\}}

PER-062-15. A multicentre, randomised, double-blind, placebocontrolled, phase 3 study evaluating the efficacy and safety of two doses of anifrolumab in adult subjects with active proliferative lupus nephritis. www.ins.gob.pe/ensayosclinicos/ $\mathrm{rpec} /$ recuperarECPBNuevoEN.asp? numec=062-15 (first received 4 May 2016).

\section{RING 2015 \{published data only\}}

Houssiau FA. RING, an investigator-initiated trial aimed at testing the efficacy of rituximab in refractory lupus nephritis: rationale, trial design and call for participation [abstract]. Clinical \& Experimental Rheumatology 2015;33(3 Suppl 90):S30. [EMBASE: 71976757; NCT01673295]

RITUXILUP 2013 \{published data only\}

Lightstone L. Phase 3 open label randomised multicentre controlled trial of rituximab and mycophenolate mofetil without oral steroids for the treatment of lupus nephritis (RITUXILUP). www.clinicaltrials.gov/ct2/show/NCT01773616 (first received 23 January 2013).

\section{Additional references}

\section{Adler 2006}

Adler M, Chambers S, Edwards C, Neild G, Isenberg D. An assessment of renal failure in an SLE cohort with special reference to ethnicity, over a 25-year period. Rheumatology 2006;45(9):1144-7. [MEDLINE: 16527882]

\section{ANZDATA 2016}

Australia, New Zealand Dialysis, Transplant Registry. ANZDATA Registry. 39th Report, Chapter 1: Incidence of End Stage Kidney Disease. www.anzdata.org.au/anzdata/ AnzdataReport/39thReport/c01_incidence_v5.0_20170418.pdf (accessed 17 May 2018)

\section{Bansal 1997}

Bansal VK, Beto JA. Treatment of lupus nephritis: a metaanalysis of clinical trials. American Journal of Kidney Diseases 1997;29(2):193-9.

\section{Begg 1996}

Begg C, Cho M, Eastwood S, Horton R, Moher D, Olkin I, et al. Improving the quality of reporting of randomized controlled trials. The CONSORT statement. JAMA 1996;276(8):637-9. [MEDLINE: 8773637]

\section{Cao 2015}

Cao H, Rao Y, Liu L, Lin J, Yang H, Zhang X, et al. The efficacy and safety of leflunomide for the treatment of lupus nephritis in Chinese patients: systematic review and meta-analysis. PLOS ONE [Electronic Resource] 2015;10(12):e0144548. [MEDLINE: 26670616] 


\section{Cervera 2009}

Cervera R, Khamashta MA, Hughes GR. The Euro-lupus project: epidemiology of systemic lupus erythematosus in Europe. Lupus 2009;18(10):869-74. [MEDLINE: 19671784]

\section{Chen 2017}

Chen Y, Sun J, Zou K, Yang Y, Lin G. Treatment for lupus nephritis: an overview of systematic reviews and metaanalyses. Rheumatology International 2017;37(7):1089-99. [MEDLINE: 28493175]

\section{Contreras 2006}

Contreras G, Lenz O, Pardo V, Borja E, Cely C, Iqbal K, et al. Outcomes in African Americans and Hispanics with lupus nephritis. Kidney International 2006;69(10):1846-51. [MEDLINE: 16598205]

\section{Costenbader 2011}

Costenbader KH, Desai A, Alarcon GS, Hiraki LT, Shaykevich T, Brookhart MA, et al. Trends in the incidence, demographics, and outcomes of end-stage renal disease due to lupus nephritis in the US from 1995 to 2006. Arthritis \& Rheumatism 2011;63(6):1681-8. [MEDLINE: 21445962]

\section{Daleboudt 2011}

Daleboudt GM, Berger SP, Broadbent E, Kaptein AA. Healthrelated quality of life in patients with systemic lupus erythematosus and proliferative lupus nephritis. Psychology Health \& Medicine 2011;16(4):393-404. [MEDLINE: 21749237]

\section{Deeks 2001}

Deeks JJ. Systematic reviews in health care: Systematic review of evaluations of diagnostic and screening tests. BMJ 2001;323(7305):157-62. [PUBMED: 11463691]

\section{Deng 2012}

Deng J, Huo D, Wu Q, Yang Z, Liao Y. A meta-analysis of randomized controlled trials comparing tacrolimus with intravenous cyclophosphamide in the induction treatment for lupus nephritis. Tohoku Journal of Experimental Medicine 2012;227(4):281-8. [MEDLINE: 22820853]

\section{Dooley 1997}

Dooley MA, Hogan S, Jenette C, Falk R. Cyclophosphamide therapy for lupus nephritis: poor renal survival in black Americans. Glomerular Disease Collaborative Network. Kidney International 1997;51(4):1188-95. [MEDLINE: 9083285]

\section{Feng 2013}

Feng L, Deng J, Huo DM, Wu QY, Liao YH. Mycophenolate mofetil versus azathioprine as maintenance therapy for lupus nephritis: a meta-analysis. Nephrology 2013;18(2):104-10. [MEDLINE: 23113811]

\section{Fernandes das Neves 2015}

Fernandes das Neves M, Irlapati RV, Isenberg D. Assessment of long-term remission in lupus nephritis patients: a retrospective analysis over 30 years. Rheumatology 2015;54(8):1403-7. [MEDLINE: 25725362]

\section{GRADE 2008}

Guyatt GH, Oxman AD, Vist GE, Kunz R, Falck-Ytter Y, AlonsoCoello $P$, et al. GRADE: an emerging consensus on rating quality of evidence and strength of recommendations. $B M J$ 2008;336(7650):924-6. [MEDLINE: 18436948]

\section{GRADE 2011}

Guyatt GH, Oxman AD, Schunemann HJ, Tugwell P, Knottnerus A. GRADE guidelines: a new series of articles in the Journal of Clinical Epidemiology. Journal of Clinical Epidemiology 2011;64(4):380-2. [MEDLINE: 21185693]

\section{Hanly 2016}

Hanly JG, O'Keeffe AG, Su L, Urowitz MB, Romero-Diaz, Gordon C, et al. The frequency and outcome of lupus nephritis: results from an international inception cohort study. Rheumatology 2016;55(2):252-62. [MEDLINE: 26342222]

\section{Hannah 2016}

Hannah J, Casian A, D'Cruz D. Tacrolimus use in lupus nephritis: a systematic review and meta-analysis. Autoimmunity Reviews 2016;15(1):93-101. [MEDLINE: 26427983]

\section{Higgins 2003}

Higgins JP, Thompson SG, Deeks JJ, Altman DG. Measuring inconsistency in meta-analyses. BMJ 2003;327(7414):557-60. [MEDLINE: 12958120]

\section{Higgins 2011}

Higgins JP, Green S (editors). Cochrane Handbook for Systematic Reviews of Interventions Version 5.1.0 [updated March 2011]. The Cochrane Collaboration. 2011. Available from www.cochrane-handbook.org.

\section{Houssiau 2010}

Houssiau FA, Vasconcelos C, D'Cruz D, Sebastiani GD, de Ramon Garrido E, Danieli MG, et al. The 10-year follow-up data of the Euro-Lupus Nephritis Trial comparing low-dose and highdose intravenous cyclophosphamide. Annals of the Rheumatic Diseases 2010;69(1):61-4. [MEDLINE: 19155235]

\section{Ioannidis $\mathbf{2 0 0 0}$}

Ioannidis JP, Boki KA, Katsorida ME, Drosos AA, Skopouli FN, Boletis JN, et al. Remission, relapse, and re-remission of proliferative lupus nephritis treated with cyclophosphamide. Kidney International 2000;57(1):258-64. [MEDLINE: 10620207]

\section{Isenberg $\mathbf{2 0 1 0}$}

Isenberg D, Appel GB, Contreras G, Dooley MA, Ginzler EM, Jayne $D$, et al. Influence of race/ethnicity on response to lupus nephritis treatment: the ALMS study. Rheumatology 2010;49(1):128-40. [MEDLINE: 19933596]

\section{Kamanamool 2010}

Kamanamool N, McEvoy M, Attia J, Ingsathit A, Ngamjanyaporn P, Thakkinstian A. Efficacy and adverse events of mycophenolate mofetil versus cyclophosphamide for induction therapy of lupus nephritis: systematic review and meta-analysis. Medicine 2010;89(4):227-35. [MEDLINE: 20616662] 


\section{Korbet 2007}

Korbet SM, Schwartz MM, Evans J, Lewis EJ, Collaborative Study Group. Severe lupus nephritis: racial differences in presentation and outcome. Journal of the American Society of Nephrology 2007;18(1):244-54. [MEDLINE: 17167111]

\section{Lee 2010}

Lee $Y$, Woo JH, Choi SJ, Ji JD, Song GG. Induction and maintenance therapy for lupus nephritis: a systematic review and meta-analysis. Lupus 2010;19(6):703-10. [MEDLINE: 20064907]

\section{Lee 2011}

Lee YH, Lee HS, Choi SJ, Dai Ji J, Song GG. Efficacy and safety of tacrolimus therapy for lupus nephritis: a systematic review of clinical trials. Lupus 2011;20(6):636-40. [MEDLINE: 21382917]

\section{Lee 2015}

Lee YH, Song GG. Relative efficacy and safety of tacrolimus, mycophenolate mofetil, and cyclophosphamide as induction therapy for lupus nephritis: a Bayesian network meta-analysis of randomized controlled trials. Lupus 2015;24(14):1520-8. [MEDLINE: 26162684]

\section{Liang 2006}

Renal Disease Subcommittee of the American College of Rheumatology Ad Hoc Committee on Systemic Lupus Erythematosus Response, Criteria. The American College of Rheumatology response criteria for proliferative and membranous renal disease in systemic lupus erythematosus clinical trials. Arthritis \& Rheumatism 2006;54(2):421-32. [MEDLINE: 16453282]

\section{Liu 2012}

Liu LL, Jiang Y, Wang LN, Yao L, Li ZL. Efficacy and safety of mycophenolate mofetil versus cyclophosphamide for induction therapy of lupus nephritis: a meta-analysis of randomized controlled trials. Drugs 2012;72(11):1521-33. [MEDLINE: 22818016]

\section{Mak 2009}

Mak A, Cheak AA, Tan JY, Su HC, Ho RC, Lau CS. Mycophenolate mofetil is as efficacious as, but safer than, cyclophosphamide in the treatment of proliferative lupus nephritis: a metaanalysis and meta-regression. Rheumatology 2009;48(8):944-52. [MEDLINE: 19494179]

\section{Maneiro 2014}

Maneiro JR, Lopez-Canoa N, Salgado E, Gomez-Reino JJ. Maintenance therapy of lupus nephritis with mycophenolate or azathioprine: systematic review and meta-analysis. Rheumatology 2014;53(5):834-8. [MEDLINE: 24369416]

\section{McElhone 2006}

McElhone K, Abbott J, Teh LS. A review of health related quality of life in systemic lupus erythematosus. Lupus 2006;15(10):633-43. [MEDLINE: 17120589]
Mills 2013

Mills EJ, Thorlund K, loannidis JP. Demystifying trial networks and network meta-analysis. BMJ 2013;346:f2914. [MEDLINE: 23674332]

\section{Moher 1999}

Moher D, Cook DJ, Eastwood S, Olkin I, Rennie D, Stroup DF. Improving the quality of reports of meta-analyses of randomised controlled trials: the QUOROM statement. Quality of Reporting of Meta-analyses. Lancet 1999;354(9193):1896-900. [MEDLINE: 10584742]

\section{Mok 2002}

Mok CC, Ho CT, Chan KW, Lau CS, Wong RW. Outcome and prognostic indicators of diffuse proliferative lupus glomerulonephritis treated with sequential oral cyclophosphamide and azathioprine. Arthritis \& Rheumatism 2002;46(4):1003-13. [MEDLINE: 11953978]

\section{Moore 2006}

Moore RA, Derry S. Systematic review and meta-analysis of randomised trials and cohort studies of mycophenolate mofetil in lupus nephritis. Arthritis Research \& Therapy 2006;8(6):R182. [MEDLINE: 17163990]

\section{Moroni 2007}

Moroni G, Quaglini S, Gallelli B, Banfi G, Messa P, Ponticelli C. The long-term outcome of 93 patients with proliferative lupus nephritis. Nephrology Dialysis Transplantation 2007;22(9):2531-9. [MEDLINE: 17510096]

\section{Murphy 2013}

Murphy G, Lisnevskaia L, Isenberg D. Systemic lupus erythematosus and other autoimmune rheumatic diseases: challenges to treatment. Lancet 2013;382(9894):809-18. [MEDLINE: 23972423]

\section{Palmer 2017}

Palmer SC, Tunnicliffe DJ, Singh-Grewal D, Mavridis D, Tonelli M, Johnson DW, et al. Induction and maintenance immunosuppression treatment of proliferative lupus nephritis: a network meta-analysis of randomized trials. American Journal of Kidney Disease 2017;70(3):324-36. [MEDLINE: 28233655]

\section{Ponticelli 1998}

Ponticelli C, Moroni G. Flares in lupus nephritis: incidence, impact on renal survival and management. Lupus 1998;7(9):635-8. [MEDLINE: 9884102]

\section{Radhakrishnan 2010}

Radhakrishnan J, Moutzouris DA, Ginzler EM, Solomons N, Siempos II, Appel GB. Mycophenolate mofetil and intravenous cyclophosphamide are similar as induction therapy for class V lupus nephritis. Kidney International 2010;77(2):152-60. [MEDLINE: 19890271]

\section{Rovin 2014}

Rovin BH, Parikh SV. Lupus nephritis: the evolving role of novel therapeutics. American Journal of Kidney Diseases 2014;63(4):677-90. [MEDLINE: 24411715] 


\section{Schünemann 2011a}

Schünemann HJ, Oxman AD, Higgins JP, Vist GE, Glasziou P, Guyatt GH. Chapter 11: Presenting results and 'Summary of findings' tables. In: Higgins JP, Green S (editors). Cochrane Handbook for Systematic Reviews of Interventions Version 5.1.0 [updated March 2011]. The Cochrane Collaboration, 2011. Available from www.cochrane-handbook.org.

\section{Schünemann 2011b}

Schünemann HJ, Oxman AD, Higgins JP, Deeks JJ, Glasziou P, Guyatt GH. Chapter 12: Interpreting results and drawing conclusions. In: Higgins JP, Green S (editors). Cochrane Handbook for Systematic Reviews of Interventions Version 5.1.0 [updated March 2011]. The Cochrane Collaboration, 2011. Available from www.cochrane-handbook.org.

\section{Sexton 2014}

Sexton DJ, Reule S, Solid C, Chen S, Collins AJ, Foley RN. ESRD from lupus nephritis in the United States. Clinical Journal of the American Society of Nephrology: CJASN 2014;10(2):251-9. [MEDLINE: 25534208]

\section{Singh 2016}

Singh JA, Hossain A, Kotb A, Oliveira A, Mudano AS, Grossman J, et al. Treatments for lupus nephritis: a systematic review and network metaanalysis. Journal of Rheumatology 2016;44(6):1801-15. [MEDLINE: 27585688]

\section{So 2011}

So MW, Koo BS, Kim YG, Lee CK, Yoo B. Predictive value of remission status after 6 months induction therapy in patients with proliferative lupus nephritis: a retrospective analysis. Clinical Rheumatology 2011;30(11):1399-405. [MEDLINE: 21594768]

\section{Strand 2000}

Stand V, Gladman D, Isenberg D, Petri M, Smolen J, Tugwell P. Endpoints: consensus recommendations from OMERACT IV. Outcome Measures in Rheumatology. Lupus 2000;9(5):322-7. [MEDLINE: 10878722]

\section{Sutanto 2013}

Sutanto B, Singh-Grewal D, McNeil HP, O'Neill S, Craig JC, Jones $\mathrm{J}$, et al. Experiences and perspectives of adults living with systemic lupus erythematosus: thematic synthesis of qualitative studies. Arthritis Care \& Research 2013;65(11):1752-65. [MEDLINE: 23609952]

\section{Tektonidou 2016}

Tektonidou MG, Dasgupta A, Ward MM. Risk of end-stage renal disease in patients with lupus nephritis, 1971-2015: a systematic review and Bayesian meta-analysis. Arthritis \& Rheumatology 2016;68(6):1432-41. [MEDLINE: 26815601]

\section{Tench 2000}

Tench, CM, McCurdie I, White PD, D'Cruz DP. The prevalence and associations of fatigue in systemic lupus erythematosus. Rheumatology 2000;39(11):1249-54. [MEDLINE: 11085805]

\section{Tian 2014}

Tian SY, Feldman BM, Beyene J, Brown PE, Uleryk EM, Silverman ED. Immunosuppressive therapies for the induction treatment of proliferative lupus nephritis: a systematic review and network metaanalysis. Journal of Rheumatology 2014;41(10):1998-2007. [MEDLINE: 25225281]

\section{Tian 2015}

Tian SY, Feldman BM, Beyene J, Brown PE, Uleryk EM, Silverman ED. Immunosuppressive therapies for the maintenance treatment of proliferative lupus nephritis: a systematic review and network metaanalysis. Journal of Rheumatology 2015;42(8):1392-400. [MEDLINE: 26077406]

\section{Touma 2011}

Touma Z, Gladman DD, Urowitz MB, Beyene J, Uleryk EM, Shah PS. Mycophenolate mofetil for induction treatment of lupus nephritis: a systematic review and metaanalysis. Journal of Rheumatology 2011;38(1):69-78. [MEDLINE: 20952473]

\section{Tunnicliffe 2015}

Tunnicliffe DJ, Singh-Grewal D, Kim S, Craig JC, Tong A. Diagnosis, monitoring, and treatment of systemic lupus erythematosus: a systematic review of clinical practice guidelines. Arthritis Care \& Research 2015;67(10):1440-52. [MEDLINE: 25778500]

\section{van Vollenhoven 2017}

van Vollenhoven R, Voskuyl A, Bertsias G, Aranow C, Aringer M, Arnaud L, et al. A framework for remission in SLE: consensus findings from a large international task force on definitions of remission in SLE (DORIS). Annals of the Rheumatic Diseases 2017;76(3):554-61. [MEDLINE: 27884822]

\section{Vu 1999}

Vu TV, Escalante A. A comparison of the quality of life of patients with systemic lupus erythematosus with and without endstage renal disease. Journal of Rheumatology 1999;26(12):2595-601. [MEDLINE: 10606368]

\section{Walsh 2007}

Walsh M, James M, Jayne D, Tonelli M, Manns BJ, Hemmelgarn BR. Mycophenolate mofetil for induction therapy of lupus nephritis: a systematic review and meta-analysis. Clinical Journal of the American Society of Nephrology: CJASN 2007;2(5):968-75. [MEDLINE: 17702723]

\section{Weidenbusch 2013}

Weidenbusch M, Rommele C, Schrottle A, Anders HJ. Beyond the LUNAR trial. Efficacy of rituximab in refractory lupus nephritis. Nephrology Dialysis Transplantation 2013;28(1):106-11. [MEDLINE: 22764193]

\section{Zakharova 2016}

Zakharova EV, Makarova TA, Zvonova EV, Anilina AM, Stolyarevich ES. Immunosuppressive treatment for lupus nephritis: long-term results in 178 patients. BioMed Research International 2016:7407919. [MEDLINE: 28050564] 


\section{Zhang 2016}

Zhang X, Ji L, Yang L, Tang X, Qin W. The effect of calcineurin inhibitors in the induction and maintenance treatment of lupus nephritis: a systematic review and meta-analysis. International Urology \& Nephrology 2016;48(5):731-43. [MEDLINE: 26781720]

\section{Zhou 2011}

Zhou DJ, Wu XC. Meta-analysis of calcineurin inhibitor in the treatment of lupus nephritis. Zhonghua Erke Zazh [Chinese Journal of Pediatrics] 2011;49(4):287-93. [MEDLINE: 21624207]

\section{Zhu 2007}

Zhu B, Chen N, Lin Y, Ren H, Zhang W, Wang W, et al. Mycophenolate mofetil in induction and maintenance therapy of severe lupus nephritis: a meta-analysis of randomized controlled trials. Nephrology Dialysis Transplantation 2007;22(7):1933-42. [MEDLINE: 17405792]

\section{References to other published versions of this review}

\section{Flanc 2003}

Flanc R, Roberts M, Chadban S, Kerr P, Edworthy S, Atkins R. Treatment for lupus nephritis. Cochrane Database of Systematic Reviews 2003, Issue 4. [DOI: 10.1002/14651858.CD002922]

\section{Flanc 2004a}

Flanc RS, Roberts MA, Strippoli GF, Chadban SJ, Kerr PG, Atkins RC. Treatment for lupus nephritis. Cochrane Database of Systematic Reviews 2004, Issue 1. [DOI: 10.1002/14651858.CD002922.pub2]

\section{Flanc 2004b}

Flanc RS, Roberts MA, Strippoli GF, Chadban SJ, Kerr PG, Atkins RC. Treatment of diffuse proliferative lupus nephritis: a meta-analysis of randomized controlled trials. American Journal of Kidney Diseases 2004;43(2):197-208. [MEDLINE: 14750085]

\section{Henderson 2012}

Henderson L, Masson P, Craig JC, Flanc RS, Roberts MA, Strippoli GF, et al. Treatment for lupus nephritis. Cochrane Database of Systematic Reviews 2012, Issue 12. [DOI: 10.1002/14651858.CD002922.pub3]

\section{Henderson 2013}

Henderson LK, Masson P, Craig JC, Roberts MA, Flanc RS, Strippoli GFM, et al. Induction and maintenance treatment of proliferative lupus nephritis: a meta-analysis of randomized controlled trials. American journal of kidney diseases 2013;61(1):74-87. [MEDLINE: 23182601]

* Indicates the major publication for the study

\section{CHARACTERISTICS OF STUDIES}

Characteristics of included studies [ordered by study ID]

Abedi 2007

\begin{tabular}{ll}
\hline Methods & - Study design: parallel RCT \\
& - Study timeframe: not reported \\
\hline Participants & Duration of follow-up: 18 months \\
- Country: Iran \\
- Setting: not reported \\
- proven) \\
- Number (randomised): 30 (numbers per group not reported) \\
- Sex (M/F): not reported \\
- Exclusion criteria: not reported
\end{tabular}

Interventions

Induction therapy: duration of treatment was 18 months

- Treatment group 1

* MMF: $2 \mathrm{~g} / \mathrm{d}$

- Treatment group 2

* IV CPA: 0.75 to $1 \mathrm{~g} /$ month for 6 months then every 3 months for 1 year

- Both groups

* Corticosteroids

$\begin{array}{ll}\text { Outcomes } & \text { Complete remission } \\ \text { - Partial remission } \\ \text { - Proteinuria }\end{array}$


Abedi 2007 (Continued)

- Serum albumin

- Hb, ESR, serum complement, urinary activity

- Serious infection

- Leucopenia

- Amenorrhoea

- Diarrhoea

\begin{tabular}{ll}
\hline Notes & Abstract-only publication \\
& - Funding source not reported
\end{tabular}

\section{Risk of bias}

\begin{tabular}{lll}
\hline Bias & Authors' judgement & Support for judgement \\
\hline $\begin{array}{l}\text { Random sequence genera- } \\
\text { tion (selection bias) }\end{array}$ & Unclear risk & $\begin{array}{l}\text { Study was described as randomised, method of randomisation was not report- } \\
\text { ed }\end{array}$ \\
\hline $\begin{array}{l}\text { Allocation concealment } \\
\text { (selection bias) }\end{array}$ & Unclear risk & Insufficient information to permit judgement \\
\hline $\begin{array}{l}\text { Blinding of participants } \\
\text { and personnel (perfor- } \\
\text { mance bias) }\end{array}$ & High risk & Open-label study \\
All outcomes & & \\
\hline
\end{tabular}

Blinding of outcome as- Unclear risk Insufficient information to permit judgement
sessment (detection bias)

All outcomes

\begin{tabular}{lll}
\hline $\begin{array}{l}\text { Incomplete outcome data } \\
\text { (attrition bias) } \\
\text { All outcomes }\end{array}$ & Unclear risk & Insufficient information to permit judgement \\
\hline $\begin{array}{l}\text { Selective reporting (re- } \\
\text { porting bias) }\end{array}$ & High risk & Data unable to be meta-analysed \\
\hline Other bias & Unclear risk & Abstract-only publication; insufficient information to permit judgement \\
\hline
\end{tabular}

\section{ACCESS 2014}

\begin{tabular}{ll}
\hline Methods & Study design: double-blind, parallel RCT \\
- Study timeframe: November 2008 to June 2012 \\
- Duration of follow-up: 24 and 52 weeks \\
\hline Participants & Countries: USA and Mexico \\
- Setting: multicentre (19 sites) \\
- Inclusion criteria: $\geq 16$ years; diagnosis of SLE (ACR criteria) positive ANA and/or positive anti-dou- \\
ble-stranded DNA (anti-dsDNA) antibody test result at study entry; active lupus nephritis defined by \\
kidney biopsy findings within the last 12 months of proliferative nephritis (ISN/RPS criteria (class III or \\
class IV with or without features of class V)) and UPCR of $>1$ \\
- Number (randomised/analysed): treatment group (66/66); control group (68/68) \\
- Mean age \pm SD (years): treatment group (32 \pm 10.1$) ;$ control group (32.7 \pm 12$)$ \\
- Sex (M/F): treatment group (8/58); control group (12/56) \\
- Exclusion criteria: not reported
\end{tabular}


ACCESS 2014 (Continued)

Interventions
Induction therapy: duration of treatment was 6 months

- Treatment group

* Abatacept: monthly infusions at doses that were adjusted for body weight according to the abatacept dose that is recommended for rheumatoid arthritis (for < $60 \mathrm{~kg}, 500 \mathrm{mg}$; for $60-100 \mathrm{~kg} ; 750$ $\mathrm{mg}$ for $>100 \mathrm{~kg}, 1 \mathrm{~g}$ )

- Control group

* Placebo

- Both groups

* Six IV pulses of $500 \mathrm{mg}$ of CPA at two-week intervals followed by oral AZA at $2 \mathrm{mg} / \mathrm{kg} / \mathrm{d}$ based on the ELNT regimen

* Oral glucocorticoid treatment was begun at $60 \mathrm{mg} / \mathrm{d}$ for 2 weeks in all subjects, followed by a prescribed taper to $10 \mathrm{mg} / \mathrm{d}$ over the next 10 weeks
- Death (all causes)

- Complete response: UPCR of 0.5 based on a $24 \mathrm{~h}$ urine collection, $\mathrm{SCr}$ level of $1.2 \mathrm{mg} / \mathrm{dL}$ or $125 \%$ of baseline, and adherence to the prednisone taper to $10 \mathrm{mg} / \mathrm{d}$ by week 12

- Partial response: UPCR required only 50\% improvement from baseline (rather than a decline to $<0.5$ based in complete response) on a $24 \mathrm{~h}$ urine collection, $\mathrm{SCr}$ level of $1.2 \mathrm{mg} / \mathrm{dL}$ or $125 \%$ of baseline, and adherence to the prednisone taper to $10 \mathrm{mg} / \mathrm{d}$ by week 12

- Relapse: renal flare was defined as the recurrence of proteinuria of $>1 \mathrm{~g} / 24 \mathrm{~h}$; for all others, a renal flare was defined as either of the following: SCr level at least $25 \%$ higher than baseline or above the upper limit of normal, plus proteinuria at least $75 \%$ of baseline; or doubling of the UPCR compared with the lowest previous value

- Major infection

- The ACCESS study did not use an initial IV pulse MP, but rather left that decision to the site investigator's discretion, unlike Euro-lupus nephritis treatment regimen

- Funding source: NIH National Institute of Allergy and Infectious Diseases contract N01-Al-15416, protocol number ITN034AI

\section{Risk of bias}

Bias Authors' judgement Support for judgement

Random sequence genera- Unclear risk tion (selection bias)

\begin{tabular}{l}
$\begin{array}{l}\text { Allocation concealment } \\
\text { (selection bias) }\end{array}$ \\
\hline
\end{tabular}

Blinding of participants Low risk Double-blind with identical placebo

and personnel (perfor-

mance bias)

All outcomes

\begin{tabular}{|c|c|c|}
\hline $\begin{array}{l}\text { Blinding of outcome as- } \\
\text { sessment (detection bias) } \\
\text { All outcomes }\end{array}$ & Unclear risk & Insufficient information to permit judgement \\
\hline
\end{tabular}

\begin{tabular}{l}
\hline Incomplete outcome data Low risk No missing outcome data \\
(attrition bias) \\
All outcomes
\end{tabular}

Selective reporting (re- Low risk Study protocol available and pre-specified outcomes were reported
porting bias)


ACCESS 2014 (Continued)

Other bias High risk Some authors involved in data acquisition and analysis are employees of pharmaceutical companies

\section{ALMS 2007}

Methods $\quad$ Study design: open-label, parallel RCT

- Study timeframe (enrolment): 27 July 2005 to 6 October 2006

- Duration of follow-up (median): 6 months (induction therapy) and 36 months (maintenance therapy)

Participants - Country: international (countries not reported)

- Setting: multinational ( 100 sites)

- Inclusion criteria: aged 12 to 75 years with diagnosis of SLE (ACR criteria), biopsy-proven lupus nephritis (active or chronic) within 6 months before randomisation, ISN/RPS 2003 class III, IV-S, IV-G, V, III+V, IV+V, class III or V must have proteinuria > 2 g/d; class III (22); IV (147); III/V (7); IV/V (16); V (35)

- Number (randomised/analysed)

* Induction therapy: treatment group 1 (185/185); treatment group 2 (185/185)

* Maintenance therapy: treatment group 1 (116/116); treatment group $2(111 / 111)$

- Mean age \pm SD (years)

* Induction therapy: treatment group 1 (32.4 \pm 11.2$)$; treatment group $2(31.3 \pm 10.3)$

* Maintenance therapy: treatment group 1 ( $31.8 \pm 10.6)$; treatment group $2(31 \pm 10.8)$

- $\operatorname{Sex}(M / F)$

* Induction therapy: treatment group 1 (28/157); treatment group $2(29 / 156)$

* Maintenance therapy: treatment group 1 (15/101); treatment group 2 (17/94)

- Exclusion criteria: treatment with MMF or IV CPA within the previous year; continuous dialysis for $>2$ weeks before randomisation or anticipated duration > 8 weeks; pancreatitis, GI haemorrhage within 6 months or active peptic ulcer within 3 months; severe viral infection; severe cardiovascular disease; bone marrow insufficiency with cytopenias not attributable to SLE; current infection requiring IV antibiotics

- Treatment group 1

* Oral MMF: titrated from $0.5 \mathrm{~g}$ twice daily in week 1 to $1.0 \mathrm{~g}$ twice daily in week 2, target dose $1.5 \mathrm{~g}$ twice daily in week 3

- Treatment group 2

* IV CPA: monthly pulses 0.5 to $1.0 \mathrm{~g} / \mathrm{m}^{2}$

- Both groups

* Oral prednisolone with defined taper, maximum starting dose $60 \mathrm{mg} / \mathrm{d}$

Maintenance therapy: duration of therapy was 36 months

- Treatment group 1

* Oral MMF: $2 \mathrm{~g} / \mathrm{d}$

* AZA placebo

- Treatment group 2

* Oral AZA: $2 \mathrm{mg} / \mathrm{kg} / \mathrm{d}$

* MMF placebo

- Both groups

* Oral prednisolone with defined taper, maximum starting dose $10 \mathrm{mg} / \mathrm{d}$

Outcomes Induction therapy

- Death (all causes)

- Stable kidney function: stabilisation $\pm 25 \%$ or improvement in $\mathrm{SCr}$ 
- Complete renal remission: return to normal creatinine, proteinuria $\leq 0.5 \mathrm{~g} / \mathrm{d}$ and inactive urine sediment

- Partial renal remission: prespecified decrease in UPCR (fall in $<3.0 \mathrm{~g} / \mathrm{d}$ protein if baseline $\geq 3$ or $\geq 50 \%$ reduction if $<3$ at baseline and stabilisation of $\mathrm{SCr} \pm 25 \%$ )

- Major infection

- Systemic disease activity and damage

- Adverse events (reported by $>10 \%$ participants)

Maintenance therapy

- Death

- ESKD

- doubling of $\mathrm{SCr}$

- Renal flare: proteinuric or nephritic

- Complete renal remission

- Combined renal and extra-renal remission

\begin{tabular}{ll}
\hline Notes & Funding source: Aspreva Pharmaceuticals Corporation as part of the Roche-Aspreva collaboration \\
& agreement
\end{tabular}

\section{Risk of bias}

\begin{tabular}{|c|c|c|}
\hline Bias & Authors' judgement & Support for judgement \\
\hline $\begin{array}{l}\text { Random sequence genera- } \\
\text { tion (selection bias) }\end{array}$ & Unclear risk & $\begin{array}{l}\text { Participants randomly assigned ( } 1: 1 \text {, stratified by race and biopsy class, non- } \\
\text { blocked) but sequence of generation is not reported }\end{array}$ \\
\hline $\begin{array}{l}\text { Allocation concealment } \\
\text { (selection bias) }\end{array}$ & Low risk & $\begin{array}{l}\text { Central, computerised, interactive voice response system. Method would not } \\
\text { allow investigator/participant to know or influence intervention group }\end{array}$ \\
\hline $\begin{array}{l}\text { Blinding of participants } \\
\text { and personnel (perfor- } \\
\text { mance bias) } \\
\text { All outcomes }\end{array}$ & High risk & Induction therapy - Open-label study; maintenance therapy - double-blind \\
\hline $\begin{array}{l}\text { Blinding of outcome as- } \\
\text { sessment (detection bias) } \\
\text { All outcomes }\end{array}$ & Low risk & Primary outcome assessed by blinded Clinical EndPoints Committee \\
\hline $\begin{array}{l}\text { Incomplete outcome data } \\
\text { (attrition bias) } \\
\text { All outcomes }\end{array}$ & Low risk & $\begin{array}{l}\text { No missing outcome data; Induction therapy (group 1: } 1 \text { lost to follow-up; } \\
\text { group 2: } 2 \text { lost to follow-up) }\end{array}$ \\
\hline $\begin{array}{l}\text { Selective reporting (re- } \\
\text { porting bias) }\end{array}$ & Low risk & Study protocol available and pre-specified outcomes were reported \\
\hline Other bias & High risk & $\begin{array}{l}\text { Sponsored by Aspreva Pharmaceuticals Corporation included in the data } \\
\text { analysis \& authorship }\end{array}$ \\
\hline
\end{tabular}

\section{APRIL-LN 2012}

$\begin{array}{ll}\text { Methods } & \text { Study design: double-blind, double dummy RCT } \\ \text { - Study timeframe: not reported } \\ \text { - Duration of follow-up: } 12 \text { months planned }\end{array}$


APRIL-LN 2012 (Continued)

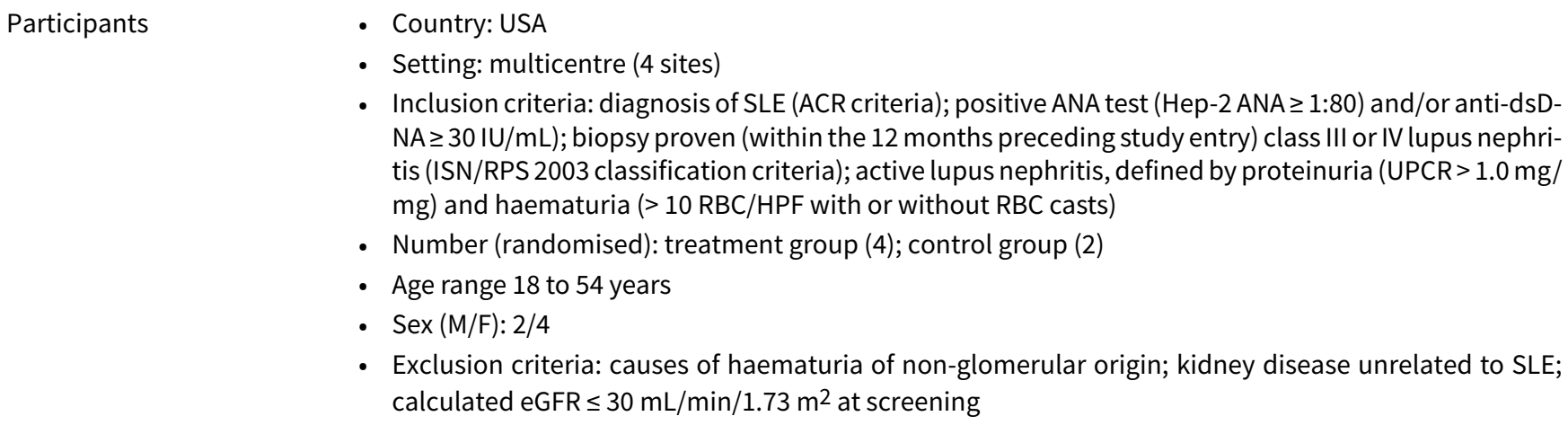

- Inclusion criteria: diagnosis of SLE (ACR criteria); positive ANA test (Hep-2 ANA $\geq 1: 80$ ) and/or anti-dsD$\mathrm{NA} \geq 30 \mathrm{IU} / \mathrm{mL}$ ); biopsy proven (within the 12 months preceding study entry) class III or IV lupus nephritis (ISN/RPS 2003 classification criteria); active lupus nephritis, defined by proteinuria (UPCR $>1.0 \mathrm{mg}$ / $\mathrm{mg}$ ) and haematuria (> $10 \mathrm{RBC} / \mathrm{HPF}$ with or without RBC casts)

- Number (randomised): treatment group (4); control group (2)

- Age range 18 to 54 years

- $\operatorname{Sex}(M / F): 2 / 4$

- Exclusion criteria: causes of haematuria of non-glomerular origin; kidney disease unrelated to SLE; calculated eGFR $\leq 30 \mathrm{~mL} / \mathrm{min} / 1.73 \mathrm{~m}^{2}$ at screening

\begin{tabular}{|c|c|}
\hline Interventions & $\begin{array}{l}\text { Induction therapy: duration of therapy was } 12 \text { months } \\
\text { - Treatment group } \\
\text { * SC atacicept: } 150 \mathrm{mg} \text { twice weekly for } 4 \text { weeks then } 150 \mathrm{mg} \text { weekly for a planned } 48 \text { weeks } \\
\text { - Control group } \\
\text { * SC placebo } \\
\text { - Both groups } \\
\text { * On study Day } 14, \text { patients commenced MMF ( } 500 \mathrm{mg} \text {, twice daily, orally) and prednisone or equiva- } \\
\text { lent (the lesser of } 0.8 \mathrm{mg} / \mathrm{kg} / \mathrm{d} \text { or } 60 \mathrm{mg} / \mathrm{d} \text {, orally). MMF dose was increased to } 1,000 \mathrm{mg} \text { twice daily } \\
\text { at Day } 7 \text {, thereafter up to a maximum of } 1.5 \mathrm{~g} \text { twice daily by Day } 1\end{array}$ \\
\hline Outcomes & $\begin{array}{l}\text { - Major infection } \\
\text { - Treatment failure }\end{array}$ \\
\hline Notes & $\begin{array}{l}\text { - Follow-up was planned for } 12 \text { months } \\
\text { - Early termination of the project } \\
\text { - Funding source: Merck Serono S.A.; ZymoGenetics Inc; EMD Serono Inc }\end{array}$ \\
\hline
\end{tabular}

\section{Risk of bias}

\begin{tabular}{lll}
\hline Bias & Authors' judgement & Support for judgement \\
\hline $\begin{array}{l}\text { Random sequence genera- } \\
\text { tion (selection bias) }\end{array}$ & Unclear risk & $\begin{array}{l}\text { Study was described as randomised, method of randomisation was not report- } \\
\text { ed }\end{array}$ \\
\hline $\begin{array}{l}\text { Allocation concealment } \\
\text { (selection bias) }\end{array}$ & Unclear risk & Insufficient information to permit judgement \\
\hline $\begin{array}{l}\text { Blinding of participants } \\
\begin{array}{l}\text { and personnel (perfor- } \\
\text { mance bias) }\end{array}\end{array}$ & Low risk & Double-blind, double-dummy placebo study \\
All outcomes & & \\
\hline
\end{tabular}

\begin{tabular}{|c|c|c|}
\hline $\begin{array}{l}\text { Blinding of outcome as- } \\
\text { sessment (detection bias) } \\
\text { All outcomes }\end{array}$ & Unclear risk & Insufficient information to permit judgement \\
\hline
\end{tabular}

\begin{tabular}{lll}
$\begin{array}{l}\text { Incomplete outcome data } \\
\text { (attrition bias) } \\
\text { All outcomes }\end{array}$ & Low risk & No missing outcome data \\
\hline $\begin{array}{l}\text { Selective reporting (re- } \\
\text { porting bias) }\end{array}$ & High risk & Study protocol available and not all prespecified outcomes were reported \\
\hline
\end{tabular}


APRIL-LN 2012 (Continued)

Other bias High risk Sponsor involved in authorship. The study was terminated early; there were differences in characteristics (for example eGFR) between groups at baseline

AURA-LV 2016

Methods

- Study design: double-blind, parallel RCT

- Study timeframe: not reported

- Duration of follow-up: 24 and 48 weeks

Participants Country: $>20$ countries (not reported)

- Setting: multinational (number of sites not reported)

- Inclusion criteria: patients aged 18 to 75 years; diagnosis of SLE (ACR criteria); biopsy proven classes III, IV-S or IV-G, (A) or (A/C); or Class V, alone or in combination with Class III or IV (ISN/RPS 2003) (within 6 months prior to screening (Visit 1); laboratory evidence of active nephritis at screening, defined as Class III, IV-S or IV-G (confirmed proteinuria $\geq 1,500 \mathrm{mg} / 24 \mathrm{~h}$, UPCR of $\geq 1.5 \mathrm{mg} / \mathrm{mg}$; Class V (alone or in combination with Class III or IV: proteinuria $\geq 2,000 \mathrm{mg} / 24 \mathrm{~h}$, a UPCR of $\geq 2 \mathrm{mg} / \mathrm{mg}$ )

- Number (randomised): 265 patients (numbers not reported for groups)

- Mean age \pm SD (years): not reported

- $\operatorname{Sex}(M / F)$ : not reported

- Exclusion criteria: eGFR of $\leq 45 \mathrm{~mL} / \mathrm{min} / 1.73 \mathrm{~m}^{2}$; currently requiring or expected to require $\mathrm{HD}$ or PD during the study period; previous kidney transplant or planned transplant within study period; in the opinion of the investigator, subject does not require long-term immunosuppressive treatment (in addition to corticosteroids); current or medical history of: pancreatitis or GI haemorrhage within 6 months prior to screening; active unhealed peptic ulcer within 3 months prior to screening; congenital or acquired immunodeficiency; clinically significant drug or alcohol abuse 2 years prior to screening; malignancy within 5 years of screening, with the exception of basal and squamous cell carcinomas treated by complete excision; cervical dysplasia that is cervical intraepithelial neoplasia 1, but have been treated with conization or loop electrosurgical excision procedure, and have had a normal repeat PAP are allowed; lymphoproliferative disease or previous total lymphoid irradiation; severe viral infection (e.g. CMV, HBV, HCV) within 3 months of screening; or known HIV infection; active TB, or known history of TB; other known clinically significant active medical conditions, such as severe cardiovascular disease including congestive heart failure, history of cardiac dysrhythmia or congenital long QT syndrome; liver dysfunction at screening and confirmed before randomisation; chronic obstructive pulmonary disease or asthma requiring oral steroids; bone marrow insufficiency unrelated to active SLE (according to Investigator judgment) with WCC $<2500 / \mathrm{mm}^{3}$; absolute neutrophil count < $1.3 \times 10^{3} / \mu \mathrm{L}$; thrombocytopenia (platelet count $<50,000 / \mathrm{mm}^{3}$ ); active bleeding disorders; current infection requiring IV antibiotics; any overlapping autoimmune condition for which the condition or the treatment of the condition may affect the study assessments or outcomes; overlapping conditions for which the condition or treatment is not expected to affect assessments or outcomes are not excluded; pregnant, breast feeding or, if of childbearing potential, not using adequate contraceptive precautions

\begin{tabular}{ll} 
Interventions & $\begin{array}{l}\text { Induction therapy: duration of therapy was } 6 \text { months } \\
\text { Treatment group } 1\end{array}$ \\
& $*$ Low-dose oral voclosporin: $23.7 \mathrm{mg}$ twice/d \\
& $*$ Treatment group 2 \\
& Control group \\
& $*$ Oral placebo \\
& Both groups \\
& $*$ Oral MMF and corticosteroids \\
& - Death \\
\hline \multirow{2}{*}{ Outcomes } & Complete remission
\end{tabular}


AURA-LV 2016 (Continued)

- Major infection

$\begin{array}{ll}\text { Notes } & \text { Abstract-only publications } \\ & \text { - Funding source: Aurinia Pharmaceuticals Inc }\end{array}$

\section{Risk of bias}

\begin{tabular}{|c|c|c|}
\hline Bias & Authors' judgement & Support for judgement \\
\hline $\begin{array}{l}\text { Random sequence genera- } \\
\text { tion (selection bias) }\end{array}$ & Unclear risk & $\begin{array}{l}\text { Study was described as randomised, method of randomisation was not report- } \\
\text { ed }\end{array}$ \\
\hline $\begin{array}{l}\text { Allocation concealment } \\
\text { (selection bias) }\end{array}$ & Unclear risk & Insufficient information to permit judgement \\
\hline $\begin{array}{l}\text { Blinding of participants } \\
\text { and personnel (perfor- } \\
\text { mance bias) } \\
\text { All outcomes }\end{array}$ & Low risk & Double-blind \\
\hline $\begin{array}{l}\text { Blinding of outcome as- } \\
\text { sessment (detection bias) } \\
\text { All outcomes }\end{array}$ & Low risk & Outcome assessor blinded according to protocol \\
\hline $\begin{array}{l}\text { Incomplete outcome data } \\
\text { (attrition bias) } \\
\text { All outcomes }\end{array}$ & Low risk & No missing outcome data \\
\hline $\begin{array}{l}\text { Selective reporting (re- } \\
\text { porting bias) }\end{array}$ & High risk & Not all pre-specified outcomes reported \\
\hline Other bias & High risk & Pharma funded; some authors involved are employees of Aurinia \\
\hline
\end{tabular}

\section{Balletta 1992}

\begin{tabular}{ll}
\hline Methods & Study design: parallel RCT \\
& - Study timeframe: not reported \\
\hline Participants & Duration of follow-up: $>12$ months \\
\hline & - Country: Italy \\
- Inclusion criteria: lupus nephritis shown on biopsy (diffuse proliferative, mesangioproliferative, mem- & branoproliferative, focal proliferative, diffuse proliferative) \\
- Number (randomised): treatment group (5); control group (5) \\
- Mean age \pm SD (years): treatment group (25.6 \pm 6.2$)$; control group $(23.4 \pm 3.7)$ \\
- Sex (M/F): treatment group (0/5); control group (1/4) \\
- Exclusion criteria: not reported
\end{tabular}

Interventions

Induction therapy

- Treatment group

* Oral CSA: $1.5 \mathrm{mg} / \mathrm{kg}$ twice $/ \mathrm{d}$

* Prednisolone: as per control 
Balletta 1992 (Continued)

- Control group

* Prednisolone: pulse, 2 to $3 \mathrm{mg} / \mathrm{kg} / \mathrm{d}$ for 3 consecutive days, then oral dose $1 \mathrm{mg} / \mathrm{kg} / \mathrm{d}$ for 2 months and tapered

\begin{tabular}{ll}
\hline Outcomes & $\mathrm{SCr}$ \\
& $\mathrm{CrCl}$ \\
& Proteinuria \\
\hline \multirow{2}{*}{ Notes } & $\bullet 6 / 10$ participants had biopsy \\
& $\bullet$ Funding source: not reported \\
\hline
\end{tabular}

\section{Risk of bias}

\begin{tabular}{|c|c|c|}
\hline Bias & Authors' judgement & Support for judgement \\
\hline $\begin{array}{l}\text { Random sequence genera- } \\
\text { tion (selection bias) }\end{array}$ & Unclear risk & $\begin{array}{l}\text { Study was described as randomised, method of randomisation was not report- } \\
\text { ed }\end{array}$ \\
\hline $\begin{array}{l}\text { Allocation concealment } \\
\text { (selection bias) }\end{array}$ & Unclear risk & Insufficient information to permit judgement \\
\hline $\begin{array}{l}\text { Blinding of participants } \\
\text { and personnel (perfor- } \\
\text { mance bias) } \\
\text { All outcomes }\end{array}$ & Unclear risk & Insufficient information to permit judgement \\
\hline $\begin{array}{l}\text { Blinding of outcome as- } \\
\text { sessment (detection bias) } \\
\text { All outcomes }\end{array}$ & Unclear risk & Insufficient information to permit judgement \\
\hline $\begin{array}{l}\text { Incomplete outcome data } \\
\text { (attrition bias) } \\
\text { All outcomes }\end{array}$ & Low risk & No missing outcome data \\
\hline $\begin{array}{l}\text { Selective reporting (re- } \\
\text { porting bias) }\end{array}$ & High risk & Not all expected outcomes are reported \\
\hline Other bias & Low risk & The study appears to be free of other sources of bias \\
\hline
\end{tabular}

Bao 2008

\begin{tabular}{|c|c|}
\hline Methods & $\begin{array}{l}\text { - Study design: open-label RCT } \\
\text { - Study timeframe: September } 2005 \text { to December } 2006 \\
\text { - Duration of follow-up: } 6 \text { months prolonged to } 9 \text { months if complete remission not achieved within } 6 \\
\text { months }\end{array}$ \\
\hline Participants & $\begin{array}{l}\text { - Country: China } \\
\text { - Setting: single centre } \\
\text { - Inclusion criteria: aged } 12 \text { to } 60 \text { years; diagnosis of SLE (ACR } 1997 \text { criteria); SLEDAI } \geq 12 \text { ', biopsy-proven } \\
\text { lupus nephritis class IV + V (ISN/RPS 2003) within } 3 \text { weeks before enrolment; overt proteinuria ( } \geq 1.5 \\
\text { g/d) } \pm \text { active urine sediment } \\
\text { - Number (randomised/analysed): treatment group } 1(20 / 20) \text {; treatment group } 2(20 / 20) \\
\text { - Mean age } \pm \text { SD (years): treatment group } 1(27.2 \pm 7.1) \text {; treatment group } 2(30.6 \pm 4.6) \\
\text { - Sex (M/F): treatment group } 1(4 / 16) \text {; treatment group } 2(2 / 18)\end{array}$ \\
\hline
\end{tabular}


Bao 2008 (Continued)

- Exclusion criteria: creatinine $>3.0 \mathrm{mg} / \mathrm{dL}(265.2 \mu \mathrm{mol} / \mathrm{L})$ or $\mathrm{CrCl}<30 \mathrm{~mL} / \mathrm{min} / 1.73 \mathrm{~m}^{2}$ on repeated testing; deranged liver function tests; abnormal glucose; known hypersensitivity or contraindication to any of the regimens; use of CPA, MMF or TAC within the past 12 weeks; pregnancy or lactation; cerebral lupus; leflunomide and methotrexate forbidden

Induction therapy
Interventions
Treatment group 1
$*$ MMF: $1.0 \mathrm{~g} / \mathrm{d}$ twice daily $(0.75 \mathrm{~g} / \mathrm{d}$ twice daily if $\leq 50 \mathrm{~kg})$
$*$ TAC: $4 \mathrm{mg} / \mathrm{d}$ twice daily $(3 \mathrm{mg} / \mathrm{d}$ twice daily if $\leq 50 \mathrm{~kg})$
- Treatment group 2
$*$ IV CPA: $0.75 \mathrm{~g} / \mathrm{m}^{2}$ of body surface area first month then adjusted to 0.5 to $1.0 \mathrm{~g} / \mathrm{m}^{2} \mathrm{monthly}$ based
on WCC $(\leq 2.5)$
Both groups
$*$ IV MP: $0.5 \mathrm{~g} / \mathrm{d}$ for 3 days then oral prednisolone $(0.6$ to $0.8 \mathrm{mg} / \mathrm{kg} / \mathrm{d}$ for 4 wh) followed by a taper
(reduced by $5 \mathrm{mg} / \mathrm{d}$ every week to $20 \mathrm{mg} / \mathrm{d}$ then $2.5 \mathrm{mg}$ every week until maintenance dosage of
$10 \mathrm{mg} / \mathrm{d})$

\begin{tabular}{ll}
\hline - Dutcomes & - Doubling of $\mathrm{SCr}$ \\
- & Deterioration of kidney function \\
- Stable kidney function (normal value $\mathrm{SCr}$ or no more than $15 \%$ above baseline) \\
- Complete remission: proteinuria $(<0.4 \mathrm{~g} / 24 \mathrm{~h}$ ), normal urine sediment, serum albumin $\geq 3.5 \mathrm{~g} / \mathrm{dL}$, \\
- normal $\mathrm{SCr}$ or not $>15 \%$ from baseline \\
- Serum albumin $\geq 3.5 \mathrm{~g} / \mathrm{dL}$, normal $\mathrm{SCr}$ or not $>15 \%$ from baseline \\
- Herpes zoster virus infection \\
- Irregular menstruation \\
- Gl syndrome \\
- Alopecia \\
- Leucopenia \\
- Proteinuria
\end{tabular}

Notes

- Funding source: Roche China and Astellas Ireland Co. Ltd

\section{Risk of bias}

\begin{tabular}{lll}
\hline Bias & Authors' judgement & Support for judgement \\
\hline $\begin{array}{l}\text { Random sequence genera- } \\
\text { tion (selection bias) }\end{array}$ & Low risk & $\begin{array}{l}\text { A computer-generated randomisation list was drawn up by a statistician with a } \\
\text { block of every four participants. They enrolled participants were allocated the } \\
\text { next available number upon entry into the study }\end{array}$ \\
\hline $\begin{array}{l}\text { Allocation concealment } \\
\text { (selection bias) }\end{array}$ & Unclear risk & $\begin{array}{l}\text { A computer-generated randomisation list was given to the pharmacy depart- } \\
\text { ment. Each patient collected medication directly from the pharmacy depart- } \\
\text { ment. Unclear whether participants and or investigators might have an oppor- } \\
\text { tunity to influence assignment }\end{array}$ \\
\hline $\begin{array}{l}\text { Blinding of participants } \\
\text { and personnel (perfor- } \\
\text { mance bias) }\end{array}$ & High risk & Open-label study \\
All outcomes &
\end{tabular}


Bao 2008 (Continued)

$\begin{array}{lll}\text { Blinding of outcome as- } & \text { Low risk } & \begin{array}{l}\text { Adjudication of primary and key secondary outcome judged at coordinating } \\ \text { sessment (detection bias) }\end{array} \\ \text { All outcomes } & \begin{array}{l}\text { centre by personnel who had no knowledge of the treatment assignment and } \\ \text { ratings were confirmed by repeat testing after a } 1 \text { month interval }\end{array}\end{array}$

\begin{tabular}{lll}
\hline $\begin{array}{l}\text { Incomplete outcome data } \\
\text { (attrition bias) } \\
\text { All outcomes }\end{array}$ & Low risk & No missing outcome data \\
\hline $\begin{array}{l}\text { Selective reporting (re- } \\
\text { porting bias) }\end{array}$ & Low risk & All expected outcomes were reported \\
\hline Other bias & Low risk & $\begin{array}{l}\text { Supported by Roche China and Astellas Ireland. Co. Ltd. Partially supported } \\
\text { but no role in design, study or analysis }\end{array}$ \\
\hline
\end{tabular}

\section{Barron 1982}

\begin{tabular}{ll}
\hline Methods & Study design: quasi-RCT \\
- Study timeframe: 1965 to 1980 \\
- Duration of follow-up: mean follow-up 59 months (range: 7 to 137 months) \\
\hline Participants & Country: USA \\
- Setting: single centre \\
- Inclusion criteria: children with SLE (ACR criteria) and severe biopsy-proven lupus nephritis, defined \\
by a nephrotic urine sediment and impaired kidney function with a CrCl between 25 and $80 \mathrm{~mL} / \mathrm{min}$. \\
If CrCl > $80 \mathrm{~mL} /$ min, the candidate had to have very active renal histology with crescents or necrosis \\
in more than $25 \%$ of glomeruli; renal biopsies were obtained during the 6 weeks before study entry \\
and were evaluated by light and electron microscopy \\
- Number (randomised): treatment group 1 (15); treatment group 2 (7) \\
- Mean age (at onset) \pm SD (years):treatment group 1 (11.9 \pm 2.9$) ;$ treatment group 2 (11.4 \pm 3.6$)$ \\
- Sex (M/F): treatment group 1 (2/13); treatment group 2 (1/6) \\
- Exclusion criteria: drug-induced SLE
\end{tabular}

\begin{tabular}{|c|c|}
\hline Interventions & $\begin{array}{l}\text { Induction therapy } \\
\text { - Treatment group } 1 \\
\text { * High dose oral corticosteroid: prednisone } 2 \mathrm{mg} / \mathrm{kg} / \mathrm{d} \text { for } 3 \text { to } 6 \text { months then tapered } \\
\text { - Treatment group } 2 \\
\text { * Pulse MP then oral prednisone: } 30 \mathrm{mg} / \mathrm{kg} \text { body weight (maximum } 1 \mathrm{~g} \text { ) IV, total of } 6 \text { treatments every } \\
\text { other day; following completion of MP, oral prednisone } 2 \mathrm{mg} / \mathrm{kg} / \mathrm{d} \text { by then tapered }\end{array}$ \\
\hline Outcomes & $\begin{array}{l}\text { - Death (all causes) } \\
\text { - } \mathrm{CrCl} \\
\text { - } \mathrm{C} 3, \mathrm{ANA} \\
\text { - Exacerbations } \\
\text { - Infection } \\
\text { - Aseptic necrosis }\end{array}$ \\
\hline Notes & - Funding source: not reported \\
\hline
\end{tabular}

\section{Risk of bias}

Bias Authors' judgement Support for judgement


Barron 1982 (Continued)

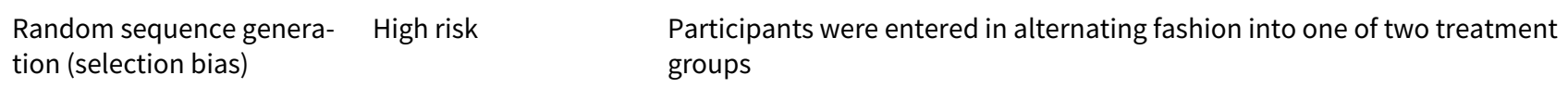

\begin{tabular}{|c|c|c|}
\hline $\begin{array}{l}\text { Allocation concealment } \\
\text { (selection bias) }\end{array}$ & High risk & $\begin{array}{l}\text { Knowledge of prior allocation due to lack of random sequence generation and } \\
\text { blinding }\end{array}$ \\
\hline
\end{tabular}

Blinding of participants High risk $\quad$ No blinding due to lack of allocation concealment
and personnel (perfor-
mance bias)
All outcomes

\begin{tabular}{lll}
\hline $\begin{array}{l}\text { Blinding of outcome as- } \\
\text { sessment (detection bias) } \\
\text { All outcomes }\end{array}$ & Unclear risk & Insufficient information to permit judgement \\
\hline $\begin{array}{l}\text { Incomplete outcome data } \\
\text { (attrition bias) }\end{array}$ & High risk & $\begin{array}{l}\text { Other patients were randomised, but only those with }>6 \text { months follow-up in- } \\
\text { cluded in analysis. It is unclear how many other patients were randomised. }\end{array}$ \\
\hline
\end{tabular}

Selective reporting (re- High risk $\quad$ Not all of the pre-specified primary outcomes were reported
porting bias)

\begin{tabular}{ll}
\hline Other bias $\quad$ Low risk & The study appears to be free of other sources of bias \\
\hline
\end{tabular}

\section{Belmont 1995}

\begin{tabular}{|c|c|}
\hline Methods & $\begin{array}{l}\text { - Study design: parallel RCT (pilot study) } \\
\text { - Study timeframe: not reported } \\
\text { - Duration of follow-up: } 18 \text { months }\end{array}$ \\
\hline Participants & $\begin{array}{l}\text { - Country: USA } \\
\text { - Setting: multicentre (number of sites not reported) } \\
\text { - Inclusion criteria: aged } 18 \text { to } 70 \text { years; SLE (ACR criteria); active kidney disease (in the absence of in- } \\
\text { fection, at least one of the following: (1) RBC casts, (2) WBC casts plus either haematuria (> 10/HPF) } \\
\text { or pyuria (> 10/HPF), ( } 3 \text { ) proteinuria at } \geq 3 \mathrm{~g} \text {, (4) proteinuria } \geq 1.5 \mathrm{~g} \text { plus (a) haematuria or (b) pyuria } \\
\text { or (c) a } 25 \% \text { decrease in C3 and/or C4 } \\
\text { - Number (randomised): treatment group (7); control group ( } 7 \text { ) } \\
\text { - Mean age } \pm \text { SD: } 35 \pm 2 \text { years } \\
\text { - Sex (M/F): } 3 / 11 \\
\text { - Proliferative lupus nephritis: } 7 / 14 \\
\text { - Exclusion criteria: not reported }\end{array}$ \\
\hline
\end{tabular}

- Treatment group

* Oral misoprostol: $20 \mu \mathrm{g}$ orally 4 times daily

- Control group

* Oral placebo: identical capsule

- Both groups

* Oral prednisone: $1 \mathrm{mg} / \mathrm{kg}$, 4 times/d

\begin{tabular}{ll}
\hline Outcomes & $\cdot \mathrm{SCr}$ \\
& $\cdot$ doubling of $\mathrm{SCr}$ \\
& $\cdot \mathrm{CrCl}$
\end{tabular}


Belmont 1995 (Continued)

- ESKD

- Complete remission of proteinuria

- C3, C4

- Anti-dsDNA

Notes - Funding source: not reported

\section{Risk of bias}

\begin{tabular}{lll}
\hline Bias & Authors' judgement & Support for judgement \\
\hline $\begin{array}{l}\text { Random sequence genera- } \\
\text { tion (selection bias) }\end{array}$ & Unclear risk & $\begin{array}{l}\text { Participants randomly assigned but methods of sequence generation are not } \\
\text { described }\end{array}$ \\
\hline $\begin{array}{l}\text { Allocation concealment } \\
\text { (selection bias) }\end{array}$ & Unclear risk & Insufficient information to permit judgement \\
\hline $\begin{array}{l}\text { Blinding of participants } \\
\text { and personnel (perfor- } \\
\text { mance bias) } \\
\text { All outcomes }\end{array}$ & Low risk & Double-blind study \\
\hline
\end{tabular}

Blinding of outcome as- Unclear risk Insufficient information to permit judgement

sessment (detection bias)

All outcomes

Incomplete outcome data Low risk Reasons for missing outcome data unlikely to be related to true outcome
(attrition bias)

All outcomes

\begin{tabular}{lll}
\hline $\begin{array}{l}\text { Selective reporting (re- } \\
\text { porting bias) }\end{array}$ & Low risk & Study protocol available and pre-specified outcomes were reported \\
\hline Other bias & Low risk & The study appears to be free of other sources of bias \\
\hline
\end{tabular}

\section{BELONG 2013}

\begin{tabular}{|c|c|}
\hline Methods & $\begin{array}{l}\text { - Study design: double-blind, parallel RCT } \\
\text { - Study timeframe: terminated } 19 \text { October } 2009 \\
\text { - Duration of follow-up: } 48 \text { weeks treatment period extended to } 96 \text { week open-label }\end{array}$ \\
\hline Participants & $\begin{array}{l}\text { - Country: } 23 \text { countries } \\
\text { - Setting: multinational (123 sites) } \\
\text { - Inclusion criteria: aged } \geq 16 \text { years; SLE (ACR criteria) including a history of anti-dsDNA positivity and } \\
\text { active lupus nephritis (defined as UPCR } \geq 1 \text { with biopsy-proven (within } 6 \text { months prior to randomi- } \\
\text { sation)); Class III or IV with coexisting class V permitted or class III or IV GN provided that } \leq 50 \% \text { of } \\
\text { glomeruli showed sclerosis or fibrosis (WHO criteria or ISN/RPS criteria) } \\
\text { - Number (randomised/analysed): treatment group } 1 \text { (127/73); treatment group } 2 \text { (126/75); control } \\
\text { group ( } 125 / 75) \\
\text { - Mean age, range (years): treatment group } 1 \text { ( } 30.6,16 \text { to } 60) \text {; treatment group } 2 \text { ( } 31.9,16 \text { to } 69) \text {; control } \\
\text { group ( } 31.3,17 \text { to } 66) \\
\text { - Sex (M/F): treatment group } 1(18 / 109) \text {; treatment group } 2(12 / 114) \text {; control group (19/106) } \\
\text { - Exclusion criteria: lupus class III (C), IV-S(C) and IV-G(C); retinitis; poorly controlled seizure disorder; } \\
\text { acute confusional state; myelitis; stroke or stroke syndrome; cerebellar ataxia or dementia; severe } \\
\text { renal impairment; estimated glomerular filtration rate <25 } \mathrm{mL} / \mathrm{min} / 1.73 \mathrm{~m} 2 \text {; ESKD requiring dialysis }\end{array}$ \\
\hline
\end{tabular}


BELONG 2013 (Continued)

or transplant; thrombocytopenia; or experiencing or at high risk of developing clinically significant bleeding or organ dysfunction

\begin{tabular}{|c|c|}
\hline Interventions & $\begin{array}{l}\text { Induction therapy: duration of treatment } 48 \text { weeks } \\
\text { - Treatment group } 1 \\
\text { * IV ocrelizumab: } 1000 \mathrm{mg} \text { infusion on days } 1 \text { and } 15 \text { followed by a single infusion at week } 16 \text { and } \\
\text { every } 16 \text { weeks } \\
\text { - Treatment group } 2 \\
\text { * IV ocrelizumab: } 400 \mathrm{mg} \text { infusion on days } 1 \text { and } 15 \text { followed by a single infusion at week } 16 \text { and every } \\
16 \text { weeks } \\
\text { - Control group } \\
\text { * Placebo } \\
\text { - All groups } \\
\text { * Groups were treated with background induction therapy at the discretion of the investigator MMF } \\
\text { (target dose } 3 \mathrm{~g} / \mathrm{d} \text { ) or CPA (ELNT regimen: } 0.5 \mathrm{~g} \text { IV every } 2 \text { weeks). Patients receiving MMF continued } \\
\text { to receive MMF, while patients receiving the ELNT CPA regimen were subsequently treated with } \\
\text { azathioprine (AZA; } 2 \mathrm{mg} / \mathrm{kg} \text { up to } 200 \mathrm{mg} / \mathrm{d} \text {, dose selected by the investigator). IV MP (up to } 3 \mathrm{~g} / \mathrm{d} \text { ) } \\
\text { was also permitted by day } 15 \text {, given in divided pulses, and oral steroids (0.5-0.75 mg/kg (60 mg/ } \\
\text { d)) were allowed with taper to } 10 \mathrm{mg} \text { over } 10 \text { weeks. Before each infusion, patients were adminis- } \\
\text { tered IV MP ( } 100 \mathrm{mg} \text { ), acetaminophen/paracetamol ( } 1 \mathrm{~g} \text { ), and an antihistamine ( } 50 \mathrm{mg} \text { IV diphen- } \\
\text { hydramine } \mathrm{HCl} \text { or equivalent) }\end{array}$ \\
\hline
\end{tabular}

\begin{tabular}{ll}
\hline Outcomes & Complete renal response (normal SCr $(25 \%$ increase from baseline) and improvement in UPCR to <0.5) \\
- Partial renal response (SCr $25 \%$ above baseline, and $50 \%$ improvement in UPCR, and if baseline ratio \\
$>3.0$, then UPCR $<3.0)$ \\
- Death \\
- Major infection \\
- Adverse events \\
- Proteinuria \\
- $\mathrm{CrCl}$
\end{tabular}

Notes Funding source: Genentech and Hoffman-La Roche

\section{Risk of bias}

\begin{tabular}{lll}
\hline Bias & Authors' judgement & Support for judgement \\
\hline $\begin{array}{l}\text { Random sequence genera- } \\
\text { tion (selection bias) }\end{array}$ & Unclear risk & $\begin{array}{l}\text { Study was described as randomised, method of randomisation was not report- } \\
\text { ed }\end{array}$ \\
\hline $\begin{array}{l}\text { Allocation concealment } \\
\text { (selection bias) }\end{array}$ & Unclear risk & Insufficient information to permit judgement \\
\hline $\begin{array}{l}\text { Blinding of participants } \\
\begin{array}{l}\text { and personnel (perfor- } \\
\text { mance bias) } \\
\text { All outcomes }\end{array}\end{array}$ & Low risk & Double-blind, placebo-controlled study \\
\hline
\end{tabular}

\begin{tabular}{lll}
\hline $\begin{array}{l}\text { Blinding of outcome as- } \\
\text { sessment (detection bias) } \\
\text { All outcomes }\end{array}$ & Unclear risk & Insufficient information to permit judgement \\
\hline $\begin{array}{l}\text { Incomplete outcome data } \\
\text { (attrition bias) }\end{array}$ & High risk & $\begin{array}{l}\text { Study was terminated before completion. Only } 36.8 \% \text { of patients completed } \\
\text { the } 48 \text {-week treatment period and were included in the analysis }\end{array}$ \\
\hline
\end{tabular}


BELONG 2013 (Continued)

Selective reporting (re- Low risk Study protocol available and pre-specified outcomes were reported porting bias)

Other bias

High risk

Genentech and Hoffman-La Roche funded the study and were involved in study design; Conflict of interest of authors relating to the pharmaceutical companies that funded the study; High drop-out rates (around 52\%) with the early termination of the study; The $1000 \mathrm{mg}$ ocrelizumab-treated group had slightly higher proportion of Caucasian patients and a lower proportion of Asian patients than the other two groups

Boedigheimer 2017

$\begin{array}{ll}\text { Methods } & \text { - Study design: double-blind, phase 1b, parallel RCT } \\ \text { - Study timeframe: } 3 \text { March } 2009 \text { to } 3 \text { June } 2014 \\ \text { - Duration of follow-up: } 6 \text { months }\end{array}$

Participants

- Country: USA, Mexico, France, Malaysia, Hong Kong

- Setting: multinational (11 sites)

- Inclusion criteria: aged 18 to 70 years; SLE (ACR criteria) with the presence of ANA at least 6 months before randomisation; any concurrent SLE medications (e.g. MMF, AZA, leflunomide, methotrexate, antimalarials) were at a stable dose for $\geq 30$ days before randomisation; concurrent prednisone was $20 \mathrm{mg} / \mathrm{d}$ (or equivalent) and for subjects without lupus nephritis could be increased or decreased once by $5 \mathrm{mg} / \mathrm{d}$ within 30 days before randomisation; subjects met current recommendations for immunisations; subjects with lupus nephritis were required to have biopsy-proven active disease within 18 months of randomisation according to WHO or ISN/RPS classification class III or IV; UPCR $>1$ or $24 \mathrm{~h}$ urine protein $>1 \mathrm{~g}$ following $\geq 12$ weeks of standard-of-care induction treatment with prednisone plus CPA or MMF, then maintained on prednisone at $20 \mathrm{mg} / \mathrm{d}$ (or equivalent) and MMF or AZA

- Number (randomised/analysed): treatment group (21/21); control group (0/21)

- Mean age \pm SD (years): treatment group (30.0 \pm 8.1$)$; control group $(36.9 \pm 11.7)$

- $\operatorname{Sex}(M / F)$ : treatment group (7/7); control group (3/4)

- Ethnicity: treatment group (Caucasian 6, African American 0, Hispanic 12, Asian 3, Other 0); control group (Caucasian 2, African American 0, Hispanic 2, Asian 3, Other 0)

- Exclusion criteria: any disorder that would interfere with study evaluations including unstable or severe disease; presence or history of vasculitis or active central nervous system lupus requiring therapy within 3 years; uncontrolled hypertension; low $\mathrm{CrCl}(<50 \mathrm{~mL} / \mathrm{min})$; low Hb levels, thrombocytopenia, neutropenia or low total WCC; poorly controlled diabetes; evidence of viral, bacterial or fungal infection within 30 days of randomisation or evidence of parasitic infestation; history of repeated infections or predisposition to infections; receipt of CPA, CSA, TAC, sirolimus, IVIG or plasmapheresis within 3 months of randomisation; or receipt of an investigational drug or device within 30 days or 5 half-lives of randomisation

Induction therapy
Interventions
- Treatment group
$*$ SC AMG $811: 20,60$ or $120 \mathrm{mg}$ administered
- Control group
$*$ SC placebo
- Both groups

$*$ Concomitant therapy could include prednisone, MMF, AZA, methotrexate and antimalarials

\begin{tabular}{ll}
\hline Outcomes & Death \\
- Major infection \\
- Adverse events \\
- Proteinuria
\end{tabular}


Boedigheimer 2017 (Continued)

- Disease activity

Notes - Study included both patients with SLE with and without lupus nephritis, we have extracted data for patients with lupus nephritis only

- Funding source: Amgen

\section{Risk of bias}

\begin{tabular}{|c|c|c|}
\hline Bias & Authors' judgement & Support for judgement \\
\hline $\begin{array}{l}\text { Random sequence genera- } \\
\text { tion (selection bias) }\end{array}$ & Unclear risk & $\begin{array}{l}\text { Study was described as randomised, method of randomisation was not report- } \\
\text { ed }\end{array}$ \\
\hline $\begin{array}{l}\text { Allocation concealment } \\
\text { (selection bias) }\end{array}$ & Unclear risk & Insufficient information to permit judgement \\
\hline $\begin{array}{l}\text { Blinding of participants } \\
\text { and personnel (perfor- } \\
\text { mance bias) } \\
\text { All outcomes }\end{array}$ & Low risk & Double-blind study \\
\hline $\begin{array}{l}\text { Blinding of outcome as- } \\
\text { sessment (detection bias) } \\
\text { All outcomes }\end{array}$ & Unclear risk & Insufficient information to permit judgement \\
\hline $\begin{array}{l}\text { Incomplete outcome data } \\
\text { (attrition bias) } \\
\text { All outcomes }\end{array}$ & Low risk & No missing outcome data \\
\hline $\begin{array}{l}\text { Selective reporting (re- } \\
\text { porting bias) }\end{array}$ & High risk & Not all expected clinical outcomes reported \\
\hline Other bias & High risk & $\begin{array}{l}\text { Phase } 1 \text { b study, study underpowered; study sponsor involved in data acquisi- } \\
\text { tion, data analysis and reporting of the study }\end{array}$ \\
\hline
\end{tabular}

\section{Boletis 1999}

\begin{tabular}{|c|c|}
\hline Methods & $\begin{array}{l}\text { - Study design: parallel RCT (pilot study) } \\
\text { - Study timeframe: not reported } \\
\text { - Duration of follow-up: } 18 \text { months }\end{array}$ \\
\hline Participants & $\begin{array}{l}\text { - Country: Greece } \\
\text { - Setting: not reported } \\
\text { - Inclusion criteria: lupus nephritis warranting CPA therapy; already received } 6 \text { months of CPA }\left(1 \mathrm{~g} / \mathrm{m}^{2}\right. \\
\text { once a month for } 6 \text { months and } 0.5 \mathrm{mg} / \mathrm{kg} \text { daily prednisone) with satisfactory response (absence of } \\
\text { major side-effects requiring interruption of therapy); inactive or substantially improved urine sedi- } \\
\text { ment, and proteinuria of less than } 1 \mathrm{~g} / \mathrm{d} \text { (for patients with baseline proteinuria }<3 \mathrm{~g} / \mathrm{d}) \text { or }<3 \mathrm{~g} / \mathrm{d} \text { (for } \\
\text { patients with baseline proteinuria }>3 \mathrm{~g} / \mathrm{d}) \\
\text { - Number (randomised/analysed): treatment group } 1(9) \text {; treatment group } 2(5) \\
\text { - Mean age } \pm \mathrm{SD} \text { (years): treatment group } 1 \text { ( } 30.4 \pm 10.9) \text {; treatment group } 2(32.4 \pm 11.7) \\
\text { - Sex (M/F): treatment group } 1(3 / 6) \text {; treatment group } 2(2 / 3) \\
\text { - Exclusion criteria: previous CPA for more than } 6 \text { months, pregnancy, aged }<18 \text { or }>75 \text { years, history } \\
\text { of malignant disorders }\end{array}$ \\
\hline
\end{tabular}


Boletis 1999 (Continued)

- Treatment group 1

* IV CPA: every 2 months for 6 months and then every 3 months for 12 months

- Treatment group 2

* IVIG: $400 \mathrm{mg} / \mathrm{kg}$ monthly for 18 months

Both groups

- Clinicians were allowed to increase the dose of prednisone if relapse or deterioration of kidney disease

\begin{tabular}{ll}
\hline Outcomes & $\cdot \mathrm{SCr}$ \\
& $\cdot \mathrm{CrCl}$ \\
& $\cdot$ Proteinuria \\
\hline Notes & $\cdot$ Funding source: not reported \\
\hline
\end{tabular}

\section{Risk of bias}

\begin{tabular}{|c|c|c|}
\hline Bias & Authors' judgement & Support for judgement \\
\hline $\begin{array}{l}\text { Random sequence genera- } \\
\text { tion (selection bias) }\end{array}$ & Unclear risk & $\begin{array}{l}\text { Study was described as randomised, method of randomisation was not report- } \\
\text { ed }\end{array}$ \\
\hline $\begin{array}{l}\text { Allocation concealment } \\
\text { (selection bias) }\end{array}$ & Low risk & Randomisation was done with sealed envelopes \\
\hline $\begin{array}{l}\text { Blinding of participants } \\
\text { and personnel (perfor- } \\
\text { mance bias) } \\
\text { All outcomes }\end{array}$ & Unclear risk & $\begin{array}{l}\text { Whether participants and investigators were blinded was not described and } \\
\text { treatment options were quite different suggesting that personnel were not } \\
\text { blinded }\end{array}$ \\
\hline $\begin{array}{l}\text { Blinding of outcome as- } \\
\text { sessment (detection bias) } \\
\text { All outcomes }\end{array}$ & Unclear risk & Insufficient information to permit judgement \\
\hline $\begin{array}{l}\text { Incomplete outcome data } \\
\text { (attrition bias) } \\
\text { All outcomes }\end{array}$ & Low risk & No missing outcome data \\
\hline $\begin{array}{l}\text { Selective reporting (re- } \\
\text { porting bias) }\end{array}$ & Low risk & Study protocol available and pre-specified outcomes were reported \\
\hline Other bias & Low risk & The study appears to be free of other sources of bias \\
\hline
\end{tabular}

Boumpas 1992

\begin{tabular}{ll}
\hline Methods & Study design: open-label, parallel RCT \\
- Study timeframe: 1981 to 1986 \\
- \\
Purticipants \\
- Country: USA \\
- Setting: not reported \\
- Inclusion criteria: age range 10 to 48 years; SLE (ACR $1982 \mathrm{criteria}$ ) and severe lupus nephritis defined \\
by a nephritic urine sediment and impaired kidney function with a CrCl between 25 to $80 \mathrm{~mL} / \mathrm{min} ;$ \\
if the CrCl was $>80 \mathrm{~mL} / \mathrm{min}$, the candidate had to have very active renal histology with $\mathrm{crescents}$ or \\
necrosis in more than $25 \%$ of glomeruli; renal biopsies were obtained during the 6 weeks before study \\
entry and were evaluated by light and electron microscopy
\end{tabular}


- Number (randomised): treatment group 1 (20); treatment group 2 (20); control group (25)

- Mean age $\pm \mathrm{SE}$ (years): treatment group $1(30 \pm 2)$; treatment group $2(30 \pm 2)$; control group $(31 \pm 2)$

- Sex (M/F): treatment group 1 (3/17); treatment group 2 (1/19); control group (1/24)

- Exclusion criteria: pregnancy; received cytotoxic drugs for more than 10 weeks; active infections; insulin-dependent DM, previous malignancy

\begin{tabular}{|c|c|}
\hline Interventions & $\begin{array}{l}\text { Induction therapy } \\
\text { - Treatment group } 1 \\
\text { * IV CPA: single doses } 0.5 \text { to } 1 \mathrm{~g} / \mathrm{m}^{2} \text { monthly for } 6 \text { months } \\
\text { - Treatment group } 2 \\
\text { * IV CPA: single doses } 0.5 \text { to } 1 \mathrm{~g} / \mathrm{m}^{2} \text { monthly for } 6 \text { months then } 3 \text { monthly for } 18 \text { months } \\
\text { - Control group } \\
\text { * IV MP: } 3 \text { doses } 1 \mathrm{~g} / \mathrm{m}^{2} \text {, then monthly single doses for } 6 \text { months } \\
\text { Other/additional treatment } \\
\text { - Patients were treated with prednisone } 0.5 \mathrm{mg} / \mathrm{kg} / \mathrm{d} \text { and continuing for } 4 \text { weeks then tapered at a rate } \\
\text { of } 5 \mathrm{mg} \text { every other day but the minimum dose to prevent extra-renal disease }\end{array}$ \\
\hline Outcomes & $\begin{array}{l}\text { - } \text { ESKD } \\
\text { - Doubling of } \mathrm{SCr} \\
\text { - Major infection } \\
\text { - Herpes zoster virus } \\
\text { - Malignancy } \\
\text { - Haemorrhagic cystitis } \\
\text { - Premature ovarian failure } \\
\text { - Osteonecrosis } \\
\text { - Relapse } \\
\text { - Stable kidney function }\end{array}$ \\
\hline Notes & $\begin{array}{l}\text { - } 2 \text { withdrawals } \\
\text { - Funding source: NIH trial }\end{array}$ \\
\hline
\end{tabular}

\section{Risk of bias}

\begin{tabular}{lll}
\hline Bias & Authors' judgement & Support for judgement \\
\hline $\begin{array}{l}\text { Random sequence genera- } \\
\text { tion (selection bias) }\end{array}$ & Unclear risk & $\begin{array}{l}\text { "Patients were assigned randomly to one of three treatment groups". No fur- } \\
\text { ther details on randomisation }\end{array}$ \\
\hline $\begin{array}{l}\text { Allocation concealment } \\
\text { (selection bias) }\end{array}$ & Low risk & Allocation drawn from a set of masked cards \\
\hline $\begin{array}{l}\text { Blinding of participants } \\
\begin{array}{l}\text { and personnel (perfor- } \\
\text { mance bias) }\end{array}\end{array}$ & High risk & Open-label study \\
All outcomes & & \\
\hline
\end{tabular}

\begin{tabular}{lll}
\hline $\begin{array}{l}\text { Blinding of outcome as- } \\
\text { sessment (detection bias) }\end{array}$ & Unclear risk & Insufficient information to permit judgement \\
All outcomes & \\
\hline $\begin{array}{l}\text { Incomplete outcome data } \\
\text { (attrition bias) }\end{array}$ & Low risk & No missing outcome data \\
All outcomes & \\
\hline
\end{tabular}


Boumpas 1992 (Continued)

Selective reporting (re- Low risk Study protocol available and pre-specified outcomes were reported porting bias)

Other bias Low risk The study appears to be free of other sources of bias

Cade 1973

$\begin{array}{ll}\text { Methods } & \text { - Study design: quasi-RCT } \\ & \text { - Study timeframe: not reported } \\ & \text { Duration of follow-up: } 36 \text { months }\end{array}$

\begin{tabular}{|c|c|}
\hline Participants & $\begin{array}{l}\text { - Country: USA } \\
\text { - Setting: single centre } \\
\text { - Inclusion criteria: Diagnosis of SLE; biopsy and functional findings of active proliferative GN due to } \\
\text { SLE; renal biopsy classification as proliferative GN closely approximates those used by Baldwin } 1970 \\
\text { - Number (randomised): treatment group } 1 \text { (15); treatment group } 2 \text { (13); treatment group } 3 \text { (13); treat- } \\
\text { ment group } 4(13) \\
\text { - Mean age, range (years): treatment group } 1(26.1,12 \text { to } 51) \text {; treatment group } 2 \text { ( } 30.5,11 \text { to } 62) \text {; treat- } \\
\text { ment group } 3 \text { ( } 22.4,12 \text { to } 51) \text {; treatment group } 4(24.8,14 \text { to } 51) \\
\text { - Sex (M/F): treatment group } 1 \text { (1/12); treatment group } 2(1 / 12) \text {; treatment group } 3(3 / 10) \text {; treatment } \\
\text { group } 4(6 / 7) \\
\text { - Exclusion criteria: lupus glomerulitis; focal proliferative disease or predominantly membranous lupus } \\
\text { nephritis }\end{array}$ \\
\hline
\end{tabular}

- Treatment group 1

* Oral prednisone: 60 to $100 \mathrm{mg} / \mathrm{d}$ for 6 months then slowly tapered to the lowest dose that controlled the patients symptoms

- Treatment group 2

* Oral AZA: started at 1.0 to $1.5 \mathrm{mg} / \mathrm{kg} / \mathrm{d}$, increased to 1.5 to $2.0 \mathrm{mg} / \mathrm{kg} / \mathrm{d}$ after 6 to 8 weeks if the patient had not improved by clinical or laboratory criteria

- Treatment group 3

* Oral prednisone: 60 to $100 \mathrm{mg} / \mathrm{d}$ for 6 months then slowly tapered to the lowest dose that controlled the patients symptoms

* Oral AZA: started at 1.0 to $1.5 \mathrm{mg} / \mathrm{kg} / \mathrm{d}$, increased to 1.5 to $2.0 \mathrm{mg} / \mathrm{kg} / \mathrm{d}$ after 6 to 8 weeks if the patient had not improved by clinical or laboratory criteria

- Treatment group 4

* Oral AZA: started at 1.0 to $1.5 \mathrm{mg} / \mathrm{kg} / \mathrm{d}$, increased to 1.5 to $2.0 \mathrm{mg} / \mathrm{kg} / \mathrm{d}$ after 6 to 8 weeks if the patient had not improved by clinical or laboratory criteria

* SC heparin: doses ranging from 20,000 units every 8 hours to 5000 units every 6 hours

\begin{tabular}{ll}
\hline Outcomes & Death (all causes) \\
& $\cdot \mathrm{ESKD}$ \\
& $\cdot \mathrm{CrCl}$
\end{tabular}

Notes - Funding source: not reported

\section{Risk of bias}


Cade 1973 (Continued)

Random sequence genera- High risk Chronological appearance
tion (selection bias)

Allocation concealment High risk
(selection bias)

(selection bias)

$\begin{array}{lll}\text { Blinding of participants } \quad \text { High risk } & \text { Open-label study }\end{array}$

and personnel (perfor-

mance bias)

All outcomes

\begin{tabular}{ll}
\hline Blinding of outcome as- & Unclear risk
\end{tabular}

All outcomes

Incomplete outcome data Low risk Insufficient information to permit judgement
(attrition bias)

All outcomes

\begin{tabular}{lll}
\hline $\begin{array}{l}\text { Selective reporting (re- } \\
\text { porting bias) }\end{array}$ & Low risk & All pre-specified outcomes are reported \\
\hline Other bias & Low risk & The study appears to be free of other sources of bias \\
\hline
\end{tabular}

\section{Chan 2000}

\begin{tabular}{|c|c|}
\hline Methods & $\begin{array}{l}\text { - Study design: parallel RCT } \\
\text { - Study timeframe: November } 1996 \text { and October } 1998 \\
\text { - Duration of follow-up: median follow-up was } 63 \text { months }\end{array}$ \\
\hline Participants & $\begin{array}{l}\text { - Country: Hong Kong } \\
\text { - Setting: multicentre } \\
\text { - Inclusion criteria: diagnosis of SLE (ACR criteria); biopsy-proven diffuse proliferative lupus nephritis } \\
\text { (class IV) (WHO classification), urinary protein excretion of } \geq 1 \mathrm{~g} / \mathrm{d} \text {, a serum albumin } \leq 3.5 \mathrm{~g} / \mathrm{dL}, \mathrm{SCr}< \\
3.4 \mathrm{mg} / \mathrm{dL}(300 \mu \mathrm{mol} / \mathrm{L}) \\
\text { - Number (randomised/analysed): treatment group } 1(33 / 32) \text {; treatment group } 2(31 / 30) \\
\text { - Mean age } \pm \mathrm{SD} \text { (years): treatment group } 1 \text { ( } 38.1 \pm 10.2) \text {; treatment group } 2(41.8 \pm 8.9) \\
\text { - Sex (M/F): treatment group } 1(6 / 26) \text {; treatment group } 2(4 / 26) \\
\text { - Exclusion criteria: } \mathrm{SCr}>4.2 \mathrm{mg} / \mathrm{dL} \text {; life-threatening complications; history of poor compliance; preg- } \\
\text { nancy; women unwilling to use contraception; CPA in the last } 6 \text { months; oral prednisolone } 0.4 \mathrm{mg} / \mathrm{kg} / \\
\text { d for more than } 2 \text { weeks }\end{array}$ \\
\hline
\end{tabular}

Interventions

Induction and maintenance therapy

- Treatment group 1

* Oral MMF $1 \mathrm{~g}$ twice daily for 6 months then $500 \mathrm{mg}$ twice daily for 6 months followed by AZA 1 to $1.5 \mathrm{mg} / \mathrm{kg} / \mathrm{d}$ for at least 1 year then tapered. From Jan 2002, protocol changed to reducing dose of MMF to $750 \mathrm{mg}$ twice daily at 6 months then $500 \mathrm{mg}$ twice daily at 12 months and continued for further 12 months before tapering

- Treatment group 2

* Oral CPA $2.5 \mathrm{mg} / \mathrm{kg} / \mathrm{d}$ for 6 months followed by AZA 1.5 to $2 \mathrm{mg} / \mathrm{kg} / \mathrm{d}$ for 6 months then 1 to $1.5 \mathrm{mg} /$ $\mathrm{kg} / \mathrm{d}$ for at least 1 year before tapering

Other information 
Chan 2000 (Continued)

- Both groups received prednisolone $0.8 \mathrm{mg} / \mathrm{kg} / \mathrm{d}$ and tapered to $10 \mathrm{mg} / \mathrm{d}$ at 6 months then maintenance dose of 5 to $7.5 \mathrm{mg} / \mathrm{kg}$ at 12 to 15 months

- MMF dosing subsequently changed from 2002: MMF $1 \mathrm{~g}$ twice daily reduced to $750 \mathrm{mg}$ twice daily after 6 months then $500 \mathrm{mg}$ twice daily for at least 1 year before tapering

\begin{tabular}{ll}
\hline Outcomes & Death \\
- & DSKD \\
- Doubling of $\mathrm{SCr}$ \\
- Relapse \\
- Major infection \\
- Herpes zoster virus infection \\
- Ovarian failure \\
- Bone toxicity \\
- Alopecia \\
- Gl upset \\
- Lymphopenia \\
- Complete remission of proteinuria: $<0.3 \mathrm{~g} / 24 \mathrm{~h}$ \\
- Partial remission of proteinuria: $>50 \%$ reduction in proteinuria, proteinuria between 0.3 and $3 \mathrm{~g} / 24 \mathrm{~h}$ \\
- SCr \\
- CrCl \\
- Daily proteinuria \\
\hline
\end{tabular}

\section{Risk of bias}

Bias Authors' judgement Support for judgement

Random sequence genera- Low risk Participants randomly assigned by drawing envelopes to one of two treatment tion (selection bias) groups in an open-label manner

\begin{tabular}{lll}
\hline $\begin{array}{l}\text { Allocation concealment } \\
\text { (selection bias) }\end{array}$ & Unclear risk & Insufficient information to permit judgement \\
\hline $\begin{array}{l}\text { Blinding of participants } \\
\text { and personnel (perfor- } \\
\text { mance bias) }\end{array}$ & Unclear risk & Insufficient information to permit judgement \\
All outcomes & \\
\hline
\end{tabular}

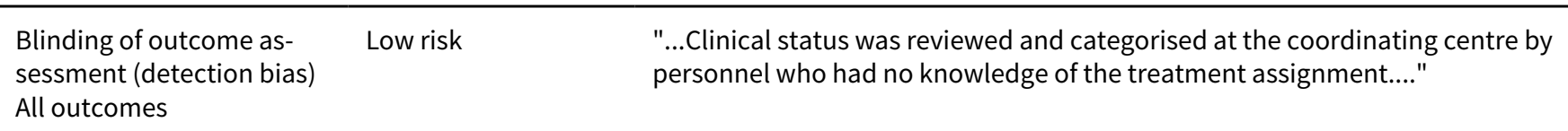

\begin{tabular}{lll}
\hline $\begin{array}{l}\text { Incomplete outcome data } \\
\text { (attrition bias) } \\
\text { All outcomes }\end{array}$ & Low risk & No missing outcome data \\
\hline $\begin{array}{l}\text { Selective reporting (re- } \\
\text { porting bias) }\end{array}$ & Low risk & Study protocol available and pre-specified outcomes were reported \\
\hline Other bias & Low risk & The study appears to be free of other sources of bias \\
\hline
\end{tabular}


Chen 2011

Methods

- Study design: open-label, parallel RCT

- Study timeframe: June 2006 to March 2008

- Duration of follow-up: 6 month follow-up; extended median follow-up was 6 months

\begin{tabular}{|c|c|}
\hline Participants & 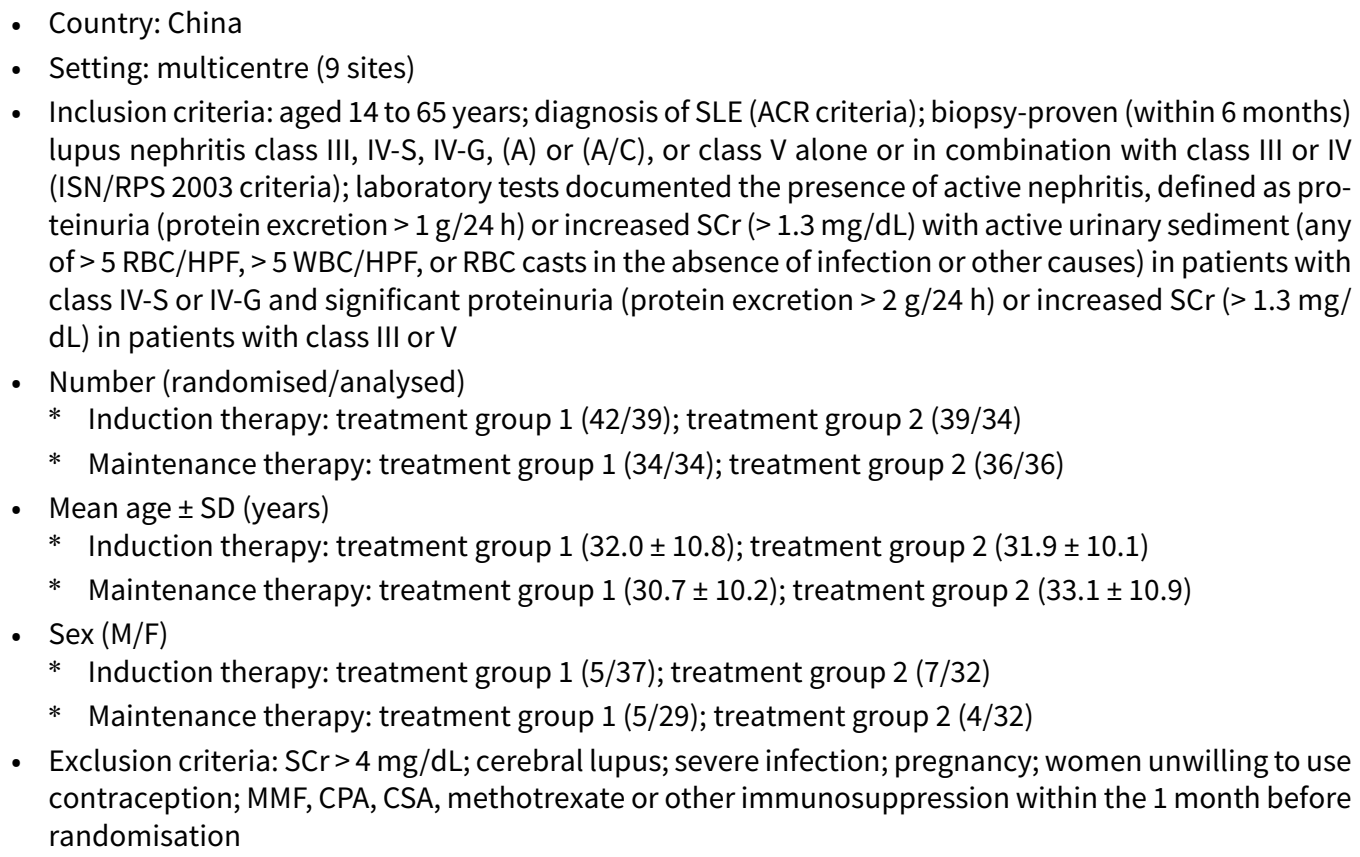 \\
\hline
\end{tabular}

Interventions

Induction therapy: duration of therapy was 6 months

- Treatment group 1

* Oral TAC: $0.05 \mathrm{mg} / \mathrm{kg}$ divided in 2 doses with target trough of 5 to $10 \mathrm{ng} / \mathrm{mL}$

- Treatment group 2

* IV CPA: $750 \mathrm{mg} / \mathrm{m}^{2}$ of body surface area every 4 weeks for a total of 6 pulses ( $25 \%$ decrease in dose if older than 60 years or creatinine $>3.4 \mathrm{mg} / \mathrm{dL}$ )

- Both groups

* Oral prednisolone: $1 \mathrm{mg} / \mathrm{kg} / \mathrm{d}$ (maximum $60 \mathrm{mg}$ ) tapered by $10 \mathrm{mg} / \mathrm{d}$ every 2 weeks to $40 \mathrm{mg}$, followed by decrease of $5 \mathrm{mg} / \mathrm{d}$ every 2 weeks until a dose of $10 \mathrm{mg} / \mathrm{d}$ achieved

Long-term maintenance therapy: duration of therapy was 6 months

- Treatment group 1

* Oral TAC: trough blood concentrations were maintained at $4-6 \mathrm{ng} / \mathrm{mL}$.

- Treatment group 2

* AZA: $2 \mathrm{mg} / \mathrm{kg} / \mathrm{d}$

- Both groups

* Oral prednisone: $10 \mathrm{mg} / \mathrm{d}$

$\begin{array}{ll}\text { Outcomes } & \text { - Death } \\ \text { - Major infection } \\ \text { - Herpes zoster virus infection } \\ \text { - Olopecia } \\ \text { - Gl upset } \\ \text { - Lymphopenia }\end{array}$


Chen 2011 (Continued)

- Complete renal remission: daily proteinuria $<0.3 \mathrm{~g} / 24 \mathrm{~h}$, normal urinary sediment, serum albumin $\geq$ $3.5 \mathrm{~g} / \mathrm{dL}$ and stable kidney function

- Partial renal remission: protein excretion of 0.3 to $2.9 \mathrm{~g} / 24 \mathrm{~h}$ and a decrease of at least $50 \%$ of baseline level), serum albumin level of at least $3.0 \mathrm{~g} / \mathrm{dL}$ and stable kidney function

- Treatment failure: failure to meet complete or partial remission

- $\mathrm{SCr}$

- Daily proteinuria

- Funding source: Scientific and Technologic Committee of Guangdong province, the Department of
Health, Guangzhou city, the Ministry of Education, Peoples' Republic of China and the 5010 Clinical
Program of Sun Yat-sen University. Astellas Pharmaceutics supplied TAC

\section{Risk of bias}

\begin{tabular}{|c|c|c|}
\hline Bias & Authors' judgement & Support for judgement \\
\hline $\begin{array}{l}\text { Random sequence genera- } \\
\text { tion (selection bias) }\end{array}$ & Low risk & $\begin{array}{l}\text { Randomisation was conducted at a central office using a computer-based ran- } \\
\text { dom allocation sequence table; randomisation not stratified by centre or base- } \\
\text { line characteristic }\end{array}$ \\
\hline $\begin{array}{l}\text { Allocation concealment } \\
\text { (selection bias) }\end{array}$ & Low risk & $\begin{array}{l}\text { Allocation concealment performed by enclosing assignments in sequentially } \\
\text { numbered, opaque, closed envelopes }\end{array}$ \\
\hline $\begin{array}{l}\text { Blinding of participants } \\
\text { and personnel (perfor- } \\
\text { mance bias) } \\
\text { All outcomes }\end{array}$ & High risk & Open-label study \\
\hline $\begin{array}{l}\text { Blinding of outcome as- } \\
\text { sessment (detection bias) } \\
\text { All outcomes }\end{array}$ & Unclear risk & Insufficient information to permit judgement \\
\hline $\begin{array}{l}\text { Incomplete outcome data } \\
\text { (attrition bias) } \\
\text { All outcomes }\end{array}$ & Low risk & $\begin{array}{l}\text { The primary outcome (complete remission) and secondary outcomes partial } \\
\text { remission and treatment failure were reported on an intention to treat bases. } \\
\text { The attrition rate for secondary safety outcomes were } 92.8 \%(39 / 42) \text { for the } \\
\text { TAC group and } 87.2 \% \text { for the IV CPA group. }\end{array}$ \\
\hline $\begin{array}{l}\text { Selective reporting (re- } \\
\text { porting bias) }\end{array}$ & Low risk & Study protocol available and prespecified outcomes were reported \\
\hline Other bias & Low risk & $\begin{array}{l}\text { Astellas Pharmaceutics supplied TAC but had no role in the design or conduct } \\
\text { of the study or analysis or interpretation of results }\end{array}$ \\
\hline
\end{tabular}

\section{Clark 1981}

\begin{tabular}{ll}
\hline Methods & Study design: open-label RCT \\
& - Study timeframe: from February 1978 \\
\hline Participants & Duration of follow-up: 12 months \\
- Country: Canada & Setting: not reported \\
- Inclusion criteria: Diagnosis of SLE (ACR criteria) and had increased DNA, low complement; presence \\
of ANA; renal biopsy showing diffuse proliferative $\mathrm{GN} ; \mathrm{CrCl}>30 \mathrm{~mL} / \mathrm{min}$ at study entry \\
- Number: treatment group 1 (6); treatment group $2(6)$ \\
- Mean age \pm SD (years): not reported
\end{tabular}


Clark 1981 (Continued)

- Sex (M/F): not reported

- Exclusion criteria: not reported

\begin{tabular}{ll}
\hline Interventions & Induction therapy \\
- Treatment group 1 & $*$ Corticosteroids \\
& $*$ AZA \\
- Treatment group 2 & $*$ Corticosteroids \\
& $*$ AZA \\
& $*$ Plasmapheresis
\end{tabular}

\begin{tabular}{ll}
\hline Outcomes & Death \\
& - ESKD \\
& - Doubling of $\mathrm{SCr}$ \\
& $-\mathrm{SCr}$ \\
& - Proteinuria \\
\hline Notes & - Funding source: Physicians' Services Incorporated Foundation \\
\hline
\end{tabular}

\section{Risk of bias}

\begin{tabular}{lll}
\hline Bias & Authors' judgement & Support for judgement \\
\hline $\begin{array}{l}\text { Random sequence genera- } \\
\text { tion (selection bias) }\end{array}$ & Unclear risk & $\begin{array}{l}\text { Study was described as randomised, method of randomisation was not report- } \\
\text { ed }\end{array}$ \\
\hline $\begin{array}{l}\text { Allocation concealment } \\
\text { (selection bias) }\end{array}$ & Unclear risk & Insufficient information to permit judgement \\
\hline $\begin{array}{l}\text { Blinding of participants } \\
\text { and personnel (perfor- } \\
\text { mance bias) }\end{array}$ & High risk & Open-label study \\
All outcomes & & \\
\hline
\end{tabular}

Blinding of outcome as- Unclear risk Insufficient information to permit judgement
sessment (detection bias)

All outcomes

\begin{tabular}{lll}
\hline $\begin{array}{l}\text { Incomplete outcome data } \\
\text { (attrition bias) } \\
\text { All outcomes }\end{array}$ & Low risk & No missing outcome data \\
\hline $\begin{array}{l}\text { Selective reporting (re- } \\
\text { porting bias) }\end{array}$ & Low risk & Study protocol available and pre-specified outcomes were reported \\
\hline Other bias & Low risk & $\begin{array}{l}\text { Supported from a grant from Physicians' Services Incorporated Foundation. } \\
\text { The study appears to be free of other sources of bias }\end{array}$ \\
\hline
\end{tabular}

Clark 1984

\begin{tabular}{ll}
\hline Methods & - Study design: parallel RCT \\
& - Study timeframe: not reported
\end{tabular}


Clark 1984 (Continued)

- Duration of follow-up: 19 months

\begin{tabular}{ll}
\hline Participants & Country: Canada. West Indies \\
- Setting: multinational (3 sites) \\
- Inclusion criteria: diagnosis of SLE (ACR criteria) and had at least one episode of ANA positivity; ele- \\
vated DNA binding and complement depression; renal biopsy showing diffuse proliferative GN \\
- Number (randomised): treatment group 1 (19); treatment group $2(20)$ \\
- Mean age $\pm \mathrm{SD}$ (years): treatment group 1 (25 \pm 2$)$; treatment group $2(26 \pm 2)$ \\
- Sex (M/F): treatment group $1(1 / 18)$; treatment group $2(5 / 15)$ \\
- Exclusion criteria: $\mathrm{CrCl}<30 \mathrm{~mL} / \mathrm{min}$ or $\mathrm{SCr}>3 \mathrm{mg} / \mathrm{dL}$
\end{tabular}

Induction therapy
Interventions
\[ \text { Treatment group } 1 \]
$*$ Treatment group 2
$*$ Conventional therapy
$*$ PEX: $4 \mathrm{~L}$ within the first two weeks, thereafter one $4 \mathrm{~L}$ PEX every $3-4$ weeks. In two centres patients
received replacement with $5 \%$ human serum albumin and in one centre replacement was with
plasma

\begin{tabular}{ll}
\hline Outcomes & Death \\
& $\cdot$ ESKD \\
& $\cdot$ Doubling of SCr \\
& $-\mathrm{SCr}$ \\
\hline
\end{tabular}

- Funding source: Physicians' Services Incorporated Foundation

\section{Risk of bias}

\begin{tabular}{lll}
\hline Bias & Authors' judgement & Support for judgement \\
\hline $\begin{array}{l}\text { Random sequence genera- } \\
\text { tion (selection bias) }\end{array}$ & Unclear risk & $\begin{array}{l}\text { Study was described as randomised, method of randomisation was not report- } \\
\text { ed }\end{array}$ \\
\hline $\begin{array}{l}\text { Allocation concealment } \\
\text { (selection bias) }\end{array}$ & Unclear risk & $\begin{array}{l}\text { "Designated non-medical person at each Centre who removed a pre-folded } \\
\text { slip of paper from a bowl" }\end{array}$ \\
\hline $\begin{array}{l}\text { Blinding of participants } \\
\text { and personnel (perfor- } \\
\text { mance bias) } \\
\text { All outcomes }\end{array}$ & High risk & Open-label study \\
\hline
\end{tabular}

\begin{tabular}{|c|c|c|}
\hline $\begin{array}{l}\text { Blinding of outcome as- } \\
\text { sessment (detection bias) } \\
\text { All outcomes }\end{array}$ & Unclear risk & Insufficient information to permit judgement \\
\hline
\end{tabular}

\begin{tabular}{lll}
\hline $\begin{array}{l}\text { Incomplete outcome data } \\
\text { (attrition bias) } \\
\text { All outcomes }\end{array}$ & Low risk & No missing outcome data \\
\hline $\begin{array}{l}\text { Selective reporting (re- } \\
\text { porting bias) }\end{array}$ & High risk & Not all relevant outcomes are reported \\
\hline Other bias & Low risk & $\begin{array}{l}\text { Supported from a grant from Physicians' Services Incorporated Foundation. } \\
\text { The study appears to be free of other sources of bias }\end{array}$ \\
\hline
\end{tabular}


Contreras 2004

$\begin{array}{ll}\text { Methods } & \text { - Study design: open-label, parallel RCT } \\ & \text { - Study timeframe: August } 1996 \text { and May } 2003 \\ & \text { - Duration of follow-up: } 72 \text { months }\end{array}$

\begin{tabular}{|c|c|}
\hline Participants & $\begin{array}{l}\text { - Country: USA } \\
\text { - Setting: single centre } \\
\text { - Inclusion criteria: diagnosis of SLE (ACR criteria); } \geq 18 \text { years; histologic diagnosis of proliferative lupus } \\
\text { nephritis (WHO class III, IV, or Vb); classes III (12), IV (46) or Vb (1) } \\
\text { - Number (randomised/analysed): treatment group } 1 \text { (19/19); treatment group } 2 \text { (20/20); treatment } \\
\text { group } 3(20 / 20) \\
\text { - Mean age } \pm \text { SD (years): treatment group } 1(33 \pm 10) \text {; treatment group } 2(33 \pm 12) \text {; treatment group } 3 \\
\text { ( } 32 \pm 11) \\
\text { - Sex (M/F): treatment group } 1(1 / 19) \text {; treatment group } 2(2 / 18) \text {; treatment group } 3(1 / 19) \\
\text { - Exclusion criteria: CrCl that was consistently< } 20 \mathrm{~mL} / \mathrm{min} \text {; any clinically significant infection; pregnan- } \\
\text { cy; the receipt of more than seven doses of IV CPA, or the receipt of AZA for longer than } 8 \text { weeks }\end{array}$ \\
\hline Interventions & $\begin{array}{l}\text { Maintenance therapy: duration of therapy } 1 \text { to } 3 \text { years } \\
\text { - Treatment group } 1 \\
\text { * IV CPA: } 0.5 \text { to } 1.0 \mathrm{~g} / \mathrm{m}^{2} \text { every } 3 \text { months } \\
\text { - Treatment group } 2 \\
\text { * AZA: } 1 \text { to } 3 \mathrm{mg} / \mathrm{kg} / \mathrm{d} \\
\text { - Treatment group } 3 \\
* \text { MMF: } 500 \text { to } 3000 \mathrm{mg} / \mathrm{d} \\
\text { - All groups } \\
\text { * Induction therapy of } 7 \text { monthly boluses of IV CPA } 0.5 \text { to } 1.0 \mathrm{~g} / \mathrm{m}^{2} \text { and corticosteroids and mainte- } \\
\text { nance therapy included prednisolone (up to } 0.5 \mathrm{mg} / \mathrm{kg} / \mathrm{d} \text { ) }\end{array}$ \\
\hline
\end{tabular}

\begin{tabular}{ll}
\hline Outcomes & ESKD \\
- Death & Doubling of SCr \\
- Stable kidney function \\
- Relapse: doubling of the UPCR (proteinuric) or an increase in SCr level of $50 \%$ or more for more than \\
& 1 month (nephritic) \\
- Major infection \\
- Herpes zoster virus infection \\
- Malignancy \\
- Ovarian failure
\end{tabular}

Notes

- Funding source: Roche

\section{Risk of bias}

\begin{tabular}{lll}
\hline Bias & Authors' judgement & Support for judgement \\
\hline $\begin{array}{l}\text { Random sequence genera- } \\
\text { tion (selection bias) }\end{array}$ & High risk & $\begin{array}{l}\text { "After induction, participants were randomly assigned, in order of enrolment } \\
\text { by means of sealed envelopes (stratified in two groups: blacks and other par- } \\
\text { ticipants)." - consecutive sequence generation }\end{array}$ \\
\hline $\begin{array}{l}\text { Allocation concealment } \\
\text { (selection bias) }\end{array}$ & Low risk & Sealed envelopes used \\
\hline
\end{tabular}


Contreras 2004 (Continued)

Blinding of participants High risk Open-label study and personnel (performance bias)

All outcomes

\begin{tabular}{lll}
\hline $\begin{array}{l}\text { Blinding of outcome as- } \\
\text { sessment (detection bias) } \\
\text { All outcomes }\end{array}$ & Unclear risk & Insufficient information to permit judgement \\
\hline $\begin{array}{l}\text { Incomplete outcome data } \\
\text { (attrition bias) } \\
\text { All outcomes }\end{array}$ & Low risk & No missing outcome data \\
\hline $\begin{array}{l}\text { Selective reporting (re- } \\
\text { porting bias) }\end{array}$ & Low risk & Study protocol available and pre-specified outcomes were reported \\
\hline $\begin{array}{l}\text { Other bias } \\
\text { High risk }\end{array}$ & $\begin{array}{l}\text { Roche pharmaceutical providing research nurse support and MMF 1999 to } \\
\text { 2003. Authors received fees for lectures and a grant from Roche Pharmaceuti- } \\
\text { cals. }\end{array}$ \\
\hline
\end{tabular}

\section{CYCLOFA-LUNE 2010}

\begin{tabular}{|c|c|}
\hline Methods & $\begin{array}{l}\text { - Study design: open-label, parallel RCT } \\
\text { - Study timeframe: January } 2002 \text { to December } 2006 \\
\text { - Duration of follow-up: median extended follow-up } 7.7 \text { years (range } 5.0 \text { to } 10.3 \text { years) }\end{array}$ \\
\hline Participants & $\begin{array}{l}\text { - Country: Czech Republic; Slovakia } \\
\text { - Setting: multinational (8 sites) } \\
\text { - Inclusion criteria: ACR criteria for SLE; biopsy-proven lupus nephritis (WHO or ISN/RPS criteria) and } \\
\text { clinical activity as defined by presence of at least two of the following: abnormal proteinuria (more } \\
\text { than } 500 \mathrm{mg} / 24 \mathrm{~h} \text { ), abnormal microscopic haematuria, or C3 hypocomplementaemia } \\
\text { - Number (analysed): treatment group } 1(21) \text {; treatment group } 2(19) \\
\text { - Mean age } \pm \text { SD (years): treatment group } 1(30 \pm 9) \text {; treatment group } 2(28 \pm 5) \\
\text { - Sex (M/F): treatment group } 1(6 / 15) \text {; treatment group } 2 \text { (5/14) } \\
\text { - Exclusion criteria: previous CPA or CSA ever before; treatment with immunosuppressive drugs or cor- } \\
\text { ticosteroids within the last } 3 \text { months; persistent elevation of SCr }>140 \mu \text { mol/L; pregnancy or lactation; } \\
\text { bone marrow insufficiency not attributable to SLE; severe co-existing conditions such as infection, } \\
\text { liver disease, or active peptic ulcer }\end{array}$ \\
\hline
\end{tabular}

Interventions

Induction and maintenance therapy: duration of therapy was 9 months induction therapy and 9 months maintenance therapy

- Treatment group 1

* Intermittent IV CPA: $10 \mathrm{mg} / \mathrm{kg}$ x 8 over 9 months followed by 4 or 5 oral pulses $(10 \mathrm{mg} / \mathrm{d}$ in 6 to 8 week intervals)

- Treatment group 2

* Daily oral CSA: 4 to $5 \mathrm{mg} / \mathrm{kg} / \mathrm{d}$ for 9 months followed by tapering dose of 3.75 to $1.25 \mathrm{mg} / \mathrm{kg} / \mathrm{d}$ for further 9 months

- Both groups

* MP $0.8 \mathrm{mg} / \mathrm{kg} / \mathrm{d}$ tapering to $0.2 \mathrm{mg} / \mathrm{kg} / \mathrm{d}$ over 8 weeks. Additional 1 to 3 doses of MP $(15 \mathrm{mg} / \mathrm{kg}$ ) were administered if felt insufficient control of kidney or extra-kidney disease, or a $30 \%$ to $50 \%$ increase in oral steroids with a change in timing of CPA or increase in dose of CSA was also allowed

\begin{tabular}{ll}
\hline Outcomes & Death \\
& - Renal relapse: signs of renal activity
\end{tabular}


CYCLOFA-LUNE 2010 (Continued)

- Major infection

- Herpes zoster virus

- Ovarian failure

- Bladder toxicity

- Alopecia

- Lymphopenia

- Complete renal remission: $\mathrm{SCr}$ within the normal range with stable or improved values as compared with baseline (no more than $15 \%$ above baseline), AND inactive urinary sediment, AND normal range proteinuria $(<0.3 \mathrm{~g} / 24 \mathrm{~h})$

- Partial renal remission: SCr within the normal range with stable or improved values as compared with baseline (no more than $15 \%$ above baseline), AND at least $50 \%$ decrease in proteinuria to less than $3 \mathrm{~g} /$ $\mathrm{d}$ if nephrotic at baseline, or to $0.5 \mathrm{~g} / \mathrm{d}$ if baseline non-nephrotic, AND either inactive urinary sediment or at least $25 \%$ improvement in C3 complement (patients with complete remission are counted within this less strict category as well

- $\mathrm{SCr}$

- Proteinuria

Notes Funding source: IGA Ministry of Health Czech Republic

\section{Risk of bias}

\begin{tabular}{|c|c|c|}
\hline Bias & Authors' judgement & Support for judgement \\
\hline $\begin{array}{l}\text { Random sequence genera- } \\
\text { tion (selection bias) }\end{array}$ & Unclear risk & $\begin{array}{l}\text { Randomisation } 1: 1 \text {, non-blocked methods for sequence generation not report- } \\
\text { ed }\end{array}$ \\
\hline $\begin{array}{l}\text { Allocation concealment } \\
\text { (selection bias) }\end{array}$ & Low risk & Central computerised system \\
\hline $\begin{array}{l}\text { Blinding of participants } \\
\text { and personnel (perfor- } \\
\text { mance bias) } \\
\text { All outcomes }\end{array}$ & High risk & Open-label study \\
\hline $\begin{array}{l}\text { Blinding of outcome as- } \\
\text { sessment (detection bias) } \\
\text { All outcomes }\end{array}$ & Unclear risk & Insufficient information to permit judgement \\
\hline $\begin{array}{l}\text { Incomplete outcome data } \\
\text { (attrition bias) } \\
\text { All outcomes }\end{array}$ & Low risk & No missing outcome data \\
\hline $\begin{array}{l}\text { Selective reporting (re- } \\
\text { porting bias) }\end{array}$ & Low risk & Study protocol available and pre-specified outcomes were reported \\
\hline Other bias & Low risk & $\begin{array}{l}\text { Research grants from the IGA Ministry of Health, Czech Republic. The study ap- } \\
\text { pears to be free of other sources of bias }\end{array}$ \\
\hline
\end{tabular}

\section{Decker 1975}

$\begin{array}{ll}\text { Methods } & \text { - Study design: open-label, parallel RCT } \\ \text { - Study timeframe: } 1969 \text { to } 1981 \\ \text { - Duration of follow-up: median } 7 \text { years }\end{array}$

Participants - Country: USA


Decker 1975 (Continued)

- Setting: multicentre (number of sites not reported)

- Inclusion criteria: Diagnosis of SLE (ACR criteria); clinical or histologic evidence of active lupus GN (mostly proliferative lesions) (WHO classification criteria)

- Number (randomised/analysed): treatment group 1 (30/28); treatment group 2 (20/19); treatment group 3 (18/18); treatment group 4 (23/22); treatment group 5 (20/20)

- Age: median age 27 years (age for individual groups not reported)

- $\operatorname{Sex}(M / F): 15 / 92$ (sex for individual groups not reported)

- Biopsy-proven lupus nephritis: $(60 / 107)$

- Exclusion criteria: $\mathrm{CrCl}<20 \mathrm{~mL} / \mathrm{min}$; major infection within 2 weeks; pregnancy; leucocyte count < $2000 / \mathrm{mm}^{3}$; cytotoxic therapy within 8 weeks; sensitivity to study drugs

Interventions

Induction therapy: duration of therapy until 18 months of remission had been achieved or 4 years of protocol therapy

- Treatment group 1

* Prednisolone alone: $1 \mathrm{mg} / \mathrm{kg}$ for 4 to 8 weeks, then tapering

- Treatment group 2

* AZA: up to $4 \mathrm{mg} / \mathrm{kg} / \mathrm{d}$

- Treatment group 3

* Oral CPA: up to $4 \mathrm{mg} / \mathrm{kg} / \mathrm{d}$

- Treatment group 4

* CPA and AZA: up to $1 \mathrm{mg} / \mathrm{kg} / \mathrm{d}$ of each

- Treatment group 4

* IV pulse CPA: IV every 3 month 0.5 to $1.0 \mathrm{~g} / \mathrm{m}^{2}$

- Additional treatment

* Groups 2 to 4 were also treated with low-dose prednisone (up to $0.5 \mathrm{mg} / \mathrm{kg} / \mathrm{d}$ )

Notes $\quad$ Funding source: NIH trial

\section{Risk of bias}

Bias Authors' judgement Support for judgement

Random sequence genera- Low risk " "...drawing marked card sequence from a table of random numbers..." tion (selection bias)

\begin{tabular}{lll}
$\begin{array}{l}\text { Allocation concealment } \\
\text { (selection bias) }\end{array}$ & Unclear risk & Insufficient information to permit judgement \\
\hline $\begin{array}{l}\text { Blinding of participants } \\
\text { and personnel (perfor- } \\
\text { mance bias) }\end{array}$ & Unclear risk & Insufficient information to permit judgement \\
All outcomes & &
\end{tabular}


Decker 1975 (Continued)

Blinding of outcome as- Unclear risk Insufficient information to permit judgement sessment (detection bias)

All outcomes

\begin{tabular}{lll}
\hline $\begin{array}{l}\text { Incomplete outcome data } \\
\text { (attrition bias) } \\
\text { All outcomes }\end{array}$ & Low risk & $\begin{array}{l}3.6 \%(4 / 111) \text { of participants excluded as they did not complete } 3 \text { months of } \\
\text { treatment }\end{array}$ \\
\hline $\begin{array}{l}\text { Selective reporting (re- } \\
\text { porting bias) }\end{array}$ & Low risk & All expected outcomes were reported \\
\hline Other bias & High risk & $\begin{array}{l}\text { Patients were assigned to treatment groups } 1,2 \text { and } 3 \text { from the beginning of } \\
\text { the study (1969). Treatment groups } 4 \text { and } 5 \text { were introduced in January } 1973 . \\
\text { Pooling of multiple studies }\end{array}$ \\
\hline
\end{tabular}

Deng 2016

\begin{tabular}{ll}
\hline Methods & - Study design: parallel RCT \\
& - Study timeframe: not reported \\
\hline Participants & - Country: China \\
- Setting: not reported \\
- Inclusion criteria: biopsy-proven proliferative lupus nephritis \\
- Mean age \pm SD (nears): not reported \\
- Sex (M/F): not reported \\
- Exclusion criteria: not reported
\end{tabular}

Induction therapy: duration of therapy was 6 months
Interventions

\begin{tabular}{ll}
\hline Outcomes & Adverse events \\
& - Proteinuria \\
& - Serum albumin \\
\hline Notes & - Abstract-only publication \\
& - Funding source: not reported \\
\hline
\end{tabular}

\section{Risk of bias}

\begin{tabular}{lll}
\hline Bias & Authors' judgement & Support for judgement \\
\hline $\begin{array}{l}\text { Random sequence genera- } \\
\text { tion (selection bias) }\end{array}$ & Unclear risk & Insufficient information to permit judgement \\
\hline
\end{tabular}


Deng 2016 (Continued)

Allocation concealment $\quad$ Unclear risk Insufficient information to permit judgement
(selection bias)

Blinding of participants Unclear risk Insufficient information to permit judgement

and personnel (perfor-

mance bias)

All outcomes

\begin{tabular}{lll}
\hline Blinding of outcome as- & Unclear risk & Insufficient information to permit judgement \\
sessment (detection bias) &
\end{tabular}

sessment (detection bias)

All outcomes

\begin{tabular}{lll}
\hline $\begin{array}{l}\text { Incomplete outcome data } \\
\text { (attrition bias) } \\
\text { All outcomes }\end{array}$ & Unclear risk & Insufficient information to permit judgement \\
\hline $\begin{array}{l}\text { Selective reporting (re- } \\
\text { porting bias) }\end{array}$ & High risk & $\begin{array}{l}\text { Not all pre-specified outcomes found on the protocol are reported; data could } \\
\text { not be meta-analysed }\end{array}$ \\
\hline Other bias & High risk & $\begin{array}{l}\text { Primary outcomes identified on clinicaltrials.gov page not reported. Focus on } \\
\text { p-values in the results, with no reporting of the continuous or categorical data }\end{array}$ \\
\hline
\end{tabular}

Derksen 1988

\begin{tabular}{|c|c|}
\hline Methods & $\begin{array}{l}\text { - Study design: parallel RCT } \\
\text { - Study timeframe: } 1981 \text { to } 1985 \\
\text { - Duration of follow-up: } 26 \text { weeks }\end{array}$ \\
\hline Participants & $\begin{array}{l}\text { - Country: Netherlands } \\
\text { - Setting: multicentre ( } 5 \text { sites) } \\
\text { - Inclusion criteria: diagnosis of SLE (ARA criteria); presence of active lupus nephritis, defined by a de- } \\
\text { creased } \mathrm{CrCl} \text {, an active urine sediment ( }>5 \mathrm{RBC} / \mathrm{HPF} \text { and cellular casts) and proteinuria > } 0.5 \mathrm{~g} / 24 \mathrm{~h} \text {; } \\
\text { biopsy-proven proliferative lupus nephritis (class III or IV WHO classification criteria); insufficient re- } \\
\text { sponse of kidney function to treatment with corticosteroids alone given in a single daily dose of } 1-1.5 \\
\mathrm{mg} / \mathrm{kg} \text { for at least } 3 \text { weeks } \\
\text { - Number (randomised): treatment group } 1 \text { (11); treatment group } 2(9) \\
\text { - Mean age, range SD (years): treatment group } 1 \text { ( } 28,15 \text { to } 55) \text {; treatment group } 2(36,18 \text { to } 60) \\
\text { - Sex (M/F): treatment group } 1 \text { (3/8); treatment group } 2(2 / 7) \\
\text { - Exclusion criteria: deterioration of kidney function could be explained by other causes, such as the } \\
\text { use of NSAIDs, infection or hypotension; patients with active renal insufficiency with oliguria/anuria } \\
\text { (dialysis indications), and patients with psychiatric manifestations }\end{array}$ \\
\hline
\end{tabular}

Interventions

Induction therapy: duration of therapy was 26 weeks

- Treatment group 1

* Prednisone \pm cytotoxics (oral AZA or CPA $2 \mathrm{mg} / \mathrm{kg}$ if kidney function and haematological functions permitted)

- Treatment group 2

* PEX alone: short course

- Both groups

- Daily oral prednisone $(1.5 \mathrm{mg} / \mathrm{kg}$ ) until the time of randomisation, the dose was gradually reduced (a decrease in daily dose of $10 \mathrm{mg}$, once a week) until a daily dose of $1 \mathrm{mg} / \mathrm{kg}$ was reached

\begin{tabular}{ll}
\hline Outcomes & Death \\
& $\cdot$ ESKD \\
\hline
\end{tabular}


Derksen 1988 (Continued)

- $\mathrm{CrCl}$

Notes - Funding source: not reported

\section{Risk of bias}

\begin{tabular}{lll}
\hline Bias & Authors' judgement & Support for judgement \\
\hline $\begin{array}{l}\text { Random sequence genera- } \\
\text { tion (selection bias) }\end{array}$ & Low risk & Drawing lots from card sequence \\
\hline $\begin{array}{l}\text { Allocation concealment } \\
\text { (selection bias) }\end{array}$ & Unclear risk & Insufficient information to permit judgement \\
\hline $\begin{array}{l}\text { Blinding of participants } \\
\text { and personnel (perfor- } \\
\text { mance bias) } \\
\text { All outcomes }\end{array}$ & Unclear risk & Insufficient information to permit judgement \\
\hline $\begin{array}{l}\text { Blinding of outcome as- } \\
\text { sessment (detection bias) }\end{array}$ & Unclear risk & \\
All outcomes & & Insufficient information to permit judgement \\
\hline $\begin{array}{l}\text { Incomplete outcome data } \\
\text { (attrition bias) } \\
\text { All outcomes }\end{array}$ & Unclear risk & Insufficient information to permit judgement \\
\hline $\begin{array}{l}\text { Selective reporting (re- } \\
\text { porting bias) }\end{array}$ & High risk & Pooling interventions in cytotoxic group \\
\hline \begin{tabular}{l} 
Other bias \\
\hline
\end{tabular} & High risk & Not all expected outcomes are reported \\
\hline
\end{tabular}

\section{Donadio 1972}

\begin{tabular}{ll}
\hline Methods & Study design: open-label, parallel RCT \\
- Study timeframe: not reported \\
- Duration of follow-up: 3 years \\
\hline Participants & Country: USA \\
- Setting: single centre \\
- Inclusion criteria: histologic evidence of kidney disease; one or more of the following: serositis, arthral- \\
gia, and arthritis, skin rash consistent with SLE and haematological abnormalities that included \\
leukopenia, thrombocytopenia or a circulating anticoagulant \\
- Number (randomised): treatment group (7); treatment group (9) \\
- Sex (M/F): $2 / 14$ \\
- Exclusion criteria: received $>7.5$ mg prednisone daily in the previous 6 months (except a dose of 20 \\
mg daily for a maximum of 2 weeks); previous cytotoxic medication other than antimalarial treatment
\end{tabular}

\footnotetext{
Interventions Induction therapy

- Treatment group

* Prednisone +AZA ( $2 \mathrm{mg} / \mathrm{kg} /$ body weight for 6 months); average duration of therapy was 26 months for AZA
} 
Donadio 1972 (Continued)

- Control group

* Prednisone: $60 \mathrm{mg} / \mathrm{d}$ for 2 months, $40 \mathrm{mg} / \mathrm{d}$ by 3 months, $30 \mathrm{mg} / \mathrm{d}$ by 4 months, $25 \mathrm{mg} / \mathrm{d}$ by 5 months and $20 \mathrm{mg} / \mathrm{d}$ by 6 months

\begin{tabular}{ll}
\hline Outcomes & Death \\
& - Complete remission \\
& - Relapse \\
& - Toxicity \\
- & $\mathrm{CrCl}$ \\
& - Proteinuria \\
& - Leucopenia $\left(\mathrm{WCC}<3000 / \mathrm{mL}^{3}\right)$ \\
\hline Notes & - Funding source: Mayo Foundation
\end{tabular}

\section{Risk of bias}

Bias Authors' judgement Support for judgement

Random sequence genera- Low risk tion (selection bias)

Participants allocated within each category to treatment group A or B according to random selection. Table of random numbers used. Each incoming set of 4 participants assigned to 2 As and 2 Bs in random order

\begin{tabular}{lll}
\hline $\begin{array}{l}\text { Allocation concealment } \\
\text { (selection bias) }\end{array}$ & Unclear risk & Insufficient information to permit judgement \\
\hline $\begin{array}{l}\text { Blinding of participants } \\
\text { and personnel (perfor- } \\
\text { mance bias) }\end{array}$ & High risk & Open-label study \\
All outcomes & \\
\hline
\end{tabular}

Blinding of outcome as- Unclear risk Insufficient information to permit judgement
sessment (detection bias)

All outcomes

Incomplete outcome data Unclear risk Insufficient information to permit judgement
(attrition bias)

All outcomes

Selective reporting (re- High risk One or more reported primary outcomes were not pre-specified
porting bias)

Other bias Low risk The study appears to be free of other sources of bias

Donadio 1976

\begin{tabular}{ll}
\hline Methods & Study design: open-label, parallel RCT \\
- Study timeframe: commenced December 1971 \\
- Duration of follow-up: 4 years \\
\hline Participants & Country: USA \\
- Setting: single centre \\
- Inclusion criteria: SLE fulfilled 4 or more criteria used for the classification of the disease; a positive \\
LE-cell preparation or rosettes of neutrophils or nucleolysis; a positive antinuclear-antibody test in \\
titres $\geq 1: 32$ or elevated levels of anti-nDNA; $\mathrm{CrCl}<80 \mathrm{~mL} / \mathrm{min} / 1.73 \mathrm{~m}^{2}$ or a reduction of $25 \%$ in the
\end{tabular}


Donadio 1976 (Continued)

$\mathrm{CrCl}$ as compared with the initial clearance of a maximal period of three months; and adequate renal biopsy showing diffuse proliferative $\mathrm{GN}$

- Number (randomised): treatment group (24); control group (26)

- Mean age, range (years): treatment group (30.2, 16 to 60$)$; control group (32.3, 17 to 50)

- Sex (M/F): treatment group (5/19); control group (4/22)

- Exclusion criteria: Previous CPA or immunosuppressive drugs in the last 6 months

\begin{tabular}{ll}
\hline Interventions & Treatment group \\
& $*$ Oral CPA: $2 \mathrm{mg} / \mathrm{kg} / \mathrm{d}$ for 6 months \\
& $*$ Maintenance dose of prednisone to control other systemic manifestations \\
- & Control group \\
& $*$ Prednisone: $60 \mathrm{mg} / \mathrm{d}$ tapered after 1 to 3 months \\
\hline Outcomes & ESKD \\
- Death & Toxicity \\
- Major infection & Treatment failure: ESKD or final CrCl increased by $25 \%$ \\
- Relapse: reappearance of systematic features, reductions in CrCl, increased proteinuria and changes & in anti-nDNA and CH50 levels \\
- Current status on kidney function \\
- Proteinuria \\
- Avascular necrosis \\
\hline - Funding source: Mayo Foundation and Constance Belden Memorial Fund
\end{tabular}

Risk of bias

\begin{tabular}{|c|c|c|}
\hline Bias & Authors' judgement & Support for judgement \\
\hline $\begin{array}{l}\text { Random sequence genera- } \\
\text { tion (selection bias) }\end{array}$ & Low risk & Random number tables used \\
\hline $\begin{array}{l}\text { Allocation concealment } \\
\text { (selection bias) }\end{array}$ & Unclear risk & Insufficient information to permit judgement \\
\hline $\begin{array}{l}\text { Blinding of participants } \\
\text { and personnel (perfor- } \\
\text { mance bias) } \\
\text { All outcomes }\end{array}$ & High risk & Open-label study \\
\hline $\begin{array}{l}\text { Blinding of outcome as- } \\
\text { sessment (detection bias) } \\
\text { All outcomes }\end{array}$ & Unclear risk & Insufficient information to permit judgement \\
\hline $\begin{array}{l}\text { Incomplete outcome data } \\
\text { (attrition bias) } \\
\text { All outcomes }\end{array}$ & Unclear risk & Insufficient information to permit judgement \\
\hline $\begin{array}{l}\text { Selective reporting (re- } \\
\text { porting bias) }\end{array}$ & Low risk & All expected outcomes are reported \\
\hline Other bias & Low risk & The study appears to be free of other sources of bias \\
\hline
\end{tabular}


Doria 1994

\begin{tabular}{|c|c|}
\hline Methods & $\begin{array}{l}\text { - Study design: parallel RCT } \\
\text { - Study timeframe: } 1988 \text { to } 1993 \\
\text { - Duration of follow-up: every } 4 \text { weeks for } 24 \text { months and then every } 8 \text { weeks thereafter }\end{array}$ \\
\hline Participants & $\begin{array}{l}\text { - Country: Italy } \\
\text { - Setting: single centre } \\
\text { - Inclusion criteria: SLE (1982 ACR criteria); biopsy-proven class IV lupus nephritis (WHO classification } \\
\text { criteria); normal kidney function ( } \mathrm{SCr} \leq 1.2 \mathrm{mg} / \mathrm{dL} \text { ) } \\
\text { - Number (randomised): treatment group } 1 \text { (7); treatment group } 2 \text { (5); control group (6) } \\
\text { - Mean age, range (years): treatment group } 1 \text { ( } 30,20 \text { to } 55) \text {; treatment group } 2 \text { (23, } 15 \text { to } 32) \text {; control } \\
\text { group ( } 25,15 \text { to } 46) \\
\text { - Sex (M/F): } 2 / 16 \text { (not reported for individual groups) } \\
\text { - Exclusion criteria: Pregnancy; aged < } 15 \text { and > } 80 \text { years; infections; insulin-dependent DM; history of } \\
\text { malignancy; immunosuppressive therapy within a } 6 \text { month period prior to renal biopsy }\end{array}$ \\
\hline Interventions & $\begin{array}{l}\text { Induction therapy } \\
\text { - Treatment group } \\
\text { * Standard therapy } \\
\text { * PEX: } 3 \text { x times weekly for } 1 \text { week then twice a week for } 2 \text { weeks then once a week for } 2 \text { months then } \\
\text { once a fortnight for } 3 \text { months. } 50 \% \text { of the patient's plasma volume was removed and replaced with } \\
\text { a } 4 \% \text { human albumin solution } \\
\text { - Treatment group } 2 \\
\text { * Standard therapy } \\
\text { * IV MP: } 500 \mathrm{mg} \text { daily for } 3 \text { consecutive days } \\
\text { Control group } \\
\text { * Standard therapy } \\
\square \text { Prednisone: } 2 \mathrm{mg} / \mathrm{kg} / \mathrm{d} \text { for } 4 \text { weeks with slow tapering ( } 5 \mathrm{mg} \text { every } 10 \text { days) } \\
\square \text { AZA: } 2 \mathrm{mg} / \mathrm{kg} / \mathrm{d}\end{array}$ \\
\hline
\end{tabular}

\begin{tabular}{ll}
\hline Outcomes & Death \\
& $\cdot$ ESKD \\
& $\cdot 24$ h urinary protein \\
& - Partial remission \\
& - Complete remission \\
& - Herpes zoster virus \\
& - Leucopenia \\
\hline Notes & - Funding source: not reported
\end{tabular}

\section{Risk of bias}

\begin{tabular}{lll}
\hline Bias & Authors' judgement & Support for judgement \\
\hline $\begin{array}{l}\text { Random sequence genera- } \\
\text { tion (selection bias) }\end{array}$ & Unclear risk & $\begin{array}{l}\text { Study was described as randomised, method of randomisation was not report- } \\
\text { ed }\end{array}$ \\
\hline $\begin{array}{l}\text { Allocation concealment } \\
\text { (selection bias) }\end{array}$ & Unclear risk & Insufficient information to permit judgement \\
\hline
\end{tabular}


Doria 1994 (Continued)

Blinding of participants High risk Open-label study and personnel (performance bias)

All outcomes

\begin{tabular}{ll}
\hline Blinding of outcome as- & Unclear risk
\end{tabular} Insufficient information to permit judgement

All outcomes

Incomplete outcome data Low risk No missing outcome data
(attrition bias)

All outcomes

\begin{tabular}{lll}
\hline $\begin{array}{l}\text { Selective reporting (re- } \\
\text { porting bias) }\end{array}$ & Low risk & All relevant outcomes reported \\
\hline Other bias & Low risk & The study appears to be free of other sources of bias \\
\hline
\end{tabular}

Dyadyk 2001

\begin{tabular}{ll}
\hline Methods & Study design: parallel RCT \\
- Study timeframe: not reported & Duration of follow-up: 19 years
\end{tabular}

\begin{tabular}{|c|c|}
\hline Participants & $\begin{array}{l}\text { - } \text { Country: Ukraine } \\
\text { - Setting: not reported } \\
\text { - Inclusion criteria: diffuse proliferative lupus nephritis class IV (WHO classification criteria) } \\
\text { - Number (randomised/analysed): treatment group } 1 \text { (21/21); treatment group } 2 \text { (38/38) } \\
\text { - Mean age: } 36 \text { years (not reported for groups) } \\
\text { - Sex (M/F): treatment group } 1 \text { (4/17); treatment group } 2(5 / 33) \\
\text { - Exclusion criteria: not reported }\end{array}$ \\
\hline Interventions & $\begin{array}{l}\text { Induction therapy } \\
\text { - Treatment group } 1 \\
\text { * AZA: } 1.5 \text { to } 2.0 \mathrm{mg} / \mathrm{kg} / \mathrm{d} \text {; mean total duration of therapy (18.9 months) } \\
\text { - Treatment group } 2 \\
\text { * CPA: } 1.5 \text { to } 3.5 \mathrm{mg} / \mathrm{kg} / \mathrm{d} \text {; mean total duration of therapy ( } 21.7 \text { months) }\end{array}$ \\
\hline Outcomes & $\begin{array}{l}\text { - Death (all causes) } \\
\text { - Complete remission } \\
\text { - Partial remission }\end{array}$ \\
\hline Notes & $\begin{array}{l}\text { - Abstract-only publications } \\
\text { - } 5 \text { and } 10 \text { year survival follow-up } \\
\text { - Funding source: not reported }\end{array}$ \\
\hline
\end{tabular}

\section{Risk of bias}

\begin{tabular}{lll}
\hline Bias & Authors' judgement & Support for judgement \\
\hline $\begin{array}{l}\text { Random sequence genera- } \\
\text { tion (selection bias) }\end{array}$ & Unclear risk & $\begin{array}{l}\text { Study was described as randomised, method of randomisation was not report- } \\
\text { ed }\end{array}$ \\
\hline
\end{tabular}


Dyadyk 2001 (Continued)

\begin{tabular}{l}
$\begin{array}{l}\text { Allocation concealment } \\
\text { (selection bias) }\end{array}$ \\
\hline
\end{tabular}

\begin{tabular}{lll}
\hline Blinding of participants & High risk & Open-label study \\
and personnel (perfor- & & \\
mance bias) & \\
All outcomes &
\end{tabular}

Blinding of outcome as- Unclear risk Insufficient information to permit judgement
sessment (detection bias)

All outcomes

\begin{tabular}{lll}
\hline $\begin{array}{l}\text { Incomplete outcome data } \\
\text { (attrition bias) } \\
\text { All outcomes }\end{array}$ & Unclear risk & Insufficient information to permit judgement \\
\hline $\begin{array}{l}\text { Selective reporting (re- } \\
\text { porting bias) }\end{array}$ & High risk & Not all relevant reported outcomes are reported \\
\hline Other bias & Low risk & The study appears to be free of other sources of bias \\
\hline
\end{tabular}

\section{El-Sehemy 2006}

\begin{tabular}{ll}
\hline Methods & Study design: parallel RCT \\
- Study timeframe: commenced January 2004 \\
- & Duration of follow-up: 6 months \\
\hline Participants & Country: Egypt \\
- Setting: single centre \\
- Inclusion criteria: all SLE patients; class III (1), class IV (10), class Vc (5), class Va or b (4), class V (1), \\
unclassified (1) \\
- Number (randomised/analysed): treatment group 1 (7/7); treatment group 2 (7/7); treatment group \\
- $3(8 / 8)$ \\
- to 27$)$ \\
- Sex (M/F): all female \\
Exclusion criteria: uncontrolled infection; CNS manifestations; known neoplastic disease; intention \\
to become pregnant; previous immunosuppressive drugs < 3 months prior to study
\end{tabular}

Induction therapy: duration of therapy not reported
- Treatment group 1
$*$ CPA: $0.75 \mathrm{mg} / \mathrm{m}^{2}$
- Treatment group 2
$*$ CSA: 1 to $2 \mathrm{mg} / \mathrm{kg} / \mathrm{d}$
- Treatment group 3
$*$ AZA: 1 to $2 \mathrm{mg} / \mathrm{kg} / \mathrm{d}$
- All groups
$*$ MP 500 to $1000 \mathrm{mg} / \mathrm{kg} / \mathrm{d}$ for 3 to 5 days then oral prednisolone $0.5 \mathrm{mg} / \mathrm{kg} / \mathrm{d}$ for 4 weeks then tapered
dose

\begin{tabular}{ll}
\hline Outcomes & Major infection \\
- Ovarian failure \\
- Proteinuria
\end{tabular}


El-Sehemy 2006 (Continued)

- $\mathrm{CrCl}$

Notes - Three participants from group 1 and one participant from group 3 shifted to group II due to side effects or no response

- Funding source: not reported

\section{Risk of bias}

\begin{tabular}{|c|c|c|}
\hline Bias & Authors' judgement & Support for judgement \\
\hline $\begin{array}{l}\text { Random sequence genera- } \\
\text { tion (selection bias) }\end{array}$ & Unclear risk & $\begin{array}{l}\text { Study was described as randomised, method of randomisation was not report- } \\
\text { ed }\end{array}$ \\
\hline $\begin{array}{l}\text { Allocation concealment } \\
\text { (selection bias) }\end{array}$ & Unclear risk & Insufficient information to permit judgement \\
\hline $\begin{array}{l}\text { Blinding of participants } \\
\text { and personnel (perfor- } \\
\text { mance bias) } \\
\text { All outcomes }\end{array}$ & Unclear risk & Insufficient information to permit judgement \\
\hline $\begin{array}{l}\text { Blinding of outcome as- } \\
\text { sessment (detection bias) } \\
\text { All outcomes }\end{array}$ & Unclear risk & Insufficient information to permit judgement \\
\hline $\begin{array}{l}\text { Incomplete outcome data } \\
\text { (attrition bias) } \\
\text { All outcomes }\end{array}$ & Low risk & No missing outcome data \\
\hline $\begin{array}{l}\text { Selective reporting (re- } \\
\text { porting bias) }\end{array}$ & High risk & Not all expected patient outcomes reported \\
\hline Other bias & High risk & $\begin{array}{l}\text { Baseline kidney function highly different between groups. Reported outcomes } \\
\text { with patients transferred to different groups }\end{array}$ \\
\hline
\end{tabular}

El-Shafey 2010

\begin{tabular}{ll}
\hline Methods & Study design: open label, RCT \\
- Study timeframe: February 2006 to December 2008 \\
- \\
\hline Participants
\end{tabular}


El-Shafey 2010 (Continued)

- Treatment group 1

* MMF: $1 \mathrm{~g}$ twice daily for 6 months

- Treatment group 2

* IV CPA: 0.5 to $1.0 \mathrm{~g} / \mathrm{m}^{2}$ for 6 months, median monthly dose $0.75 \mathrm{~g} / \mathrm{m}^{2}$

- Both groups

* Prednisolone: $60 \mathrm{mg} / \mathrm{d}$ for 4 to 6 weeks, then $40 \mathrm{mg} / \mathrm{d}$ for 2 weeks followed by tapering dose to 5 to $10 \mathrm{mg} / \mathrm{d}$

\begin{tabular}{|c|c|}
\hline Outcomes & $\begin{array}{l}\text { - Death (all causes) } \\
\text { - ESKD } \\
\text { - Remission: combined complete and partial remission at } 6 \text { months } \\
\text { - Complete renal remission: normal } \mathrm{SCr} \text {, proteinuria }<0.5 \mathrm{~g} / \mathrm{d} \text { and urine } \mathrm{RBC}<5 \text { per HPF, without RBC } \\
\text { cast } \\
\text { - Partial renal remission: improvement of } 50 \% \text { in all abnormal renal measurements without deteriora- } \\
\text { tion (within } 20 \% \text { of any measurement } \\
\text { - Major infection } \\
\text { - Herpes zoster virus } \\
\text { - Menstrual irregularities } \\
\text { - Diarrhoea } \\
\text { - Lymphopenia } \\
\text { - SCr } \\
\text { - eGFR } \\
\text { - Proteinuria }\end{array}$ \\
\hline Notes & - Funding source: not reported \\
\hline
\end{tabular}

\section{Risk of bias}

\begin{tabular}{lll}
\hline Bias & Authors' judgement & Support for judgement \\
\hline $\begin{array}{l}\text { Random sequence genera- } \\
\text { tion (selection bias) }\end{array}$ & Unclear risk & $\begin{array}{l}\text { Study was described as randomised, method of randomisation was not report- } \\
\text { ed }\end{array}$ \\
\hline $\begin{array}{l}\text { Allocation concealment } \\
\text { (selection bias) }\end{array}$ & Unclear risk & Insufficient information to permit judgement \\
\hline $\begin{array}{l}\text { Blinding of participants } \\
\text { and personnel (perfor- } \\
\text { mance bias) }\end{array}$ & High risk & Open-label study \\
$\begin{array}{l}\text { All outcomes } \\
\text { Blinding of outcome as- } \\
\text { sessment (detection bias) } \\
\text { All outcomes }\end{array}$ & Unclear risk & Insufficient information to permit judgement \\
\hline $\begin{array}{l}\text { Incomplete outcome data } \\
\text { (attrition bias) } \\
\text { All outcomes }\end{array}$ & Unclear risk & Insufficient information to permit judgement \\
\hline $\begin{array}{l}\text { Selective reporting (re- } \\
\text { porting bias) }\end{array}$ & Low risk & Study protocol available and pre-specified outcomes were reported \\
\hline \begin{tabular}{l} 
Other bias \\
\hline
\end{tabular} & Low risk & The study appears to be free of other sources of bias \\
\hline
\end{tabular}


Florez-Suarez 2004

\begin{tabular}{ll}
\hline Methods & Study design: parallel RCT \\
& - Study timeframe: not reported \\
\hline Participants & Duration of follow-up: 1year \\
\hline & - Country: Mexico \\
- Setting: not reported \\
- Number (randomised): 20 (numbers per group not reported) \\
- Mean age \pm SD (years): not reported \\
- Sex (M/F): not reported \\
- Exclusion criteria: not reported
\end{tabular}

\begin{tabular}{ll}
\hline Interventions & Induction therapy: duration of treatment was 12 months \\
& $*$ MMF: up to $2 \mathrm{~g} / \mathrm{d}$ \\
& Treatment group 1 \\
& $*$ IV CPA: monthly (does not reported) \\
& Both groups \\
& $*$ Prednisone \\
\hline Outcomes & Complete remission \\
& - Partial remission \\
- Treatment failure & Death \\
\hline Notes & Abstract-only publication; authors contact - no reply \\
& Funding source: Roche Mexico \\
\hline
\end{tabular}

\section{Risk of bias}

\section{Bias Authors' judgement Support for judgement}

Random sequence genera- Unclear risk Study was described as randomised, method of randomisation was not reporttion (selection bias) ed

\begin{tabular}{|c|c|c|}
\hline $\begin{array}{l}\text { Allocation concealment } \\
\text { (selection bias) }\end{array}$ & Unclear risk & Insufficient information to permit judgement \\
\hline $\begin{array}{l}\text { Blinding of participants } \\
\text { and personnel (perfor- } \\
\text { mance bias) } \\
\text { All outcomes }\end{array}$ & High risk & Open-label study \\
\hline $\begin{array}{l}\text { Blinding of outcome as- } \\
\text { sessment (detection bias) } \\
\text { All outcomes }\end{array}$ & Unclear risk & Insufficient information to permit judgement \\
\hline $\begin{array}{l}\text { Incomplete outcome data } \\
\text { (attrition bias) } \\
\text { All outcomes }\end{array}$ & Unclear risk & Insufficient information to permit judgement \\
\hline $\begin{array}{l}\text { Selective reporting (re- } \\
\text { porting bias) }\end{array}$ & High risk & Data unable to be meta-analysed \\
\hline
\end{tabular}


Florez-Suarez 2004 (Continued)

Other bias High risk abstract-only publication; funded by Roche Mexico

Fries 1973

\begin{tabular}{ll} 
Methods & Study design: open-label, RCT \\
& - Study timeframe: not reported \\
\hline Participants & - Country: USA \\
- Setting: single centre \\
- Inclusion criteria: SLE with antinuclear antibodies; involvement of two or more organs \\
- Number (randomised/lupus nephritis): treatment group (10/5); control group (12/5) \\
- Mean age \pm SD (years): not reported \\
- Sex (M/F): not reported \\
- Exclusion criteria: not reported
\end{tabular}

\begin{tabular}{ll}
\hline Interventions & $\begin{array}{l}\text { Induction therapy } \\
\text { - Treatment group } \\
* \quad \text { CPA: adjusted on the basis of weekly WCC, attempting to maintain a WCC between } 3500 \text { and } 4000 \\
\text { cells/cu mm } \\
\text { - Control group } \\
* \text { Prednisone: } 1 \mathrm{mg} / \mathrm{kg} / \mathrm{d}\end{array}$ \\
\hline Outcomes & Relapse \\
& - Failure or response of treatment \\
\hline Notes & Significant cross-over \\
& tional Institutes of Health RR00311-04 \\
\end{tabular}

\section{Risk of bias}

\begin{tabular}{lll}
\hline Bias & Authors' judgement & Support for judgement \\
\hline $\begin{array}{l}\text { Random sequence genera- } \\
\text { tion (selection bias) }\end{array}$ & Unclear risk & $\begin{array}{l}\text { Study was described as randomised, method of randomisation was not report- } \\
\text { ed }\end{array}$ \\
\hline $\begin{array}{l}\text { Allocation concealment } \\
\text { (selection bias) }\end{array}$ & Unclear risk & Insufficient information to permit judgement \\
\hline $\begin{array}{l}\text { Blinding of participants } \\
\text { and personnel (perfor- } \\
\text { mance bias) }\end{array}$ & High risk & Open-label study \\
All outcomes & \\
\hline $\begin{array}{l}\text { Blinding of outcome as- } \\
\text { sessment (detection bias) } \\
\text { All outcomes }\end{array}$ & Unclear risk & Insufficient information to permit judgement \\
\hline $\begin{array}{l}\text { Incomplete outcome data } \\
\text { (attrition bias) } \\
\text { All outcomes }\end{array}$ & Unclear risk & Insufficient information to permit judgement \\
\hline
\end{tabular}


Fries 1973 (Continued)

Selective reporting (re- High risk Not all relevant reported outcomes are reported
porting bias)

Other bias High risk Heavy cross-over between groups

\section{Fu 1997}

\begin{tabular}{ll}
\hline Methods & Study design: parallel RCT \\
& - Study timeframe: July 1994 to December 1995 \\
& - Duration of follow-up: 1 year \\
\hline Participants & Country: Taiwan \\
- Setting: single centre \\
- Inclusion criteria: diagnosis of SLE (ACR 1982 revised criteria); class III-IV lupus nephritis proven by \\
- biopsy (WHO classification criteria) with heavy proteinuria and normal SCr \\
- Number (randomised): treatment group 1 (20); treatment group 2 (20) \\
- Sean age \pm SD (years): treatment group 1 (10.2 \pm 3.4$) ;$ treatment group $2(10.4 \pm 3.1)$ \\
- Exclusion criteria: not reported
\end{tabular}

\begin{tabular}{ll}
\hline Interventions & Maintenance therapy: duration of treatment was 12 months \\
- & Treatment group 1 \\
& $*$ Oral CPA: $2 \mathrm{mg} / \mathrm{kg} / \mathrm{d}$ \\
& $*$ Prednisolone: $2 \mathrm{mg} / \mathrm{kg} / \mathrm{d}$ \\
- Treatment group 2 & $*$ CSA: $5 \mathrm{mg} / \mathrm{kg} / \mathrm{d}$ every $12 \mathrm{~h}$ \\
- Both groups & $*$ Oral prednisolone $2 \mathrm{mg} / \mathrm{kg} / \mathrm{d}$ for 4 weeks \pm pulsed MP (if unresponsive). Dose of prednisolone ta- \\
& pered to 0.5 to $1 \mathrm{mg} / \mathrm{kg}$ as maintenance therapy for $>1$ year before randomisation \\
\hline Outcomes & Proteinuria \\
- SCr & CrCl \\
- Height velocity & Height SDS \\
\hline Notes & Funding source: not reported \\
\hline
\end{tabular}

\section{Risk of bias}

\begin{tabular}{lll}
\hline Bias & Authors' judgement & Support for judgement \\
\hline $\begin{array}{l}\text { Random sequence genera- } \\
\text { tion (selection bias) }\end{array}$ & Low risk & $\begin{array}{l}\text { Participants randomly assigned (1:1, stratified by race and biopsy class, non- } \\
\text { blocked) by a central computerised, interactive voice response system random } \\
\text { number table }\end{array}$ \\
\hline $\begin{array}{l}\text { Allocation concealment } \\
\text { (selection bias) }\end{array}$ & Low risk & $\begin{array}{l}\text { Used sealed, completely opaque, envelopes numbered in sequence according } \\
\text { to a table of random numbers }\end{array}$ \\
\hline $\begin{array}{l}\text { Blinding of participants } \\
\text { and personnel (perfor- } \\
\text { mance bias) }\end{array}$ & High risk & Open-label study \\
\hline
\end{tabular}


Fu 1997 (Continued)

All outcomes

\begin{tabular}{|c|c|c|}
\hline $\begin{array}{l}\text { Blinding of outcome as- } \\
\text { sessment (detection bias) }\end{array}$ & Unclear risk & Insufficient information to permit judgement \\
\hline
\end{tabular}

All outcomes

\begin{tabular}{lll}
\hline $\begin{array}{l}\text { Incomplete outcome data } \\
\text { (attrition bias) } \\
\text { All outcomes }\end{array}$ & Low risk & No missing outcome data \\
\hline $\begin{array}{l}\text { Selective reporting (re- } \\
\text { porting bias) }\end{array}$ & High risk & Not all of the study's prespecified primary outcomes were reported \\
\hline Other bias & Low risk & $\begin{array}{l}\text { Funding source not declared. The study appears to be free of other sources of } \\
\text { bias }\end{array}$
\end{tabular}

Furie 2014

$\begin{array}{ll}\text { Methods } & \text { Study design: double-blind, parallel RCT } \\ \text { - Study timeframe: not reported } \\ \text { - Duration of follow-up: } 12 \text { months }\end{array}$

Participants

- Countries: North America, Europe, South America, Asia, Australia, India, South Africa, Turkey

- Setting: multinational (85 sites)

- Inclusion criteria: aged $\geq 18$ years; diagnosis of SLE (ACR criteria); class III or IV GN (ISN/RPS 2003 criteria or WHO 1982 classification), complement C3 or C4 levels below the lower limit of normal or elevated anti-dsDNA antibody titres at the time of screening were further requirements for eligibility as were UPCR of $\geq 0.44 \mathrm{mg} / \mathrm{mg}(50 \mathrm{mg} / \mathrm{mmol})$ at the time of screening and active urinary sediment $>5$ $\mathrm{RBC}$ or $>8 \mathrm{WBC} / \mathrm{HPF}$ or cylinduria at time of screening or the current flare

- Number (randomised/analysed): treatment group 1 (99/99); treatment group 2 (99/99); control group $(100 / 100)$

- Mean age \pm SD (years): treatment group 1 (30.5 \pm 10.6$)$; treatment group 2 (31 \pm 9.5$)$; control group $(31.8 \pm 9)$

- Sex (M/F): treatment group 1 (13/86); treatment group 2 (15/84); control group (19/81)

- Exclusion criteria: evidence of severe, rapidly advancing kidney failure (i.e. increase in SCr levels of $\geq$ $1 \mathrm{mg} / \mathrm{dL}$ within 1 month prior to screening or a SCr level of $>3 \mathrm{mg} / \mathrm{dL}$ ); evidence of severe unstable and or progressive central nervous system lupus; use of immunosuppressive or immunomodulatory agents during the study except for antimalarial agents and protocol defined MMF and glucocorticoids

Interventions

Induction therapy: duration of therapy was 12 months

- Treatment group 1

* Abatacept 10/10 regimen: weight tiered ( $500 \mathrm{mg}$ for patients weighing $<60 \mathrm{~kg}, 750 \mathrm{mg}$ for patients $60-100 \mathrm{~kg}, 1,000 \mathrm{mg}$ for patients $>100 \mathrm{~kg}$ ) on days $1,15,29,57,85,113,141,169,197,225,253$, 281,309 , and 337

- Treatment group 2

- Abatacept 30/10 regimen: $30 \mathrm{mg} / \mathrm{kg}$ on days $1,15,29$, and 57, followed by abatacept approximating $10 \mathrm{mg} / \mathrm{kg}$ (weight tiered: $500 \mathrm{mg}$ for patients weighing <60 kg, $750 \mathrm{mg}$ for patients $60-100 \mathrm{~kg}, 1,000$ $\mathrm{mg}$ for patients $>100 \mathrm{~kg}$ ) on days $85,113,141,169,197,225,253,281,309$, and 337

- Control group

* Placebo: consisted of dextrose $5 \%$ in water or normal saline on days 1, 15, 29, 57, 85, 113, 141, 169, $197,225,253,281,309$, and 337

- All groups

* MMF (dosage based on race and prior treatment) and prednisone (or prednisone equivalent), followed by adjustment or taper 
Furie 2014 (Continued)

\begin{tabular}{|c|c|}
\hline Outcomes & $\begin{array}{l}\text { - Death (all causes) } \\
\text { - ESKD } \\
\text { - Complete response: } 1 \text { ) eGFR } 90 \% \text { of screening level if normal at screening visit, or eGFR } 90 \% \text { of } 6 \text { - } \\
\text { month, pre-flare value if abnormal at screening, 2) UPCR } 0.26 \mathrm{~g} / \mathrm{g}(30 \mathrm{mg} / \mathrm{mmol} \text { ), and } 3 \text { ) inactive uri- } \\
\text { nary sediment (RBC and WBC/HPF within normal limits of central laboratory assessments; no RBC or } \\
\text { WBC casts) } \\
\text { - Partial response: } \mathrm{SCr} \text { level normal or } 125 \% \text { of baseline; UPCR } 50 \% \text { of baseline and } 3.0 \mathrm{~g} / \mathrm{g}(339 \mathrm{mg} / \\
\text { mmol) if nephrotic, or } 1.0 \mathrm{~g} / \mathrm{g}(133 \mathrm{mg} / \mathrm{mmol} \text { ) if non-nephrotic; urinary sediment inactive or } 50 \% \text { re- } \\
\text { duction in RBC/HPF from baseline; for confirmation, assessed on day } 337 \text { and confirmed on day } 365 \\
\text { - Major infection } \\
\text { - Herpes zoster virus }\end{array}$ \\
\hline Notes & ristol Myers Squibb \\
\hline
\end{tabular}

\section{Risk of bias}

\begin{tabular}{lll}
\hline Bias & Authors' judgement & Support for judgement \\
\hline $\begin{array}{l}\text { Random sequence genera- } \\
\text { tion (selection bias) }\end{array}$ & Unclear risk & $\begin{array}{l}\text { Study was described as randomised, method of randomisation was not report- } \\
\text { ed, however patients were stratified according to prior treatment }\end{array}$ \\
\hline $\begin{array}{l}\text { Allocation concealment } \\
\text { (selection bias) }\end{array}$ & Unclear risk & Insufficient information to permit judgement \\
\hline $\begin{array}{l}\text { Blinding of participants } \\
\text { and personnel (perfor- } \\
\text { mance bias) }\end{array}$ & Low risk & Double-blind, double dummy placebo study \\
All outcomes & \\
\hline
\end{tabular}

Blinding of outcome as- Unclear risk Insufficient information to permit judgement
sessment (detection bias)

All outcomes

Incomplete outcome data Low risk Not all relevant reported outcomes are reported
(attrition bias)

All outcomes

Selective reporting (re- Low risk Study protocol available and pre-specified outcomes were reported
porting bias)

Other bias High risk Sponsor included in data analysis/authorship

\section{Ginzler 1976}

\begin{tabular}{ll}
\hline Methods & Study design: cross-over RCT \\
- Study timeframe: not reported \\
- Duration of follow-up: 4 months then crossed over \\
\hline Participants & Country: USA \\
- Setting: single centre \\
- Inclusion criteria: diagnosis of SLE (ARA criteria); active kidney disease as manifested by either 1$)$ the \\
new appearance of hypocomplementaemia, azotaemia (SCr $>1.2$ mg\%), urinary protein excretion \\
$>200 \mathrm{mg} / 24$ h; cellular casts or more than $10 \mathrm{RBC} / \mathrm{HPF}$ in the urine sediment, or hypertension, or 2$)$ \\
deterioration in renal status in a patient with previously known renal disease, including either the
\end{tabular}


Ginzler 1976 (Continued)

new development of any of the above manifestations, or a $50 \%$ increase in $\mathrm{SCr}$, or a $200 \%$ increase in urinary protein excretion; a renal biopsy demonstrating diffuse proliferative or membranous GN

- Number (randomised): treatment group 1 (8); treatment group 2 (6)

- Mean age \pm SD (years): treatment group 1 (28.2 \pm 8.5$)$; treatment group 2 (25.8 \pm 6.2 )

- $\operatorname{Sex}(M / F)$ : not reported

- Exclusion criteria: $\mathrm{SCr}>3 \mathrm{mg} / \mathrm{dL}$, previous exposure to cytotoxic drugs

Interventions

Induction therapy: duration of treatment was 4 months

- Treatment group 1

* Oral AZA: $1.25 \mathrm{mg} / \mathrm{kg} / \mathrm{d}$

* CPA: $1.25 \mathrm{mg} / \mathrm{kg} / \mathrm{d}$

- Treatment group 2

* AZA: $2.5 \mathrm{mg} / \mathrm{kg} / \mathrm{d}$

- Both groups

* Prednisone prior to randomisation (minimum dose of $1 \mathrm{mg} / \mathrm{kg} / \mathrm{d}$ for 3 weeks); steroid dose was tapered throughout the study by a maximum of $5 \mathrm{mg}$ decrements at each clinic visit, in accordance with parameters of clinical disease activity

\begin{tabular}{ll}
\hline Outcomes & Death \\
& - ESKD \\
& - Toxicity \\
& Proteinuria \\
& - OrCl \\
& - Infection \\
\hline Notes & - Funding source: Supported by a grant from Lupus Erythematosus Foundation
\end{tabular}

\section{Risk of bias}

\begin{tabular}{lll}
\hline Bias & Authors' judgement & Support for judgement \\
\hline $\begin{array}{l}\text { Random sequence genera- } \\
\text { tion (selection bias) }\end{array}$ & Unclear risk & $\begin{array}{l}\text { Study was described as randomised, method of randomisation was not report- } \\
\text { ed }\end{array}$ \\
\hline $\begin{array}{l}\text { Allocation concealment } \\
\text { (selection bias) }\end{array}$ & Unclear risk & Insufficient information to permit judgement \\
\hline $\begin{array}{l}\text { Blinding of participants } \\
\begin{array}{l}\text { and personnel (perfor- } \\
\text { mance bias) }\end{array}\end{array}$ & Low risk & $\begin{array}{l}\text { Double-blind with a cross-over to other treatment under certain conditions } \\
\text { (predetermined therapeutic failures) }\end{array}$ \\
\hline
\end{tabular}

$\begin{array}{lll}\text { Blinding of outcome as- } & \text { Unclear risk } & \text { Insufficient information to permit judgement } \\ \text { sessment (detection bias) } & \end{array}$
sessment (detection bias)

All outcomes

Incomplete outcome data Low risk No missing outcome data
(attrition bias)
All outcomes

Selective reporting (re- Low risk Study protocol available and prespecified outcomes were reported
porting bias)


Ginzler 1976 (Continued)

Other bias High risk Cross-over design and reporting of results, difficult to separate treatment effects

Ginzler 2005

Methods $\quad$ Study design: open-label, non-inferiority RCT

- Study timeframe: December 1999 to October 2003

- Duration of follow-up: 24 weeks

\section{Participants}

- Country: USA

- Setting: multicentre (19 sites)

- Inclusion criteria: diagnosis of SLE (ACR criteria), biopsy-proven lupus nephritis class III, IV or V, clinical activity defined by one of; incident decrease in kidney function, proteinuria (>0.5 g/24 h), microscopic haematuria (> $5 \mathrm{RBC} / \mathrm{HPF}$ ); participants with class III or V required to have $\mathrm{SCr}>1.0 \mathrm{mg} / \mathrm{dL}$ or proteinuria $>2 \mathrm{~g} / 24 \mathrm{~h}$

- Number (randomised/analysed): treatment group 1 (71/71); treatment group 2 (69/69)

* 113 had diffuse proliferative lupus nephritis; 27 had pure membranous

- Mean age \pm SD (years): treatment group $1(32.5 \pm 10)$; treatment group $2(31.0 \pm 9.0)$

- Sex (M/F): treatment group 1 (10/61); treatment group 2 (4/65)

- Ethnicity (Black/white/Hispanic/Asian/other): treatment group 1(43/12/10/6/0); treatment group 2 $(36 / 12 / 18 / 2 / 1)$

- Exclusion criteria: $\mathrm{CrCl}<30 \mathrm{~mL} / \mathrm{min}, \mathrm{SCr}>3.0 \mathrm{mg} / \mathrm{dL}$; severe co-existing conditions precluding immunosuppression or requiring IV antibiotics; prior treatment with MMF; treatment with IV CPA in last 12 months; treatment within last 30 days; pregnancy or lactation

Interventions

Induction therapy: duration of therapy was 24 weeks

- Treatment group 1

* MMF: 0.5 g twice daily to increase to $\max 1 \mathrm{~g} 3$ times/d

- Treatment group 2

* IV CPA: $0.5 \mathrm{~g} / \mathrm{m}^{2} \mathrm{BSA}$ increased to $1.0 \mathrm{~g} / \mathrm{m}^{2}$

- Both groups

* Prednisone at a dose of $1 \mathrm{mg} / \mathrm{kg} / \mathrm{d}$, with tapering by 10 to $20 \%$ at 1 week or 2 week intervals, on the basis of clinical improvement

* The new appearance or worsening of manifestations of extrarenal disease could be treated with one 3-day pulse of IV MP or increased dose of prednisone to a maximum of $2 \mathrm{mg} / \mathrm{kg} / \mathrm{d}$

- Death
- ESKD
- Doubling of $\mathrm{SCr}$
- Relapse
- Stable kidney function
- Major infection
- Herpes zoster
- Ovarian failure
- Gl upset
- Diarrhoea
- Lymphopenia (<800 lymphocytes/mm 3 )
- Complete remission in proteinuria
- Partial remission in proteinuria
- Complete renal remission: defined at 24 weeks as return to within $10 \%$ of normal values of SCr levels,
proteinuria, and urine sediment


- Partial renal remission: defined at 24 weeks as improvement of 50\% in all abnormal renal measurements, without worsening (within 10 percent) of any measurement

- Treatment failure: patients in whom treatment failed included all those without complete or partial remission at 24 weeks, plus those who stopped treatment for any reason

- $\mathrm{SCr}$

- Daily proteinuria

$\begin{array}{ll}\text { Notes } & 1 \text { participant on MMF crossed-over to CPA and } 2 \text { participants on IV CPA crossed over to MMF } \\ \text { - Funding source: FDA's Orphan Products Development program and a supplemental grant from Roche } \\ \text { Laboratories }\end{array}$

\section{Risk of bias}

\begin{tabular}{|c|c|c|}
\hline Bias & Authors' judgement & Support for judgement \\
\hline $\begin{array}{l}\text { Random sequence genera- } \\
\text { tion (selection bias) }\end{array}$ & Low risk & Treatment assigned at central site with the use of sealed envelopes \\
\hline $\begin{array}{l}\text { Allocation concealment } \\
\text { (selection bias) }\end{array}$ & Low risk & Sealed envelopes used \\
\hline $\begin{array}{l}\text { Blinding of participants } \\
\text { and personnel (perfor- } \\
\text { mance bias) } \\
\text { All outcomes }\end{array}$ & High risk & Open-label study \\
\hline $\begin{array}{l}\text { Blinding of outcome as- } \\
\text { sessment (detection bias) } \\
\text { All outcomes }\end{array}$ & Unclear risk & Insufficient information to permit judgement \\
\hline $\begin{array}{l}\text { Incomplete outcome data } \\
\text { (attrition bias) } \\
\text { All outcomes }\end{array}$ & Low risk & No missing outcome data \\
\hline $\begin{array}{l}\text { Selective reporting (re- } \\
\text { porting bias) }\end{array}$ & High risk & $\begin{array}{l}\text { Due to early termination, primary outcome as per protocol not reported; Not } \\
\text { all expected outcomes reported }\end{array}$ \\
\hline Other bias & High risk & $\begin{array}{l}\text { The study was terminated early and there was heavy cross-over between study } \\
\text { arms. Funding provided by a supplemental grant from Roche laboratories }\end{array}$ \\
\hline
\end{tabular}

Gourley 1996

\begin{tabular}{ll}
\hline Methods & Study design: open-label, parallel RCT \\
& - Study timeframe: from mid 1990 \\
& - Duration of follow-up: > 5 years \\
\hline Participants & Country: USA \\
- Setting: single centre & Inclusion criteria: SLE; GN was defined as a sediment on 2 or more urinalysis that showed either 10 \\
& or more RBC/HPF or erythrocyte or leukocyte casts (without evidence of infection) or both plus biop- \\
& sy-proven active proliferative lupus GN (within 3 months of study entry); $79 / 82$ class III/IV on biopsy; \\
& $3 / 82$ no biopsy \\
- Number (randomised): treatment group 1 (27); treatment group 2 (28); control group (27) \\
- Mean age (years): treatment group 1 (30); treatment group 2 (31); control group (30) \\
- Sex (M/F): treatment group 1 (6/21); treatment group 2 (3/25); control group (5/22)
\end{tabular}


Gourley 1996 (Continued)

- Exclusion criteria: cytotoxic drug treatment $>2$ weeks and with 6 weeks of start date; 10 weeks of CPA therapy; pulse therapy of corticosteroids within 6 weeks of start of study; oral corticosteroids $>0.5$ $\mathrm{mg} / \mathrm{kg} / \mathrm{d}$; active or chronic infection; pregnancy; insulin-dependent DM; allergy to study medication

\begin{tabular}{|c|c|}
\hline Interventions & $\begin{array}{l}\text { Induction therapy } \\
\text { - Treatment group } 1 \\
\text { * IV CPA: } 0.75 \mathrm{~g} / \mathrm{m}^{2} \text { boluses monthly for } 6 \text { months then } 3 \text { monthly for at least } 2 \text { years } \\
\text { - Treatment group } 2 \\
\text { * IV MP: as per control group } \\
\text { * IV CPA: as per treatment group } 1 \\
\text { - Control group } \\
\text { * IV MP: } 3 \text { doses }\left(1 \mathrm{~g} / \mathrm{m}^{2}\right) \text { over } 3 \text { consecutive days then one dose monthly for } 12 \text { months } \\
\text { - All groups } \\
\text { * Initially given oral prednisone }(0.5 \mathrm{mg} / \mathrm{kg} / \mathrm{d}) \text { for } 4 \text { weeks. The prednisone dose was then tapered } \\
\quad \text { by } 5 \mathrm{mg} \text { every other day each week to the minimal dose required to control extrarenal disease or } \\
0.25 \mathrm{mg} / \mathrm{kg} \text { every other day, whichever was greater } \\
\text { * For severe extrarenal flares of lupus, patients were permitted to receive prednisone, } 1.0 \mathrm{mg} / \mathrm{kg} \text { per } \\
\text { day for } 2 \text { weeks }\end{array}$ \\
\hline Outcomes & $\begin{array}{l}\text { - } \text { Death } \\
\text { - } \mathrm{ESKD} \\
\text { - } \text { Doubling of } \mathrm{SCr} \\
\text { - Renal remission } \\
\text { - Treatment failure: } \geq 10 \mathrm{RBC} / \mathrm{HPF} \text {, cellular casts, proteinuria (>1 } \mathrm{g} \text { of protein/d) } \\
\text { - Relapse: reactivation of renal disease after } 6 \text { or more months of remission } \\
\text { - One or more infections } \\
\text { - Herpes zoster virus infection } \\
\text { - Amenorrhoea } \\
\text { - Avascular necrosis }\end{array}$ \\
\hline Notes & $\begin{array}{l}\text { - } 2 \text { participants lost to follow-up } \\
\text { - Funding source: Arthritis Foundation }\end{array}$ \\
\hline
\end{tabular}

\section{Risk of bias}

\begin{tabular}{lll}
\hline Bias & Authors' judgement & Support for judgement \\
\hline $\begin{array}{l}\text { Random sequence genera- } \\
\text { tion (selection bias) }\end{array}$ & Low risk & Masked cards from table of random numbers \\
\hline $\begin{array}{l}\text { Allocation concealment } \\
\text { (selection bias) }\end{array}$ & Unclear risk & Using masked card but no description methods of allocation concealment \\
\hline $\begin{array}{l}\text { Blinding of participants } \\
\text { and personnel (perfor- } \\
\text { mance bias) }\end{array}$ & High risk & Open-label study \\
All outcomes & & \\
\hline
\end{tabular}

\begin{tabular}{|c|c|c|}
\hline $\begin{array}{l}\text { Blinding of outcome as- } \\
\text { sessment (detection bias) } \\
\text { All outcomes }\end{array}$ & Low risk & $\begin{array}{l}\text { Outcome data with the exception of adverse events, were collected in a blind- } \\
\text { ed manner }\end{array}$ \\
\hline
\end{tabular}

\begin{tabular}{ll}
\hline $\begin{array}{l}\text { Incomplete outcome data } \\
\text { (attrition bias) }\end{array}$ & Low risk \\
All outcomes & $\begin{array}{l}\text { No missing outcome data; participants at endpoints censored but considered } \\
\text { in final analysis }\end{array}$
\end{tabular}


Gourley 1996 (Continued)

Selective reporting (re- Low risk Study protocol available and prespecified outcomes were reported porting bias)

Other bias Low risk The study appears to be free of other sources of bias

Grootscholten 2006

\begin{tabular}{|c|c|}
\hline Methods & $\begin{array}{l}\text { - Study design: parallel RCT } \\
\text { - Study timeframe: September } 1995 \text { to September } 2001 \\
\text { - Duration of follow-up: median follow-up } 5.7 \text { years (interquartile range } 4.1 \text { to } 7.2 \text { years); unintentional } \\
\text { skewed distribution (resulting from stratification per centre and small contribution of some centres). } \\
\text { Median extended follow-up was } 9.6 \text { years (range } 0.1 \text { to } 13.2 \text { years) }\end{array}$ \\
\hline Participants & $\begin{array}{l}\text { - Country: Netherlands } \\
\text { - Setting: multicentre (number of sites not reported) } \\
\text { - Inclusion criteria: biopsy-proven lupus nephritis (PALGA), diagnosis of SLE (ACR criteria); } 18 \text { to } 60 \\
\text { years; } \mathrm{CrCl}>25 \mathrm{~mL} / \mathrm{min} \text {; if already known to have proliferative lupus nephritis, renal biopsy < } 1 \text { year } \\
\text { before; WHO class IV or Vd must have signs of active nephritis or deterioration of kidney function; class } \\
\text { III or Vc lupus nephritis had to meet both criteria } \\
\text { - Number (randomised/analysed): treatment group } 1(50 / 50) \text {; treatment group } 2(37 / 37) \\
\text { - Mean age, range (years): treatment group } 1 \text { ( } 30,24 \text { to } 47) \text {; treatment group } 2(33,26 \text { to } 39) \\
\text { - Sex (M/F): treatment group } 1 \text { ( } 6 / 44) \text {; treatment group } 2(9 / 28) \\
\text { - Exclusion criteria: decline in kidney function (> } 30 \% \text { increase in } \mathrm{SCr} \text { ) in month before inclusion; active } \\
\text { infection; malignancy < }<\text { years before randomisation; pregnancy or no contraceptives during first } 2.5 \\
\text { years of treatment; hepatitis or cirrhosis of liver; active peptic ulcer; leucocytopenia }(<3 \times 109 / \mathrm{L} \text { ) or } \\
\text { thrombocytopenia (< } 100 \times 109 / \mathrm{L} \text { with suppressed bone marrow; allergy to AZA or CPA }\end{array}$ \\
\hline
\end{tabular}

\begin{tabular}{|c|c|}
\hline Interventions & $\begin{array}{l}\text { Induction and maintenance therapy } \\
\text { - Treatment group } 1 \\
\text { * IV CPA: } 750 \mathrm{mg} / \mathrm{m}^{2}, 13 \text { pulses in } 2 \text { years, oral prednisolone cumulative corticosteroid dose (11 g) } \\
\text { - Treatment group } 2 \\
\text { * Oral AZA: } 2 \mathrm{mg} / \mathrm{kg} / \mathrm{d} \text { in } 2 \text { years, IV MP ( } 3 \times 3 \text { pulses of } 1000 \mathrm{mg} \text { ) and oral prednisolone (initially } 1 \mathrm{mg} / \\
\mathrm{kg} / \mathrm{d} \text { for } 4 \text { weeks, } 0.75 \mathrm{mg} / \mathrm{kg} / \mathrm{d} \text { for } 4 \text { weeks, } 0.50 \mathrm{mg} / \mathrm{kg} / \mathrm{d} \text { for } 4 \text { weeks and thereafter tapered by } \\
5 \mathrm{mg} \text { every } 4 \text { weeks to a final dose of } 10 \mathrm{mg} \text { daily after } 6 \text { months) } \\
\text { - Both groups } \\
\text { * Switched to long-term AZA ( } 2 \mathrm{mg} / \mathrm{kg} \text { ) plus prednisolone }(10 \mathrm{mg} / \mathrm{d}) \text { after } 2 \text { years }\end{array}$ \\
\hline Outcomes & $\begin{array}{l}\text { - Death } \\
\text { - } \mathrm{ESKD} \\
\text { - Doubling of } \mathrm{SCr} \\
\text { - Deterioration of kidney function } \\
\text { - major infection } \\
\text { - Ovarian failure } \\
\text { - Daily proteinuria } \\
\text { - Renal relapse: could occur after week } 12 \text {, and was defined as doubling of the lowest obtained } \mathrm{SCr} \text { so } \\
\text { far and/ or development of either a nephrotic syndrome (proteinuria }>3.5 \mathrm{~g} / \mathrm{d} \text { and serum albumin }< \\
30 \mathrm{~g} / \mathrm{L} \text { ), while the lowest protein excretion so far had been } \leq 2.0 \mathrm{~g} / \mathrm{d} \text { repeatedly, or proteinuria }<1.5 \mathrm{~g} / \\
\mathrm{d} \text { without other causes, in a previously non-proteinuric patient }\end{array}$ \\
\hline Notes & $\begin{array}{l}\text { - } 8 / 87 \text { class III or Vc class IV or Vd 79/97 13/87 given previous cytotoxics IV CPA:7/50 (14\%) AZA: 6/37 } \\
\text { (16\%) If } 1 \text { Y failure (DSC) switched to other arm of study } 1 \text { lost to follow-up in each group } \\
\text { - Funding source: Dutch Kidney Foundation, Dutch League against Rheumatism }\end{array}$ \\
\hline
\end{tabular}


Grootscholten 2006 (Continued)

Risk of bias

\begin{tabular}{|c|c|c|}
\hline Bias & Authors' judgement & Support for judgement \\
\hline $\begin{array}{l}\text { Random sequence genera- } \\
\text { tion (selection bias) }\end{array}$ & Low risk & $\begin{array}{l}\text { Randomisation performed at a central office with a computer program, us- } \\
\text { ing the minimisation determinants: centre, } \mathrm{SCr}(<150 \text { or }>150 \mu \mathrm{mol} / \mathrm{L}) \text {, WHO } \\
\text { class III or IV, previous treatment with immunosuppressive medication for lu- } \\
\text { pus nephritis }\end{array}$ \\
\hline $\begin{array}{l}\text { Allocation concealment } \\
\text { (selection bias) }\end{array}$ & Unclear risk & Central office with computer program. Not sufficiently clear to determine risk \\
\hline $\begin{array}{l}\text { Blinding of participants } \\
\text { and personnel (perfor- } \\
\text { mance bias) } \\
\text { All outcomes }\end{array}$ & High risk & Open-label study \\
\hline
\end{tabular}

$\begin{array}{lll}\text { Blinding of outcome as- } & \text { Unclear risk } & \text { Insufficient information to permit judgement } \\ \text { sessment (detection bias) } & \end{array}$

sessment (detection bias)

All outcomes

\begin{tabular}{lll}
$\begin{array}{l}\text { Incomplete outcome data } \\
\text { (attrition bias) } \\
\text { All outcomes }\end{array}$ & Low risk & No missing outcome data \\
\hline $\begin{array}{l}\text { Selective reporting (re- } \\
\text { porting bias) }\end{array}$ & Low risk & Study protocol available and pre-specified outcomes were reported \\
\hline Other bias & Low risk & $\begin{array}{l}\text { Funding from Dutch Kidney Foundation and Dutch League against Rheuma- } \\
\text { tism. One author disclosed speaking fees from Novartis. The study appears to } \\
\text { be free of other sources of bias }\end{array}$
\end{tabular}

Hahn 1975

\begin{tabular}{ll}
\hline Methods & Study design: open-label, RCT \\
- Study timeframe: not reported \\
- \\
\hline Puration of follow-up: 2 years \\
\hline Participants & Country: USA \\
- Setting: single centre \\
Inclusion criteria: SLE diagnosed using established specific criteria (positive antinuclear antibodies, a \\
teria for SLE (ARA); active life-threatening disease (severe nephritis, central nervous system involve- \\
ment, haemolytic anaemia, thrombocytopenia, myocarditis, lupus crisis) \\
- Number (randomised): treatment group (11); control group (13) \\
- Mean age \pm SD (years): treatment group (33.5 \pm 13.2$) ;$ control group (31.7 \pm 13.9$)$ \\
- Sex (M/F): treatment group (2/9); control group (2/11) \\
- Exclusion criteria: prior treatment with cytotoxic drugs; 20 mg prednisone/d during the preceding 6 \\
weeks
\end{tabular}

Interventions

Induction therapy: duration of therapy was 24 months

- treatment group

* Oral AZA: 3 to $4 \mathrm{mg} / \mathrm{kg} / \mathrm{d}$

* Prednisone: as per control group 
Hahn 1975 (Continued)

\section{- Control group}

* Prednisone: daily oral dose of 40 to $60 \mathrm{mg}$ was maintained for 4 to 6 months. After prednisone was maintained at 40 to $60 \mathrm{mg}$ daily for 6 months in both groups, it was tapered slowly (by $5 \mathrm{mg}$ increments every 2 weeks to a level of $30 \mathrm{mg}$ daily, then by $2.5 \mathrm{mg}$ increments every 2 weeks)

\begin{tabular}{ll}
\hline Outcomes & Death \\
- & Toxicity \\
- & Major infection \\
- Infection & Proteinuria \\
- & Remission of proteinuria \\
- & $\mathrm{CrCl}$ \\
- $\mathrm{SCr}$ & \\
\hline - & $2 / 24$ lost to follow-up \\
- Funding source: US Public Health Service grants AM17469 and AM05548 and Public Health Service \\
\\
Research grant FR-36 from the General Clinical Research centre Branch, Division of Research Facilities \\
and Resources; and the Arthritis Foundation
\end{tabular}

\section{Risk of bias}

\begin{tabular}{lll}
\hline Bias & Authors' judgement & Support for judgement \\
\hline $\begin{array}{l}\text { Random sequence genera- } \\
\text { tion (selection bias) }\end{array}$ & Low risk & $\begin{array}{l}\text { Slips of paper bearing letters A or B sealed in envelopes then placed in a draw- } \\
\text { er. On randomising patient, envelopes drawn randomly from drawer }\end{array}$ \\
\hline $\begin{array}{l}\text { Allocation concealment } \\
\text { (selection bias) }\end{array}$ & Low risk & Sealed envelopes used in randomisation \\
\hline $\begin{array}{l}\text { Blinding of participants } \\
\text { and personnel (perfor- } \\
\text { mance bias) }\end{array}$ & High risk & Open-label study \\
All outcomes & & \\
\hline
\end{tabular}

Blinding of outcome as- Unclear risk Insufficient information to permit judgement
sessment (detection bias)

All outcomes

\begin{tabular}{l}
\hline Incomplete outcome data Low risk No missing outcome data \\
(attrition bias) \\
All outcomes
\end{tabular}

Selective reporting (re- High risk $\quad$ Not all expected clinical outcomes were reported
porting bias)

Other bias Low risk The study appears to be free of other sources of bias

Hong 2007

\begin{tabular}{ll}
\hline Methods & - Study design: open-label, parallel RCT \\
& - Study timeframe: not reported \\
& Duration of follow-up: 6 months \\
\hline Participants & - Country: China \\
& - Setting: not reported \\
\hline
\end{tabular}


Hong 2007 (Continued)

- Inclusion criteria: diffuse proliferative lupus nephritis on renal biopsy; all > $2 \mathrm{~g} / \mathrm{d}$ proteinuria and $\mathrm{SCr}$ $<3 \mathrm{mg} / \mathrm{dL}$

- Number (randomised/analysed): treatment group 1 (13/13); treatment group $2(12 / 12)$

- Mean age \pm SD: 30.7 \pm 5.1 years

- $\operatorname{Sex}(\mathrm{M} / \mathrm{F}): 2 / 23$

- Exclusion criteria: not reported

\begin{tabular}{|c|c|}
\hline Interventions & $\begin{array}{l}\text { Induction therapy } \\
\text { - Treatment group } 1 \\
\text { * Oral FK506 (TAC): } 0.1 \mathrm{mg} / \mathrm{kg} / \mathrm{d} \\
\text { - Treatment group } 2 \\
\text { * IV CPA: } 0.5 \text { to } 0.75 \mathrm{~g} / \mathrm{m}^{2} \text { monthly } \\
\text { - Both groups } \\
\text { * Prednisolone: } 0.8 \mathrm{mg} / \mathrm{kg} / \mathrm{d}\end{array}$ \\
\hline Outcomes & $\begin{array}{l}\text { - Stable kidney function } \\
\text { - No response } \\
\text { - Infection } \\
\text { - Complete remission (urinary protein excretion }<0.4 \mathrm{~g} / 24 \mathrm{~h} \text {, no active urinary sediment (urinary } \mathrm{RBC}< \\
10 \times 10^{4} / \mathrm{mL} \text { ), serum albumin }>35 \mathrm{~g} / \mathrm{L}, \mathrm{SCr} \text { in normal ranges) } \\
\text { - Partial remission (between complete remission and no response - referred to urinary protein excretion } \\
>2 \mathrm{~g} \text { or the reduction less than the baseline value, serum albumin }<30 \mathrm{~g} / \mathrm{L} \text {, or increment of } \mathrm{SCr}>50 \% \\
\text { of the baseline value) } \\
\text { - Proteinuria }\end{array}$ \\
\hline Notes & $\begin{array}{l}\text { - Abstract-only publication } \\
\text { - Funding source: not reported }\end{array}$ \\
\hline
\end{tabular}

\section{Risk of bias}

\begin{tabular}{lll}
\hline Bias & Authors' judgement & Support for judgement \\
\hline $\begin{array}{l}\text { Random sequence genera- } \\
\text { tion (selection bias) }\end{array}$ & Unclear risk & Insufficient information to permit judgement \\
\hline $\begin{array}{l}\text { Allocation concealment } \\
\text { (selection bias) }\end{array}$ & Unclear risk & Insufficient information to permit judgement \\
\hline $\begin{array}{l}\text { Blinding of participants } \\
\text { and personnel (perfor- } \\
\text { mance bias) }\end{array}$ & High risk & Open-label study \\
All outcomes & & \\
\hline
\end{tabular}

\begin{tabular}{|c|c|c|}
\hline $\begin{array}{l}\text { Blinding of outcome as- } \\
\text { sessment (detection bias) } \\
\text { All outcomes }\end{array}$ & Unclear risk & Insufficient information to permit judgement \\
\hline
\end{tabular}

Incomplete outcome data Unclear risk Insufficient information to permit judgement
(attrition bias)

All outcomes

\begin{tabular}{lll}
\hline $\begin{array}{l}\text { Selective reporting (re- } \\
\text { porting bias) }\end{array}$ & High risk & Not all expected outcomes reported \\
\hline Other bias & Unclear risk & Abstract-only publication; insufficient information to permit judgement \\
\hline
\end{tabular}


Houssiau 2002

\begin{tabular}{|c|c|}
\hline Methods & $\begin{array}{l}\text { - Study design: RCT } \\
\text { - Study timeframe: September } 1996 \text { to September } 2000 \\
\text { - Duration of follow-up: } 10 \text { years }\end{array}$ \\
\hline Participants & $\begin{array}{l}\text { - Country: Europe (countries not reported) } \\
\text { - Setting: multinational (19 sites) } \\
\text { - Inclusion criteria: diagnosis of SLE (ACR criteria); age } \geq 14 \text { years; biopsy-proven proliferative lupus GN } \\
\text { (WHO class III, IV, Vc, or Vd); proteinuria } 500 \mathrm{mg} / 24 \mathrm{~h} ; 69 / 90 \text { class IV or Vc/Vd } \\
\text { - Number (randomised): treatment group } 1 \text { (46); treatment group } 2(44) \\
\text { - Mean age } \pm \mathrm{SD} \text { (years): treatment group } 1 \text { ( } 30 \pm 11) \text {; treatment group } 2(33 \pm 12) \\
\text { - Sex (M/F): treatment group } 1 \text { ( } 3 / 43) \text {; treatment group } 2 \text { (3/41) } \\
\text { - Exclusion criteria: CPA or AZA in previous year; > } 15 \mathrm{mg} / \mathrm{d} \text { prednisolone during preceding month; renal } \\
\text { thrombotic microangiopathy; pre-existing CKD; pregnancy; previous malignancy - except skin or cer- } \\
\text { vical intraepithelial neoplasia's; DM; severe toxicity or immunosuppressive drugs; anticipated poor } \\
\text { compliance }\end{array}$ \\
\hline
\end{tabular}

- Treatment group 1

* High dose IV CPA: received 8 pulses within a year ( 6 monthly pulses followed by 2 quarterly pulses. The initial IV CPA dose was $0.5 \mathrm{~g} / \mathrm{m}^{2}$ of body surface area; subsequent doses were increased by 250 $\mathrm{mg}$ according to the WBC count nadir measured on day 14 , with a maximum of $1,500 \mathrm{mg}$ per pulse

- Treatment group 2

* Low dose IV CPA: received 6 fortnightly IV CPA pulses at a fixed dose of $500 \mathrm{mg}$

- Both groups

* All patients received 3 daily pulses of $750 \mathrm{mg}$ of IV MP, followed by oral prednisolone (or equivalent) at an initial dosage of $0.5 \mathrm{mg} / \mathrm{kg} / \mathrm{d}$ for 4 weeks. A dosage of $1 \mathrm{mg} / \mathrm{kg} / \mathrm{d}$ was allowed in critically ill patients (those with renal impairment or severe extrarenal disease), glucocorticoid therapy (5-7.5 $\mathrm{mg}$ of prednisolone per day) was maintained at least until month 30 after inclusion; after 4 weeks, prednisolone (or equivalent) dosages were tapered by $2.5 \mathrm{mg}$ every 2 weeks.

* Both treatment arms, AZA ( $2 \mathrm{mg} / \mathrm{kg} / \mathrm{d}$ ) was started 2 weeks after the last CPA injection and continued at least until month 30 after study inclusion

- ESKD

- Renal remission: defined as $10 \mathrm{RBC} / \mathrm{HPF}$ and a 24-hour urinary protein level $<1 \mathrm{~g}$, in the absence of a doubling of the SCr level; and the number of severe flares

- Treatment failure: defined as any of the following 3 features: 1 . Absence of a primary response A. For patients with a baseline $\mathrm{SCr} \geq 1.3 \mathrm{mg} / \mathrm{dL}$ but $\leq 2.6 \mathrm{mg} / \mathrm{dL}$, absence of a primary response was defined as failure of the $\mathrm{SCr}$ to decrease to $<1.3 \mathrm{mg} / \mathrm{dL}$ at 6 months; B. For patients with a baseline $\mathrm{SCr}>2.6$ $\mathrm{mg} / \mathrm{dL}$, absence of a primary response was defined as failure of the $\mathrm{SCr}$ level to improve by $50 \%$ at 6 months; C. For patients with nephrotic syndrome at baseline (serum albumin level $<3.5 \mathrm{~g} / \mathrm{dL}$ and 24-hour urinary protein level $\geq 3 \mathrm{~g} / \mathrm{d}$ ), but without renal impairment $(\mathrm{SCr}<1.3 \mathrm{mg} / \mathrm{dL}$ ), absence of a primary response was defined as the persistence of nephrotic syndrome at 6 months; 2 . A glucocorticoid-resistant flare (defined as a severe flare that did not respond to a 1-month increase in the glucocorticoid dosage); 3 . A doubling of the $\mathrm{SCr}$ over the lowest value reached at any time during the follow-up and confirmed on 2 consecutive visits 1 month apart

- Doubling of $\mathrm{SCr}$

- Relapse: severe renal flare was defined as 1 of the following 3 features: renal impairment, increase in proteinuria, or severe systemic disease. Renal impairment was defined as an SLE-related increase of $33 \%$ in the SCr within a 1-month period; An increase in proteinuria defined as recurrence or appearance of nephrotic syndrome (albuminaemia $\leq 3.5 \mathrm{~g} / \mathrm{dL}$ and proteinuria $\geq 3 \mathrm{~g} / 24 \mathrm{~h}$ ); In patients with low-grade proteinuria at baseline ( $\geq 0.5 \mathrm{~g}$ but $\leq 1 \mathrm{~g}$ in $24 \mathrm{~h}$ ); a 3 -fold increase in 24-hour urinary protein levels within a 3-month period was also considered a severe flare, provided that it was accompanied by microscopic haematuria and a 33\% reduction of serum C3 levels within a 3-month period 
Houssiau 2002 (Continued)

- Toxicity

- Proteinuria

- Infection

- Herpes zoster virus

- Ovarian failure

- Leucopenia: $\leq 4000 / \mu \mathrm{L}$

\begin{tabular}{ll}
\hline Notes & Follow-up: median 41 month follow-up; 1 patient lost to follow-up. 73 month follow-up; 5 participants \\
& lost to follow-up, 10 year follow-up \\
- Funding source: supported by the European League against Rheumatism
\end{tabular}

\section{Risk of bias}

\begin{tabular}{|c|c|c|}
\hline Bias & Authors' judgement & Support for judgement \\
\hline $\begin{array}{l}\text { Random sequence genera- } \\
\text { tion (selection bias) }\end{array}$ & Low risk & Randomisation by minimisation \\
\hline $\begin{array}{l}\text { Allocation concealment } \\
\text { (selection bias) }\end{array}$ & Unclear risk & Insufficient information to permit judgement \\
\hline $\begin{array}{l}\text { Blinding of participants } \\
\text { and personnel (perfor- } \\
\text { mance bias) } \\
\text { All outcomes }\end{array}$ & Unclear risk & Insufficient information to permit judgement \\
\hline $\begin{array}{l}\text { Blinding of outcome as- } \\
\text { sessment (detection bias) } \\
\text { All outcomes }\end{array}$ & Unclear risk & Insufficient information to permit judgement \\
\hline $\begin{array}{l}\text { Incomplete outcome data } \\
\text { (attrition bias) } \\
\text { All outcomes }\end{array}$ & Low risk & No missing outcome data \\
\hline $\begin{array}{l}\text { Selective reporting (re- } \\
\text { porting bias) }\end{array}$ & Low risk & Study protocol available and pre-specified outcomes were reported \\
\hline Other bias & Low risk & $\begin{array}{l}\text { Supported by the European League Against Rheumatism. The study appears to } \\
\text { be free of other sources of bias }\end{array}$ \\
\hline
\end{tabular}

Jayne 2013

\begin{tabular}{ll}
\hline Methods & Study design: double-blind double-dummy RCT \\
& - Study timeframe: not reported \\
\hline Participants & - Couration of follow-up: 6 months \\
- Setting: multicentre (number of sites not reported) \\
- Inclusion criteria: active lupus nephritis \\
- Number (randomised): treatment group 1 (16); treatment group 2 (16); control group (15) \\
- Mean age \pm SD (years): not reported \\
- Sex (M/F): not reported \\
- Exclusion criteria: not reported
\end{tabular}


Jayne 2013 (Continued)
Interventions
Induction therapy: duration of treatment was 6 months
- Treatment group 1
* High-dose laquinimod: oral $1 \mathrm{mg} / \mathrm{d}$
- Treatment group 2
* Low-dose laquinimod: oral $0.5 \mathrm{mg} / \mathrm{d}$
- Control group
* Placebo
- All groups
* All patients received MMF and prednisone (or equivalent)

\begin{tabular}{ll}
\hline Outcomes & Death \\
& - Remission \\
- Kidney function & - Adverse events
\end{tabular}

\begin{tabular}{ll}
\hline Notes & Abstract-only publication \\
- Funding source: not reported
\end{tabular}

\section{Risk of bias}

Bias Authors' judgement Support for judgement

Random sequence genera- Unclear risk Insufficient information to permit judgement
tion (selection bias)

Allocation concealment $\quad$ Unclear risk Insufficient information to permit judgement
(selection bias)

\begin{tabular}{|c|c|c|}
\hline $\begin{array}{l}\text { Blinding of participants } \\
\text { and personnel (perfor- } \\
\text { mance bias) } \\
\text { All outcomes }\end{array}$ & Low risk & Double-blind, double dummy placebo study \\
\hline $\begin{array}{l}\text { Blinding of outcome as- } \\
\text { sessment (detection bias) } \\
\text { All outcomes }\end{array}$ & Unclear risk & Insufficient information to permit judgement \\
\hline $\begin{array}{l}\text { Incomplete outcome data } \\
\text { (attrition bias) } \\
\text { All outcomes }\end{array}$ & Low risk & No missing outcome data \\
\hline $\begin{array}{l}\text { Selective reporting (re- } \\
\text { porting bias) }\end{array}$ & High risk & Not all prespecified outcomes were reported \\
\hline Other bias & Unclear risk & Abstract-only publication; insufficient information to permit judgement \\
\hline
\end{tabular}

Kaballo 2016

\begin{tabular}{ll}
\hline Methods & - Study design: parallel RCT \\
& - Study timeframe: March 2008 to August 2011 \\
& - Duration of follow-up: 36 months
\end{tabular}

Participants

- Country: Sudan 
Kaballo 2016 (Continued)

- Setting: multicentre (2 sites)

- Inclusion criteria: aged 12 to 75 years and have been diagnosed with SLE (ACR revised criteria); lupus nephritis criteria included persistent proteinuria $>0.5 \mathrm{~g} / \mathrm{d}$ and presence of active urine sediment; renal biopsies were performed at presentation, only patients who had a histological diagnosis of severe proliferative Class III and IV and/or membranous Class V lupus nephritis (ISN/RPS 2003 classification) were enrolled

- Number (randomised): treatment group 1 (41); treatment group 2 (40)

- Mean age \pm SD (years): treatment group 1 (27.1 \pm 9.8$)$; treatment group $2(29.4 \pm 11.6)$

- $\operatorname{Sex}(M / F)$ : treatment group 1 (3/38); treatment group 2 (3/37)

- Exclusion criteria: ESKD; malignancy; severe cardiovascular or liver disease; severe infection

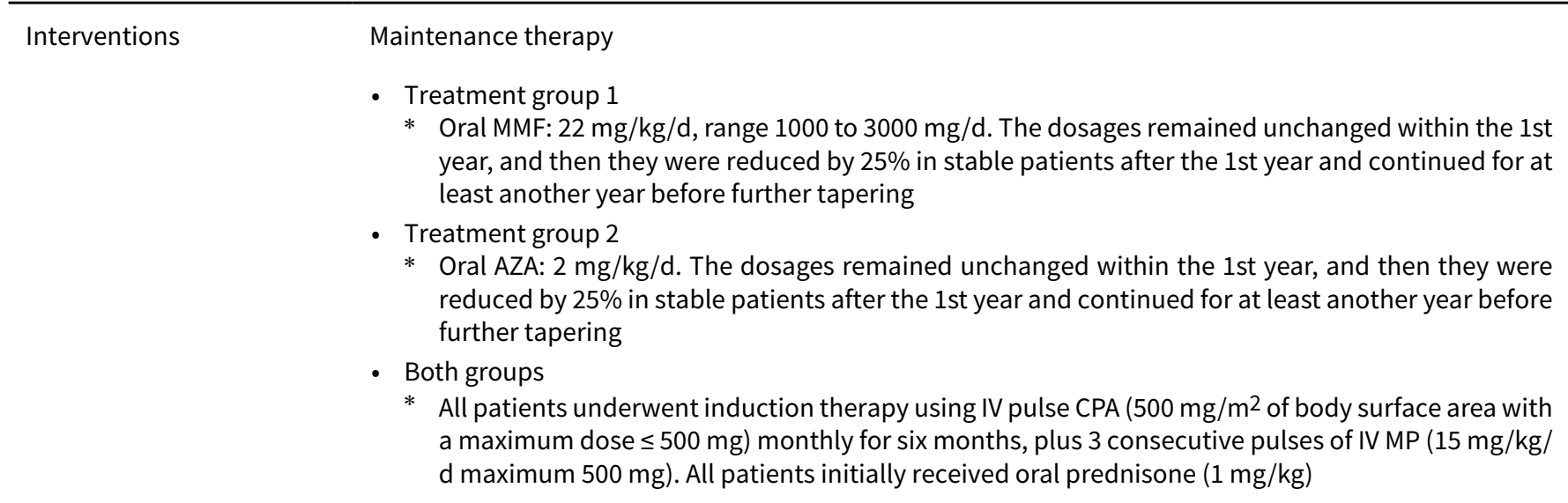

\begin{tabular}{ll}
\hline - & Death \\
- & ESKD \\
- & Pomplete remission: defined as reduction in proteinuria to $\leq 0.2 \mathrm{~g} / \mathrm{d}$ with normal $\mathrm{SCr}$ \\
& and $2.0 \mathrm{~g} / \mathrm{d}$ or reduction of proteinuria more than $50 \%$ with normal $\mathrm{SCr}$ \\
- Relapse: patients in complete or partial remission, defined by an increase in $\mathrm{SCr}$ levels $50 \%$ or more \\
over the last value besides a nephritic urinary sediment and generally increased proteinuria (nephritic \\
flare) or by an increase in proteinuria without modification of $\mathrm{SCr}$ (proteinuric flare). Proteinuria had \\
to increase by at least $2 \mathrm{~g} / \mathrm{d}$ if the basal proteinuria was $<3.0 \mathrm{~g} / \mathrm{d}$, or double if the patient had already \\
nephrotic range proteinuria \\
- Doubling of SCr \\
- Major infection \\
- Alopecia \\
- Leucopenia \\
- Nausea \\
- Vomiting \\
- Diarrhoea \\
- Proteinuria
\end{tabular}

Notes $\quad$ Funding source: not reported

\section{Risk of bias}

\begin{tabular}{lll}
\hline Bias & Authors' judgement & Support for judgement \\
\hline $\begin{array}{l}\text { Random sequence genera- } \\
\text { tion (selection bias) }\end{array}$ & Low risk & $\begin{array}{l}\text { Patients stratified by block randomisation (stratification factors were gender, } \\
\text { age and weight) }\end{array}$ \\
\hline $\begin{array}{l}\text { Allocation concealment } \\
\text { (selection bias) }\end{array}$ & Unclear risk & Insufficient information to permit judgement \\
\hline
\end{tabular}




\section{Kaballo 2016 (Continued)}

Blinding of participants and personnel (perfor-

Insufficient information to permit judgement mance bias)

All outcomes

\begin{tabular}{|c|c|c|}
\hline $\begin{array}{l}\text { Blinding of outcome as- } \\
\text { sessment (detection bias) } \\
\text { All outcomes }\end{array}$ & Unclear risk & Insufficient information to permit judgement \\
\hline $\begin{array}{l}\text { Incomplete outcome data } \\
\text { (attrition bias) } \\
\text { All outcomes }\end{array}$ & Low risk & No missing outcome data \\
\hline $\begin{array}{l}\text { Selective reporting (re- } \\
\text { porting bias) }\end{array}$ & Low risk & Trial registration was not reported, all expected outcomes were reported \\
\hline Other bias & Low risk & The study appears to be free of other sources of bias \\
\hline
\end{tabular}

Kamanamool 2017

\begin{tabular}{ll}
\hline Methods & - Study design: open-label, parallel RCT \\
& - Study timeframe: 1 April 2012 to 31 March 2016 \\
& - Duration of follow-up: 12 months
\end{tabular}

\section{Participants}

- Country: Thailand

- Setting: multicentre (number of sites not reported)

- Inclusion criteria: patients with active, biopsy-proven lupus nephritis Class III, IV or V (ISN/RPS) 2003 criteria within 24 weeks of randomisation and who were ANA (ANA) or anti-double stranded DNA (anti-dsDNA) positive

- Number (randomised/analysed): treatment group 1 (42/42); treatment group 2 (41/41)

* Treatment group 1: class III or IV (29), class V or III/IV + V (13)

* Treatment group 2: class III or IV (28), class V or III/IV + V (13)

- Mean age \pm SD (years): treatment group 1 (34.1 \pm 11.1$)$; treatment group 2 (31.7 \pm 10.5$)$

- Sex (M/F): treatment group 1 (1/40); treatment group 2 (3/38)

- Exclusion criteria: Severe extra-renal manifestations; previous therapy with calcineurin inhibitor or MMF or CPA within the previous four months before randomisation; allergy to macrolide antibiotics; uncontrolled hypertension (SBP > $160 \mathrm{~mm} \mathrm{Hg}$ or DBP > $100 \mathrm{~mm} \mathrm{Hg}$ ); severely deteriorated kidney function or rapid progressive crescentic $\mathrm{GN}$; severe myocarditis or cardiomyopathy; requiring plasmapheresis or IVIG; severe infection or active TB; active hepatitis and evidence of chronic liver disease; HIV infection; MD; pregnancy; hypersensitivity or contraindication to MMF, mycophenolic acid, TAC, corticosteroids or any components of these drug products
Induction therapy

- Treatment group 1

* Oral MMF: initiated at a dose of $500 \mathrm{mg}$ twice daily (patients $>50 \mathrm{~kg}$ and eGFR $>60 \mathrm{~mL} / \mathrm{min}$ ) for 2 weeks. It was then advanced to $750 \mathrm{mg}$ twice daily in lupus nephritis patients weighing less than $50 \mathrm{~kg}$, or $1000 \mathrm{mg}$ twice daily in lupus nephritis patients weighing $50 \mathrm{~kg}$ or more. Dosage of MMF was prescribed according to the ACR recommendations, which suggest MMF $2 \mathrm{~g} / \mathrm{d}$ for Asians

- Treatment group 2

* Oral TAC: started at a dosage of $0.1 \mathrm{mg} / \mathrm{kg} / \mathrm{d}$ divided into two daily doses at 12-hour intervals, and the dosage was titrated to achieve trough blood concentrations of $6-10 \mathrm{ng} / \mathrm{mL}$ in the first and second month and then $4-8 \mathrm{ng} / \mathrm{mL}$ thereafter 
Kamanamool 2017 (Continued)

- Both groups

* All patients received prednisone at a dose of 0.5 to $0.7 \mathrm{mg} / \mathrm{kg} / \mathrm{d}$ (maximum $60 \mathrm{mg} / \mathrm{d}$ ), with tapering by 5 to $10 \mathrm{mg} / \mathrm{d}$ every two weeks until a dose of $5 \mathrm{mg} / \mathrm{d}$ had been achieved, and this dosage was maintained until the end of 24 weeks

* All patients who had remission received AZA 1 to $2 \mathrm{mg} / \mathrm{kg} / \mathrm{d}$ for 24 weeks as standard treatment. For patients who did not respond to the induction therapy, treatment depended on physician decision

\begin{tabular}{ll}
\hline Outcomes & Death \\
& - Complete remission \\
& - $\mathrm{SCr}$ \\
\hline Notes & Disease activity \\
\hline
\end{tabular}

\section{Risk of bias}

\begin{tabular}{|c|c|c|}
\hline Bias & Authors' judgement & Support for judgement \\
\hline $\begin{array}{l}\text { Random sequence genera- } \\
\text { tion (selection bias) }\end{array}$ & Low risk & $\begin{array}{l}\text { "We stratified patients into two strata according to the classification of renal } \\
\text { pathology (Class III-IV LN or Class V III/IV LN). Patients were randomly assigned } \\
\text { 1:1 to a TAC group or an MMF group." }\end{array}$ \\
\hline $\begin{array}{l}\text { Allocation concealment } \\
\text { (selection bias) }\end{array}$ & Low risk & $\begin{array}{l}\text { To preserve the allocation concealment, the generation of blocks of four to six } \\
\text { randomisation lists was electronically produced at Ramathibodi Hospital and } \\
\text { web-based randomizations was used. }\end{array}$ \\
\hline $\begin{array}{l}\text { Blinding of participants } \\
\text { and personnel (perfor- } \\
\text { mance bias) } \\
\text { All outcomes }\end{array}$ & High risk & Open-label study \\
\hline
\end{tabular}

Blinding of outcome as- Unclear risk Insufficient information to permit judgement
sessment (detection bias)

All outcomes

\begin{tabular}{lll}
$\begin{array}{l}\text { Incomplete outcome data } \\
\text { (attrition bias) } \\
\text { All outcomes }\end{array}$ & Low risk & No missing outcome data \\
\hline $\begin{array}{l}\text { Selective reporting (re- } \\
\text { porting bias) }\end{array}$ & Low risk & Study protocol available and pre-specified outcomes were reported \\
\hline Other bias & Low risk & $\begin{array}{l}\text { "Astellas Pharma (Thailand) Co., Ltd. provided study drug and funded the } \\
\text { study but had no role in study design, data collection, data analysis, data inter- } \\
\text { pretation or conclusions." The study appears to be free of other sources of bias }\end{array}$
\end{tabular}

Lewis 1992

\begin{tabular}{ll}
\hline Methods & - Study design: open-label, parallel RCT \\
& - Study timeframe: 1 April 1981 to 30 September 1986 \\
& - Duration of follow-up: mean follow-up 2.5 years with termination of study \\
\hline Participants & Country: USA \\
& - Setting: multicentre \\
& Inclusion criteria: $\geq 16$ years; SLE (ARA criteria); qualifying biopsy; 35 participants with class IV disease
\end{tabular}


Lewis 1992 (Continued)

- Number (randomised): treatment group 1 (40); treatment group 2 (46)

- Mean age \pm SD (years): treatment group $1(31 \pm 11)$; treatment group $2(33 \pm 14)$

- $\operatorname{Sex}(M / F)$ : treatment group 1 (7/33); treatment group 2 (7/39)

- Exclusion criteria: pregnancy; $\mathrm{SCr}>6 \mathrm{mg} / \mathrm{dL}$; previous plasmapheresis; history of primary myocardial disease; cancer within last 5 years; prednisone-associated psychosis; peptic ulcer; active liver disease

\begin{tabular}{ll}
\hline Interventions & Induction therapy \\
- Treatment group 1 \\
$*$ Oral CPA \\
$*$ Corticosteroids \\
$*$ PEX: $3 \times$ weekly for 4 weeks \\
- Treatment group 2 \\
$*$ Oral CPA \\
$*$ Corticosteroids
\end{tabular}

\begin{tabular}{|c|c|}
\hline Outcomes & $\begin{array}{l}\text { - Death } \\
\text { - ESKD } \\
\text { - Remission: } \mathrm{SCr} \leq 1.2 \mathrm{mg} / \mathrm{dL} \text { and a } 24 \text {-hour urinary protein of } \leq 0.2 \mathrm{~g} / \mathrm{d} \\
\text { - Toxicity } \\
\text { - Infection } \\
\text { - Herpes zoster virus infection } \\
\text { - SCr } \\
\text { - Proteinuria }\end{array}$ \\
\hline Notes & - Funding source: Public health service \\
\hline
\end{tabular}

\section{Risk of bias}

\begin{tabular}{lll}
\hline Bias & Authors' judgement & Support for judgement \\
\hline $\begin{array}{l}\text { Random sequence genera- } \\
\text { tion (selection bias) }\end{array}$ & Low risk & Stratified according to clinic by central coordination centre \\
\hline $\begin{array}{l}\text { Allocation concealment } \\
\text { (selection bias) }\end{array}$ & Low risk & $\begin{array}{l}\text { Generated by Biostatistical Coordinating centre which issued treatment as- } \\
\text { signments by telephone after confirmation of patient eligibility }\end{array}$ \\
\hline $\begin{array}{l}\text { Blinding of participants } \\
\text { and personnel (perfor- } \\
\begin{array}{l}\text { mance bias) } \\
\text { All outcomes }\end{array}\end{array}$ & High risk & Open-label study \\
\hline
\end{tabular}

\begin{tabular}{lll}
\hline $\begin{array}{l}\text { Blinding of outcome as- } \\
\text { sessment (detection bias) } \\
\text { All outcomes }\end{array}$ & Unclear risk & Insufficient information to permit judgement \\
\hline $\begin{array}{l}\text { Incomplete outcome data } \\
\begin{array}{l}\text { (attrition bias) } \\
\text { All outcomes }\end{array}\end{array}$ & Low risk & No missing outcome data; 1 patient lost-to follow-up \\
\hline $\begin{array}{l}\text { Selective reporting (re- } \\
\text { porting bias) }\end{array}$ & Low risk & Study protocol available and pre-specified outcomes were reported \\
\hline Other bias & High risk & The study was terminated early \\
\hline
\end{tabular}




\begin{tabular}{ll}
\hline Methods & Study design: open-label, pilot RCT \\
- & Study timeframe: not reported \\
- & Duration of follow-up: 48 weeks \\
\hline Participants & Country: Hong Kong \\
- Setting: single centre \\
- Inclusion criteria: diagnosis of SLE (revised ACR criteria); biopsy-proven lupus nephritis class III or IV \\
(WHO classification criteria), clinical activity index $\geq 6 / 24$, proteinuria $\geq 1.5 \mathrm{~g} / 24 \mathrm{~h}$, albumin $\leq 35 \mathrm{~g} / \mathrm{L} ;$ \\
- $3 / 19$ participants with class IV disease \\
- Mean age \pm SD (years): treatment group 1 (40.3 \pm 13.9$) ;$ treatment group 2 (39.6 \pm 8.6$)$ \\
- Sex (M/F): treatment group 1 (0/9); treatment group 2 (1/9) \\
- Exclusion criteria: severe infection in last 3 months; HIV; HBV or HCV; active TB; pregnancy; on oral/ \\
IV CPA, AZA or MMF within 8 weeks or prednisolone $\geq 0.5 \mathrm{mg} / \mathrm{kg} / \mathrm{d}$ within 4 weeks; history of cancer; \\
DM or kidney failure leading to dialysis
\end{tabular}

\begin{tabular}{|c|c|}
\hline Interventions & $\begin{array}{l}\text { Induction therapy } \\
\text { - Treatment group } 1 \\
\text { * RTX: } 1000 \mathrm{mg} \text {, treatment repeated on day } 15 \\
\text { - Treatment group } 2 \\
\text { * RTX: } 1000 \mathrm{mg}, 250 \mathrm{mg} \text { MP day } 1 \text {, followed by IV CPA } 750 \mathrm{mg} \text {, treatment repeated once on day } 15 \\
\text { - Both groups } \\
\text { * All participants received } 250 \mathrm{mg} \text { IV MP on day } 1 \text {, oral prednisolone } 30 \mathrm{mg} / \mathrm{d} \text { from day } 2 \text { to day } 5 \text {, } \\
\text { then } 0.5 \mathrm{mg} / \mathrm{kg} \text { for } 4 \text { weeks, then dose reduction } 5 \mathrm{mg} \text { every } 2 \text { weeks } \\
\text { * Patients were pre-medicated with chlorpheniramine (10 mg IV) and paracetamol (1 g orally) } 30 \mathrm{~min} \\
\text { before IV infusions } \\
\text { * All participants on ACEi before the study and continued on same dose }\end{array}$ \\
\hline
\end{tabular}

\begin{tabular}{ll}
\hline Outcomes & Major infection \\
- Herpes zoster virus infection & Complete response: if the baseline (at week 0) SLEDAI scores were greater than 0 and the follow-up \\
score was equal to 0 \\
- Partial response: if the baseline SLEDAI scores were greater than the follow-up score but the follow-up \\
score was not equal to 0 \\
- Treatment failure: worse disease activity \\
- CrCl \\
- Proteinuria
\end{tabular}

Notes $\quad$ Funding source: Roche provided the study drug

\section{Risk of bias}

\begin{tabular}{lll}
\hline Bias & Authors' judgement & Support for judgement \\
\hline $\begin{array}{l}\text { Random sequence genera- } \\
\text { tion (selection bias) }\end{array}$ & Low risk & Randomisation according to a randomisation table kept by a third party \\
\hline $\begin{array}{l}\text { Allocation concealment } \\
\text { (selection bias) }\end{array}$ & Low risk & Randomisation table kept by a third party \\
\hline $\begin{array}{l}\text { Blinding of participants } \\
\text { and personnel (perfor- } \\
\text { mance bias) }\end{array}$ & High risk & Open-label study \\
\end{tabular}


Li 2009c (Continued)

All outcomes

Blinding of outcome as-
sessment (detection bias) $\quad$ Unclear risk Insufficient information to permit judgement

sessment (detection bias)

All outcomes

\begin{tabular}{lll}
$\begin{array}{l}\text { Incomplete outcome data } \\
\text { (attrition bias) } \\
\text { All outcomes }\end{array}$ & Low risk & No missing outcome data \\
\hline $\begin{array}{l}\text { Selective reporting (re- } \\
\text { porting bias) }\end{array}$ & High risk & Not all expected outcomes were reported \\
\hline Other bias & Low risk & $\begin{array}{l}\text { "...Roche provided study drug but had no role in study design, data collection, } \\
\text { data analysis, data interpretation or writing of the report..." The study appears } \\
\text { to be free of other sources of bias }\end{array}$
\end{tabular}

\section{Li 2012}

$\begin{array}{ll}\text { Methods } & \text { - Study design: open-label, parallel RCT } \\ & \text { - Study timeframe: } \\ \text { - Duration of follow-up: }\end{array}$

\begin{tabular}{|c|c|}
\hline Participants & 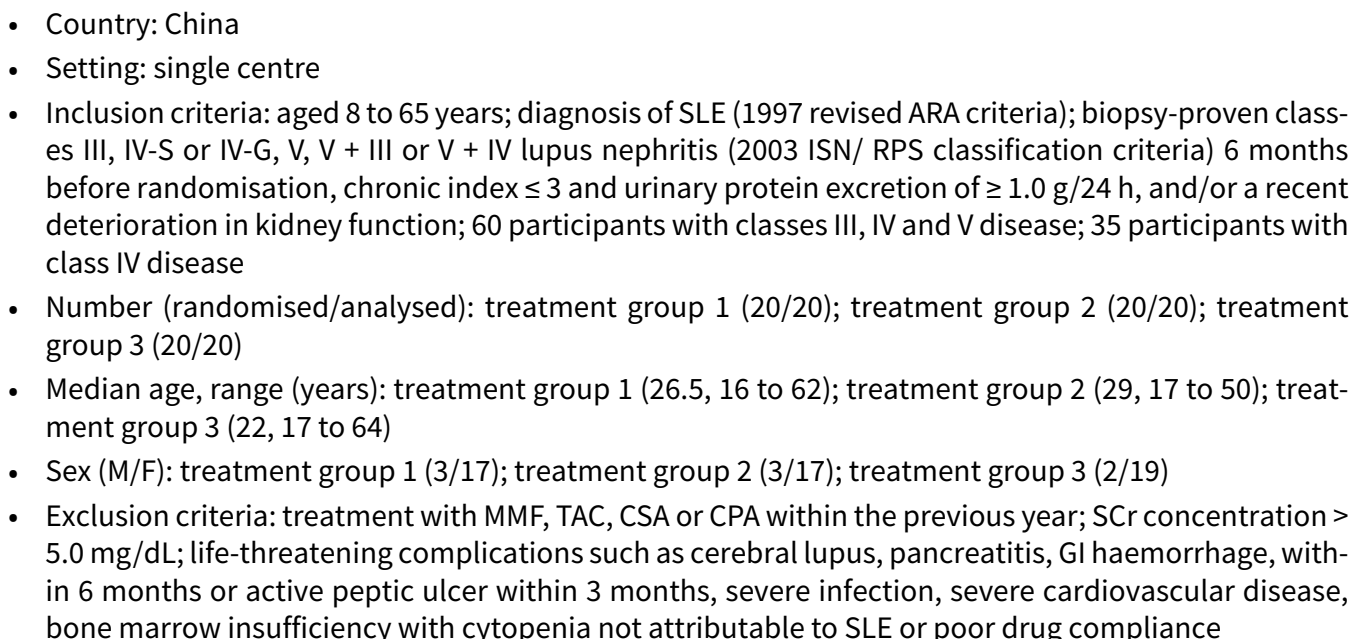 \\
\hline
\end{tabular}

Induction therapy: duration of treatment was 6 months
- Treatment group 1
$*$ Oral MMF: 1.5 to $2.0 \mathrm{~g} / \mathrm{d}$
- Treatment group 2
$*$ Oral TAC: 0.08 to $0.1 \mathrm{mg} / \mathrm{kg} / \mathrm{d}$, target 12 hour trough 6 to $8 \mathrm{ng} / \mathrm{mL}$
- Treatment group 3
$*$ IV CPA: 0.5 to $0.75 \mathrm{~g} / 1.73 \mathrm{~m}^{2}$
- All groups
$*$ All patients received corticosteroids 0.8 to $1 \mathrm{mg} / \mathrm{kg} / \mathrm{d}$ (max dose $60 \mathrm{mg} / \mathrm{d}$ ). Reduced by $10 \mathrm{mg}$ every

$\quad 2$ weeks until at $40 \mathrm{mg} / \mathrm{d}$, then reduced by $5 \mathrm{mg} / \mathrm{d}$ every 2 weeks to maintenance dose of $10 \mathrm{mg} / \mathrm{d}$


Li 2012 (Continued)

- Leucopenia

- Complete renal remission: urinary protein excretion $<0.3 \mathrm{~g} / 24 \mathrm{~h}$ with normal urine sediment, serum albumin concentration $>35 \mathrm{~g} / \mathrm{L}$ and $\mathrm{SCr}$ above baseline values by $\leq 15 \%$

- Partial renal remission: urinary protein excretion between 0.3 to $2.9 \mathrm{~g} / 24 \mathrm{~h}$, having decreased by at least $50 \%$ from baseline values, with a serum albumin concentration of at least $30 \mathrm{~g} / \mathrm{L}$ and relative stabilisation $( \pm 30 \%)$ in $\mathrm{SCr}$

- Complete remission in proteinuria

- Doubling of $\mathrm{SCr}$

- Proteinuria

- Serum albumin

Notes

- Funding source: Shanghai Institutes of Health and Chinese National Natural Science Foundation

\section{Risk of bias}

\begin{tabular}{|c|c|c|}
\hline Bias & Authors' judgement & Support for judgement \\
\hline $\begin{array}{l}\text { Random sequence genera- } \\
\text { tion (selection bias) }\end{array}$ & Unclear risk & $\begin{array}{l}\text { Study was described as randomised, method of randomisation was not report- } \\
\text { ed }\end{array}$ \\
\hline $\begin{array}{l}\text { Allocation concealment } \\
\text { (selection bias) }\end{array}$ & Unclear risk & Insufficient information to permit judgement \\
\hline $\begin{array}{l}\text { Blinding of participants } \\
\text { and personnel (perfor- } \\
\text { mance bias) } \\
\text { All outcomes }\end{array}$ & High risk & Open-label study \\
\hline $\begin{array}{l}\text { Blinding of outcome as- } \\
\text { sessment (detection bias) } \\
\text { All outcomes }\end{array}$ & Unclear risk & Insufficient information to permit judgement \\
\hline $\begin{array}{l}\text { Incomplete outcome data } \\
\text { (attrition bias) } \\
\text { All outcomes }\end{array}$ & Low risk & No missing outcome data \\
\hline $\begin{array}{l}\text { Selective reporting (re- } \\
\text { porting bias) }\end{array}$ & Low risk & Study protocol available and pre-specified outcomes were reported \\
\hline Other bias & Low risk & The study appears to be free of other sources of bias \\
\hline
\end{tabular}

Liou 2007

\begin{tabular}{ll}
\hline Methods & - Study design: open-label, parallel RCT \\
& - Study timeframe: not reported \\
& - Duration of follow-up: 18 months
\end{tabular}

\begin{tabular}{ll}
\hline Participants & - Country: China \\
- Setting: single centre \\
- Inclusion criteria: biopsy-proven lupus nephritis \\
- Number (randomised): treatment group 1 (19); treatment group 2 (21) \\
- Mean age \pm SD (years): not reported \\
- Sex (M/F): not reported \\
- Exclusion criteria: not reported
\end{tabular}


Liou 2007 (Continued)

Interventions
Induction and maintenance therapy: 6 months induction therapy and 12 months maintenance therapy

- Treatment group 1

* Oral leflunomide: $30 \mathrm{mg} / \mathrm{d}$; after 6 months of induction therapy, leflunomide was reduced to 20 $\mathrm{mg} / \mathrm{d}$

- Treatment group 2

* IV CPA: 1 g per month; after 6 months IV CPA was given 1g/3 months for maintenance therapy

- Both groups

* All patients received prednisolone 0.8 to $1 \mathrm{mg} / \mathrm{kg} / \mathrm{d}$ tapered to $10 \mathrm{mg} / \mathrm{d}$

\begin{tabular}{ll}
\hline Outcomes & Complete renal remission (not defined) \\
& - Herpes zoster virus infection \\
& - Proteinuria \\
& - Scrum albumin \\
\hline Notes & - Abstract-only publication \\
& - Only induction therapy (6 months) reported \\
& Funding source: not reported \\
\hline
\end{tabular}

\section{Risk of bias}

\section{Bias}

Random sequence genera- Unclear risk tion (selection bias)

Allocation concealment $\quad$ Unclear risk Insufficient information to permit judgement
(selection bias)

\section{Authors' judgement Support for judgement}

Insufficient information to permit judgement
High risk
Open-label study

and personnel (perfor-

mance bias)

All outcomes

\begin{tabular}{lll}
$\begin{array}{l}\text { Blinding of outcome as- } \\
\text { sessment (detection bias) } \\
\text { All outcomes }\end{array}$ & Unclear risk & Insufficient information to permit judgement \\
\hline $\begin{array}{l}\text { Incomplete outcome data } \\
\text { (attrition bias) }\end{array}$ & Unclear risk & Insufficient information to permit judgement \\
All outcomes & &
\end{tabular}

Selective reporting (re- High risk Not all expected clinical outcomes are reported
porting bias)

\begin{tabular}{ll}
\hline Other bias $\quad$ Unclear risk $\quad$ Abstract-only publication; insufficient information to permit judgement \\
\hline
\end{tabular}

Liu 2015

\begin{tabular}{ll}
\hline Methods & Study design: open-label, parallel RCT \\
& - Study timeframe: April 2009 to June 2011 \\
& Duration of follow-up: 24 weeks \\
\hline
\end{tabular}


Liu 2015 (Continued)

\section{Participants}

- Country: China

- Setting: multicentre (number of sites not reported)

- Inclusion criteria: patients aged 18 to 65 years; diagnosis of SLE (ACR criteria); biopsy-proven class III, IV, V, III+V, and IV+V lupus nephritis (ISN/RPS 2003 classification criteria) within 6 months before study entry; proteinuria ( $\geq 1.5 \mathrm{~g} / \mathrm{d}$ ) with a $\mathrm{SCr} \leq 3.0 \mathrm{mg} / \mathrm{dL}$ )

- Number (randomised/analysed): treatment group 1 (181/175); treatment group 2 (181/181)

* Treatment group 1: class III (10), class IV (74), class V (32); class III+IV or IV+V (65)

* Treatment group 2: class III (9), class IV (76), class V (37); class III+IV or IV+V (52)

- Median age, IQR (years): treatment group 1 (33.6, 24.2 to 41.5); treatment group 2 (30.3, 23.3 to 38.6)

- Sex (M/F): treatment group 1 (20/161); treatment group 2 (13/168)

- Exclusion criteria: treatment with MMF, CPA, TAC, or high-dose MP; current RRT; plasmapheresis, or IVIG within the 12 weeks before randomisation; abnormal liver function or serum glucose test results; and pathologic chronicity index $>3$ \begin{tabular}{l} 
Induction therapy: duration of therapy was 6 months \\
$\begin{array}{l}\text { Interventions } \\
* \text { IV CPA: initiated at a dose of } 0.75 \mathrm{~g} / \mathrm{m}^{2} \text { body surface area and then adjusted to a dose of } 0.5 \text { to } 1.0 \\
\mathrm{~g} / \mathrm{m}^{2} \text { body surface area every } 4 \text { weeks for } 6 \text { doses } \\
\text { - Treatment group } 2 \\
* \text { Oral MMF: } 0.5 \mathrm{~g} \text { twice } / \mathrm{d} \\
* \text { Oral TAC: } 2 \mathrm{mg} \text { twice } / \mathrm{d} \\
\text { Both groups } \\
* \text { IV MP pulse therapy }(0.5 \mathrm{~g} / \mathrm{d}) \text { for } 3 \text { days, followed by oral prednisone }(0.6 \mathrm{mg} / \mathrm{kg} / \mathrm{d}) \mathrm{every} \mathrm{morning} \\
\text { for } 4 \text { weeks. The daily dose of prednisone was tapered by } 5 \mathrm{mg} / \mathrm{d} \text { every } 2 \mathrm{weeks} \text { to } 20 \mathrm{mg} / \mathrm{d} \text { and } \\
\text { then by } 2.5 \mathrm{mg} / \mathrm{d} \text { every } 2 \text { weeks to a maintenance dose of } 10 \mathrm{mg} / \mathrm{d}\end{array}$ \\
\hline
\end{tabular}

Outcomes

- Death

- Complete remission: $24 \mathrm{~h}$ urinary protein excretion $\leq 0.4 \mathrm{~g}$, the absence of active urine sediments, serum albumin level $\geq 35 \mathrm{~g} / \mathrm{L}$, and normal SCr

- Partial remission: $\geq 50 \%$ reduction in proteinuria and urine protein $<3.5 \mathrm{~g} / 24 \mathrm{~h}$, serum albumin level $\geq 30 \mathrm{~g} / \mathrm{L}$, and normal or $\leq 25 \%$ increase in SCr level from baseline

- Doubling of $\mathrm{SCr}$

- Major infection

- Herpes zoster virus infection

- Menstrual disorder

- Avascular necrosis

- Alopecia

- Leucopenia

- Upper GI symptoms

- Diarrhoea

Notes

- Funding source: National Basic Research Program of China (973 Program, No. 2012CB517600, No. 2012CB517606), National Key Technology R\&D Program (2011BAI10B04, 2013BAI09B04).

\section{Risk of bias}

\begin{tabular}{lll}
\hline Bias & Authors' judgement & Support for judgement \\
\hline $\begin{array}{l}\text { Random sequence genera- } \\
\text { tion (selection bias) }\end{array}$ & Low risk & $\begin{array}{l}\text { Randomisation list, stratified by centre was created by Rundo International } \\
\text { Pharmaceutical Research \& Development (Shanghai) Co. Ltd. by using com- } \\
\text { puter generated random-number sequences }\end{array}$ \\
\hline $\begin{array}{l}\text { Allocation concealment } \\
\text { (selection bias) }\end{array}$ & Low risk & $\begin{array}{l}\text { Sequentially numbered, concealed envelopes containing group assignment } \\
\text { were provided to the investigators. After eligible patients provided written in- }\end{array}$
\end{tabular}


Liu 2015 (Continued)

formed consent, the envelopes were opened in sequence and patients were randomly assigned, in a 1:1 ratio, to the multi-target regimen or IV CPA

Blinding of participants $\quad$ High risk
and personnel (perfor-
mance bias)

\begin{tabular}{|c|c|c|}
\hline $\begin{array}{l}\text { Blinding of outcome as- } \\
\text { sessment (detection bias) }\end{array}$ & Low risk & $\begin{array}{l}\text { The outcomes were adjudicated by the Clinical Endpoints Committee, blinded } \\
\text { to treatment regimen. }\end{array}$ \\
\hline
\end{tabular}

All outcomes

\begin{tabular}{|c|c|c|}
\hline $\begin{array}{l}\text { Incomplete outcome data } \\
\text { (attrition bias) } \\
\text { All outcomes }\end{array}$ & High risk & $\begin{array}{l}\text { Unclear why } 6 \text { patients }(3 \%) \text { in the IV CPA group were not given therapy and } \\
\text { not included in the analysis and why patients in the IV CPA group were seen at } \\
\text { twice the follow-up rate then patients in the multi-target therapy group }\end{array}$ \\
\hline
\end{tabular}

\begin{tabular}{lll}
\hline $\begin{array}{l}\text { Selective reporting (re- } \\
\text { porting bias) }\end{array}$ & High risk & Not all prespecified outcomes were reported \\
\hline Other bias & Low risk & This study appears to be free of other sources of bias \\
\hline
\end{tabular}

Loo 2010

\begin{tabular}{|c|c|}
\hline Methods & $\begin{array}{l}\text { - Study design: open-label, parallel RCT } \\
\text { - Study timeframe: not reported } \\
\text { - Duration of follow-up: } 6 \text { months }\end{array}$ \\
\hline Participants & $\begin{array}{l}\text { - Country: Malaysia } \\
\text { - Setting: single centre } \\
\text { - Inclusion criteria: aged } \geq 12 \text { years; diagnosis of SLE (ARA } 1982 \text { criteria) and biopsy proven severe class- } \\
\text { es III or IV } \pm \text { V lupus nephritis (ISN/RPS } 2003 \text { classification criteria) } \\
\text { - Number (randomised/analysed): treatment group } 1(14 / 14) \text {; treatment group } 2(14 / 14) \\
\text { - Mean age } \pm \text { SD (years): treatment group } 1 \text { ( } 31.9 \pm 11.6) \text {; treatment group } 2(30.2 \pm 7.5) \\
\text { - Sex (M/F): treatment group } 1(4 / 10) \text {; treatment group } 2(0 / 14) \\
\text { - Ethnicity: treatment group } 1 \text { (Chinese (5), Malay ( } 7) \text {, Indian (2)); treatment group } 2 \text { (Chinese (5), Malay } \\
\text { ( } 7) \text {, Indian ( } 2) \text { ) } \\
\text { - Exclusion criteria: not reported }\end{array}$ \\
\hline
\end{tabular}

Interventions Induction therapy: duration of therapy was 6 months

- Treatment group 1

* PEX: 3 sessions ( $3 \mathrm{~L}$ per session) following MP treatment. For PEX, the plasma removed was replaced with 2 litres of human albumin $5 \%$ and the balance with Hartman's solution

- Treatment group 2

* Immunoadsorption: 3 sessions carried out on a daily or every other day basis for 3 days. Three litres of plasma or 1 plasma volume, whichever was greater was processed at each session

- Both groups

* All patients received standard induction IV pulse MP at $250 \mathrm{mg} / \mathrm{d}$ for 3 days followed by PEX or immunoadsorption. Followed by IVIG $10 \mathrm{~g} / \mathrm{d}$ for 3 days. Patients subsequently proceed to the consolidation phase with pulse IV CPA at 10 to $12 \mathrm{mg} / \mathrm{kg} / \mathrm{dose}$ 2-weekly for 4 doses, then monthly for four more doses. Patients were then randomised to receive maintenance therapy with either oral CSA or MMF in conjunction with low dose steroid, for a further 12 to 18 months 
Loo 2010 (Continued)
Notes
- Funding source: not reported

\section{Risk of bias}

\begin{tabular}{|c|c|c|}
\hline Bias & Authors' judgement & Support for judgement \\
\hline $\begin{array}{l}\text { Random sequence genera- } \\
\text { tion (selection bias) }\end{array}$ & High risk & Consecutive enrolment \\
\hline $\begin{array}{l}\text { Allocation concealment } \\
\text { (selection bias) }\end{array}$ & Unclear risk & Insufficient information to permit judgement \\
\hline $\begin{array}{l}\text { Blinding of participants } \\
\text { and personnel (perfor- } \\
\text { mance bias) } \\
\text { All outcomes }\end{array}$ & High risk & Open-label study \\
\hline $\begin{array}{l}\text { Blinding of outcome as- } \\
\text { sessment (detection bias) } \\
\text { All outcomes }\end{array}$ & Unclear risk & Insufficient information to permit judgement \\
\hline $\begin{array}{l}\text { Incomplete outcome data } \\
\text { (attrition bias) } \\
\text { All outcomes }\end{array}$ & Unclear risk & Insufficient information to permit judgement \\
\hline $\begin{array}{l}\text { Selective reporting (re- } \\
\text { porting bias) }\end{array}$ & High risk & Not all expected outcomes were reported \\
\hline Other bias & High risk & $\begin{array}{l}\text { Marked differences (demographics and clinical characteristics) between } \\
\text { groups at baseline }\end{array}$ \\
\hline
\end{tabular}

\section{Lui 1997}

\begin{tabular}{ll} 
Methods & - Study design: parallel RCT \\
& - Study timeframe: not reported \\
\hline Participants & Duration of follow-up: 12 months \\
\hline & Country: Hong Kong \\
- Setting: not reported \\
- Number (randomised/analysed): treatment group 1 (17/17); treatment group 2 (17/17) \\
- Mean age \pm SD (years): not reported \\
- Sex (M/F): not reported \\
- Exclusion criteria: not reported
\end{tabular}

Interventions

Induction therapy

- Treatment group 1

* Oral CSA: $5 \mathrm{mg} / \mathrm{kg} / \mathrm{d}$, reduced to $2.5 \mathrm{mg} / \mathrm{kg} / \mathrm{d}$

- Treatment group 2

* Oral CPA: $1 \mathrm{mg} / \mathrm{kg} / \mathrm{d}$

- Both groups

* All patients received prednisolone $(0.5 \mathrm{mg} / \mathrm{kg} / \mathrm{d})$ and AZA $(1 \mathrm{mg} / \mathrm{kg} / \mathrm{d})$ 
Lui 1997 (Continued)

- Futcomes
- Partial response
- Complete response
- Proteinuria
- $\mathrm{CrCl}$
- Infection
- Herpes zoster virus infection
- Leucopenia
- Amenorrhoea

\begin{tabular}{ll}
\hline Notes & Abstract-only publication \\
& - Funding source: not reported
\end{tabular}

\section{Risk of bias}

\begin{tabular}{|c|c|c|}
\hline Bias & Authors' judgement & Support for judgement \\
\hline $\begin{array}{l}\text { Random sequence genera- } \\
\text { tion (selection bias) }\end{array}$ & Unclear risk & Insufficient information to permit judgement \\
\hline $\begin{array}{l}\text { Allocation concealment } \\
\text { (selection bias) }\end{array}$ & Unclear risk & Insufficient information to permit judgement \\
\hline $\begin{array}{l}\text { Blinding of participants } \\
\text { and personnel (perfor- } \\
\text { mance bias) } \\
\text { All outcomes }\end{array}$ & High risk & Open-label study \\
\hline $\begin{array}{l}\text { Blinding of outcome as- } \\
\text { sessment (detection bias) } \\
\text { All outcomes }\end{array}$ & Unclear risk & Insufficient information to permit judgement \\
\hline $\begin{array}{l}\text { Incomplete outcome data } \\
\text { (attrition bias) } \\
\text { All outcomes }\end{array}$ & Unclear risk & Insufficient information to permit judgement \\
\hline $\begin{array}{l}\text { Selective reporting (re- } \\
\text { porting bias) }\end{array}$ & Unclear risk & Insufficient information to permit judgement \\
\hline Other bias & Unclear risk & Abstract-only publication; insufficient information to permit judgement \\
\hline
\end{tabular}

\section{LUNAR 2012}

\begin{tabular}{ll}
\hline Methods & Study design: phase III, double-blind double-dummy RCT \\
& - Study timeframe: January 2006 to January 2008 \\
\hline Participants & Duration of follow-up: 12 months \\
\hline - Countries: USA, Latin America & Setting: multinational (52 sites) \\
- Inclusion criteria: aged 16 to 75 years of age; diagnosis of SLE (ACR criteria); history of ANA positivity; \\
diagnosis of class III or IV lupus nephritis (ISN/RPS 2003 Classification) with either active or active \\
chronic disease; proteinuria (urine polymerase chain reaction $>1.0)$; If the biopsy was performed $>3$ \\
months before screening; an active urinary sediment $(>10 \mathrm{RBC} / \mathrm{HPF}$ or the presence of RBC casts) \\
- Number (randomised/analysed): treatment group (72/72); control group (72/72)
\end{tabular}


LUNAR 2012 (Continued)

- Mean age \pm SD (years): treatment group $(31.8 \pm 9.6)$; control group $(29.4 \pm 9.3)$

- Sex (M/F): treatment group (9/63); control group (5/67)

- Exclusion criteria: active infection; recurrent or chronic infection,; CPA or CNI treatment within 90 days prior to screening; MMF $>2 \mathrm{~g}$ daily $>90 \mathrm{~d}$ prior to screening; use of prednisolone $>20 \mathrm{mg} / \mathrm{d}>14$ days prior to screening; previous treatment with CAMPATH-1H; B-cell targeted therapy; pregnancy or lactation; history of cancer

\begin{tabular}{|c|c|}
\hline Interventions & $\begin{array}{l}\text { Induction therapy: duration of therapy was } 12 \text { months } \\
\text { - Treatment group } \\
\text { * IV RTX: } 1000 \mathrm{mg} \text { (days } 1,15,168,182 \text { ) } \\
\text { - Control group } \\
\text { * Placebo } \\
\text { - Both groups } \\
\text { * MMF: initial dosage of } 1.5 \mathrm{~g} / \mathrm{d} \text { in } 3 \text { divided doses, and the dosage was increased to } 3 \mathrm{~g} / \mathrm{d} \text { by week } 4 \\
\text { * IV MP: } 1,000 \mathrm{mg} \text { was administered } 30-60 \text { minutes prior to the administration of study drug on day } \\
1 \text { and again within } 3 \text { days. } \\
\text { * Oral prednisone: } 0.75 \mathrm{mg} / \mathrm{kg} / \mathrm{d} \text { (maximum } 60 \mathrm{mg} \text { ) was administered until day } 16 \text { and tapered to } 10 \\
\text { mg/d by week } 16\end{array}$ \\
\hline Outcomes & $\begin{array}{l}\text { - Death (all causes) } \\
\text { - Stable creatinine } \\
\text { - Major infection } \\
\text { - Herpes zoster virus infection } \\
\text { - Complete response: } \mathrm{SCr} \leq 115 \% \text { of baseline if it was normal at baseline; inactive urinary sediment (< } \\
5 \mathrm{RBC} / \mathrm{HPF} \text { and absence of } \mathrm{RBC} \text { casts); and UPCR }<0.5 \\
\text { - Partial response: } \mathrm{SCr} \leq 115 \% \text { of baseline; } \mathrm{RBCS} / \mathrm{HPF} \leq 50 \% \text { above baseline and no RBC casts; and at } \\
\text { least a } 50 \% \text { decrease in the UPCR to }<1.0 \text { (if the baseline UPCR was } \leq 3.0 \text { ) or to } \leq 3.0 \text { (if the baseline } \\
\text { UPCR was }>3.0 \text { ) } \\
\text { - Treatment failure (if criteria for complete response or partial response were not met, for early termi- } \\
\text { nation from the study or inability to assess the end point due to missing data, or for initiation of a new } \\
\text { immunosuppressant agent prior to week } 52 \\
\text { - Complete response in proteinuria } \\
\text { - Partial response in proteinuria } \\
\text { - Serious adverse events } \\
\text { - Nausea } \\
\text { - Diarrhoea }\end{array}$ \\
\hline
\end{tabular}

Notes

- Funding source: Genentech and Biogen Idec

\section{Risk of bias}

\begin{tabular}{lll}
\hline Bias & Authors' judgement & Support for judgement \\
\hline $\begin{array}{l}\text { Random sequence genera- } \\
\text { tion (selection bias) }\end{array}$ & Unclear risk & $\begin{array}{l}\text { Study was described as randomised, method of randomisation was not report- } \\
\text { ed }\end{array}$ \\
\hline $\begin{array}{l}\text { Allocation concealment } \\
\text { (selection bias) }\end{array}$ & Unclear risk & Insufficient information to permit judgement \\
\hline $\begin{array}{l}\text { Blinding of participants } \\
\text { and personnel (perfor- } \\
\text { mance bias) }\end{array}$ & Low risk & Double-blind, double-dummy placebo study \\
$\begin{array}{l}\text { All outcomes } \\
\text { Blinding of outcome as- } \\
\text { sessment (detection bias) }\end{array}$ & Unclear risk & Insufficient information to permit judgement \\
\hline
\end{tabular}


LUNAR 2012 (Continued)

All outcomes

\begin{tabular}{lll}
$\begin{array}{l}\text { Incomplete outcome data } \\
\text { (attrition bias) } \\
\text { All outcomes }\end{array}$ & Low risk & No missing outcome data \\
\hline $\begin{array}{l}\text { Selective reporting (re- } \\
\text { porting bias) }\end{array}$ & Low risk & Study protocol available and pre-specified outcomes were reported \\
\hline Other bias & High risk & $\begin{array}{l}\text { Some authors declared grants/research support from Genentech and Aspreva, } \\
\text { and sponsor included in data analysis and authorship }\end{array}$
\end{tabular}

\section{MAINTAIN Nephritis 2010}

\begin{tabular}{ll}
\hline Methods & Study design: open-label, parallel RCT \\
- Study timeframe: July 2002 and March 2006 \\
- \\
Duration of follow-up: median follow-up 53 months; extended median follow-up was 9.16 years (range \\
1.5 to 13 years) \\
\hline - Country: European (countries not reported) \\
- Setting: multinational ( 27 sites) \\
- Inclusion criteria: SLE $\geq 14$ years, diagnosis of SLE (ACR criteria), proteinuria $\geq 0.5 \mathrm{~g} / \mathrm{d}$, biopsy-proven \\
lupus nephritis Class III, IV, Vc or Vd lupus nephritis (WHO classification criteria) \\
- - Mumber (randomised/analysed): treatment group 1 (52/52); treatment group 2 (53/53) \\
- Sex (M/F): treatment group 1 (4/48); treatment group 2 (5/48) \\
- Exclusion criteria: recent treatment with high dose corticosteroids or immunosuppressive drugs; non- \\
lupus related renal disease (such as microthrombotic disease associated with antiphospholipid syn- \\
drome); pre-existing chronic kidney failure (defined as a SCr value above the upper normal value for \\
the local laboratory) due to a previous episode of lupus nephritis or other cause; pregnancy or breast \\
feeding; previous malignancy (except skin and cervical intraepithelial neoplasia's); DM; previously \\
documented severe toxicity of immunosuppressants, anticipated non-compliance with the protocol
\end{tabular}

Maintenance therapy
Interventions $\quad$ Treatment group 1
$*$ AZA: $2 \mathrm{mg} / \mathrm{kg} / \mathrm{d}$
- Treatment group 2
$*$ MMF: $2 \mathrm{~g} / \mathrm{d}$
- Both groups
$*$ Induction therapy of $3 \times 750 \mathrm{mg}$ IV MP followed by oral glucocorticoids $0.5 \mathrm{mg} / \mathrm{kg} / \mathrm{d}$ and 6 fortnightly
pulses IV CPA $500 \mathrm{mg}$

$\begin{array}{ll}\text { - } & \text { Death } \\ \text { - } & \text { ESKD } \\ \text { - } & \text { Relapse: (i) recurrence or the development of nephrotic syndrome (serum albumin } \leq 3.5 \mathrm{~g} / \mathrm{dL} \text { and } \\ \text { proteinuria } \geq 3 \mathrm{~g} / 24 \mathrm{~h} \text { ); (ii) renal impairment }(\geq 33 \% \text { increase of } \mathrm{SCr} \text { within a } 1 \text {-month period directly } \\ \text { attributed to lupus nephritis and confirmed } 1 \text { week later; flare referred to as 'renal impairment') or } \\ \text { (iii) a threefold increase of } 24 \mathrm{~h} \text { proteinuria within a 3-month period accompanied by microscopic } \\ \text { haematuria (defined as a number of } \mathrm{RBC} / \mathrm{HPF} \text { superior to upper normal limit for the local laboratory) } \\ \text { and } \geq 33 \% \text { reduction of serum C3 level within a 3-month period (this definition of renal flare was only } \\ \text { applicable to those patients with low-grade baseline } 24 \mathrm{~h} \text { proteinuria }(\geq 0.5 \mathrm{~g} \text { and }<1 \mathrm{~g} \text { ); this type of } \\ \text { renal flare is further referred to as 'proteinuria increase' } \\ \text { - Time to renal flare }\end{array}$


MAINTAIN Nephritis 2010 (Continued)

- Doubling of $\mathrm{SCr}$

- Number of withdrawals due to toxicity

- Number of treatment failures

- Major infection

- Herpes zoster virus infection

- Avascular necrosis

- Malignancy

- Alopecia

- Leucopenia

- Kidney function over time

- 24 hour proteinuria over time

Notes - Funding source: no external funding

\section{Risk of bias}

\begin{tabular}{|c|c|c|}
\hline Bias & Authors' judgement & Support for judgement \\
\hline $\begin{array}{l}\text { Random sequence genera- } \\
\text { tion (selection bias) }\end{array}$ & Unclear risk & Randomisation by minimisation \\
\hline $\begin{array}{l}\text { Allocation concealment } \\
\text { (selection bias) }\end{array}$ & Unclear risk & Insufficient information to permit judgement \\
\hline $\begin{array}{l}\text { Blinding of participants } \\
\text { and personnel (perfor- } \\
\text { mance bias) } \\
\text { All outcomes }\end{array}$ & High risk & Open-label study \\
\hline $\begin{array}{l}\text { Blinding of outcome as- } \\
\text { sessment (detection bias) } \\
\text { All outcomes }\end{array}$ & Unclear risk & Insufficient information to permit judgement \\
\hline $\begin{array}{l}\text { Incomplete outcome data } \\
\text { (attrition bias) } \\
\text { All outcomes }\end{array}$ & Low risk & No missing outcome data \\
\hline $\begin{array}{l}\text { Selective reporting (re- } \\
\text { porting bias) }\end{array}$ & Low risk & Study protocol available and pre-specified outcomes were reported \\
\hline Other bias & Low risk & $\begin{array}{l}\text { No competing interests declared. The study appears to be free of other sources } \\
\text { of bias }\end{array}$ \\
\hline
\end{tabular}

\section{Mehra 2018}

\begin{tabular}{ll}
\hline Methods & Study design: open-label, parallel RCT \\
& - Study timeframe: December 2015 to December 2016 \\
& - Duration of follow-up: 12 months \\
\hline Participants & Country: India \\
- Setting: single centre & Inclusion criteria: diagnosis of SLE (ACR criteria); aged $>16$ years; proteinuria $\geq 500 \mathrm{mg} / 24 \mathrm{~h}$ and $/$ or \\
& urine routine microscopy showing active cellular casts/sediments ( $>5 \mathrm{RBC} / \mathrm{HPF}$ and $>5 \mathrm{WBC} / \mathrm{HPF}$ and \\
& cellular casts); biopsy-proven proliferative class III, IV lupus GN (ISN/RPS) criteria
\end{tabular}


Mehra 2018 (Continued)

- Number (randomised/analysed): treatment group 1 (37/37); treatment group 2 (38/38)

* Treatment group 1: class III (11), class IV (26); had crescents (14; 38\%)

* Treatment group 2: class III (17), class IV (21); had crescents (8; 21\%)

- Mean age \pm SD (years): not reported

- Sex (M/F): treatment group 1 (3/34); treatment group 2 (4/34)

- Exclusion criteria: ever treated previously with IV or oral cyclophosphamide, MMF, cyclosporine or steroids $>15 \mathrm{mg} / \mathrm{d}$ in the last 3 months; renal thrombotic microangiopathy, pre-existing chronic kidney failure, previous malignancy (except skin and cervical intraepithelial neoplasia); DM or coronary heart disease; previously documented severe toxicity to immunosuppressive drugs; patients with active acute or chronic infections; pregnancy

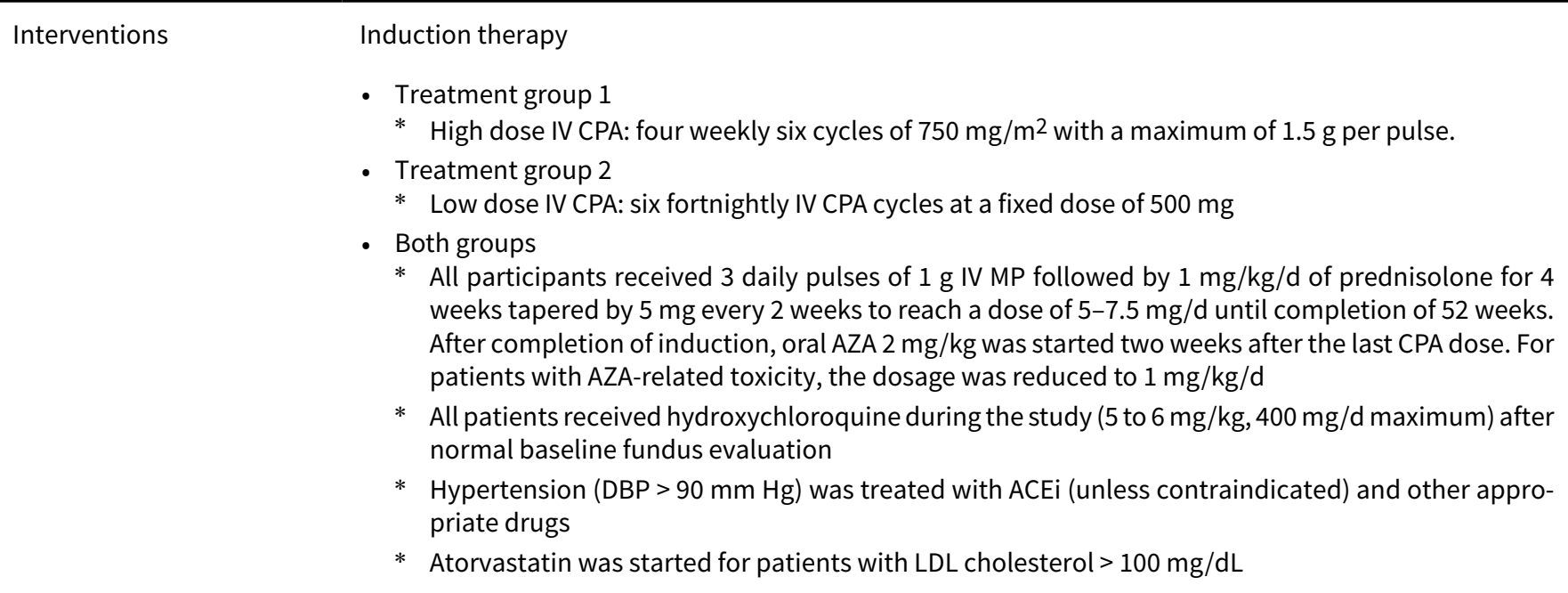

\section{Outcomes}

- Death

- Complete remission: UPCR $<0.5 \mathrm{~g}$ and normal GFR $(>90 \mathrm{~mL} / \mathrm{min}$ ) or stable $(<10 \%$ deterioration from baseline if GFR was previously abnormal) kidney function and inactive urinary sediments.

- Partial remission: $\geq 50 \%$ reduction in proteinuria to sub-nephrotic levels, normal GFR (>90 mL/ min) or stable $(<10 \%)$ deterioration from baseline if GFR was previously abnormal

- Renal relapse (not defined)

- Treatment failure

- Major infection

- Herpes zoster virus infection

- Ovarian failure

- Bone toxicity: avascular necrosis

- Alopecia

- Leucopenia

- GI disturbance

- $\mathrm{CrCl}$

\section{Risk of bias}

\section{Bias} Authors' judgement Support for judgement

Random sequence genera- Low risk tion (selection bias)

"Patients were randomised, using block randomization, eight blocks of 10 patients each with 1:1 random allocation was performed using a computer generated random number table." 
Mehra 2018 (Continued)

Allocation concealmen (selection bias)

Low risk

"Fellow researcher had given random block and number to patients sequentially, who was unaware of treatment allocation and had no other role in the study."

Blinding of participants High risk
and personnel (perfor-
mance bias)

Blinding of outcome as-

Unclear risk

Insufficient information to permit judgement sessment (detection bias) All outcomes

Incomplete outcome data Low risk No missing outcome data
(attrition bias)
All outcomes

\begin{tabular}{|c|c|c|}
\hline $\begin{array}{l}\text { Selective reporting (re- } \\
\text { porting bias) }\end{array}$ & High risk & $\begin{array}{l}\text { Not all expected outcomes were reported and partial remission listed in proto- } \\
\text { col not reported. }\end{array}$ \\
\hline
\end{tabular}

Other bias Low risk The study appears to be free of other sources of bias

Mendonca 2017

\begin{tabular}{ll}
\hline Methods & Study design: open-label, parallel RCT \\
- & Study timeframe: November 2014 to November 2015 \\
- & Duration of follow-up: 6 months \\
\hline Participants & Country: India \\
- Setting: single centre \\
- Inclusion criteria: SLE according to the SLICC 2012 and the ACR criteria; all biopsy-proven class III, IV \\
or III/IV +V lupus nephritis was diagnosed based on biopsy findings as per the ISN/RPS \\
Number (randomised/analysed): treatment group 1 (18/17); treatment group 2 (23/23) \\
* Treatment group 1: class III (1); class IV (11); class V (2); class III+V or class IV+V (3) \\
* Treatment group 2: class III (1); class IV (15); class V (3); class III+V or class IV+V (4) \\
- Mean age \pm SD (years): treatment group 1 (26.0 \pm 10.8$) ;$ treatment group 2 (25.7 \pm 10.3$)$ \\
- Sex (M/F): treatment group 1 (3/14); treatment group 2 (5/18) \\
- Exclusion criteria: CKD stage-3 and above; crescentic lupus nephritis; pancreatitis, GI haemorrhage \\
within six months or active peptic ulcer disease within last three months; ongoing infection; bone \\
marrow insufficiency with cytopenias not attributable to SLE; and prior treatment with CPA or MM
\end{tabular}

- Treatment group 1

* Oral MMF: twice daily, titrated from $750 \mathrm{mg}$ twice daily in the 1st week, and $1.0 \mathrm{~g}$ twice daily in the 2 nd week, to a target dosage of $1.5 \mathrm{~g}$ twice daily, if required, based on the disease activity and response. Reduction was permitted to $2 \mathrm{~g} / \mathrm{d}$ in response to any adverse events

- Treatment group 2

* Low dose IV CPA: Pulse CPA ( $750 \mathrm{mg} / \mathrm{m}^{2}$ ), which was adjusted to 500 to $1000 \mathrm{mg} / \mathrm{m}^{2}$ every 4 weeks to maintain a nadir leukocyte count of 2.5 to $4.0 \times 109 / \mathrm{L}$ for a total of 6 pulses. A $25 \%$ decrease in dosage for age older than 60 years, and $\mathrm{SCr}>3.4 \mathrm{mg} / \mathrm{dL}$ was followed

- Both groups

* All participants had received unified concomitant corticosteroid therapy according to protocol that consisted of three doses of IV pulse MP $500 \mathrm{mg}$ followed by oral prednisone (or equivalent) at an initial dose of $0.5 \mathrm{mg} / \mathrm{kg} / \mathrm{d}$. Prednisolone dosage was tapered by a decrease of $5 \mathrm{mg} / \mathrm{d}$ every 
two weeks until a dose of $10 \mathrm{mg} /$ day was achieved, and this dosage was maintained till the end of six months.

* Doses of ACEi and/or ARB had been unchanged during the 6 month follow-up period

* Target blood pressure was kept at $130 / 80 \mathrm{~mm} \mathrm{Hg}$

* Hyperlipidaemia was treated using statins and/or fibric acid derivatives as required

\begin{tabular}{ll}
\hline Outcomes & Death \\
- & Complete remission: urinary protein excretion $<0.3 \mathrm{~g} / 24 \mathrm{~h}$ was accomplished with normal serum al- \\
& bumin levels and/or an improvement in the baseline $\mathrm{SCr}$ levels of $>50 \%$ \\
- Partial remission: improvement of $>50 \%$ from baseline proteinuria, serum albumin levels of at least & $30 \mathrm{~g} / \mathrm{L}$, and $\mathrm{SCr}$ level of $\geq 25 \%$ from baseline or stable $\mathrm{SCr}$ level within $25 \%$ of the baseline \\
- Treatment failure & - Major infection \\
- Herpes zoster virus infection \\
- Diarrhoea \\
- Nausea \\
- Vomiting \\
- CrCl \\
- SCr \\
- Daily proteinuria
\end{tabular}

\section{Risk of bias}

\begin{tabular}{lll}
\hline Bias & Authors' judgement & Support for judgement \\
\hline $\begin{array}{l}\text { Random sequence genera- } \\
\text { tion (selection bias) }\end{array}$ & Unclear risk & $\begin{array}{l}\text { Study was described as randomised, method of randomisation was not report- } \\
\text { ed }\end{array}$ \\
\hline $\begin{array}{l}\text { Allocation concealment } \\
\text { (selection bias) }\end{array}$ & Unclear risk & Insufficient information to permit judgement \\
\hline $\begin{array}{l}\text { Blinding of participants } \\
\text { and personnel (perfor- } \\
\text { mance bias) }\end{array}$ & High risk & Open-label study \\
All outcomes & & \\
\hline
\end{tabular}

Blinding of outcome as- Unclear risk Insufficient information to permit judgement
sessment (detection bias)

All outcomes

Incomplete outcome data Low risk No missing outcome data

(attrition bias)

All outcomes

Selective reporting (re- High risk No protocol available, some expected outcomes not reported
porting bias)

Other bias Low risk The study appears to be free of other sources of bias

Mitwalli 2011

Methods - Study design: double-blind, parallel RCT


Mitwalli 2011 (Continued)

- Study timeframe: December 1997 to January 2007

- Duration of follow-up: mean follow-up $6.77 \pm 3.3$ years

\begin{tabular}{ll}
\hline Participants & Country: Saudi Arabia \\
- Setting: single centre \\
- Inclusion criteria: adult patients with newly diagnosed biopsy-proven lupus nephritis (WHO class IV) \\
- Number (randomised/analysed): treatment group 1 (73/73); treatment group 2 (44/44) \\
- Mean age \pm SD (years): treatment group 1 (36.4 \pm 12.7$)$; treatment group $2(30.3 \pm 10.4)$ \\
- Sex (M/F): treatment group 1 (12/61); treatment group 2 (5/39) \\
- Exclusion criteria: not reported
\end{tabular}

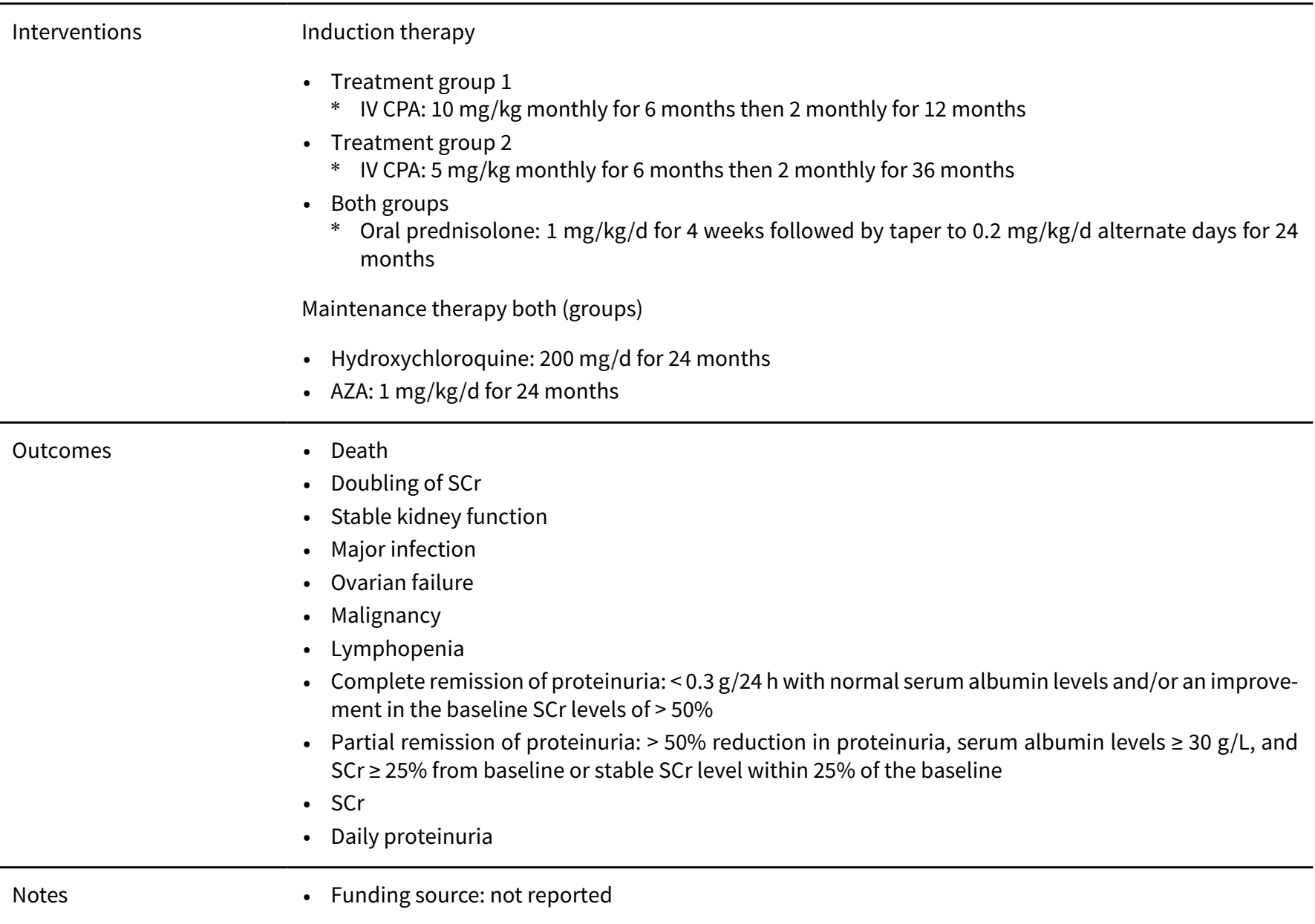

\section{Risk of bias}

\begin{tabular}{lll}
\hline Bias & Authors' judgement & Support for judgement \\
\hline $\begin{array}{l}\text { Random sequence genera- } \\
\text { tion (selection bias) }\end{array}$ & Unclear risk & $\begin{array}{l}\text { Study was described as randomised, method of randomisation was not report- } \\
\text { ed }\end{array}$ \\
\hline $\begin{array}{l}\text { Allocation concealment } \\
\text { (selection bias) }\end{array}$ & Unclear risk & Insufficient information to permit judgement \\
\hline $\begin{array}{l}\text { Blinding of participants } \\
\text { and personnel (perfor- } \\
\text { mance bias) }\end{array}$ & Low risk & Double-blind study \\
All outcomes & \\
\hline
\end{tabular}


Mitwalli 2011 (Continued)

Blinding of outcome as- Low risk Outcome assessor was blinded according to the protocol sessment (detection bias)

All outcomes

\begin{tabular}{lll}
$\begin{array}{l}\text { Incomplete outcome data } \\
\text { (attrition bias) } \\
\text { All outcomes }\end{array}$ & Low risk & No missing outcome data \\
\hline $\begin{array}{l}\text { Selective reporting (re- } \\
\text { porting bias) }\end{array}$ & Low risk & All outcomes on clinicaltrials.gov are reported \\
\hline Other bias & High risk & $\begin{array}{l}\text { Marked differences in clinical characteristics between the groups - median cu- } \\
\text { mulative dose of CPA between the groups, high rates of leucopenia in the low } \\
\text { dose compared to the high dose CPA group at baseline }\end{array}$
\end{tabular}

Mok 2016

\begin{tabular}{|c|c|}
\hline Methods & $\begin{array}{l}\text { - Study design: open-label, parallel RCT } \\
\text { - Study timeframe: } 2005 \text { to } 2012 \\
\text { - Duration of follow-up: median } 30 \text { months }\end{array}$ \\
\hline Participants & $\begin{array}{l}\text { - Countries: Hong Kong, China } \\
\text { - Setting: multicentre (number of centres not reported) } \\
\text { - Inclusion criteria: aged } \geq 18 \text { years; diagnosis of } \mathrm{SLE}(\mathrm{ACR} \text { criteria); biopsy-proven active lupus class III/ } \\
\text { IV/ V(ISN/RPS } 2003 \text { classification) within } 4 \text { weeks; } \mathrm{SCr}<2.3 \mathrm{mg} / \mathrm{dL} \\
\text { - Number (randomised/analysed): treatment group } 1(74 / 74) \text {; treatment group } 2(76 / 76) \\
\text { - Mean age } \pm \mathrm{SD} \text { (years): treatment group } 1(36.2 \pm 14) \text {; treatment group } 2(36.1 \pm 13.1) \\
\text { - Sex (M/F): treatment group } 1 \text { (4/70); treatment group } 2(8 / 68) \\
\text { - Exclusion criteria: refusal to be randomised; preference for treatment with conventional regimens } \\
\text { such as CPA; planning for pregnancy within } 12 \text { months after randomisation }\end{array}$ \\
\hline
\end{tabular}

- Treatment group 1

* TAC: initial dosage $0.1 \mathrm{mg} / \mathrm{kg} / \mathrm{d}$ in two divided doses, reduced to $0.06 \mathrm{mg} / \mathrm{kg} / \mathrm{d}$ if clinical response was satisfactory at month in two divided doses for 6 months

- Treatment group 2

* MMF: $2 \mathrm{~g} / \mathrm{d}$ initially, augmented to up to $3 \mathrm{~g} / \mathrm{d}$ if clinical response was suboptimal in two divided doses for 6 months

- Both groups

* Prednisolone: $0.6 \mathrm{mg} / \mathrm{kg} / \mathrm{d}$ for 6 weeks then tapered by $5 \mathrm{mg} / \mathrm{d}$ every week to $<10 \mathrm{mg} / \mathrm{d}$. At end of intervention, if complete clinical response or good partial response, changed to AZA $(2 \mathrm{mg} / \mathrm{kg} / \mathrm{d})$ for maintenance. Poor responders re-induced with oral CPA $2 \mathrm{mg} / \mathrm{kg} / \mathrm{d}$

\begin{tabular}{ll}
\hline Outcomes & Death \\
& - ESKD \\
& - Doubling of $\mathrm{SCr}$ \\
& - Stable kidney function \\
- Relapse & - Major infection \\
& - Herpes zoster virus \\
& - Diarrhoea \\
& - Nausea
\end{tabular}


- Complete renal remission: stabilisation (within 25\%) or improvement in SCr with reduction of proteinuria to $<1 \mathrm{~g} / \mathrm{d}$ (or UPCR $<1.0$ ), resolution of urinary sediment abnormalities (urine RBC $<5 / \mathrm{HPF}$ and absence of cellular casts) and persistent improvement in C3 and anti-dsDNA levels

- Partial renal remission: stabilisation (within 25\%) or improvement in $\mathrm{SCr}$ with persistent reduction of proteinuria (if nephrotic range at baseline, a $\geq 50 \%$ decrease in proteinuria but $<3 \mathrm{~g} / \mathrm{d}$ (or UPCR $<3.0$ ); if non-nephrotic at baseline, a decrease to $\leq 50 \%$ of the pre-treatment value but $>1 \mathrm{~g} / \mathrm{d}$ (or UPCR $>$ 1.0 ) and improvement in urinary sediment abnormalities ( $\geq 50 \%$ reduction in haematuria and urine $\mathrm{RBC}<10 / \mathrm{HPF}$ )

- Treatment failure: deterioration of $\mathrm{SCr}(>25 \%)$, an increase in proteinuria, or a reduction in proteinuria but not to the extent of complete renal remission or partial renal remission)

- Renal flare: proteinuric flare - an increase in proteinuria to more than $2 \mathrm{~g} / \mathrm{d}$ (or UPCR >2.0), with or without deterioration in $\mathrm{SCr}(<30 \%)$, after a complete remission; or doubling of proteinuria (or UPCR), with or without deterioration in $\mathrm{SCr}(<30 \%)$, in patients who achieved partial remission. Nephrotic flare - an increase or recurrence of active urinary sediments (RBC $\geq 10 / \mathrm{HPF}$ or active cellular casts) with a concomitant increase in proteinuria (or UPCR) or deterioration in $\mathrm{SCr}(\geq 30 \%)$ after excluding other causes (e.g. sepsis, over diuresis, nephrotoxic agents, renal vein thrombosis)

- Alopecia

- Proteinuria

- $\mathrm{CrCl}$

- Serum albumin

Notes - Funding source: no support from any organisation including industry (Roche and Astella)

\section{Risk of bias}

\begin{tabular}{lll}
\hline Bias & Authors' judgement & Support for judgement \\
\hline $\begin{array}{l}\text { Random sequence genera- } \\
\text { tion (selection bias) }\end{array}$ & Low risk & $\begin{array}{l}\text { Participants were randomised by computer-generated blocks of four in a 1:1 } \\
\text { ratio }\end{array}$ \\
\hline $\begin{array}{l}\text { Allocation concealment } \\
\text { (selection bias) }\end{array}$ & Unclear risk & Central research assistant was responsible for treatment allocation \\
\hline $\begin{array}{l}\text { Blinding of participants } \\
\text { and personnel (perfor- } \\
\text { mance bias) } \\
\text { All outcomes }\end{array}$ & High risk & Open-label study \\
\hline
\end{tabular}

Blinding of outcome as- Unclear risk Insufficient information to permit judgement

sessment (detection bias)

All outcomes

Incomplete outcome data Low risk No missing outcome data
(attrition bias)

All outcomes

Selective reporting (re- Low risk Study protocol available and pre-specified outcomes were reported porting bias)

Other bias Low risk The study appears to be free of other sources of bias

Moroni 2006

$\begin{array}{ll}\text { Methods } & \text { - Study design: open label, parallel RCT } \\ \text { - Study timeframe (recruitment): March } 1999 \text { to March } 2001\end{array}$


Moroni 2006 (Continued)

- Duration of follow-up: a least 1 year follow-up, invited to continue to 4 years

\begin{tabular}{|c|c|}
\hline Participants & $\begin{array}{l}\text { - Country: Italy } \\
\text { - Setting: multicentre } \\
\text { - Inclusion criteria: aged at least } 16 \text { years; diagnosis of } \mathrm{SLE} \text { (ACR criteria) and biopsy-proven class IV, Vc } \\
\text { or Vd lupus nephritis with a chronicity index of } \leq 4 \text { (WHO classification); patients with a new diagnosis } \\
\text { of lupus nephritis or were experiencing a new flare of a previously quiescent disease were enrolled } \\
\text { if they had active urine sediment ( } \geq 5 \mathrm{RBC} / \mathrm{HPF} \text { ); proteinuria }>1 \mathrm{~g} / \mathrm{d} \text { in case of new diagnosis or }>2 \\
\mathrm{~g} \text { if new renal flare; } \mathrm{SCr}<4 \mathrm{mg} / \mathrm{dL} \text {; after induction therapy those with no major extrarenal signs or } \\
\text { symptoms of lupus requiring aggressive therapy; } \mathrm{SCr} \leq 1.5 \mathrm{mg} / \mathrm{dL} \text {, proteinuria }>0.5 \mathrm{~g} / \mathrm{d} \text {; } \mathrm{CrCl}>60 \mathrm{~mL} / \\
\text { min; diastolic } \mathrm{BP}<90 \mathrm{~mm} \mathrm{Hg} \text { with a maximum of two antihypertensive drugs and the oral prednisone } \\
\text { dose } \leq 0.5 \mathrm{mg} / \mathrm{kg} / \mathrm{d} \\
\text { - Number (randomised/analysed): treatment group } 1(36 / 36) \text {; treatment group } 2(33 / 33) \\
\text { - Mean age } \pm \mathrm{SD} \text { (years): treatment group } 1 \text { ( } 31.7 \pm 9.1) \text {; treatment group } 2(31.2 \pm 11.7) \\
\text { - Sex (M/F): treatment group } 1 \text { (3/33); treatment group } 2 \text { (4/29) } \\
\text { - Exclusion criteria: potential silent nephritis; renal diseases unrelated to } \mathrm{SLE} \text {; treatment with CSA or } \\
\text { AZA in the } 6 \text { months preceding the screening visit; cumulative CPA dose }>200 \mathrm{mg} / \mathrm{kg} \text {; any contraindi- } \\
\text { cation to the study drugs; previous malignancy }\end{array}$ \\
\hline Interventions & $\begin{array}{l}\text { Maintenance therapy: duration of therapy was } 24 \text { months } \\
\text { - Treatment group } 1 \\
\text { * CSA: } 4 \mathrm{mg} / \mathrm{kg} / \mathrm{d} \text { and reduced to maintenance dose }(2.5 \text { to } 3.0 \mathrm{mg} / \mathrm{kg} / \mathrm{d}) \text { if proteinuria }<1 \mathrm{~g} / \mathrm{d} \text {, if } \\
\text { proteinuria was higher the dose was reduced more slowly } \\
\text { - Treatment group } 2 \\
\text { * AZA: } 2 \mathrm{mg} / \mathrm{kg} / \mathrm{d} \text { optional reduction at } 1 \text { month to } 1.5 \mathrm{mg} / \mathrm{kg} / \mathrm{d} \text { if proteinuria }<1 \mathrm{~g} / \mathrm{d} \text { and SCr stable } \\
\text { - Both groups } \\
\text { * Induction therapy: } 3 \times \text { IV MP } 0.5 \mathrm{~g} \text { if } \leq 50 \mathrm{~kg} \text { and } 1 \mathrm{~g} \text { if }>50 \mathrm{~kg} \text {. followed by prednisolone } 1 \mathrm{mg} / \mathrm{kg} / \\
\text { d for } 10 \text { to } 15 \text { days then tapered } \\
\text { * During maintenance therapy both groups received oral prednisone which had to be reduced from } \\
0.5 \text { to } 0.2 \mathrm{mg} / \mathrm{kg} / \mathrm{d} \text { by the end of the } 6 \text { months, in the case of normal levels of } \mathrm{SCr} \text { and proteinuria } \\
\text { of }<0.5 \mathrm{~g} / \mathrm{d} \text { and in absence of extrarenal symptoms. A further reduction or complete withdrawal } \\
\text { could be attempted at the investigators discretion }\end{array}$ \\
\hline
\end{tabular}

\begin{tabular}{ll}
\hline Outcomes & 1. Death \\
2. ESKD \\
3. Major infection \\
4. Lymphopenia \\
5. Gl disorders \\
6. Complete remission proteinuria \\
7. Proteinuria at 2 and 4 years \\
8. CrCl at 2 and 4 years \\
9. 24 hour proteinuria \\
10.Renal flare
\end{tabular}

Notes

- Funding source: educational grant from Novartis Pharma AG

\section{Risk of bias}

\begin{tabular}{lll}
\hline Bias & Authors' judgement & Support for judgement \\
\hline $\begin{array}{l}\text { Random sequence genera- } \\
\text { tion (selection bias) }\end{array}$ & Low risk & Randomisation according to a coin-based design \\
\hline $\begin{array}{l}\text { Allocation concealment } \\
\text { (selection bias) }\end{array}$ & Low risk & $\begin{array}{l}\text { Stratified by centre and performed centrally. Phone calls to randomisation } \\
\text { centre-computer program assigned participants }\end{array}$ \\
\hline
\end{tabular}


Moroni 2006 (Continued)

Blinding of participants High risk $\quad$ Open-label study
and personnel (perfor-
mance bias)

All outcomes

Blinding of outcome as-
sessment (detection bias) $\quad$ Low risk Blinded endpoint study

All outcomes

Incomplete outcome data Low risk No missing outcome data
(attrition bias)

All outcomes

\begin{tabular}{lll}
\hline $\begin{array}{l}\text { Selective reporting (re- } \\
\text { porting bias) }\end{array}$ & Low risk & Study protocol available and pre-specified outcomes were reported \\
\hline Other bias & High risk & $\begin{array}{l}\text { Sponsor included in data management and analysis: Novartis Pharma and au- } \\
\text { thorship }\end{array}$ \\
\hline
\end{tabular}

\section{Mulic-Bacic 2008}

\begin{tabular}{ll} 
Methods & Study design: open-label, parallel RCT \\
& - Study timeframe: not reported \\
\hline Participants & - Country: Bosnia Herzegovina \\
- Setting: not reported \\
- Inclusion criteria: active lupus nephritis class III, IV or V (WHO classification criteria) \\
- Number (randomised/analysed): treatment group 1 (20/20); treatment group 2 (25/25) \\
- Mean age \pm SD (years): not reported \\
- Sex (M/F): not reported \\
- Exclusion criteria: not reported
\end{tabular}

\begin{tabular}{ll}
\hline Interventions & Induction therapy: duration of therapy was 24 months \\
- Treatment group 1 & $*$ MMF: $2 \mathrm{~g} / \mathrm{d}$ for 6 months then $1 \mathrm{~g} / \mathrm{d}$ for 18 months, administer orally \\
- Treatment group 2 & $*$ IV CPA: $0.5 \mathrm{~g} / \mathrm{m}^{2}$ monthly \\
- Both groups & $*$ Prednisolone: 0.75 to $1 \mathrm{mg} / \mathrm{kg} / \mathrm{d}$ with determined tapering \\
\hline Outcomes & Death \\
- Stable kidney function \\
- Complete remission proteinuria \\
- Partial remission proteinuria \\
- Complete remission: normalisation of abnormal renal measurements and maintenance of baseline \\
- Partial remission \\
\hline - Abstract-only publication \\
- Funding source: not reported
\end{tabular}


Mulic-Bacic 2008 (Continued)

Risk of bias

\begin{tabular}{lll}
\hline Bias & Authors' judgement & Support for judgement \\
\hline $\begin{array}{l}\text { Random sequence genera- } \\
\text { tion (selection bias) }\end{array}$ & Unclear risk & $\begin{array}{l}\text { Study was described as randomised, method of randomisation was not report- } \\
\text { ed }\end{array}$ \\
\hline $\begin{array}{l}\text { Allocation concealment } \\
\text { (selection bias) }\end{array}$ & Unclear risk & Insufficient information to permit judgement \\
\hline $\begin{array}{l}\text { Blinding of participants } \\
\text { and personnel (perfor- } \\
\text { mance bias) }\end{array}$ & High risk & Open-label study \\
All outcomes & & \\
\hline
\end{tabular}

Blinding of outcome as- Unclear risk Insufficient information to permit judgement

sessment (detection bias)

All outcomes

Incomplete outcome data Low risk No missing outcome data
(attrition bias)

All outcomes

\begin{tabular}{lll}
\hline $\begin{array}{l}\text { Selective reporting (re- } \\
\text { porting bias) }\end{array}$ & High risk & $\begin{array}{l}\text { Not all expected clinical outcomes reported and no protocol available; ab- } \\
\text { stract-only publication }\end{array}$
\end{tabular}

Other bias Unclear risk Abstract-only publication; insufficient information to permit judgement

\section{MyLupus 2011}

\begin{tabular}{|c|c|}
\hline Methods & $\begin{array}{l}\text { - Study design: open-label, parallel RCT } \\
\text { - Study timeframe: February } 2007 \text { to November } 2009 \\
\text { - Duration of follow-up: } 6 \text { months }\end{array}$ \\
\hline Participants & $\begin{array}{l}\text { - Countries: France, Germany, Italy, Spain, UK, Hungary, Greece, Colombia, Taiwan } \\
\text { - Setting: multinational (19 sites) } \\
\text { - Inclusion criteria: aged } \geq 18 \text { years, (i) diagnosis of SLE (ACR criteria); biopsy-proven (within previous } 24 \\
\text { months) proliferative lupus nephritis (class III or IV) (ISN/RPS } 2003 \text { classification criteria); proteinuria } \\
\text { defined as UPCR > } 0.5 \text { at screening and baseline; and clinical activity defined by one or more of the } \\
\text { following: } \mathrm{SCr}>1 \mathrm{mg} / \mathrm{dL} \text {; microscopic haematuria (> } 5 \mathrm{RBC} / \mathrm{HPF} \text { ) and presence of cellular casts } \\
\text { - Number (randomised/analysed): treatment group } 1(42 / 42) \text {; treatment group } 2(39 / 39) \\
\text { - Mean age } \pm \mathrm{SD} \text { (years): treatment group } 1 \text { ( } 32.2 \pm 8.5 \text { ); treatment group } 2(34.2 \pm 10.7) \\
\text { - Sex (M/F): treatment group } 1(5 / 37) \text {; treatment group } 2(10 / 29) \\
\text { - Exclusion criteria: } \mathrm{CrCl}<30 \mathrm{~mL} / \mathrm{min} \text {; IV glucocorticoids, oral or IV CPA or MMF during the previous } 3 \\
\text { months; antibody therapy within the previous } 6 \text { months }\end{array}$ \\
\hline
\end{tabular}

Interventions

Induction therapy: duration of therapy was 6 months

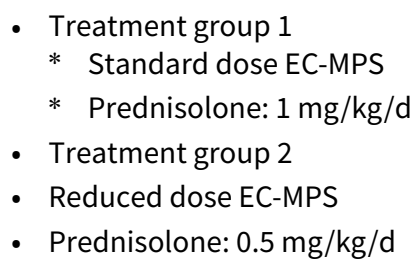


MyLupus 2011 (Continued)

- Both groups

* MP: $0.5 \mathrm{~g}$ IV/d for 3 days

* EC-MPS started at $1440 \mathrm{mg} / \mathrm{d}$ for first 2 weeks then $2160 \mathrm{mg}$ in remaining 22 weeks

* Prednisolone tapered in both groups according to guidelines

\begin{tabular}{ll}
\hline Outcomes & Death \\
- & Infection \\
- & Herpes zoster virus infection \\
- & Vomiting \\
- & Diarrhoea \\
- & Partial remission: reduction in UPCR of $50 \%$ compared with baseline, and SCr improved or stable (i.e. \\
& within $10 \%$ of baseline value) \\
- Renal flare: A mild SLE flare was diagnosed if SLE increased after partial or complete response, defined & as the presence of 1 or 2 BILAG B scores and no A scores and intention by the investigator to increase \\
& the glucocorticoid dose; a moderate to severe SLE flare was diagnosed if increased lupus activity after \\
& partial or complete response resulted in 1 BILAG A score or 3 BILAG B scores \\
- UPCR & Creatinine \\
- & Funding source: Novartis Pharma AG
\end{tabular}

\section{Risk of bias}

\begin{tabular}{lll}
\hline Bias & Authors' judgement & Support for judgement \\
\hline $\begin{array}{l}\text { Random sequence genera- } \\
\text { tion (selection bias) }\end{array}$ & Unclear risk & $\begin{array}{l}\text { Study was described as randomised, method of randomisation was not report- } \\
\text { ed }\end{array}$ \\
\hline $\begin{array}{l}\text { Allocation concealment } \\
\text { (selection bias) }\end{array}$ & High risk & Insufficient information to permit judgement \\
\hline $\begin{array}{l}\text { Blinding of participants } \\
\text { and personnel (perfor- } \\
\text { mance bias) } \\
\text { All outcomes }\end{array}$ & High risk & Open-label study \\
\hline
\end{tabular}

\begin{tabular}{|c|c|c|}
\hline $\begin{array}{l}\text { Blinding of outcome as- } \\
\text { sessment (detection bias) } \\
\text { All outcomes }\end{array}$ & Unclear risk & Insufficient information to permit judgement \\
\hline
\end{tabular}

\begin{tabular}{lll}
\hline $\begin{array}{l}\text { Incomplete outcome data } \\
\text { (attrition bias) } \\
\text { All outcomes }\end{array}$ & Low risk & No missing outcome data \\
\hline $\begin{array}{l}\text { Selective reporting (re- } \\
\text { porting bias) }\end{array}$ & High risk & Not all expected outcomes were reported \\
\hline Other bias & High risk & $\begin{array}{l}\text { Novartis Pharma AG funded. Sponsor involved in authorship, Disclosure of } \\
\text { consulting fees from Novartis Pharma, Amgen, BMS and Roche }\end{array}$ \\
\hline
\end{tabular}

\section{Nakamura 2002e}

\begin{tabular}{ll}
\hline Methods & Study design: open-label, parallel RCT \\
& - Study timeframe: not reported \\
\hline
\end{tabular}


Nakamura 2002e (Continued)

- Duration of follow-up: 6 months

\begin{tabular}{ll}
\hline Participants & Country: Japan \\
- Setting: not reported \\
- Inclusion criteria: diagnosis of SLE (ACR criteria); biopsy-proven diffuse proliferative, class IV lupus \\
nephritis (WHO classification criteria); oral corticosteroid with or without cytotoxic drugs for at least \\
6 months with treatment resistance \\
- Number (randomised): treatment group 1 (10); treatment group 2 (10) \\
- Mean age (years): treatment group 1 (30.5); treatment group $2(29.5)$ \\
- Sex (M/F): treatment group $1(2 / 8) ;$ treatment group $2(2 / 8)$ \\
- Exclusion criteria: not reported
\end{tabular}

Interventions

Induction therapy: duration of therapy was 6 months

- Treatment group 1

* PEX: double filtration 1 to 2 weekly

- Treatment group 2

* IV CPA: 0.75 to $1.0 \mathrm{~g} / \mathrm{m}^{2}$ once a month for 6 months

- Both groups

- Oral prednisone (or equivalent): $1 \mathrm{mg} / \mathrm{kg} / \mathrm{d}$ tapered to the minimum dose needed to control extrarenal diseases

\begin{tabular}{ll}
\hline Outcomes & Proteinuria \\
& $\cdot$ Urinary podocyte number \\
\hline Notes & Funding source: not reported \\
\hline
\end{tabular}

\section{Risk of bias}

\begin{tabular}{lll}
\hline Bias & Authors' judgement & Support for judgement \\
\hline $\begin{array}{l}\text { Random sequence genera- } \\
\text { tion (selection bias) }\end{array}$ & Unclear risk & $\begin{array}{l}\text { Study was described as randomised, method of randomisation was not report- } \\
\text { ed }\end{array}$ \\
\hline $\begin{array}{l}\text { Allocation concealment } \\
\text { (selection bias) }\end{array}$ & Unclear risk & Insufficient information to permit judgement \\
\hline $\begin{array}{l}\text { Blinding of participants } \\
\text { and personnel (perfor- } \\
\text { mance bias) }\end{array}$ & High risk & Open-label study \\
All outcomes & & \\
\hline
\end{tabular}

Blinding of outcome as- Unclear risk Insufficient information to permit judgement
sessment (detection bias)

All outcomes

\begin{tabular}{lll}
\hline $\begin{array}{l}\text { Incomplete outcome data } \\
\text { (attrition bias) } \\
\text { All outcomes }\end{array}$ & Unclear risk & Insufficient information to permit judgement \\
\hline $\begin{array}{l}\text { Selective reporting (re- } \\
\text { porting bias) }\end{array}$ & High risk & Not all expected outcomes were reported \\
\hline Other bias & Low risk & The study appears to be free of other sources of bias \\
\hline
\end{tabular}


Ong 2005

\begin{tabular}{|c|c|}
\hline Methods & $\begin{array}{l}\text { - Study design: open-label, parallel RCT } \\
\text { - Study timeframe: January } 2001 \text { to December } 2002 \\
\text { - Duration of follow-up: } 6 \text { months }\end{array}$ \\
\hline Participants & $\begin{array}{l}\text { - Country: Malaysia } \\
\text { - Setting: multicentre ( } 8 \text { sites) } \\
\text { - Inclusion criteria: aged > } 16 \text { years; diagnosis of SLE (ACR criteria); class III or IV lupus nephritis (WHO } \\
\text { classification criteria) } \\
\text { - Number (randomised/analysed): treatment group } 1(28 / 25) \text {; treatment group } 2(26 / 19) \\
\text { - Mean age } \pm \text { SD (years): treatment group } 1(30.5 \pm 8.7) \text {; treatment group } 2(31.3 \pm 9.9) \\
\text { - Sex (M/F): treatment group } 1(3 / 23) \text {; treatment group } 2(4 / 15) \\
\text { - Exclusion criteria: SCr > } 200 \mu \mathrm{mol} / \mathrm{L}, \mathrm{WCC}<3.5 \times 109 / \mathrm{L} \text {; major infection; history of cancer; alcohol or } \\
\text { substance misuse; pregnancy; active peptic ulcer disease; allergy to MMF or CPA; use of study drugs } \\
\text { in preceding } 6 \text { months }\end{array}$ \\
\hline
\end{tabular}

Interventions Induction therapy: duration of therapy was 6 months

- Treatment group 1

* IV CPA: 0.75 to $1 \mathrm{~g} / \mathrm{m}^{2}$ monthly for 6 months

- Treatment group 2

* MMF: $1 \mathrm{~g}$ orally twice daily for 6 months

- Both groups

* Prednisolone: $60 \mathrm{mg} / \mathrm{d}$ for 4 to 6 weeks then tapering dose to 5 to $10 \mathrm{mg} / \mathrm{d}$

\begin{tabular}{ll}
\hline Outcomes & Death \\
- ESKD & Stable kidney function \\
- Major infection \\
- Herpes zoster virus \\
- Leucopenia $(<3.5 \times 109 / \mathrm{L})$ \\
- Oligomenorrhoea \\
- Gl side effects \\
- Complete renal remission: stabilisation or improvement in kidney function, RCC $<10$, proteinuria $<3 \mathrm{~g}$ \\
- Combined partial remission: stabilisation or improvement in kidney function, RCC $<10$, proteinuria $<$ \\
- 3 g if was $>3$ g or at least $50 \%$ reduction or $<1.0$ g if subnephrotic \\
\hline - Funding source: not reported; MMF supplied by Roche Malaysia
\end{tabular}

Risk of bias

\begin{tabular}{lll}
\hline Bias & Authors' judgement & Support for judgement \\
\hline $\begin{array}{l}\text { Random sequence genera- } \\
\text { tion (selection bias) }\end{array}$ & Low risk & $\begin{array}{l}\text { Randomisation code generated separately for each centre using random per- } \\
\text { mutated block method with randomly varying block size (1:1) }\end{array}$ \\
\hline $\begin{array}{l}\text { Allocation concealment } \\
\text { (selection bias) }\end{array}$ & Low risk & Randomisation performed centrally \\
\hline $\begin{array}{l}\text { Blinding of participants } \\
\begin{array}{l}\text { and personnel (perfor- } \\
\text { mance bias) } \\
\text { All outcomes }\end{array}\end{array}$ & High risk & Open-label study \\
\hline
\end{tabular}


Ong 2005 (Continued)

Blinding of outcome as- Unclear risk Insufficient information to permit judgement sessment (detection bias)

All outcomes

Incomplete outcome data Low risk No missing outcome data
(attrition bias)

All outcomes

Selective reporting (re- Low risk Study protocol available and pre-specified outcomes were reported
porting bias)

Other bias Low risk The study appears to be free of other sources of bias

Pal 2017

\begin{tabular}{ll}
\hline Methods & - Study design: open-label, parallel RCT \\
& - Study timeframe: not reported \\
\hline Participants & - Couration of follow-up: not reported \\
- Setting: not reported \\
- Inclusion criteria: lupus nephritis class III and IV or III/IV + V \\
- Number (randomised/analysed): 58 (number per group not reported) \\
- Mean age \pm SD (years): not reported \\
- Sex (M/F): not reported \\
- Exclusion criteria: not reported
\end{tabular}

Interventions

Induction therapy: duration of therapy not reported

- Treatment group 1

* Oral TAC: $0.75 \mathrm{mg} / \mathrm{kg}$

* Oral AZA: $2 \mathrm{mg} / \mathrm{kg}$

- Treatment group 2

* IV CPA: $500 \mathrm{mg} / \mathrm{m}^{2}$ monthly

- Both groups

* MP: 3 pulsed doses and subsequently, prednisolone was given at doses of $0.5 \mathrm{mg} / \mathrm{kg} / \mathrm{d}$ for the next 1 month and then tapered as tolerated to $10 \mathrm{mg}$ or less by 3 months

\begin{tabular}{ll}
\hline Outcomes & Complete renal remission \\
& - Partial renal remission \\
- Daily proteinuria & Adverse events \\
& - Disease activity \\
\hline Notes & - Abstract-only publication \\
& - Funding source: not reported \\
\hline
\end{tabular}

\section{Risk of bias}

\section{Bias}

Random sequence generation (selection bias)

\section{Authors' judgement Support for judgement}

Unclear risk Study was described as randomised, method of randomisation was not reported 
Pal 2017 (Continued)

\begin{tabular}{l}
$\begin{array}{l}\text { Allocation concealment } \\
\text { (selection bias) }\end{array}$ Unclear risk Insufficient information to permit judgement \\
\hline
\end{tabular}

Blinding of participants High risk Likely to be an open-label study
and personnel (perfor-

\begin{tabular}{ll}
\hline Blinding of outcome as- & Unclear risk
\end{tabular}

(detection bias)

All outcomes

Incomplete outcome data Unclear risk Insufficient information to permit judgement
(attrition bias)

All outcomes

Selective reporting (re- High risk Not all expected outcomes have been reported
porting bias)

\begin{tabular}{ll}
\hline Other bias $\quad$ Unclear risk $\quad$ Abstract-only publication; insufficient information to permit judgement \\
\hline
\end{tabular}

\section{Rathi 2016}

\begin{tabular}{|c|c|}
\hline Methods & $\begin{array}{l}\text { - Study design: open-label, proof-of-concept RCT } \\
\text { - Study timeframe: not reported } \\
\text { - Duration of follow-up: } 6 \text { months }\end{array}$ \\
\hline Participants & $\begin{array}{l}\text { - Country: India } \\
\text { - Setting: single centre } \\
\text { - Inclusion criteria: aged } 12 \text { to } 65 \text { years; diagnosis of SLE (ACR criteria); biopsy-proven class III, IV, V, III } \\
+\mathrm{V} \text {, or IV+V lupus nephritis (ISN/RPS } 2003 \text { classification criteria) } \\
\text { - Number (randomised/analysed): treatment group } 1(50 / 50) \text {; treatment group } 2(50 / 50) \\
\text { - Mean age } \pm \text { SD (years): treatment group } 1 \text { ( } 30.6 \pm 9.5) \text {; treatment group } 2(28.3 \pm 9.5) \\
\text { - Sex (M/F): treatment group } 1 \text { (5/45); treatment group } 2 \text { (3/47) } \\
\text { - Exclusion criteria: crescentic lupus nephritis (> 50\% crescents in biopsy); SCr of > } 265 \mu \mathrm{mol} / \mathrm{L} \text {; neuro- } \\
\text { logical or pulmonary lupus; ongoing infection; pregnancy; prior treatment with CPA or MMF }\end{array}$ \\
\hline
\end{tabular}

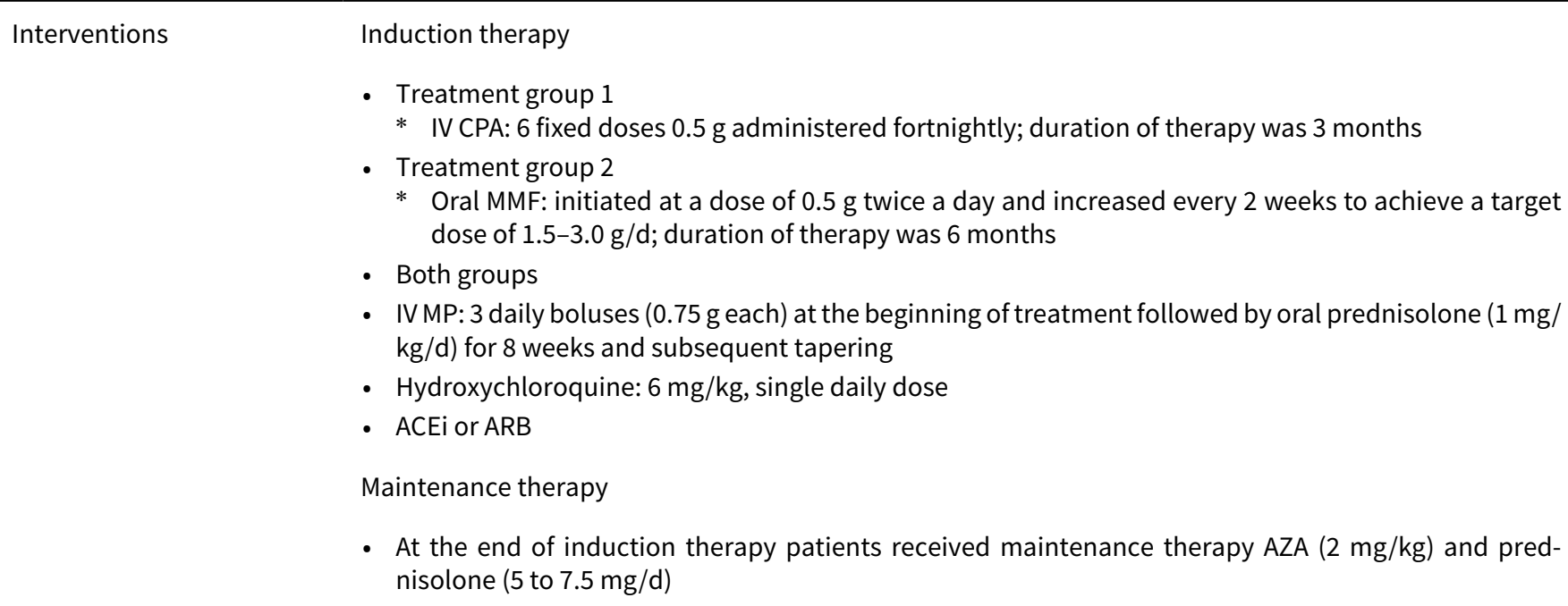

- At the end of induction therapy patients received maintenance therapy AZA ( $2 \mathrm{mg} / \mathrm{kg})$ and prednisolone $(5$ to $7.5 \mathrm{mg} / \mathrm{d}$ ) 
Rathi 2016 (Continued)
Outcomes
- Death
- Complete remission: return to normal $\mathrm{SCr}$ along with proteinuria $\leq 0.5 \mathrm{~g} / \mathrm{d}$ and inactive urine sediment
- Partial remission: defined as treatment response, as a decrease in the UPCR to $<3$ in subjects with a baseline ratio $\geq 3$ or a decrease in UPCR by $\geq 50 \%$ in those with a baseline ratio $<3$, along with stabil- isation or improvement in $\mathrm{SCr}$ (a 24-week SCr level within $25 \%$ of baseline).
- Herpes zoster virus infection
- Ovarian failure
- Alopecia
- Leucopenia

Notes Funding source: not reported

\section{Risk of bias}

\begin{tabular}{lll}
\hline Bias & Authors' judgement & Support for judgement \\
\hline $\begin{array}{l}\text { Random sequence genera- } \\
\text { tion (selection bias) }\end{array}$ & Unclear risk & $\begin{array}{l}\text { Study was described as randomised, method of randomisation was not report- } \\
\text { ed }\end{array}$ \\
\hline $\begin{array}{l}\text { Allocation concealment } \\
\text { (selection bias) }\end{array}$ & Unclear risk & Insufficient information to permit judgement \\
\hline $\begin{array}{l}\text { Blinding of participants } \\
\text { and personnel (perfor- } \\
\text { mance bias) } \\
\text { All outcomes }\end{array}$ & High risk & Open-label study \\
\hline
\end{tabular}

Blinding of outcome as- Unclear risk Insufficient information to permit judgement
sessment (detection bias) All outcomes

\begin{tabular}{lll}
\hline $\begin{array}{l}\text { Incomplete outcome data } \\
\text { (attrition bias) } \\
\text { All outcomes }\end{array}$ & Unclear risk & Insufficient information to permit judgement \\
\hline $\begin{array}{l}\text { Selective reporting (re- } \\
\text { porting bias) }\end{array}$ & Low risk & $\begin{array}{l}\text { Study protocol available from Indian clinical trials registry and pre-specified } \\
\text { outcomes were reported }\end{array}$ \\
\hline Other bias & High risk & $\begin{array}{l}\text { High dropout rate; baseline characteristics different between the two groups } \\
\text { with UPCR significantly higher in the CPA group }\end{array}$ \\
\hline
\end{tabular}

Rovin 2016

\begin{tabular}{ll}
\hline Methods & Study design: double-blind, parallel, proof-of-concept RCT \\
- Study timeframe: not reported \\
- \\
\hline Puration of follow-up: placebo mean 40.1 weeks; sirukumab mean 36.1 weeks \\
\hline - Countries: 6 (countries not reported) \\
- Setting: multinational (18 sites) \\
Inclusion criteria: adults (18 to 70 years); diagnosis of SLE (ACR or SLICC criteria), including seroposi- \\
Class III or IV lupus nephritis (ISN/RPS 2003 classification criteria), and persistently active (proteinuria \\
$>0.5 \mathrm{~g} / \mathrm{d}$ or at least one of the following criteria: haematuria ( $\geq 5$ RBC/HPF), anti-dsDNA-positive test,
\end{tabular}


Rovin 2016 (Continued)

or C3 or C4 complement levels below the lower limit of normal; plus disease despite standard-of-care induction and maintenance immunosuppressive treatment

- Number (randomised/analysed): treatment group (21/21); control group (4/4)

* Treatment group: class III (7); class IV (14)

* Control group: class III (2); class IV (2)

- Mean age \pm SD (years): treatment group (30.6 \pm 7.7$)$; control group $(37.8 \pm 11.4)$

- Sex (M/F): treatment group (4/17); control group (0/4)

- Exclusion criteria: received CPA within 3 months of randomisation; unless intolerant, patients were required to be on a stable dose of an ACEi and/or an ARB; poorly controlled hypertension (mean SBP $>150 \mathrm{~mm} \mathrm{Hg}$ ) or a pattern of worsening or unstable kidney disease during the 8-week screening period

Induction therapy: duration of therapy was 6 months
Interventions
- Treatment group
$*$ Sirukumab (IL- 6 antibody): $10 \mathrm{mg} / \mathrm{kg}$ administered IV every 4 weeks
- Control group
$*$ Placebo: administered IV every 4 weeks
Both groups
$*$ MMF $(1$ to $3 \mathrm{~g} / \mathrm{d}$; or the equivalent dose of mycophenolic acid/mycophenolate sodium) or AZA (1 to
$3 \mathrm{mg} / \mathrm{kg} / \mathrm{d})$, with or without oral corticosteroids $(\leq 20 \mathrm{mg} / \mathrm{d}$ prednisone or equivalent)

\begin{tabular}{ll}
\hline Outcomes & - Death \\
& - Major infection \\
& - Malignancy \\
- Kiarrhoea & Kidney function
\end{tabular}

Notes

- Funding source: Janssen Research \& development LLC

\section{Risk of bias}

Bias Authors' judgement Support for judgement

Random sequence genera- Unclear risk tion (selection bias)

Allocation concealment $\quad$ Unclear risk Insufficient information to permit judgement
(selection bias)

Blinding of participants Low risk Double-blind, placebo-controlled study
and personnel (performance bias)

All outcomes

\begin{tabular}{|c|c|c|}
\hline $\begin{array}{l}\text { Blinding of outcome as- } \\
\text { sessment (detection bias) } \\
\text { All outcomes }\end{array}$ & Unclear risk & Insufficient information to permit judgement \\
\hline $\begin{array}{l}\text { Incomplete outcome data } \\
\text { (attrition bias) } \\
\text { All outcomes }\end{array}$ & Low risk & No missing outcome data \\
\hline $\begin{array}{l}\text { Selective reporting (re- } \\
\text { porting bias) }\end{array}$ & High risk & Not all pre-specified outcomes were reported \\
\hline Other bias & High risk & $\begin{array}{l}\text { Marked differences (demographics and clinical characteristics) between } \\
\text { groups at baseline. Sponsor involved in authorship }\end{array}$ \\
\hline
\end{tabular}


Sabry 2009

\begin{tabular}{|c|c|}
\hline Methods & $\begin{array}{l}\text { - Study design: quasi-RCT } \\
\text { - Study timeframe: not reported } \\
\text { - } \quad \text { Duration of follow-up: } 1 \text { year }\end{array}$ \\
\hline Participants & $\begin{array}{l}\text { - Country: Egypt } \\
\text { - Setting: single centre } \\
\text { - Inclusion criteria: ACR criteria for SLE; } \geq 18 \text { years; biopsy-proven proliferative lupus nephritis (WHO } \\
\text { class IV), urine protein >0.5 g/d } \\
\text { - Number (randomised/analysed): treatment group } 1(26 / 26) \text {; treatment group } 2(20 / 20) \\
\text { - Mean age } \pm \text { SD (years): treatment group } 1(26.4 \pm 9) \text {; treatment group } 2(25.7 \pm 7) \\
\text { - Sex (M/F): treatment group } 1(4 / 22) \text {; treatment group } 2(2 / 18) \\
\text { - Exclusion criteria: CSA or AZA in previous year or }>15 \mathrm{mg} / \mathrm{d} \text { prednisolone in previous month; renal } \\
\text { thrombotic microangiopathy; pre-existing CKD; pregnancy; previous malignancy; DM, documented } \\
\text { toxicity; anticipated poor compliance }\end{array}$ \\
\hline
\end{tabular}

\begin{tabular}{|c|c|}
\hline Interventions & $\begin{array}{l}\text { Induction therapy: duration of therapy was } 12 \text { months } \\
\text { - Treatment group } 1 \\
\text { * High dose CPA: } 6 \text { x monthly pulses }+2 \times \text { quarterly pulses. Initial dose }\left(0.5 \mathrm{~g} / 1.73 \mathrm{~m}^{2}\right) \text { then dose in- } \\
\text { creased by } 250 \mathrm{mg} \text { according to WCC on day } 14 \text { with final increment to maximum dose of } 1 \mathrm{~g} / 1.73 \mathrm{~m}^{2} \\
\text { - Treatment group } 2 \\
\text { * Low dose CPA: } 6 \text { x monthly pulses }+2 \times \text { quarterly pulses fixed dose of } 0.5 \mathrm{~g} / \mathrm{d} \\
\text { - Both groups } \\
\text { * Prednisolone }(0.5 \mathrm{mg} / \mathrm{kg}) \text { and AZA }(2 \mathrm{mg} / \mathrm{kg} / \mathrm{d}) \text { given in both treatment arms. Prednisolone given } \\
\text { at high dose for } 4 \text { weeks then given alternate days after being tapered by } 5 \mathrm{mg} \text { each week to mini- } \\
\text { mal dose to control extrarenal SLE manifestations or } 0.25 \mathrm{mg} / \mathrm{kg} / \mathrm{d} \text {. AZA started } 2 \text { weeks after last } \\
\text { infusion and continued until the end of the study }\end{array}$ \\
\hline
\end{tabular}

\begin{tabular}{ll}
\hline Outcomes & Death \\
- & ESKD \\
- & Roubling of $\mathrm{SCr}$ \\
& $50 \%$ or more for more than 1 month \\
- Treatment failure: defined as urinary protein excretion $\geq 3 \mathrm{~g} / 24 \mathrm{~h}$; and/or doubling of SCr or severe \\
flare that was resistant to increased glucocorticoid dose; patients who did not meet complete or par- \\
- tial remission criteria were considered as having treatment failure \\
- Major infection \\
- Ovarian failure \\
- Anaemia \\
- Leucopenia \\
- - Proteinuria effects \\
- SCr \\
- Serum albumin \\
- Six participants with most severe form of lupus nephritis allocated to high-dose arm \\
- Funding source: not reported
\end{tabular}

\section{Risk of bias}

Bias Authors' judgement Support for judgement


Sabry 2009 (Continued)

Random sequence genera- High risk All participants meeting inclusion criteria randomised. Manual randomisation tion (selection bias)

to allocate every other patient to either group and then assigned to one of 2 regimens. Six participants with most severe form of lupus nephritis allocated to high dose arm

\begin{tabular}{lll}
\hline $\begin{array}{l}\text { Allocation concealment } \\
\text { (selection bias) }\end{array}$ & High risk & Use of alternation to allocate \\
\hline $\begin{array}{l}\text { Blinding of participants } \\
\text { and personnel (perfor- } \\
\text { mance bias) }\end{array}$ & Unclear risk & Insufficient information to permit judgement \\
All outcomes &
\end{tabular}

\begin{tabular}{|c|c|c|}
\hline $\begin{array}{l}\text { Blinding of outcome as- } \\
\text { sessment (detection bias) } \\
\text { All outcomes }\end{array}$ & Unclear risk & Insufficient information to permit judgement \\
\hline $\begin{array}{l}\text { Incomplete outcome data } \\
\text { (attrition bias) } \\
\text { All outcomes }\end{array}$ & Low risk & No missing outcome data \\
\hline $\begin{array}{l}\text { Selective reporting (re- } \\
\text { porting bias) }\end{array}$ & Unclear risk & No study protocol available, all expected outcomes were reported \\
\hline Other bias & Unclear risk & $\begin{array}{l}\text { Differences in baseline characteristics between the groups (more severe pro- } \\
\text { teinuria and lower serum albumin in high dose CPA }\end{array}$ \\
\hline
\end{tabular}

\section{Sedhain 2016}

\begin{tabular}{ll}
\hline Methods & Study design: open-label, parallel RCT \\
& - Study timeframe: January 2014 to June 2015 \\
\hline Participants & Duration of follow-up: 6 months \\
\hline & - Country: Nepal \\
- Setting: single centre & Inclusion criteria: biopsy-proven proliferative lupus nephritis \\
& - Number (randomised/analysed): 49/42; treatment group 1 (21); treatment group 2 (21) \\
& - Sex (M/F): not reported \\
& - Exclusion criteria: not reported
\end{tabular}

\begin{tabular}{ll}
\hline Interventions & Induction therapy: duration of therapy was 6 months \\
& Treatment group 1 \\
& - Treatment group 2 \\
& $*$ IV CPA: administered monthly \\
\hline Outcomes & Complete remission: normal SCr and proteinuria $\leq 0.5 \mathrm{~g} / \mathrm{d}$ \\
& - Partial remission \\
& - Preatment failure: no response to therapy \\
\hline Notes & Abstract-only publication
\end{tabular}


Sedhain 2016 (Continued)

- Funding source: not reported

\section{Risk of bias}

\begin{tabular}{|c|c|c|}
\hline Bias & Authors' judgement & Support for judgement \\
\hline $\begin{array}{l}\text { Random sequence genera- } \\
\text { tion (selection bias) }\end{array}$ & Unclear risk & $\begin{array}{l}\text { Study was described as randomised, method of randomisation was not report- } \\
\text { ed }\end{array}$ \\
\hline $\begin{array}{l}\text { Allocation concealment } \\
\text { (selection bias) }\end{array}$ & Unclear risk & Insufficient information to permit judgement \\
\hline $\begin{array}{l}\text { Blinding of participants } \\
\text { and personnel (perfor- } \\
\text { mance bias) } \\
\text { All outcomes }\end{array}$ & High risk & Open-label study \\
\hline $\begin{array}{l}\text { Blinding of outcome as- } \\
\text { sessment (detection bias) } \\
\text { All outcomes }\end{array}$ & Unclear risk & Insufficient information to permit judgement \\
\hline $\begin{array}{l}\text { Incomplete outcome data } \\
\text { (attrition bias) } \\
\text { All outcomes }\end{array}$ & Unclear risk & Insufficient information to provide judgement \\
\hline $\begin{array}{l}\text { Selective reporting (re- } \\
\text { porting bias) }\end{array}$ & Unclear risk & Insufficient information to provide judgement \\
\hline Other bias & High risk & $\begin{array}{l}\text { Characteristics of the six patients unable to complete the study period are not } \\
\text { provided and these patients were not included in the analysis; abstract-only } \\
\text { publication }\end{array}$ \\
\hline
\end{tabular}

\section{Sesso 1994a}

\begin{tabular}{|c|c|}
\hline Methods & $\begin{array}{l}\text { - Study design: parallel RCT } \\
\text { - Study timeframe: September } 1990 \text { to December } 1992 \\
\text { - Duration of follow-up: } 15 \text { months }\end{array}$ \\
\hline Participants & $\begin{array}{l}\text { - Country: Brazil } \\
\text { - Setting: single centre } \\
\text { - Inclusion criteria: aged } \geq 16 \text { years; diagnosis of SLE (ARA criteria); severe lupus nephritis (defined as } \\
\text { nephritic urine sediment or urinary protein of }>3.0 \mathrm{~g} / \mathrm{d} \text { and impaired kidney function }(\mathrm{CrCl}<80 \mathrm{~mL} / \\
\text { min or a recent reduction of at least } 30 \%) ; \text { if } \mathrm{CrCl} \text { was stable the patient had to have histology of diffuse } \\
\text { proliferative } \mathrm{GN}(\mathrm{WHO} \text { classification criteria); } 23 / 29 \text { diffuse proliferative lupus nephritis } \\
\text { - Number (randomised): treatment group } 1(14) \text {; treatment group } 2(15) \\
\text { - Mean age } \pm \mathrm{SE} \text { (years): treatment group } 1(30.0 \pm 2.7) \text {; treatment group } 2(24.3 \pm 1.5) \\
\text { - Sex (M/F): treatment group } 1(2 / 12) ; \text { treatment group } 2(2 / 13) \\
\text { - Exclusion criteria: } \mathrm{CrCl}<20 \mathrm{~mL} / \mathrm{min} ; \mathrm{SCr}>6 \mathrm{mg} / \mathrm{dL} \text {; major infection within } 2 \text { weeks of study entry; } \\
\text { pregnancy; low leucocyte count; pulse MP or CPA within } 1 \text { year }\end{array}$ \\
\hline
\end{tabular}

Interventions

Induction therapy: duration of therapy was 10 months

- Treatment group 1

* IV CPA: 0.5 to $1.0 \mathrm{~g} / \mathrm{m}^{2}$, monthly pulse for 4 months, bimonthly for 4 months then quarterly for 6 months 
Sesso 1994a (Continued)

- Treatment group 2

* IV MP: 10 to $20 \mathrm{mg} / \mathrm{kg}$; $\max 1.0 \mathrm{~g} \times 3$ daily, then monthly for 4 months, bimonthly for 4 months then quarterly for 6 months

- Both groups

* Low dose oral prednisolone: $0.5 \mathrm{mg} / \mathrm{kg} / \mathrm{d}$ initially then tapered to control extra-renal manifestations

\begin{tabular}{ll}
\hline Outcomes & Death \\
- ESKD \\
- Doubling of SCr \\
- Bone toxicity \\
- Malignancy \\
- Major infection \\
- Proteinuria \\
- Complete remission: improvement of SCr and of urine sediment or proteinuria \\
- Partial remission: trend of improvement of SCr and of urine sediment or proteinuria \\
- Relapse: worsening of urine sediment, proteinuria and kidney function after having reached initial \\
improvement with therapy, requiring reinstitution of therapy \\
- 2 participants lost to follow-up \\
- Funding source: Instituto Paulista de Estudos e Pesquisas em Nefrologia e Hipertensao
\end{tabular}

\section{Risk of bias}

\begin{tabular}{lll}
\hline Bias & Authors' judgement & Support for judgement \\
\hline $\begin{array}{l}\text { Random sequence genera- } \\
\text { tion (selection bias) }\end{array}$ & Unclear risk & $\begin{array}{l}\text { Study was described as randomised, method of randomisation was not report- } \\
\text { ed }\end{array}$ \\
\hline $\begin{array}{l}\text { Allocation concealment } \\
\text { (selection bias) }\end{array}$ & Unclear risk & Insufficient information to permit judgement \\
\hline $\begin{array}{l}\text { Blinding of participants } \\
\text { and personnel (perfor- } \\
\text { mance bias) } \\
\text { All outcomes }\end{array}$ & Unclear risk & Insufficient information to permit judgement \\
\hline
\end{tabular}

\begin{tabular}{lll}
\hline $\begin{array}{l}\text { Blinding of outcome as- } \\
\text { sessment (detection bias) } \\
\text { All outcomes }\end{array}$ & Unclear risk & Insufficient information to permit judgement \\
\hline $\begin{array}{l}\text { Incomplete outcome data } \\
\begin{array}{l}\text { (attrition bias) } \\
\text { All outcomes }\end{array}\end{array}$ & Low risk & No missing outcome data \\
\hline $\begin{array}{l}\text { Selective reporting (re- } \\
\text { porting bias) }\end{array}$ & Low risk & All expected outcomes were reported \\
\hline Other bias & High risk & Proteinuria between groups at baseline was different \\
\hline
\end{tabular}

\section{SIMPL 2014}

Methods $\quad$ Study design: double-blind, pilot RCT


SIMPL 2014 (Continued)

- Study timeframe: not reported

- Duration of follow-up: 36 months

Participants

- Country: Canada

- Setting: single-centre

- Inclusion criteria: aged $\geq 18$ years; had a history of SLE according to ACR criteria; class III or class IV or class III/IV + V lupus nephritis by the ISN/RPS classification criteria; must have had an index biopsy within the 3 years previous to study enrolment, and could have been induced with CPA, MMF or another immunosuppressant as seen as appropriate by their physician; to be in at least partial remission at the time of randomisation, defined as having a) 0.3 to $2.9 \mathrm{~g} / \mathrm{d}$ proteinuria, b) serum albumin at least $30 \mathrm{~g} / \mathrm{L}$ and c) stable kidney function), be receiving between 5 and $20 \mathrm{mg} / \mathrm{d}$ of prednisone and provide informed consent

- Number (randomised/analysed): treatment group 1 (7/7); treatment group 2 (8/8)

* Treatment group 1: class III (1), class IV (6), class V (5)

* Treatment group 2: class III (3), class IV (4), class V (3)

- Mean age \pm SD (years): treatment group $1(28.4 \pm 5.6)$; treatment group $2(39.2 \pm 12.8)$

- Sex (M/F): treatment group 1 (0/7); treatment group 2 (2/6)

- Exclusion criteria: pregnant; required prednisone for treatment of another medical condition other than SLE; were receiving or expected to receive RRT within the next 6 months

Interventions

Maintenance therapy: duration of therapy was 36 months

- Treatment group 1

* Prednisone withdrawal: tapered the dose of prednisone contained in the capsules at a rate of 5 $\mathrm{mg} / \mathrm{d}$ every 2 weeks until the dose was $10 \mathrm{mg} / \mathrm{d}$, then by $2.5 \mathrm{mg} / \mathrm{d}$ every 2 weeks until the dose was $5 \mathrm{mg} / \mathrm{d}$ and then by $1 \mathrm{mg} / \mathrm{d}$ every 2 weeks until no prednisone and only placebo was contained in the capsules. A capsule containing placebo only was then continued for the duration of the study.

- Treatment group 2

* Prednisone: Low-dose maintenance glucocorticoids were tapered from their steroid dose at the time of randomisation, if necessary, to a target dose of $7.5 \mathrm{mg} / \mathrm{d}$ using the same algorithm as the prednisone withdrawal group. Patients who were already on 5 to $7.5 \mathrm{mg} / \mathrm{d}$ of prednisone therapy were maintained on their current dose with no changes made to the dose

- Both groups

* Hydroxychloroquine, and antihypertensives, NSAIDs and statins were left to the discretion of the patient's usual care providers. Vitamin D and calcium were recommended for all patients in the study as osteoporosis prophylaxis

Outcomes

- Relapse (composite of renal and major non-renal flare)

* Renal flare: defined as the occurrence of any one of the three following events: (1) Increased proteinuria, measured by either 24 hour urine collection or by a urine protein to creatinine ratio, by at least a) $1 \mathrm{~g} / \mathrm{d}$ if the baseline proteinuria was less than $0.2 \mathrm{~g} / \mathrm{d}$ or, b) $2 \mathrm{~g} / \mathrm{d}$ if the baseline proteinuria was between 0.2 and $1 \mathrm{~g} / \mathrm{d}$ (inclusive), or c) more than double the baseline proteinuria if the baseline proteinuria was greater than $1 \mathrm{~g} / \mathrm{d}$; (2) A sustained (i.e. for two consecutive measures) increase in SCr by at least 30\% over baseline that was not due to institution of antihypertensive therapy or angiotensin converting enzyme inhibitor therapy and with new haematuria attributable to active SLE; (3) New sustained haematuria attributable to active SLE, and exclusive of menses, infection or medications, that was associated with an increase in proteinuria by at least $0.8 \mathrm{~g} / \mathrm{d}$ )

* Major non-renal flare

- Major infection

- Quality of life: SF-36

\begin{tabular}{ll}
\hline Notes & 2 participants lost to follow-up \\
- & Funding source: centre for Advancement of Health, Calgary
\end{tabular}

\section{Risk of bias}

\section{Bias}

\section{Authors' judgement Support for judgement}


SIMPL 2014 (Continued)

Random sequence genera- Low risk tion (selection bias)
"Patients were randomly allocated to either the prednisone or placebo group using a random number list generated by an independent statistician. Randomization was blocked and stratified according to the duration of steroid treatment at the time of enrollment ( $\leq 12$ months or $>12$ months) and remission status (partial or complete)."

Allocation concealment Low risk
(selection bias)

"Allocation was concealed using sealed, opaque, sequentially numbered envelopes maintained by an independent physician. When a participant was randomised, the independent physician faxed the study number and assigned treatment to the study pharmacy."

\begin{tabular}{|c|c|c|}
\hline $\begin{array}{l}\text { Blinding of participants } \\
\text { and personnel (perfor- }\end{array}$ & Low risk & $\begin{array}{l}\text { Double-blind study "Patients, investigators, care providers and data analysts } \\
\text { remained blinded to study treatment throughout the trial." }\end{array}$ \\
\hline
\end{tabular}
and personnel (perforDouble-blind study "Patients, investigators, care providers
remained blinded to study treatment throughout the trial."

mance bias)

All outcomes

\begin{tabular}{|c|c|c|}
\hline $\begin{array}{l}\text { Blinding of outcome as- } \\
\text { sessment (detection bias) } \\
\text { All outcomes }\end{array}$ & Low risk & $\begin{array}{l}\text { "Patients, investigators, care providers and data analysts remained blinded to } \\
\text { study treatment throughout the trial." }\end{array}$ \\
\hline
\end{tabular}

\begin{tabular}{lll}
\hline $\begin{array}{l}\text { Incomplete outcome data } \\
\text { (attrition bias) } \\
\text { All outcomes }\end{array}$ & Low risk & No missing outcome data \\
\hline $\begin{array}{l}\text { Selective reporting (re- } \\
\text { porting bias) }\end{array}$ & High risk & $\begin{array}{l}\text { All prespecified outcomes are reported, but not all expected outcomes are re- } \\
\text { ported }\end{array}$ \\
\hline Other bias & High risk & Pilot study - underpowered \\
\hline
\end{tabular}

\section{Steinberg 1971}

\begin{tabular}{|c|c|}
\hline Methods & $\begin{array}{l}\text { - Study design: double-blind, parallel RCT } \\
\text { - Study timeframe: not reported } \\
\text { - Duration of follow-up: } 10 \text { weeks }\end{array}$ \\
\hline Participants & $\begin{array}{l}\text { - Country: USA } \\
\text { - Setting: single centre } \\
\text { - Inclusion criteria: diagnosis of SLE (ARA criteria); a positive lupus erythematosus cell test in the course } \\
\text { of the disease; kidney disease unaccounted for by other pathological processes, with at least one of } \\
\text { the following: RBC casts in a fresh centrifuged urine sediment; cellular casts and either haematuria ( } \geq \\
20 \mathrm{RBC} / \mathrm{HPF} \text { ) or pyuria (e } \geq 20 \mathrm{WBC} / \mathrm{HPF}) ; \text { proteinuria } \geq 1 \mathrm{~g} / 24 \mathrm{~h} ; \mathrm{CrCl}<50 \mathrm{~mL} / \mathrm{min} ; 8 / 15 \text { diffuse prolif- } \\
\text { erative lupus nephritis } \\
\text { - Number (randomised/analysed): treatment group (7/9); control group }(6 / 6) \\
\text { - Mean age, range (years): treatment group ( } 23,11 \text { to } 36) \text {; control group }(23,11 \text { to } 36) \\
\text { - Sex (M/F): treatment group (0/7); control group (2/6) } \\
\text { - Exclusion criteria: major infection within the preceding } 2 \text { weeks; pregnancy; granulocyte count }<1500 / \\
\mathrm{mm}^{3}, \text { immunosuppressive therapy within } 3 \text { months; severe liver disease }\end{array}$ \\
\hline
\end{tabular}

Interventions Induction therapy: duration of treatment was 10 weeks

- Treatment group

* Oral CPA: initial dose of $3 \mathrm{mg} / \mathrm{kg} / \mathrm{d}$ could be increased to $4 \mathrm{mg} / \mathrm{kg} / \mathrm{d}$ after 2 weeks

* Prednisone: $30 \mathrm{mg} / \mathrm{d}$

- Control group

* Prednisone: $30 \mathrm{mg} / \mathrm{d}$ 
Steinberg 1971 (Continued)

- Both groups

* Aspirin: $30 \mathrm{mg} / \mathrm{d}$

\begin{tabular}{ll}
\hline Outcomes & Death \\
- Toxicity \\
- Alopecia \\
- Complete remission of proteinuria \\
- Relapse: major SLE flare (criteria not reported) \\
- Proteinuria \\
- CrCl \\
\hline Notes \\
- 2 participants crossed-over to CPA therapy following placebo treatment period and were included in \\
Johnson Laboratories, Evansville, Ind
\end{tabular}

\section{Risk of bias}

\begin{tabular}{lll}
\hline Bias & Authors' judgement & Support for judgement \\
\hline $\begin{array}{l}\text { Random sequence genera- } \\
\text { tion (selection bias) }\end{array}$ & Low risk & $\begin{array}{l}\text { Used consecutively numbered envelopes, each containing a randomly as- } \\
\text { signed prescription for placebo or CPA }\end{array}$ \\
\hline $\begin{array}{l}\text { Allocation concealment } \\
\text { (selection bias) }\end{array}$ & Low risk & $\begin{array}{l}\text { As each patient entered the study, the next sequential envelope was opened in } \\
\text { the pharmacy }\end{array}$ \\
\hline $\begin{array}{l}\text { Blinding of participants } \\
\begin{array}{l}\text { and personnel (perfor- } \\
\text { mance bias) } \\
\text { All outcomes }\end{array}\end{array}$ & Low risk & Double-blind study \\
\hline
\end{tabular}

Blinding of outcome as-
$\begin{aligned} & \text { sessment (detection bias) } \\ & \text { All outcomes }\end{aligned}$ Unclear risk Insufficient information to permit judgement

Incomplete outcome data Low risk No missing outcome data

(attrition bias)

All outcomes

\begin{tabular}{lll}
\hline $\begin{array}{l}\text { Selective reporting (re- } \\
\text { porting bias) }\end{array}$ & Low risk & Study protocol available and pre-specified outcomes were reported \\
\hline Other bias & High risk & $\begin{array}{l}\text { Cross-over of two participants from the placebo to CPA arm were included in } \\
\text { the analysis }\end{array}$ \\
\hline
\end{tabular}

\section{Sun 2015}

\begin{tabular}{ll}
\hline Methods & Study design: open-label, parallel RCT \\
& - Study timeframe: September 2007 to February 2012 \\
\hline Participants & Duration of follow-up: 6 months \\
\hline & - Country: China \\
- Setting: single centre \\
- Inclusion criteria: aged 14 to 60 years; SLEDAI $\geq 12 ;$ renal-biopsy-proven diffuse segment or global (IV- \\
S of IV-G) lupus nephritis (ISN/RPS 2003 classification criteria) \\
\hline
\end{tabular}


Sun 2015 (Continued)

- Number (randomised/analysed): treatment group 1 (40/40); treatment group 2 (42/42)

- Mean age \pm SD (years): treatment group $1(33.3 \pm 11)$; treatment group 2 (31.9 \pm 8.7$)$

- $\operatorname{Sex}(M / F)$ : treatment group 1 (3/37); treatment group 2 (4/38)

- Exclusion criteria: complicated by uncontrolled severe infections or neuropsychiatric SLE; abnormal liver or kidney function (defined as $>2$ times of the normal values of transaminases or $>265.2 \mu \mathrm{mol} / \mathrm{L}$ of SCr level); patients with $<3 \times 10^{9} / \mathrm{L}$ of WBC or $<50 \times 10^{9} / \mathrm{L}$ of platelets; patients who received any cytotoxic or immunosuppressive drugs like CPA, TAC, MMF, or CSA within 3 months; pregnant or lactating women; patients with cerebrovascular disease, glucose metabolism disorder, or severe cardiopulmonary dysfunction

Induction therapy: duration of therapy was 6 months
Interventions
- Treatment group 1
- Treatment group 2
$*$ IV CPA: monthly dose of $0.4 \mathrm{~g} / \mathrm{m}^{2}$
$*$ Oral MMF: $1.0 \mathrm{~g} / \mathrm{d}$
- Both groups
- Prednisolone was started at a daily dose of $1.0 \mathrm{mg} / \mathrm{kg}$ for both groups, and then the dose was reduced
gradually after 4 to 8 weeks until completion of the treatment
1. Death
2. Major infection
3. Leucopenia: WCC $<4000 / \mathrm{mm} \mathrm{m}^{3}$
4. Complete remission: $<0.3 \mathrm{~g} / 24 \mathrm{~h}$ proteinuria with $\geq 35 \mathrm{~g} / \mathrm{L}$ of serum albumin and normal SCr level
5. Partial remission: proteinuria range 0.3 to $2.9 \mathrm{~g} / 24 \mathrm{~h}$ with an albumin concentration of $\geq 30 \mathrm{~g} / \mathrm{L}$, stable
or improved kidney function with reduction of proteinuria by $>50 \%$
6. Serum albumin
7. Proteinuria

Notes

- Funding source: This study was in part supported by the Natural Science Foundation of Hunan Province (No. 13JJ3033)

\section{Risk of bias}

\begin{tabular}{lll}
\hline Bias & Authors' judgement & Support for judgement \\
\hline $\begin{array}{l}\text { Random sequence genera- } \\
\text { tion (selection bias) }\end{array}$ & Unclear risk & $\begin{array}{l}\text { Study was described as randomised, method of randomisation was not report- } \\
\text { ed }\end{array}$ \\
\hline $\begin{array}{l}\text { Allocation concealment } \\
\text { (selection bias) }\end{array}$ & Unclear risk & Insufficient information to permit judgement \\
\hline $\begin{array}{l}\text { Blinding of participants } \\
\text { and personnel (perfor- } \\
\text { mance bias) } \\
\text { All outcomes }\end{array}$ & High risk & Open-label study \\
\hline
\end{tabular}

$\begin{array}{ll}\text { Blinding of outcome as- } & \text { Unclear risk }\end{array}$ Insufficient information to permit judgement

All outcomes

Incomplete outcome data Low risk No missing outcome data
(attrition bias)

All outcomes 
Sun 2015 (Continued)

Selective reporting (re- Low risk $\quad$ No study protocol available but expected outcomes are reported
porting bias)

Other bias Low risk The study appears to be free of other sources of bias

Wallace 1998

\begin{tabular}{|c|c|}
\hline Methods & $\begin{array}{l}\text { - Study design: open-label, parallel RCT } \\
\text { - Study timeframe: not reported } \\
\text { - Duration of follow-up: > } 24 \text { months }\end{array}$ \\
\hline Participants & $\begin{array}{l}\text { - Country: USA } \\
\text { - Setting: single centre } \\
\text { - Inclusion criteria: diagnosis of SLE (ACR criteria); aged } \geq 16 \text { years; class III or IV lupus nephritis on renal } \\
\text { biopsy and chronicity index }<6 ; 2 / 19 \text { class IV } \\
\text { - Number (randomised): treatment group } 1(9) \text {; treatment group } 2 \text { (9) } \\
\text { - Mean age } \pm \mathrm{SD} \text { (years): treatment group } 1(33.0 \pm 10.0) \text {; treatment group } 2(32.0 \pm 14.0) \\
\text { - Sex (M/F): treatment group } 1(1 / 8) \text {; treatment group } 2(0 / 9) \\
\text { - Exclusion criteria: } \mathrm{SCr}>3 \mathrm{mg} / \mathrm{dL} \text {; renal biopsy chronicity index } \geq 6 \text {; pregnancy; < } 16 \text { years; immuno- } \\
\text { suppression in last } 3 \text { months }\end{array}$ \\
\hline
\end{tabular}

\begin{tabular}{|c|c|}
\hline Interventions & $\begin{array}{l}\text { Induction therapy: duration of treatment was } 8 \text { months } \\
\text { - Treatment group } 1 \\
\text { * PEX: } 3 \text { x daily preceding CPA } \\
\text { * IV CPA: }\left(750 \mathrm{mg} / \mathrm{m}^{2} \times 6\right) \\
\text { - Treatment group } 2 \\
\text { - IV CPA: } 750 \mathrm{mg} / \mathrm{m}^{2} \times 6 \text { over } 8 \text { months } \\
\text { - Both groups } \\
\text { * Prednisolone: } 1 \mathrm{mg} / \mathrm{kg} / \mathrm{d} \text { for } 6 \text { weeks then tapering dose }\end{array}$ \\
\hline Outcomes & $\begin{array}{l}\text { - Death } \\
\text { - } \text { ESKD } \\
\text { - Complete remission: } \mathrm{SCr}<1.4 \mathrm{mg} / \mathrm{dL} \text {, a } 24-\mathrm{h} \text { urine protein }<500 \mathrm{mg} \text {, absence of urinary casts; normal } \\
\text { BP and serum albumin }>4.0 \mathrm{mg} / \mathrm{dL} \\
\text { - } \mathrm{SCr} \\
\text { - Serum albumin } \\
\text { - Proteinuria }\end{array}$ \\
\hline Notes & $\begin{array}{l}\text { - } 1 \text { patient lost to follow-up } \\
\text { - Funding source: not reported }\end{array}$ \\
\hline
\end{tabular}

\section{Risk of bias}

\begin{tabular}{lll}
\hline Bias & Authors' judgement & Support for judgement \\
\hline $\begin{array}{l}\text { Random sequence genera- } \\
\text { tion (selection bias) }\end{array}$ & Unclear risk & $\begin{array}{l}\text { Study was described as randomised, method of randomisation was not report- } \\
\text { ed }\end{array}$ \\
\hline $\begin{array}{l}\text { Allocation concealment } \\
\text { (selection bias) }\end{array}$ & Unclear risk & Insufficient information to permit judgement \\
\hline
\end{tabular}


Wallace 1998 (Continued)

Blinding of participants High risk Open-label study
and personnel (perfor-
mance bias)

All outcomes

Blinding of outcome as- Unclear risk Insufficient information to permit judgement

sessment (detection bias)

All outcomes

Incomplete outcome data Low risk No missing outcome data
(attrition bias)

All outcomes

\begin{tabular}{lll}
\hline $\begin{array}{l}\text { Selective reporting (re- } \\
\text { porting bias) }\end{array}$ & High risk & Not all expected outcomes were reported \\
\hline Other bias & Low risk & The study appears to be free of other sources of bias \\
\hline
\end{tabular}

Yap 2017

\begin{tabular}{|c|c|}
\hline Methods & $\begin{array}{l}\text { - Study design: parallel RCT } \\
\text { - Study timeframe: not reported } \\
\text { - Duration of follow-up: } 6 \text { months }\end{array}$ \\
\hline Participants & $\begin{array}{l}\text { - Country: Hong Kong } \\
\text { - Setting: not reported } \\
\text { - Inclusion criteria: active lupus nephritis } \\
\text { - Number (randomised): treatment group } 1(7) \text {; treatment group } 2(7) \\
\text { - Mean age } \pm \text { SD (years): not reported } \\
\text { - Sex (M/F): not reported } \\
\text { - Exclusion criteria: not reported }\end{array}$ \\
\hline Interventions & $\begin{array}{l}\text { Induction therapy: duration of treatment was } 6 \text { months } \\
\text { - Treatment group } 1 \\
\text { * MMF: no details provided } \\
\text { - Treatment group } 2 \\
\text { * CPA: no details provided } \\
\text { - Both groups } \\
* \text { Prednisone or prednisone equivalent: no details provided }\end{array}$ \\
\hline Outcomes & - Immunological function \\
\hline Notes & - Funding source: Bristol Myers Squibb \\
\hline
\end{tabular}

\section{Risk of bias}

\begin{tabular}{lll}
\hline Bias & Authors' judgement & Support for judgement \\
\hline $\begin{array}{l}\text { Random sequence genera- } \\
\text { tion (selection bias) }\end{array}$ & Unclear risk & $\begin{array}{l}\text { Study was described as randomised, method of randomisation was not report- } \\
\text { ed }\end{array}$ \\
\hline $\begin{array}{l}\text { Allocation concealment } \\
\text { (selection bias) }\end{array}$ & Unclear risk & Insufficient information to permit judgement \\
\hline
\end{tabular}


Yap 2017 (Continued)

Blinding of participants Unclear risk Insufficient information to permit judgement and personnel (performance bias)

All outcomes

\begin{tabular}{lll}
\hline Blinding of outcome as- & Unclear risk & Insufficient information to permit judgement \\
sessment (detection bias) &
\end{tabular}

All outcomes

Incomplete outcome data Unclear risk Insufficient information to permit judgement

(attrition bias)

All outcomes

Selective reporting (re- High risk $\quad$ Not all relevant clinical outcomes reported
porting bias)

Other bias Unclear risk Insufficient information to permit judgement; abstract-only publication

Yee 2004

\begin{tabular}{ll}
\hline Methods & Study design: open label, parallel RCT \\
& - Study timeframe: June 1992 to May 1996 \\
- Duration of follow-up: intended to be 5 to 10 years
\end{tabular}

Participants

- Countries: Austria, Czech Republic, Lithuania, Slovenia, Sweden, UK

- Setting: multinational (8 sites)

- Inclusion criteria: diagnosis of SLE (ACR criteria); biopsy-proven proliferative lupus nephritis (WHO classification criteria), aged 16 to 65 years

- Number (randomised/analysed): treatment group 1 (16/13); treatment group 2 (16/16)

* Treatment group 1: class III (6), class IV (10)

* Treatment group 2: class III (5), class IV (8)

- Mean age \pm SD (years): treatment group 1 (42.4 \pm 11.8$)$; treatment group 2 (32.2 \pm 11.7$)$

- $\operatorname{Sex}(M / F)$ : treatment group 1 (2/11); treatment group 2 (2/14)

- Exclusion criteria: previous CPA or AZA in preceding 3 weeks; pure membranous or mesangial proliferative GN on biopsy; previous treatment with CPA for > 3 months; allergy to study drugs; previous malignancy; primary immunodeficiency (except complement components); non-lupus-related kidney disease

Induction therapy: duration of treatment was 24 months
$\begin{aligned} & \text { Interventions } \\ & \text { Treatment group } 1 \\ & * \text { Intermittent IV CPA: } 10 \mathrm{mg} / \mathrm{kg} 3 \mathrm{x} / \mathrm{wk}, \mathrm{max} 1 \mathrm{~g} \text { for } 4 \text { doses, then orally (same dose split over } 2 / 7) 4 \\ & \text { weekly for } 9 \text { months and } 6 \text { weekly for } 12 \mathrm{months} \\ & * \text { IV MP } 6.6 \mathrm{mg} / \mathrm{kg} \text { before each pulse of CPA then orally at same dose split over } 2 \text { days before each } \\ & \text { oral dose } \mathrm{plus} \text { oral prednisolone } 0.3 \mathrm{mg} / \mathrm{kg} / \mathrm{d} \text { reducing to } 0.1 \mathrm{mg} / \mathrm{kg} / \mathrm{d} \text { to maintenance dose of } 0.05 \\ & \text { to } 0.1 \mathrm{mg} / \mathrm{kg} / \mathrm{d}\end{aligned}$
$\begin{aligned} & \text { Treatment group } 2 \\ & *\end{aligned}$
Oral CPA: $2 \mathrm{mg} / \mathrm{kg} / \mathrm{d}$ for $3 \mathrm{months}$ then $1.5 \mathrm{mg} / \mathrm{kg} / \mathrm{d}$
Oral prednisolone: $0.85 \mathrm{mg} / \mathrm{kg} / \mathrm{d}$ (max dose $60 \mathrm{mg}$ ) reducing to $0.11 \mathrm{mg} / \mathrm{kg} / \mathrm{d}$ by week 53
Both groups
H2 receptor antagonist (ranitidine $150 \mathrm{mg}$ at night or cimetidine $400 \mathrm{mg}$ at night) and amphotericin
lozenges $(10 \mathrm{mg}$ four times a day) as prophylaxis while on daily CPA and for two weeks with each
pulse of CPA


Yee 2004 (Continued)

- ESKD

- Doubling of SCr

- Major infection

- Ovarian failure

- Malignancy

- Bladder toxicity

- Nausea/vomiting

- Treatment failure: failure to respond to treatment

\section{Notes}

- Study terminated after 4 years due to poor recruitment and high withdrawal rate

- Funding source: European League Against Rheumatism (EULAR) Standing Committee on International Clinical Studies including Therapeutic Trials (ESCIST); Lupus UK; and the Swedish Medical Research Council (grant 13489).

\section{Risk of bias}

\begin{tabular}{lll}
\hline Bias & Authors' judgement & Support for judgement \\
\hline $\begin{array}{l}\text { Random sequence genera- } \\
\text { tion (selection bias) }\end{array}$ & Low risk & $\begin{array}{l}\text { Participants were stratified according to the presence of kidney failure and un- } \\
\text { derwent block randomisation to either therapy }\end{array}$ \\
\hline $\begin{array}{l}\text { Allocation concealment } \\
\text { (selection bias) }\end{array}$ & Unclear risk & Insufficient information to permit judgement \\
\hline $\begin{array}{l}\text { Blinding of participants } \\
\text { and personnel (perfor- } \\
\text { mance bias) } \\
\text { All outcomes }\end{array}$ & High risk & Open-label study \\
\hline
\end{tabular}

\begin{tabular}{|c|c|c|}
\hline $\begin{array}{l}\text { Blinding of outcome as- } \\
\text { sessment (detection bias) } \\
\text { All outcomes }\end{array}$ & Unclear risk & Insufficient information to permit judgement \\
\hline $\begin{array}{l}\text { Incomplete outcome data } \\
\text { (attrition bias) } \\
\text { All outcomes }\end{array}$ & Low risk & No missing outcome data \\
\hline $\begin{array}{l}\text { Selective reporting (re- } \\
\text { porting bias) }\end{array}$ & High risk & Not all pre-specified outcomes reported: alopecia \\
\hline Other bias & High risk & $\begin{array}{l}\text { Study was terminated after four years as patient recruitment was disappoint- } \\
\text { ing and many patients had been withdrawn; Many physicians became reluc- } \\
\text { tant to enter patients because of concerns that the oral regimen was slower to } \\
\text { work and more toxic than the pulse regimen, following development of severe } \\
\text { neutropenia in the continuous group; This led to the premature termination of } \\
\text { the study }\end{array}$ \\
\hline
\end{tabular}

\section{Zhang 1995a}

\begin{tabular}{ll}
\hline Methods & - Study design: open-label, parallel RCT \\
& - Study timeframe: not reported \\
& Duration of follow-up: 12 to 39 months \\
\hline Participants & - Country: China \\
& - Setting: single centre
\end{tabular}


Zhang 1995a (Continued)

- Inclusion criteria: biopsy-proven active lupus nephritis

- Number (randomised): 36 (numbers per group not reported)

- Mean age \pm SD (years): not reported

- Sex (M/F): not reported

- Exclusion criteria: not reported

\begin{tabular}{ll}
\hline Interventions & Induction therapy \\
& Treatment group 1 \\
& $*$ CPA: monthly pulse 0.5 to $0.8 \mathrm{~g} / \mathrm{m}^{2}$ until remission \\
& Treatment group 2 \\
& $*$ CPA: monthly pulse 0.5 to $0.8 \mathrm{~g} / \mathrm{m}^{2}$ for 1 year \\
& Both groups \\
& $*$ Minimum necessary dose of steroids \\
\hline Outcomes & Remission \\
& - Relapse \\
- Urinalysis & Serology \\
\hline Notes & Abstract-only publications \\
& - Funding source: not reported
\end{tabular}

\section{Risk of bias}

\begin{tabular}{lll}
\hline Bias & Authors' judgement & Support for judgement \\
\hline $\begin{array}{l}\text { Random sequence genera- } \\
\text { tion (selection bias) }\end{array}$ & Unclear risk & $\begin{array}{l}\text { Study was described as randomised, method of randomisation was not report- } \\
\text { ed }\end{array}$ \\
\hline $\begin{array}{l}\text { Allocation concealment } \\
\text { (selection bias) }\end{array}$ & Unclear risk & Insufficient information to permit judgement \\
\hline $\begin{array}{l}\text { Blinding of participants } \\
\text { and personnel (perfor- } \\
\text { mance bias) } \\
\text { All outcomes }\end{array}$ & High risk & Open-label study \\
\hline
\end{tabular}

Blinding of outcome as- Unclear risk Insufficient information to permit judgement
sessment (detection bias)

All outcomes

Incomplete outcome data Unclear risk Insufficient information to permit judgement
(attrition bias)

All outcomes

\begin{tabular}{lll}
\hline $\begin{array}{l}\text { Selective reporting (re- } \\
\text { porting bias) }\end{array}$ & High risk & Data unable to be meta-analysed \\
\hline Other bias & Unclear risk & Abstract-only publication; insufficient information to permit judgement \\
\hline
\end{tabular}

ACEi - angiotensin-converting enzyme inhibitor; ACR - American College of Rheumatology; ANA - antinuclear antibody; ARA - American Rheumatology Association; ARB - angiotensin receptor blocker; AZA - azathioprine; BILAG - British Isles Lupus Assessment Group; CKD chronic kidney disease; CMV - cytomegalovirus; CNI - calcineurin; CNS - central nervous system; CPA - cyclophosphamide; $\mathrm{CrCl}$ - creatinine clearance; CSA - cyclosporin A; DM - diabetes mellitus; EC-MPS - enteric-coated mycophenolate sodium; eGFR - estimated glomerular filtration rate; ELNT - Euro-lupus nephritis treatment; ESKD - end-stage kidney disease; GI - gastrointestinal: GN - glomerulonephritis; 
Hb - haemoglobin; HBV - hepatitis B virus; HCV - hepatitis C virus; HIV - human immunodeficiency virus; HPF - high power field; IA - immunoadsorption; ISN/RPS - International Society of Nephrology/Renal Pathology Society; IV - intravenous; IVIG - intravenous immunoglobulin; M/F - male/female; MMF - mycophenolate mofetil; MP - methylprednisolone; NSAID/s - nonsteroidal anti-inflammatory drug/s; PEX - plasma exchange or plasmapheresis; PALGA - Dutch Pathology Registry; RBC - red blood cell/s; RCC - red cell count; RCT randomised controlled trial; RTX - rituximab; SC - subcutaneous; SCr - serum creatinine; SD - standard deviation; SDS - standard deviation score; SLE - systemic lupus erythematosus; SLEDAI - SLE Disease Activity Index; SLICC - Systemic Lupus Collaborating Clinics; TAC tacrolimus; TB - tuberculosis; WHO - World Health Organization; ISN/RPS - International Society of Nephrology/ Renal Pathology Society; UPCR - urine protein-to-creatinine ratio; WBC - white blood cell/s; WCC - white cell count

\section{Characteristics of excluded studies [ordered by study ID]}

\begin{tabular}{|c|c|}
\hline Study & Reason for exclusion \\
\hline Andrade-Ortega 2010 & Wrong population: not biopsy-proven lupus nephritis \\
\hline Antunes 2001 & Wrong intervention: not comparing immunosuppression \\
\hline ASPEN 2008 & Wrong population: not biopsy-proven lupus nephritis \\
\hline ATLAS 2016 & $\begin{array}{l}\text { Wrong population: diagnosis of biopsy-proven proliferative lupus nephritis at randomisation un- } \\
\text { clear }\end{array}$ \\
\hline Austin 2009 & Wrong population: not biopsy-proven lupus nephritis but membranous \\
\hline Balow 1981 & Wrong population: not biopsy-proven lupus nephritis \\
\hline Balow 1984 & No relevant outcomes \\
\hline Ble 2011 & Wrong intervention: not immunosuppressive intervention \\
\hline Chanchairujira 2009 & No relevant clinical outcomes \\
\hline Clark 1993 & Wrong population: not biopsy-proven lupus nephritis \\
\hline Clark 2001a & Wrong population: not biopsy-proven lupus nephritis \\
\hline CONTROL 2016 & $\begin{array}{l}\text { Wrong population: diagnosis of biopsy-proven proliferative lupus nephritis at randomisation was } \\
\text { unclear }\end{array}$ \\
\hline Davis 1999 & $\begin{array}{l}\text { Wrong population and intervention: not biopsy-proven lupus nephritis or comparing immunosup- } \\
\text { pression }\end{array}$ \\
\hline Daza 2005 & Wrong intervention: not comparing immunosuppression \\
\hline Deng 2017a & Wrong intervention: not comparing immunosuppression \\
\hline Feng 2014 & Wrong population: not biopsy-proven lupus nephritis \\
\hline Frutos 1997 & Insufficient information to determine if the study is randomised \\
\hline Hebert 1987 & Wrong population: not biopsy-proven lupus nephritis \\
\hline Khajehdehi 2012 & Wrong intervention: not immunosuppressive intervention \\
\hline Kuo 2001 & Wrong intervention: not comparing immunosuppression \\
\hline Li 2005 & Insufficient information to determine if the study is randomised \\
\hline
\end{tabular}




\begin{tabular}{|c|c|}
\hline Study & Reason for exclusion \\
\hline Li 2014a & Wrong intervention: not immunosuppressive intervention \\
\hline LJP 394-90-05 2003 & Wrong population: not biopsy-proven lupus nephritis \\
\hline LJP 394-90-09 2005 & Wrong population: not biopsy-proven lupus nephritis \\
\hline Lu 2002 & Wrong population: not biopsy-proven lupus nephritis \\
\hline Miyasaka 2009 & Wrong population: included class II and class V lupus nephritis \\
\hline NCT00001212 & Wrong population: membranous lupus nephritis \\
\hline NCT00404157 & The study has been terminated \\
\hline NCT00429377 & $\begin{array}{l}\text { The recruitment status of this study is unknown (registered 2007). The completion date of this } \\
\text { study has passed and the status has not been verified in more than two years. }\end{array}$ \\
\hline NCT00436438 & Study terminated early for administrative reasons \\
\hline NCT00539799 & $\begin{array}{l}\text { This study was withdrawn prior to enrolment, as the local pharmacy were unwilling to comply with } \\
\text { the study protocol }\end{array}$ \\
\hline NCT00659217 & $\begin{array}{l}\text { The recruitment status of this study is unknown (registered 2008). The completion date of this } \\
\text { study has passed and the status has not been verified in more than two years }\end{array}$ \\
\hline NCT01299922 & This study was withdrawn prior to recruitment \\
\hline NCT01342016 & This study has been terminated due to safety concerns of active control drug \\
\hline NCT01930890 & $\begin{array}{l}\text { Study was terminated because results from previous studies did not demonstrate sufficient effica- } \\
\text { cy }\end{array}$ \\
\hline NCT02176486 & Study was terminated, insufficient enrolment \\
\hline Pierucci 1989 & Wrong population: not comparing immunosuppression \\
\hline Schaumann 1992 & Unclear if biopsy-proven lupus nephritis \\
\hline Su 2007 & Wrong population: not biopsy-proven lupus nephritis \\
\hline Sztejnbok 1971 & Wrong population: not biopsy-proven lupus nephritis \\
\hline Wallace 2006 & Wrong population: not biopsy-proven lupus nephritis \\
\hline Wang 2007 & $\begin{array}{l}\text { Wrong population: non-invasive necrotising vasculopathy-severe variant not usually responsive to } \\
\text { standard therapy }\end{array}$ \\
\hline Witte 1993 & Unclear if biopsy-proven lupus nephritis \\
\hline Yap 2012 & Wrong population: not biopsy-proven lupus nephritis \\
\hline Ye 2001 & Wrong population: not biopsy-proven lupus nephritis \\
\hline Yoshida 1996 & Wrong intervention: not comparing immunosuppression \\
\hline
\end{tabular}




\begin{tabular}{ll}
\hline Study & Reason for exclusion \\
\hline Zhang 2015c & Wrong population: biopsy-proven proliferative lupus nephritis were excluded \\
\hline Zheng 2005a & Unclear if biopsy-proven lupus nephritis \\
\hline
\end{tabular}

Characteristics of ongoing studies [ordered by study ID]

\section{2nd Dutch Lupus Trial}

\begin{tabular}{ll}
\hline Trial name or title & $\begin{array}{l}\text { Comparison of short course cyclophosphamide followed by mycophenolate mofetil versus long } \\
\text { course cyclophosphamide in the treatment of proliferative lupus nephritis }\end{array}$ \\
\hline Methods & Multicentre RCT \\
\hline Participants & Adult, proliferative lupus nephritis, biopsy-proven, active urinary sediment, proteinuria \\
\hline Interventions & $\begin{array}{l}6 \text { months IV CPA induction followed by either } 3 \text { monthly IV CPA or MMF for } 18 \text { months, then } 2 \text { years } \\
\text { AZA in both arms }\end{array}$ \\
\hline Outcomes & Renal relapse \\
\hline Starting date & January 2003 \\
\hline Contact information & Marc Bijl, University Medical Centre Groningen \\
\hline Notes &
\end{tabular}

\section{ChicTR-TRC-09000587}

Trial name or title

The intensive therapy of severe lupus nephritis: a multicenter, randomised, controlled prospective clinical trial

\begin{tabular}{ll}
\hline Methods & Multicentre, randomised controlled \\
\hline Participants & Adult, SLE according to ACR criteria, renal biopsy-proven lupus nephritis: 24 hours proteinuria ( $\geq$ \\
& $3.0 \mathrm{~g} / \mathrm{d}$ or +++ ), erythrocyturia $>5 / \mathrm{HPF}$, leucocyturia or cast (RBC, Hb, tubuli or mixed); SLEDAI score \\
$\geq 10$
\end{tabular}

\begin{tabular}{l} 
Interventions \\
$\begin{array}{l}\text { 1. NIH IV CPA standard program (Induction period, follow-up once every four weeks; consolidation } \\
\text { therapy: follow-up once every twelve weeks, maintenance therapy: follow-up once every twelve } \\
\text { weeks. }\end{array}$ \\
$\begin{array}{l}\text { 2. Intensive group: mini-pulse of CPA, hydroxychloroquine and another immunosuppressive agent, } \\
\text { such as MMF, leflunomide, AZA or methotrexate }\end{array}$ \\
\hline Outcomes \\
Starting date
\end{tabular}

\section{Notes}


ChicTR-TRC-10000931

\begin{tabular}{ll}
\hline Trial name or title & Treatment of severe lupus nephritis with tacrolimus (FK 506) based immunosuppression \\
\hline Methods & Multicentre RCT \\
\hline Participants & $\begin{array}{l}\text { Adult; SLE (ACR criteria); SLEDAI > } 10 \text { points; biopsy-proven lupus nephritis severe type III, IV type, } \mathrm{V} \\
+ \text { III type and } \mathrm{V}+\mathrm{IV} \text {-type lupus nephritis (WHO2004 criteria), heavy-III, with severe segmental lesions } \\
\text { that have loop necrosis or crescent formation of the III-type lupus nephritis); significant renal dis- } \\
\text { ease, proteinuria } \geq 2 \mathrm{~g} / 24 \mathrm{~h}, \text { with active urine sediment (urine RBC }>400,000 / \mathrm{mL}, \text { tube urine, leuko- } \\
\text { cytes in urine), SCr }<3 \mathrm{mg} / \mathrm{dL}(265 \mu \text { mol/L) }\end{array}$ \\
\hline Interventions & Tacrolimus (0.5 $\mathrm{mg}$ and $1 \mathrm{mg})$ \\
\hline Outcomes & Serum albumin, SCr, proteinuria, immunological function, renal biopsy, adverse events \\
\hline Starting date & 2009 \\
\hline Contact information & Changlin Mei, Shanghai Changzheng Hospital, Beijing China \\
\hline Notes & Sponsor - Astellas Pharma China Inc. \\
\hline
\end{tabular}

\section{CTRI/2016/01/006488}

\begin{tabular}{|c|c|}
\hline Trial name or title & Comparison of two steroid dose regimen in lupus nephritis: a randomised controlled trial \\
\hline Methods & $\mathrm{RCT}$ \\
\hline Participants & $\begin{array}{l}12 \text { to } 70 \text { years of age, SLE (ACR criteria); biopsy-proven lupus nephritis (ISN/RPS class III, IV, III+V or } \\
\text { IV+V) }\end{array}$ \\
\hline \multirow[t]{3}{*}{ Interventions } & 1. Low dose oral prednisolone $(0.5 \mathrm{mg} / \mathrm{kg} / \mathrm{d})$ \\
\hline & 2. Oral prednisolone $(1 \mathrm{mg} / \mathrm{kg} / \mathrm{d})$ \\
\hline & $\begin{array}{l}\text { Patients in both groups will receive IV MP }(750 \mathrm{mg}) \text { for } 3 \text { days, followed by oral prednisolone for a } \\
\text { period of } 8 \text { weeks followed by a taper. All patients will receive MMF }\end{array}$ \\
\hline Outcomes & $\begin{array}{l}\text { Complete remission, partial remission, SELENA-SLEDAI, quality of life, immunological function, ad- } \\
\text { verse events }\end{array}$ \\
\hline Starting date & January 2016 \\
\hline Contact information & $\begin{array}{l}\text { Krishan Lal Gupta, Department of nephrology, Postgraduate Institute of Medical Education and Re- } \\
\text { search, Chandigarh, India }\end{array}$ \\
\hline Notes & \\
\hline
\end{tabular}

\section{CTRI/2017/05/008697}

$\begin{array}{ll}\text { Trial name or title } & \begin{array}{l}\text { Randomised controlled trial of multi-targetted therapy versus low-dose intravenous cyclophos- } \\ \text { phamide in the treatment of lupus nephritis }\end{array}\end{array}$

Methods Parallel RCT


CTRI/2017/05/008697 (Continued)

Participants

Adults, SLE ACR criteria; lupus nephritis class III, IV, V, a combination of III+V or IV+V; $\mathrm{SCr}<3.0 \mathrm{mg}$ / $\mathrm{dL}$

Interventions

1. MMF: $1 \mathrm{~g} / \mathrm{d}$ in 2 divided doses and TAC $0.1 \mathrm{mg} / \mathrm{kg} / \mathrm{d}$ to target a trough level of 4 to $7 \mathrm{ng} / \mathrm{mL}$. MMF and TAC will be taken morning and evening, before meals, and with a glass of water

2. CPA: Euro-lupus Nephritis trial group regimen of six fortnightly IV infusions of a fixed dose of 500 mg CPA

- All the patients will be given 3 IV infusion of MP $(750 \mathrm{mg})$ followed by $1 \mathrm{mg} / \mathrm{kg} / \mathrm{d}$ of oral prednisolone for a period of 8 weeks followed by taper to $7.5 \mathrm{mg} / \mathrm{d}$ at the end of 6 months

- All the patients will be given maintenance treatment after completion of induction treatment, in the form of AZA ( $2 \mathrm{mg} / \mathrm{kg}$ ) plus low-dose steroids

Outcomes

1. Decrease in $24 \mathrm{~h}$ proteinuria, defined as decrease in the UPCR to 3 in subjects with baseline nephrotic range proteinuria ( $\geq 3$ UPCR) or decrease in the UPCR by $\geq 50 \%$ in subjects with subnephrotic proteinuria (3 UPCR)

2. Stabilization of SCr (i.e., a week $24 \mathrm{SCr}$ level $\pm 25 \%$ of baseline) or improvement

\begin{tabular}{ll}
\hline Starting date & July 2016 \\
\hline Contact information & $\begin{array}{l}\text { Krishan Lal Gupta, Department of Nephrology, Nehru Hospital, Post Graduate Institute of Medical } \\
\text { Education and Research, Chandigarh, India }\end{array}$ \\
\hline Notes & Follow-up: 6 months \\
\hline
\end{tabular}

\section{ISRCTN66475575}

Trial name or title

Enteric coat mycophenolate sodium versus intravenous cyclophosphamide for severe paediatric lupus nephritis

\begin{tabular}{ll}
\hline Methods & Multicentre RCT \\
\hline Participants & Paediatric lupus nephritis \\
\hline Interventions & 1. EC-MPS (myfortic $\left.{ }^{\circledR}\right): 720$ to $860 \mathrm{mg} / \mathrm{m}^{2} / \mathrm{d}$ (max \\
& 2. CPA: 750 to $1000 \mathrm{months}$ + oral steroid \\
& \\
\hline Outcomes & 1. Death \\
& 2. ESKD \\
3. Complete remission & 4. Partial remission \\
5. Relapse (renal and non-renal) & 6. Disease activity: SLEDAl \\
7. Infection & 8. Gl symptoms
\end{tabular}

Starting date July 2009

Contact information

Wattana Chartapisak, Department of Pediatrics, Chiang Mai, Thailand 


\begin{tabular}{ll}
\hline Trial name or title & To compare the efficacy and safety of FK506 vs IVC in the treatment of class III-IV lupus nephritis \\
\hline Methods & Multicentre RCT \\
\hline Participants & $\begin{array}{l}\text { Adult (18 to } 65 \text { years) female patients with SLE according to ACR criteria, SLEDAI > 10; biop- } \\
\text { sy-proven } \\
\text { class III or IV lupus nephritis according to the WHO classification criteria within } 3 \text { month and have } \\
\text { significant active pathological lesion; proteinuria } \geq 2 \text { g/24 h, and an active urine sediment (haema- } \\
\text { turia with white cells and casts in urine) }\end{array}$ \\
\hline Interventions & $\begin{array}{l}\text { 1. TAC: } 0.1 \text { mg/kg/d } \\
\text { 2. IV CPA }\end{array}$ \\
\hline Outcomes & Safety and efficacy \\
\hline Starting date & May 2004 \\
\hline Contact information & Lei-shi Li, Research Institute of Nephrology, Jinling Hospital, Nanjing University School of Medicine, \\
Nanjing, Jiangsu, China
\end{tabular}

\section{NCT00705367}

\section{Trial name or title}

A single centre, randomised, placebo-controlled, double blind, parallel group study to evaluate the tolerability of a single dose of Abatacept $30 \mathrm{mg} / \mathrm{kg}$ via intravenous infusion in Chinese SLE subjects with lupus nephritis

\begin{tabular}{ll}
\hline Methods & Single-centre, double blind and open-label extension RCT \\
\hline Participants & $\begin{array}{l}\text { Adult; } \geq 18 \text { years of age; SLE and with lupus nephritis currently stable for the last } 3 \text { months without } \\
\text { change in treatment for lupus nephritis; stable renal disease; no flaring of other organ systems in a } \\
\text { minimum of the last } 3 \text { months }\end{array}$ \\
\hline Interventions & $\begin{array}{l}\text { 1. Abatacept: IV } 30 \mathrm{mg} / \mathrm{kg} \text {, single dose at day } 1 \text { and IV } 10 \mathrm{mg} / \mathrm{kg} \text { on days } 15 \text { and } 29 \text { followed by doses } \\
\text { every } 4 \text { weeks until the end of the study }\end{array}$ \\
\hline 2. Placebo: IV \\
\hline $\begin{array}{l}\text { 1. Death } \\
\text { 2. Adverse events } \\
\text { 3. Clinical characteristics: e.g. blood pressure, heart rate }\end{array}$ \\
\hline Starting date & August 2008 \\
\hline Contact information & Study includes short-term follow-up period and long-term extension period
\end{tabular}


NCT00881309

Trial name or title

To compare the efficacy and safety of tripterygium vs azathioprine in the maintenance therapy for lupus nephritis

\begin{tabular}{ll}
\hline Methods & RCT \\
\hline Participants & Adults, class III-V lupus nephritis (biopsy-proven) \\
\hline Interventions & $\begin{array}{l}\text { Induction with MMF, CPA, TAC or multi-target therapy followed by randomisation to either AZA } \\
\text { maintenance therapy or tripterygium } 90 \text { mg once/d }\end{array}$ \\
\hline Outcomes & Complete remission \\
\hline Starting date & March 2009 \\
\hline Contact information & Weixin Hu, Nanjing University School of Medicine, China \\
\hline Notes & \\
\hline
\end{tabular}

NCT01056237

\begin{tabular}{ll}
\hline Trial name or title & Long-term study of multi-target therapy as maintenance treatment for lupus nephritis \\
\hline Methods & Open-label RCT \\
\hline Participants & $\begin{array}{l}\text { Adults ( } 18 \text { to } 65 \text { years); SLE; diagnosed class } \mathbb{I}, \mathbb{N}, \mathbb{N}+\mathrm{V}, \mathbb{I}+\mathrm{V} \text { or } \vee \text { lupus nephritis (ISN/RPS 2003 } \\
\text { criteria) by renal biopsy; all patients had received induction therapy for } 6 \text { months with multi-thera- } \\
\text { py (FK506 + MMF) or IV CPA pulses. Patients were recruited when received partial remission or com- } \\
\text { plete remission after } 6 \text { months induction therapy }\end{array}$ \\
\hline Interventions & $\begin{array}{l}\text { 1. Multi-target therapy: TAC }(1 \text { to } 3 \mathrm{mg} / \mathrm{d}) \text { and MMF }(0.5 \text { to } 0.75 \mathrm{~g} / \mathrm{d}) \\
\text { 2. AZA: } 1.0 \text { to } 2.0 \mathrm{mg} / \mathrm{kg} / \mathrm{d}\end{array}$ \\
\hline Outcomes & Safety and efficacy \\
\hline Contact information date & February 2010 \\
\hline Notes & Zhi-Hong Liu, Nanjing University School of Medicine \\
\hline
\end{tabular}

\section{NCT01172002}

\begin{tabular}{ll}
\hline Trial name or title & Leflunomide versus AZA for maintenance therapy of lupus nephritis \\
\hline Methods & Open-label RCT \\
\hline Participants & Adults, biopsy-proven proliferative lupus nephritis \\
\hline Interventions & Leflunomide versus AZA (maintenance therapy) \\
\hline Outcomes & Lupus nephritis flare \\
\hline
\end{tabular}


NCT01172002 (Continued)

Starting date

March 2010

Contact information

Bao Chun De, Renji Hospital

Notes

NCT01284725

\begin{tabular}{ll}
\hline Trial name or title & Weaning of Immunosuppression in Nephritis of Lupus \\
\hline Methods & Open-label RCT \\
\hline Participants & Adult, biopsy-proven proliferative lupus nephritis \\
\hline Interventions & Immunosuppressive treatment discontinuation versus continuation of MMF or AZA \\
\hline Outcomes & Discontinuation of maintenance immunosuppressive therapy \\
\hline Starting date & January 2011 \\
\hline Contact information & Noemie Jourde Chiche, Assistance Publique hôpitaux de Marseille \\
\hline Notes & \\
\hline
\end{tabular}

\section{NCT01639339}

Trial name or title A phase 3, randomised, double-blind, placebo-controlled study to evaluate the efficacy and safety of Belimumab plus standard of care versus placebo plus standard of care in adult subjects with active lupus nephritis

\begin{tabular}{ll}
\hline Methods & Double-blind, placebo controlled RCT \\
\hline Participants & $\begin{array}{l}\text { Adult, diagnosis of SLE (ACR criteria), biopsy-proven lupus nephritis, clinically active lupus nephri- } \\
\text { tis, autoantibody positive }\end{array}$ \\
\hline Interventions & Belimumab versus placebo and standard therapy \\
\hline Outcomes & Renal response, complete renal response, adverse events \\
\hline Starting date & July 2012 \\
\hline Contact information & GlaxoSmithKline \\
\hline
\end{tabular}

\section{Notes}

\section{NCT01714817}

\begin{tabular}{ll}
\hline Trial name or title & A phase 3 randomised, double-blind, placebo-controlled study to evaluate the efficacy and safe- \\
ty of BMS-188667 (Abatacept) or placebo on a background of mycophenolate mofetil and corticos- \\
teroids in the treatment of subjects with active class III or IV lupus nephritis
\end{tabular}


NCT01714817 (Continued)

Methods Double-blind RCT
Participants
Age > 16 years; biopsy-proven class III or IV lupus nephritis within 12 months; UPCR $\geq 1$; $\mathrm{SCr} \leq 3 \mathrm{mg}$ / $\mathrm{dL}$ (i.e., $\leq 265 \mu \mathrm{mol} / \mathrm{L}$ ); active disease within 3 months - based on one of the following (1) worsening of lupus nephritis - UPCR $\geq 3$ (2) active urine sediment (3) biopsy within 3 months indicating active class III or IV

Inclusion criteria for the long-term extension period: achieved complete or partial renal response after completing 2 years of double-blind treatment
1. BMS-188667 + MMF + Prednisone: BMS- $18866730 \mathrm{mg} / \mathrm{kg}$ injection by IV on days $1,15,29$, and 57 , followed by a weight-tiered dose approximating $10 \mathrm{mg} / \mathrm{kg}$ injection by IV every 4 weeks, MMF 1.5 $\mathrm{g}$ tablet by mouth and prednisone up to $60 \mathrm{mg}$ tablet by mouth daily for 104 weeks

2. Placebo matching with BMS-188667 injection by IV on Days $1,15,29$, and 57 , followed by every 4 weeks, MMF $1.5 \mathrm{~g}$ tablet by mouth and prednisone up to $60 \mathrm{mg}$ tablet by mouth daily for 104 weeks

\begin{tabular}{ll}
\hline Outcomes & Renal response \\
\hline Starting date & January 2013 \\
\hline Contact information & Bristol-Myers Squibb \\
\hline Notes & \\
\hline
\end{tabular}

\section{NCT01845740}

$\begin{array}{ll}\text { Trial name or title } & \begin{array}{l}\text { A phase lb study of milatuzumab administered subcutaneously in patients with active systemic lu- } \\ \text { pus Erythematosus }\end{array}\end{array}$

\begin{tabular}{|c|c|}
\hline Methods & Double-blind RCT \\
\hline Participants & $\begin{array}{l}\text { Adult } \geq 18 \text { years; SLE (ACR criteria); positive ANA (titre } \geq 1: 80 \text { ); at least } 1 \text { BILAG A or } 2 \text { BILAG B scores } \\
\text { in any organ/body system and } \geq 6 \text { SELENA-SLEDAI score; receiving at least } 5.0 \mathrm{mg} / \mathrm{d} \text { oral pred- } \\
\text { nisone (or equivalent) at stable doses for at least } 4 \text { weeks prior to study entry If receiving immuno- } \\
\text { suppressives or antimalarial agents, at stable doses for at least } 4 \text { weeks prior to study entry }\end{array}$ \\
\hline
\end{tabular}

\begin{tabular}{ll}
\hline Interventions & 1. High dose milatuzumab SC $250 \mathrm{mg}$ \\
& 2. Low dose milatuzumab SC $150 \mathrm{mg}$ \\
3. Placebo SC
\end{tabular}

\begin{tabular}{ll}
\hline Outcomes & Safety and efficacy \\
\hline Starting date & January 2015 \\
\hline Contact information & $\begin{array}{l}\text { Heather Horne, Cedars Sinai Medical Center-Wallace Rheumatic Study centre, California, United } \\
\text { States of America }\end{array}$ \\
\hline Notes & \\
\hline
\end{tabular}

\section{NCT01861561}

\section{Trial name or title}

Efficacy and infectious complications of induction therapy with low-dose versus high-dose intravenous cyclophosphamide for proliferative lupus nephritis in children 
NCT01861561 (Continued)

\begin{tabular}{ll} 
Methods & Open-label RCT \\
\hline Participants & $\begin{array}{l}\text { Children ( } \leq 15 \text { years), diagnosis of SLE (ACR } 1997 \text { criteria), biopsy-proven class III or IV lupus nephri- } \\
\text { tis (ISN/RPS } 2003 \text { classification criteria) }\end{array}$ \\
\hline Interventions & High-dose IV CPA versus low-dose IV CPA (induction therapy) \\
\hline Outcomes & Complete renal response, partial renal response, infection, quality of life, disease activity \\
\hline Starting date & May 2013 \\
\hline Contact information & Nuntawan Piyaphanee, Siriraj Hospital, Thailand \\
\hline Notes &
\end{tabular}

\section{NCT02226341}

\begin{tabular}{ll}
\hline Trial name or title & $\begin{array}{l}\text { Open-label prospective randomised study to determine the efficacy and safety of two dosing regi- } \\
\text { mens of ACTHar in the treatment of proliferative lupus nephritis }\end{array}$ \\
\hline Methods & Open-label RCT \\
\hline Participants & $\begin{array}{l}\geq 16 \text { years, diagnosis of SLE (ACR/SLICC criteria), biopsy-proven class III or IV } \pm V \text { lupus nephritis } \\
\text { (ISN/RPS } 2003 \text { classification criteria) }\end{array}$ \\
\hline Interventions & CellCept daily \& ACTHar gel biweekly versus CellCept daily \& ACTHar gel every other day \\
\hline Outcomes & $\begin{array}{l}\text { Complete response, partial response, renal flares, adverse events, cortisol levels, urinary lympho- } \\
\text { cytes }\end{array}$
\end{tabular}

\begin{tabular}{ll}
\hline Starting date & October 2014 \\
\hline Contact information & Anca D Askanase, Columbia University, USA \\
\hline
\end{tabular}

Notes

\section{NCT02256150}

\section{Trial name or title}

\begin{tabular}{ll}
\hline Methods & Open-label RCT \\
\hline Participants & $\begin{array}{l}\text { Adult, diagnosis of SLE (ACR 1997 criteria), biopsy-proven class III, III+V, IV, IV+V or V (ISN/RPS 2003 } \\
\text { classification criteria), proteinuria > 1 g/d, SLEDAl > 8, patient body weight 40-80kg at screening }\end{array}$ \\
\hline Interventions & Mizoribine versus CPA \\
\hline Outcomes & $\begin{array}{l}\text { Complete remission, partial remission, treatment failure, ESKD, doubling of SCr, SCr, eGFR, C3, an- } \\
\text { ti-dsDNA, anti-phospholipid, anti-Sm, SLEDAI }\end{array}$
\end{tabular}

A multi-center, randomised, controlled, open-label clinical study to evaluate the efficacy and safety of mizoribine in comparison with cyclophosphamide in the treatment of lupus nephritis 
NCT02256150 (Continued)

Contact information

Asahi Kasei Pharma Corporation

\section{Notes}

\section{NCT02260934}

Trial name or title

Rituximab plus cyclophosphamide followed by belimumab for the treatment of lupus nephritis (ITN055Al)

\begin{tabular}{ll}
\hline Methods & Open-label RCT \\
\hline Participants & $\begin{array}{l}\text { Adult, diagnosis of SLE (ACR criteria), biopsy-proven proliferative lupus nephritis (ISN/RPS classi- } \\
\text { fication criteria), }>5 \text { RBC/HPF in absence of menses and infection, }>\text { WBC/HPF in absence of infec- } \\
\text { tion or cellular casts, UPCR }>1\end{array}$
\end{tabular}

\begin{tabular}{ll}
\hline Interventions & RTX, CPA and belimumab versus RTX and CPA \\
\hline Outcomes & $\begin{array}{l}\text { Major infection, hypogammaglobulinaemia, complete response, partial response, treatment fail- } \\
\text { ure, relapse anti-dsDNA, C3 and C4, death, leucopenia, ovarian failure, malignancy, thrombocy- } \\
\text { topenia, adverse advents }\end{array}$
\end{tabular}

\begin{tabular}{ll}
\hline Starting date & October 2014 \\
\hline Contact information & $\begin{array}{l}\text { Betty Diamond, Feinstein Institute for Medical Research: centre for Autoimmune and Musculoskele- } \\
\text { tal Diseases, USA }\end{array}$
\end{tabular}

\section{Notes}

\section{NCT02457221}

Trial name or title A phase III, randomised, open, parallel-controlled, multi-center study to compare the efficacy and safety of tacrolimus capsules and cyclophosphamide injection in treatment of lupus nephritis

\begin{tabular}{|c|c|}
\hline Methods & Open-label RCT \\
\hline Participants & $\begin{array}{l}\text { 18-60 years, } 18.5 \leq \mathrm{BMI}<27 \text {, diagnosis of SLE (ACR } 1997 \text { criteria), biopsy-proven class III, IV, V, III+V, } \\
\text { IV+V lupus nephritis (ISN/RPS } 2003 \text { classification criteria) within } 24 \text { weeks of study entry, protein- } \\
\text { uria }>1.5 \mathrm{~g} / \mathrm{d}, \mathrm{SCr}<3 \mathrm{mg} / \mathrm{dL}\end{array}$ \\
\hline Interventions & TAC versus CPA (induction therapy) \\
\hline Outcomes & $\begin{array}{l}\text { Complete remission, partial remission, proteinuria, serum albumin, } \mathrm{SCr} \text {, eGFR, anti-dsDNA and } \\
\text { ANA, SLEDAI, C3 and C4, renal biopsy active index and chronic index }\end{array}$ \\
\hline Starting date & March 2015 \\
\hline Contact information & Astellas Pharma China, Inc. \\
\hline
\end{tabular}

\section{Notes}


NCT02547922

Trial name or title

\section{Methods}

Participants

Interventions

Outcomes

Starting date

Contact information

A multicentre, randomised, double-blind, placebo-controlled, phase 2 study evaluating the efficacy and safety of Anifrolumab in adult subjects with active proliferative lupus nephritis

\section{Double-blind RCT}

18 to 70 years, fulfil four or more of the ACR 1982 criteria which must include positive ANA, elevated anti-dsDNA, anti-Smith; biopsy-proven class III $\pm \mathrm{V}$, IV $\pm \mathrm{V}$, UPCR $1 \mathrm{~g} / \mathrm{d}$, eGFR $\geq 35 \mathrm{~mL} / \mathrm{min} / 1.73 \mathrm{~m}^{2}$, women of childbearing potential must have negative serum beta-hCG
High-dose anifrolumab, low-dose anifrolumab versus placebo

Complete renal response, partial renal response, eGFR, proteinuria, urine sediment, adverse events

AstraZeneca Clinical Study Information centre

Notes

\section{NCT02550652}

\section{Trial name or title}

Methods

Participants

A randomised, double-blind, placebo-controlled, multi-center study to evaluate the safety and efficacy of Obinutuzumab in patients with ISN/RPS 2003 Class III or IV lupus nephritis 
NCT02630628 (Continued)

Participants

Adult, biopsy-proven lupus nephritis Class III/IV \pm V (ISN/RPS 2003 classification criteria), positive anti-dsDNA, UPCR $>1.0 \mathrm{~g}$ or $24 \mathrm{~h}$ urine protein $>1.0 \mathrm{~g} / \mathrm{d}$ at baseline), with or without haematuria, new or flaring patients

\begin{tabular}{ll}
\hline Interventions & TAC versus MMF \\
\hline Outcomes & Renal response \\
\hline Starting date & September 2015 \\
\hline Contact information & Tak-Mao Daniel Chan, The University of Hong Kong
\end{tabular}

Notes

\section{NCT02770170}

Trial name or title

A double-blind, randomised, placebo-controlled trial evaluating the effect of BI 655064 administered as sub-cutaneous injections, on renal response after one year treatment in patients with lupus nephritis

\begin{tabular}{ll}
\hline Methods & Double-blind, placebo-controlled RCT \\
\hline Participants & $\begin{array}{l}\text { 18-70 years, diagnosis of SLE (ACR criteria), biopsy-proven class III or IV lupus nephritis (ISN/RPS } \\
2003 \text { classification criteria), proteinuria } \geq 1.0 \mathrm{~g} / \mathrm{d} \text { (UPCR } \geq 100 \mathrm{mg} / \mathrm{mmol})\end{array}$ \\
\hline Interventions & BI 655064 (anti-CD-40 antibody) versus placebo \\
\hline Outcomes & Complete renal response, partial response \\
\hline Starting date & January 2016 \\
\hline Contact information & Boehringer Ingelheim \\
\hline
\end{tabular}

Notes

\begin{tabular}{ll}
\hline NCT02936375 & \\
\hline Trial name or title & Opuratimod as treatment for active lupus nephritis \\
\hline Methods & $\begin{array}{l}\text { Diagnosis of SLE (ACR criteria), biopsy-proven class III, IV, V, III+IV or IV+V active lupus nephritis, pro- } \\
\text { teinuria 1g/d, body weight } \geq 40 \mathrm{~kg}, \text { SLEDAl-2K } \geq 8 \text {, agreement of contraception }\end{array}$ \\
\hline Interventions & Iguratimod versus CPA and AZA \\
\hline Outcomes & $\begin{array}{l}\text { Renal remission, renal flare, adverse events, disease activity (SLEDAl-2K, BILAG), patient general as- } \\
\text { sessment }\end{array}$ \\
\hline Starting date & March 2017 \\
\hline Contact information & Chunde Bao, RenJi Hospital \\
\hline
\end{tabular}


NCT02936375 (Continued)

Notes

\begin{tabular}{ll}
\hline Methods & Open-label RCT \\
\hline Participants & $\begin{array}{l}18 \text { to } 80 \text { years, biopsy-proven class III or IV } \pm \text { V lupus nephritis lupus nephritis (ISN/RPS 2003 classifi- } \\
\text { cation criteria), active lupus nephritis indicated by proteinuria }>1 \mathrm{~g} / \mathrm{d} \text { and/or rise in SCr by } 15 \%\end{array}$ \\
\hline Interventions & $\begin{array}{l}\text { MMF (induction and maintenance therapy) versus CPA (induction therapy) and AZA (maintenance } \\
\text { therapy) }\end{array}$ \\
\hline Outcomes & $\begin{array}{l}\text { Lymphocyte subset profile (CD8+ T cells, CD4+ Th1, Th2, Th17 \& Treg), Naïve \& memory B cells, } \\
\text { plasma cells, serum cytokine profile (IL-2, IL-5, IL-6, IL-7, IL-10, IL-17, IL-21, IL-23, IFN-alpha, IFN- } \\
\text { gamma, TGF-beta) }\end{array}$ \\
\hline Starting date & March 2012 \\
\hline Contact information & Desmond Yap, Queen Mary Hospital, Hong Kong \\
\hline Notes &
\end{tabular}

\section{NCT03021499}

Trial name or title
( $23.7 \mathrm{mg}$ twice daily) with placebo in achieving renal response in subjects with active lupus nephri-
tis

\begin{tabular}{ll}
\hline Methods & Double-blind RCT \\
\hline Participants & Subjects with evidence of active nephritis, defined as follows: Kidney biopsy result within 2 years \\
& prior to screening indicating Class III, IV-S, IV-G (alone or in combination with Class V), or Class V lu- \\
pus nephritis with a doubling or greater increase of UPCR within the last 6 months to a minimum \\
of $\geq 1.5 \mathrm{mg} / \mathrm{mg}$ for Class III/IV or to a minimum of $\geq 2 \mathrm{mg} / \mathrm{mg}$ for Class V at screening. Biopsy results \\
over $6 \mathrm{months}$ prior to screening must be reviewed with a medical monitor to confirm eligibility. \\
Or kidney biopsy result within 6 months prior to screening indicating Class III, IV-S or IV-G (alone \\
or in combination with Class V) lupus nephritis with a UPCR of $\geq 1.5 \mathrm{mg} / \mathrm{mg}$ at screening. Or kidney \\
biopsy result within 6 months prior to screening indicating Class V lupus nephritis and a UPCR of \\
$\geq 2 \mathrm{mg} / \mathrm{mg}$ at screening. Women of childbearing potential must have a negative serum pregnancy \\
test at screening and a negative urine pregnancy test at baseline.
\end{tabular}

Interventions $\quad$ 1. Voclosporin oral, $23.7 \mathrm{mg}$ BID

2. Voclosporin placebo, oral, 3 capsules BID

\begin{tabular}{ll}
\hline Outcomes & 1. Renal response \\
2. Partial renal response \\
3. kidney function \\
4. Disease activity - SELENA-SLEDAI \\
5. Quality of life
\end{tabular}


NCT03021499 (Continued)

Starting date May 2017

\section{Contact information}

Notes
Mary Anne Dooley, University of North Carolina

Sponsor - Aurinia Pharmaceuticals Inc.

\section{NCT03214731}

\begin{tabular}{|c|c|}
\hline Trial name or title & Efficacy and safety of artesunate plus standard of care in active lupus nephritis (AURORA) \\
\hline Methods & Multicentre, double-blind RCT \\
\hline Participants & $\begin{array}{l}14 \text { to } 65 \text { years; SLE (ACR criteria); renal biopsy within } 6 \text { months prior to randomisation with a histo- } \\
\text { logical diagnosis (ISN/RPS } 2003 \text { classification of lupus nephritis) - class III, IV, V, III+V and IV+V (ex- } \\
\text { cluding Class III(C), IV-S(C), and IV-G(C)); class IV or IV+V lupus nephritis: proteinuria } \geq 1 \mathrm{~g} / 24 \mathrm{~h} \text { (or } \\
\mathrm{UPCR} \geq 1.0 \text { ) or } \mathrm{SCr}>1.3 \mathrm{mg} / \mathrm{dL} \text {, with active urinary sediment ( }>5 \mathrm{RBC} / \mathrm{HPF} \text { or }>5 \mathrm{WBC} / \mathrm{HPF} \text { (or with- } \\
\text { in the reference range of the laboratory) in absence of menses and genitourinary tract infection, } \\
\text { or presence of cellular casts (RBC or WBC casts)); Class III, III+V or V lupus nephritis: proteinuria } \geq \\
2 \mathrm{~g} / 24 \mathrm{~h} \text { (or UPCR } \geq 2.0 \text { ) or SCr }>1.3 \mathrm{mg} / \mathrm{dL} \text {; Provision of written informed consent by subject or } \\
\text { guardian }\end{array}$ \\
\hline
\end{tabular}

\begin{tabular}{ll}
\hline Interventions & $\begin{array}{l}\text { 1. High-dose artesunate: } 50 \mathrm{mg} \\
\text { 2. Low-dose artesunate: } 25 \mathrm{mg} \\
\text { 3. Placebo } \\
\text { - All patients received standard of care }\end{array}$ \\
\hline Outcomes & $\begin{array}{l}\text { 1. Complete remission } \\
\text { 2. Partial remission }\end{array}$ \\
\hline Starting date & September 2017 \\
\hline Nontact information & Xue Qing Yu, The 1st Affiliated Hospital, Sun Yet-sen University, Guangzhou, Guangdong, China \\
\hline
\end{tabular}

\section{PER-062-15}

Trial name or title

\section{Methods}

Participants
A multicentre, randomised, double-blind, placebo-controlled, phase 3 study evaluating the efficacy and safety of two doses of anifrolumab in adult subjects with active systemic lupus erythematosus

\section{Multicentre, double-blind RCT}

Aged 18 - 70 years; weight $\geq 40.0 \mathrm{~kg}$; adequate peripheral venous access; SLE (ACR criteria); currently receiving at least 1 of the following: (a) a dose of oral prednisone $(\leq 40 \mathrm{mg} / \mathrm{d})$ for a minimum of 2 weeks, the dose of oral prednisone the subject is taking must be stable for a minimum of 2 weeks prior to Week 0 (Day 1) (b) Any of the following medications administered for a minimum of 12 weeks prior to signing the informed consent, and at a stable dose for a minimum of 8 weeks prior to Day 1. 
PER-062-15 (Continued)

- Investigational product will be administered every 4 weeks from Week 0 to Week 48 for a total of 13 doses.
1. SLE Responder Index

2. Disease activity - SLEDAI, BILAG

3. Cutaneous Lupus Erythematosus Disease Area and Severity Index (CLASI) activity

4. Immunological function

\begin{tabular}{ll}
\hline Starting date & May 2015 \\
\hline Contact information & Luis Fernando Bellatin Vargas, Hogar Clínica, San Juan De Dios-Arequipa \\
\hline Notes & \\
\hline
\end{tabular}

\section{RING 2015}

\begin{tabular}{ll}
\hline Trial name or title & $\begin{array}{l}\text { RING, an investigator-initiated trial aimed at testing the efficacy of rituximab in refractory lupus } \\
\text { nephritis: Rationale, trial design and call for participation (abstract) }\end{array}$ \\
\hline Methods & RCT \\
\hline Participants & $\begin{array}{l}\text { SLE, age }>15 \text { years old, ISN/RPS Class III, IV or V lupus nephritis (biopsy within } 24 \text { months), refrac- } \\
\text { tory lupus nephritis with previous treatment with Euro-lupus/NIH CPA or AZA or MMF, maximum } 10 \\
\text { mg prednisolone/d, UPCR }>1(\mathrm{mg} / \mathrm{mg}), \text { and female patients on contraception }\end{array}$
\end{tabular}

\begin{tabular}{ll}
\hline Interventions & 1. RTX \\
& 2. Standard of care
\end{tabular}

\begin{tabular}{ll}
\hline Outcomes & Complete response (UPCR $\leq 0.5$ (expressed in $\mathrm{mg} / \mathrm{mg}$ ) measured in a 24 h urine collection; and \\
eGFR $\geq 60 \mathrm{~mL} / \mathrm{min}$ or, if $<60 \mathrm{~mL} / \mathrm{min}$ at screening, not fallen by $>20 \%$ compared to screening; and \\
no increase of glucocorticoids throughout the study (except for two limited courses as per proto- \\
col; vide infra); and no introduction of another immunosuppressant.)
\end{tabular}

\begin{tabular}{ll}
\hline Starting date & August 2012 \\
\hline Contact information & Frédéric A. Houssiau, Université Catholique de Louvain, Belgium \\
\hline
\end{tabular}

\section{Notes}

\section{RITUXILUP 2013}

Trial name or title Phase 3 open label randomised multicentre controlled trial of rituximab and mycophenolate mofetil without oral steroids for the treatment of lupus nephritis

\begin{tabular}{ll}
\hline Methods & Open-label RCT \\
\hline Participants & $\begin{array}{l}12 \text { to } 75 \text { years, biopsy-proven lupus nephritis (ISN/RPS } 2003 \text { classification criteria), active lupus } \\
\text { nephritis UPCR }>1000 \mathrm{mg} / \mathrm{mmol}, \text { not planning pregnancy during study period }\end{array}$ \\
\hline Interventions & RTX versus prednisolone \\
\hline
\end{tabular}


RITUXILUP 2013 (Continued)

Outcomes

Complete renal response, major infections, serious adverse and adverse events, disease activity scores, renal flare, serum C3, C4, anti-dsDNA, quality of life

\begin{tabular}{ll}
\hline Starting date & April 2015 \\
\hline Contact information & Liz Lightstone, Hammersmith Hospital, Imperial College Healthcare NHS Trust, United Kingdom
\end{tabular}

Notes

ACEi - angiotensin-converting enzyme inhibitor; ACR - American College of Rheumatology; ARA - American Rheumatology Association; ARB - angiotensin receptor blocker; AZA - azathioprine; BILAG - British Isles Lupus Assessment Group; CKD - chronic kidney disease; CMV cytomegalovirus; CNI - calcineurin; CNS - central nervous system; CPA - cyclophosphamide; $\mathrm{CrCl}$ - creatinine clearance; CSA - cyclosporin A; DM - diabetes mellitus; EC-MPS - enteric-coated mycophenolate sodium; eGFR - estimated glomerular filtration rate; ELNT - Euro-lupus nephritis treatment; ESKD - end-stage kidney disease; ESR - erythrocyte sedimentation rate; GI - gastrointestinal: GN - glomerulonephritis; HBV - hepatitis B virus; HCV - hepatitis C virus; HIV - human immunodeficiency virus; HPF - high power field; IA - immunoadsorption; ISN/RPS - International Society of Nephrology/Renal Pathology Society; IV - intravenous; IVIG - intravenous immunoglobulin; M/F - male/ female; MMF - mycophenolate mofetil; MP - methylprednisolone; NSAID/s - nonsteroidal anti-inflammatory drug/s; PEX - plasma exchange or plasmapheresis; PLAGA - Dutch Pathology Registry; RBC - red blood cell/s; RCC - red cell count; RCT - randomised controlled trial; RTX - rituximab; SC - subcutaneous; SCr - serum creatinine; SD - standard deviation; SELENA - Safety of Estrogens in Lupus Erythematosus National Assessment; SLE - systemic lupus erythematosus; SLEDAI - SLE Disease Activity Index; SLICC - Systemic Lupus Collaborating Clinics; TAC - tacrolimus; TB - tuberculosis; WHO - World Health Organization; ISN/RPS - International Society of Nephrology/ Renal Pathology Society; UPCR - urine protein-to-creatinine ratio; WBC - white blood cell/s; WCC - white cell count

\section{DATA AND ANALYSES}

\section{Comparison 1. Mycophenolate mofetil (MMF) versus IV cyclophosphamide (CPA)}

\begin{tabular}{|c|c|c|c|c|}
\hline $\begin{array}{l}\text { Outcome or subgroup ti- } \\
\text { tle }\end{array}$ & No. of studies & $\begin{array}{l}\text { No. of partici- } \\
\text { pants }\end{array}$ & Statistical method & Effect size \\
\hline 1 Death & 8 & 826 & Risk Ratio (M-H, Random, 95\% Cl) & $1.12[0.61,2.06]$ \\
\hline 2 Remission & 9 & & Risk Ratio (M-H, Random, 95\% CI) & Subtotals only \\
\hline $\begin{array}{l}2.1 \text { Complete renal remis- } \\
\text { sion: MMF versus IV CPA }\end{array}$ & 9 & 868 & Risk Ratio (M-H, Random, 95\% Cl) & $1.17[0.97,1.42]$ \\
\hline $\begin{array}{l}\text { 2.2 Partial renal remission: } \\
\text { MMF versus IV CPA }\end{array}$ & 9 & 868 & Risk Ratio (M-H, Random, 95\% Cl) & $1.02[0.89,1.18]$ \\
\hline $\begin{array}{l}2.3 \text { Complete remission in } \\
\text { proteinuria: MMF versus IV } \\
\text { CPA }\end{array}$ & 6 & 686 & Risk Ratio (M-H, Random, 95\% Cl) & $1.16[0.85,1.58]$ \\
\hline $\begin{array}{l}\text { 2.4 Partial remission in } \\
\text { proteinuria: MMF versus IV } \\
\text { CPA }\end{array}$ & 6 & 744 & Risk Ratio (M-H, Random, 95\% Cl) & $1.03[0.91,1.18]$ \\
\hline 3 Adverse renal outcomes & 4 & & Risk Ratio (M-H, Random, 95\% Cl) & Subtotals only \\
\hline 3.1 ESKD & 3 & 231 & Risk Ratio (M-H, Random, 95\% CI) & $0.71[0.27,1.84]$ \\
\hline 3.2 Renal relapse & 1 & 140 & Risk Ratio (M-H, Random, 95\% Cl) & $0.97[0.39,2.44]$ \\
\hline
\end{tabular}




\begin{tabular}{|c|c|c|c|c|}
\hline $\begin{array}{l}\text { Outcome or subgroup ti- } \\
\text { tle }\end{array}$ & No. of studies & $\begin{array}{l}\text { No. of partici- } \\
\text { pants }\end{array}$ & Statistical method & Effect size \\
\hline $\begin{array}{l}\text { 3.3 Doubling of serum cre- } \\
\text { atinine }\end{array}$ & 1 & 40 & Risk Ratio (M-H, Random, 95\% Cl) & $0.0[0.0,0.0]$ \\
\hline 4 Stable kidney function & 6 & 641 & Risk Ratio (M-H, Random, 95\% Cl) & $1.05[0.94,1.17]$ \\
\hline 5 Ovarian failure & 3 & 539 & Risk Ratio (M-H, Random, 95\% Cl) & $0.36[0.06,2.18]$ \\
\hline 6 Menstrual irregularities & 2 & 87 & Risk Ratio (M-H, Random, 95\% Cl) & $0.33[0.07,1.59]$ \\
\hline 7 Infection & 7 & 1452 & Risk Ratio (M-H, Random, 95\% Cl) & $1.13[0.81,1.58]$ \\
\hline 7.1 Major infection & 6 & 699 & Risk Ratio (M-H, Random, 95\% Cl) & $1.02[0.67,1.54]$ \\
\hline 7.2 Herpes zoster virus & 6 & 753 & Risk Ratio (M-H, Random, 95\% Cl) & $1.39[0.78,2.46]$ \\
\hline 8 Malignancy & 1 & 364 & Risk Ratio (M-H, Random, 95\% Cl) & $0.65[0.11,3.86]$ \\
\hline 9 Leucopenia & 6 & 753 & Risk Ratio (M-H, Random, 95\% Cl) & $0.59[0.33,1.08]$ \\
\hline 10 Bladder toxicity & 1 & 364 & Risk Ratio (M-H, Random, 95\% Cl) & $0.33[0.01,7.95]$ \\
\hline 11 Alopecia & 3 & 622 & Risk Ratio (M-H, Random, 95\% Cl) & $0.29[0.19,0.46]$ \\
\hline $\begin{array}{l}12 \text { Gastrointestinal (GI) ad- } \\
\text { verse events }\end{array}$ & 4 & & Risk Ratio (M-H, Random, 95\% Cl) & Subtotals only \\
\hline 12.1 Diarrhoea & 4 & 609 & Risk Ratio (M-H, Random, 95\% Cl) & $2.42[1.64,3.58]$ \\
\hline 12.2 Vomiting & 3 & 562 & Risk Ratio (M-H, Random, 95\% Cl) & $0.48[0.24,0.97]$ \\
\hline 12.3 Nausea & 3 & 562 & Risk Ratio (M-H, Random, 95\% Cl) & $0.48[0.23,0.98]$ \\
\hline $12.4 \mathrm{GI}$ upset & 3 & 569 & Risk Ratio (M-H, Random, 95\% Cl) & $0.91[0.78,1.06]$ \\
\hline 13 Daily proteinuria & 4 & 271 & Mean Difference (IV, Random, 95\% Cl) & $-0.08[-0.43,0.26]$ \\
\hline 14 Serum creatinine & 6 & 759 & Mean Difference (IV, Random, 95\% CI) & $2.14[-3.09,7.37]$ \\
\hline
\end{tabular}

Analysis 1.1. Comparison 1 Mycophenolate mofetil (MMF) versus IV cyclophosphamide (CPA), Outcome 1 Death.

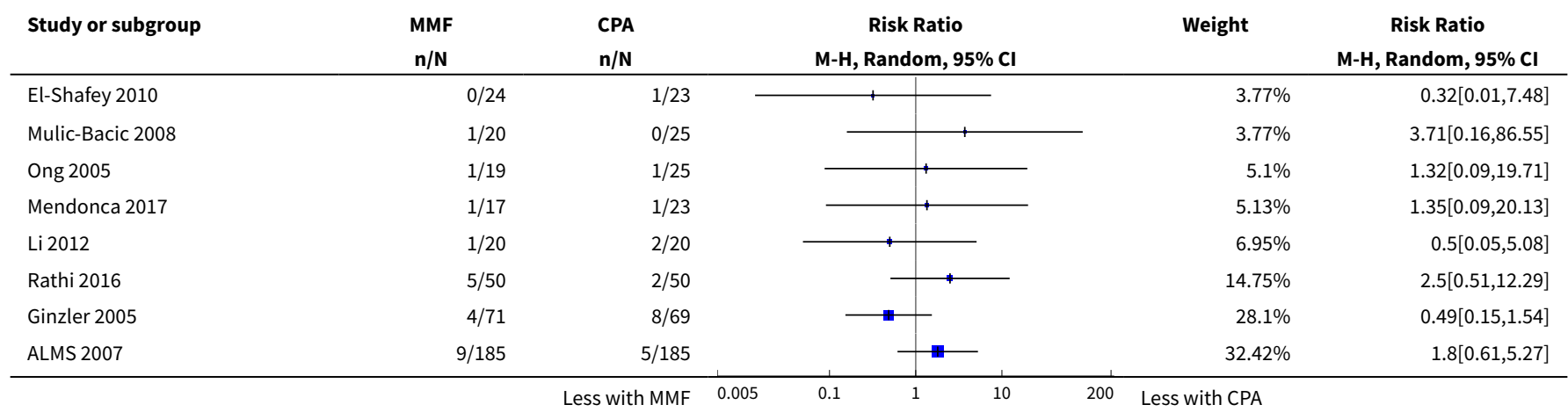




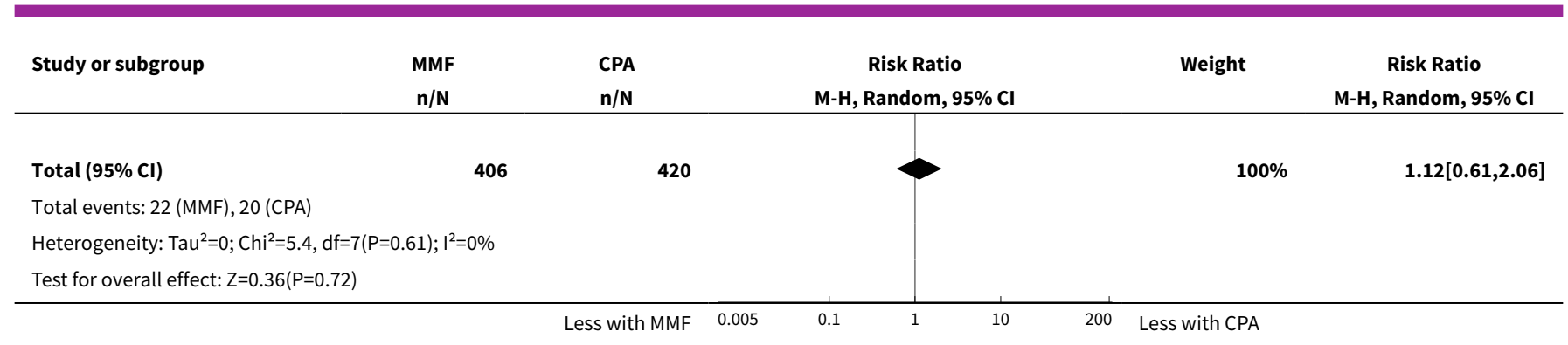

Analysis 1.2. Comparison 1 Mycophenolate mofetil (MMF) versus IV cyclophosphamide (CPA), Outcome 2 Remission.

\begin{tabular}{|c|c|c|c|c|c|}
\hline Study or subgroup & $\begin{array}{l}\text { MMF } \\
\text { n/N }\end{array}$ & $\begin{array}{l}\mathrm{CPA} \\
\mathrm{n} / \mathrm{N}\end{array}$ & $\begin{array}{c}\text { Risk Ratio } \\
\text { M-H, Random, 95\% Cl }\end{array}$ & Weight & $\begin{array}{c}\text { Risk Ratio } \\
\text { M-H, Random, 95\% Cl }\end{array}$ \\
\hline \multicolumn{6}{|c|}{ 1.2.1 Complete renal remission: MMF versus IV CPA } \\
\hline Ong 2005 & $5 / 19$ & $3 / 25$ & - & $2.22 \%$ & $2.19[0.6,8.06]$ \\
\hline Ginzler 2005 & $16 / 71$ & $4 / 69$ & & $3.44 \%$ & $3.89[1.37,11.05]$ \\
\hline El-Shafey 2010 & $6 / 24$ & $5 / 23$ & + & $3.47 \%$ & $1.15[0.41,3.25]$ \\
\hline Li 2012 & $9 / 20$ & $6 / 20$ & & $5.5 \%$ & $1.5[0.66,3.43]$ \\
\hline Mendonca 2017 & $9 / 17$ & $11 / 23$ & - & $9.81 \%$ & $1.11[0.6,2.06]$ \\
\hline Mulic-Bacic 2008 & $14 / 20$ & $15 / 25$ & & $20.34 \%$ & $1.17[0.76,1.79]$ \\
\hline Sedhain 2016 & $14 / 21$ & $14 / 21$ & $\longrightarrow$ & $20.54 \%$ & $1[0.65,1.53]$ \\
\hline Rathi 2016 & $27 / 50$ & $25 / 50$ & & $26.41 \%$ & $1.08[0.74,1.57]$ \\
\hline Subtotal $(95 \% \mathrm{Cl})$ & 427 & 441 & & $100 \%$ & $1.17[0.97,1.42]$ \\
\hline \multicolumn{6}{|c|}{ Total events: 116 (MMF), 98 (CPA) } \\
\hline \multicolumn{6}{|c|}{ Test for overall effect: $Z=1.6(P=0.11)$} \\
\hline \multicolumn{6}{|c|}{ 1.2.2 Partial renal remission: MMF versus IV CPA } \\
\hline Sedhain 2016 & $6 / 21$ & $4 / 21$ & & $1.62 \%$ & $1.5[0.49,4.56]$ \\
\hline Li 2012 & $6 / 20$ & $6 / 20$ & & $2.23 \%$ & $1[0.39,2.58]$ \\
\hline Mulic-Bacic 2008 & $5 / 20$ & $10 / 25$ & & $2.47 \%$ & $0.63[0.25,1.53]$ \\
\hline El-Shafey 2010 & $8 / 24$ & $7 / 23$ & & $2.84 \%$ & $1.1[0.47,2.53]$ \\
\hline Mendonca 2017 & $6 / 17$ & $9 / 23$ & & $2.96 \%$ & $0.9[0.4,2.05]$ \\
\hline Ong 2005 & $6 / 19$ & $10 / 25$ & + & $2.99 \%$ & $0.79[0.35,1.79]$ \\
\hline Ginzler 2005 & $21 / 71$ & $17 / 69$ & - & $6.67 \%$ & $1.2[0.69,2.07]$ \\
\hline Rathi 2016 & $37 / 50$ & $37 / 50$ & & $36.98 \%$ & $1[0.79,1.26]$ \\
\hline ALMS 2007 & $88 / 185$ & $83 / 185$ & & $41.24 \%$ & $1.06[0.85,1.32]$ \\
\hline Subtotal $(95 \% \mathrm{Cl})$ & 427 & 441 & & $100 \%$ & $1.02[0.89,1.18]$ \\
\hline \multicolumn{6}{|c|}{ Test for overall effect: $\mathrm{Z}=0.33(\mathrm{P}=0.74)$} \\
\hline \multicolumn{6}{|c|}{ 1.2.3 Complete remission in proteinuria: MMF versus IV CPA } \\
\hline Ginzler 2005 & $16 / 71$ & $4 / 69$ & & $7.36 \%$ & $3.89[1.37,11.05]$ \\
\hline El-Shafey 2010 & $6 / 24$ & $5 / 23$ & - & $7.41 \%$ & $1.15[0.41,3.25]$ \\
\hline Li 2012 & $9 / 20$ & $6 / 20$ & $\rightarrow$ & $10.74 \%$ & $1.5[0.66,3.43]$ \\
\hline Ong 2005 & $11 / 19$ & $15 / 25$ & $\rightarrow$ & $20.97 \%$ & $0.96[0.59,1.59]$ \\
\hline Mulic-Bacic 2008 & $14 / 20$ & $15 / 25$ & $\rightarrow-$ & $24.45 \%$ & $1.17[0.76,1.79]$ \\
\hline ALMS 2007 & $44 / 185$ & $50 / 185$ & - & $29.08 \%$ & $0.88[0.62,1.25]$ \\
\hline Subtotal $(95 \% \mathrm{CI})$ & 339 & 347 & & $100 \%$ & $1.16[0.85,1.58]$ \\
\hline
\end{tabular}




\begin{tabular}{|c|c|c|c|c|c|}
\hline Study or subgroup & $\begin{array}{l}\text { MMF } \\
\text { n/N }\end{array}$ & $\begin{array}{l}\mathrm{CPA} \\
\mathrm{n} / \mathrm{N} \\
\end{array}$ & $\begin{array}{c}\text { Risk Ratio } \\
\text { M-H, Random, } 95 \% \text { CI }\end{array}$ & Weight & $\begin{array}{c}\text { Risk Ratio } \\
\text { M-H, Random, } 95 \% \mathrm{Cl}\end{array}$ \\
\hline \multicolumn{6}{|c|}{ Total events: 100 (MMF), 95 (CPA) } \\
\hline \multicolumn{6}{|c|}{ Heterogeneity: $\mathrm{Tau}^{2}=0.05 ; \mathrm{Chi}^{2}=8.15, \mathrm{df}=5(\mathrm{P}=0.15) ; \mathrm{I}^{2}=38.66 \%$} \\
\hline \multicolumn{6}{|c|}{ Test for overall effect: $Z=0.93(P=0.35)$} \\
\hline Mulic-Bacic 2008 & $5 / 20$ & $10 / 25$ & - & $2.1 \%$ & $0.63[0.25,1.53]$ \\
\hline El-Shafey 2010 & $8 / 24$ & $7 / 23$ & & $2.42 \%$ & $1.1[0.47,2.53]$ \\
\hline Ginzler 2005 & $21 / 71$ & $17 / 69$ & $\longrightarrow$ & $5.68 \%$ & $1.2[0.69,2.07]$ \\
\hline Sedhain 2016 & $14 / 21$ & $14 / 21$ & $\longrightarrow$ & $9.28 \%$ & $1[0.65,1.53]$ \\
\hline Rathi 2016 & $37 / 50$ & $37 / 50$ & 1 & $31.45 \%$ & $1[0.79,1.26]$ \\
\hline ALMS 2007 & $104 / 185$ & $98 / 185$ & $\theta$ & $49.07 \%$ & $1.06[0.88,1.28]$ \\
\hline \multicolumn{6}{|c|}{ Total events: 189 (MMF), 183 (CPA) } \\
\hline \multicolumn{6}{|c|}{ Heterogeneity: $\mathrm{Tau}^{2}=0 ; \mathrm{Chi}^{2}=1.69, \mathrm{df}=5(\mathrm{P}=0.89) ; \mathrm{I}^{2}=0 \%$} \\
\hline Test for overall effec & & & & & \\
\hline
\end{tabular}

Analysis 1.3. Comparison 1 Mycophenolate mofetil (MMF) versus IV cyclophosphamide (CPA), Outcome 3 Adverse renal outcomes.

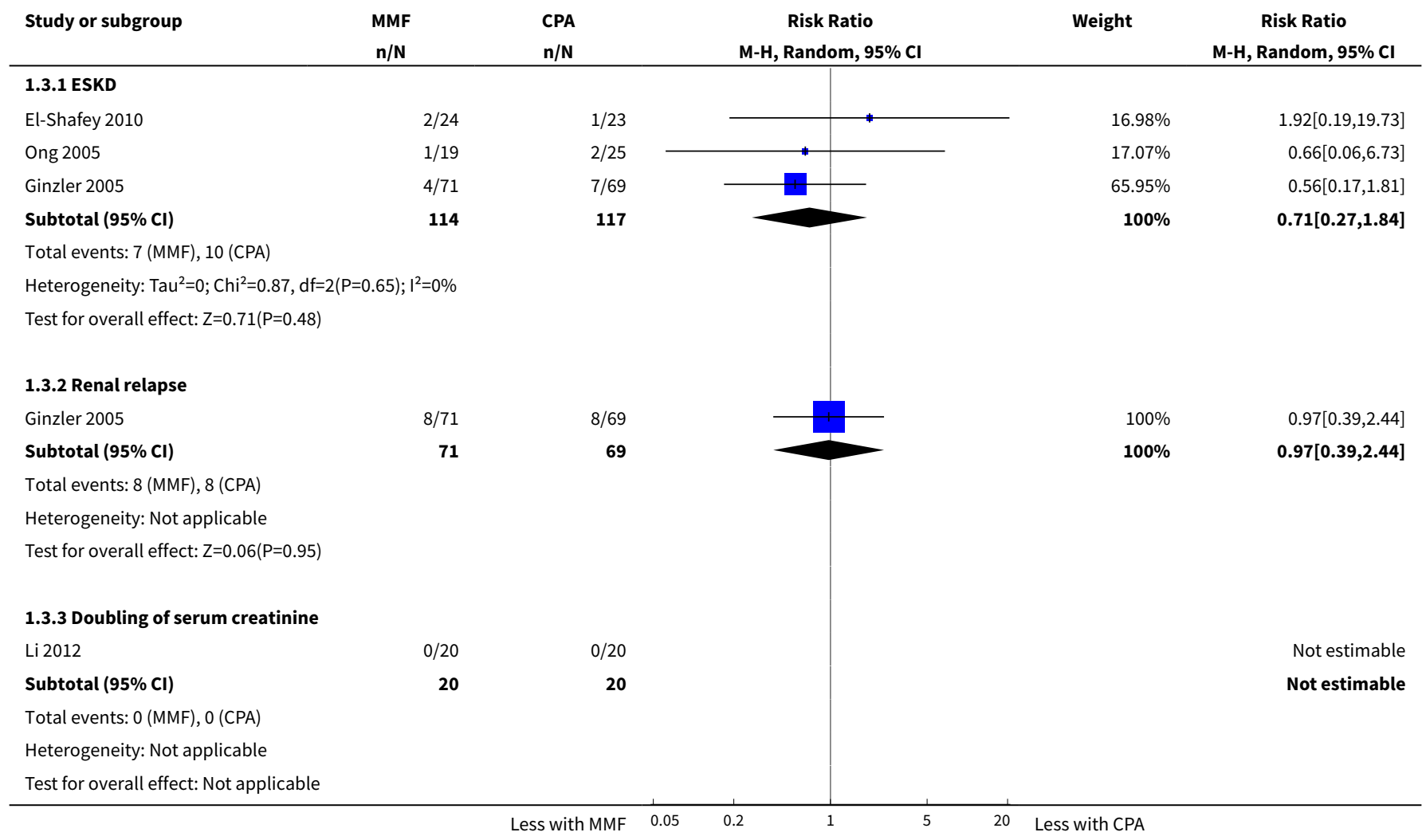


Analysis 1.4. Comparison 1 Mycophenolate mofetil (MMF) versus IV cyclophosphamide (CPA), Outcome 4 Stable kidney function.

\begin{tabular}{|c|c|c|c|c|c|}
\hline Study or subgroup & $\begin{array}{l}\text { MMF } \\
\text { n/N }\end{array}$ & $\begin{array}{l}\text { CPA } \\
\mathrm{n} / \mathrm{N}\end{array}$ & $\begin{array}{c}\text { Risk Ratio } \\
\text { M-H, Random, } 95 \% \mathrm{CI}\end{array}$ & Weight & $\begin{array}{c}\text { Risk Ratio } \\
\text { M-H, Random, 95\% Cl }\end{array}$ \\
\hline Li 2012 & $9 / 20$ & $6 / 20$ & 1 & $1.82 \%$ & $1.5[0.66,3.43]$ \\
\hline Ong 2005 & $11 / 19$ & $13 / 25$ & & $4.31 \%$ & $1.11[0.65,1.91]$ \\
\hline Sedhain 2016 & $14 / 21$ & $14 / 21$ & - & $6.81 \%$ & $1[0.65,1.53]$ \\
\hline Mulic-Bacic 2008 & $16 / 20$ & $14 / 25$ & $\longrightarrow$ & $7.38 \%$ & $1.43[0.95,2.15]$ \\
\hline Rathi 2016 & $27 / 50$ & $25 / 50$ & 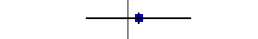 & $8.76 \%$ & $1.08[0.74,1.57]$ \\
\hline ALMS 2007 & $130 / 185$ & $130 / 185$ & & $70.91 \%$ & $1[0.88,1.14]$ \\
\hline Total $(95 \% \mathrm{CI})$ & 315 & 326 & 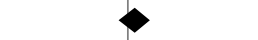 & $100 \%$ & $1.05[0.94,1.17]$ \\
\hline \multicolumn{6}{|c|}{ Total events: 207 (MMF), 202 (CPA) } \\
\hline \multicolumn{6}{|c|}{ Heterogeneity: $\mathrm{Tau}^{2}=0 ; \mathrm{Chi}^{2}=3.55, \mathrm{df}=5(\mathrm{P}=0.62) ; \mathrm{I}^{2}=0 \%$} \\
\hline Test for overall effect & & & & & \\
\hline
\end{tabular}

Analysis 1.5. Comparison 1 Mycophenolate mofetil (MMF) versus IV cyclophosphamide (CPA), Outcome 5 Ovarian failure.

\begin{tabular}{|c|c|c|c|c|c|}
\hline Study or subgroup & $\begin{array}{l}\text { MMF } \\
\text { n/N }\end{array}$ & $\begin{array}{l}\mathrm{CPA} \\
\mathrm{n} / \mathrm{N}\end{array}$ & $\begin{array}{c}\text { Risk Ratio } \\
\text { M-H, Random, 95\% Cl }\end{array}$ & Weight & $\begin{array}{c}\text { Risk Ratio } \\
\text { M-H, Random, 95\% Cl }\end{array}$ \\
\hline Ginzler 2005 & $0 / 65$ & $2 / 61$ & - & $25.17 \%$ & $0.19[0.01,3.84]$ \\
\hline Rathi 2016 & $2 / 50$ & $1 / 50$ & $\rightarrow$ & $34.54 \%$ & $2[0.19,21.36]$ \\
\hline ALMS 2007 & $1 / 157$ & $8 / 156$ & - & $40.28 \%$ & $0.12[0.02,0.98]$ \\
\hline Total $(95 \% \mathrm{Cl})$ & 272 & 267 & & $100 \%$ & $0.36[0.06,2.18]$ \\
\hline \multicolumn{6}{|c|}{ Heterogeneity: $\mathrm{Tau}^{2}=0.98 ; \mathrm{Chi}^{2}=3.26, \mathrm{df}=2(\mathrm{P}=0.2) ; \mathrm{I}^{2}=38.59 \%$} \\
\hline \multicolumn{6}{|c|}{ Test for overall effect: $Z=1.11(P=0.27)$} \\
\hline
\end{tabular}

Analysis 1.6. Comparison 1 Mycophenolate mofetil (MMF) versus IV cyclophosphamide (CPA), Outcome 6 Menstrual irregularities.

\begin{tabular}{|c|c|c|c|c|c|}
\hline Study or subgroup & $\begin{array}{l}\text { MMF } \\
\text { n/N }\end{array}$ & $\begin{array}{l}\mathrm{CPA} \\
\mathrm{n} / \mathrm{N}\end{array}$ & $\begin{array}{c}\text { Risk Ratio } \\
\text { M-H, Random, 95\% Cl }\end{array}$ & Weight & $\begin{array}{c}\text { Risk Ratio } \\
\text { M-H, Random, } 95 \% \text { CI }\end{array}$ \\
\hline El-Shafey 2010 & $1 / 24$ & $2 / 23$ & $\rightarrow-$ & $44.84 \%$ & $0.48[0.05,4.93]$ \\
\hline Li 2012 & $1 / 20$ & $4 / 20$ & 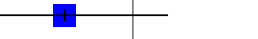 & $55.16 \%$ & $0.25[0.03,2.05]$ \\
\hline Total $(95 \% \mathrm{Cl})$ & 44 & 43 & - & $100 \%$ & $0.33[0.07,1.59]$ \\
\hline \multicolumn{6}{|c|}{ Total events: 2 (MMF), 6 (CPA) } \\
\hline Test for overall effect & & & & & \\
\hline
\end{tabular}


Analysis 1.7. Comparison 1 Mycophenolate mofetil (MMF) versus IV cyclophosphamide (CPA), Outcome 7 Infection.

\begin{tabular}{|c|c|c|c|c|c|}
\hline \multirow{2}{*}{$\begin{array}{l}\text { Study or subgroup } \\
\text { 1.7.1 Major infection }\end{array}$} & \multirow[t]{2}{*}{$\begin{array}{l}\text { MMF } \\
\mathrm{n} / \mathrm{N}\end{array}$} & \multirow[t]{2}{*}{$\begin{array}{l}\text { CPA } \\
n / N\end{array}$} & $\begin{array}{c}\text { Risk Ratio } \\
\text { M-H, Random, } 95 \% \text { Cl } \\
\end{array}$ & \multirow[t]{2}{*}{ Weight } & \multirow[t]{2}{*}{$\begin{array}{c}\text { Risk Ratio } \\
\text { M-H, Random, } 95 \% \text { Cl }\end{array}$} \\
\hline & & & & & \\
\hline Mendonca 2017 & $1 / 17$ & $3 / 23$ & & $2.39 \%$ & $0.45[0.05,3.97]$ \\
\hline Ginzler 2005 & $1 / 83$ & $6 / 75$ & & $2.58 \%$ & $0.15[0.02,1.22]$ \\
\hline El-Shafey 2010 & $2 / 24$ & $2 / 23$ & & $3.21 \%$ & $0.96[0.15,6.25]$ \\
\hline Ong 2005 & $3 / 19$ & $3 / 25$ & + & $5.12 \%$ & $1.32[0.3,5.81]$ \\
\hline ALMS 2007 & $22 / 185$ & $18 / 185$ & & $32.6 \%$ & $1.22[0.68,2.2]$ \\
\hline Subtotal $(95 \% \mathrm{Cl})$ & 348 & 351 & & $65.51 \%$ & $1.02[0.67,1.54]$ \\
\hline \multicolumn{6}{|c|}{ Total events: 37 (MMF), 40 (CPA) } \\
\hline \multicolumn{6}{|c|}{ Heterogeneity: $\mathrm{Tau}^{2}=0 ; \mathrm{Chi}^{2}=4.33, \mathrm{df}=5(\mathrm{P}=0.5) ; \mathrm{I}^{2}=0 \%$} \\
\hline \multicolumn{6}{|c|}{ Test for overall effect: $Z=0.07(P=0.94)$} \\
\hline \multicolumn{6}{|c|}{ 1.7.2 Herpes zoster virus } \\
\hline Rathi 2016 & $2 / 50$ & $1 / 50$ & & $2.01 \%$ & $2[0.19,21.36]$ \\
\hline El-Shafey 2010 & $2 / 24$ & $3 / 23$ & & $3.93 \%$ & $0.64[0.12,3.48]$ \\
\hline Ong 2005 & $3 / 19$ & $3 / 25$ & & $5.12 \%$ & $1.32[0.3,5.81]$ \\
\hline Mendonca 2017 & $3 / 17$ & $3 / 23$ & - & $5.21 \%$ & $1.35[0.31,5.9]$ \\
\hline Ginzler 2005 & $3 / 83$ & $4 / 75$ & + & $5.27 \%$ & $0.68[0.16,2.93]$ \\
\hline ALMS 2007 & $14 / 184$ & $6 / 180$ & & $12.95 \%$ & $2.28[0.9,5.81]$ \\
\hline Subtotal $(95 \% \mathrm{Cl})$ & 377 & 376 & & $34.49 \%$ & $1.39[0.78,2.46]$ \\
\hline \multicolumn{6}{|c|}{ Total events: 27 (MMF), 20 (CPA) } \\
\hline \multicolumn{6}{|c|}{ Heterogeneity: $\operatorname{Tau}^{2}=0 ; \mathrm{Chi}^{2}=2.92, \mathrm{df}=5(\mathrm{P}=0.71) ; \mathrm{I}^{2}=0 \%$} \\
\hline \multicolumn{6}{|c|}{ Test for overall effect: $Z=1.12(P=0.26)$} \\
\hline Total $(95 \% \mathrm{Cl})$ & 725 & 727 & & $100 \%$ & $1.13[0.81,1.58]$ \\
\hline \multicolumn{6}{|c|}{ Total events: 64 (MMF), 60 (CPA) } \\
\hline Test for subgroup diff & $f=1(P=0.39)$, & & & & \\
\hline
\end{tabular}

Analysis 1.8. Comparison 1 Mycophenolate mofetil (MMF) versus IV cyclophosphamide (CPA), Outcome 8 Malignancy.

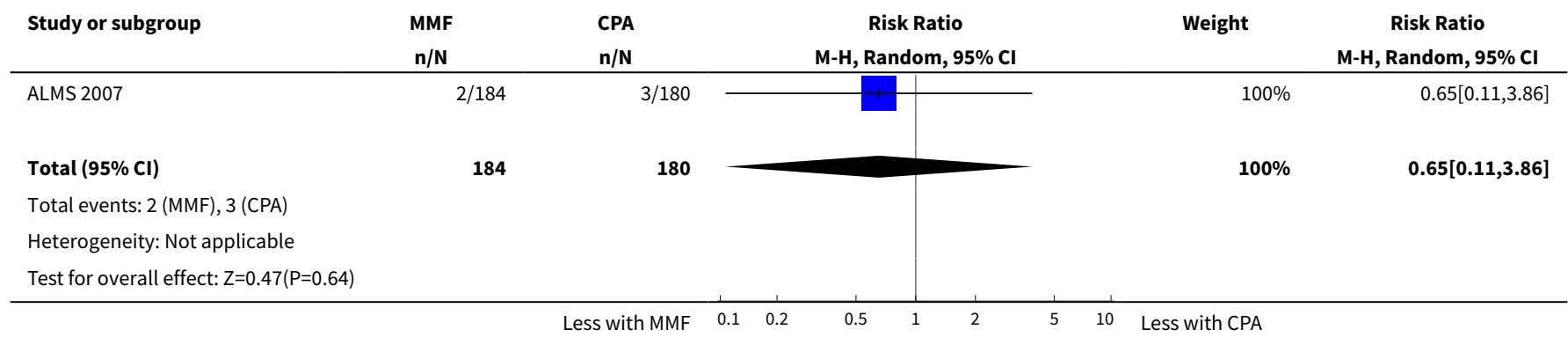


Analysis 1.9. Comparison 1 Mycophenolate mofetil (MMF) versus IV cyclophosphamide (CPA), Outcome 9 Leucopenia.

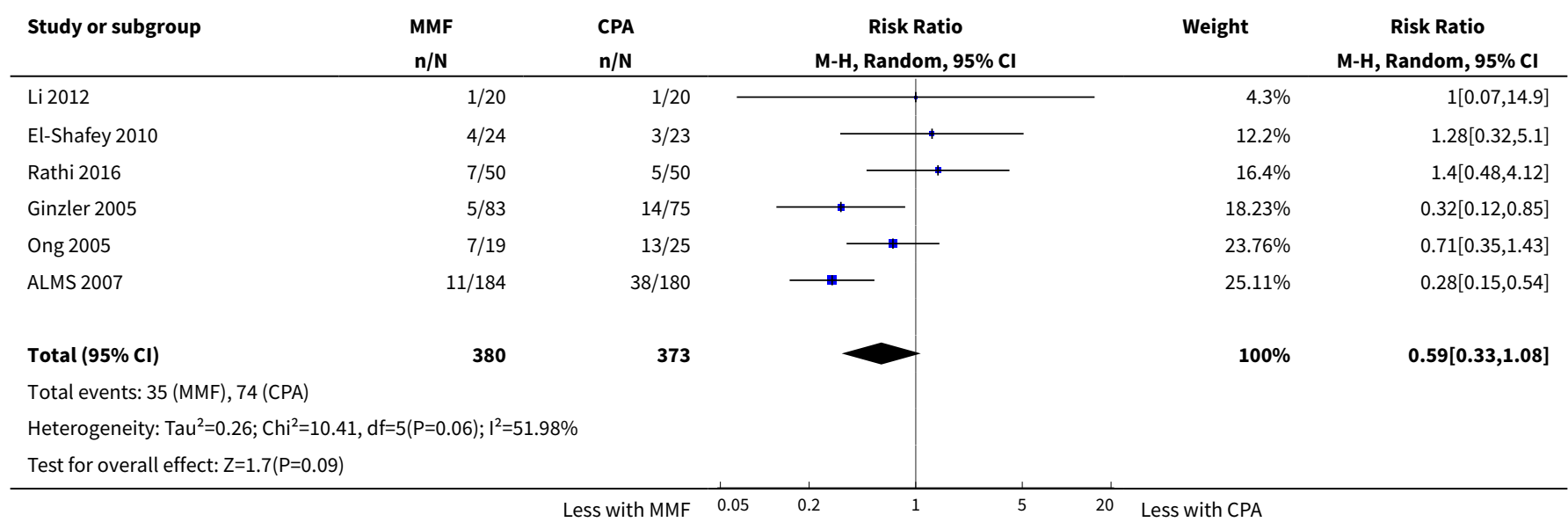

Analysis 1.10. Comparison 1 Mycophenolate mofetil (MMF) versus IV cyclophosphamide (CPA), Outcome 10 Bladder toxicity.

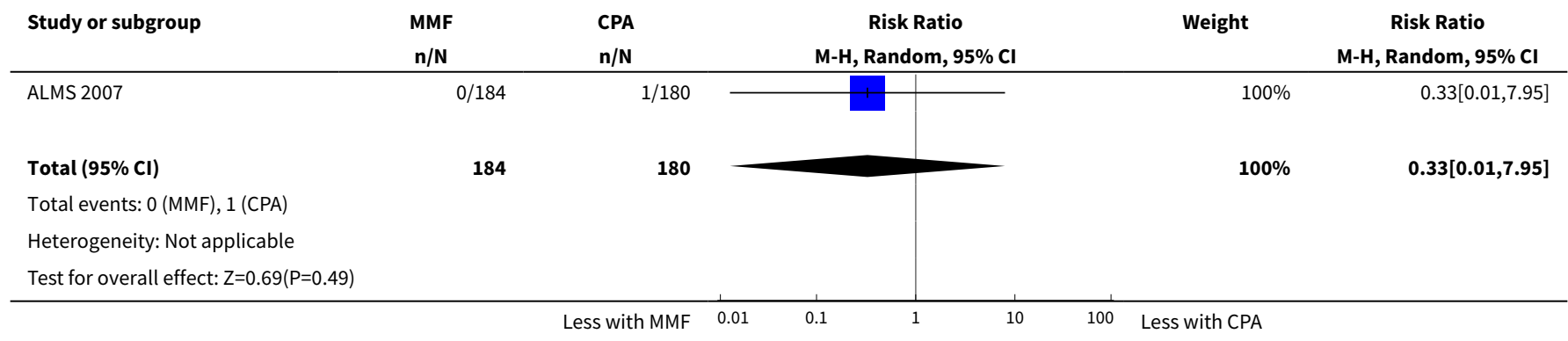

\section{Analysis 1.11. Comparison 1 Mycophenolate mofetil (MMF)} versus IV cyclophosphamide (CPA), Outcome 11 Alopecia.

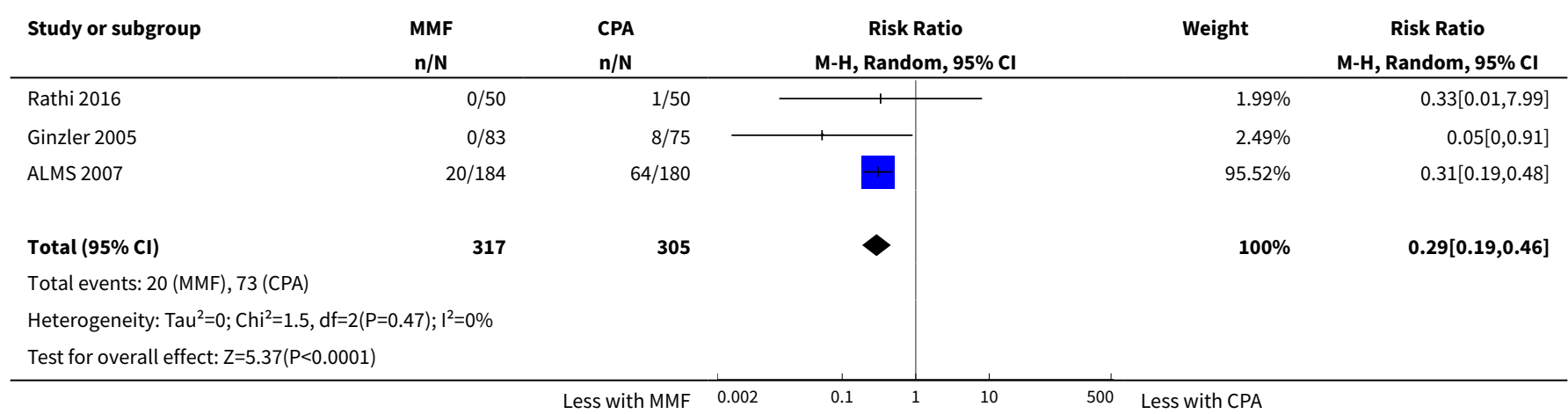


Analysis 1.12. Comparison 1 Mycophenolate mofetil (MMF) versus IV cyclophosphamide (CPA), Outcome 12 Gastrointestinal (GI) adverse events.

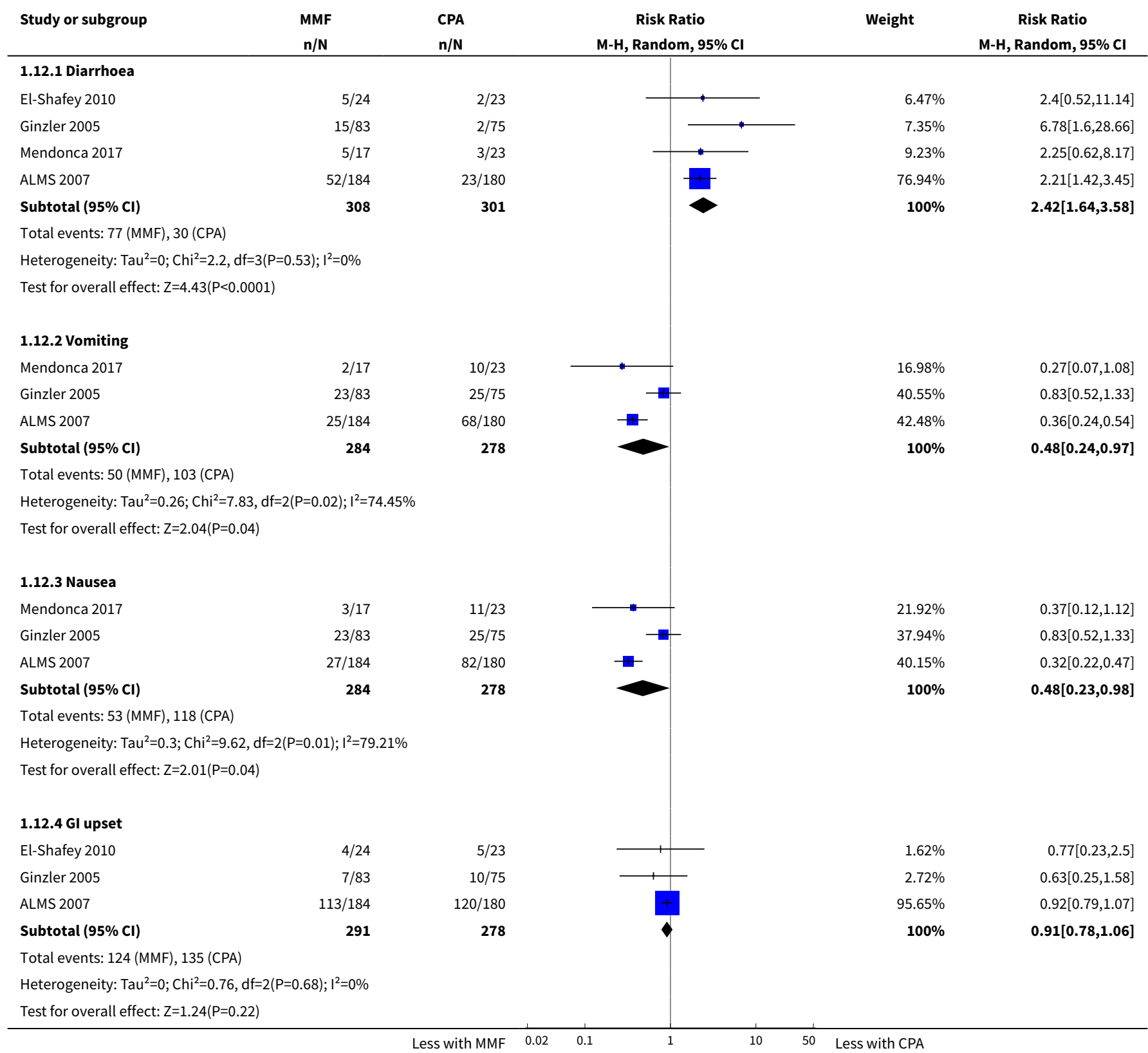

Analysis 1.13. Comparison 1 Mycophenolate mofetil (MMF) versus IV cyclophosphamide (CPA), Outcome 13 Daily proteinuria.

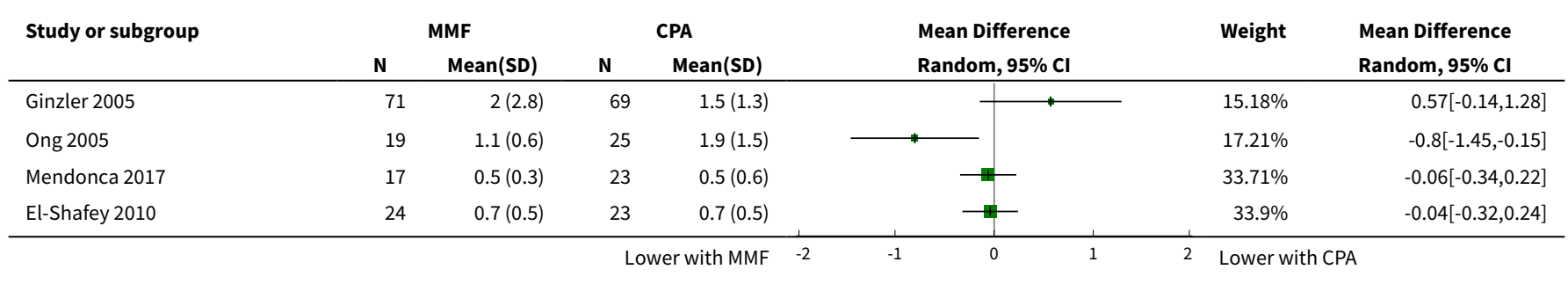




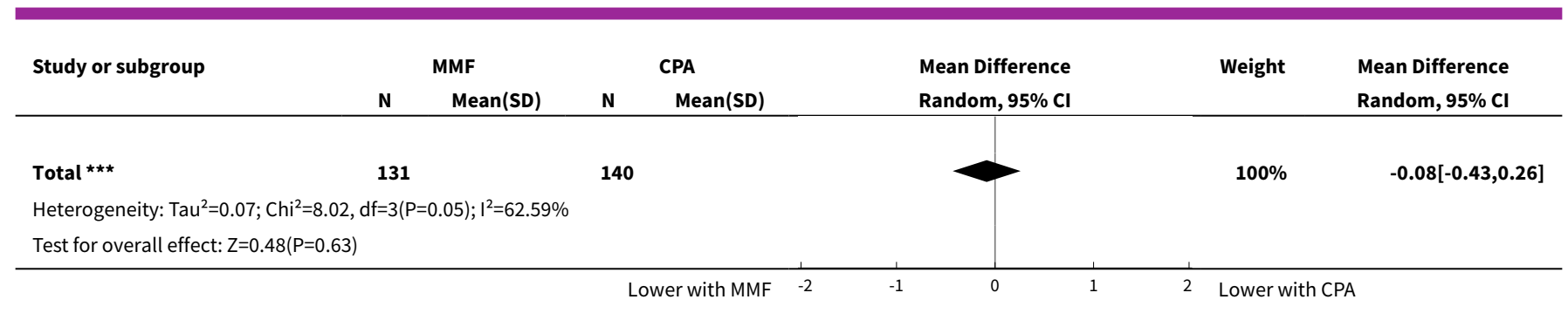

Analysis 1.14. Comparison 1 Mycophenolate mofetil (MMF) versus IV cyclophosphamide (CPA), Outcome 14 Serum creatinine.

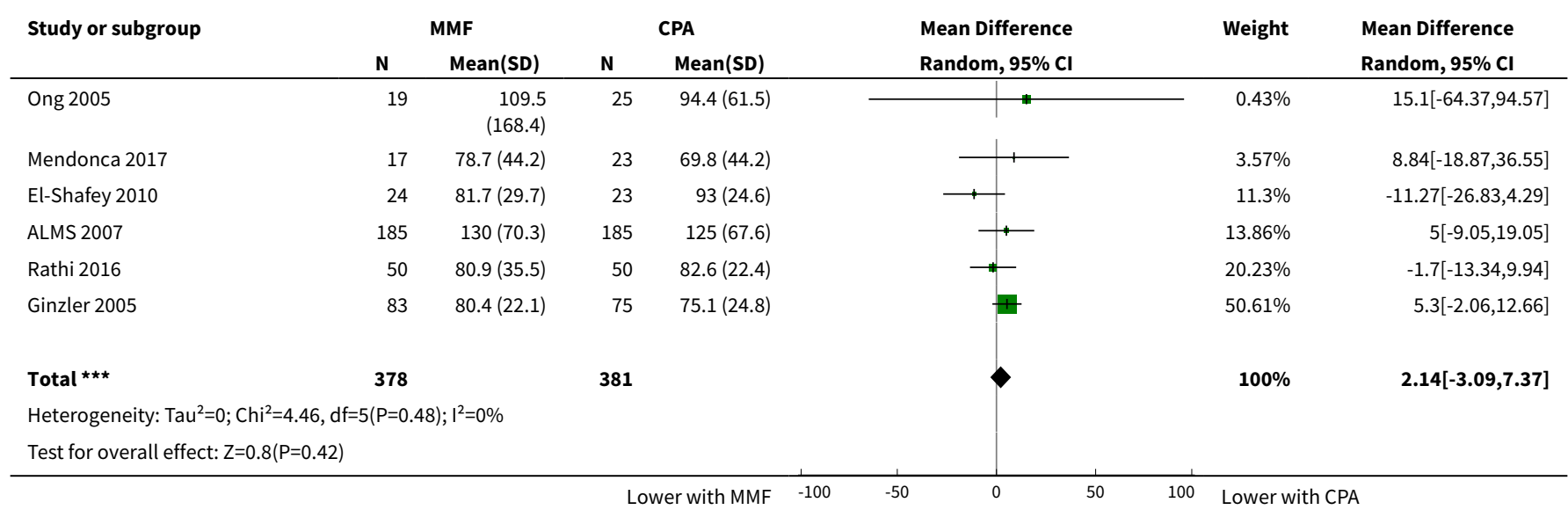

\section{Comparison 2. Mycophenolate mofetil (MMF) versus oral cyclophosphamide (CPA)}

\begin{tabular}{lllll}
\hline $\begin{array}{l}\text { Outcome or subgroup } \\
\text { title }\end{array}$ & No. of studies & $\begin{array}{l}\text { No. of partici- } \\
\text { pants }\end{array}$ & Statistical method & Effect size \\
\hline 1 Death & 1 & 62 & Risk Ratio (M-H, Random, 95\% Cl) & $0.19[0.01,3.76]$ \\
\hline 2 Remission & 1 & Risk Ratio (M-H, Random, 95\% Cl) & Subtotals only \\
\hline $\begin{array}{l}\text { 2.1 Complete remission } \\
\text { in proteinuria }\end{array}$ & 1 & 62 & Risk Ratio (M-H, Random, 95\% Cl) & $0.98[0.74,1.30]$ \\
\hline $\begin{array}{l}2.2 \text { Partial remission in } \\
\text { proteinuria }\end{array}$ & 1 & Risk Ratio (M-H, Random, 95\% Cl) & $1.07[0.44,2.59]$ \\
\hline $\begin{array}{l}\text { 3 Adverse renal out- } \\
\text { comes }\end{array}$ & 1 & 62 & Risk Ratio (M-H, Random, 95\% Cl) & Subtotals only \\
\hline $\begin{array}{l}\text { 3.1 ESKD } \\
\text { 3.2 Renal relapse }\end{array}$ & 1 & & Risk Ratio (M-H, Random, 95\% Cl) & $0.19[0.01,3.76]$ \\
\hline $\begin{array}{l}3.3 \text { Doubling of serum } \\
\text { creatinine }\end{array}$ & 1 & 62 & Risk Ratio (M-H, Random, 95\% Cl) & $1.15[0.55,2.37]$ \\
\hline
\end{tabular}




\begin{tabular}{lllll}
\hline $\begin{array}{l}\text { Outcome or subgroup } \\
\text { title }\end{array}$ & No. of studies & $\begin{array}{l}\text { No. of partici- } \\
\text { pants }\end{array}$ & Statistical method & Effect size \\
\hline 4 Ovarian failure & 1 & 53 & Risk Ratio (M-H, Random, 95\% Cl) & $0.10[0.01,0.73]$ \\
\hline 5 Infection & 1 & Risk Ratio (M-H, Random, 95\% Cl) & Subtotals only \\
\hline 5.1 Major infection & 1 & 62 & Risk Ratio (M-H, Random, 95\% Cl) & $0.21[0.05,0.89]$ \\
\hline 5.2 Herpes zoster virus & 1 & Risk Ratio (M-H, Random, 95\% Cl) & $0.38[0.08,1.79]$ \\
\hline 6 Leucopenia & 1 & 62 & Risk Ratio (M-H, Random, 95\% Cl) & $0.06[0.00,0.92]$ \\
\hline 7 Bone toxicity & 1 & 62 & Risk Ratio (M-H, Random, 95\% Cl) & $0.0[0.0,0.0]$ \\
\hline 8 Alopecia & 1 & 62 & Risk Ratio (M-H, Random, 95\% Cl) & $0.05[0.00,0.81]$ \\
\hline 9 Gastrointestinal (GI) & 1 & 62 & Risk Ratio (M-H, Random, 95\% Cl) & Subtotals only \\
\hline \begin{tabular}{l} 
adverse events \\
\hline 10 Daily proteinuria
\end{tabular} & 1 & 62 & Risk Ratio (M-H, Random, 95\% Cl) & $2.81[0.31,25.58]$ \\
\hline
\end{tabular}

Analysis 2.1. Comparison 2 Mycophenolate mofetil (MMF) versus oral cyclophosphamide (CPA), Outcome 1 Death.

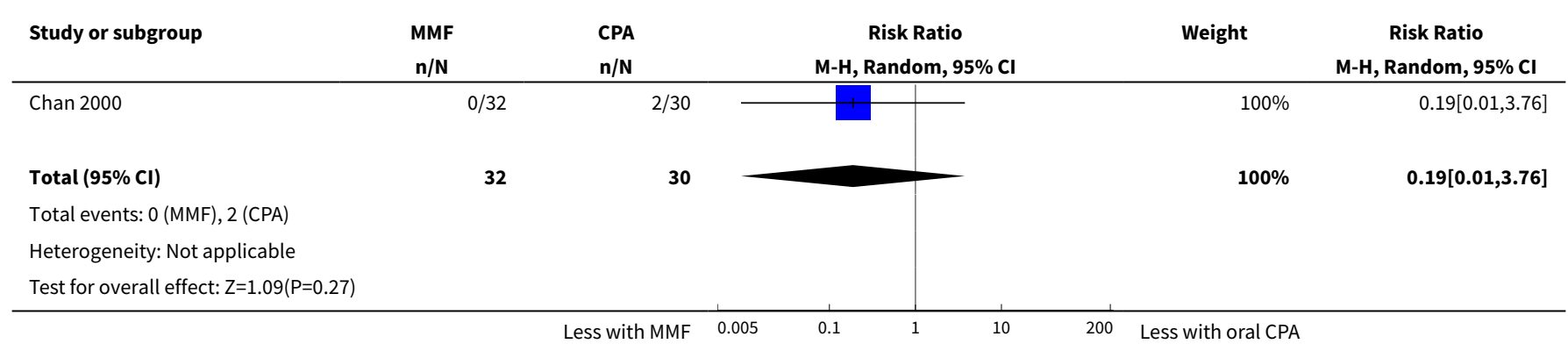

Analysis 2.2. Comparison 2 Mycophenolate mofetil (MMF)
versus oral cyclophosphamide (CPA), Outcome 2 Remission.

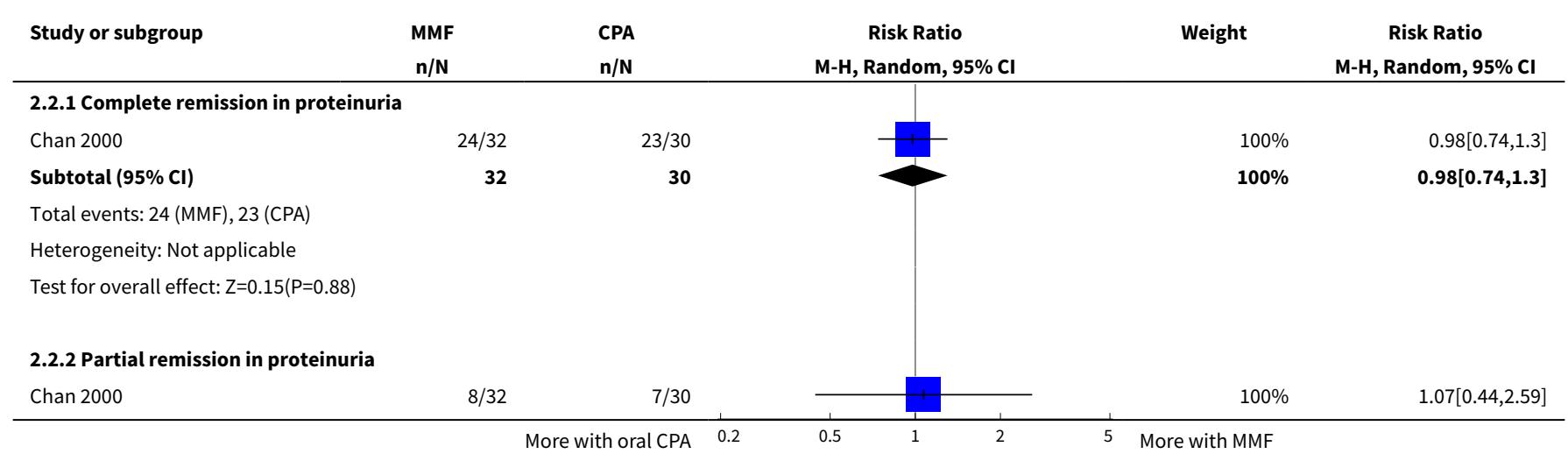




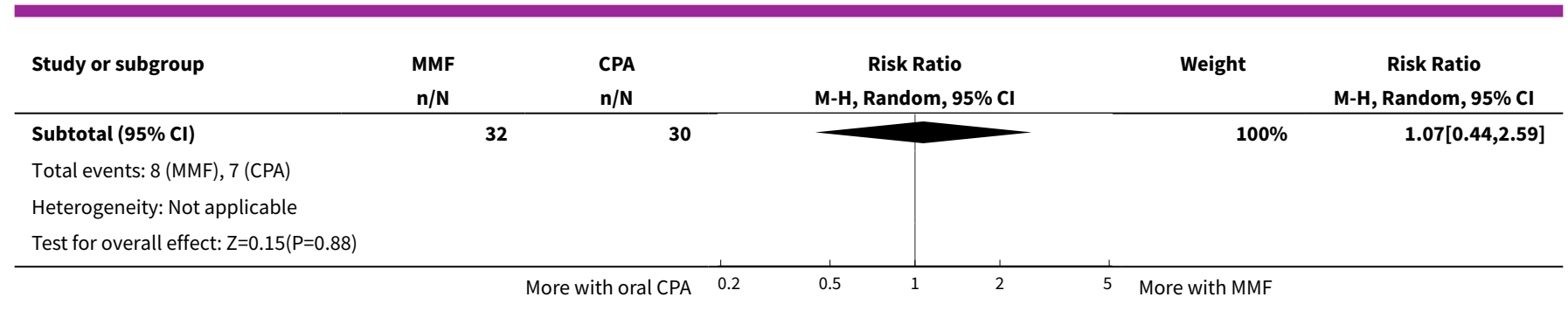

Analysis 2.3. Comparison 2 Mycophenolate mofetil (MMF) versus oral cyclophosphamide (CPA), Outcome 3 Adverse renal outcomes.

\begin{tabular}{|c|c|c|c|c|c|}
\hline Study or subgroup & \multirow[t]{2}{*}{$\begin{array}{c}\text { MMF } \\
\mathrm{n} / \mathrm{N}\end{array}$} & $\begin{array}{l}\mathrm{CPA} \\
\mathrm{n} / \mathrm{N}\end{array}$ & $\begin{array}{c}\text { Risk Ratio } \\
\text { M-H, Random, } 95 \% \mathrm{Cl} \\
\end{array}$ & Weight & $\begin{array}{c}\text { Risk Ratio } \\
\text { M-H, Random, } 95 \% \mathrm{Cl} \\
\end{array}$ \\
\hline \multicolumn{5}{|l|}{ 2.3.1 ESKD } & \\
\hline Chan 2000 & $0 / 32$ & $2 / 30$ & - & $100 \%$ & $0.19[0.01,3.76]$ \\
\hline Subtotal $(95 \% \mathrm{Cl})$ & 32 & 30 & & $100 \%$ & $0.19[0.01,3.76]$ \\
\hline \multicolumn{6}{|c|}{ Total events: 0 (MMF), 2 (CPA) } \\
\hline \multicolumn{6}{|c|}{ Heterogeneity: Not applicable } \\
\hline \multicolumn{6}{|c|}{ Test for overall effect: $Z=1.09(P=0.27)$} \\
\hline \multicolumn{6}{|l|}{ 2.3.2 Renal relapse } \\
\hline Chan 2000 & $11 / 32$ & $9 / 30$ & & $100 \%$ & $1.15[0.55,2.37]$ \\
\hline Subtotal $(95 \% \mathrm{Cl})$ & 32 & 30 & & $100 \%$ & $1.15[0.55,2.37]$ \\
\hline \multicolumn{6}{|c|}{ Total events: 11 (MMF), 9 (CPA) } \\
\hline \multicolumn{6}{|c|}{ Heterogeneity: Not applicable } \\
\hline \multicolumn{6}{|c|}{ Test for overall effect: $Z=0.37(P=0.71)$} \\
\hline \multicolumn{6}{|c|}{ 2.3.3 Doubling of serum creatinine } \\
\hline Chan 2000 & $2 / 32$ & $3 / 30$ & & $100 \%$ & $0.63[0.11,3.48]$ \\
\hline Subtotal $(95 \% \mathrm{Cl})$ & 32 & 30 & & $100 \%$ & $0.63[0.11,3.48]$ \\
\hline \multicolumn{6}{|c|}{ Total events: 2 (MMF), 3 (CPA) } \\
\hline \multicolumn{6}{|c|}{ Heterogeneity: Not applicable } \\
\hline Test for overall effect & & & & & \\
\hline
\end{tabular}

Analysis 2.4. Comparison 2 Mycophenolate mofetil (MMF) versus oral cyclophosphamide (CPA), Outcome 4 Ovarian failure.

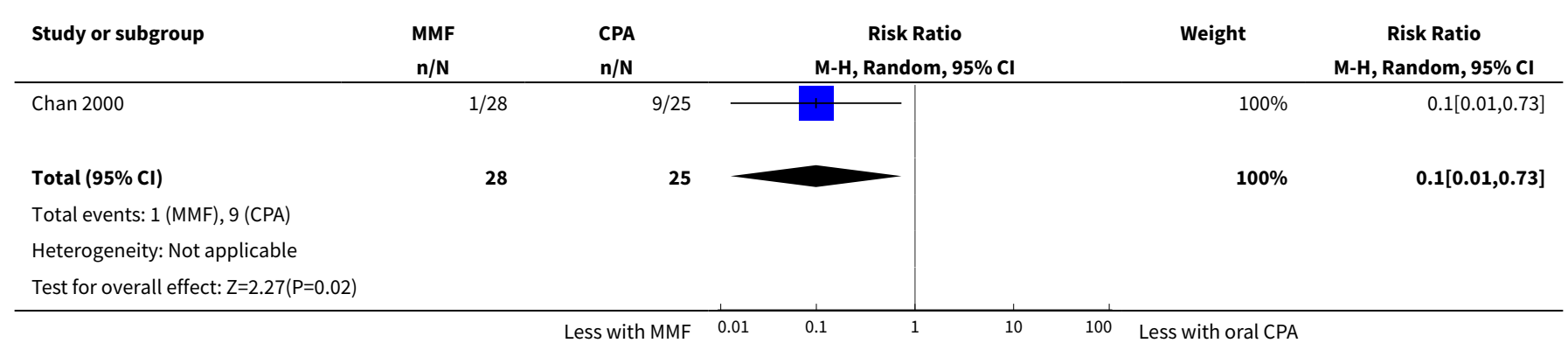


Analysis 2.5. Comparison 2 Mycophenolate mofetil (MMF) versus oral cyclophosphamide (CPA), Outcome 5 Infection.

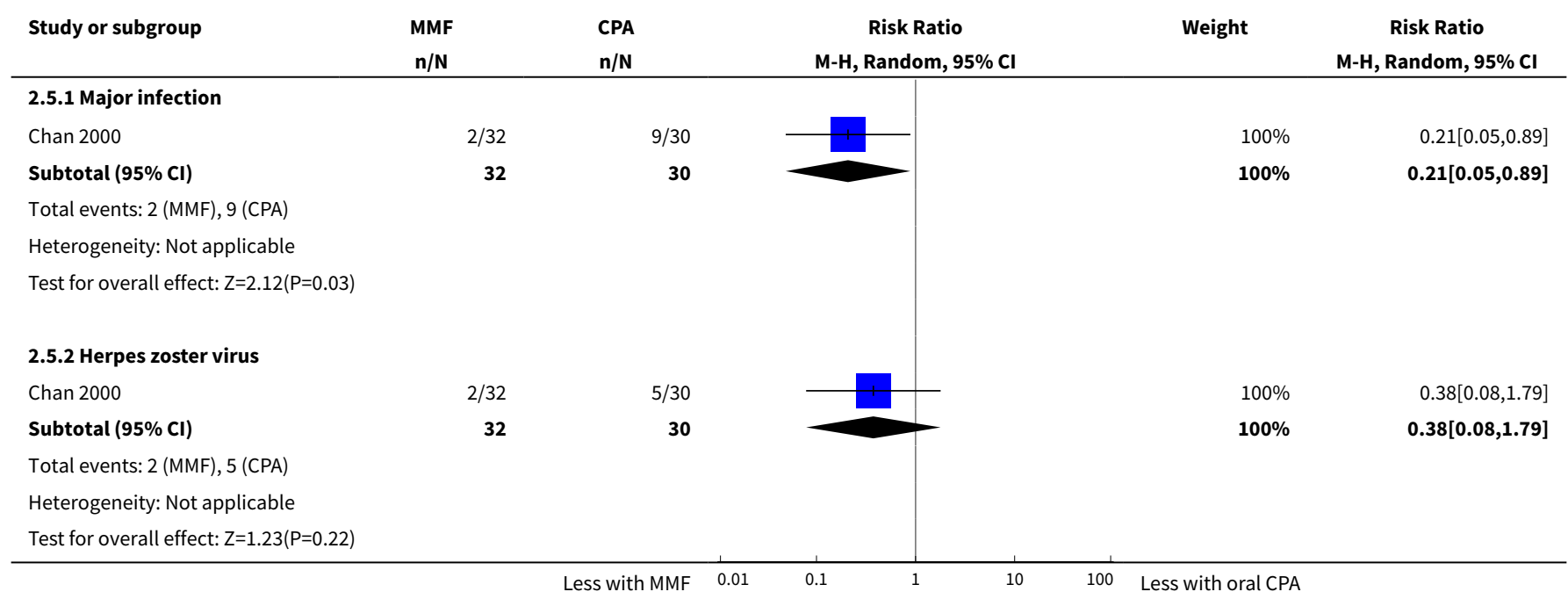

Analysis 2.6. Comparison 2 Mycophenolate mofetil (MMF) versus oral cyclophosphamide (CPA), Outcome 6 Leucopenia.

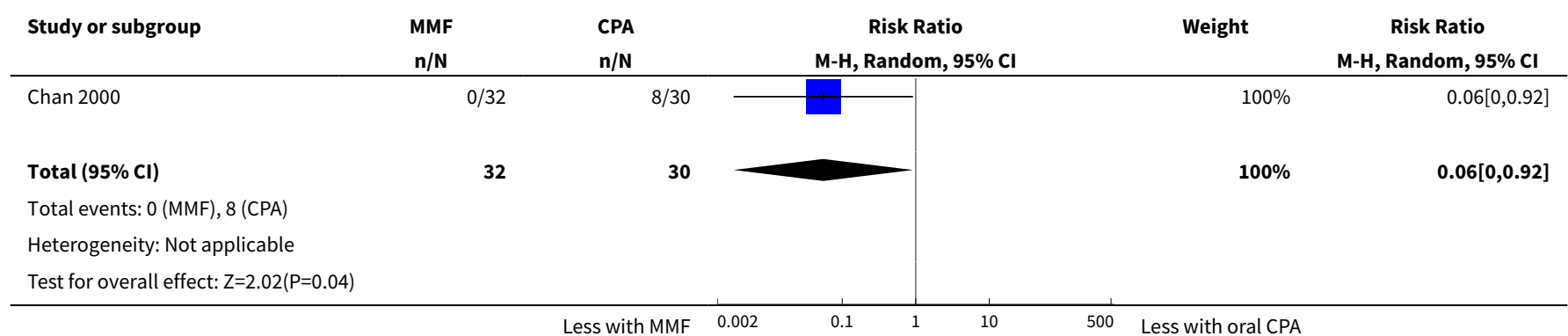

Analysis 2.7. Comparison 2 Mycophenolate mofetil (MMF) versus oral cyclophosphamide (CPA), Outcome 7 Bone toxicity.

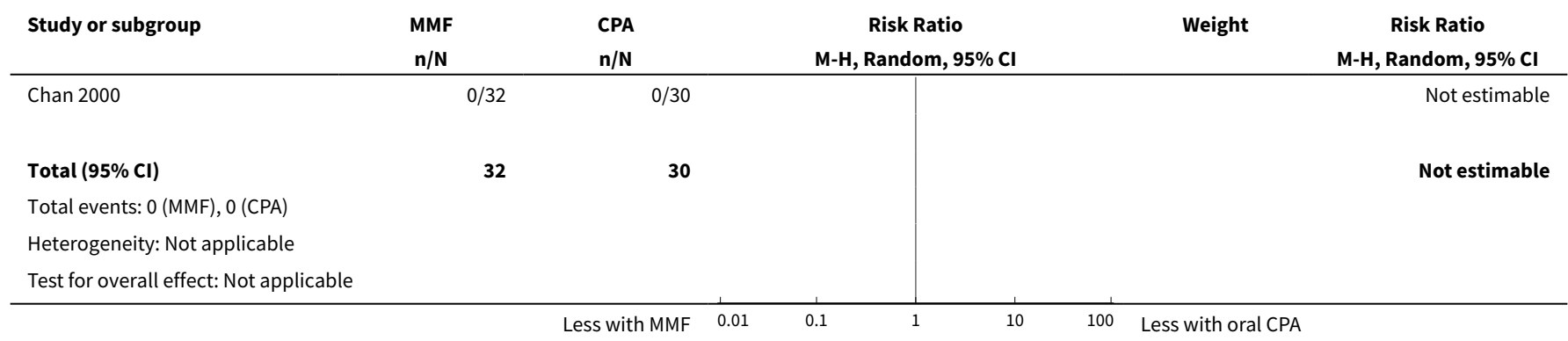


Analysis 2.8. Comparison 2 Mycophenolate mofetil (MMF) versus oral cyclophosphamide (CPA), Outcome 8 Alopecia.

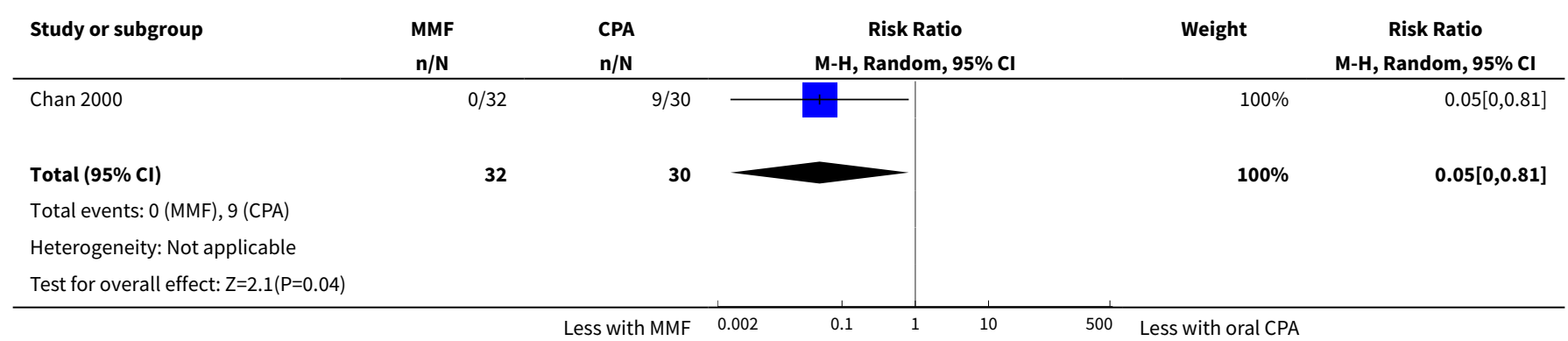

Analysis 2.9. Comparison 2 Mycophenolate mofetil (MMF) versus oral cyclophosphamide (CPA), Outcome 9 Gastrointestinal (GI) adverse events.

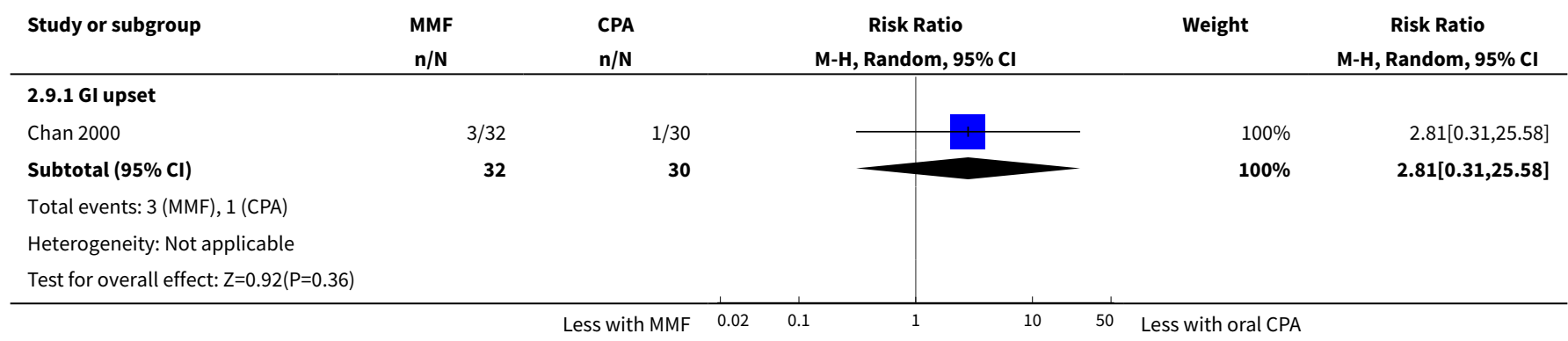

Analysis 2.10. Comparison 2 Mycophenolate mofetil (MMF) versus oral cyclophosphamide (CPA), Outcome 10 Daily proteinuria.

\begin{tabular}{|c|c|c|c|c|c|c|c|}
\hline \multirow[t]{2}{*}{ Study or subgroup } & \multicolumn{2}{|c|}{ MMF } & \multicolumn{2}{|c|}{ CPA } & \multirow{2}{*}{$\begin{array}{l}\text { Mean Difference } \\
\text { Random, 95\% Cl }\end{array}$} & \multirow[t]{2}{*}{ Weight } & \multirow{2}{*}{$\begin{array}{l}\text { Mean Difference } \\
\text { Random, } 95 \% \mathrm{CI}\end{array}$} \\
\hline & $\mathbf{N}$ & $\operatorname{Mean}(\mathrm{SD})$ & $\mathbf{N}$ & Mean(SD) & & & \\
\hline Chan 2000 & 21 & $0.5(1.1)$ & 21 & $0.2(0.3)$ & $7+1$ & $100 \%$ & $0.3[-0.19,0.79]$ \\
\hline 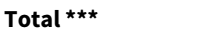 & 21 & & 21 & & & $100 \%$ & $0.3[-0.19,0.79]$ \\
\hline \multicolumn{8}{|c|}{ Heterogeneity: Not applicable } \\
\hline \multicolumn{8}{|c|}{ Test for overall effect: $Z=1.21(P=0.23)$} \\
\hline
\end{tabular}

\section{Comparison 3. Mycophenolate mofetil (MMF) + tacrolimus (TAC) versus IV cyclophosphamide (CPA)}

\begin{tabular}{lllll}
\hline $\begin{array}{l}\text { Outcome or subgroup ti- } \\
\text { tle }\end{array}$ & No. of studies & $\begin{array}{l}\text { No. of partici- } \\
\text { pants }\end{array}$ & Statistical method & Effect size \\
\hline 1 Death & 2 & 402 & Risk Ratio (M-H, Random, 95\% Cl) & $0.0[0.0,0.0]$ \\
\hline 2 Remission & 2 & Risk Ratio (M-H, Random, 95\% Cl) & Subtotals only \\
\hline
\end{tabular}




\begin{tabular}{|c|c|c|c|c|}
\hline $\begin{array}{l}\text { Outcome or subgroup ti- } \\
\text { tle }\end{array}$ & No. of studies & $\begin{array}{l}\text { No. of partici- } \\
\text { pants }\end{array}$ & Statistical method & Effect size \\
\hline $\begin{array}{l}2.1 \text { Complete renal remis- } \\
\text { sion }\end{array}$ & 2 & 402 & Risk Ratio (M-H, Random, 95\% Cl) & $2.38[1.07,5.30]$ \\
\hline 2.2 Partial renal remission & 2 & 402 & Risk Ratio (M-H, Random, 95\% Cl) & $1.0[0.78,1.28]$ \\
\hline $\begin{array}{l}2.3 \text { Complete remission in } \\
\text { proteinuria }\end{array}$ & 2 & 402 & Risk Ratio (M-H, Random, 95\% Cl) & $2.38[1.07,5.30]$ \\
\hline $\begin{array}{l}2.4 \text { Partial remission in } \\
\text { proteinuria }\end{array}$ & 2 & 402 & Risk Ratio (M-H, Random, 95\% Cl) & $0.98[0.76,1.26]$ \\
\hline 3 Adverse renal outcomes & 2 & & Risk Ratio (M-H, Random, 95\% Cl) & Subtotals only \\
\hline $\begin{array}{l}3.1 \text { Doubling of serum cre- } \\
\text { atinine }\end{array}$ & 2 & 402 & Risk Ratio (M-H, Random, 95\% Cl) & $0.98[0.10,9.23]$ \\
\hline 4 Stable kidney function & 2 & 402 & Risk Ratio (M-H, Random, 95\% Cl) & $1.78[1.40,2.26]$ \\
\hline 5 Ovarian failure & 1 & 34 & Risk Ratio (M-H, Random, 95\% Cl) & $0.0[0.0,0.0]$ \\
\hline 6 Menstrual irregularities & 1 & 323 & Risk Ratio (M-H, Random, 95\% Cl) & $0.28[0.06,1.35]$ \\
\hline 7 Infection & 2 & & Risk Ratio (M-H, Random, 95\% Cl) & Subtotals only \\
\hline 7.1 Major infection & 2 & 402 & Risk Ratio (M-H, Random, 95\% Cl) & $1.65[0.11,24.44]$ \\
\hline 7.2 Herpes zoster virus & 2 & 402 & Risk Ratio (M-H, Random, $95 \% \mathrm{Cl}$ ) & $0.80[0.22,2.94]$ \\
\hline 8 Leucopenia & 2 & 402 & Risk Ratio (M-H, Random, 95\% Cl) & $0.23[0.04,1.44]$ \\
\hline 9 Bone toxicity & 1 & 362 & Risk Ratio (M-H, Random, 95\% Cl) & $3.0[0.12,73.16]$ \\
\hline 10 Alopecia & 2 & 402 & Risk Ratio (M-H, Random, 95\% Cl) & $0.78[0.36,1.72]$ \\
\hline $\begin{array}{l}11 \text { Gastrointestinal (GI) ad- } \\
\text { verse events }\end{array}$ & 2 & & Risk Ratio (M-H, Random, 95\% Cl) & Subtotals only \\
\hline 11.1 Diarrhoea & 1 & 362 & Risk Ratio (M-H, Random, 95\% Cl) & $2.33[0.92,5.94]$ \\
\hline $11.2 \mathrm{GI}$ upset & 2 & 402 & Risk Ratio (M-H, Random, 95\% Cl) & $0.21[0.10,0.41]$ \\
\hline 12 Daily proteinuria & 1 & 40 & Mean Difference (IV, Random, 95\% CI) & $-1.69[-2.81,-0.57]$ \\
\hline
\end{tabular}

Analysis 3.1. Comparison 3 Mycophenolate mofetil (MMF) + tacrolimus (TAC) versus IV cyclophosphamide (CPA), Outcome 1 Death.

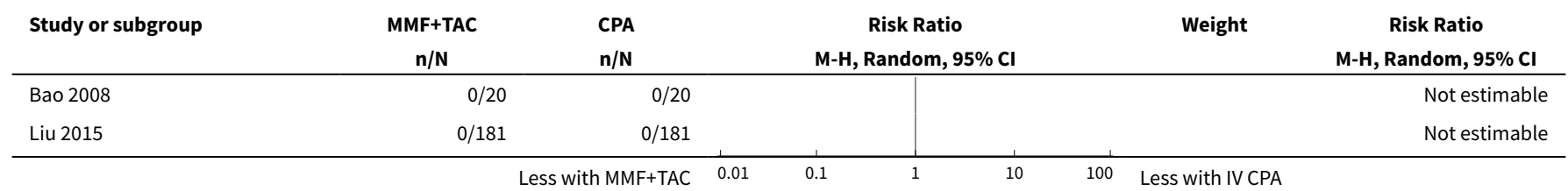




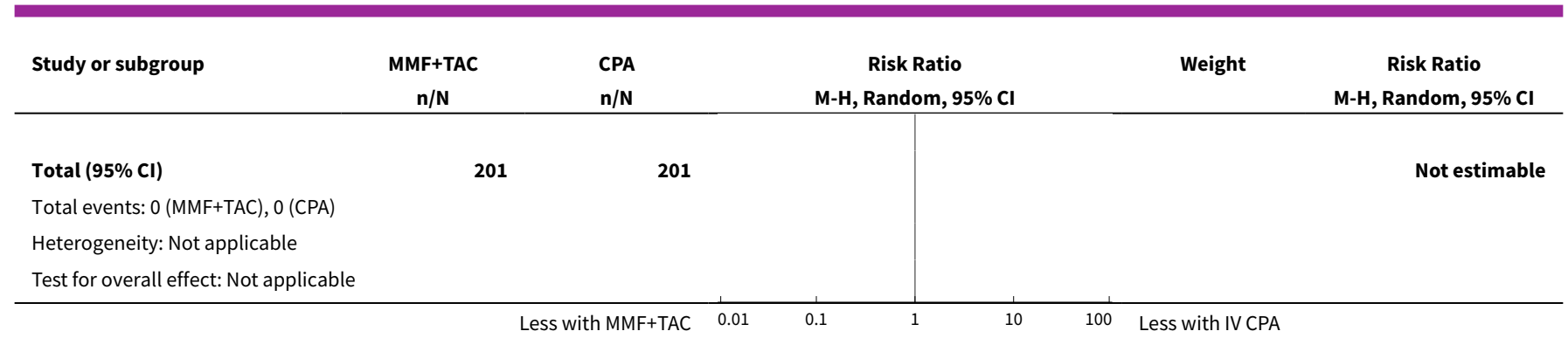

Analysis 3.2. Comparison 3 Mycophenolate mofetil (MMF) + tacrolimus (TAC) versus IV cyclophosphamide (CPA), Outcome 2 Remission.

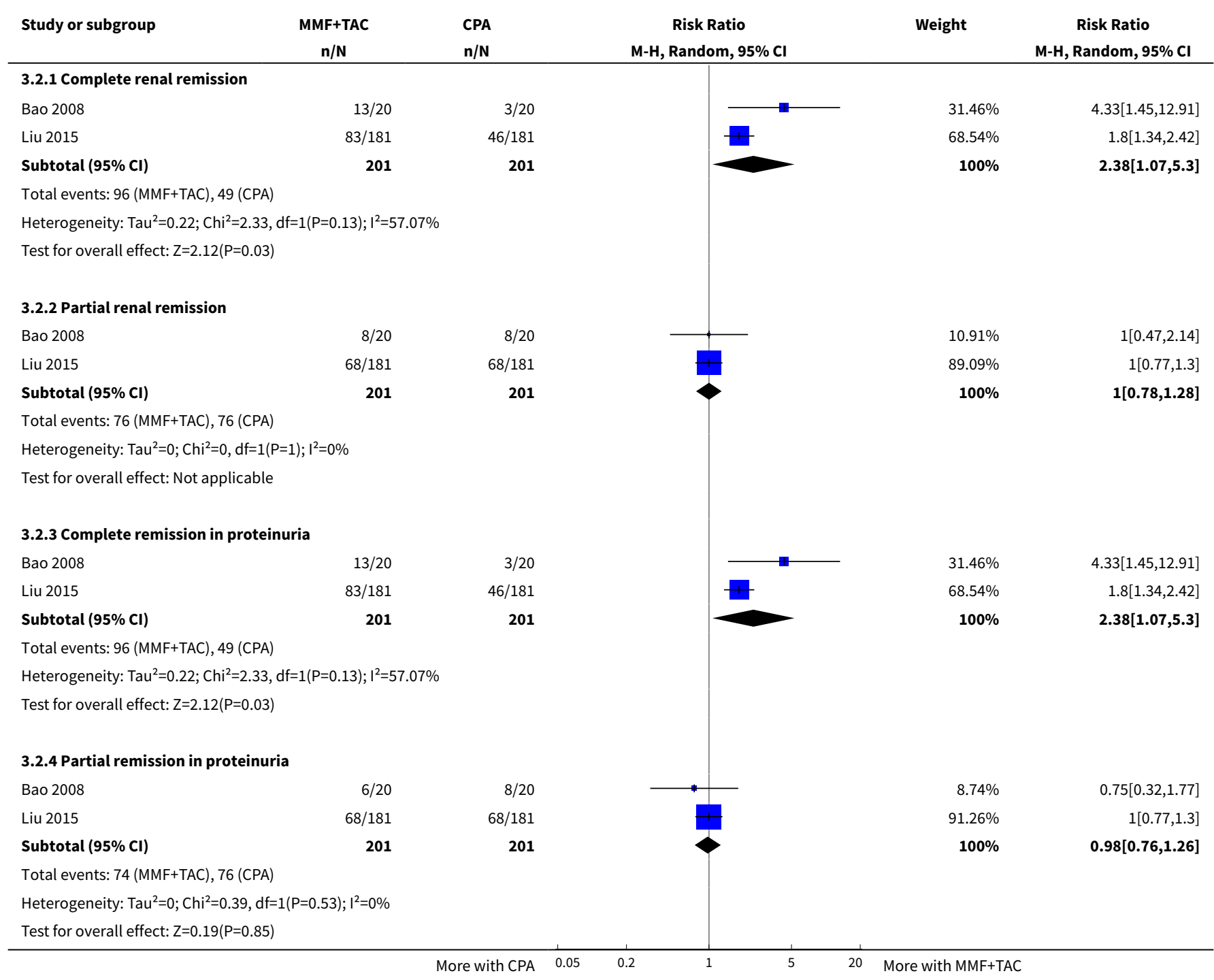


Analysis 3.3. Comparison 3 Mycophenolate mofetil (MMF) + tacrolimus (TAC) versus IV cyclophosphamide (CPA), Outcome 3 Adverse renal outcomes.

\begin{tabular}{|c|c|c|c|c|c|}
\hline Study or subgroup & $\begin{array}{c}\text { MMF+TAC } \\
\mathbf{n} / \mathbf{N}\end{array}$ & $\begin{array}{l}\mathrm{CPA} \\
\mathrm{n} / \mathrm{N}\end{array}$ & $\begin{array}{c}\text { Risk Ratio } \\
\text { M-H, Random, 95\% Cl }\end{array}$ & Weight & $\begin{array}{c}\text { Risk Ratio } \\
\text { M-H, Random, } 95 \% \mathrm{CI}\end{array}$ \\
\hline \multicolumn{6}{|c|}{ 3.3.1 Doubling of serum creatinine } \\
\hline Liu 2015 & $1 / 181$ & $0 / 181$ & 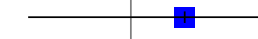 & $49.19 \%$ & $3[0.12,73.16]$ \\
\hline Bao 2008 & $0 / 20$ & $1 / 20$ & & $50.81 \%$ & $0.33[0.01,7.72]$ \\
\hline Subtotal $(95 \% \mathrm{CI})$ & 201 & 201 & & $100 \%$ & $0.98[0.1,9.23]$ \\
\hline \multicolumn{6}{|c|}{ Total events: 1 (MMF+TAC), 1 (CPA) } \\
\hline \multicolumn{6}{|c|}{ Heterogeneity: $\operatorname{Tau}^{2}=0 ; \mathrm{Chi}^{2}=0.92, \mathrm{df}=1(\mathrm{P}=0.34) ; \mathrm{I}^{2}=0 \%$} \\
\hline \multicolumn{6}{|c|}{ Test for overall effect: $Z=0.02(P=0.99)$} \\
\hline
\end{tabular}

Analysis 3.4. Comparison 3 Mycophenolate mofetil (MMF) + tacrolimus (TAC) versus IV cyclophosphamide (CPA), Outcome 4 Stable kidney function.

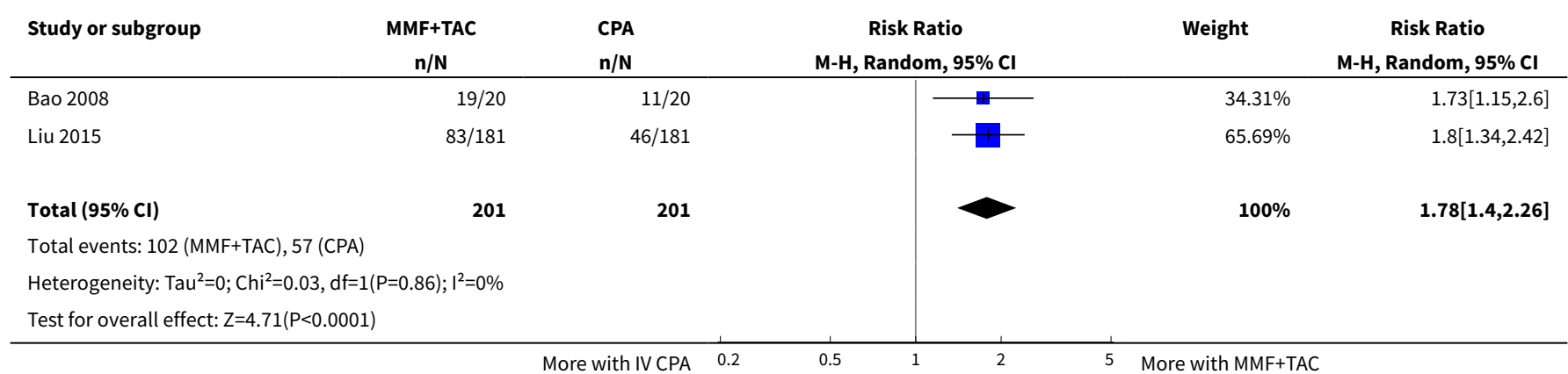

Analysis 3.5. Comparison 3 Mycophenolate mofetil (MMF) + tacrolimus (TAC) versus IV cyclophosphamide (CPA), Outcome 5 Ovarian failure.

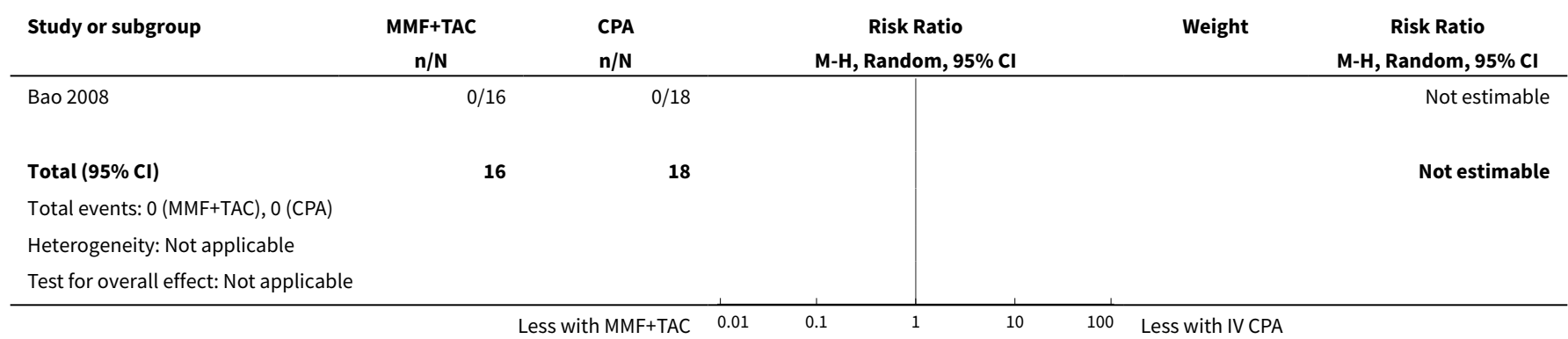

Analysis 3.6. Comparison 3 Mycophenolate mofetil (MMF) + tacrolimus (TAC) versus IV cyclophosphamide (CPA), Outcome 6 Menstrual irregularities.

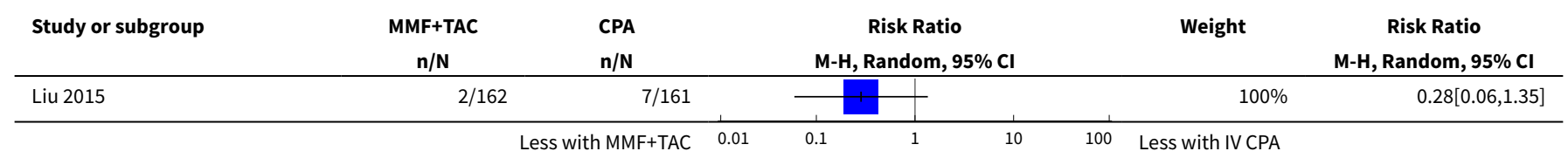




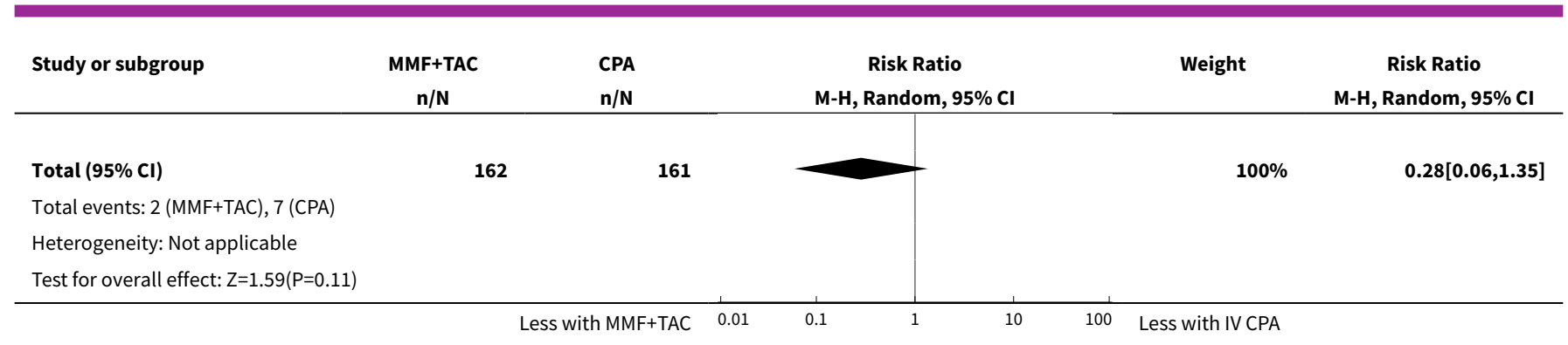

Analysis 3.7. Comparison 3 Mycophenolate mofetil (MMF) + tacrolimus (TAC) versus IV cyclophosphamide (CPA), Outcome 7 Infection.

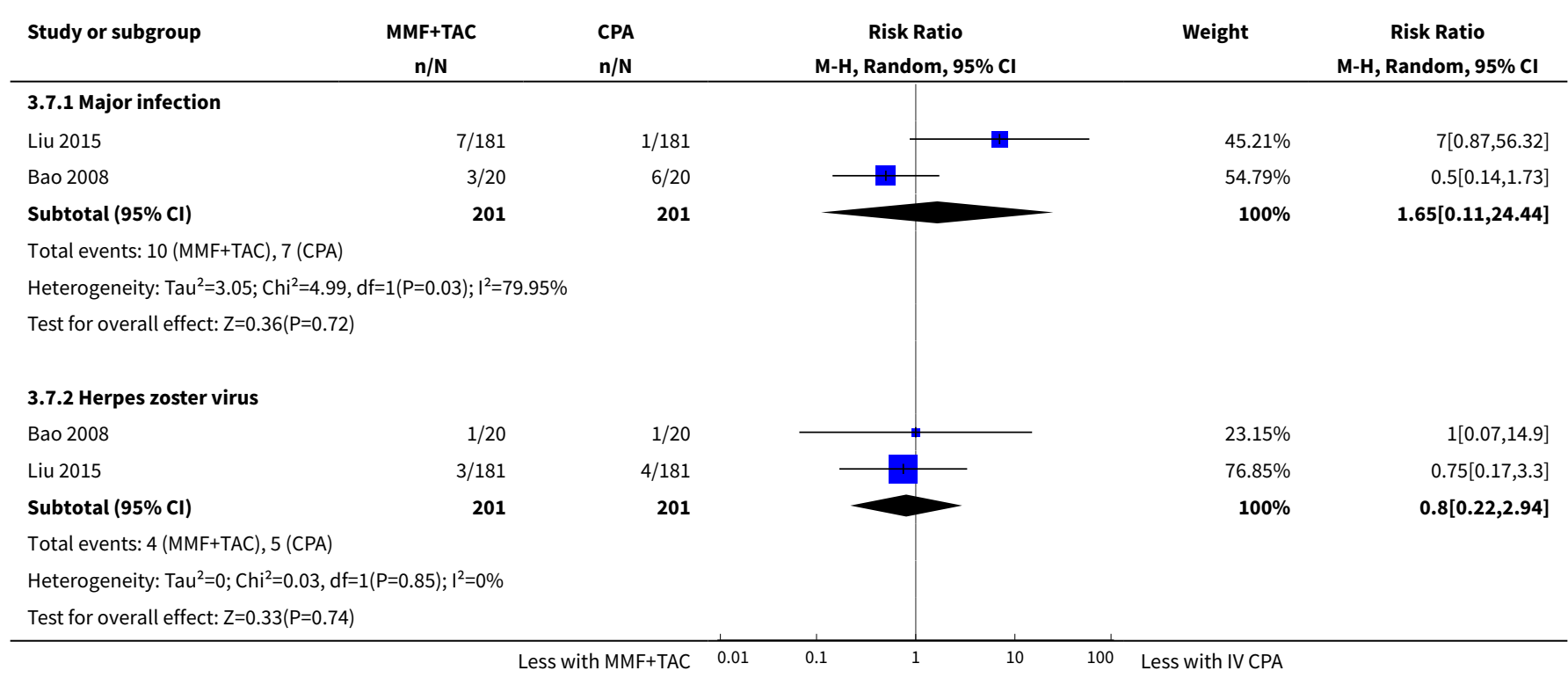

Analysis 3.8. Comparison 3 Mycophenolate mofetil (MMF) + tacrolimus (TAC) versus IV cyclophosphamide (CPA), Outcome 8 Leucopenia.

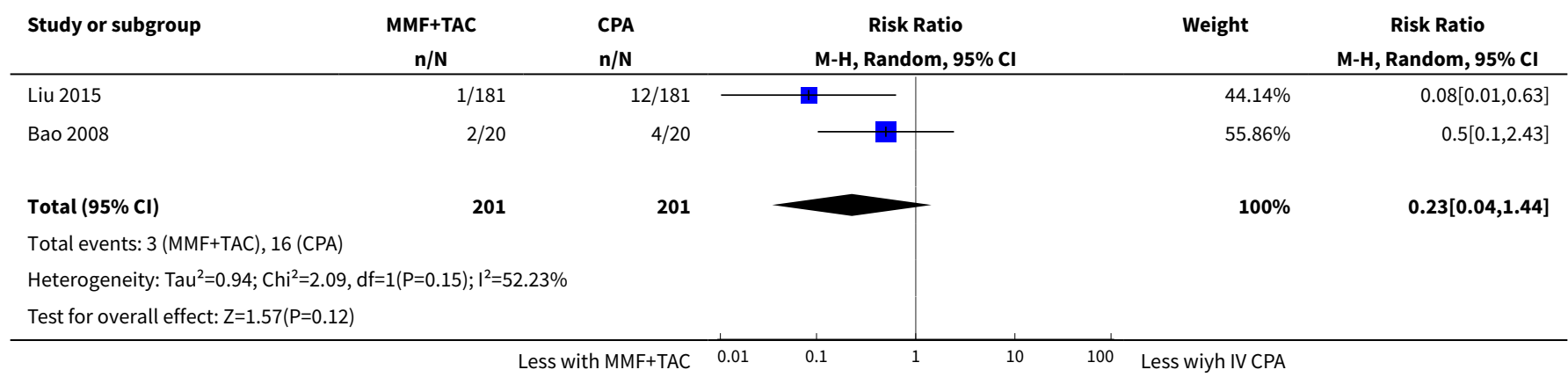


Analysis 3.9. Comparison 3 Mycophenolate mofetil (MMF) + tacrolimus

(TAC) versus IV cyclophosphamide (CPA), Outcome 9 Bone toxicity.

\begin{tabular}{|c|c|c|c|c|c|}
\hline Study or subgroup & $\begin{array}{c}\text { MMF+TAC } \\
n / N\end{array}$ & $\begin{array}{l}\mathrm{CPA} \\
\mathrm{n} / \mathrm{N}\end{array}$ & $\begin{array}{c}\text { Risk Ratio } \\
\text { M-H, Random, } 95 \% \mathrm{Cl}\end{array}$ & Weight & $\begin{array}{c}\text { Risk Ratio } \\
\text { M-H, Random, } 95 \% \mathrm{Cl}\end{array}$ \\
\hline Liu 2015 & $1 / 181$ & $0 / 181$ & + & $100 \%$ & $3[0.12,73.16]$ \\
\hline Total $(95 \% \mathrm{Cl})$ & 181 & 181 & & $100 \%$ & $3[0.12,73.16]$ \\
\hline \multicolumn{6}{|c|}{ Total events: 1 (MMF+TAC), 0 (CPA) } \\
\hline \multicolumn{6}{|c|}{ Heterogeneity: Not applicable } \\
\hline \multicolumn{6}{|c|}{ Test for overall effect: $Z=0.67(P=0.5)$} \\
\hline
\end{tabular}

Analysis 3.10. Comparison 3 Mycophenolate mofetil (MMF) + tacrolimus (TAC) versus IV cyclophosphamide (CPA), Outcome 10 Alopecia.

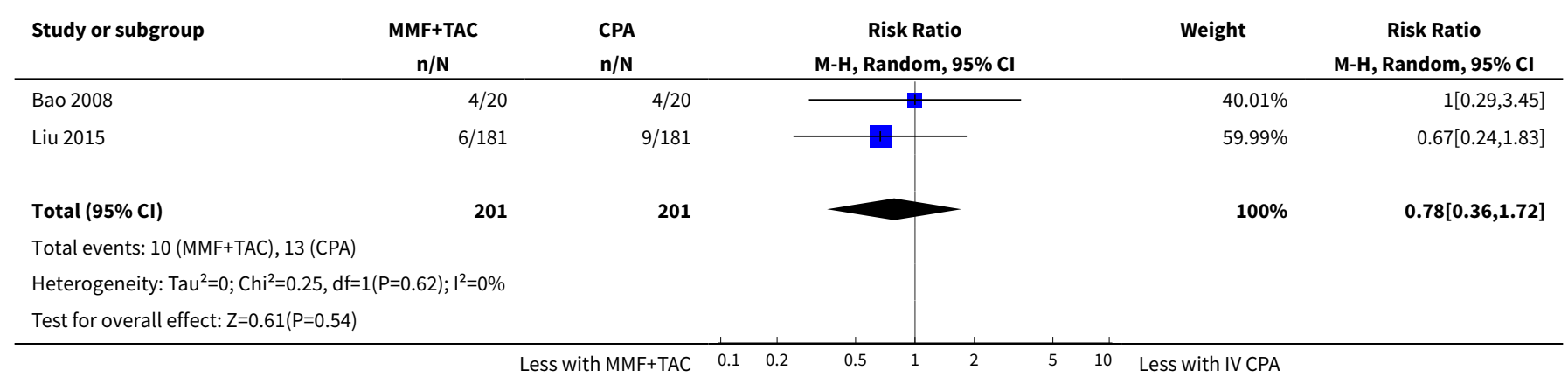

Analysis 3.11. Comparison 3 Mycophenolate mofetil (MMF) + tacrolimus (TAC) versus IV cyclophosphamide (CPA), Outcome 11 Gastrointestinal (GI) adverse events.

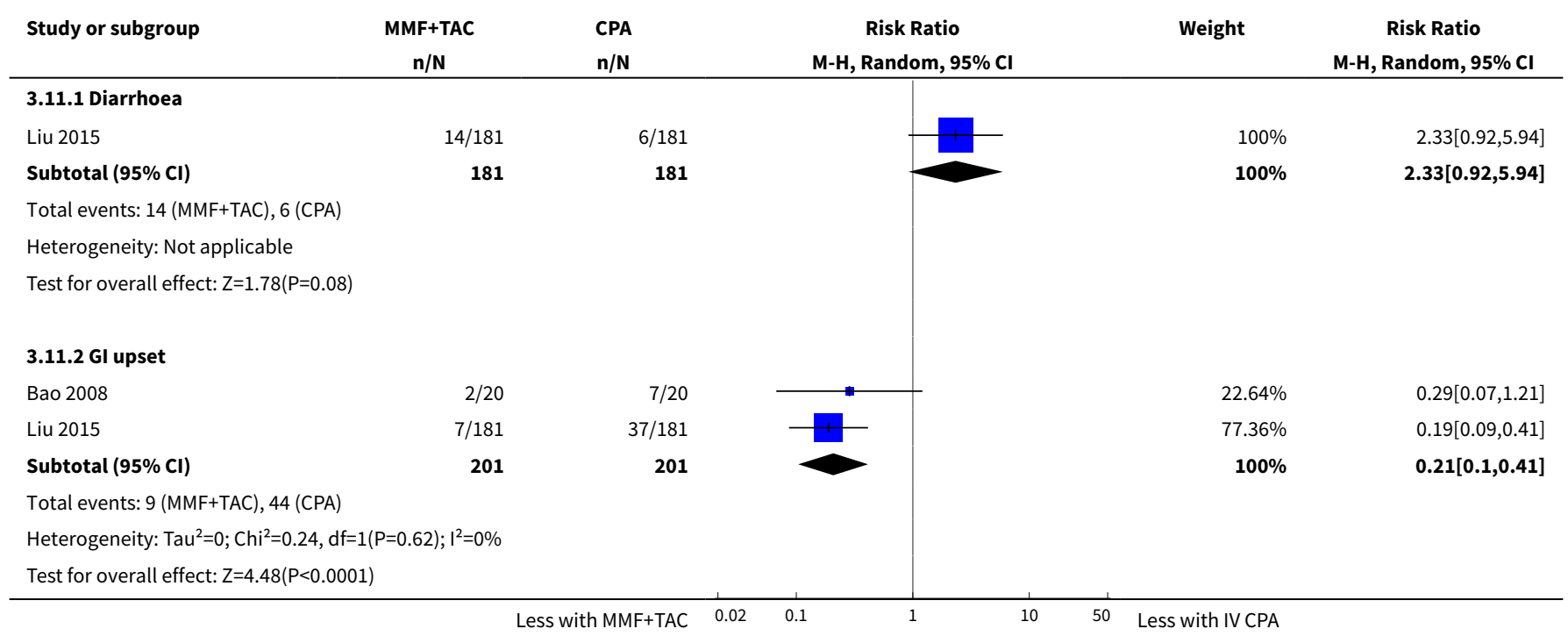


Analysis 3.12. Comparison 3 Mycophenolate mofetil (MMF) + tacrolimus (TAC) versus IV cyclophosphamide (CPA), Outcome 12 Daily proteinuria.

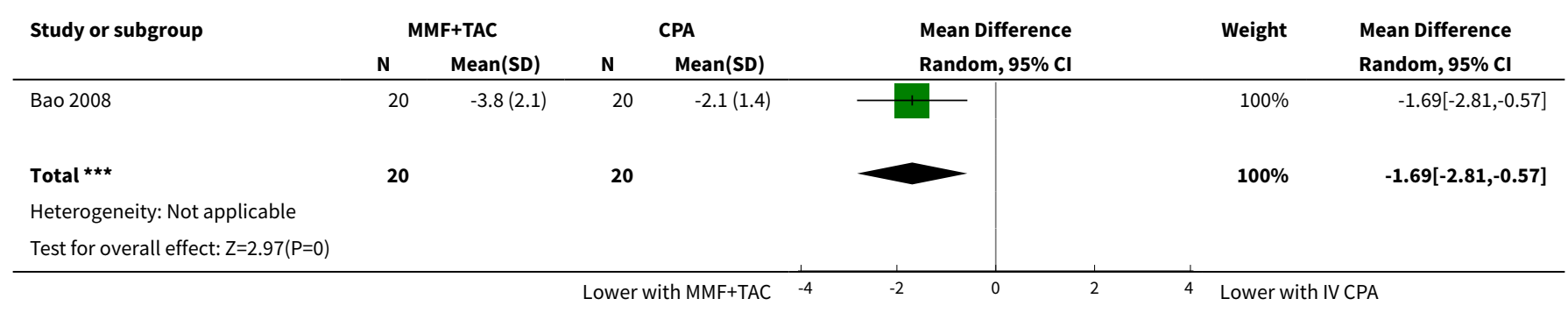

\section{Comparison 4. Mycophenolate mofetil (MMF) + IV cyclophosphamide (CPA) versus IV CPA}

\begin{tabular}{|c|c|c|c|c|}
\hline $\begin{array}{l}\text { Outcome or subgroup } \\
\text { title }\end{array}$ & No. of studies & $\begin{array}{l}\text { No. of partici- } \\
\text { pants }\end{array}$ & Statistical method & Effect size \\
\hline 1 Death & 1 & 82 & Risk Ratio (M-H, Random, 95\% Cl) & $0.95[0.06,14.72]$ \\
\hline 2 Remission & 1 & & Risk Ratio (M-H, Random, 95\% Cl) & Subtotals only \\
\hline $\begin{array}{l}2.1 \text { Complete renal re- } \\
\text { mission }\end{array}$ & 1 & 82 & Risk Ratio (M-H, Random, 95\% Cl) & $1.22[0.78,1.89]$ \\
\hline $\begin{array}{l}2.2 \text { Partial renal remis- } \\
\text { sion }\end{array}$ & 1 & 82 & Risk Ratio (M-H, Random, 95\% Cl) & $1.03[0.55,1.90]$ \\
\hline $\begin{array}{l}3 \text { Menstrual irregulari- } \\
\text { ties }\end{array}$ & 1 & 75 & Risk Ratio (M-H, Random, 95\% Cl) & $0.49[0.16,1.48]$ \\
\hline 4 Infection & 1 & 82 & Risk Ratio (M-H, Random, 95\% Cl) & $0.37[0.14,0.93]$ \\
\hline 4.1 Major infection & 1 & 82 & Risk Ratio (M-H, Random, 95\% Cl) & $0.37[0.14,0.93]$ \\
\hline 5 Leucopenia & 1 & 82 & Risk Ratio (M-H, Random, 95\% Cl) & $0.63[0.11,3.60]$ \\
\hline 6 Daily proteinuria & 1 & 77 & Mean Difference (IV, Random, 95\% CI) & $-0.54[-1.12,0.04]$ \\
\hline
\end{tabular}

Analysis 4.1. Comparison 4 Mycophenolate mofetil (MMF) + IV cyclophosphamide (CPA) versus IV CPA, Outcome 1 Death.

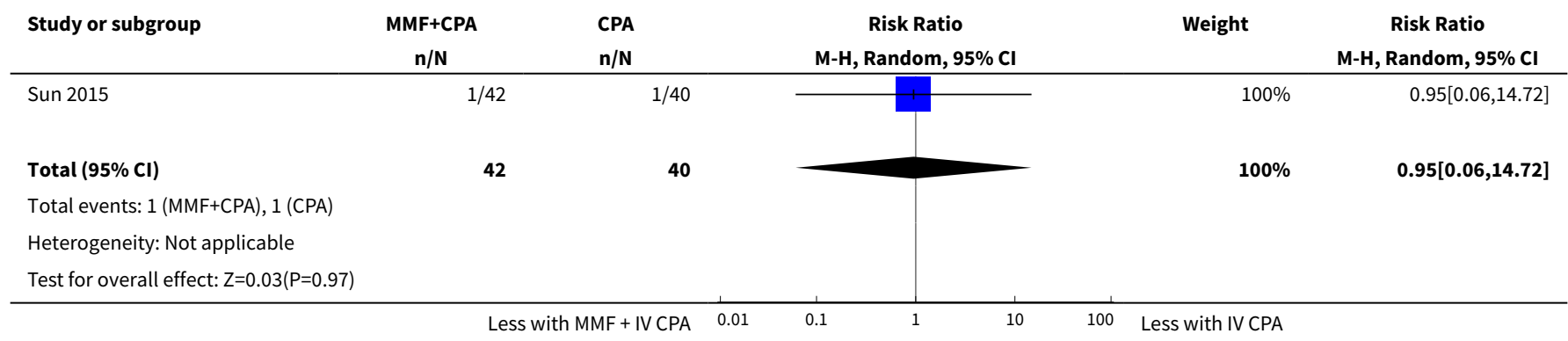


Analysis 4.2. Comparison 4 Mycophenolate mofetil (MMF) + IV cyclophosphamide (CPA) versus IV CPA, Outcome 2 Remission.

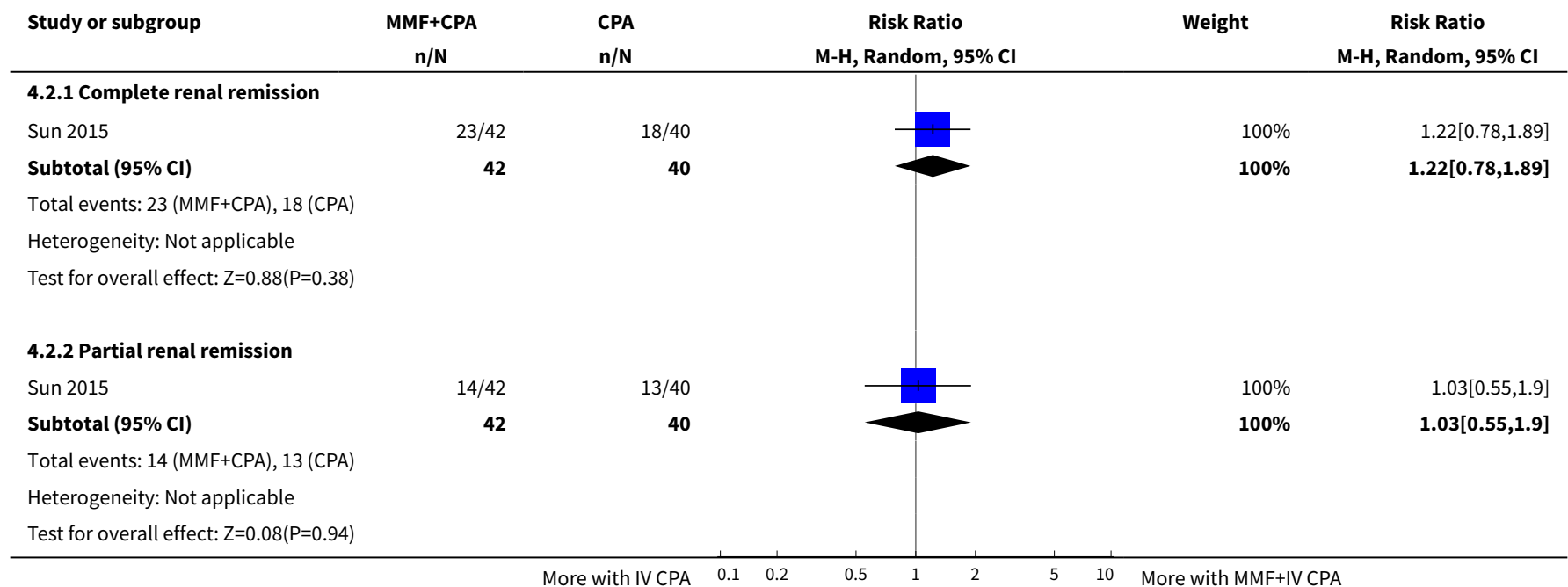

Analysis 4.3. Comparison 4 Mycophenolate mofetil (MMF) + IV cyclophosphamide (CPA) versus IV CPA, Outcome 3 Menstrual irregularities.

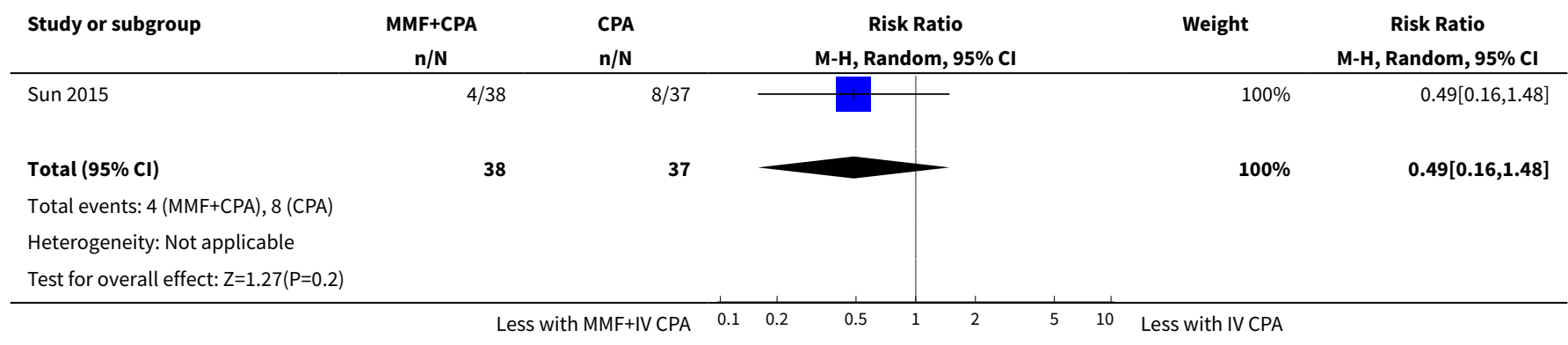

Analysis 4.4. Comparison 4 Mycophenolate mofetil (MMF) + IV cyclophosphamide (CPA) versus IV CPA, Outcome 4 Infection.

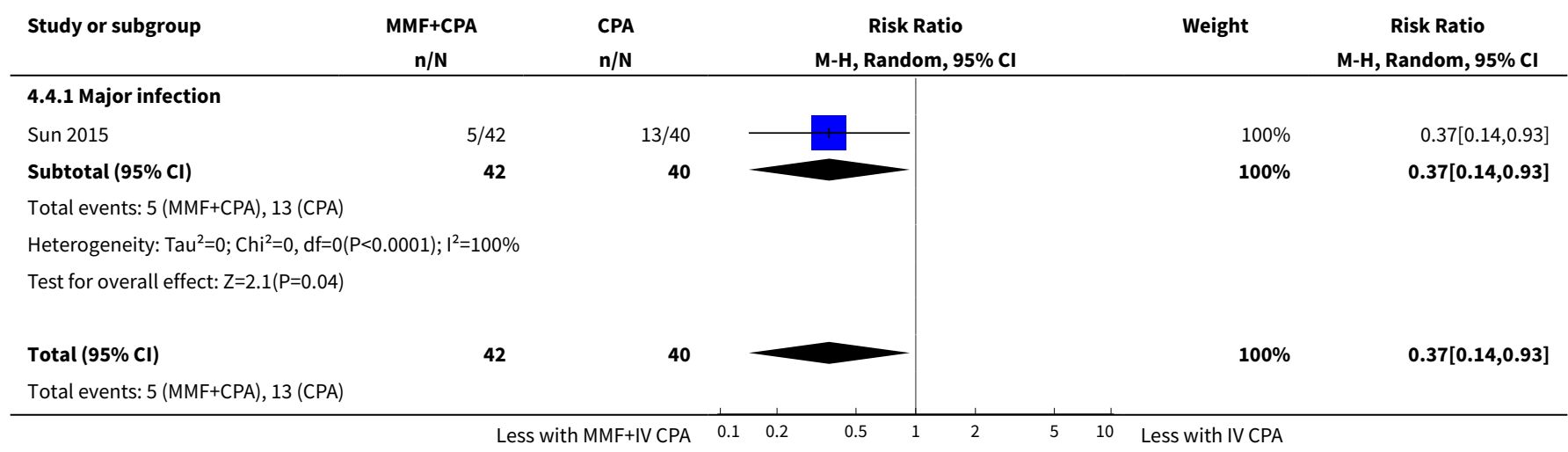




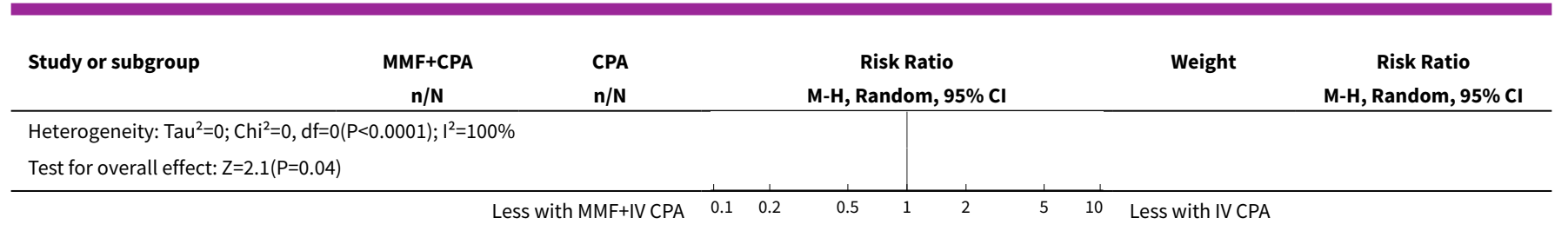

Analysis 4.5. Comparison 4 Mycophenolate mofetil (MMF) + IV cyclophosphamide (CPA) versus IV CPA, Outcome 5 Leucopenia.

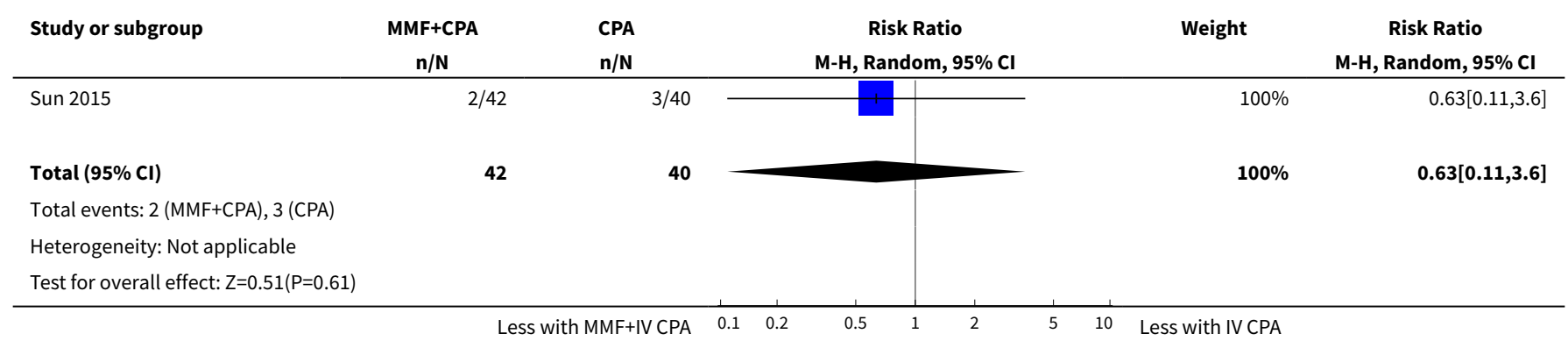

Analysis 4.6. Comparison 4 Mycophenolate mofetil (MMF) + IV cyclophosphamide (CPA) versus IV CPA, Outcome 6 Daily proteinuria.

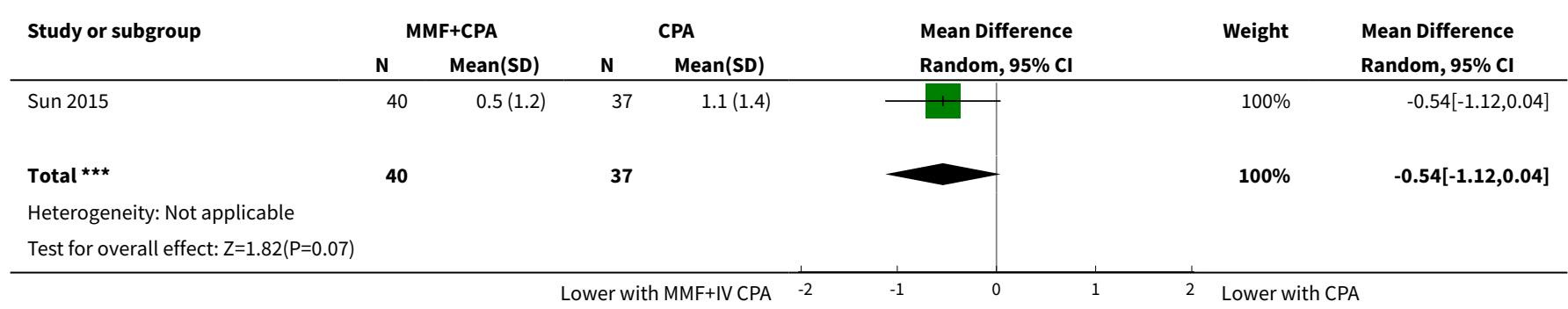

\section{Comparison 5. Mycophenolate mofetil (MMF) versus tacrolimus (TAC)}

\begin{tabular}{lllll}
\hline $\begin{array}{l}\text { Outcome or subgroup ti- } \\
\text { tle }\end{array}$ & No. of studies & $\begin{array}{l}\text { No. of partici- } \\
\text { pants }\end{array}$ & Statistical method & Effect size \\
\hline 1 Death & 3 & 273 & Risk Ratio (M-H, Random, 95\% Cl) & $1.10[0.44,2.77]$ \\
\hline 2 Remission & 3 & Risk Ratio (M-H, Random, 95\% Cl) & Subtotals only \\
\hline $\begin{array}{l}2.1 \text { Complete renal remis- } \\
\text { sion }\end{array}$ & 3 & 273 & Risk Ratio (M-H, Random, 95\% Cl) & $1.02[0.83,1.26]$ \\
\hline $\begin{array}{l}2.2 \text { Partial renal remission } \\
2.3 \text { Complete remission in }\end{array}$ & 1 & 190 & Risk Ratio (M-H, Random, 95\% Cl) & $0.83[0.51,1.36]$ \\
\hline \begin{tabular}{l} 
proteinuria \\
\hline
\end{tabular} & 40 & Risk Ratio (M-H, Random, 95\% Cl) & $1.0[0.50,1.98]$
\end{tabular}




\begin{tabular}{|c|c|c|c|c|}
\hline $\begin{array}{l}\text { Outcome or subgroup ti- } \\
\text { tle }\end{array}$ & No. of studies & $\begin{array}{l}\text { No. of partici- } \\
\text { pants }\end{array}$ & Statistical method & Effect size \\
\hline $\begin{array}{l}2.4 \text { Partial remission in } \\
\text { proteinuria }\end{array}$ & 2 & 190 & Risk Ratio (M-H, Random, 95\% Cl) & $0.90[0.79,1.03]$ \\
\hline 3 Adverse renal outcomes & 1 & & Risk Ratio (M-H, Random, 95\% Cl) & Subtotals only \\
\hline 3.1 ESKD & 1 & 150 & Risk Ratio (M-H, Random, 95\% Cl) & $1.22[0.51,2.91]$ \\
\hline 3.2 Renal relapse & 1 & 150 & Risk Ratio (M-H, Random, 95\% Cl) & $0.67[0.48,0.93]$ \\
\hline $\begin{array}{l}\text { 3.3 Renal relapse (nephrit- } \\
\text { ic flare) }\end{array}$ & 1 & 152 & Risk Ratio (M-H, Random, 95\% Cl) & $0.68[0.36,1.28]$ \\
\hline $\begin{array}{l}\text { 3.4 Renal relapse (protein- } \\
\text { uric flare) }\end{array}$ & 1 & 150 & Risk Ratio (M-H, Random, 95\% Cl) & $0.67[0.41,1.12]$ \\
\hline $\begin{array}{l}\text { 3.5 Deterioration in kidney } \\
\text { function }\end{array}$ & 1 & 150 & Risk Ratio (M-H, Random, 95\% Cl) & $0.54[0.27,1.09]$ \\
\hline 4 Stable kidney function & 1 & 40 & Risk Ratio (M-H, Random, 95\% Cl) & $1.0[0.50,1.98]$ \\
\hline 5 Menstrual irregularities & 1 & 40 & Risk Ratio (M-H, Random, 95\% Cl) & $3.0[0.13,69.52]$ \\
\hline 6 Infection & 2 & & Risk Ratio (M-H, Random, 95\% Cl) & Subtotals only \\
\hline 6.1 Major infection & 2 & 190 & Risk Ratio (M-H, Random, 95\% Cl) & $2.14[0.93,4.92]$ \\
\hline 6.2 Herpes zoster virus & 1 & 150 & Risk Ratio (M-H, Random, 95\% Cl) & $6.82[1.60,28.96]$ \\
\hline 7 Leucopenia & 1 & 40 & Risk Ratio (M-H, Random, 95\% Cl) & $1.0[0.07,14.90]$ \\
\hline 8 Alopecia & 1 & 150 & Risk Ratio (M-H, Random, 95\% Cl) & $0.07[0.00,1.31]$ \\
\hline $\begin{array}{l}9 \text { Daily proteinuria (at } 24 \\
\text { weeks) }\end{array}$ & 1 & 150 & Mean Difference (IV, Random, 95\% CI) & $0.18[-0.25,0.61]$ \\
\hline 10 Disease activity & 2 & & Mean Difference (IV, Random, 95\% CI) & Subtotals only \\
\hline 10.1 Renal SLEDAI & 2 & 233 & Mean Difference (IV, Random, 95\% CI) & $-0.21[-2.05,1.63]$ \\
\hline 10.2 Extrarenal SLEDAI & 2 & 233 & Mean Difference (IV, Random, 95\% CI) & $-0.26[-0.74,0.22]$ \\
\hline 11 Serum creatinine & 1 & 83 & Mean Difference (IV, Random, 95\% CI) & $-0.01[-0.16,0.14]$ \\
\hline 12 Creatinine clearance & 1 & 40 & Mean Difference (IV, Random, 95\% CI) & $-1.93[-7.77,3.91]$ \\
\hline
\end{tabular}

Analysis 5.1. Comparison 5 Mycophenolate mofetil (MMF) versus tacrolimus (TAC), Outcome 1 Death.

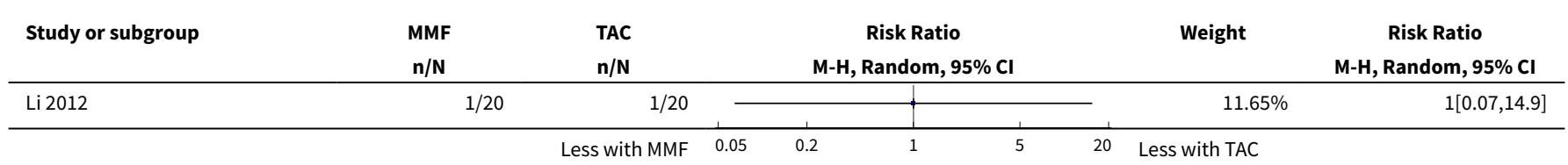




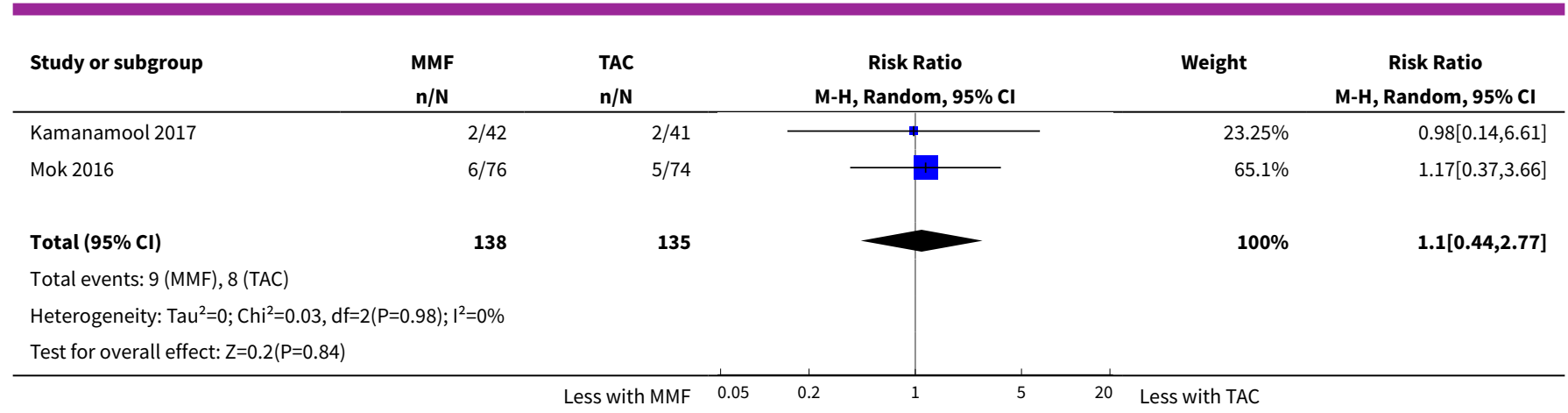

\section{Analysis 5.2. Comparison 5 Mycophenolate mofetil (MMF) versus tacrolimus (TAC), Outcome 2 Remission.}

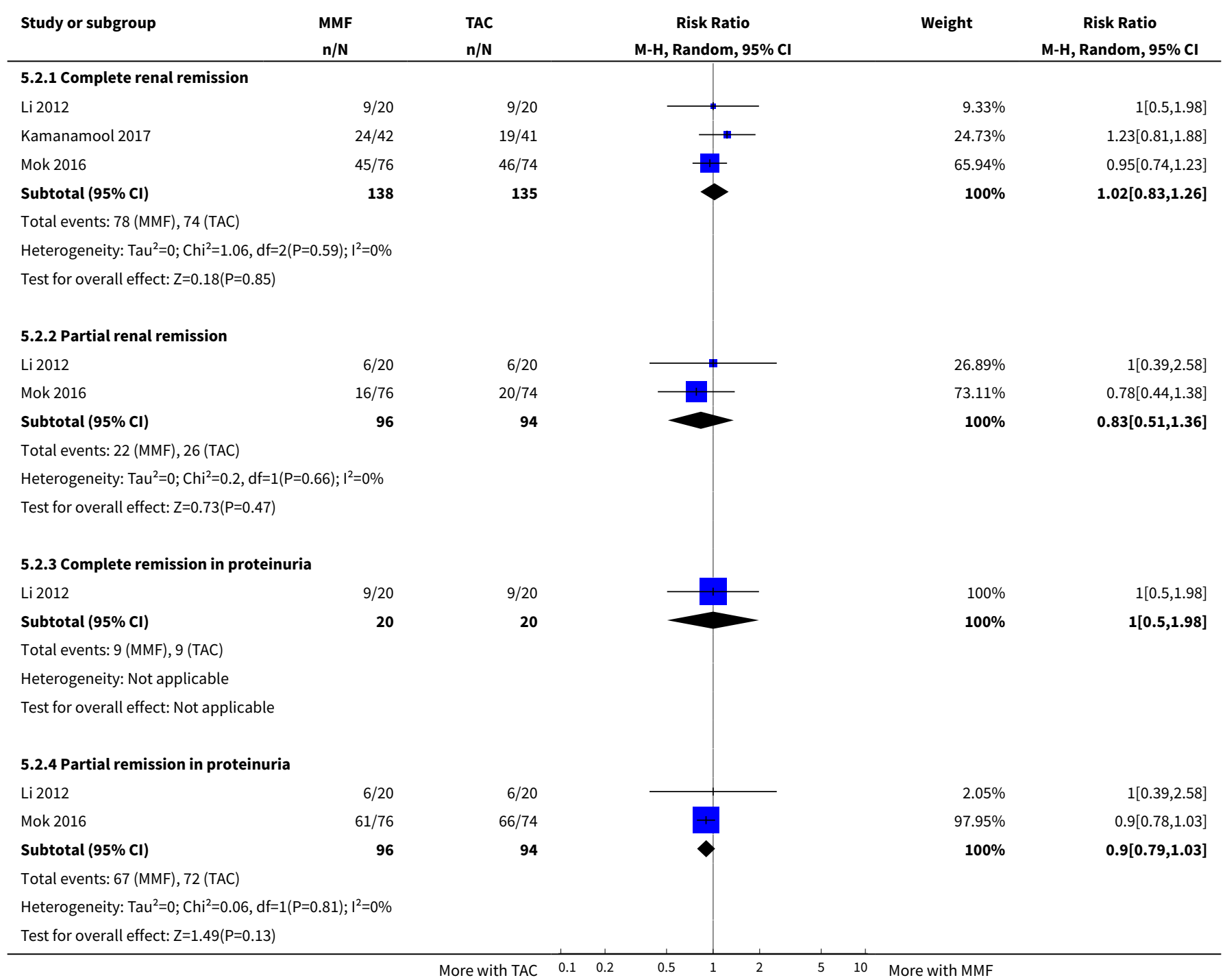


Analysis 5.3. Comparison 5 Mycophenolate mofetil (MMF) versus tacrolimus (TAC), Outcome 3 Adverse renal outcomes.

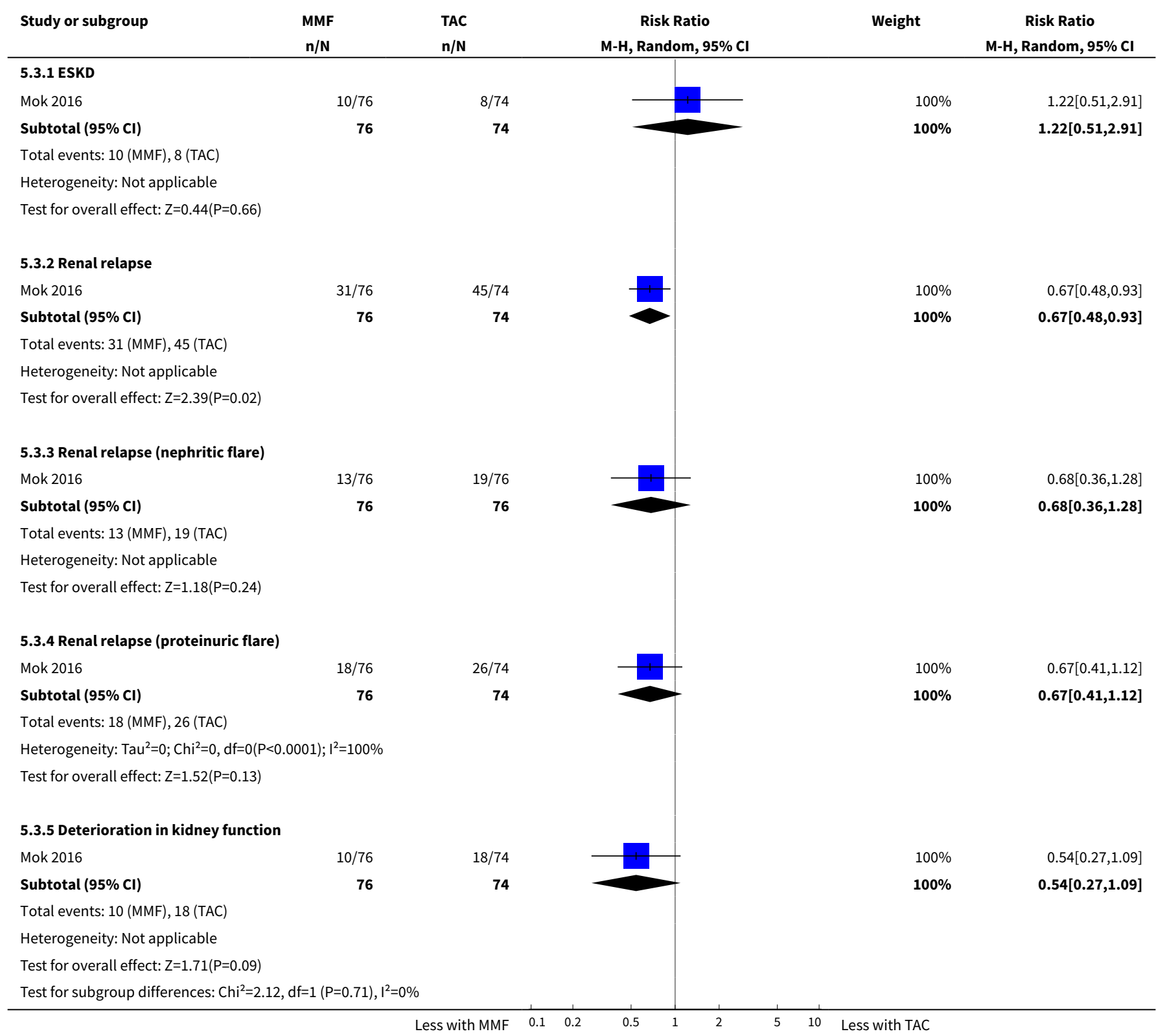

Analysis 5.4. Comparison 5 Mycophenolate mofetil (MMF) versus tacrolimus (TAC), Outcome 4 Stable kidney function.

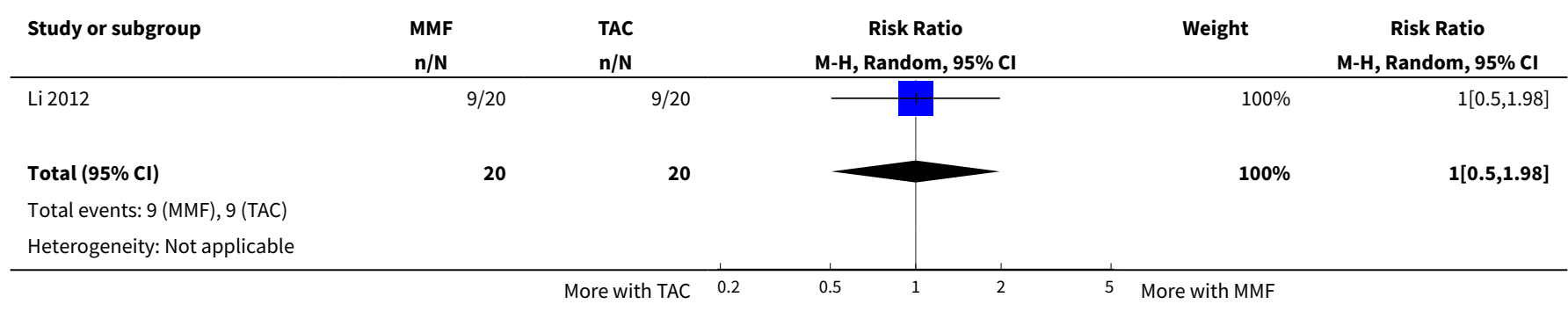




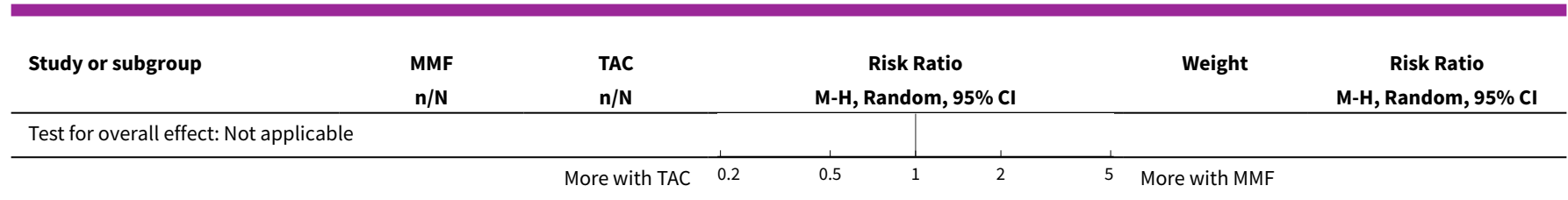

Analysis 5.5. Comparison 5 Mycophenolate mofetil (MMF) versus tacrolimus (TAC), Outcome 5 Menstrual irregularities.

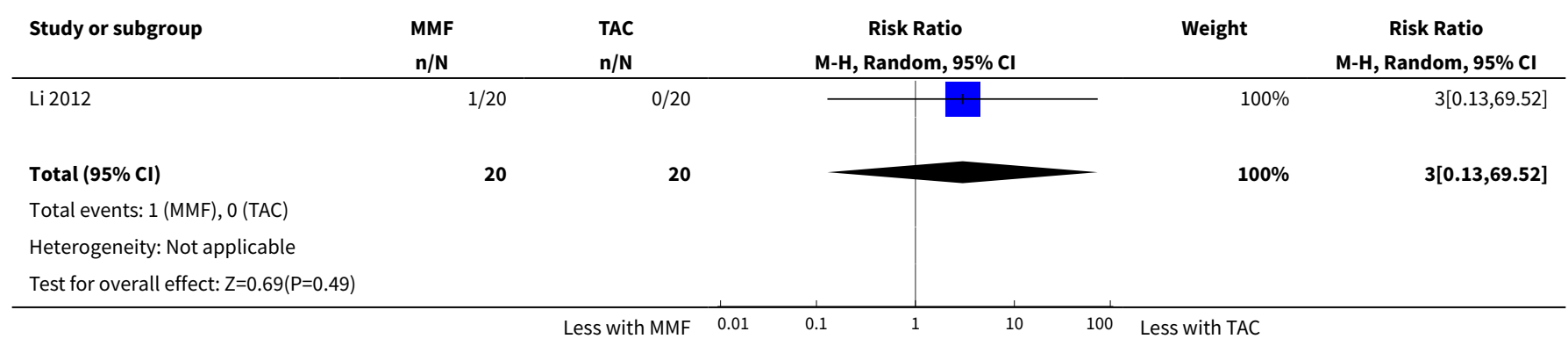

Analysis 5.6. Comparison 5 Mycophenolate mofetil (MMF) versus tacrolimus (TAC), Outcome 6 Infection.

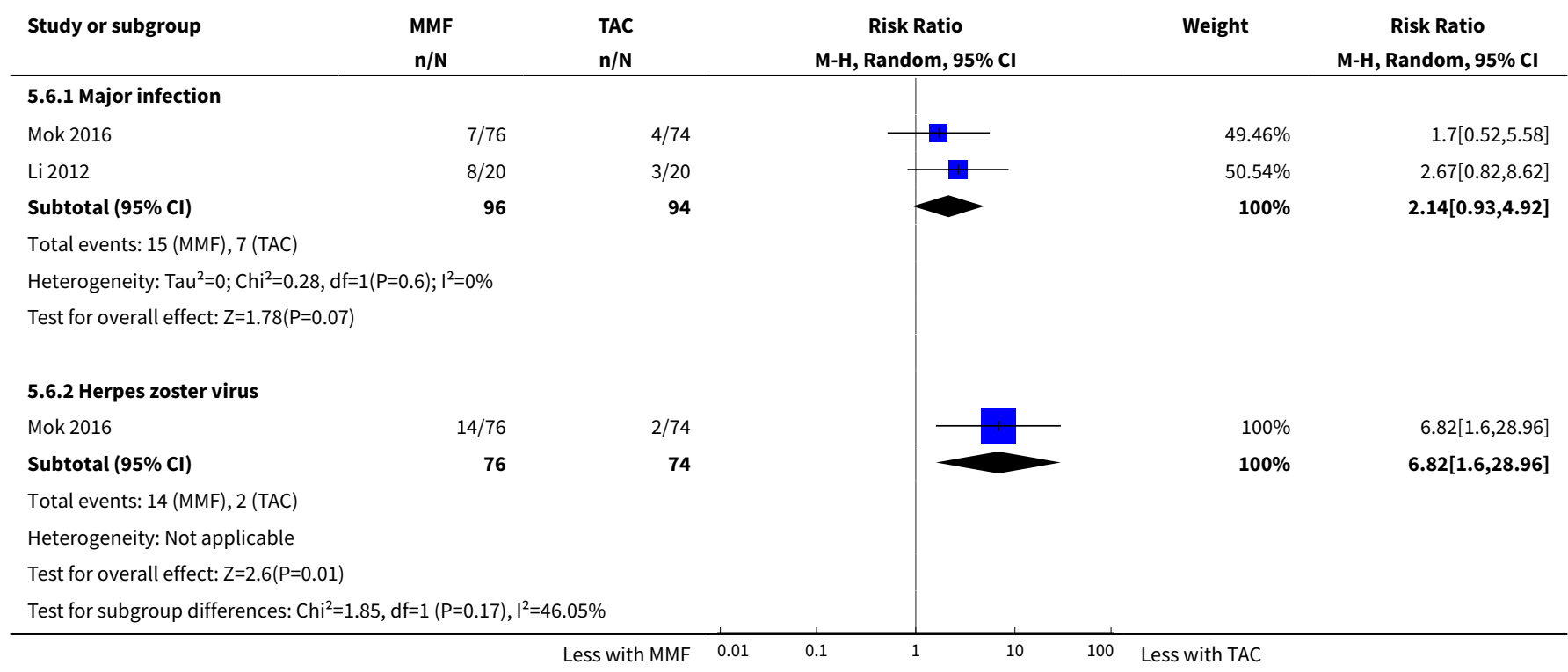

Analysis 5.7. Comparison 5 Mycophenolate mofetil (MMF) versus tacrolimus (TAC), Outcome 7 Leucopenia.

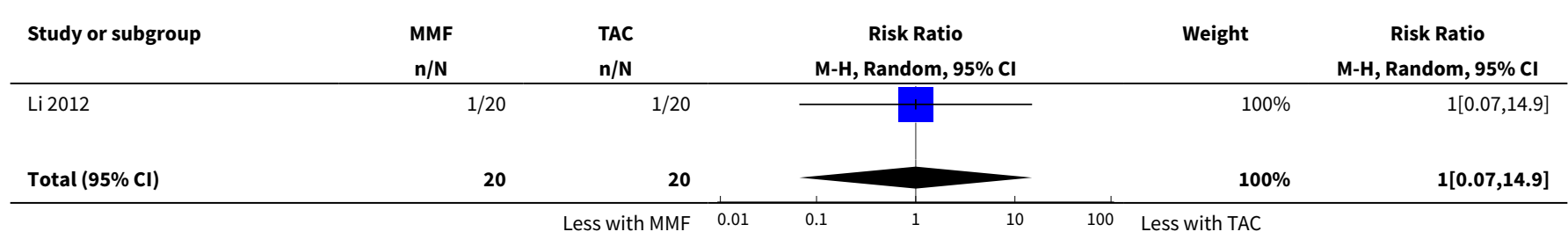




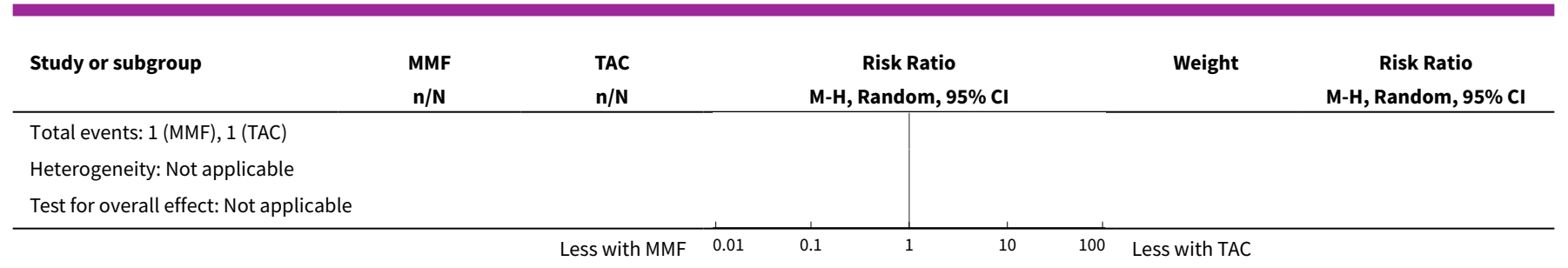

Analysis 5.8. Comparison 5 Mycophenolate mofetil (MMF) versus tacrolimus (TAC), Outcome 8 Alopecia.

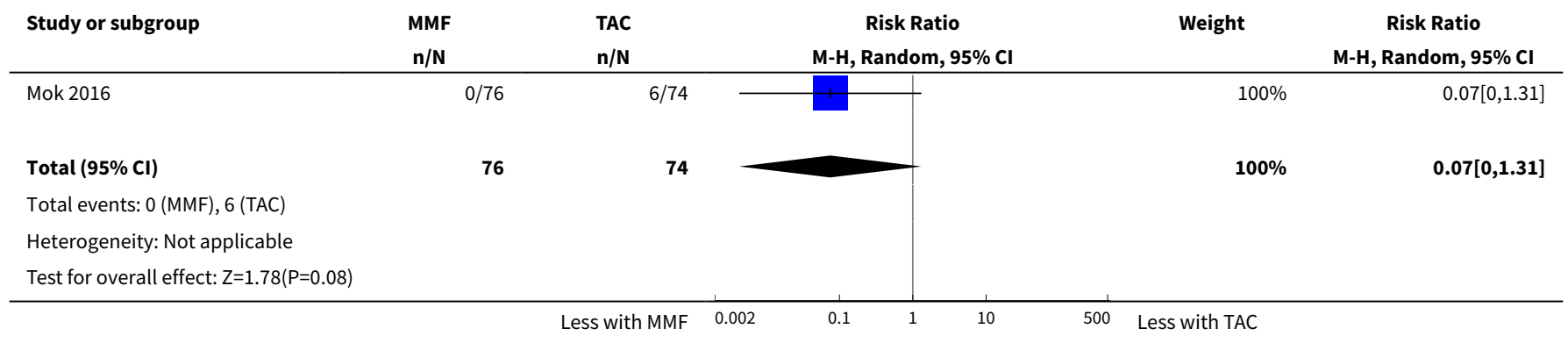

\section{Analysis 5.9. Comparison 5 Mycophenolate mofetil (MMF) versus} tacrolimus (TAC), Outcome 9 Daily proteinuria (at 24 weeks).

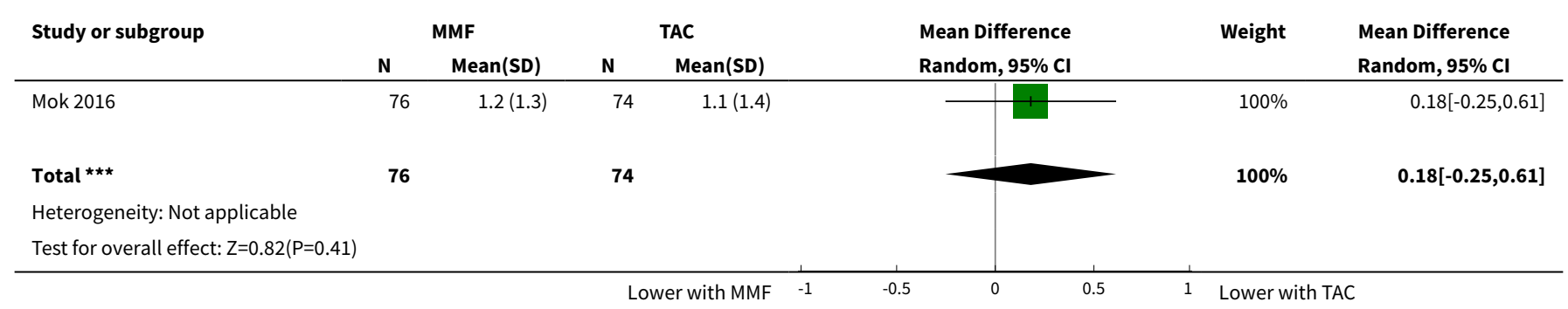

Analysis 5.10. Comparison 5 Mycophenolate mofetil (MMF) versus tacrolimus (TAC), Outcome 10 Disease activity.

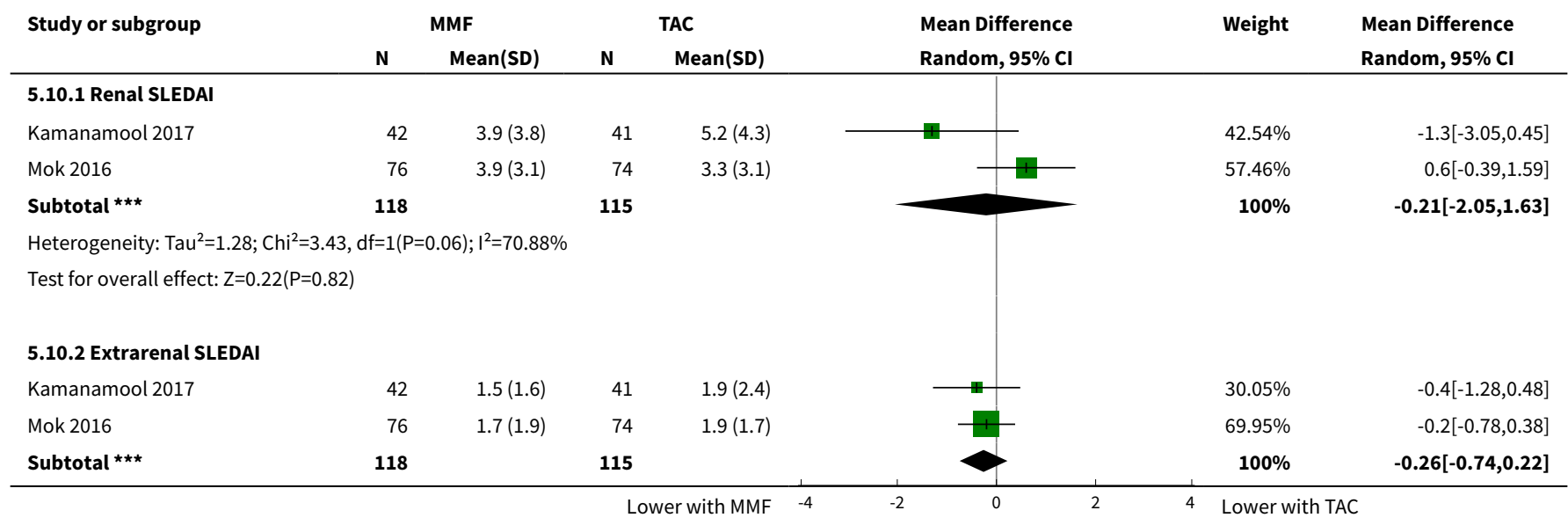




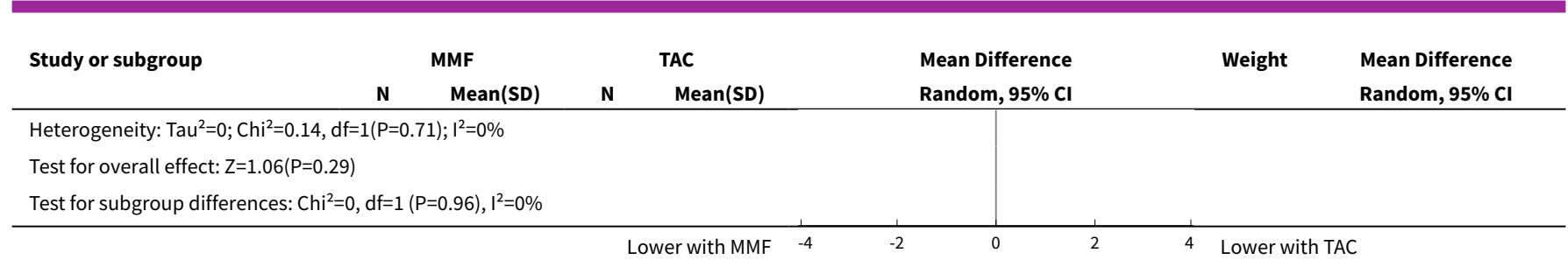

Analysis 5.11. Comparison 5 Mycophenolate mofetil (MMF) versus tacrolimus (TAC), Outcome 11 Serum creatinine.

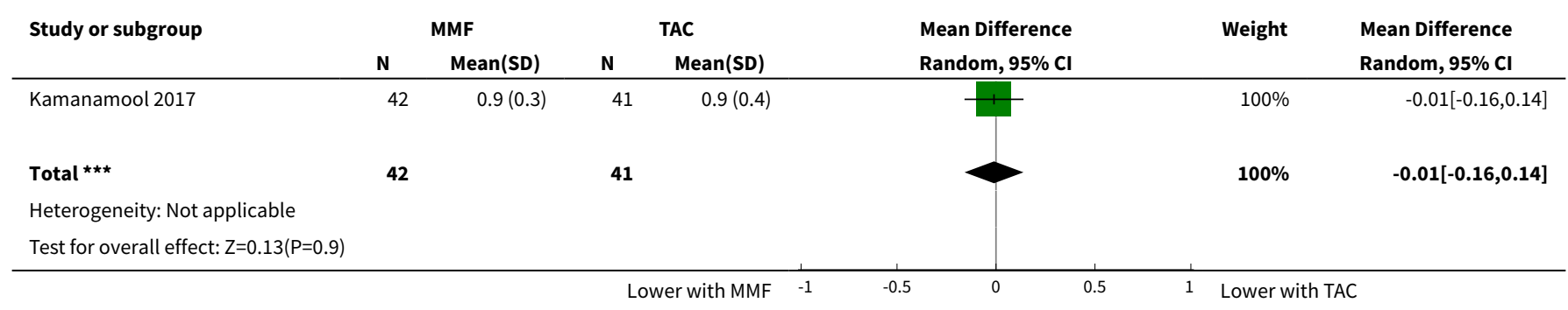

\section{Analysis 5.12. Comparison 5 Mycophenolate mofetil (MMF)} versus tacrolimus (TAC), Outcome 12 Creatinine clearance.

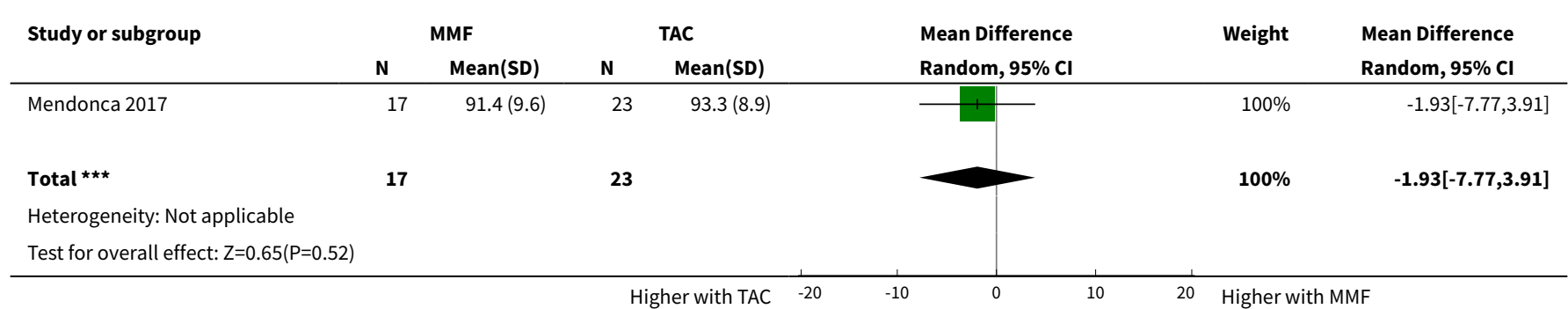

\section{Comparison 6. Calcineurin inhibitors (CNI) versus IV cyclophosphamide (CPA)}

\begin{tabular}{lllll}
\hline $\begin{array}{l}\text { Outcome or subgroup ti- } \\
\text { tle }\end{array}$ & No. of studies & $\begin{array}{l}\text { No. of partici- } \\
\text { pants }\end{array}$ & Statistical method & Effect size \\
\hline 1 Death & 3 & & Risk Ratio (M-H, Random, 95\% Cl) & Subtotals only \\
\hline $\begin{array}{lllll}1.1 \text { Death } \\
\text { 1.2 Death: extended fol- }\end{array}$ & 1 & 153 & Risk Ratio (M-H, Random, 95\% Cl) & $0.41[0.06,2.69]$ \\
\hline $\begin{array}{l}\text { low-up } \\
\text { Remission }\end{array}$ & 48 & Risk Ratio (M-H, Random, 95\% Cl) & $0.0[0.0,0.0]$ \\
\hline $\begin{array}{l}2.1 \text { Complete renal remis- } \\
\text { sion }\end{array}$ & 4 & & Risk Ratio (M-H, Random, 95\% Cl) & Subtotals only \\
\hline \begin{tabular}{l}
2.2 Partial renal remission \\
\hline
\end{tabular} & 4 & 178 & Risk Ratio (M-H, Random, 95\% Cl) & $1.35[0.94,1.93]$ \\
\hline
\end{tabular}




\begin{tabular}{|c|c|c|c|c|}
\hline $\begin{array}{l}\text { Outcome or subgroup ti- } \\
\text { tle }\end{array}$ & No. of studies & $\begin{array}{l}\text { No. of partici- } \\
\text { pants }\end{array}$ & Statistical method & Effect size \\
\hline $\begin{array}{l}2.3 \text { Complete remission in } \\
\text { proteinuria }\end{array}$ & 3 & 105 & Risk Ratio (M-H, Random, 95\% Cl) & $1.71[1.08,2.70]$ \\
\hline 3 Adverse renal outcomes & 2 & & Risk Ratio (M-H, Random, 95\% Cl) & Subtotals only \\
\hline $\begin{array}{l}\text { 3.1 ESKD: extended fol- } \\
\text { low-up }\end{array}$ & 1 & 38 & Risk Ratio (M-H, Random, 95\% Cl) & $1.0[0.07,14.85]$ \\
\hline $\begin{array}{l}3.2 \text { Doubling of serum cre- } \\
\text { atinine }\end{array}$ & 1 & 40 & Risk Ratio (M-H, Random, 95\% Cl) & $0.33[0.01,7.72]$ \\
\hline $\begin{array}{l}\text { 3.3 Doubling of serum } \\
\text { creatinine: extended fol- } \\
\text { low-up }\end{array}$ & 1 & 38 & Risk Ratio (M-H, Random, 95\% Cl) & $1.0[0.16,6.38]$ \\
\hline 4 Stable kidney function & 4 & 186 & Risk Ratio (M-H, Random, 95\% Cl) & $1.11[0.61,2.00]$ \\
\hline 5 Ovarian failure & 2 & & Risk Ratio (M-H, Random, 95\% Cl) & Subtotals only \\
\hline 5.1 Ovarian failure & 2 & 113 & Risk Ratio (M-H, Random, 95\% Cl) & $0.25[0.03,2.18]$ \\
\hline $\begin{array}{l}\text { 5.2 Premature ovarian fail- } \\
\text { ure: extended follow-up }\end{array}$ & 1 & 27 & Risk Ratio (M-H, Random, 95\% Cl) & $0.31[0.01,7.02]$ \\
\hline 6 Menstrual irregularities & 2 & 54 & Risk Ratio (M-H, Random, 95\% Cl) & $0.41[0.04,4.05]$ \\
\hline 7 Infection & 4 & & Risk Ratio (M-H, Random, 95\% Cl) & Subtotals only \\
\hline 7.1 Major infection & 3 & 138 & Risk Ratio (M-H, Random, 95\% Cl) & $0.73[0.33,1.63]$ \\
\hline 7.2 Herpes zoster virus & 2 & 113 & Risk Ratio (M-H, Random, 95\% Cl) & $1.41[0.38,5.20]$ \\
\hline $\begin{array}{l}8 \text { Malignancy: extended } \\
\text { follow-up }\end{array}$ & 1 & 38 & Risk Ratio (M-H, Random, 95\% Cl) & $5.0[0.26,97.70]$ \\
\hline 9 Leucopenia & 3 & 153 & Risk Ratio (M-H, Random, 95\% Cl) & $0.44[0.13,1.49]$ \\
\hline 10 Alopecia & 2 & 113 & Risk Ratio (M-H, Random, 95\% Cl) & $0.21[0.02,1.76]$ \\
\hline $\begin{array}{l}11 \text { Gastrointestinal (GI) ad- } \\
\text { verse events }\end{array}$ & 1 & 73 & Risk Ratio (M-H, Random, 95\% Cl) & $0.35[0.12,1.01]$ \\
\hline 12 Daily proteinuria & 2 & 156 & Mean Difference (IV, Random, 95\% CI) & $-0.37[-0.67,-0.07]$ \\
\hline 12.1 At 9 months & 1 & 40 & Mean Difference (IV, Random, 95\% CI) & $-0.83[-1.37,-0.29]$ \\
\hline 12.2 At 12 months & 1 & 38 & Mean Difference (IV, Random, 95\% Cl) & $-0.27[-0.43,-0.11]$ \\
\hline 12.3 At 18 months & 1 & 40 & Mean Difference (IV, Random, 95\% CI) & $-1.0[-2.26,0.26]$ \\
\hline 12.4 Extended follow-up & 1 & 38 & Mean Difference (IV, Random, 95\% CI) & $-0.10[-0.49,0.29]$ \\
\hline 13 Creatinine clearance & 3 & & Mean Difference (IV, Random, 95\% CI) & Subtotals only \\
\hline
\end{tabular}




\begin{tabular}{|c|c|c|c|c|}
\hline $\begin{array}{l}\text { Outcome or subgroup ti- } \\
\text { tle }\end{array}$ & No. of studies & $\begin{array}{l}\text { No. of partici- } \\
\text { pants }\end{array}$ & Statistical method & Effect size \\
\hline 13.1 At 6 months & 1 & 150 & Mean Difference (IV, Random, 95\% CI) & $11.70[1.61,21.79]$ \\
\hline 13.2 At 9 months & 1 & 40 & Mean Difference (IV, Random, 95\% CI) & $14.90[1.35,28.45]$ \\
\hline 13.3 At 12 months & 1 & 38 & Mean Difference (IV, Random, 95\% CI) & $-15.70[-23.71,-7.69]$ \\
\hline 13.4 At 18 months & 1 & 40 & Mean Difference (IV, Random, 95\% CI) & $-1.40[-17.25,14.45]$ \\
\hline 14 Serum creatinine & 1 & & Mean Difference (IV, Random, 95\% CI) & Subtotals only \\
\hline 14.1 At 9 months & 1 & 40 & Mean Difference (IV, Random, 95\% CI) & $12.70[1.88,23.52]$ \\
\hline 14.2 At 18 months & 1 & 40 & Mean Difference (IV, Random, 95\% CI) & $2.70[-11.50,16.90]$ \\
\hline 14.3 Extended follow-up & 1 & 38 & Mean Difference (IV, Random, 95\% CI) & $-8.0[-20.35,4.35]$ \\
\hline
\end{tabular}

\section{Analysis 6.1. Comparison 6 Calcineurin inhibitors (CNI) versus IV cyclophosphamide (CPA), Outcome 1 Death.}

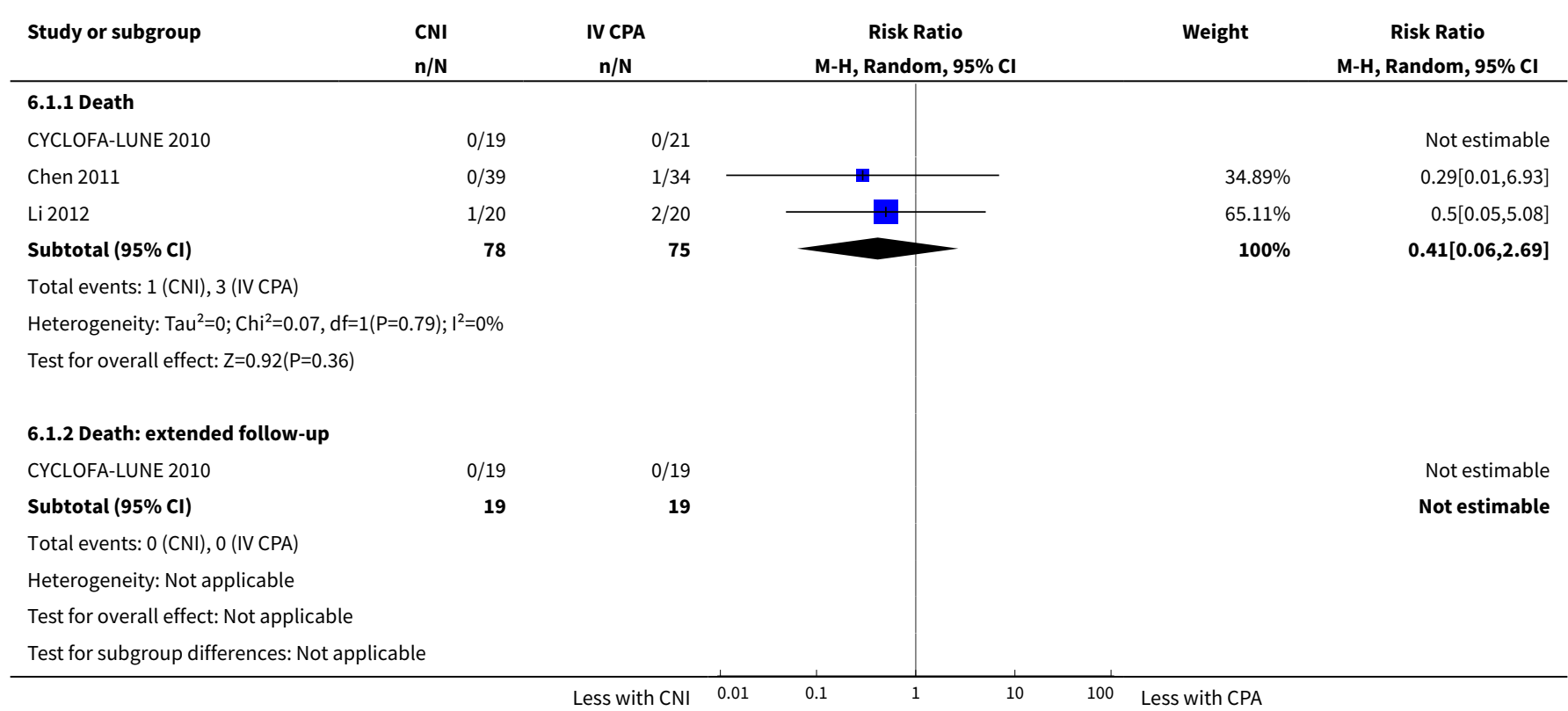

Analysis 6.2. Comparison 6 Calcineurin inhibitors (CNI) versus IV cyclophosphamide (CPA), Outcome 2 Remission.

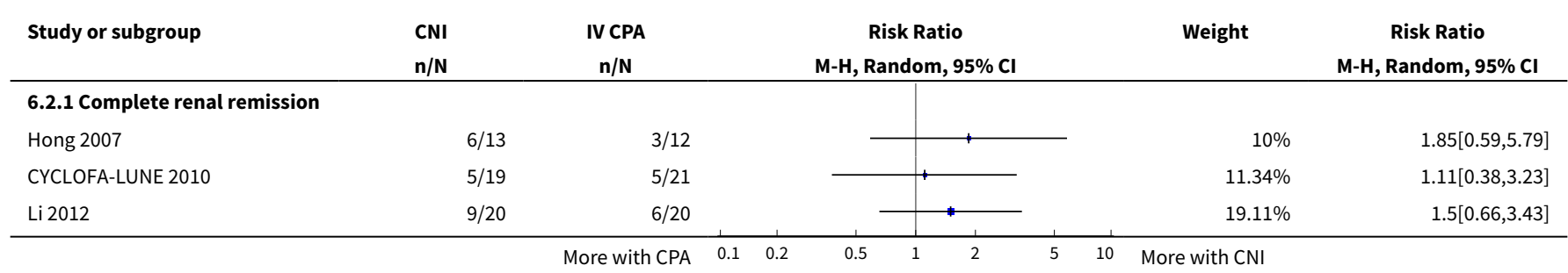




\begin{tabular}{|c|c|c|c|c|c|}
\hline Study or subgroup & $\begin{array}{l}\text { CNI } \\
\mathrm{n} / \mathrm{N}\end{array}$ & $\begin{array}{l}\text { IV CPA } \\
\mathbf{n} / \mathbf{N}\end{array}$ & $\begin{array}{c}\text { Risk Ratio } \\
\text { M-H, Random, } 95 \% \mathrm{Cl}\end{array}$ & Weight & $\begin{array}{c}\text { Risk Ratio } \\
\text { M-H, Random, } 95 \% \mathrm{Cl}\end{array}$ \\
\hline Chen 2011 & $22 / 39$ & $15 / 34$ & 71 & $59.55 \%$ & $1.28[0.8,2.04]$ \\
\hline Subtotal $(95 \% \mathrm{CI})$ & 91 & 87 & & $100 \%$ & $1.35[0.94,1.93]$ \\
\hline \multicolumn{6}{|c|}{ Total events: 42 (CNI), 29 (IV CPA) } \\
\hline \multicolumn{6}{|c|}{ Heterogeneity: $\mathrm{Tau}^{2}=0 ; \mathrm{Chi}^{2}=0.54, \mathrm{df}=3(\mathrm{P}=0.91) ; \mathrm{I}^{2}=0 \%$} \\
\hline \multicolumn{6}{|c|}{ Test for overall effect: $Z=1.61(P=0.11)$} \\
\hline \multicolumn{6}{|c|}{ 6.2.2 Partial renal remission } \\
\hline Hong 2007 & $4 / 13$ & $2 / 12$ & & $5.71 \%$ & $1.85[0.41,8.32]$ \\
\hline Li 2012 & $6 / 20$ & $6 / 20$ & & $14.42 \%$ & $1[0.39,2.58]$ \\
\hline CYCLOFA-LUNE 2010 & $8 / 19$ & $11 / 21$ & $=$ & $29.09 \%$ & $0.8[0.41,1.57]$ \\
\hline Chen 2011 & $16 / 39$ & $17 / 34$ & & $50.78 \%$ & $0.82[0.5,1.36]$ \\
\hline Subtotal $(95 \% \mathrm{CI})$ & 91 & 87 & & $100 \%$ & $0.88[0.61,1.26]$ \\
\hline \multicolumn{6}{|c|}{ Total events: 34 (CNI), 36 (IV CPA) } \\
\hline \multicolumn{6}{|c|}{ Heterogeneity: $\operatorname{Tau}^{2}=0 ; \mathrm{Chi}^{2}=1.17, \mathrm{df}=3(\mathrm{P}=0.76) ; \mathrm{I}^{2}=0 \%$} \\
\hline \multicolumn{6}{|c|}{ Test for overall effect: $Z=0.7(P=0.48)$} \\
\hline \multicolumn{6}{|c|}{ 6.2.3 Complete remission in proteinuria } \\
\hline Hong 2007 & $6 / 13$ & $3 / 12$ & & $15.99 \%$ & $1.85[0.59,5.79]$ \\
\hline Li 2012 & $9 / 20$ & $6 / 20$ & $=$ & $30.56 \%$ & $1.5[0.66,3.43]$ \\
\hline CYCLOFA-LUNE 2010 & $13 / 19$ & $8 / 21$ & & $53.44 \%$ & $1.8[0.96,3.36]$ \\
\hline Subtotal $(95 \% \mathrm{Cl})$ & 52 & 53 & & $100 \%$ & $1.71[1.08,2.7]$ \\
\hline \multicolumn{6}{|c|}{ Total events: 28 (CNI), 17 (IV CPA) } \\
\hline \multicolumn{6}{|c|}{ Heterogeneity: $\mathrm{Tau}^{2}=0 ; \mathrm{Chi}^{2}=0.14, \mathrm{df}=2(\mathrm{P}=0.93) ; \mathrm{I}^{2}=0 \%$} \\
\hline \multicolumn{6}{|c|}{ Test for overall effect: $Z=2.29(P=0.02)$} \\
\hline Test for subgroup diff & $(P=0.06)$, & & & & \\
\hline
\end{tabular}

Analysis 6.3. Comparison 6 Calcineurin inhibitors (CNI) versus IV cyclophosphamide (CPA), Outcome 3 Adverse renal outcomes.

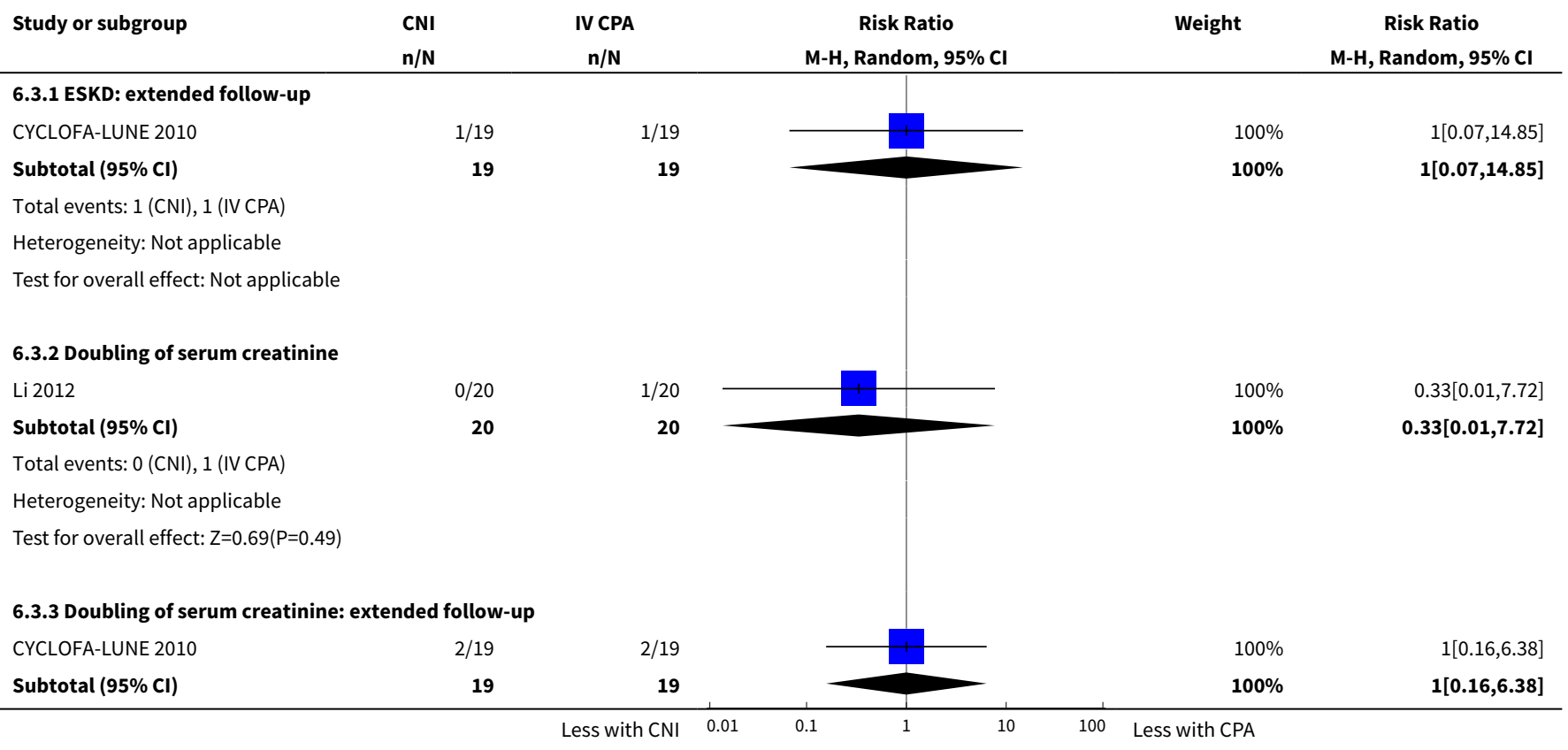




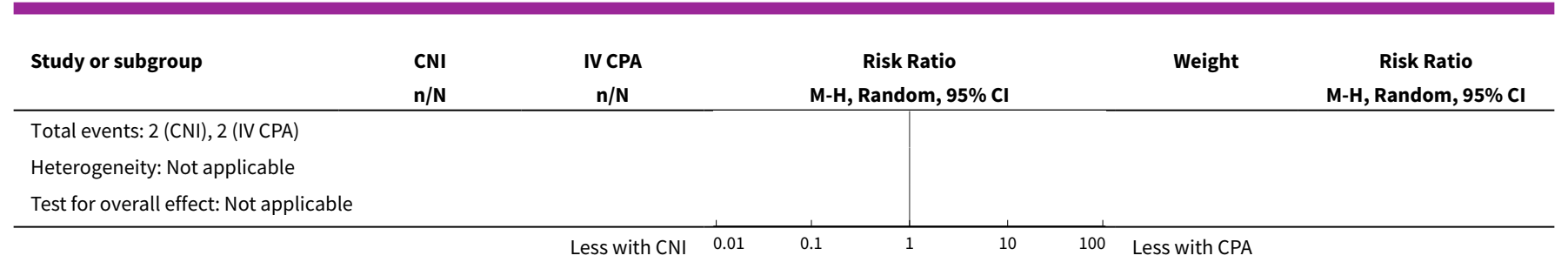

Analysis 6.4. Comparison 6 Calcineurin inhibitors (CNI) versus IV cyclophosphamide (CPA), Outcome 4 Stable kidney function.

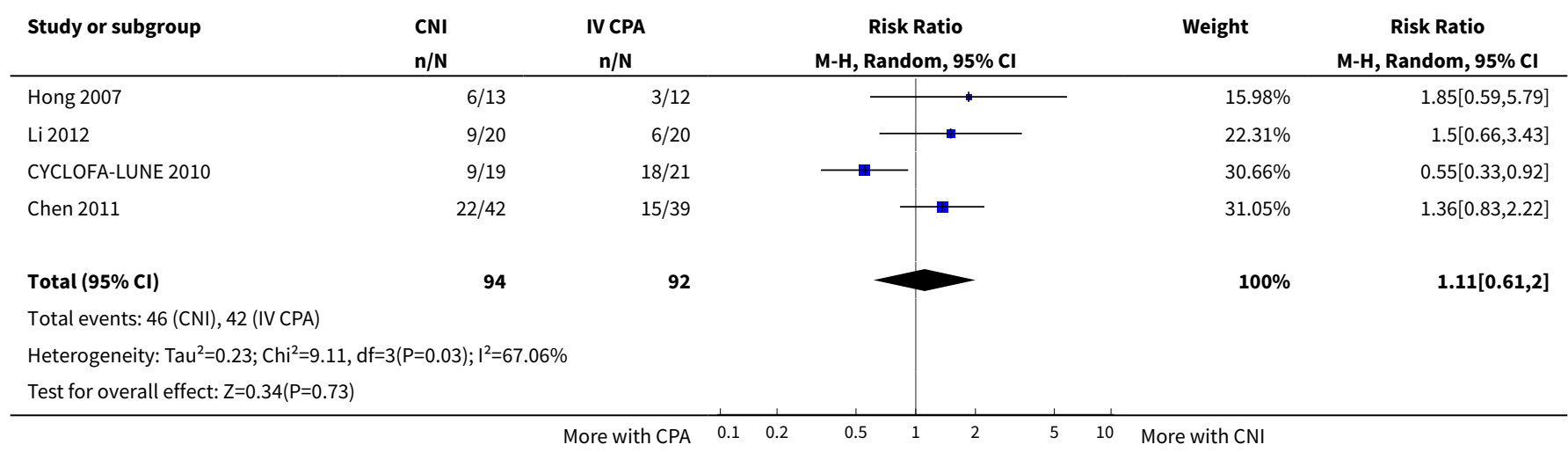

Analysis 6.5. Comparison 6 Calcineurin inhibitors (CNI) versus IV cyclophosphamide (CPA), Outcome 5 Ovarian failure.

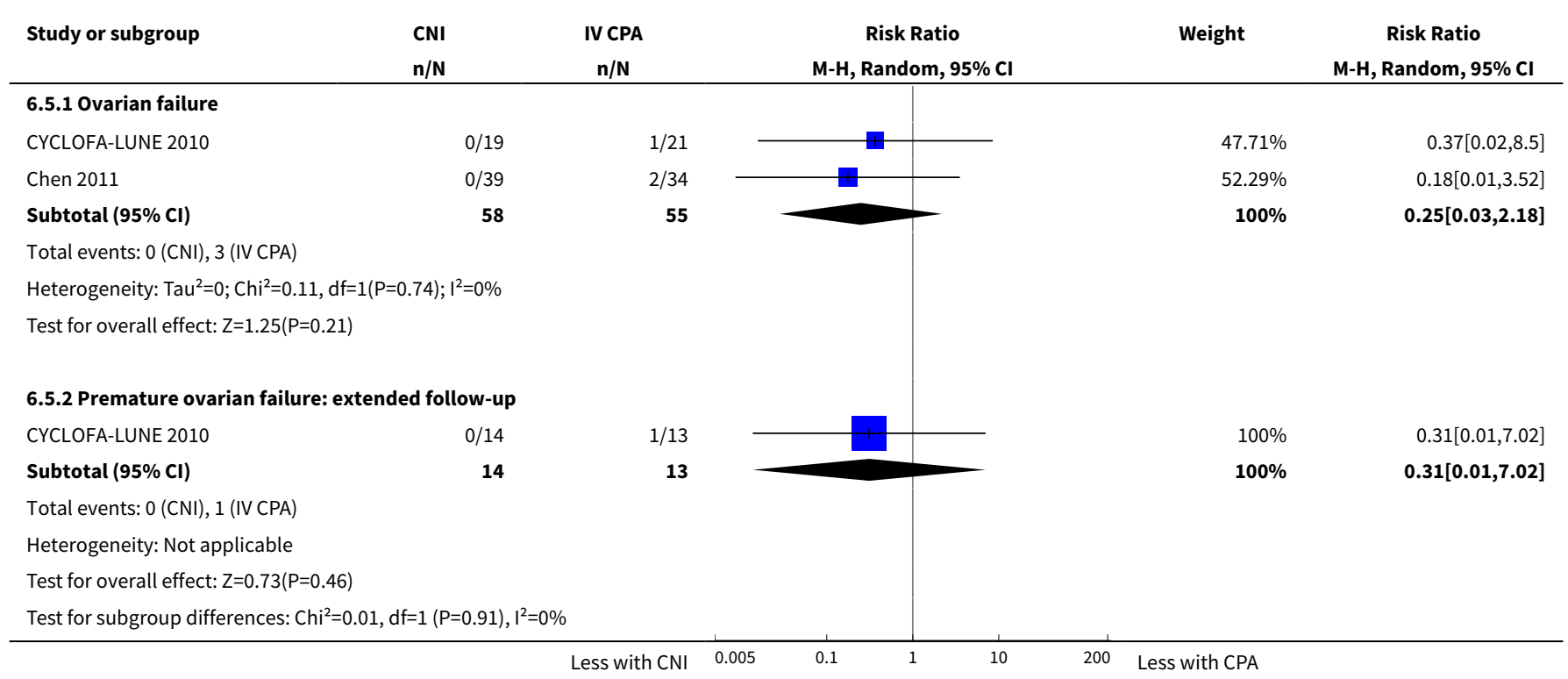


Analysis 6.6. Comparison 6 Calcineurin inhibitors (CNI) versus IV cyclophosphamide (CPA), Outcome 6 Menstrual irregularities.

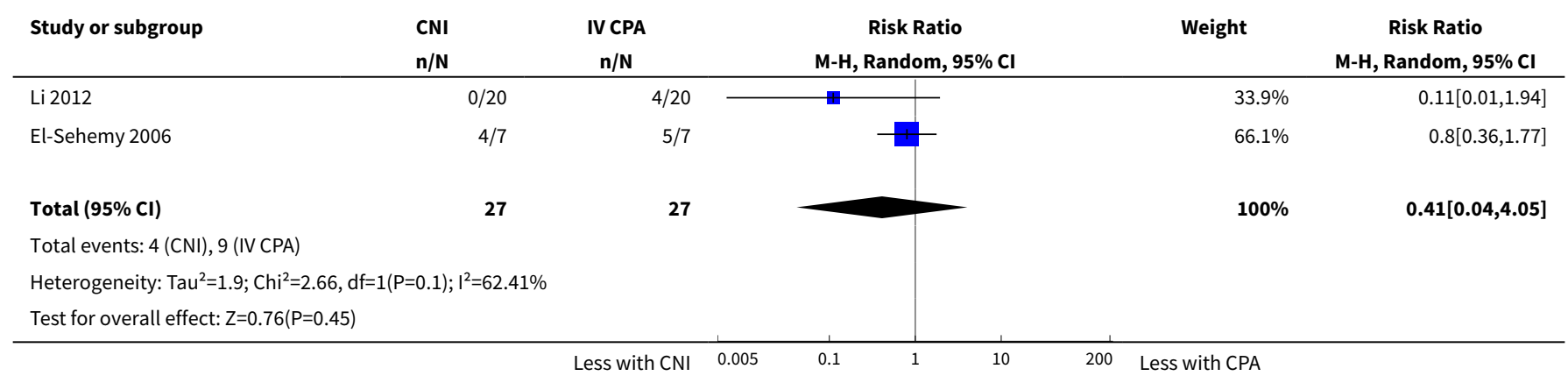

Analysis 6.7. Comparison 6 Calcineurin inhibitors (CNI) versus IV cyclophosphamide (CPA), Outcome 7 Infection.

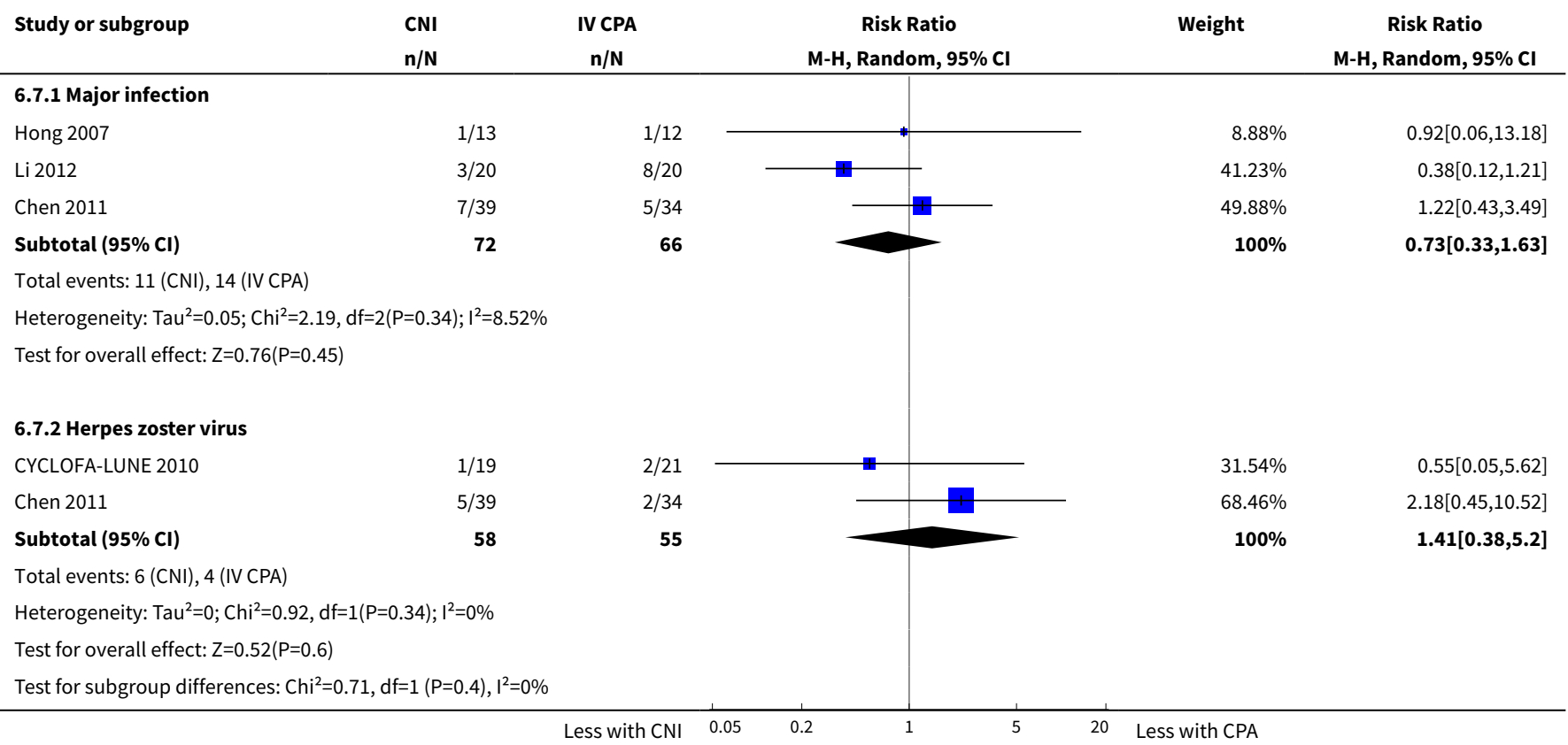

Analysis 6.8. Comparison 6 Calcineurin inhibitors (CNI) versus IV cyclophosphamide (CPA), Outcome 8 Malignancy: extended follow-up.

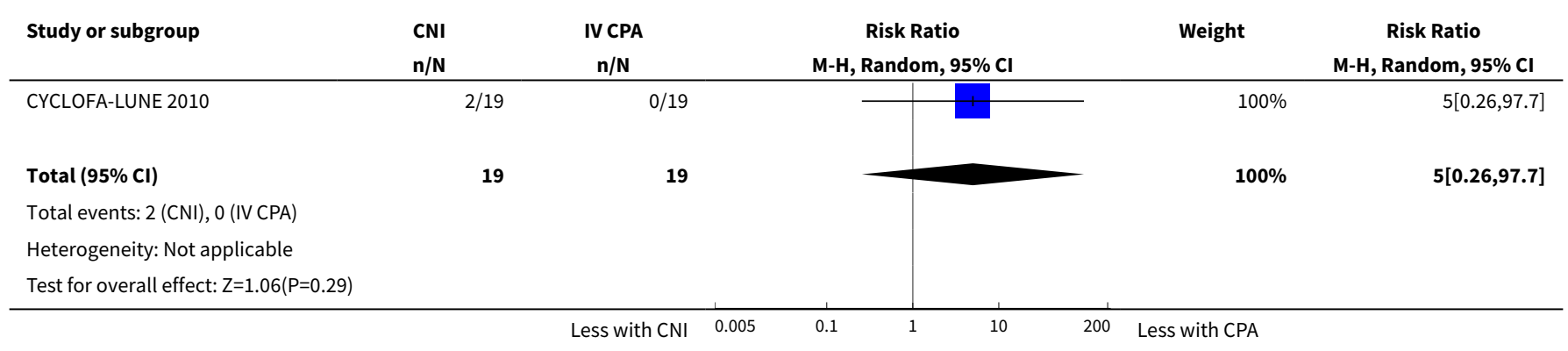


Analysis 6.9. Comparison 6 Calcineurin inhibitors (CNI) versus IV cyclophosphamide (CPA), Outcome 9 Leucopenia.

\begin{tabular}{|c|c|c|c|c|c|}
\hline Study or subgroup & $\begin{array}{l}\mathrm{CNI} \\
\mathrm{n} / \mathrm{N}\end{array}$ & $\begin{array}{l}\text { IV CPA } \\
\mathrm{n} / \mathrm{N}\end{array}$ & $\begin{array}{c}\text { Risk Ratio } \\
\text { M-H, Random, 95\% CI }\end{array}$ & Weight & $\begin{array}{c}\text { Risk Ratio } \\
\text { M-H, Random, } 95 \% \text { Cl }\end{array}$ \\
\hline Chen 2011 & $0 / 39$ & $5 / 34$ & $\rightarrow$ & $18.53 \%$ & $0.08[0,1.39]$ \\
\hline Li 2012 & $1 / 20$ & $1 / 20$ & & $20.76 \%$ & $1[0.07,14.9]$ \\
\hline CYCLOFA-LUNE 2010 & $2 / 19$ & $4 / 21$ & & $60.71 \%$ & $0.55[0.11,2.68]$ \\
\hline Total $(95 \% \mathrm{Cl})$ & 78 & 75 & & $100 \%$ & $0.44[0.13,1.49]$ \\
\hline \multicolumn{6}{|c|}{ Heterogeneity: $\mathrm{Tau}^{2}=0 ; \mathrm{Chi}^{2}=1.99, \mathrm{df}=2(\mathrm{P}=0.37) ; \mathrm{I}^{2}=0 \%$} \\
\hline \multicolumn{6}{|c|}{ Test for overall effect: $Z=1.32(P=0.19)$} \\
\hline
\end{tabular}

Analysis 6.10. Comparison 6 Calcineurin inhibitors (CNI) versus IV cyclophosphamide (CPA), Outcome 10 Alopecia.

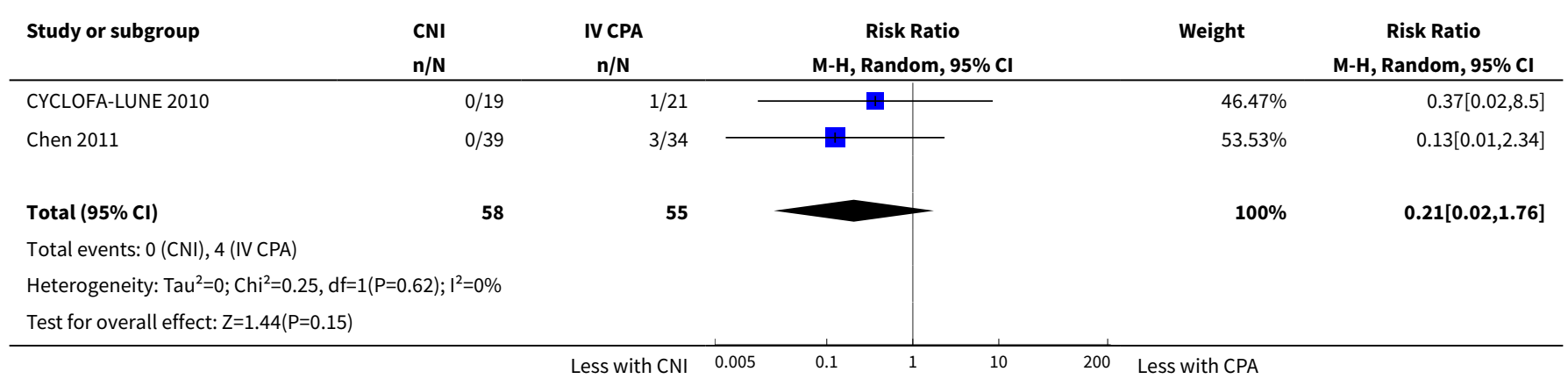

Analysis 6.11. Comparison 6 Calcineurin inhibitors (CNI) versus IV cyclophosphamide (CPA), Outcome 11 Gastrointestinal (GI) adverse events.

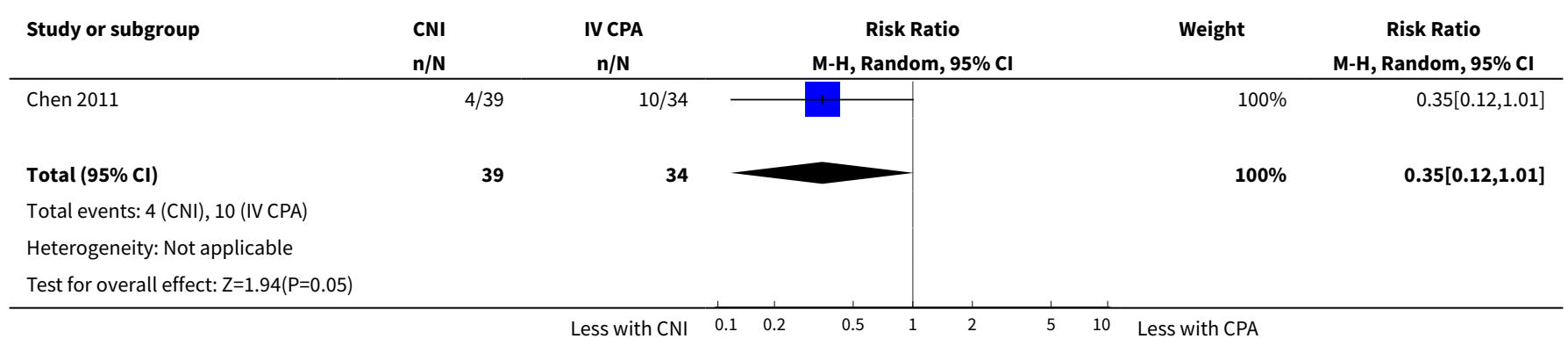


Analysis 6.12. Comparison 6 Calcineurin inhibitors (CNI) versus IV cyclophosphamide (CPA), Outcome 12 Daily proteinuria.

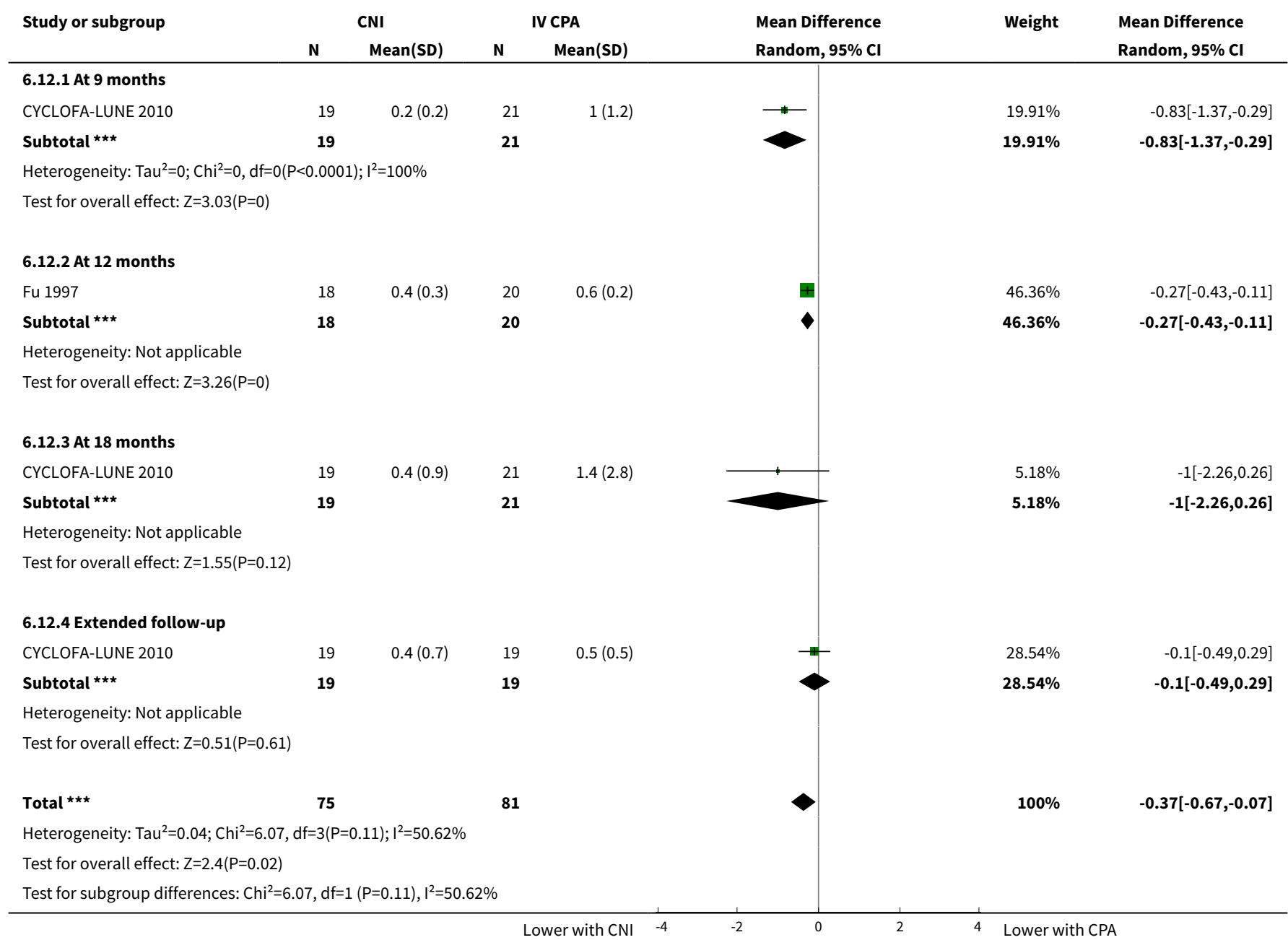

\section{Analysis 6.13. Comparison 6 Calcineurin inhibitors (CNI) versus IV cyclophosphamide (CPA), Outcome 13 Creatinine clearance.}

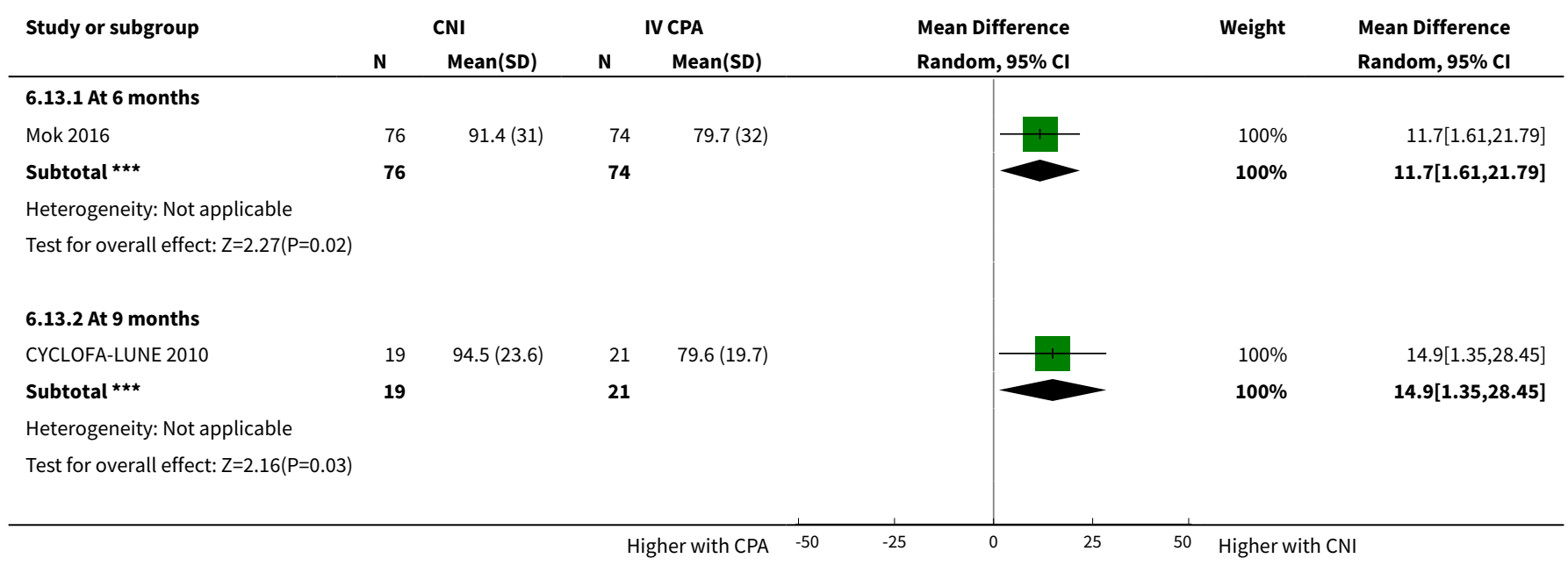




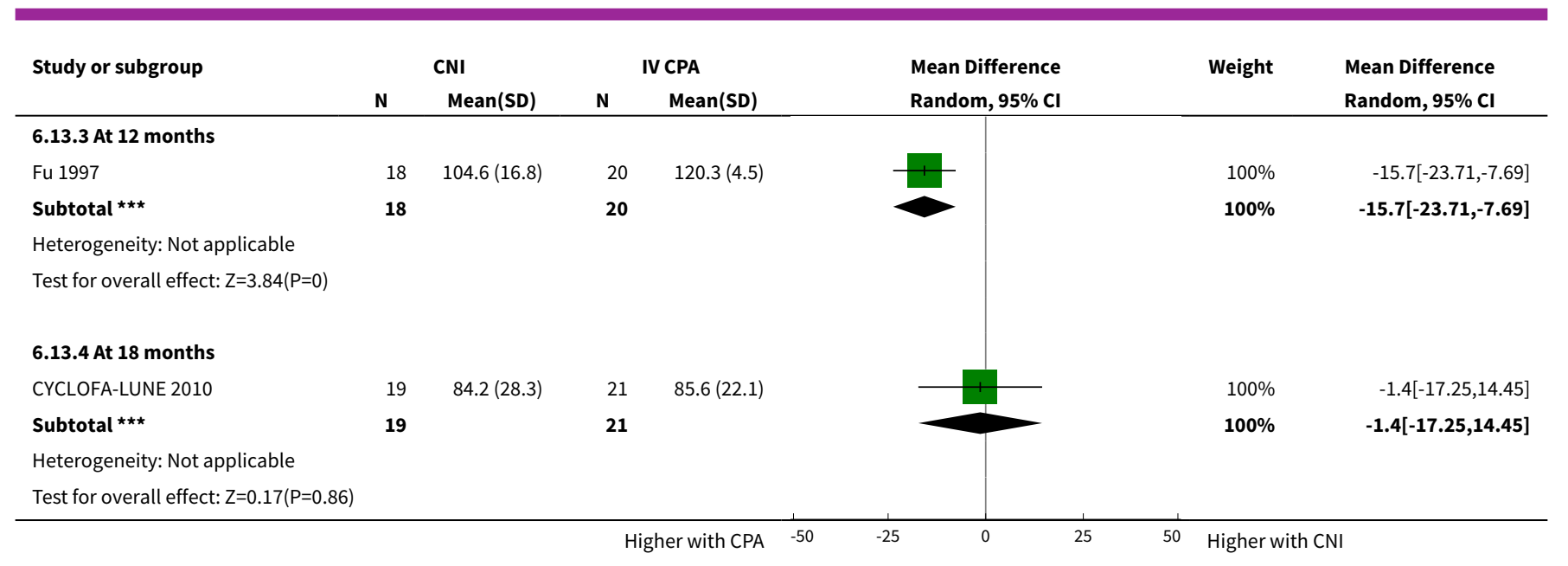

\section{Analysis 6.14. Comparison 6 Calcineurin inhibitors (CNI) versus IV cyclophosphamide (CPA), Outcome 14 Serum creatinine.}

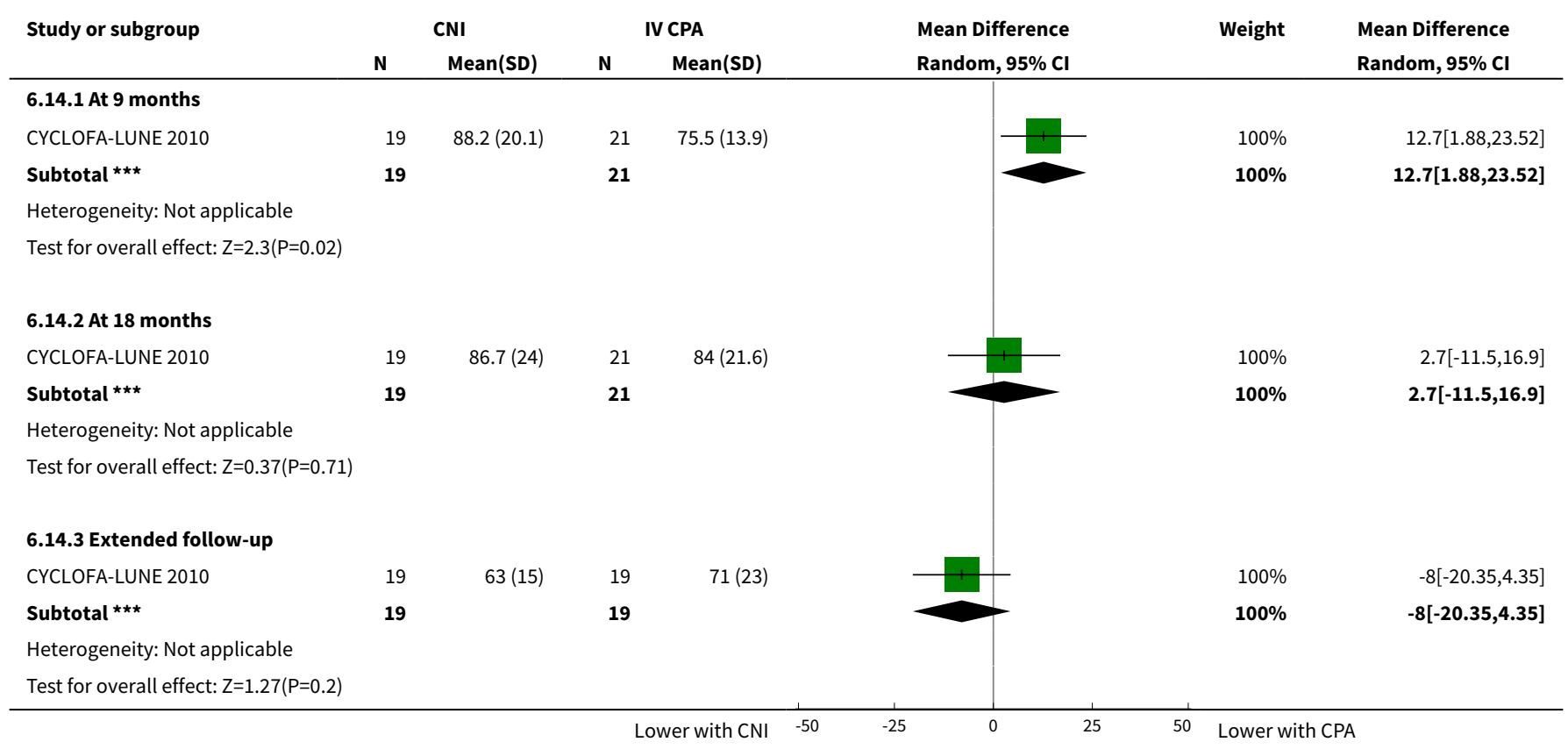

\section{Comparison 7. Cyclophosphamide (CPA) versus azathioprine (AZA)}

\begin{tabular}{lllll}
\hline $\begin{array}{l}\text { Outcome or subgroup ti- } \\
\text { tle }\end{array}$ & No. of studies & $\begin{array}{l}\text { No. of partici- } \\
\text { pants }\end{array}$ & Statistical method & Effect size \\
\hline 1 Death & 2 & & Risk Ratio (M-H, Random, 95\% Cl) & Subtotals only \\
\hline 1.1 At 5 years & 2 & 146 & Risk Ratio (M-H, Random, 95\% Cl) & $1.39[0.25,7.77]$ \\
\hline 1.2 At 10 years & 1 & 59 & Risk Ratio (M-H, Random, 95\% Cl) & $1.93[1.22,3.06]$ \\
\hline
\end{tabular}




\begin{tabular}{|c|c|c|c|c|}
\hline $\begin{array}{l}\text { Outcome or subgroup ti- } \\
\text { tle }\end{array}$ & No. of studies & $\begin{array}{l}\text { No. of partici- } \\
\text { pants }\end{array}$ & Statistical method & Effect size \\
\hline 2 Remission in proteinuria & 1 & & Risk Ratio (M-H, Random, 95\% Cl) & Subtotals only \\
\hline 2.1 Complete remission & 1 & 59 & Risk Ratio (M-H, Random, 95\% Cl) & $2.03[0.64,6.46]$ \\
\hline 2.2 Partial remission & 1 & 59 & Risk Ratio (M-H, Random, 95\% Cl) & $1.80[0.67,4.81]$ \\
\hline 3 Adverse renal outcomes & 2 & & Risk Ratio (M-H, Random, 95\% Cl) & Subtotals only \\
\hline 3.1 ESKD & 2 & 144 & Risk Ratio (M-H, Random, 95\% Cl) & $0.40[0.15,1.07]$ \\
\hline $\begin{array}{l}\text { 3.2 ESKD at } 9.6 \text { years (me- } \\
\text { dian) }\end{array}$ & 1 & 100 & Risk Ratio (M-H, Random, $95 \% \mathrm{Cl}$ ) & $1.0[0.15,6.82]$ \\
\hline 3.3 Renal relapse & 1 & 87 & Risk Ratio (M-H, Random, 95\% Cl) & $0.15[0.03,0.64]$ \\
\hline $\begin{array}{l}\text { 3.4 Renal relapse at } 9.6 \\
\text { years (median) }\end{array}$ & 1 & 87 & Risk Ratio (M-H, Random, 95\% Cl) & $0.26[0.10,0.67]$ \\
\hline $\begin{array}{l}3.5 \text { Doubling of serum cre- } \\
\text { atinine }\end{array}$ & 2 & 144 & Risk Ratio (M-H, Random, 95\% Cl) & $0.48[0.24,0.95]$ \\
\hline $\begin{array}{l}\text { 3.6 Deterioration of kidney } \\
\text { function }\end{array}$ & 1 & 30 & Risk Ratio (M-H, Random, 95\% Cl) & $0.67[0.18,2.42]$ \\
\hline 4 Stable kidney function & 1 & 57 & Risk Ratio (M-H, Random, 95\% Cl) & $1.32[0.86,2.01]$ \\
\hline 5 Ovarian failure & 2 & 126 & Risk Ratio (M-H, Random, 95\% Cl) & $2.11[0.59,7.53]$ \\
\hline 6 Menstrual irregularities & 1 & 15 & Risk Ratio (M-H, Random, 95\% Cl) & $1.90[0.69,5.23]$ \\
\hline 7 Infection & 1 & & Risk Ratio (M-H, Random, 95\% Cl) & Subtotals only \\
\hline 7.1 Major infection & 1 & 57 & Risk Ratio (M-H, Random, 95\% Cl) & $1.25[0.27,5.86]$ \\
\hline 7.2 Herpes zoster virus & 1 & 57 & Risk Ratio (M-H, Random, 95\% Cl) & $2.75[0.68,11.18]$ \\
\hline 8 Malignancy & 2 & & Risk Ratio (M-H, Random, 95\% Cl) & Subtotals only \\
\hline 8.1 CPA versus $A Z A$ & 2 & 144 & Risk Ratio (M-H, Random, 95\% Cl) & $0.59[0.13,2.63]$ \\
\hline 8.210 year follow-up & 1 & 87 & Risk Ratio (M-H, Random, 95\% Cl) & $0.74[0.11,5.01]$ \\
\hline 9 Bone toxicity & 1 & 87 & Risk Ratio (M-H, Random, 95\% Cl) & $0.0[0.0,0.0]$ \\
\hline 10 Bladder toxicity & 2 & 144 & Risk Ratio (M-H, Random, 95\% Cl) & $3.59[0.19,66.14]$ \\
\hline
\end{tabular}


Analysis 7.1. Comparison 7 Cyclophosphamide (CPA) versus azathioprine (AZA), Outcome 1 Death.

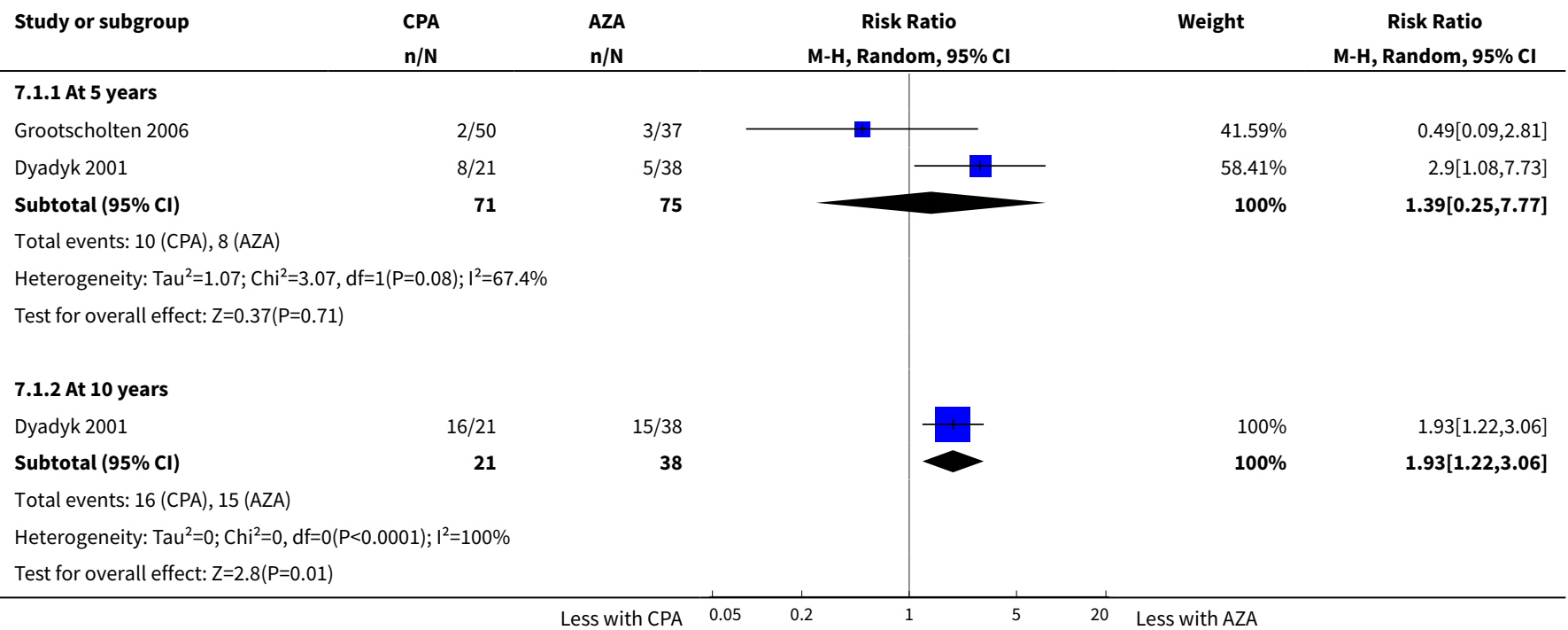

Analysis 7.2. Comparison 7 Cyclophosphamide (CPA) versus azathioprine (AZA), Outcome 2 Remission in proteinuria.

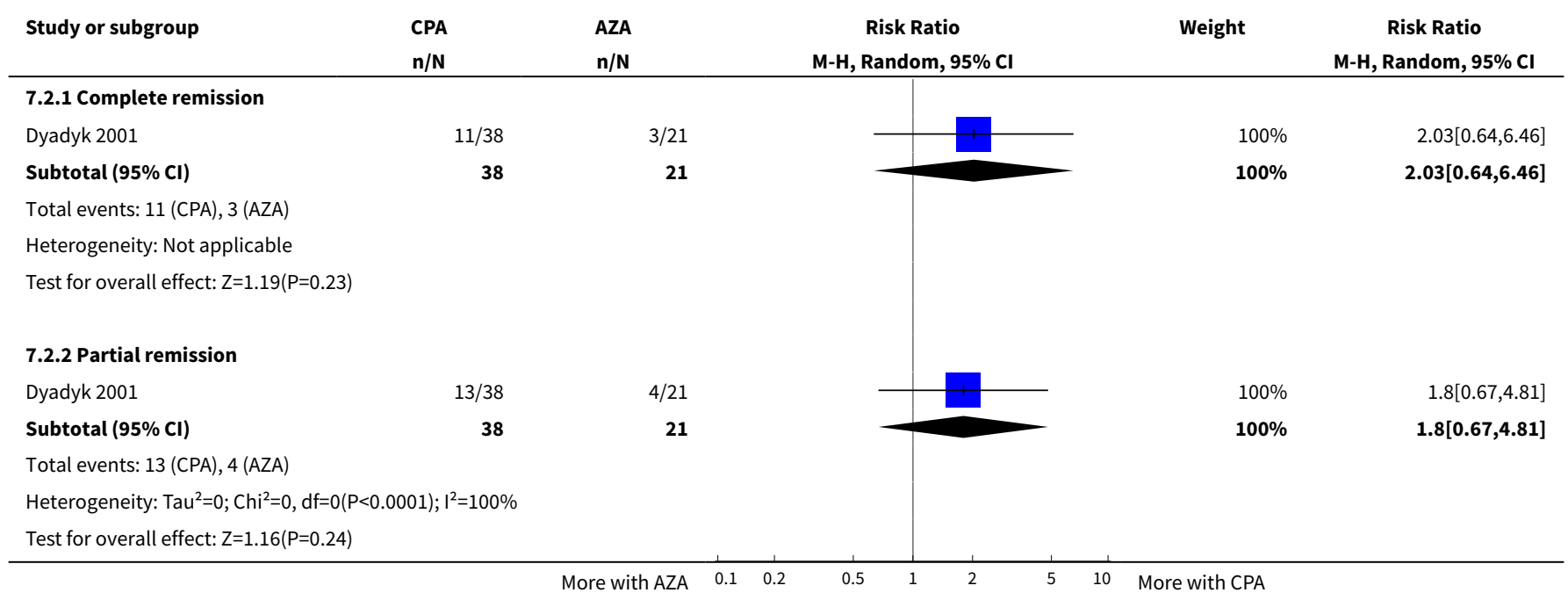

Analysis 7.3. Comparison 7 Cyclophosphamide (CPA) versus azathioprine (AZA), Outcome 3 Adverse renal outcomes.

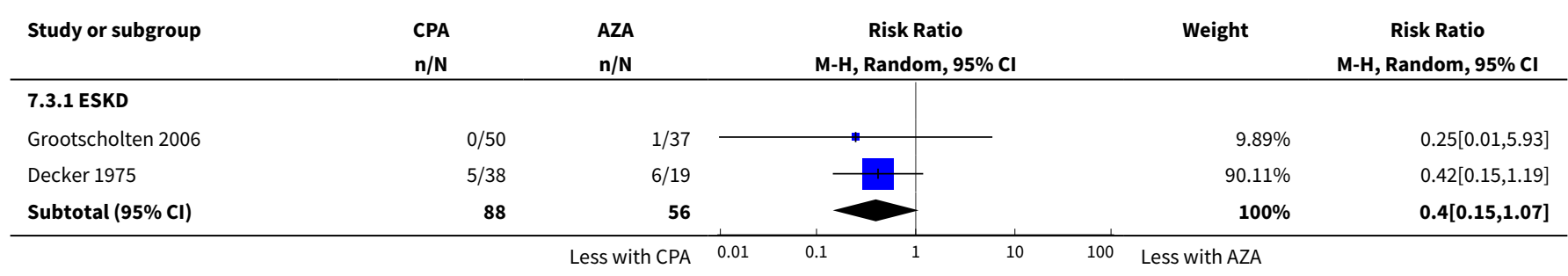




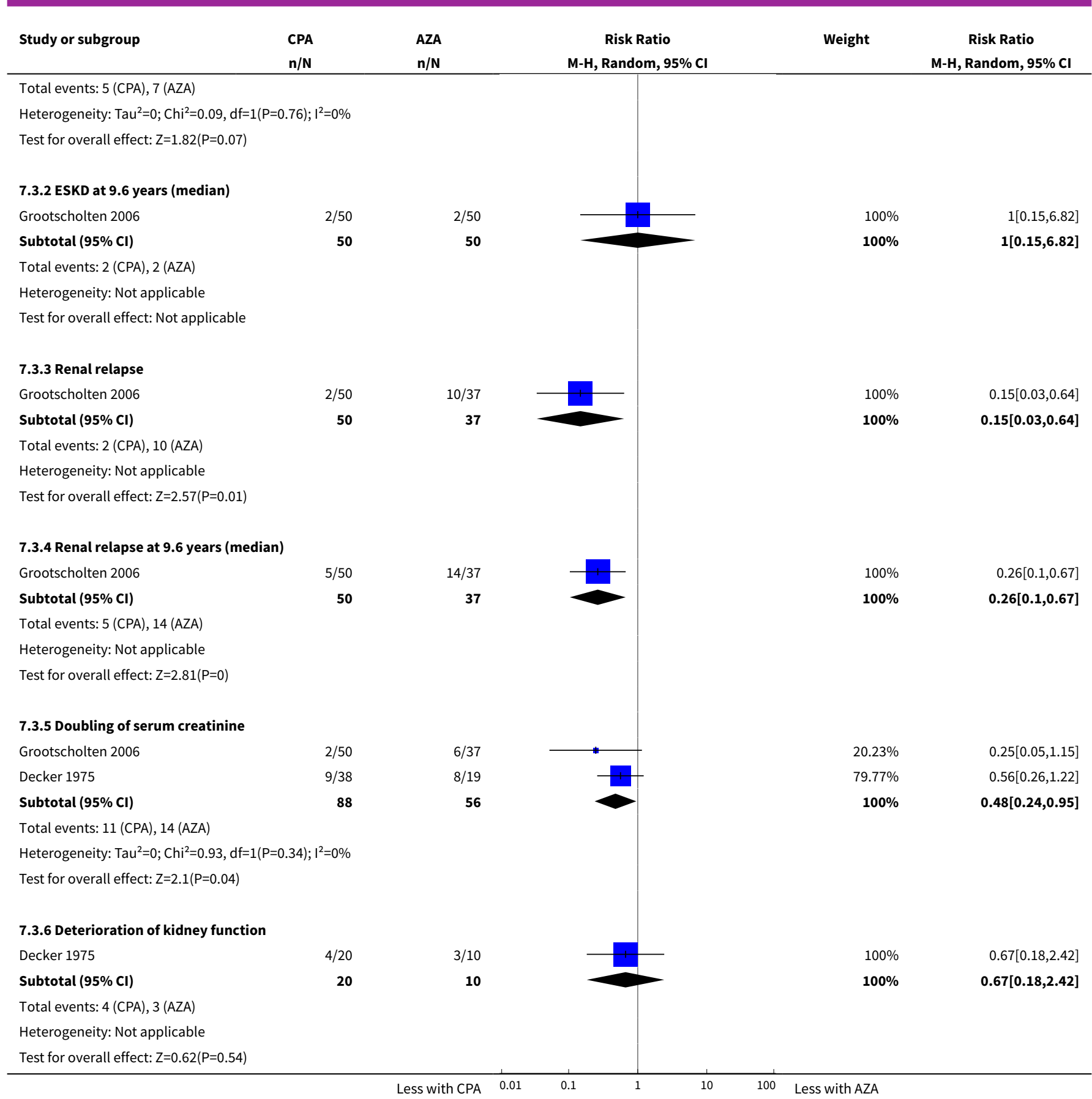

Analysis 7.4. Comparison 7 Cyclophosphamide (CPA) versus azathioprine (AZA), Outcome 4 Stable kidney function.

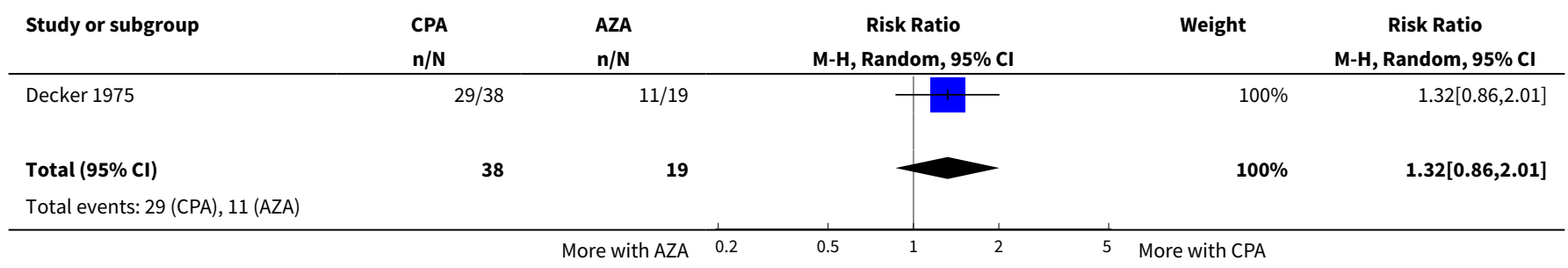




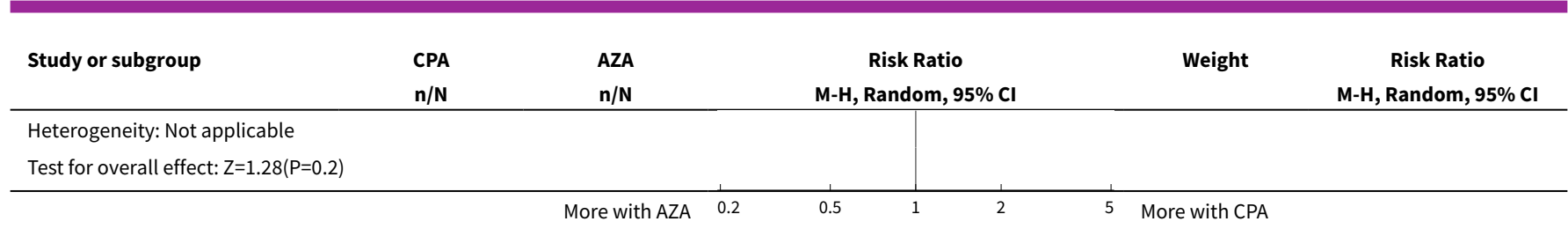

Analysis 7.5. Comparison 7 Cyclophosphamide (CPA) versus azathioprine (AZA), Outcome 5 Ovarian failure.

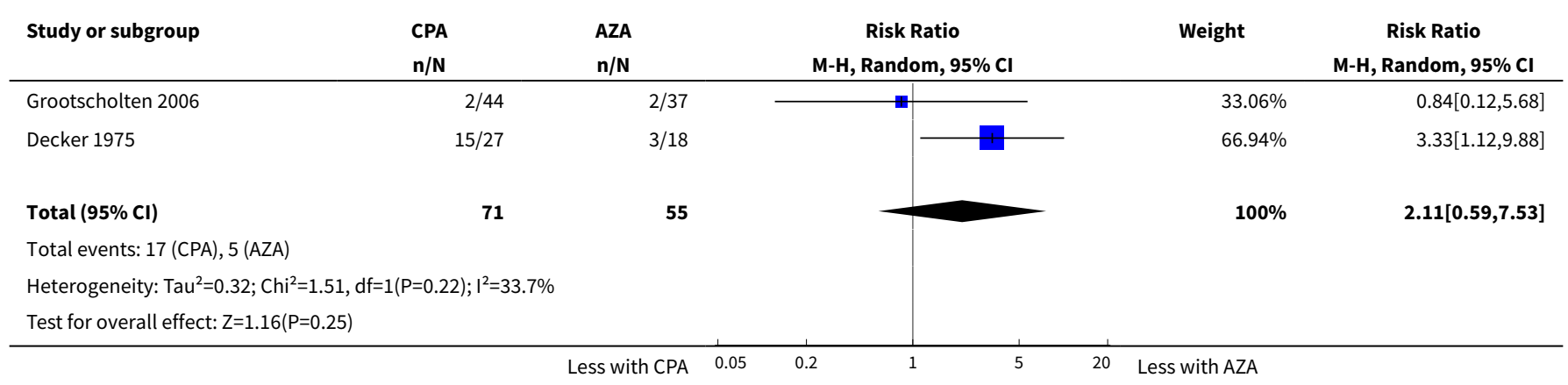

Analysis 7.6. Comparison 7 Cyclophosphamide (CPA) versus azathioprine (AZA), Outcome 6 Menstrual irregularities.

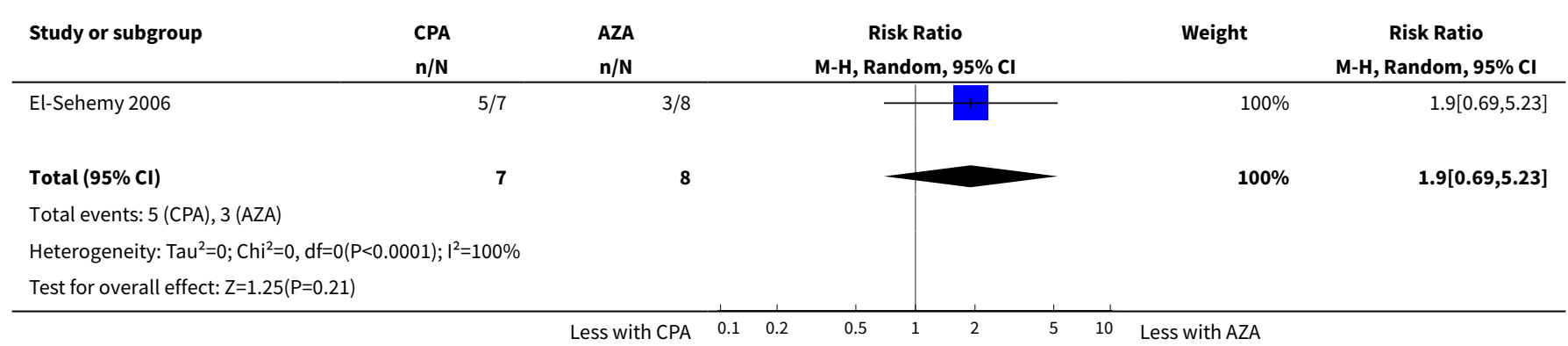

Analysis 7.7. Comparison 7 Cyclophosphamide (CPA) versus azathioprine (AZA), Outcome 7 Infection.

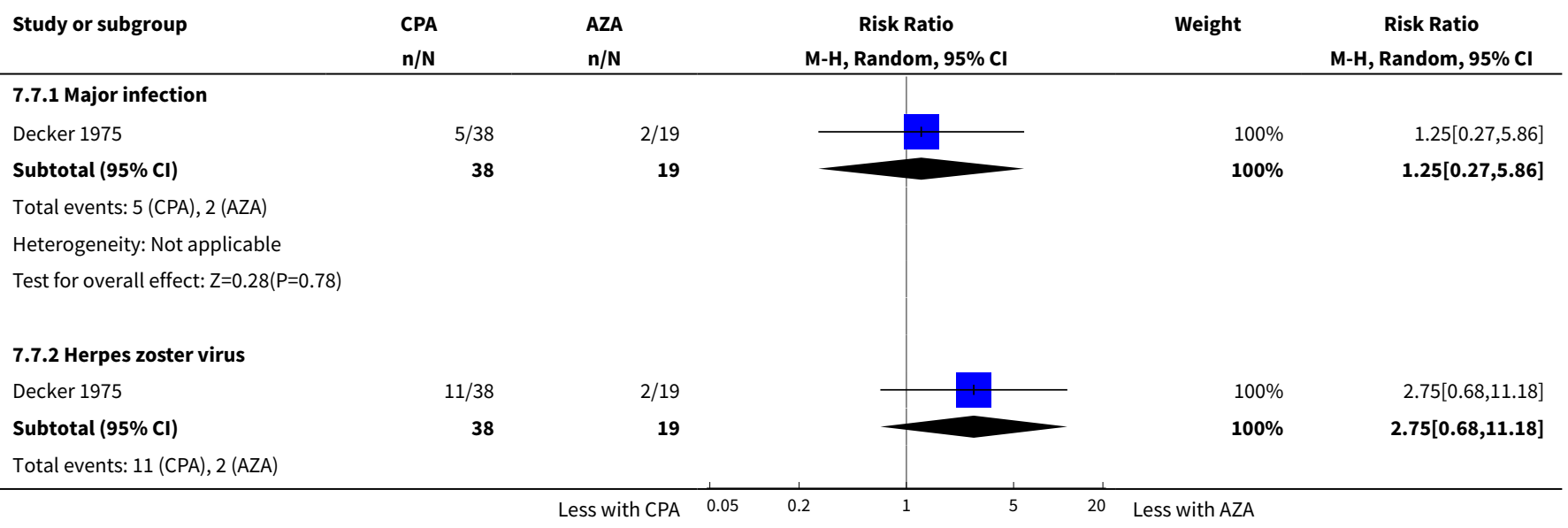




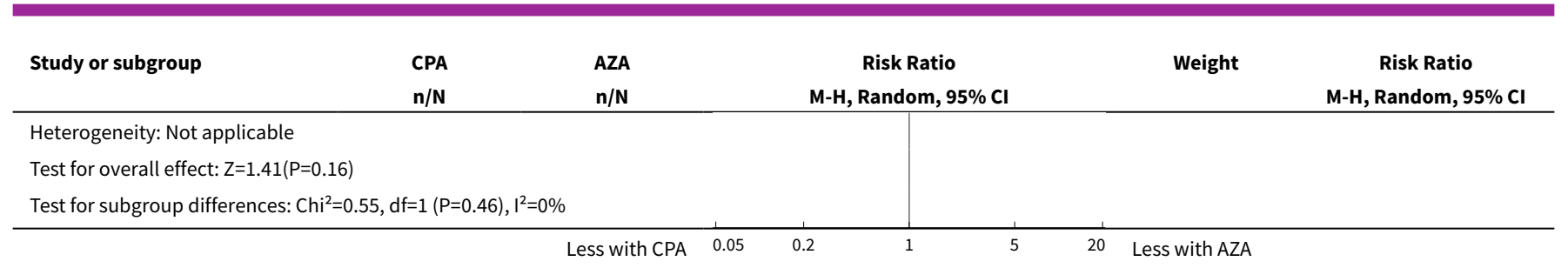

Analysis 7.8. Comparison 7 Cyclophosphamide (CPA) versus azathioprine (AZA), Outcome 8 Malignancy.

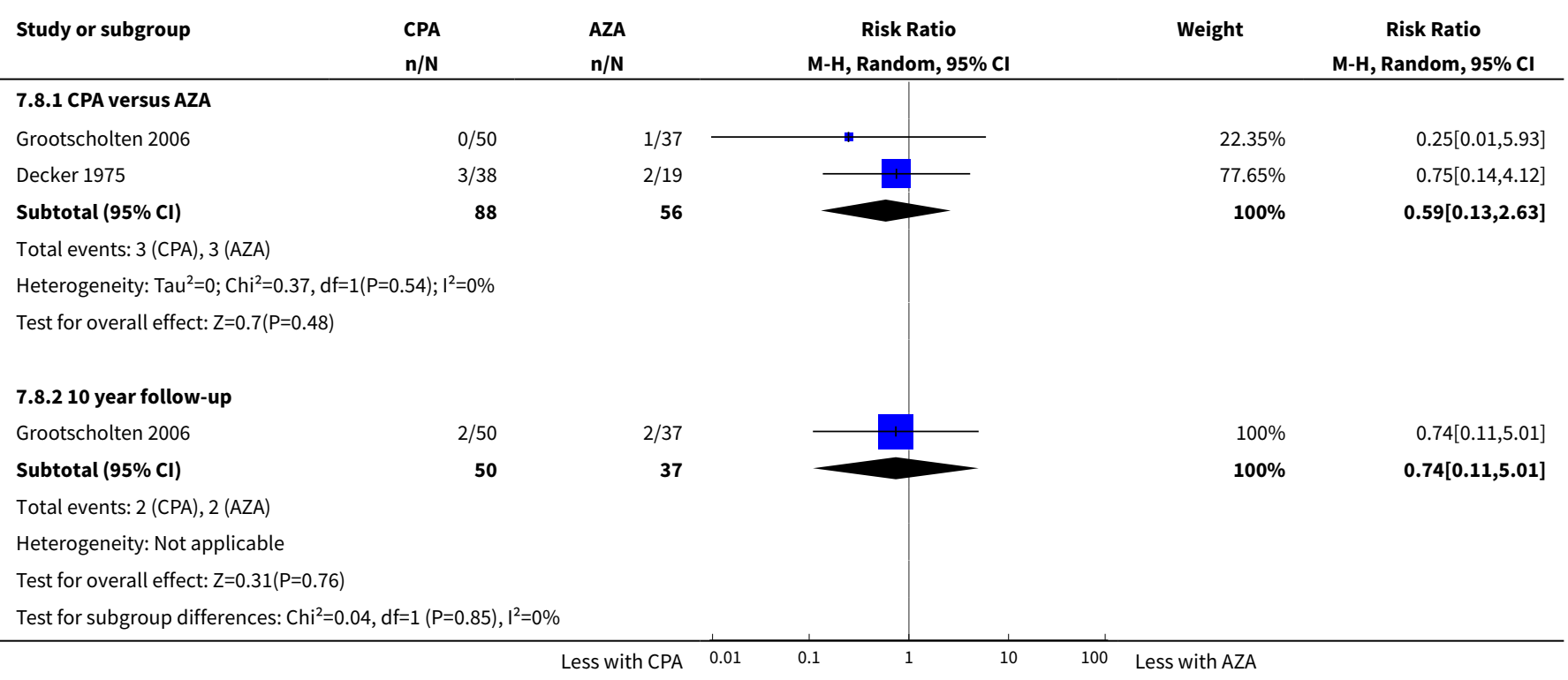

Analysis 7.9. Comparison 7 Cyclophosphamide (CPA) versus azathioprine (AZA), Outcome 9 Bone toxicity.

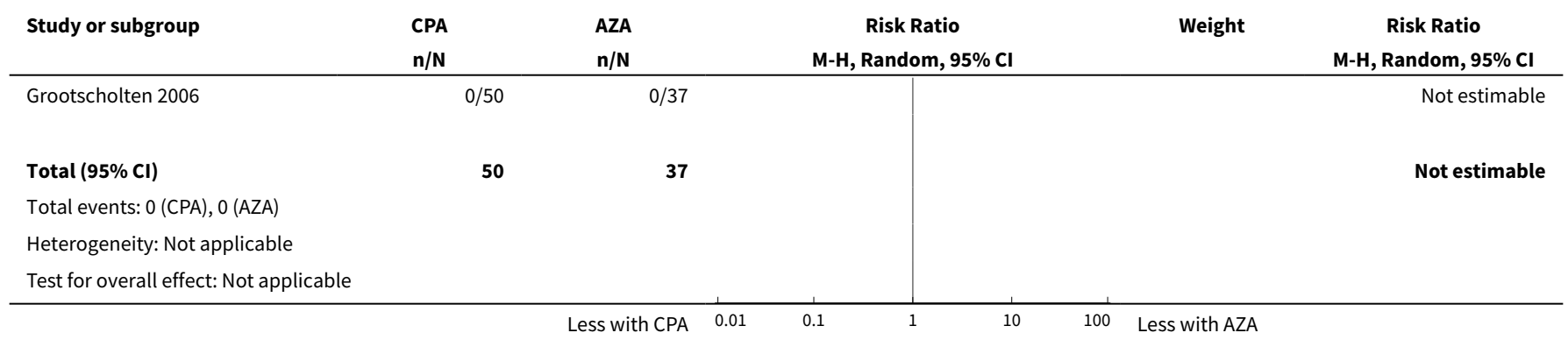

Analysis 7.10. Comparison 7 Cyclophosphamide (CPA) versus azathioprine (AZA), Outcome 10 Bladder toxicity.

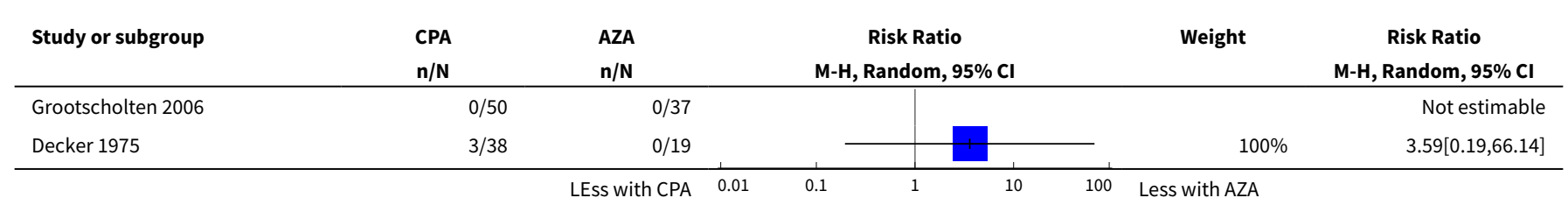




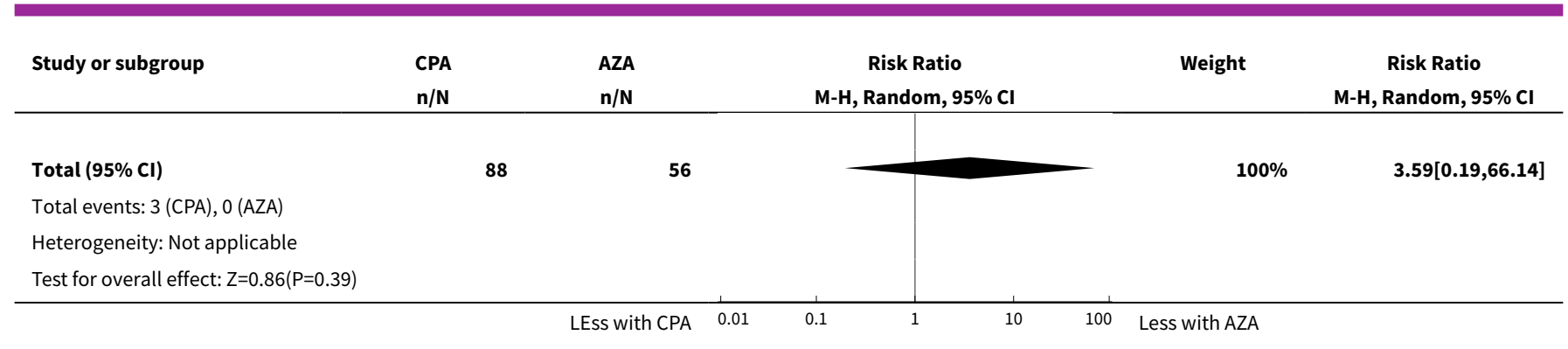

Comparison 8. Rituximab (RTX) + mycophenolate mofetil (MMF) versus placebo + MMF

\begin{tabular}{|c|c|c|c|c|}
\hline $\begin{array}{l}\text { Outcome or subgroup } \\
\text { title }\end{array}$ & No. of studies & $\begin{array}{l}\text { No. of partici- } \\
\text { pants }\end{array}$ & Statistical method & Effect size \\
\hline 1 Death & 1 & 144 & Risk Ratio (M-H, Random, 95\% Cl) & $5.0[0.24,102.35]$ \\
\hline 2 Remission & 1 & & Risk Ratio (M-H, Random, 95\% Cl) & Subtotals only \\
\hline $\begin{array}{l}2.1 \text { Complete renal re- } \\
\text { sponse }\end{array}$ & 1 & 144 & Risk Ratio (M-H, Random, 95\% Cl) & $0.86[0.51,1.45]$ \\
\hline 2.2 Partial renal response & 1 & 144 & Risk Ratio (M-H, Random, 95\% Cl) & $2.0[1.05,3.82]$ \\
\hline $\begin{array}{l}2.3 \text { Complete remission } \\
\text { in proteinuria }\end{array}$ & 1 & 144 & Risk Ratio (M-H, Random, 95\% Cl) & $0.87[0.63,1.21]$ \\
\hline 3 Stable kidney function & 1 & 144 & Risk Ratio (M-H, Random, 95\% Cl) & $1.24[0.90,1.71]$ \\
\hline 4 Infection & 1 & & Risk Ratio (M-H, Random, 95\% Cl) & Subtotals only \\
\hline 4.1 Major infection & 1 & 144 & Risk Ratio (M-H, Random, 95\% Cl) & $1.0[0.48,2.08]$ \\
\hline 4.2 Herpes zoster virus & 1 & 144 & Risk Ratio (M-H, Random, 95\% Cl) & $0.82[0.36,1.85]$ \\
\hline 5 Leucopenia & 1 & 144 & Risk Ratio (M-H, Random, 95\% Cl) & $3.0[0.85,10.63]$ \\
\hline
\end{tabular}

Analysis 8.1. Comparison 8 Rituximab (RTX) + mycophenolate mofetil (MMF) versus placebo + MMF, Outcome 1 Death.

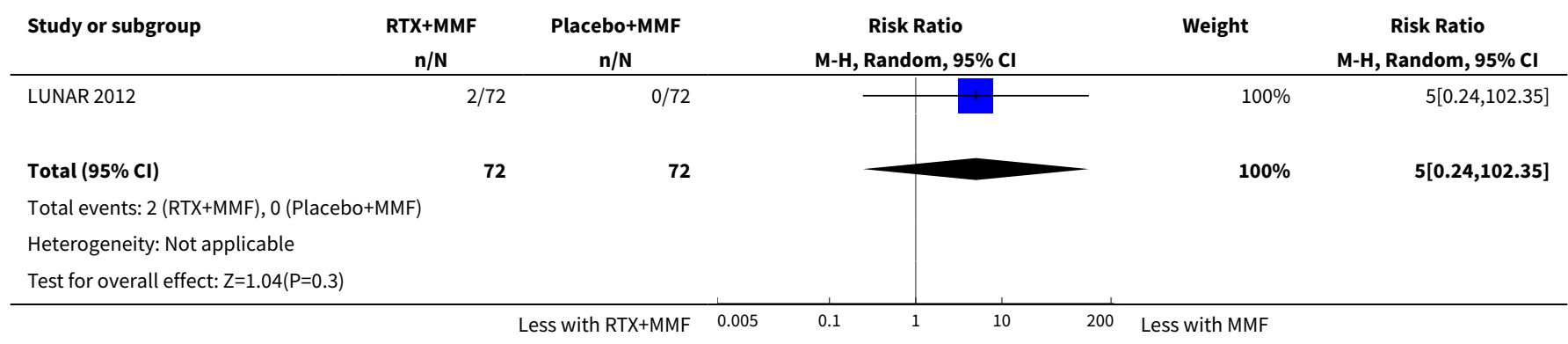


Analysis 8.2. Comparison 8 Rituximab (RTX) + mycophenolate mofetil (MMF) versus placebo + MMF, Outcome 2 Remission.

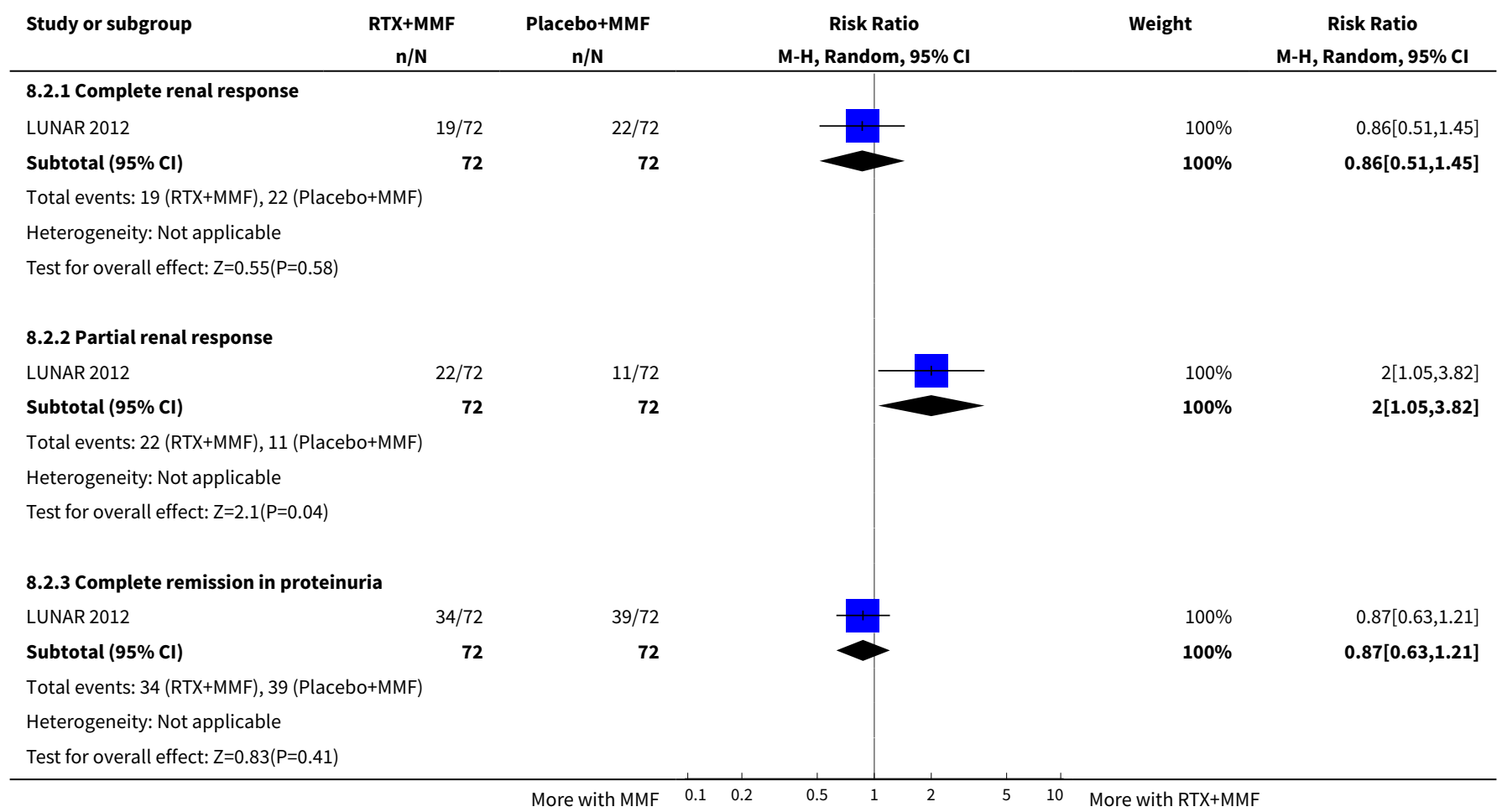

Analysis 8.3. Comparison 8 Rituximab (RTX) + mycophenolate mofetil (MMF) versus placebo + MMF, Outcome 3 Stable kidney function.

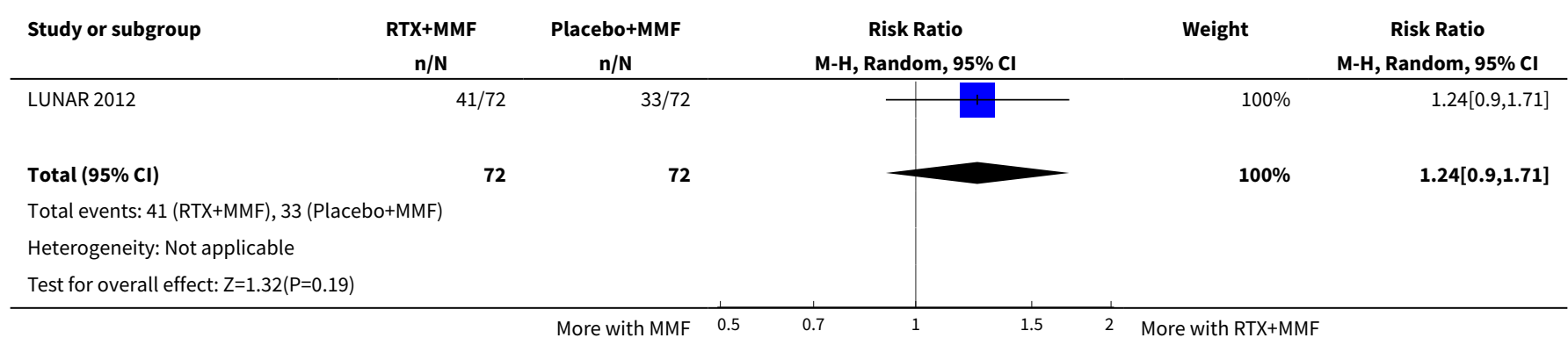

Analysis 8.4. Comparison 8 Rituximab (RTX) + mycophenolate mofetil (MMF) versus placebo + MMF, Outcome 4 Infection.

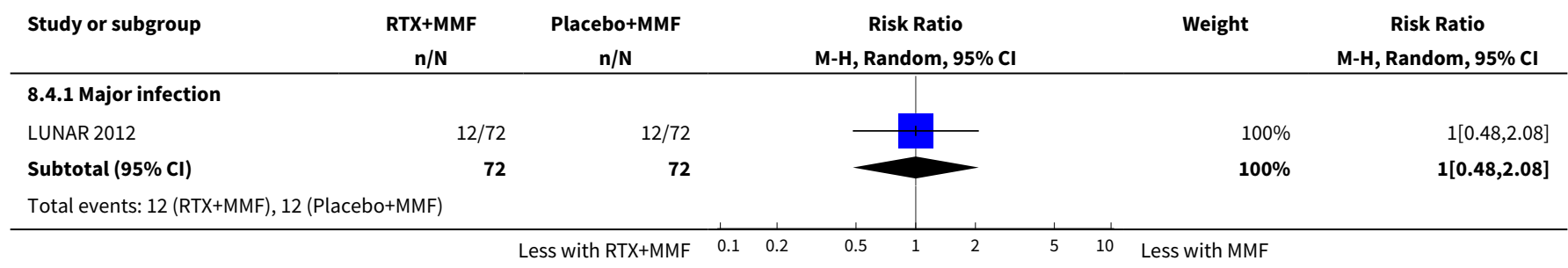




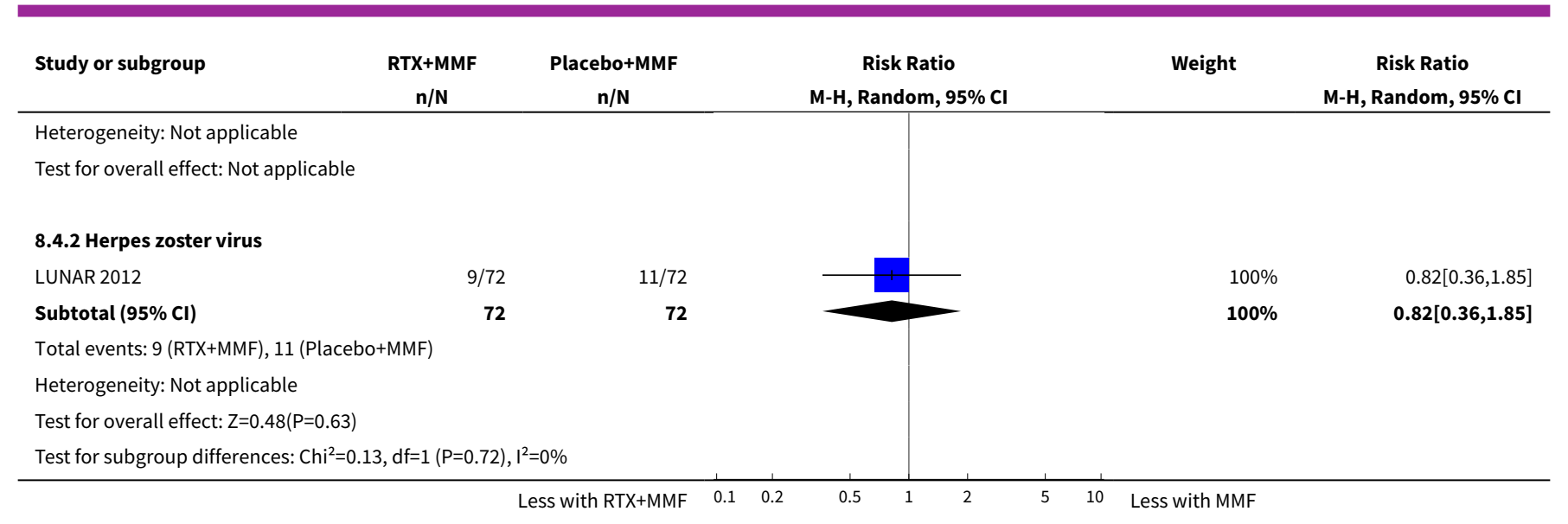

Analysis 8.5. Comparison 8 Rituximab (RTX) + mycophenolate mofetil (MMF) versus placebo + MMF, Outcome 5 Leucopenia.

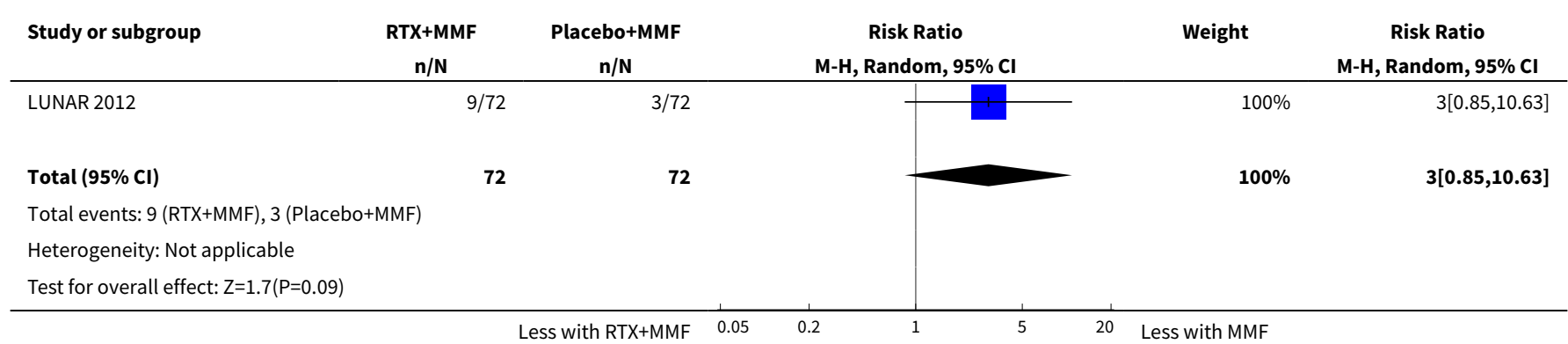

\section{Comparison 9. Rituximab (RTX) + cyclophosphamide (CPA) versus RTX}

\begin{tabular}{lllll}
\hline $\begin{array}{l}\text { Outcome or subgroup } \\
\text { title }\end{array}$ & No. of studies & $\begin{array}{l}\text { No. of partici- } \\
\text { pants }\end{array}$ & Statistical method & Effect size \\
\hline 1 Remission & 1 & & Risk Ratio (M-H, Random, 95\% Cl) & Subtotals only \\
\hline $\begin{array}{l}1.1 \text { Complete renal re- } \\
\text { sponse }\end{array}$ & 1 & 19 & Risk Ratio (M-H, Random, 95\% Cl) & $0.9[0.16,5.13]$ \\
\hline $\begin{array}{lllll}1.2 \text { Partial renal response } \\
\text { 2 Infection }\end{array}$ & 1 & 19 & Risk Ratio (M-H, Random, 95\% Cl) & $0.75[0.35,1.62]$ \\
\hline 2.1 Major infection & 1 & 38 & Risk Ratio (M-H, Random, 95\% Cl) & $0.57[0.08,4.20]$ \\
\hline 2.2 Herpes zoster virus & 1 & 19 & Risk Ratio (M-H, Random, 95\% Cl) & $0.9[0.07,12.38]$ \\
\hline 3 Daily proteinuria & 1 & 19 & Mean Difference (IV, Random, 95\% Cl) & $-0.30[-2.29,1.69]$ \\
\hline 4 Creatinine clearance & 1 & 19 & Mean Difference (IV, Random, 95\% Cl) & $-17.20[-50.66,16.26]$ \\
\hline 5 Serum creatinine & 1 & 19 & Mean Difference (IV, Random, 95\% Cl) & $35.00[-27.14,97.14]$ \\
\hline
\end{tabular}


Analysis 9.1. Comparison 9 Rituximab (RTX) + cyclophosphamide (CPA) versus RTX, Outcome 1 Remission.

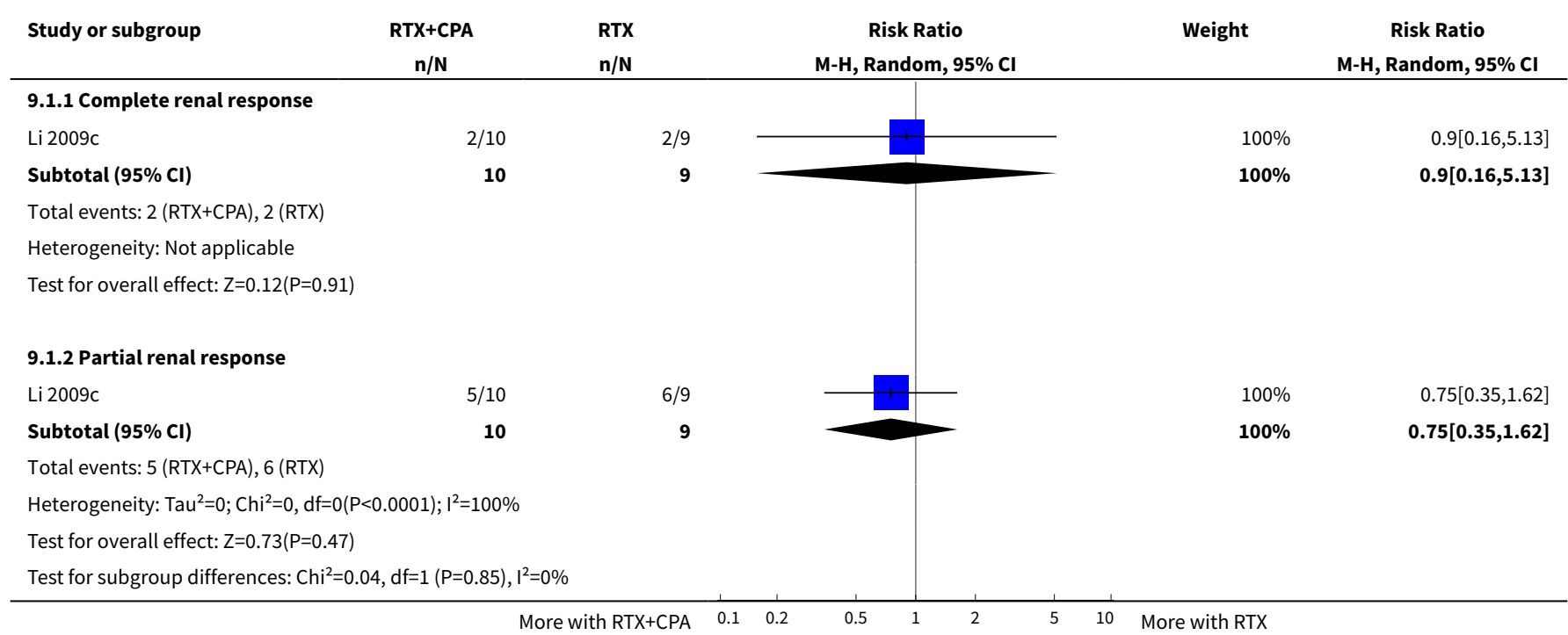

\section{Analysis 9.2. Comparison 9 Rituximab (RTX) + cyclophosphamide (CPA) versus RTX, Outcome 2 Infection.}

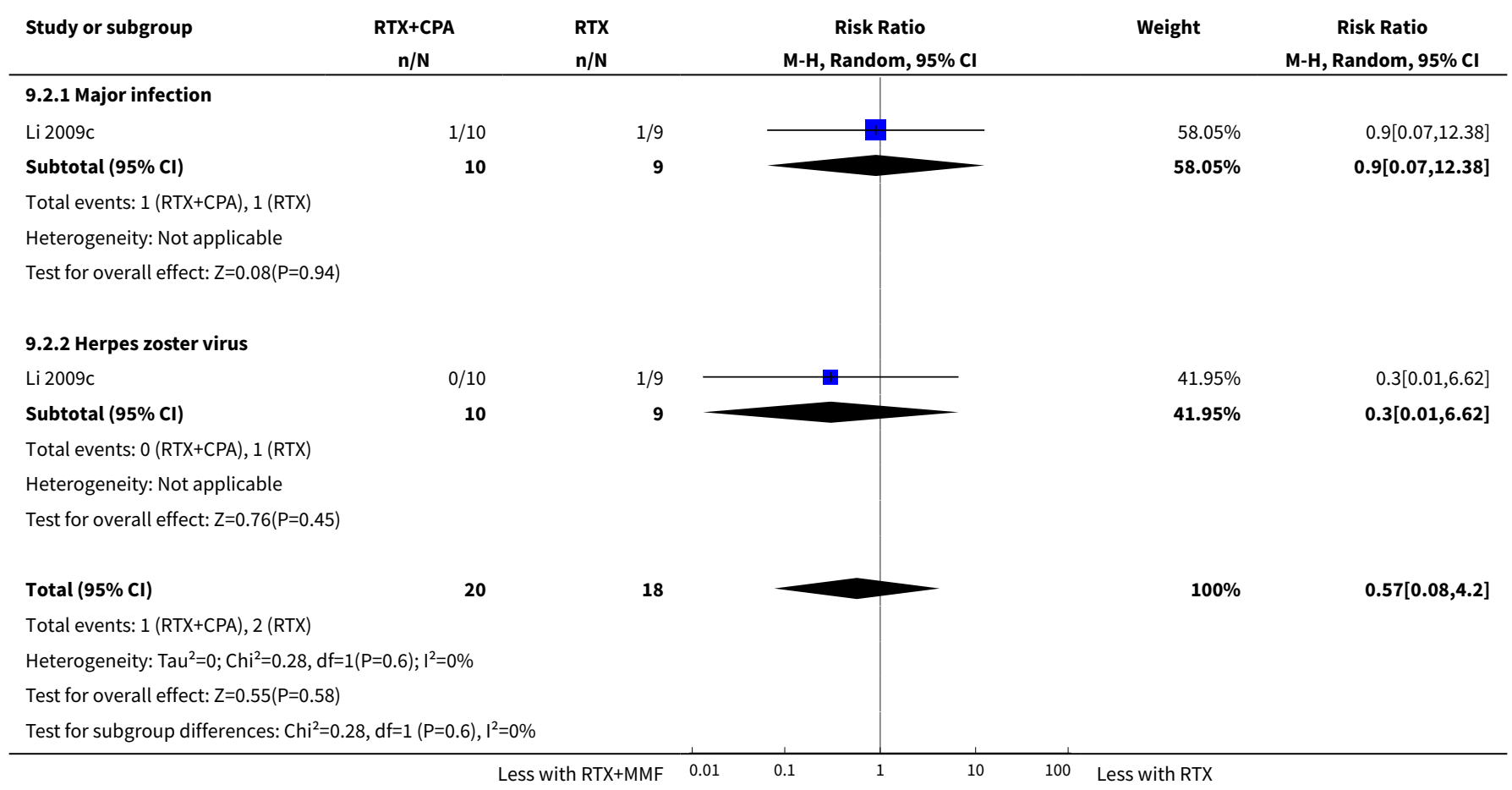


Analysis 9.3. Comparison 9 Rituximab (RTX) + cyclophosphamide (CPA) versus RTX, Outcome 3 Daily proteinuria.

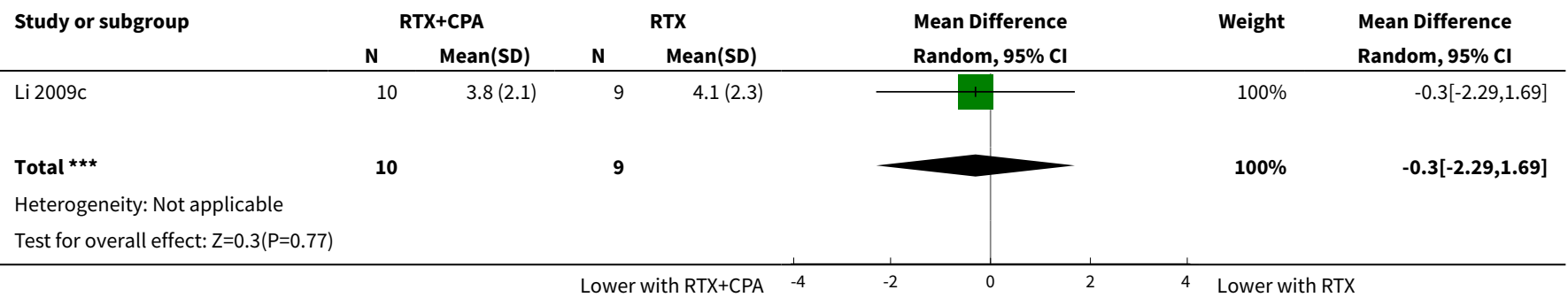

Analysis 9.4. Comparison 9 Rituximab (RTX) + cyclophosphamide

(CPA) versus RTX, Outcome 4 Creatinine clearance.

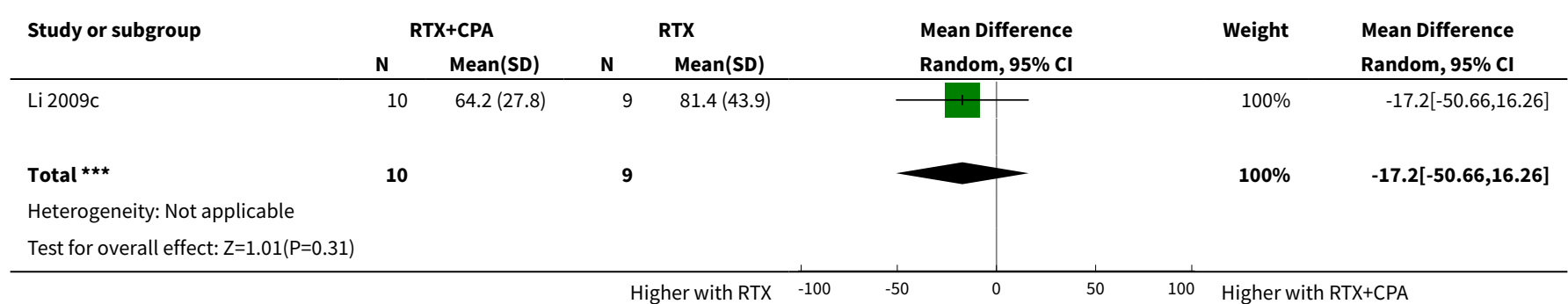

Analysis 9.5. Comparison 9 Rituximab (RTX) + cyclophosphamide (CPA) versus RTX, Outcome 5 Serum creatinine.

\begin{tabular}{|c|c|c|c|c|c|c|c|}
\hline \multirow[t]{2}{*}{ Study or subgroup } & \multicolumn{2}{|c|}{ RTX+CPA } & \multicolumn{2}{|c|}{ RTX } & \multirow{2}{*}{$\begin{array}{l}\text { Mean Difference } \\
\text { Random, } 95 \% \mathrm{Cl}\end{array}$} & \multirow[t]{2}{*}{ Weight } & \multirow{2}{*}{$\begin{array}{l}\text { Mean Difference } \\
\text { Random, } 95 \% \mathrm{CI}\end{array}$} \\
\hline & $\mathbf{N}$ & Mean(SD) & $\mathbf{N}$ & Mean(SD) & & & \\
\hline Li 2009c & 10 & $134.8(84.7)$ & 9 & $99.8(50.9)$ & & $100 \%$ & $35[-27.14,97.14]$ \\
\hline Total $\star \star \star ~$ & 10 & & 9 & & & $100 \%$ & $35[-27.14,97.14]$ \\
\hline \multicolumn{8}{|c|}{ Heterogeneity: Not applicable } \\
\hline \multicolumn{8}{|c|}{ Test for overall effect: $Z=1.1(P=0.27)$} \\
\hline
\end{tabular}

\section{Comparison 10. Abatacept + other immunosuppressive agent (IS) + versus placebo + other IS}

\begin{tabular}{lllll}
\hline Outcome or subgroup title & No. of studies & $\begin{array}{l}\text { No. of partici- } \\
\text { pants }\end{array}$ & Statistical method & Effect size \\
\hline 1 Death & 2 & & $\begin{array}{l}\text { Risk Ratio (M-H, Random, 95\% } \\
\text { Cl) }\end{array}$ & Subtotals only \\
\hline 1.1 Abatacept versus placebo & 2 & 432 & $\begin{array}{l}\text { Risk Ratio (M-H, Random, 95\% } \\
\text { Cl) }\end{array}$ & $0.29[0.10,0.91]$ \\
\hline $\begin{array}{l}1.2 \text { High dose abatacept versus } \\
\text { placebo }\end{array}$ & 1 & 199 & $\begin{array}{l}\text { Risk Ratio (M-H, Random, 95\% } \\
\text { Cl) }\end{array}$ & $0.29[0.06,1.36]$ \\
\hline
\end{tabular}




\begin{tabular}{|c|c|c|c|c|}
\hline Outcome or subgroup title & No. of studies & $\begin{array}{l}\text { No. of partici- } \\
\text { pants }\end{array}$ & Statistical method & Effect size \\
\hline $\begin{array}{l}1.3 \text { Low dose abatacept versus } \\
\text { placebo }\end{array}$ & 1 & 199 & $\begin{array}{l}\text { Risk Ratio (M-H, Random, 95\% } \\
\mathrm{Cl} \text { ) }\end{array}$ & $0.29[0.06,1.36]$ \\
\hline 2 Remission & 2 & & $\begin{array}{l}\text { Risk Ratio (M-H, Random, 95\% } \\
\mathrm{Cl} \text { ) }\end{array}$ & Subtotals only \\
\hline $\begin{array}{l}2.1 \text { Complete remission: abata- } \\
\text { cept versus placebo }\end{array}$ & 2 & 432 & $\begin{array}{l}\text { Risk Ratio (M-H, Random, 95\% } \\
\mathrm{Cl})\end{array}$ & $1.13[0.74,1.71]$ \\
\hline $\begin{array}{l}2.2 \text { Complete remission: high } \\
\text { dose abatacept versus placebo }\end{array}$ & 1 & 199 & $\begin{array}{l}\text { Risk Ratio (M-H, Random, 95\% } \\
\mathrm{Cl} \text { ) }\end{array}$ & $1.14[0.46,2.83]$ \\
\hline $\begin{array}{l}2.3 \text { Complete remission: low dose } \\
\text { abatacept versus placebo }\end{array}$ & 1 & 199 & $\begin{array}{l}\text { Risk Ratio (M-H, Random, 95\% } \\
\mathrm{Cl} \text { ) }\end{array}$ & $1.39[0.58,3.31]$ \\
\hline $\begin{array}{l}\text { 2.4 Partial remission: abatacept } \\
\text { versus placebo }\end{array}$ & 2 & 432 & $\begin{array}{l}\text { Risk Ratio (M-H, Random, 95\% } \\
\mathrm{Cl} \text { ) }\end{array}$ & $0.88[0.58,1.33]$ \\
\hline $\begin{array}{l}2.5 \text { Partial remission: high dose } \\
\text { abatacept versus placebo }\end{array}$ & 1 & 199 & $\begin{array}{l}\text { Risk Ratio (M-H, Random, 95\% } \\
\mathrm{Cl} \text { ) }\end{array}$ & $1.01[0.51,2.01]$ \\
\hline $\begin{array}{l}\text { 2.6 Partial remission: low dose } \\
\text { abatacept versus placebo }\end{array}$ & 1 & 199 & $\begin{array}{l}\text { Risk Ratio (M-H, Random, 95\% } \\
\mathrm{Cl} \text { ) }\end{array}$ & $0.65[0.29,1.43]$ \\
\hline 3 Adverse renal outcomes & 2 & & $\begin{array}{l}\text { Risk Ratio (M-H, Random, 95\% } \\
\mathrm{Cl})\end{array}$ & Subtotals only \\
\hline $\begin{array}{l}\text { 3.1 ESKD: Abatacept versus } \\
\text { placebo }\end{array}$ & 1 & 298 & $\begin{array}{l}\text { Risk Ratio (M-H, Random, 95\% } \\
\text { Cl) }\end{array}$ & $0.84[0.21,3.45]$ \\
\hline $\begin{array}{l}\text { 3.2 ESKD: high dose abatacept } \\
\text { versus placebo }\end{array}$ & 1 & 199 & $\begin{array}{l}\text { Risk Ratio (M-H, Random, 95\% } \\
\text { Cl) }\end{array}$ & $1.01[0.21,4.88]$ \\
\hline $\begin{array}{l}3.3 \text { ESKD: low dose abatacept } \\
\text { versus placebo }\end{array}$ & 1 & 199 & $\begin{array}{l}\text { Risk Ratio (M-H, Random, 95\% } \\
\mathrm{Cl} \text { ) }\end{array}$ & $0.67[0.11,3.94]$ \\
\hline $\begin{array}{l}\text { 3.4 Renal relapse: abatacept ver- } \\
\text { sus placebo }\end{array}$ & 1 & 134 & $\begin{array}{l}\text { Risk Ratio (M-H, Random, 95\% } \\
\text { Cl) }\end{array}$ & $1.03[0.22,4.92]$ \\
\hline 4 Major Infection & 2 & & $\begin{array}{l}\text { Risk Ratio (M-H, Random, 95\% } \\
\text { Cl) }\end{array}$ & Subtotals only \\
\hline 4.1 Abatacept versus placebo & 2 & 432 & $\begin{array}{l}\text { Risk Ratio (M-H, Random, 95\% } \\
\mathrm{Cl} \text { ) }\end{array}$ & $1.29[0.81,2.04]$ \\
\hline $\begin{array}{l}4.2 \text { High dose abatacept versus } \\
\text { placebo }\end{array}$ & 1 & 199 & $\begin{array}{l}\text { Risk Ratio (M-H, Random, 95\% } \\
\mathrm{Cl} \text { ) }\end{array}$ & $1.37[0.78,2.40]$ \\
\hline $\begin{array}{l}4.3 \text { Low dose abatacept versus } \\
\text { placebo }\end{array}$ & 1 & 199 & $\begin{array}{l}\text { Risk Ratio (M-H, Random, 95\% } \\
\text { Cl) }\end{array}$ & $1.07[0.59,1.95]$ \\
\hline 5 Herpes zoster virus & 1 & & $\begin{array}{l}\text { Risk Ratio (M-H, Random, 95\% } \\
\mathrm{Cl} \text { ) }\end{array}$ & Subtotals only \\
\hline
\end{tabular}




\begin{tabular}{|c|c|c|c|c|}
\hline Outcome or subgroup title & No. of studies & $\begin{array}{l}\text { No. of partici- } \\
\text { pants }\end{array}$ & Statistical method & Effect size \\
\hline 5.1 Abatacept versus placebo & 1 & 298 & $\begin{array}{l}\text { Risk Ratio (M-H, Random, 95\% } \\
\mathrm{Cl} \text { ) }\end{array}$ & $9.64[0.57,164.02]$ \\
\hline $\begin{array}{l}5.2 \text { High dose abatacept versus } \\
\text { placebo }\end{array}$ & 1 & 199 & $\begin{array}{l}\text { Risk Ratio (M-H, Random, 95\% } \\
\text { Cl) }\end{array}$ & $7.07[0.37,135.11]$ \\
\hline $\begin{array}{l}5.3 \text { Low dose abatacept versus } \\
\text { placebo }\end{array}$ & 1 & 199 & $\begin{array}{l}\text { Risk Ratio (M-H, Random, 95\% } \\
\mathrm{Cl} \text { ) }\end{array}$ & $13.13[0.75,229.99]$ \\
\hline 6 Health-related quality of life & 1 & & $\begin{array}{l}\text { Mean Difference (IV, Random, } \\
95 \% \mathrm{CI} \text { ) }\end{array}$ & Subtotals only \\
\hline 6.1 Physical component & 1 & 134 & $\begin{array}{l}\text { Mean Difference (IV, Random, } \\
95 \% \mathrm{CI} \text { ) }\end{array}$ & $0.0[-3.73,3.73]$ \\
\hline 6.2 Mental component & 1 & 134 & $\begin{array}{l}\text { Mean Difference (IV, Random, } \\
95 \% \mathrm{CI} \text { ) }\end{array}$ & $-0.60[-4.50,3.30]$ \\
\hline 7 Disease activity (BILAG) & 1 & 134 & $\begin{array}{l}\text { Mean Difference (IV, Random, } \\
95 \% \mathrm{CI})\end{array}$ & $-0.40[-1.23,0.43]$ \\
\hline
\end{tabular}

\section{Analysis 10.1. Comparison 10 Abatacept + other immunosuppressive} agent (IS) + versus placebo + other IS, Outcome 1 Death.

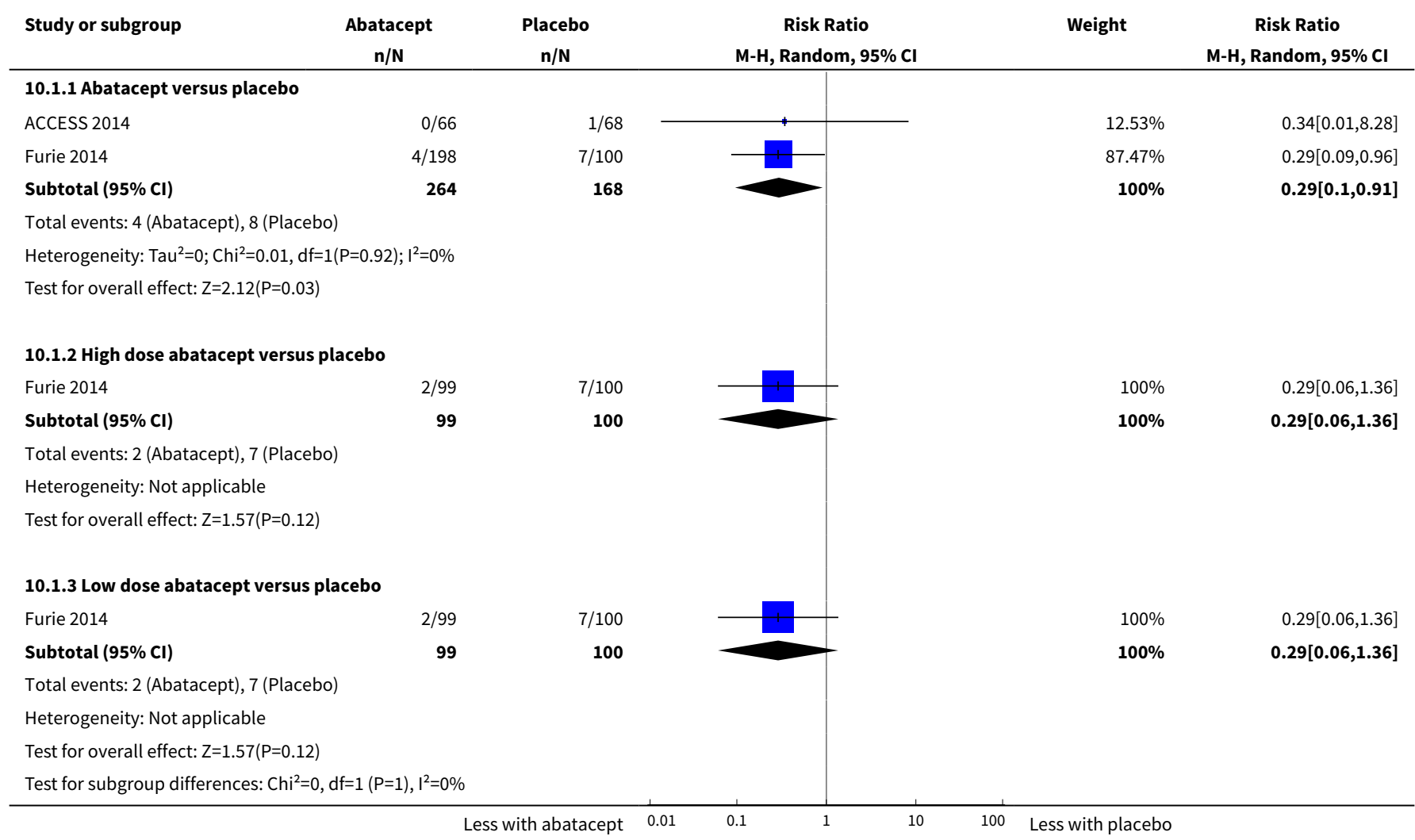




\section{Analysis 10.2. Comparison 10 Abatacept + other immunosuppressive agent (IS) + versus placebo + other IS, Outcome 2 Remission.}

$\begin{array}{cc}\begin{array}{c}\text { Abatacept } \\ \mathbf{n} / \mathbf{N}\end{array} & \text { Placebo } \\ \mathbf{n} / \mathbf{N}\end{array}$

Risk Ratio

Weight

Risk Ratio

10.2.1 Complete remission: abatacept versus placebo

M-H, Random, $95 \% \mathrm{Cl}$

M-H, Random, $95 \% \mathrm{Cl}$

$\begin{array}{lrr}\text { 10.2.1 Complete remission: abatacept versus placebo } & \\ \text { Furie } 2014 & 20 / 198 & 8 / 100 \\ \text { ACCESS } 2014 & 22 / 66 & 21 / 68 \\ \text { Subtotal (95\% CI) } & \mathbf{2 6 4} & \mathbf{1 6 8}\end{array}$

Total events: 42 (Abatacept), 29 (Placebo)

Test for overall effect: $\mathrm{Z}=0.57(\mathrm{P}=0.57)$

10.2.2 Complete remission: high dose abatacept versus placebo

Furie 2014

Subtotal $(95 \% \mathrm{Cl})$

100

Total events: 9 (Abatacept), 8 (Placebo)

Heterogeneity: Not applicable

Test for overall effect: $\mathrm{Z}=0.28(\mathrm{P}=0.78)$

10.2.3 Complete remission: low dose abatacept versus placebo

Furie 2014

99

Subtotal $(95 \% \mathrm{CI})$

100

Total events: 11 (Abatacept), 8 (Placebo)

Heterogeneity: Not applicable

Test for overall effect: $Z=0.74(P=0.46)$

10.2.4 Partial remission: abatacept versus placebo

Furie 2014

23/198

ACCESS 2014

$17 / 66$

Subtotal $(95 \% \mathrm{Cl})$

264

$14 / 100$

$19 / 68$

168

Total events: 40 (Abatacept), 33 (Placebo)

Heterogeneity: $\mathrm{Tau}^{2}=0 ; \mathrm{Chi}^{2}=0.06, \mathrm{df}=1(\mathrm{P}=0.8) ; \mathrm{I}^{2}=0 \%$

Test for overall effect: $Z=0.61(P=0.54)$

10.2.5 Partial remission: high dose abatacept versus placebo

Furie 2014

$14 / 99$

99

$14 / 100$

100

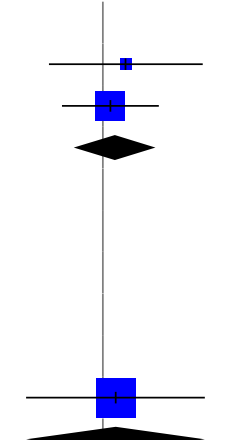

$100 \%$

$1.14[0.46,2.83]$

$100 \%$

$1.14[0.46,2.83]$

Subtotal $(95 \% \mathrm{Cl})$

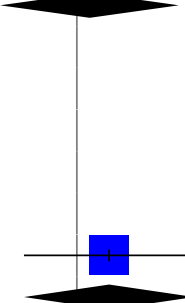

$100 \%$

$1.39[0.58,3.31]$

$100 \%$

$1.39[0.58,3.31]$

$44.97 \%$

$0.83[0.45,1.54]$

$55.03 \%$

$0.92[0.53,1.61]$

$100 \%$

$0.88[0.58,1.33]$

Total events: 14 (Abatacept), 14 (Placebo)

Heterogeneity: Not applicable

Test for overall effect: $Z=0.03(P=0.98)$

10.2.6 Partial remission: low dose abatacept versus placebo

Furie 2014

9/99

99

$14 / 100$

Subtotal $(95 \% \mathrm{Cl})$

100

Total events: 9 (Abatacept), 14 (Placebo)

Heterogeneity: Not applicable

Test for overall effect: $\mathrm{Z}=1.07(\mathrm{P}=0.28)$

Test for subgroup differences: $\mathrm{Chi}^{2}=2.46, \mathrm{df}=1(\mathrm{P}=0.78), \mathrm{I}^{2}=0 \%$ 
Analysis 10.3. Comparison 10 Abatacept + other immunosuppressive agent (IS) + versus placebo + other IS, Outcome 3 Adverse renal outcomes.

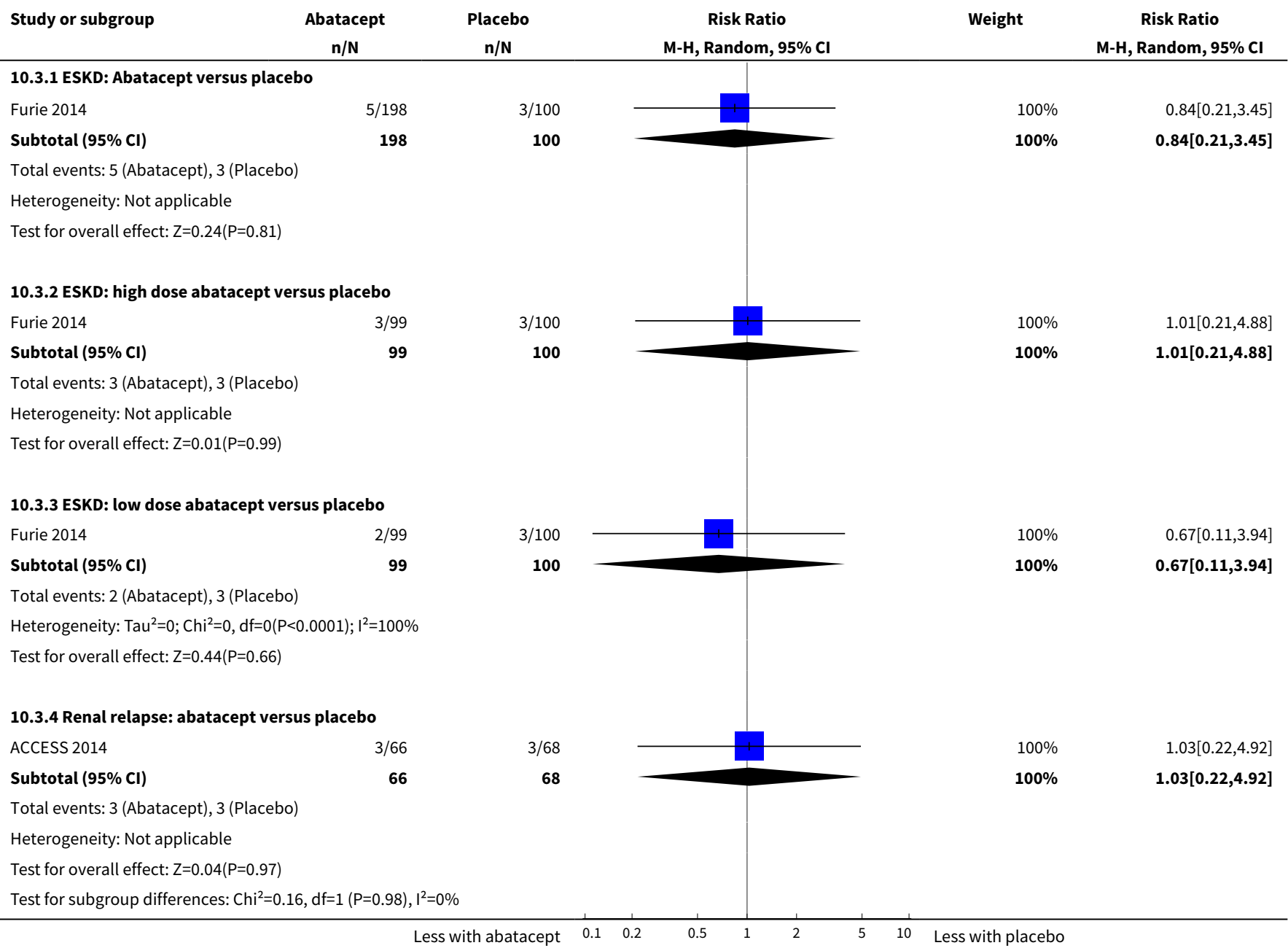

\section{Analysis 10.4. Comparison 10 Abatacept + other immunosuppressive agent (IS) + versus placebo + other IS, Outcome 4 Major Infection.}

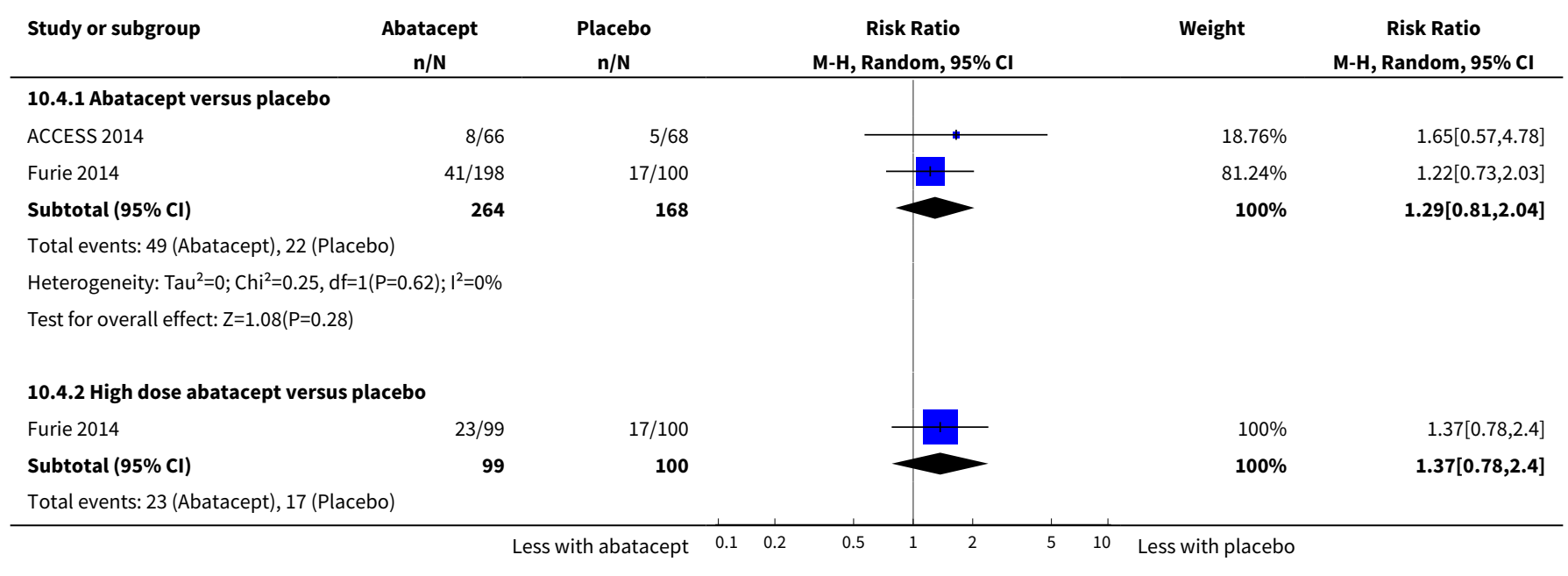




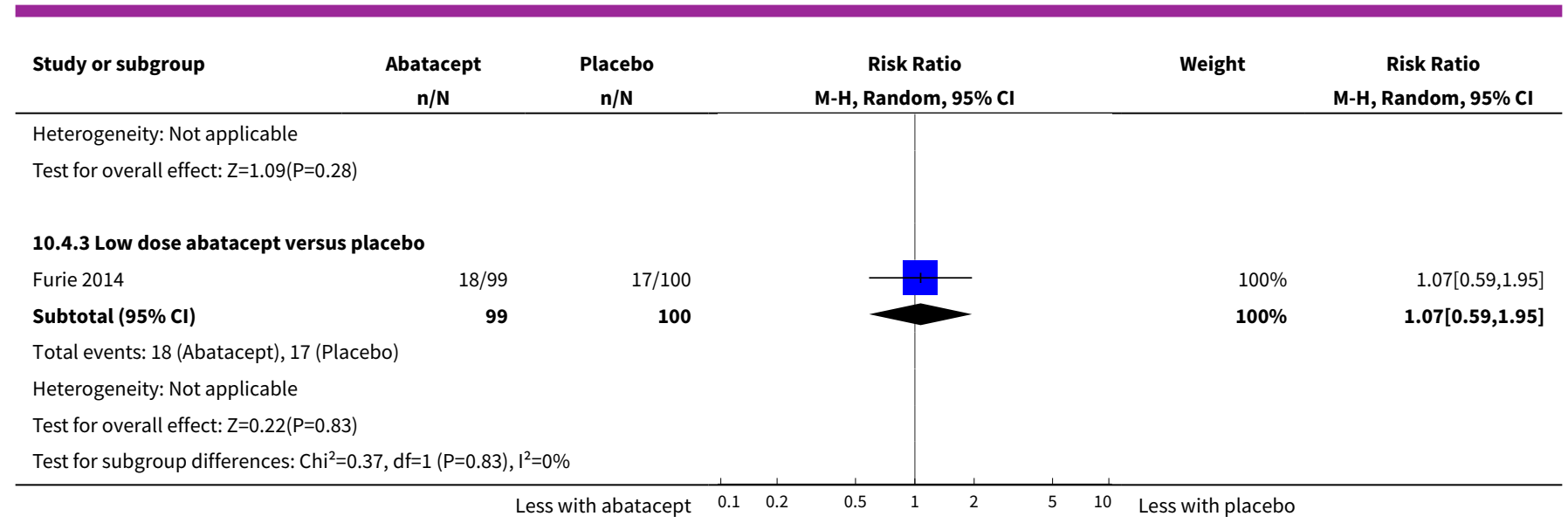

Analysis 10.5. Comparison 10 Abatacept + other immunosuppressive agent (IS) + versus placebo + other IS, Outcome 5 Herpes zoster virus.

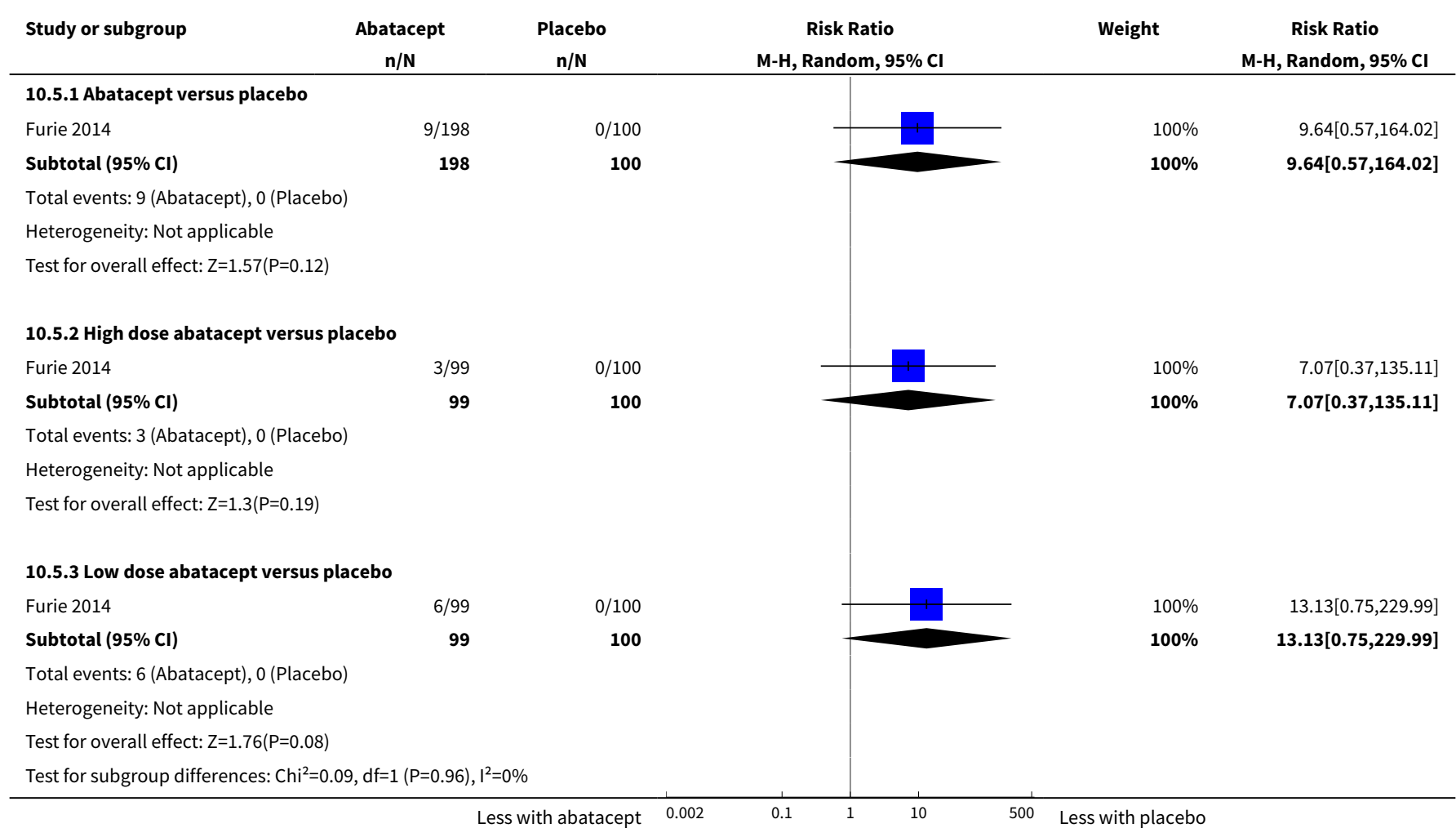

Analysis 10.6. Comparison 10 Abatacept + other immunosuppressive agent (IS) + versus placebo + other IS, Outcome 6 Health-related quality of life.

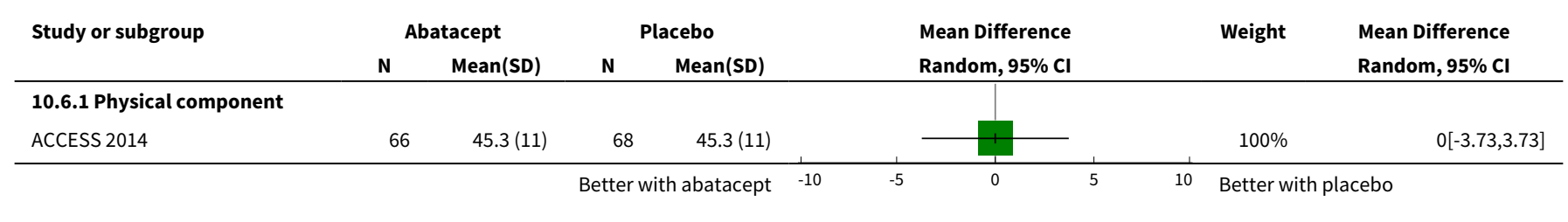




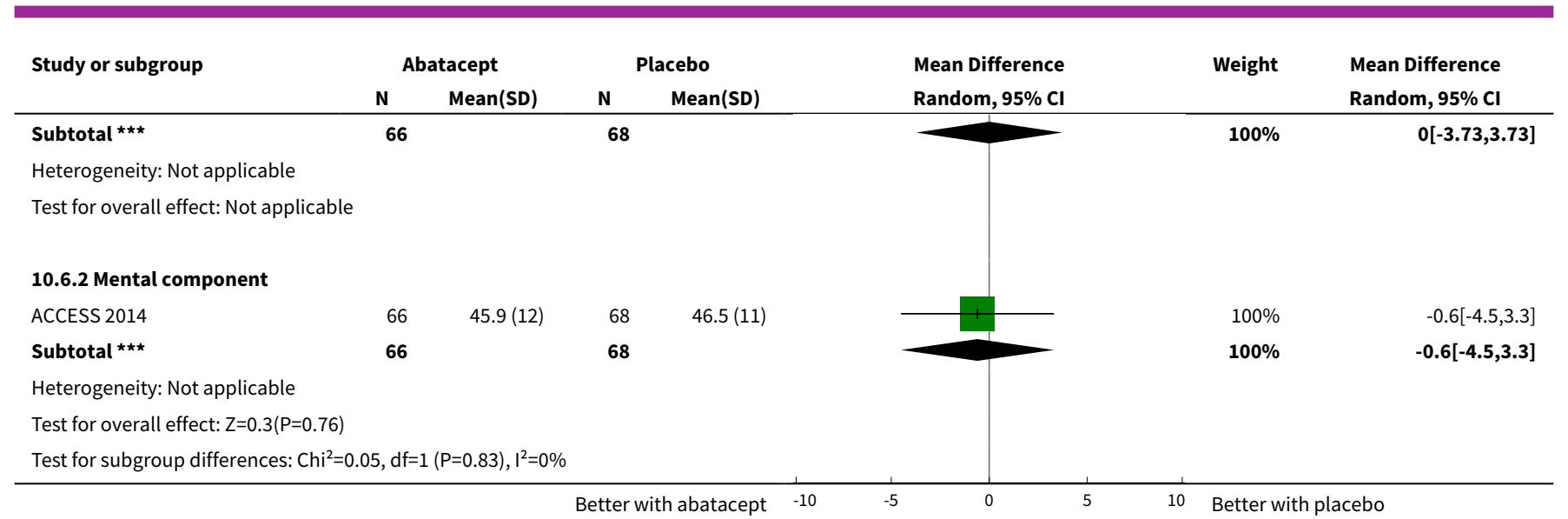

Analysis 10.7. Comparison 10 Abatacept + other immunosuppressive agent (IS) + versus placebo + other IS, Outcome 7 Disease activity (BILAG).

\begin{tabular}{|c|c|c|c|c|c|c|c|}
\hline \multirow{3}{*}{$\begin{array}{l}\text { Study or subgroup } \\
\text { ACCESS } 2014\end{array}$} & \multicolumn{2}{|c|}{ Abatacept } & \multicolumn{2}{|c|}{ Placebo } & \multirow{2}{*}{$\begin{array}{l}\text { Mean Difference } \\
\text { Random, } 95 \% \mathrm{Cl}\end{array}$} & \multirow[t]{2}{*}{ Weight } & \multirow{2}{*}{$\begin{array}{l}\text { Mean Difference } \\
\text { Random, 95\% Cl }\end{array}$} \\
\hline & $\mathbf{N}$ & $\operatorname{Mean}(S D)$ & $\mathbf{N}$ & $\operatorname{Mean}(S D)$ & & & \\
\hline & 66 & $3.4(1.8)$ & 68 & $3.8(3)$ & \begin{tabular}{l|l|l}
1 & & \\
\end{tabular} & $100 \%$ & $-0.4[-1.23,0.43]$ \\
\hline 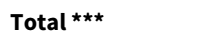 & 66 & & 68 & & & $100 \%$ & $-0.4[-1.23,0.43]$ \\
\hline \multicolumn{8}{|c|}{ Heterogeneity: Not applicable } \\
\hline \multicolumn{8}{|c|}{ Test for overall effect: $Z=0.94(P=0.35)$} \\
\hline
\end{tabular}

Comparison 11. Laquinimod + other immunosuppressive agent (IS) versus placebo + other IS

\begin{tabular}{|c|c|c|c|c|}
\hline Outcome or subgroup title & No. of studies & $\begin{array}{l}\text { No. of partici- } \\
\text { pants }\end{array}$ & Statistical method & Effect size \\
\hline 1 Death & 1 & & $\begin{array}{l}\text { Risk Ratio (M-H, Random, 95\% } \\
\mathrm{Cl})\end{array}$ & Subtotals only \\
\hline 1.1 Laquinimod versus placebo & 1 & 46 & $\begin{array}{l}\text { Risk Ratio (M-H, Random, 95\% } \\
\mathrm{Cl})\end{array}$ & $1.5[0.06,34.79]$ \\
\hline $\begin{array}{l}1.2 \text { High dose laquinimod versus } \\
\text { placebo }\end{array}$ & 1 & 30 & $\begin{array}{l}\text { Risk Ratio (M-H, Random, 95\% } \\
\mathrm{Cl})\end{array}$ & $3.0[0.13,68.26]$ \\
\hline $\begin{array}{l}1.3 \text { Low dose laquinimod versus } \\
\text { placebo }\end{array}$ & 1 & 31 & $\begin{array}{l}\text { Risk Ratio (M-H, Random, 95\% } \\
\mathrm{Cl})\end{array}$ & $0.0[0.0,0.0]$ \\
\hline 2 Complete remission & 1 & & $\begin{array}{l}\text { Risk Ratio (M-H, Random, 95\% } \\
\mathrm{Cl})\end{array}$ & Subtotals only \\
\hline $\begin{array}{l}2.1 \text { Complete remission: laquinimod } \\
\text { versus placebo }\end{array}$ & 1 & 46 & $\begin{array}{l}\text { Risk Ratio (M-H, Random, 95\% } \\
\mathrm{Cl})\end{array}$ & $1.55[0.70,3.42]$ \\
\hline $\begin{array}{l}\text { 2.2 Complete remission: high dose } \\
\text { laquinimod versus placebo }\end{array}$ & 1 & 30 & $\begin{array}{l}\text { Risk Ratio (M-H, Random, 95\% } \\
\mathrm{Cl} \text { ) }\end{array}$ & $1.2[0.47,3.09]$ \\
\hline
\end{tabular}




\begin{tabular}{lllll}
\hline Outcome or subgroup title & No. of studies & $\begin{array}{l}\text { No. of partici- } \\
\text { pants }\end{array}$ & Statistical method & Effect size \\
\hline $\begin{array}{l}\text { 2.3 Complete remission: low dose } \\
\text { laquinimod versus placebo }\end{array}$ & 1 & 31 & $\begin{array}{l}\text { Risk Ratio (M-H, Random, 95\% } \\
\text { Cl) }\end{array}$ & $1.88[0.83,4.22]$ \\
\hline
\end{tabular}

Analysis 11.1. Comparison 11 Laquinimod + other immunosuppressive agent (IS) versus placebo + other IS, Outcome 1 Death.

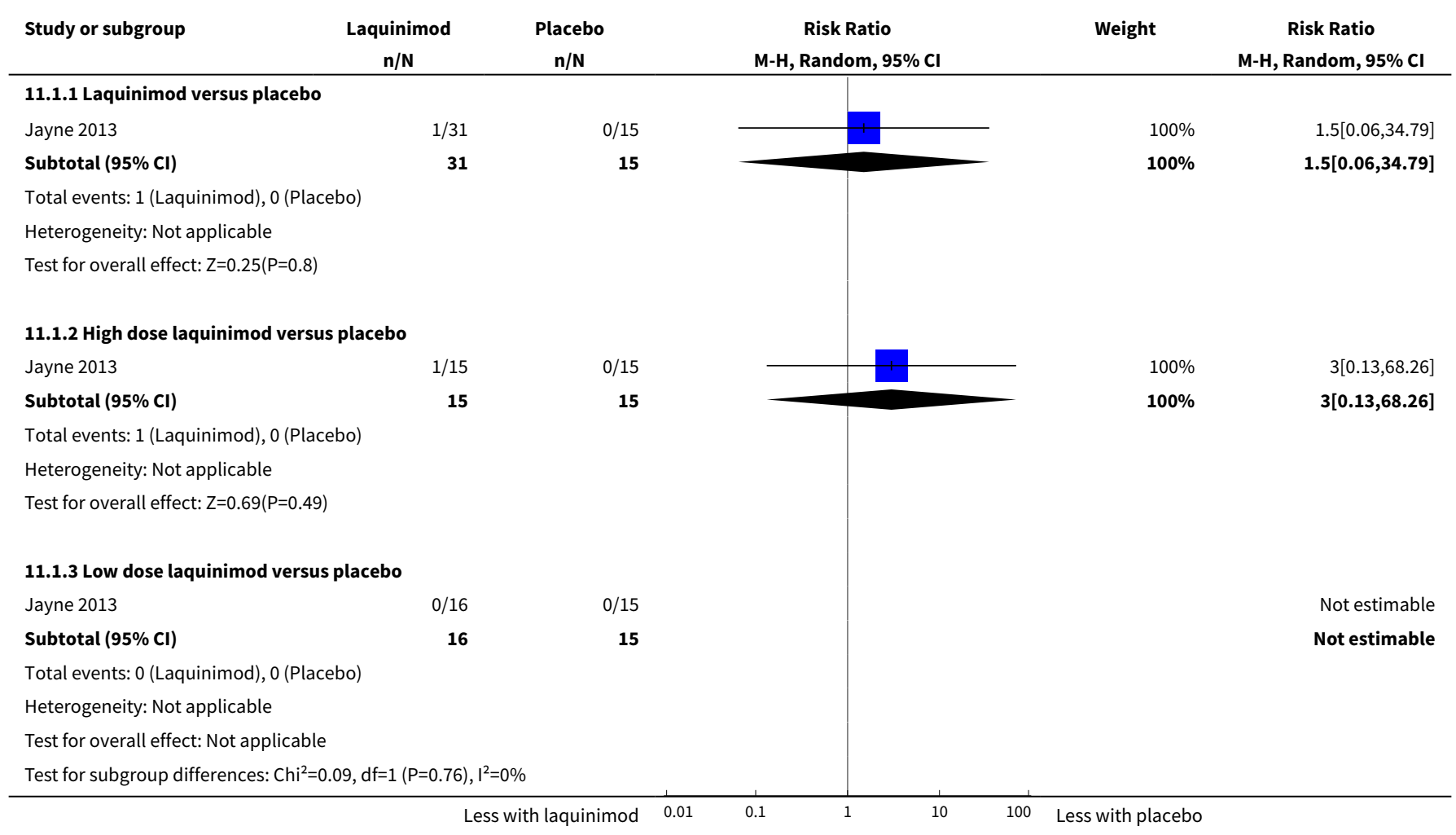

\section{Analysis 11.2. Comparison 11 Laquinimod + other immunosuppressive agent (IS) versus placebo + other IS, Outcome 2 Complete remission.}

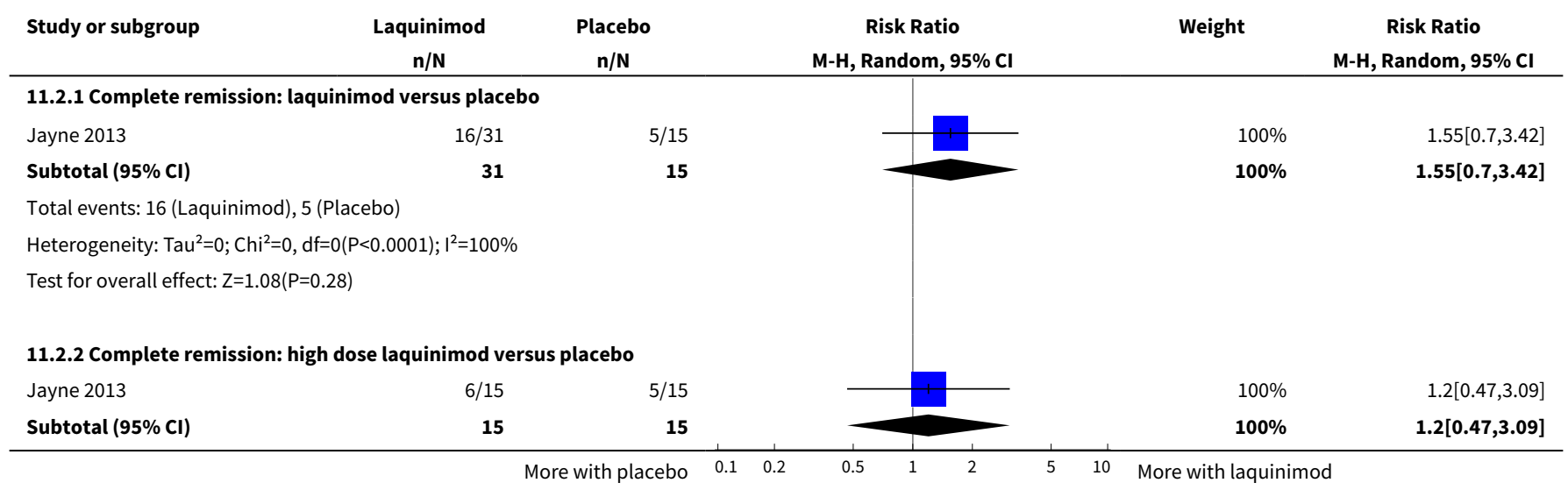




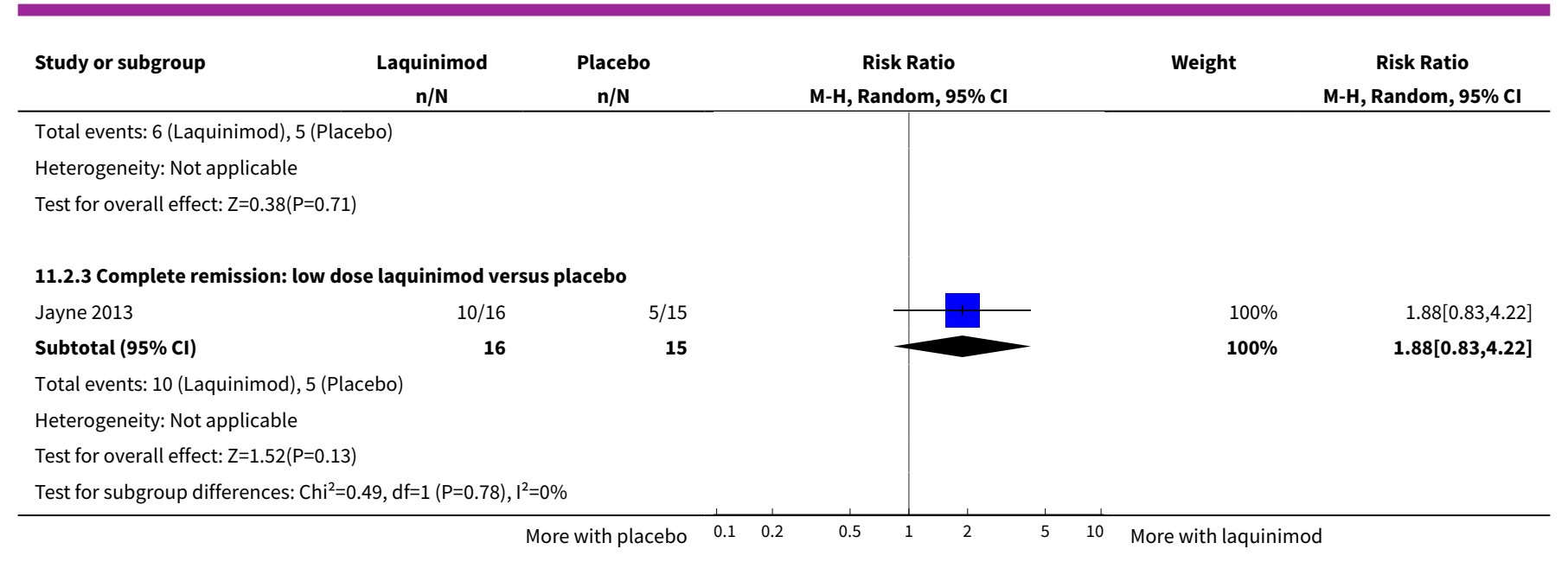

\section{Comparison 12. Ocrelizumab + other immunosuppressive agent (IS) versus placebo + other IS}

\begin{tabular}{|c|c|c|c|c|}
\hline Outcome or subgroup title & No. of studies & $\begin{array}{l}\text { No. of partici- } \\
\text { pants }\end{array}$ & Statistical method & Effect size \\
\hline 1 Death & 1 & & $\begin{array}{l}\text { Risk Ratio (M-H, Random, } \\
95 \% \mathrm{Cl})\end{array}$ & Subtotals only \\
\hline 1.1 Ocrelizumab versus placebo & 1 & 379 & $\begin{array}{l}\text { Risk Ratio (M-H, Random, } \\
95 \% \mathrm{Cl})\end{array}$ & $0.66[0.23,1.85]$ \\
\hline $\begin{array}{l}1.2 \text { High dose ocrelizumab versus } \\
\text { placebo }\end{array}$ & 1 & 253 & $\begin{array}{l}\text { Risk Ratio (M-H, Random, } \\
95 \% \mathrm{Cl})\end{array}$ & $0.81[0.25,2.60]$ \\
\hline $\begin{array}{l}1.3 \text { Low dose ocrelizumab versus } \\
\text { placebo }\end{array}$ & 1 & 251 & $\begin{array}{l}\text { Risk Ratio (M-H, Random, } \\
95 \% \mathrm{Cl})\end{array}$ & $0.50[0.13,1.94]$ \\
\hline 2 Remission & 1 & & $\begin{array}{l}\text { Risk Ratio (M-H, Random, } \\
95 \% \mathrm{Cl})\end{array}$ & Subtotals only \\
\hline $\begin{array}{l}2.1 \text { Complete remission: ocrelizumab } \\
\text { versus placebo }\end{array}$ & 1 & 223 & $\begin{array}{l}\text { Risk Ratio (M-H, Random, } \\
95 \% \mathrm{Cl})\end{array}$ & $1.07[0.74,1.56]$ \\
\hline $\begin{array}{l}\text { 2.2 Complete remission: high dose } \\
\text { ocrelizumab versus placebo }\end{array}$ & 1 & 148 & $\begin{array}{l}\text { Risk Ratio (M-H, Random, } \\
95 \% \mathrm{Cl} \text { ) }\end{array}$ & $0.91[0.57,1.44]$ \\
\hline $\begin{array}{l}2.3 \text { Complete remission: low dose } \\
\text { ocrelizumab versus placebo }\end{array}$ & 1 & 150 & $\begin{array}{l}\text { Risk Ratio (M-H, Random, } \\
95 \% \mathrm{Cl})\end{array}$ & $1.23[0.82,1.85]$ \\
\hline $\begin{array}{l}\text { 2.4 Partial remission: ocrelizumab } \\
\text { versus placebo }\end{array}$ & 1 & 223 & $\begin{array}{l}\text { Risk Ratio (M-H, Random, } \\
95 \% \mathrm{Cl})\end{array}$ & $1.49[0.89,2.49]$ \\
\hline $\begin{array}{l}2.5 \text { Partial remission: high dose ocre- } \\
\text { lizumab versus placebo }\end{array}$ & 1 & 148 & $\begin{array}{l}\text { Risk Ratio (M-H, Random, } \\
95 \% \mathrm{Cl})\end{array}$ & $1.78[1.03,3.08]$ \\
\hline $\begin{array}{l}\text { 2.6 Partial remission: low dose ocre- } \\
\text { lizumab versus placebo }\end{array}$ & 1 & 150 & $\begin{array}{l}\text { Risk Ratio (M-H, Random, } \\
95 \% \mathrm{Cl} \text { ) }\end{array}$ & $1.2[0.65,2.20]$ \\
\hline
\end{tabular}




\begin{tabular}{lllll}
\hline Outcome or subgroup title & No. of studies & $\begin{array}{l}\text { No. of partici- } \\
\text { pants }\end{array}$ & Statistical method & Effect size \\
\hline 3 Major Infection & 1 & $\begin{array}{l}\text { Risk Ratio (M-H, Random, } \\
95 \% \text { Cl) }\end{array}$ & Subtotals only \\
\hline 3.1 Ocrelizumab versus placebo & 1 & 378 & $\begin{array}{l}\text { Risk Ratio (M-H, Random, } \\
95 \% \text { Cl) }\end{array}$ & $1.14[0.95,1.36]$ \\
\hline $\begin{array}{l}\text { 3.2 High dose ocrelizumab versus } \\
\text { placebo }\end{array}$ & 1 & 252 & $\begin{array}{l}\text { Risk Ratio (M-H, Random, } \\
95 \% \text { Cl) }\end{array}$ & $1.05[0.85,1.30]$ \\
\hline $\begin{array}{l}\text { 3.3 Low dose ocrelizumab versus } \\
\text { placebo }\end{array}$ & 1 & 251 & $\begin{array}{l}\text { Risk Ratio (M-H, Random, } \\
95 \% \text { Cl) }\end{array}$ & $1.22[1.00,1.48]$ \\
\hline
\end{tabular}

Analysis 12.1. Comparison 12 Ocrelizumab + other immunosuppressive agent (IS) versus placebo + other IS, Outcome 1 Death.

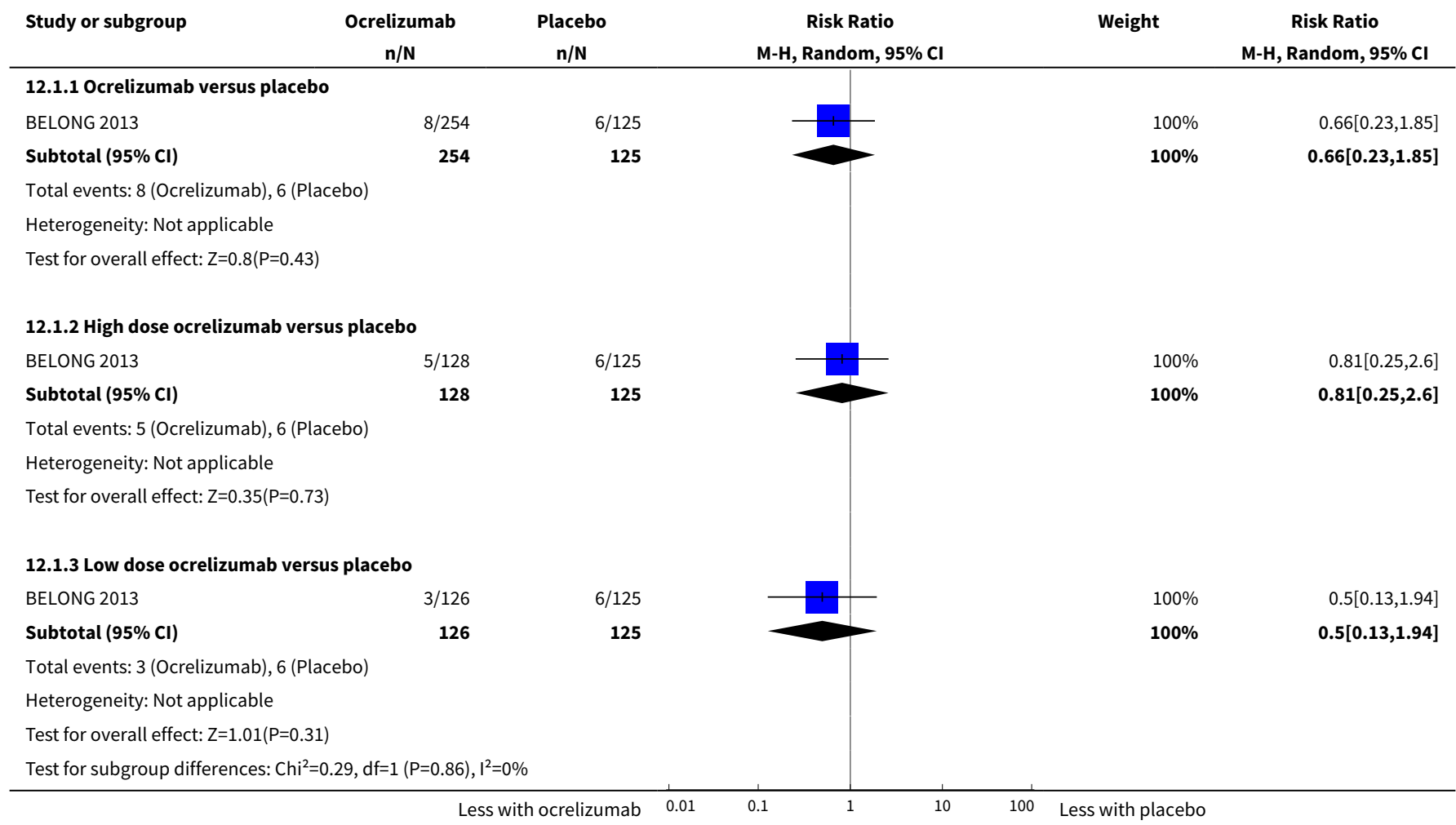

Analysis 12.2. Comparison 12 Ocrelizumab + other immunosuppressive agent (IS) versus placebo + other IS, Outcome 2 Remission.

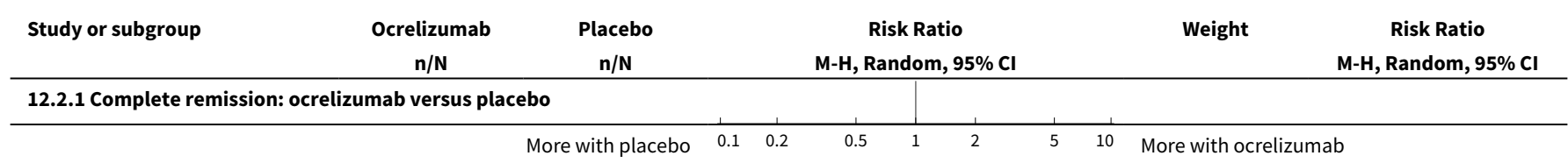




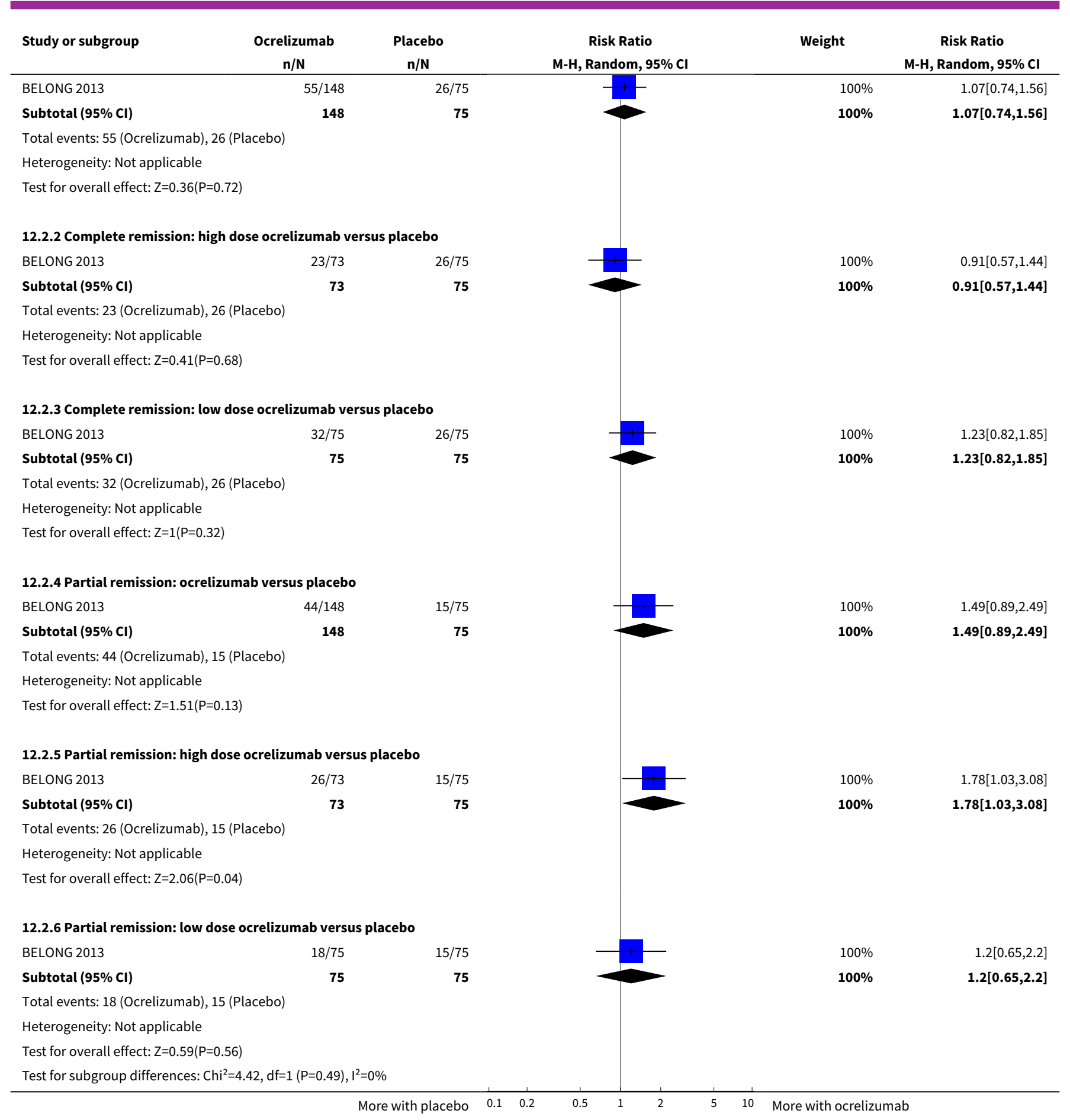

\section{Analysis 12.3. Comparison 12 Ocrelizumab + other immunosuppressive agent (IS) versus placebo + other IS, Outcome 3 Major Infection.}

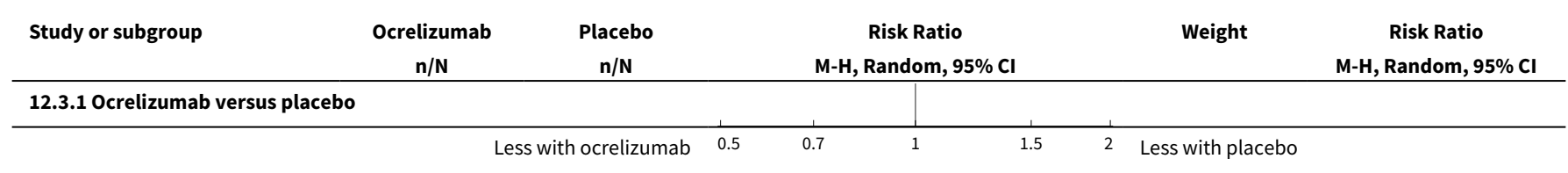




\begin{tabular}{|c|c|c|c|c|c|}
\hline Study or subgroup & $\begin{array}{l}\text { Ocrelizumab } \\
n / N\end{array}$ & $\begin{array}{c}\text { Placebo } \\
n / N\end{array}$ & $\begin{array}{c}\text { Risk Ratio } \\
\text { M-H, Random, } 95 \% \mathrm{Cl}\end{array}$ & Weight & $\begin{array}{c}\text { Risk Ratio } \\
\text { M-H, Random, } 95 \% \mathrm{Cl}\end{array}$ \\
\hline BELONG 2013 & $161 / 253$ & $70 / 125$ & + & $100 \%$ & $1.14[0.95,1.36]$ \\
\hline Subtotal $(95 \% \mathrm{Cl})$ & 253 & 125 & & $100 \%$ & $1.14[0.95,1.36]$ \\
\hline \multicolumn{6}{|c|}{ Total events: 161 (Ocrelizumab), 70 (Placebo) } \\
\hline \multicolumn{6}{|c|}{ Heterogeneity: Not applicable } \\
\hline \multicolumn{6}{|c|}{ Test for overall effect: $Z=1.38(P=0.17)$} \\
\hline \multicolumn{6}{|c|}{ 12.3.2 High dose ocrelizumab versus placebo } \\
\hline BELONG 2013 & $75 / 127$ & $70 / 125$ & & $100 \%$ & $1.05[0.85,1.3]$ \\
\hline Subtotal $(95 \% \mathrm{Cl})$ & 127 & 125 & & $100 \%$ & $1.05[0.85,1.3]$ \\
\hline \multicolumn{6}{|c|}{ Total events: 75 (Ocrelizumab), 70 (Placebo) } \\
\hline \multicolumn{6}{|c|}{ Heterogeneity: Not applicable } \\
\hline \multicolumn{6}{|c|}{ Test for overall effect: $Z=0.49(P=0.62)$} \\
\hline \multicolumn{6}{|c|}{ 12.3.3 Low dose ocrelizumab versus placebo } \\
\hline BELONG 2013 & $86 / 126$ & $70 / 125$ & & $100 \%$ & $1.22[1,1.48]$ \\
\hline Subtotal $(95 \% \mathrm{CI})$ & 126 & 125 & - & $100 \%$ & $1.22[1,1.48]$ \\
\hline \multicolumn{6}{|c|}{ Total events: 86 (Ocrelizumab), 70 (Placebo) } \\
\hline \multicolumn{6}{|c|}{ Heterogeneity: Not applicable } \\
\hline \multicolumn{6}{|c|}{ Test for overall effect: $Z=1.98(P=0.05)$} \\
\hline \multicolumn{6}{|c|}{ Test for subgroup differences: $\mathrm{Chi}^{2}=0.97, \mathrm{df}=1(\mathrm{P}=0.62), \mathrm{I}^{2}=0 \%$} \\
\hline
\end{tabular}

\section{Comparison 13. Sirukumab + other immunosuppressive agent (IS) versus placebo + other IS}

\begin{tabular}{lllll}
\hline $\begin{array}{l}\text { Outcome or subgroup } \\
\text { title }\end{array}$ & No. of studies & $\begin{array}{l}\text { No. of partici- } \\
\text { pants }\end{array}$ & Statistical method & Effect size \\
\hline 1 Death & 1 & 25 & Risk Ratio (M-H, Random, 95\% Cl) & $0.0[0.0,0.0]$ \\
\hline 2 Infection & 1 & 25 & Risk Ratio (M-H, Random, 95\% Cl) & $0.93[0.66,1.32]$ \\
\hline 2.1 Major infection & 1 & 25 & Risk Ratio (M-H, Random, 95\% Cl) & $0.93[0.66,1.32]$ \\
\hline 3 Malignancy & 1 & Risk Ratio (M-H, Random, 95\% Cl) & $0.0[0.0,0.0]$ \\
\hline $\begin{array}{l}4 \text { Gastrointestinal (GI) } \\
\text { adverse events }\end{array}$ & 1 & Risk Ratio (M-H, Random, 95\% Cl) & Subtotals only \\
\hline \begin{tabular}{l}
4.1 Diarrhoea \\
\hline
\end{tabular} & 1 & 25 & Risk Ratio (M-H, Random, 95\% Cl) & $1.59[0.10,26.15]$ \\
\hline
\end{tabular}

Analysis 13.1. Comparison 13 Sirukumab + other immunosuppressive agent (IS) versus placebo + other IS, Outcome 1 Death.

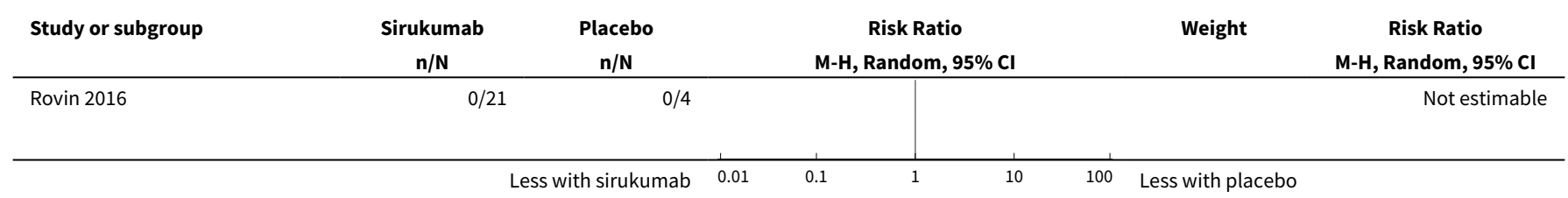




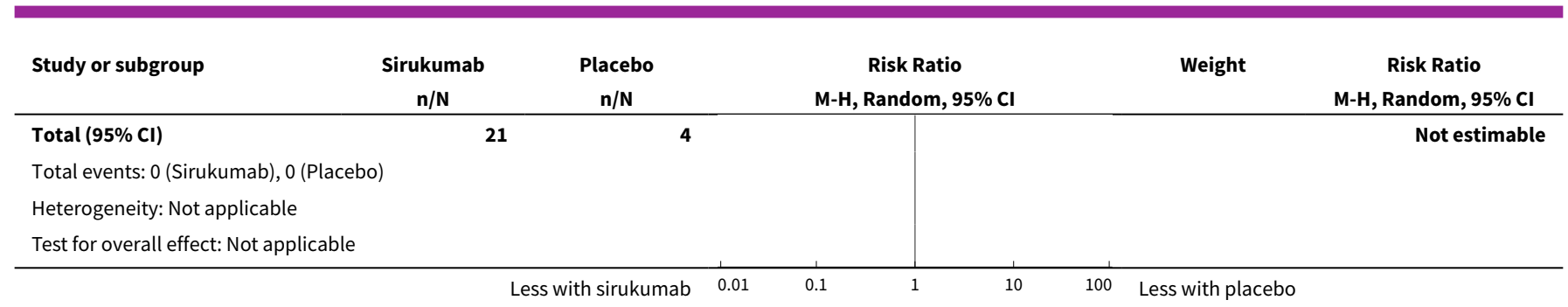

Analysis 13.2. Comparison 13 Sirukumab + other immunosuppressive agent (IS) versus placebo + other IS, Outcome 2 Infection.

\begin{tabular}{|c|c|c|c|c|c|}
\hline Study or subgroup & $\begin{array}{c}\text { Sirukumab } \\
\mathrm{n} / \mathrm{N}\end{array}$ & $\begin{array}{c}\text { Placebo } \\
\mathrm{n} / \mathrm{N}\end{array}$ & $\begin{array}{c}\text { Risk Ratio } \\
\text { M-H, Random, } 95 \% \mathrm{Cl} \\
\end{array}$ & Weight & $\begin{array}{c}\text { Risk Ratio } \\
\text { M-H, Random, } 95 \% \text { Cl }\end{array}$ \\
\hline \multicolumn{6}{|c|}{ 13.2.1 Major infection } \\
\hline Rovin 2016 & $18 / 21$ & $4 / 4$ & — & $100 \%$ & $0.93[0.66,1.32]$ \\
\hline Subtotal $(95 \% \mathrm{CI})$ & 21 & 4 & & $100 \%$ & $0.93[0.66,1.32]$ \\
\hline \multicolumn{6}{|c|}{ Total events: 18 (Sirukumab), 4 (Placebo) } \\
\hline \multicolumn{6}{|c|}{ Heterogeneity: Not applicable } \\
\hline \multicolumn{6}{|c|}{ Test for overall effect: $Z=0.39(P=0.7)$} \\
\hline Total $(95 \% \mathrm{Cl})$ & 21 & 4 & & $100 \%$ & $0.93[0.66,1.32]$ \\
\hline \multicolumn{6}{|c|}{ Total events: 18 (Sirukumab), 4 (Placebo) } \\
\hline \multicolumn{6}{|c|}{ Heterogeneity: Not applicable } \\
\hline \multicolumn{6}{|c|}{ Test for overall effect: $Z=0.39(P=0.7)$} \\
\hline & & sirukumab 0.5 & 0.7 & 2 Less with placebo & \\
\hline
\end{tabular}

Analysis 13.3. Comparison 13 Sirukumab + other immunosuppressive agent (IS) versus placebo + other IS, Outcome 3 Malignancy.

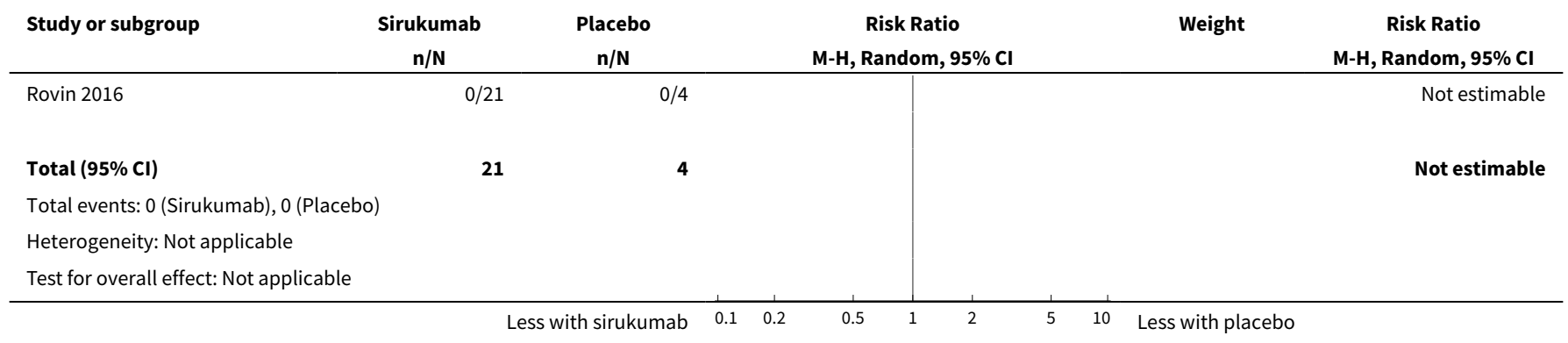

Analysis 13.4. Comparison 13 Sirukumab + other immunosuppressive agent (IS) versus placebo + other IS, Outcome 4 Gastrointestinal (GI) adverse events.

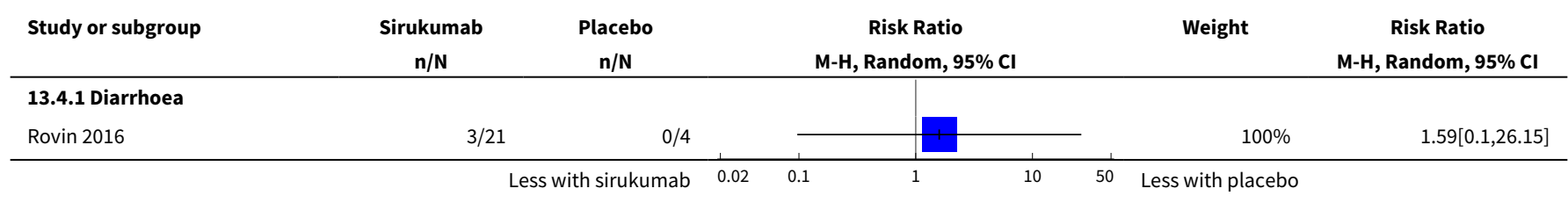




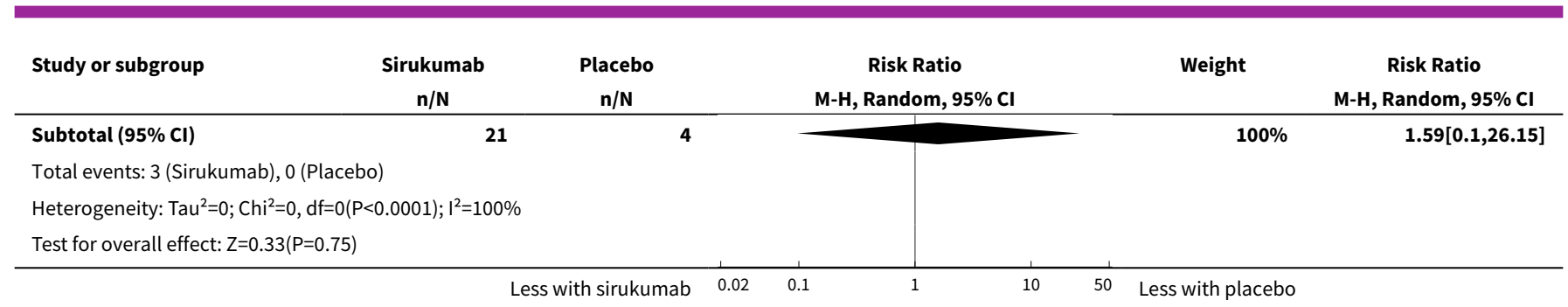

\section{Comparison 14. IV versus oral cyclophosphamide (CPA)}

\begin{tabular}{|c|c|c|c|c|}
\hline $\begin{array}{l}\text { Outcome or subgroup } \\
\text { title }\end{array}$ & No. of studies & $\begin{array}{l}\text { No. of partici- } \\
\text { pants }\end{array}$ & Statistical method & Effect size \\
\hline 1 Death & 2 & 67 & Risk Ratio (M-H, Random, 95\% Cl) & $0.80[0.20,3.24]$ \\
\hline $\begin{array}{l}2 \text { Adverse renal out- } \\
\text { comes }\end{array}$ & 2 & & Risk Ratio (M-H, Random, 95\% Cl) & Subtotals only \\
\hline 2.1 ESKD & 2 & 67 & Risk Ratio (M-H, Random, 95\% Cl) & $0.23[0.04,1.28]$ \\
\hline $\begin{array}{l}2.2 \text { Doubling of serum } \\
\text { creatinine }\end{array}$ & 2 & 67 & Risk Ratio (M-H, Random, 95\% Cl) & $0.67[0.23,1.98]$ \\
\hline $\begin{array}{l}\text { 2.3 Deterioration of kid- } \\
\text { ney function }\end{array}$ & 1 & 38 & Risk Ratio (M-H, Random, 95\% Cl) & $0.72[0.23,2.27]$ \\
\hline 3 Stable kidney function & 1 & 38 & Risk Ratio (M-H, Random, 95\% Cl) & $1.11[0.77,1.59]$ \\
\hline 4 Ovarian failure & 2 & 56 & Risk Ratio (M-H, Random, 95\% Cl) & $0.70[0.37,1.30]$ \\
\hline 5 Infection & 2 & & Risk Ratio (M-H, Random, 95\% Cl) & Subtotals only \\
\hline 5.1 Major infection & 2 & 67 & Risk Ratio (M-H, Random, 95\% Cl) & $1.16[0.47,2.90]$ \\
\hline 5.2 Herpes zoster virus & 1 & 38 & Risk Ratio (M-H, Random, 95\% Cl) & $0.75[0.28,2.04]$ \\
\hline 6 Malignancy & 2 & 67 & Risk Ratio (M-H, Random, 95\% Cl) & $1.43[0.41,4.96]$ \\
\hline 7 Bladder toxicity & 2 & 67 & Risk Ratio (M-H, Random, 95\% Cl) & $0.22[0.03,1.83]$ \\
\hline $\begin{array}{l}8 \text { Gastrointestinal (GI) } \\
\text { adverse events }\end{array}$ & 1 & & Risk Ratio (M-H, Random, 95\% Cl) & Subtotals only \\
\hline $8.1 \mathrm{Gl}$ upset & 1 & 29 & Risk Ratio (M-H, Random, 95\% Cl) & $3.69[0.43,31.43]$ \\
\hline
\end{tabular}

Analysis 14.1. Comparison 14 IV versus oral cyclophosphamide (CPA), Outcome 1 Death.

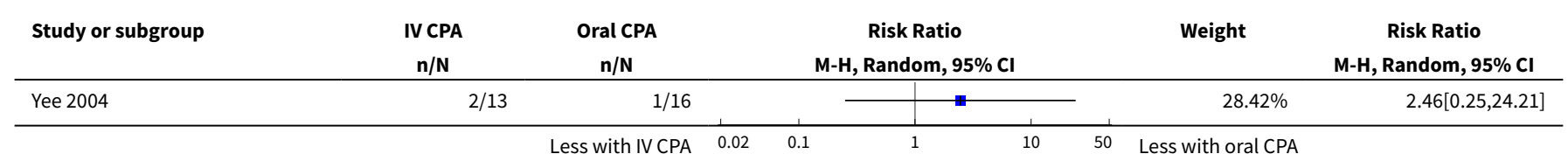




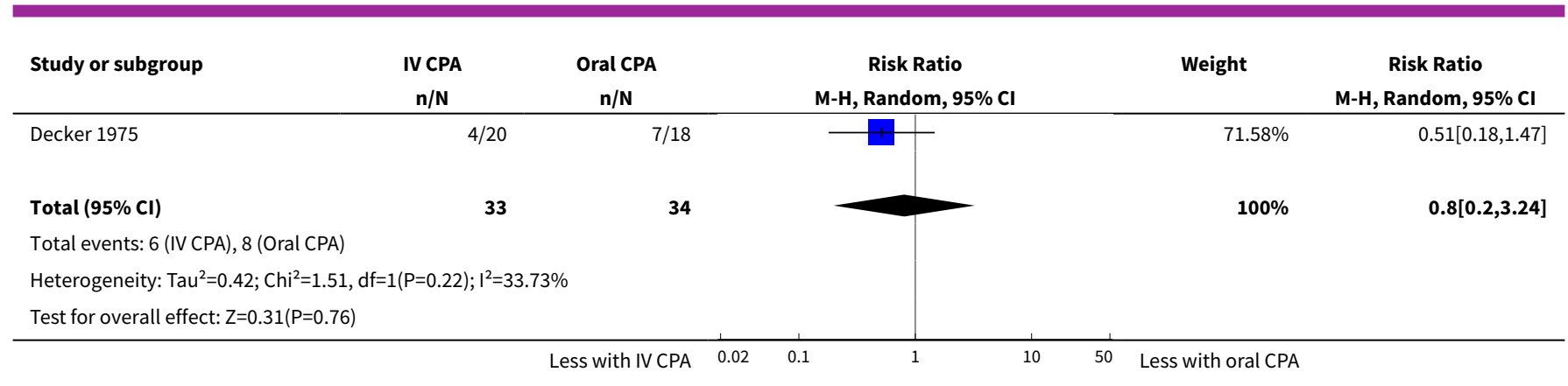

\section{Analysis 14.2. Comparison 14 IV versus oral cyclophosphamide (CPA), Outcome 2 Adverse renal outcomes.}

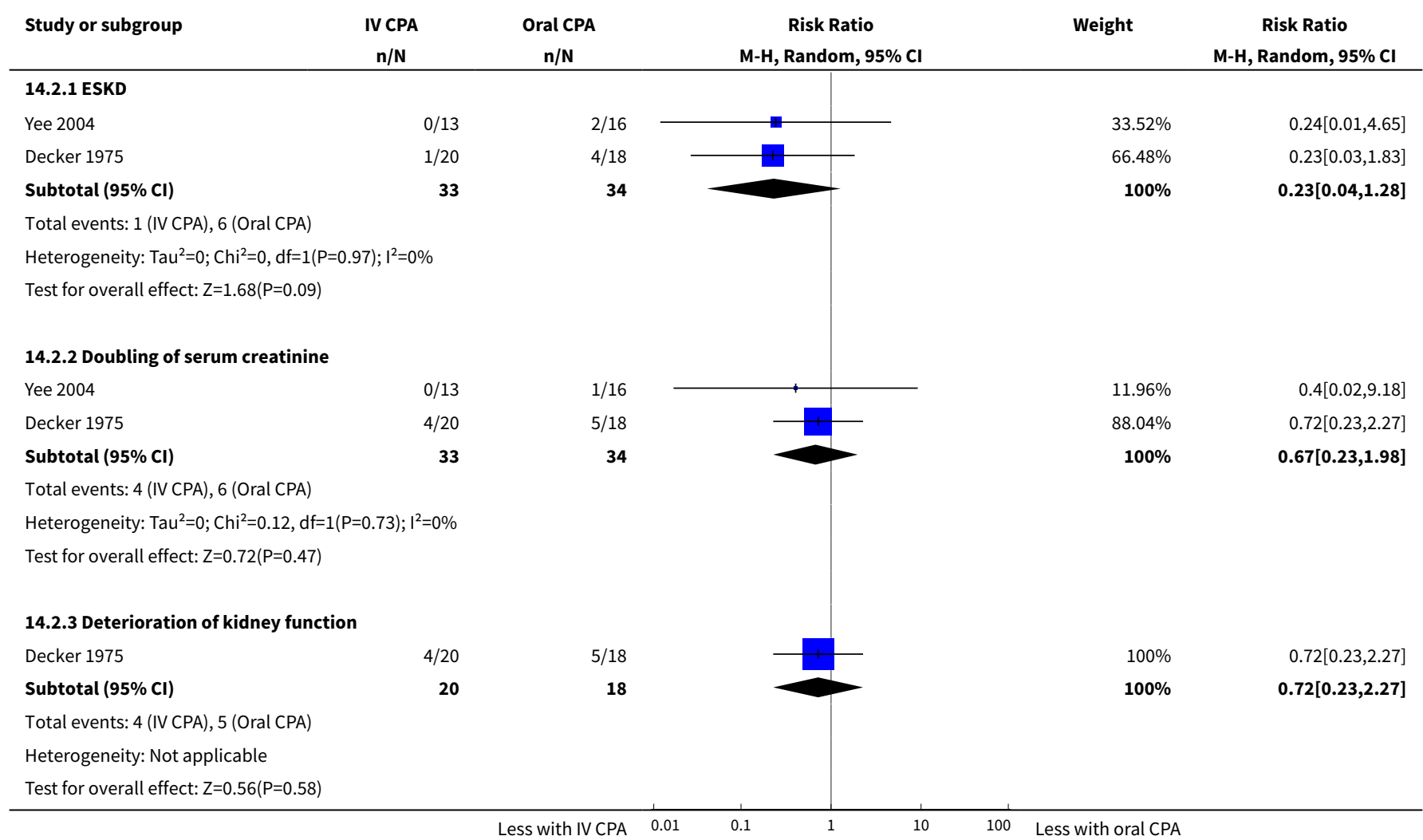

Analysis 14.3. Comparison 14 IV versus oral cyclophosphamide (CPA), Outcome 3 Stable kidney function.

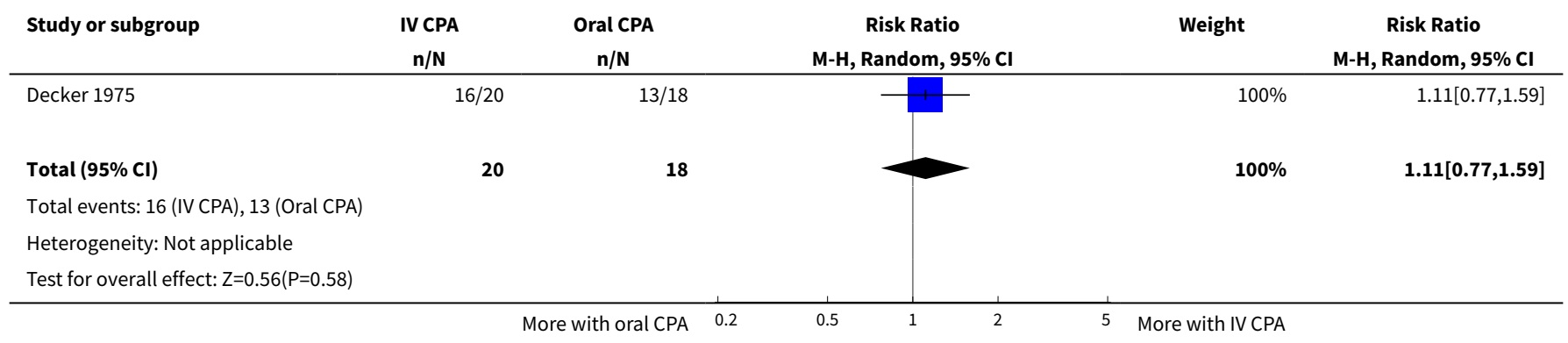


Analysis 14.4. Comparison 14 IV versus oral cyclophosphamide (CPA), Outcome 4 Ovarian failure.

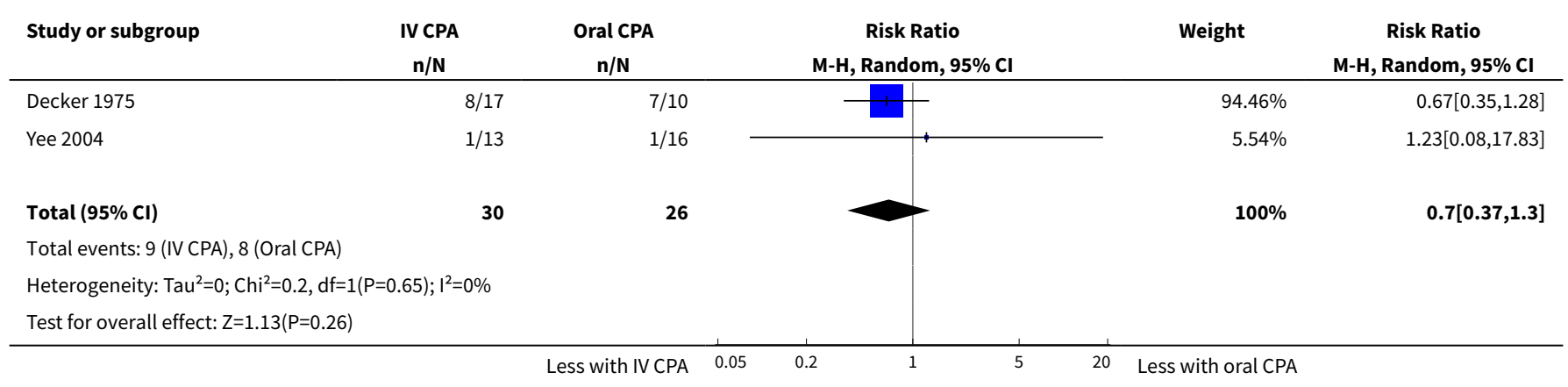

Analysis 14.5. Comparison 14 IV versus oral cyclophosphamide (CPA), Outcome 5 Infection.

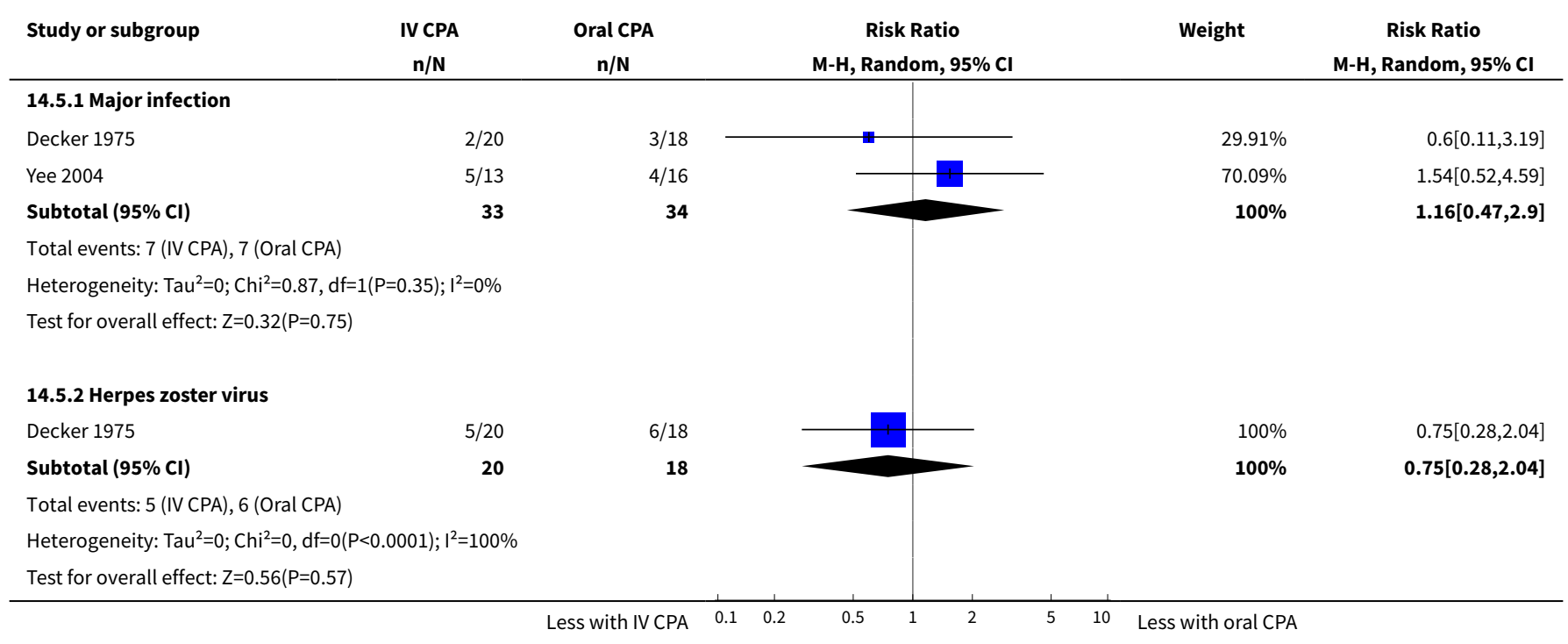

Analysis 14.6. Comparison 14 IV versus oral cyclophosphamide (CPA), Outcome 6 Malignancy.

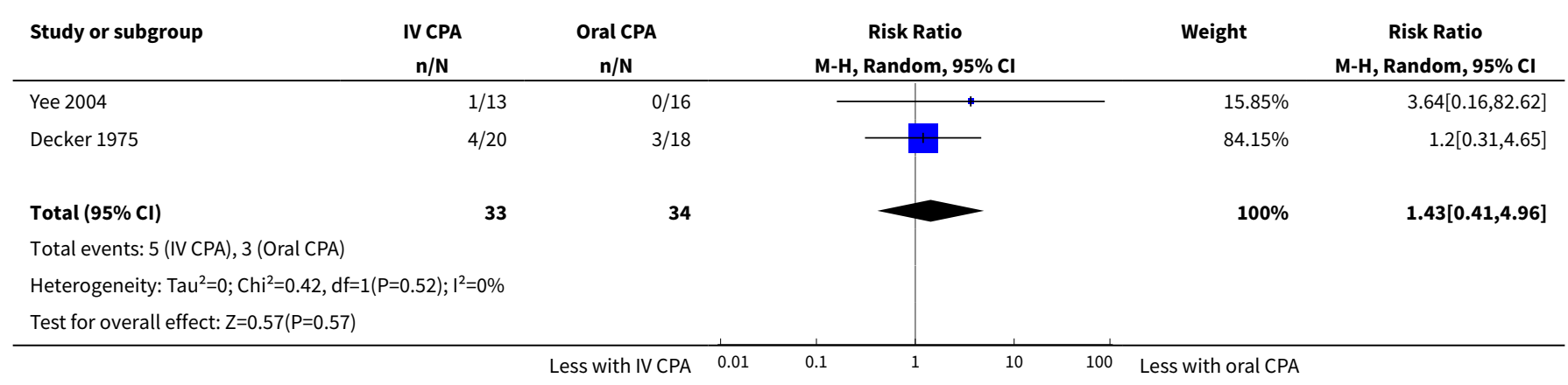


Analysis 14.7. Comparison 14 IV versus oral cyclophosphamide (CPA), Outcome 7 Bladder toxicity.

\begin{tabular}{|c|c|c|c|c|c|}
\hline Study or subgroup & $\begin{array}{c}\text { IV CPA } \\
\text { n/N }\end{array}$ & $\begin{array}{c}\text { Oral CPA } \\
n / N\end{array}$ & $\begin{array}{c}\text { Risk Ratio } \\
\text { M-H, Random, } 95 \% \mathrm{Cl}\end{array}$ & Weight & $\begin{array}{c}\text { Risk Ratio } \\
\text { M-H, Random, 95\% CI }\end{array}$ \\
\hline Yee 2004 & $0 / 13$ & $1 / 16$ & \begin{tabular}{l|l}
$\operatorname{m}$ & \\
\end{tabular} & $46.28 \%$ & $0.4[0.02,9.18]$ \\
\hline Decker 1975 & $0 / 20$ & $3 / 18$ & 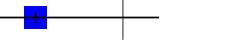 & $53.72 \%$ & $0.13[0.01,2.34]$ \\
\hline Total $(95 \% \mathrm{Cl})$ & 33 & 34 & & $100 \%$ & $0.22[0.03,1.83]$ \\
\hline \multicolumn{6}{|c|}{ Total events: 0 (IV CPA), 4 (Oral CPA) } \\
\hline Test for overall effect & & & & & \\
\hline
\end{tabular}

Analysis 14.8. Comparison 14 IV versus oral cyclophosphamide (CPA), Outcome 8 Gastrointestinal (GI) adverse events.

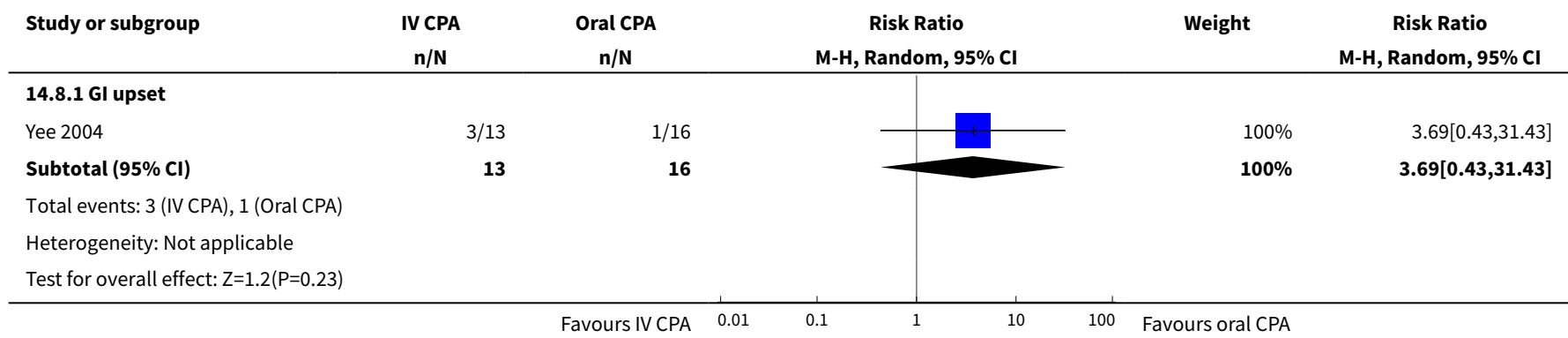

\section{Comparison 15. Low versus high dose cyclophosphamide (CPA)}

\begin{tabular}{|c|c|c|c|c|}
\hline $\begin{array}{l}\text { Outcome or subgroup } \\
\text { title }\end{array}$ & No. of studies & $\begin{array}{l}\text { No. of partici- } \\
\text { pants }\end{array}$ & Statistical method & Effect size \\
\hline 1 Death & 4 & & Risk Ratio (M-H, Random, 95\% Cl) & Subtotals only \\
\hline 1.1 At 6 months & 1 & 117 & Risk Ratio (M-H, Random, 95\% Cl) & $1.81[0.19,16.85]$ \\
\hline 1.2 At 12 months & 2 & 121 & Risk Ratio $(\mathrm{M}-\mathrm{H}$, Random, $95 \% \mathrm{Cl})$ & $0.97[0.14,6.56]$ \\
\hline 1.3 At 5 years & 1 & 85 & Risk Ratio (M-H, Random, 95\% Cl) & $0.13[0.01,2.51]$ \\
\hline 1.4 At 10 years & 1 & 90 & Risk Ratio (M-H, Random, 95\% Cl) & $0.38[0.08,1.87]$ \\
\hline 2 Remission & 3 & & Risk Ratio (M-H, Random, 95\% Cl) & Subtotals only \\
\hline $\begin{array}{l}2.1 \text { Complete renal re- } \\
\text { mission }\end{array}$ & 3 & 267 & Risk Ratio (M-H, Random, 95\% Cl) & $1.09[0.63,1.86]$ \\
\hline $\begin{array}{l}2.2 \text { Partial renal remis- } \\
\text { sion }\end{array}$ & 3 & 267 & Risk Ratio $(\mathrm{M}-\mathrm{H}$, Random, $95 \% \mathrm{Cl})$ & $0.88[0.69,1.14]$ \\
\hline $\begin{array}{l}3 \text { Adverse renal out- } \\
\text { comes }\end{array}$ & 3 & & Risk Ratio $(\mathrm{M}-\mathrm{H}$, Random, $95 \% \mathrm{Cl})$ & Subtotals only \\
\hline
\end{tabular}




\begin{tabular}{|c|c|c|c|c|}
\hline $\begin{array}{l}\text { Outcome or subgroup } \\
\text { title }\end{array}$ & No. of studies & $\begin{array}{l}\text { No. of partici- } \\
\text { pants }\end{array}$ & Statistical method & Effect size \\
\hline 3.1 ESKD & 2 & 135 & Risk Ratio (M-H, Random, 95\% Cl) & $0.49[0.05,5.20]$ \\
\hline 3.2 ESKD at 5 years & 1 & 85 & Risk Ratio (M-H, Random, 95\% Cl) & $2.80[0.30,25.81]$ \\
\hline 3.3 ESKD at 10 years & 1 & 90 & Risk Ratio (M-H, Random, 95\% Cl) & $1.91[0.37,9.92]$ \\
\hline 3.4 Renal relapse & 3 & 211 & Risk Ratio (M-H, Random, 95\% Cl) & $2.75[0.47,15.98]$ \\
\hline $\begin{array}{l}\text { 3.5 Doubling of serum } \\
\text { creatinine }\end{array}$ & 2 & 135 & Risk Ratio (M-H, Random, 95\% Cl) & $0.33[0.04,3.02]$ \\
\hline $\begin{array}{l}\text { 3.6 Doubling of serum } \\
\text { creatinine at } 5 \text { years }\end{array}$ & 1 & 85 & Risk Ratio (M-H, Random, 95\% Cl) & $0.13[0.02,1.04]$ \\
\hline $\begin{array}{l}\text { 3.7 Doubling of serum } \\
\text { creatinine at } 10 \text { years }\end{array}$ & 1 & 90 & Risk Ratio (M-H, Random, 95\% Cl) & $0.80[0.26,2.42]$ \\
\hline 4 Stable kidney function & 1 & & Risk Ratio (M-H, Random, 95\% Cl) & Subtotals only \\
\hline 4.1 At 3 years & 1 & 89 & Risk Ratio (M-H, Random, 95\% Cl) & $0.72[0.50,1.03]$ \\
\hline 4.2 At 5 years & 1 & 85 & Risk Ratio (M-H, Random, 95\% Cl) & $0.96[0.77,1.20]$ \\
\hline 5 Ovarian failure & 4 & 299 & Risk Ratio (M-H, Random, 95\% Cl) & $1.73[0.70,4.31]$ \\
\hline 6 Infection & 4 & & Risk Ratio (M-H, Random, 95\% Cl) & Subtotals only \\
\hline 6.1 Major infection & 4 & 327 & Risk Ratio (M-H, Random, 95\% Cl) & $1.44[0.83,2.49]$ \\
\hline 6.2 Herpes zoster virus & 3 & 281 & Risk Ratio (M-H, Random, 95\% Cl) & $1.58[0.41,6.05]$ \\
\hline 7 Malignancy & 2 & 206 & Risk Ratio (M-H, Random, 95\% Cl) & $1.44[0.09,23.31]$ \\
\hline 8 Leucopenia & 3 & 281 & Risk Ratio (M-H, Random, 95\% Cl) & $0.82[0.13,5.15]$ \\
\hline 9 Bone toxicity & 2 & 164 & Risk Ratio (M-H, Random, 95\% Cl) & $2.93[0.48,18.02]$ \\
\hline 10 Alopecia & 1 & 75 & Risk Ratio (M-H, Random, 95\% Cl) & $0.28[0.06,1.25]$ \\
\hline $\begin{array}{l}11 \text { Gastrointestinal (GI) } \\
\text { adverse events }\end{array}$ & 1 & & Risk Ratio (M-H, Random, 95\% Cl) & Subtotals only \\
\hline $11.1 \mathrm{GI}$ disturbance & 1 & 75 & Risk Ratio (M-H, Random, 95\% Cl) & $0.11[0.01,1.94]$ \\
\hline 12 Daily proteinuria & 3 & 242 & Mean Difference (IV, Random, 95\% CI) & $-0.10[-0.65,0.46]$ \\
\hline 13 Creatinine clearance & 1 & 117 & Mean Difference (IV, Random, 95\% CI) & $-12.60[-23.63,-1.57]$ \\
\hline 14 Serum creatinine & 3 & 247 & Mean Difference (IV, Random, 95\% CI) & $2.85[-7.61,13.31]$ \\
\hline $\begin{array}{l}15 \text { Disease activity } \\
\text { (SLEDAI) }\end{array}$ & 1 & 75 & Mean Difference (IV, Random, 95\% CI) & $-1.50[-3.04,0.04]$ \\
\hline
\end{tabular}


Analysis 15.1. Comparison 15 Low versus high dose cyclophosphamide (CPA), Outcome 1 Death.

\begin{tabular}{|c|c|c|c|c|c|}
\hline Study or subgroup & $\begin{array}{c}\text { Low dose CPA } \\
n / N\end{array}$ & $\begin{array}{l}\text { High dose CPA } \\
n / N\end{array}$ & $\begin{array}{c}\text { Risk Ratio } \\
\text { M-H, Random, 95\% CI }\end{array}$ & Weight & $\begin{array}{c}\text { Risk Ratio } \\
\text { M-H, Random, } 95 \% \mathrm{CI}\end{array}$ \\
\hline \multicolumn{6}{|l|}{ 15.1.1 At 6 months } \\
\hline Mitwalli 2011 & $3 / 73$ & $1 / 44$ & & $100 \%$ & $1.81[0.19,16.85]$ \\
\hline Subtotal $(95 \% \mathrm{Cl})$ & 73 & 44 & & $100 \%$ & $1.81[0.19,16.85]$ \\
\hline \multicolumn{6}{|c|}{ Total events: 3 (Low dose CPA), 1 (High dose CPA) } \\
\hline \multicolumn{6}{|c|}{ Heterogeneity: Not applicable } \\
\hline \multicolumn{6}{|c|}{ Test for overall effect: $\mathrm{Z}=0.52(\mathrm{P}=0.6)$} \\
\hline \multicolumn{6}{|l|}{ 15.1.2 At 12 months } \\
\hline Sabry 2009 & $0 / 20$ & $0 / 26$ & & & Not estimable \\
\hline Mehra 2018 & $2 / 38$ & $2 / 37$ & & $100 \%$ & $0.97[0.14,6.56]$ \\
\hline Subtotal $(95 \% \mathrm{Cl})$ & 58 & 63 & & $100 \%$ & $0.97[0.14,6.56]$ \\
\hline \multicolumn{6}{|c|}{ Total events: 2 (Low dose CPA), 2 (High dose CPA) } \\
\hline \multicolumn{6}{|c|}{ Test for overall effect: $\mathrm{Z}=0.03(\mathrm{P}=0.98)$} \\
\hline \multicolumn{6}{|l|}{ 15.1.3 At 5 years } \\
\hline Houssiau 2002 & $0 / 44$ & $3 / 41$ & & $100 \%$ & $0.13[0.01,2.51]$ \\
\hline Subtotal $(95 \% \mathrm{Cl})$ & 44 & 41 & & $100 \%$ & $0.13[0.01,2.51]$ \\
\hline \multicolumn{6}{|c|}{ Total events: 0 (Low dose CPA), 3 (High dose CPA) } \\
\hline \multicolumn{6}{|c|}{ Heterogeneity: Not applicable } \\
\hline \multicolumn{6}{|c|}{ Test for overall effect: $\mathrm{Z}=1.35(\mathrm{P}=0.18)$} \\
\hline \multicolumn{6}{|l|}{ 15.1.4 At 10 years } \\
\hline Houssiau 2002 & $2 / 46$ & $5 / 44$ & & $100 \%$ & $0.38[0.08,1.87]$ \\
\hline Subtotal $(95 \% \mathrm{Cl})$ & 46 & 44 & & $100 \%$ & $0.38[0.08,1.87]$ \\
\hline \multicolumn{6}{|c|}{ Total events: 2 (Low dose CPA), 5 (High dose CPA) } \\
\hline Test for overall effect & & & & & \\
\hline
\end{tabular}

\section{Analysis 15.2. Comparison 15 Low versus high dose cyclophosphamide (CPA), Outcome 2 Remission.}

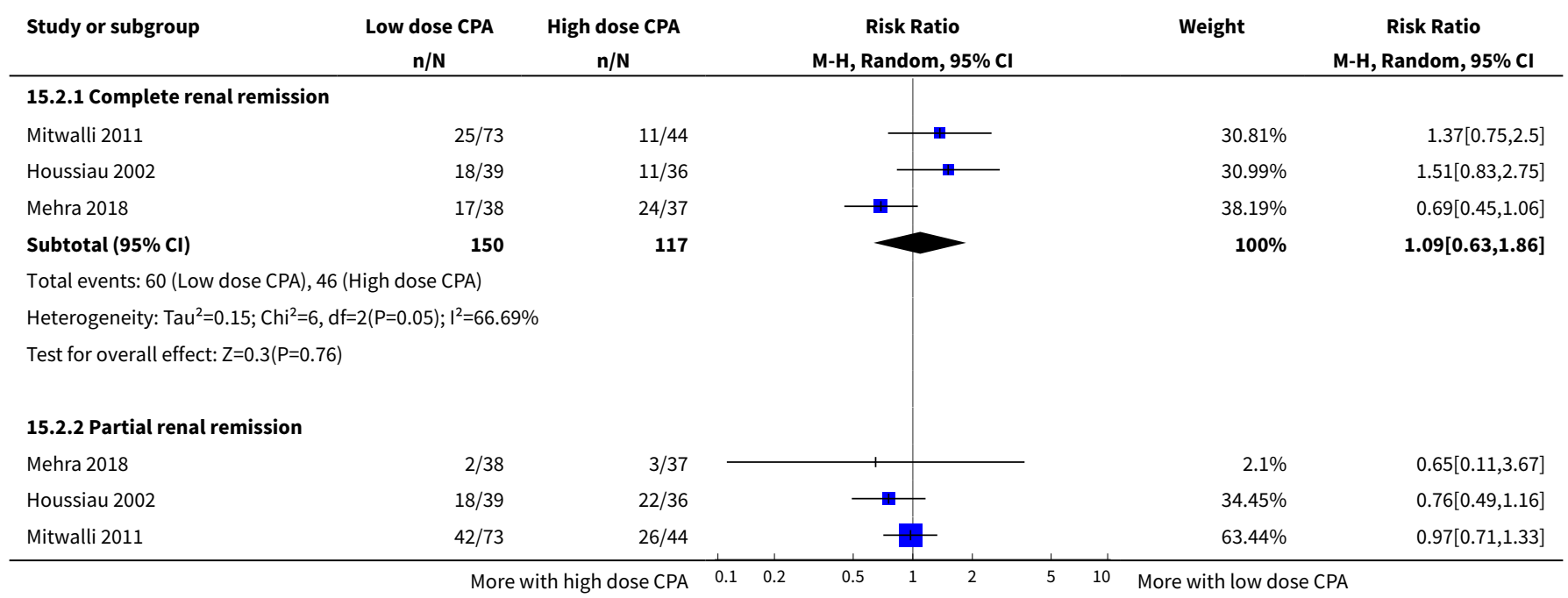




\begin{tabular}{|c|c|c|c|c|c|c|}
\hline Study or subgroup & $\begin{array}{c}\text { Low dose CPA } \\
n / N\end{array}$ & $\begin{array}{l}\text { High dose CPA } \\
n / N\end{array}$ & $\begin{array}{c}\text { Risk Ratio } \\
\text { M-H, Random, 95\% Cl }\end{array}$ & & Weight & $\begin{array}{c}\text { Risk Ratio } \\
\text { M-H, Random, } 95 \% \mathrm{Cl}\end{array}$ \\
\hline Subtotal $(95 \% \mathrm{Cl})$ & 150 & 117 & & & $100 \%$ & $0.88[0.69,1.14]$ \\
\hline \multicolumn{7}{|c|}{ Total events: 62 (Low dose CPA), 51 (High dose CPA) } \\
\hline \multicolumn{7}{|c|}{ Heterogeneity: $\mathrm{Tau}^{2}=0 ; \mathrm{Chi}^{2}=1.02, \mathrm{df}=2(\mathrm{P}=0.6) ; \mathrm{I}^{2}=0 \%$} \\
\hline \multicolumn{7}{|c|}{ Test for overall effect: $\mathrm{Z}=0.96(\mathrm{P}=0.34)$} \\
\hline \multicolumn{7}{|c|}{ Test for subgroup differences: $\mathrm{Chi}^{2}=0.46, \mathrm{df}=1(\mathrm{P}=0.5), \mathrm{I}^{2}=0 \%$} \\
\hline & & $\mathrm{h}$ high dose CPA & $0.1 \quad 0.2$ & 10 & More with low dos & CPA \\
\hline
\end{tabular}

Analysis 15.3. Comparison 15 Low versus high dose cyclophosphamide (CPA), Outcome 3 Adverse renal outcomes.

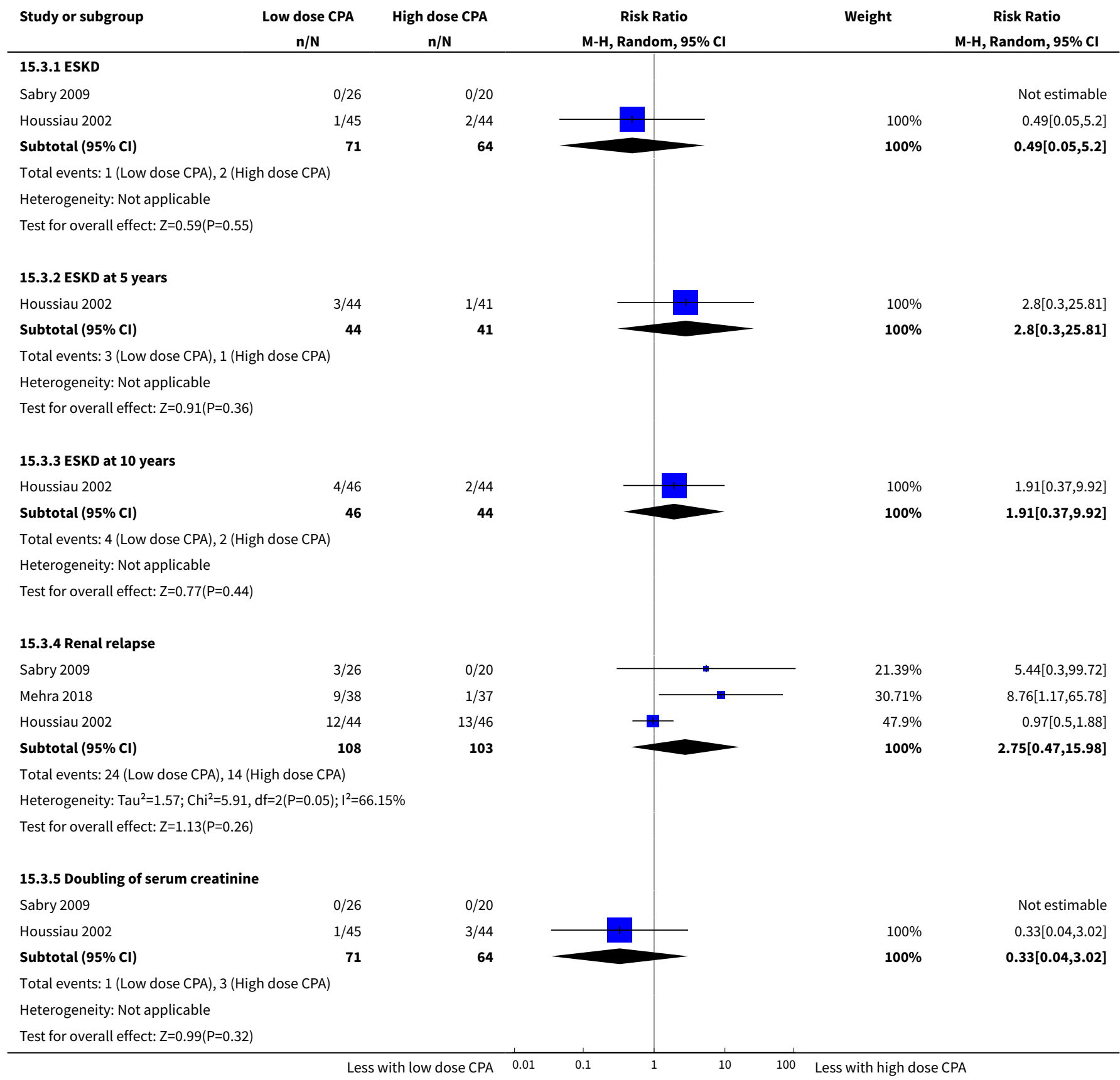




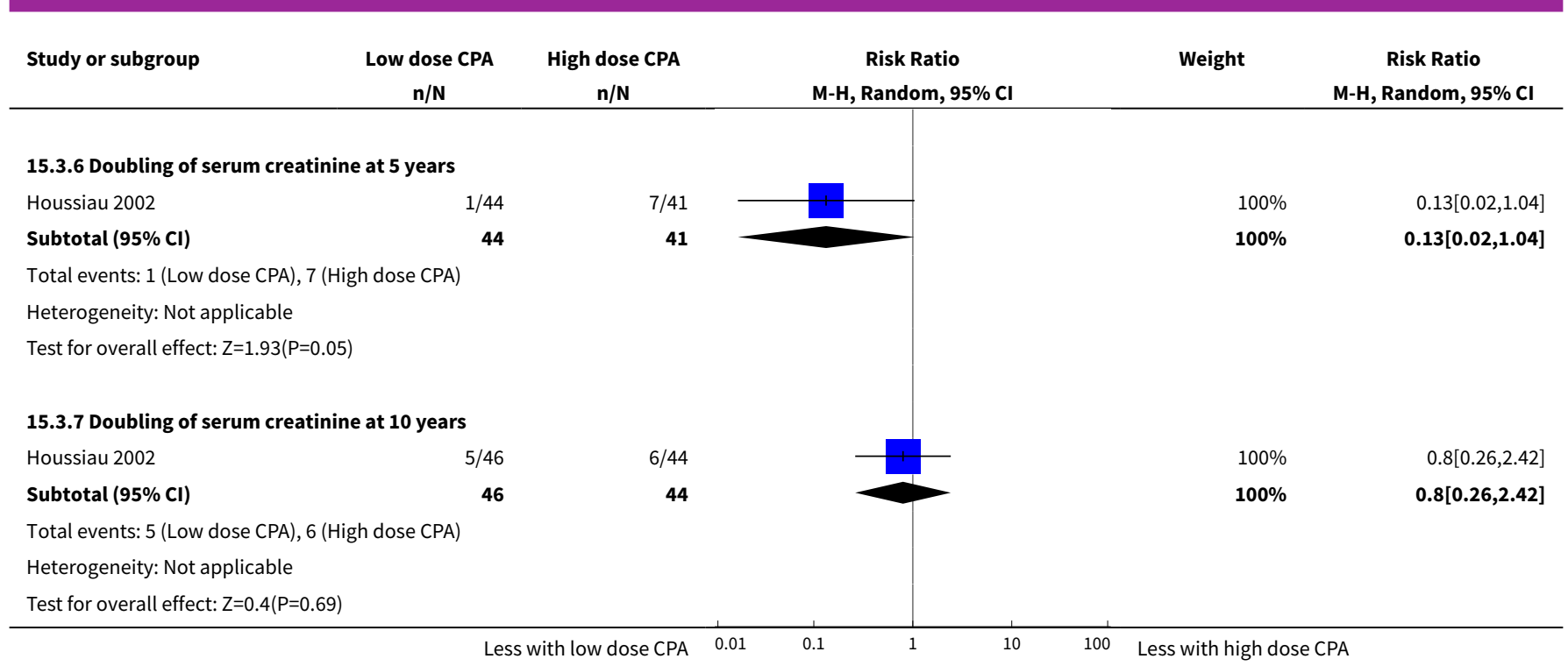

\section{Analysis 15.4. Comparison 15 Low versus high dose cyclophosphamide (CPA), Outcome 4 Stable kidney function.}

\begin{tabular}{|c|c|c|c|c|c|}
\hline Study or subgroup & $\begin{array}{c}\text { Low dose CPA } \\
n / N\end{array}$ & $\begin{array}{l}\text { High dose CPA } \\
n / N\end{array}$ & $\begin{array}{c}\text { Risk Ratio } \\
\text { M-H, Random, 95\% Cl }\end{array}$ & Weight & $\begin{array}{c}\text { Risk Ratio } \\
\text { M-H, Random, } 95 \% \mathrm{CI}\end{array}$ \\
\hline \multicolumn{6}{|l|}{ 15.4.1 At 3 years } \\
\hline Houssiau 2002 & $22 / 45$ & $30 / 44$ & & $100 \%$ & $0.72[0.5,1.03]$ \\
\hline Subtotal $(95 \% \mathrm{CI})$ & 45 & 44 & & $100 \%$ & $0.72[0.5,1.03]$ \\
\hline \multicolumn{6}{|c|}{ Total events: 22 (Low dose CPA), 30 (High dose CPA) } \\
\hline \multicolumn{6}{|c|}{ Heterogeneity: Not applicable } \\
\hline \multicolumn{6}{|l|}{ 15.4.2 At 5 years } \\
\hline Houssiau 2002 & $34 / 44$ & $33 / 41$ & - & $100 \%$ & $0.96[0.77,1.2]$ \\
\hline Subtotal $(95 \% \mathrm{Cl})$ & 44 & 41 & & $100 \%$ & $0.96[0.77,1.2]$ \\
\hline \multicolumn{6}{|c|}{ Total events: 34 (Low dose CPA), 33 (High dose CPA) } \\
\hline \multicolumn{6}{|c|}{ Heterogeneity: Not applicable } \\
\hline Test for subgroup dif & $.83, \mathrm{df}=1(\mathrm{P}=0.18)$, & $45.49 \%$ & & & \\
\hline
\end{tabular}

Analysis 15.5. Comparison 15 Low versus high dose cyclophosphamide (CPA), Outcome 5 Ovarian failure.

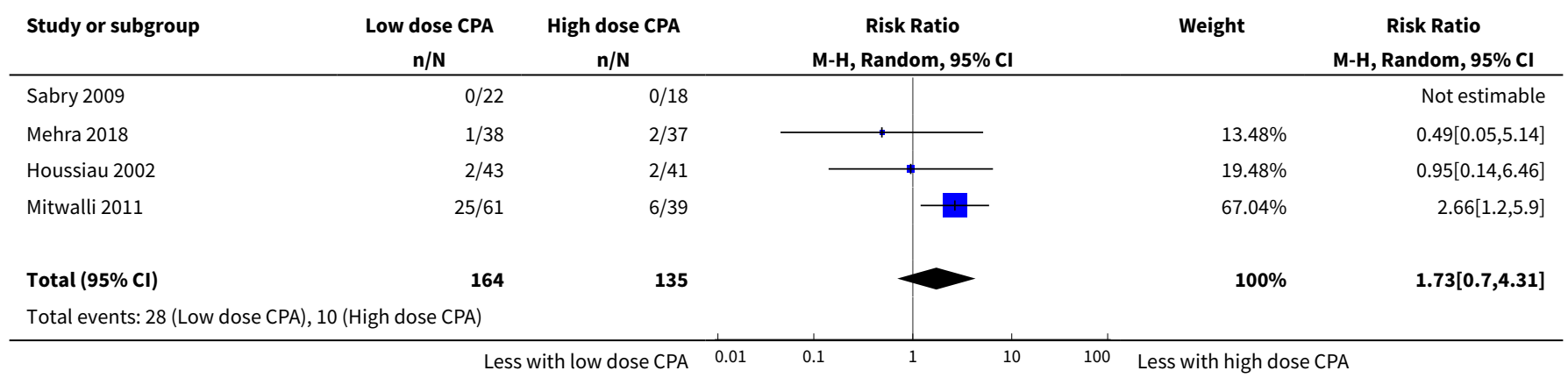




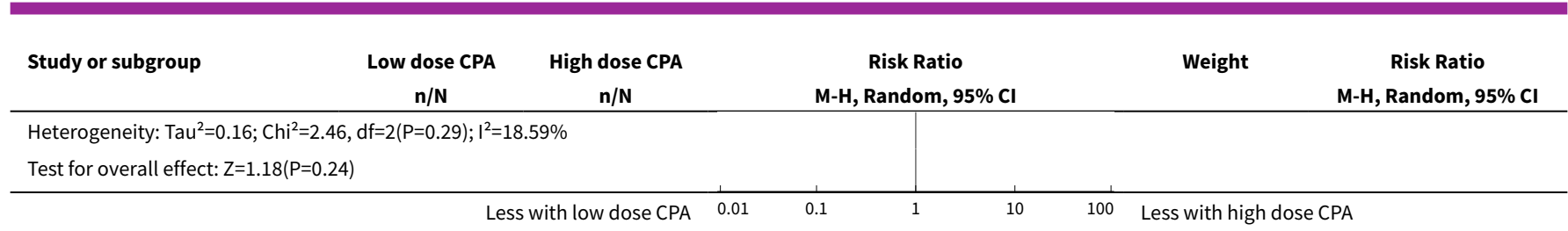

\section{Analysis 15.6. Comparison 15 Low versus high dose cyclophosphamide (CPA), Outcome 6 Infection.}

\begin{tabular}{|c|c|c|c|c|c|}
\hline Study or subgroup & $\begin{array}{c}\text { Low dose CPA } \\
n / N\end{array}$ & $\begin{array}{l}\text { High dose CPA } \\
n / N \\
\end{array}$ & $\begin{array}{c}\text { Risk Ratio } \\
\text { M-H, Random, } 95 \% \mathrm{Cl} \\
\end{array}$ & Weight & $\begin{array}{c}\text { Risk Ratio } \\
\text { M-H, Random, } 95 \% \mathrm{Cl} \\
\end{array}$ \\
\hline \multicolumn{6}{|c|}{ 15.6.1 Major infection } \\
\hline Sabry 2009 & $4 / 26$ & $5 / 20$ & $\longrightarrow-1$ & $17.95 \%$ & $0.62[0.19,2]$ \\
\hline Houssiau 2002 & $10 / 45$ & $5 / 44$ & 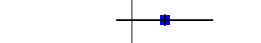 & $23.7 \%$ & $1.96[0.73,5.26]$ \\
\hline Mehra 2018 & $8 / 38$ & $7 / 37$ & & $26.98 \%$ & $1.11[0.45,2.76]$ \\
\hline Mitwalli 2011 & $23 / 73$ & $6 / 44$ & . & $31.36 \%$ & $2.31[1.02,5.23]$ \\
\hline Subtotal $(95 \% \mathrm{Cl})$ & 182 & 145 & & $100 \%$ & $1.44[0.83,2.49]$ \\
\hline \multicolumn{6}{|c|}{ Total events: 45 (Low dose CPA), 23 (High dose CPA) } \\
\hline \multicolumn{6}{|c|}{ Heterogeneity: $\mathrm{Tau}^{2}=0.08 ; \mathrm{Chi}^{2}=3.98, \mathrm{df}=3(\mathrm{P}=0.26) ; \mathrm{I}^{2}=24.56 \%$} \\
\hline \multicolumn{6}{|c|}{ Test for overall effect: $Z=1.29(P=0.2)$} \\
\hline \multicolumn{6}{|c|}{ 15.6.2 Herpes zoster virus } \\
\hline Mehra 2018 & $1 / 38$ & $0 / 37$ & & $17.36 \%$ & $2.92[0.12,69.54]$ \\
\hline Mitwalli 2011 & $0 / 44$ & $3 / 73$ & $\rightarrow$ & $20.06 \%$ & $0.23[0.01,4.44]$ \\
\hline Houssiau 2002 & $5 / 45$ & $2 / 44$ & 7 & $62.58 \%$ & $2.44[0.5,11.94]$ \\
\hline Subtotal $(95 \% \mathrm{Cl})$ & 127 & 154 & & $100 \%$ & $1.58[0.41,6.05]$ \\
\hline \multicolumn{6}{|c|}{ Total events: 6 (Low dose CPA), 5 (High dose CPA) } \\
\hline \multicolumn{6}{|c|}{ Heterogeneity: $\mathrm{Tau}^{2}=0.1 ; \mathrm{Chi}^{2}=2.12, \mathrm{df}=2(\mathrm{P}=0.35) ; 1^{2}=5.59 \%$} \\
\hline \multicolumn{6}{|c|}{ Test for overall effect: $\mathrm{Z}=0.66(\mathrm{P}=0.51)$} \\
\hline
\end{tabular}

\section{Analysis 15.7. Comparison 15 Low versus high dose cyclophosphamide (CPA), Outcome 7 Malignancy.}

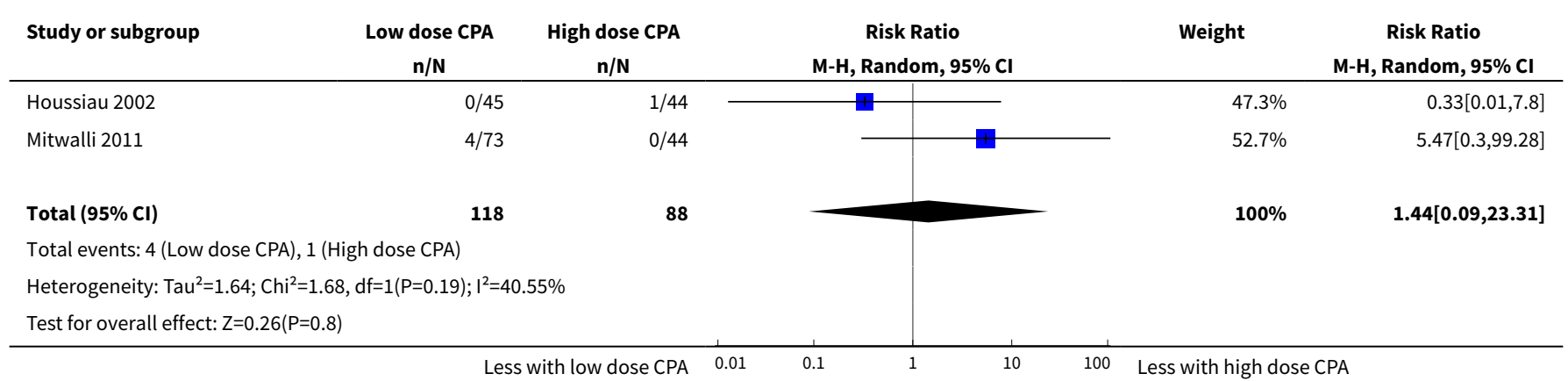


Analysis 15.8. Comparison 15 Low versus high dose cyclophosphamide (CPA), Outcome 8 Leucopenia.

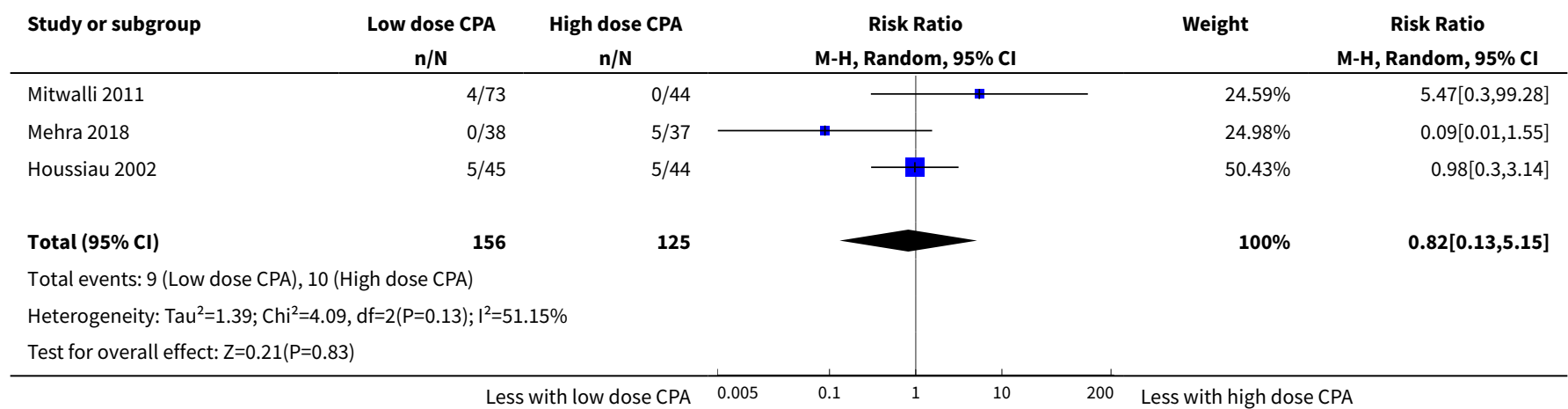

Analysis 15.9. Comparison 15 Low versus high dose cyclophosphamide (CPA), Outcome 9 Bone toxicity.

\begin{tabular}{|c|c|c|c|c|c|}
\hline Study or subgroup & $\begin{array}{c}\text { Low dose CPA } \\
n / N\end{array}$ & $\begin{array}{c}\text { High dose CPA } \\
n / N\end{array}$ & $\begin{array}{c}\text { Risk Ratio } \\
\text { M-H, Random, 95\% Cl }\end{array}$ & Weight & $\begin{array}{c}\text { Risk Ratio } \\
\text { M-H, Random, } 95 \% \text { CI }\end{array}$ \\
\hline Houssiau 2002 & $1 / 45$ & $0 / 44$ & 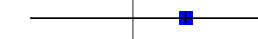 & $32.8 \%$ & $2.93[0.12,70.16]$ \\
\hline Mehra 2018 & $3 / 38$ & $1 / 37$ & 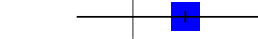 & $67.2 \%$ & $2.92[0.32,26.83]$ \\
\hline Total $(95 \% \mathrm{CI})$ & 83 & 81 & & $100 \%$ & $2.93[0.48,18.02]$ \\
\hline \multicolumn{6}{|c|}{ Total events: 4 (Low dose CPA), 1 (High dose CPA) } \\
\hline Test for overall effect & & & & & \\
\hline
\end{tabular}

Analysis 15.10. Comparison 15 Low versus high dose cyclophosphamide (CPA), Outcome 10 Alopecia.

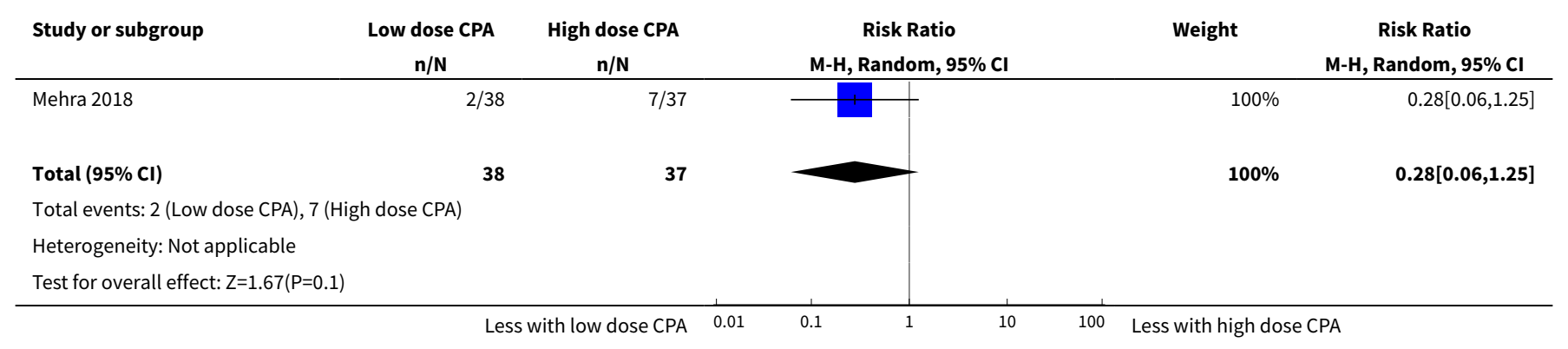

Analysis 15.11. Comparison 15 Low versus high dose cyclophosphamide (CPA), Outcome 11 Gastrointestinal (GI) adverse events.

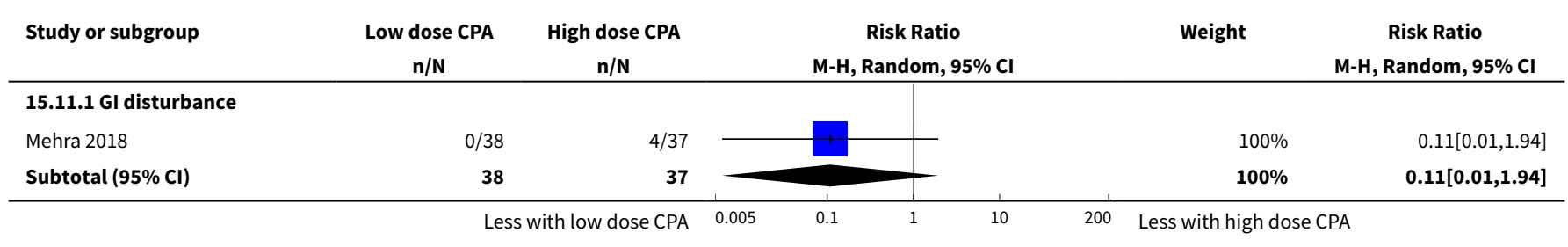




\begin{tabular}{|c|c|c|c|c|c|c|}
\hline Study or subgroup & $\begin{array}{c}\text { Low dose CPA } \\
n / N \\
\end{array}$ & $\begin{array}{c}\text { High dose CPA } \\
n / N \\
\end{array}$ & \multicolumn{2}{|r|}{$\begin{array}{c}\text { Risk Ratio } \\
\text { M-H, Random, 95\% CI }\end{array}$} & Weight & \multirow[t]{2}{*}{$\begin{array}{c}\text { Risk Ratio } \\
\text { M-H, Random, } 95 \% \mathrm{Cl} \\
\end{array}$} \\
\hline \multicolumn{6}{|c|}{ Total events: 0 (Low dose CPA), 4 (High dose CPA) } & \\
\hline \multicolumn{7}{|c|}{ Heterogeneity: Not applicable } \\
\hline Test for overall effec & & & & & & \\
\hline
\end{tabular}

\section{Analysis 15.12. Comparison 15 Low versus high dose cyclophosphamide (CPA), Outcome 12 Daily proteinuria.}

\begin{tabular}{|c|c|c|c|c|c|c|c|}
\hline \multirow[t]{2}{*}{ Study or subgroup } & \multicolumn{2}{|c|}{ Low dose CPA } & \multicolumn{2}{|c|}{ High dose CPA } & \multirow{2}{*}{$\begin{array}{l}\text { Mean Difference } \\
\text { Random, } 95 \% \mathrm{Cl}\end{array}$} & \multirow[t]{2}{*}{ Weight } & \multirow{2}{*}{$\begin{array}{l}\text { Mean Difference } \\
\text { Random, } 95 \% \mathrm{CI}\end{array}$} \\
\hline & $\mathbf{N}$ & $\operatorname{Mean}(S D)$ & $\mathbf{N}$ & $\operatorname{Mean}(\mathrm{SD})$ & & & \\
\hline Sabry 2009 & 26 & $2.9(1.5)$ & 20 & $2.1(1.6)$ & & $22.13 \%$ & $0.8[-0.11,1.71]$ \\
\hline Houssiau 2002 & 39 & $0.7(1)$ & 36 & $1.1(1.3)$ & - & $36.05 \%$ & $-0.42[-0.95,0.11]$ \\
\hline Mitwalli 2011 & 44 & $0.9(1.1)$ & 77 & $1.2(1.1)$ & 1 & $41.81 \%$ & $-0.29[-0.7,0.12]$ \\
\hline 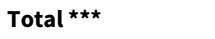 & 109 & & 133 & & & $100 \%$ & $-0.1[-0.65,0.46]$ \\
\hline \multicolumn{8}{|c|}{ Test for overall effect: $Z=0.34(P=0.74)$} \\
\hline
\end{tabular}

Analysis 15.13. Comparison 15 Low versus high dose cyclophosphamide (CPA), Outcome 13 Creatinine clearance.

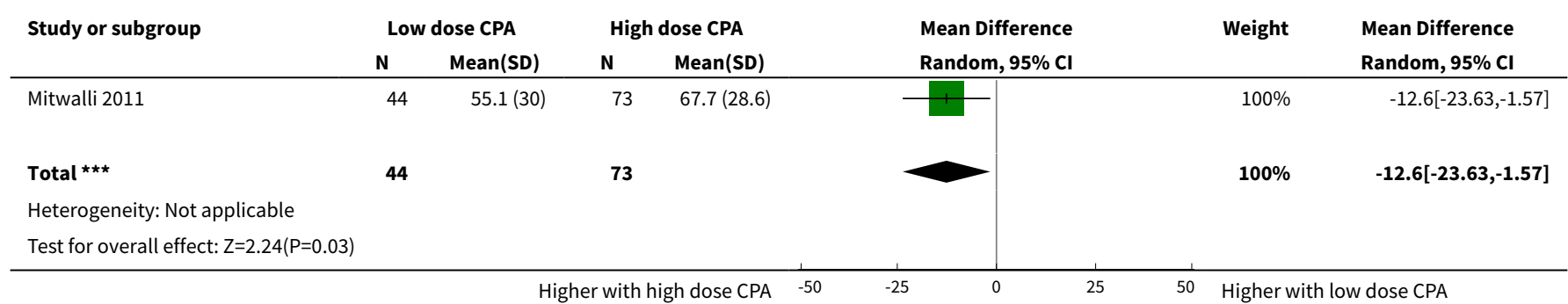

Analysis 15.14. Comparison 15 Low versus high dose cyclophosphamide (CPA), Outcome 14 Serum creatinine.

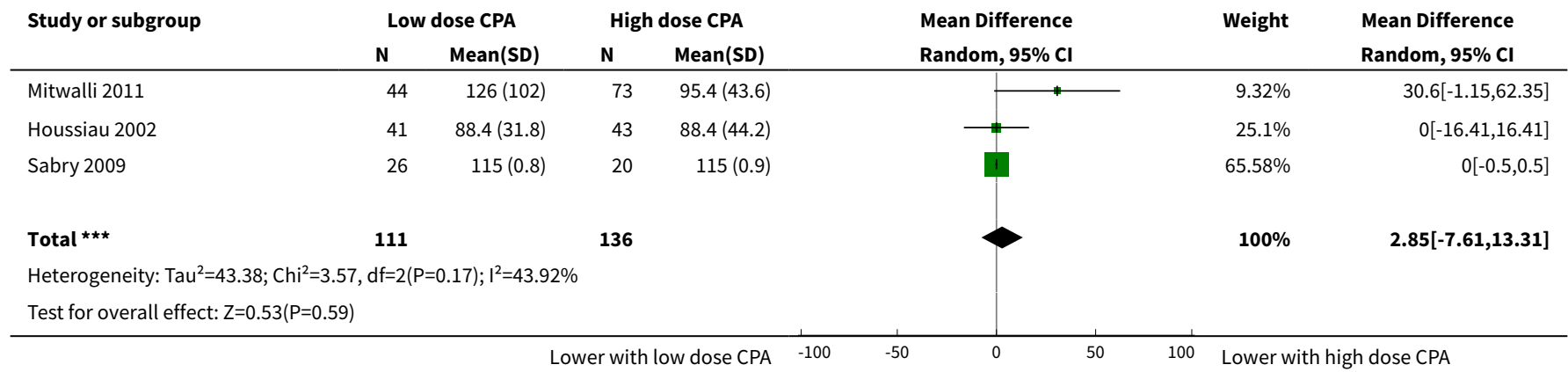




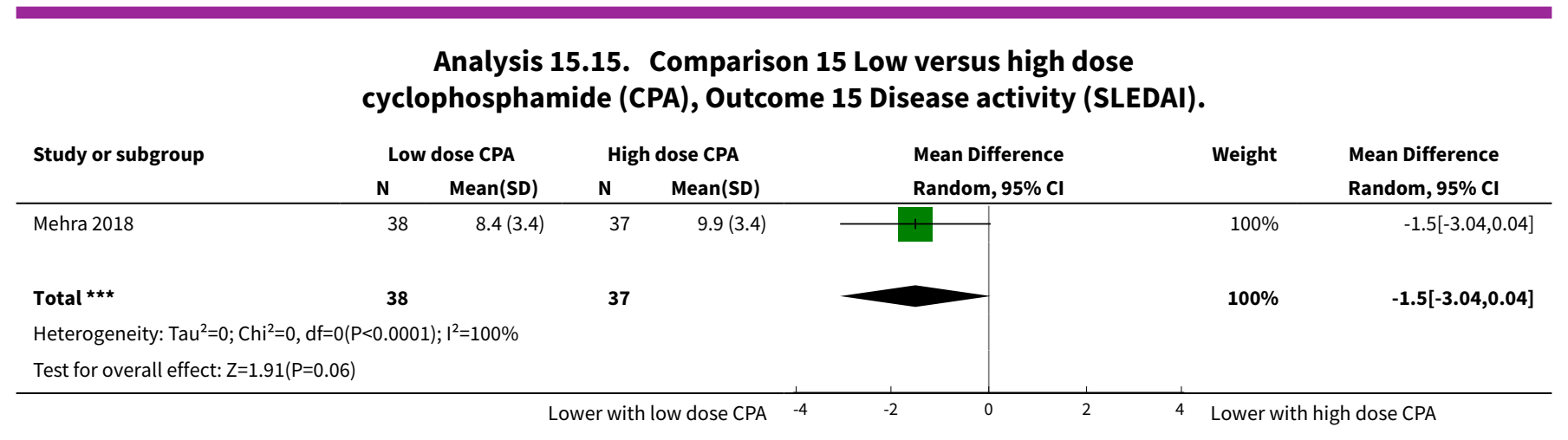

\section{Comparison 16. Standard versus reduced dose oral corticosteroids}

\begin{tabular}{|c|c|c|c|c|}
\hline $\begin{array}{l}\text { Outcome or subgroup } \\
\text { title }\end{array}$ & No. of studies & $\begin{array}{l}\text { No. of partici- } \\
\text { pants }\end{array}$ & Statistical method & Effect size \\
\hline 1 Death & 1 & 81 & Risk Ratio (M-H, Random, 95\% Cl) & $4.65[0.23,93.95]$ \\
\hline 2 Remission & 1 & & Risk Ratio (M-H, Random, 95\% Cl) & Subtotals only \\
\hline $\begin{array}{l}2.1 \text { Complete renal re- } \\
\text { mission }\end{array}$ & 1 & 81 & Risk Ratio (M-H, Random, 95\% Cl) & $0.93[0.39,2.23]$ \\
\hline $\begin{array}{l}\text { 2.2 Partial renal remis- } \\
\text { sion }\end{array}$ & 1 & 81 & Risk Ratio (M-H, Random, 95\% Cl) & $1.33[0.78,2.24]$ \\
\hline 3 Relapse & 1 & 50 & Risk Ratio (M-H, Random, 95\% Cl) & $2.38[0.10,55.72]$ \\
\hline 4 Infection & 1 & & Risk Ratio (M-H, Random, 95\% Cl) & Subtotals only \\
\hline 4.1 Major infection & 1 & 81 & Risk Ratio (M-H, Random, 95\% Cl) & $4.64[0.57,38.00]$ \\
\hline 4.2 Herpes zoster virus & 1 & 81 & Risk Ratio (M-H, Random, 95\% Cl) & $13.95[0.82,236.48]$ \\
\hline $\begin{array}{l}5 \text { Gastrointestinal (GI) } \\
\text { adverse events }\end{array}$ & 1 & & Risk Ratio (M-H, Random, 95\% Cl) & Subtotals only \\
\hline 5.1 Diarrhoea & 1 & 81 & Risk Ratio (M-H, Random, 95\% Cl) & $1.16[0.51,2.64]$ \\
\hline 5.2 Vomiting & 1 & 81 & Risk Ratio (M-H, Random, 95\% Cl) & $0.93[0.25,3.46]$ \\
\hline 5.3 Nausea & 1 & 81 & Risk Ratio (M-H, Random, 95\% Cl) & $2.79[0.30,25.67]$ \\
\hline 6 Creatinine clearance & 1 & 74 & Mean Difference (IV, Random, 95\% CI) & $-5.80[-21.08,9.48]$ \\
\hline 7 Serum creatinine & 1 & 81 & Mean Difference (IV, Random, 95\% CI) & $-2.40[-15.98,11.18]$ \\
\hline
\end{tabular}


Analysis 16.1. Comparison 16 Standard versus reduced dose oral corticosteroids, Outcome 1 Death.

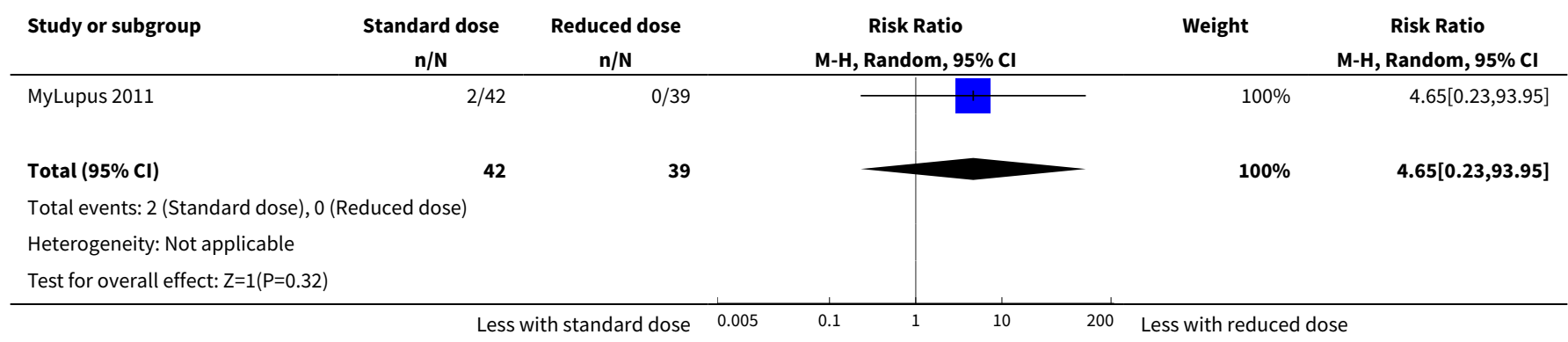

Analysis 16.2. Comparison 16 Standard versus reduced dose oral corticosteroids, Outcome 2 Remission.

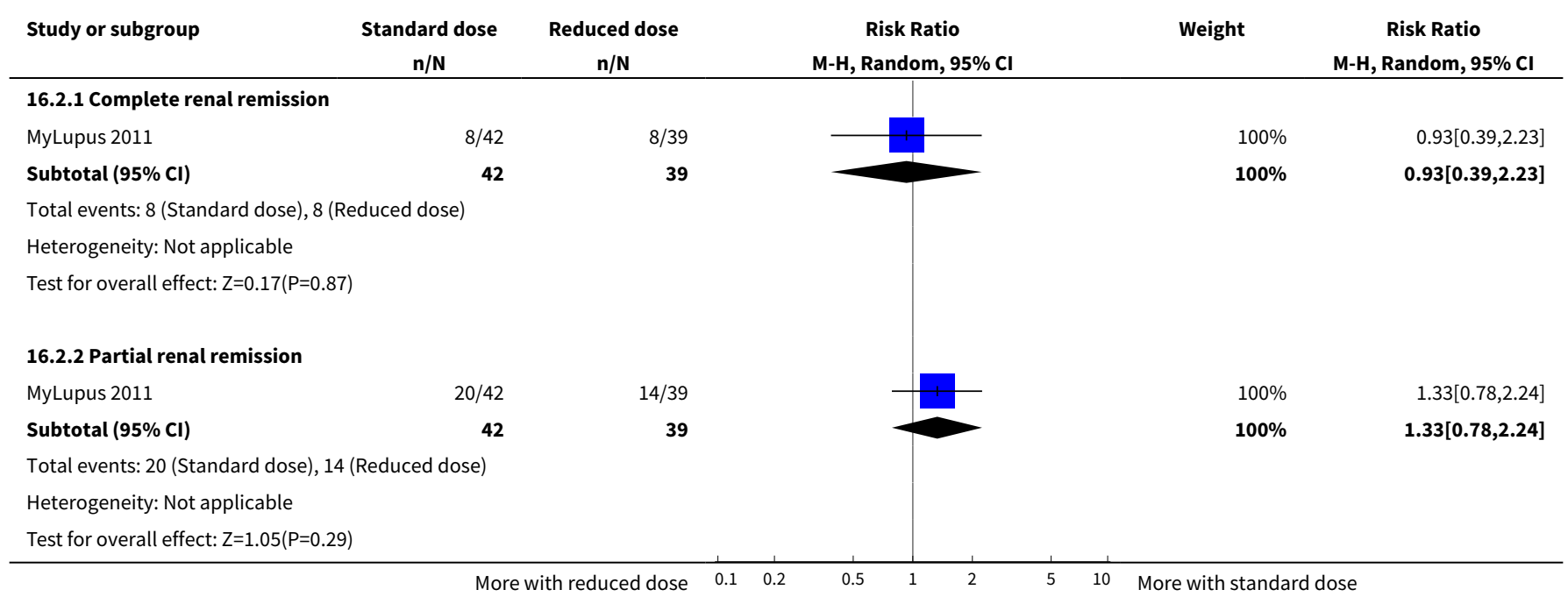

Analysis 16.3. Comparison 16 Standard versus reduced dose oral corticosteroids, Outcome 3 Relapse.

\begin{tabular}{|c|c|c|c|c|c|}
\hline Study or subgroup & $\begin{array}{c}\text { Standard dose } \\
\mathrm{n} / \mathrm{N}\end{array}$ & $\begin{array}{c}\text { Reduced dose } \\
n / N\end{array}$ & $\begin{array}{c}\text { Risk Ratio } \\
\text { M-H, Random, } 95 \% \mathrm{CI}\end{array}$ & Weight & $\begin{array}{c}\text { Risk Ratio } \\
\text { M-H, Random, } 95 \% \mathrm{Cl}\end{array}$ \\
\hline MyLupus 2011 & $1 / 28$ & $0 / 22$ & & $100 \%$ & $2.38[0.1,55.72]$ \\
\hline Total $(95 \% \mathrm{Cl})$ & 28 & 22 & & $100 \%$ & $2.38[0.1,55.72]$ \\
\hline \multicolumn{6}{|c|}{ Total events: 1 (Standard dose), 0 (Reduced dose) } \\
\hline \multicolumn{6}{|c|}{ Heterogeneity: Not applicable } \\
\hline Test for overall effec & & & & & \\
\hline
\end{tabular}


Analysis 16.4. Comparison 16 Standard versus reduced dose oral corticosteroids, Outcome 4 Infection.

\begin{tabular}{|c|c|c|c|c|c|}
\hline Study or subgroup & $\begin{array}{c}\text { Standard dose } \\
n / \mathbf{N}\end{array}$ & $\begin{array}{c}\text { Reduced dose } \\
n / N\end{array}$ & $\begin{array}{c}\text { Risk Ratio } \\
\text { M-H, Random, } 95 \% \mathrm{CI}\end{array}$ & Weight & $\begin{array}{c}\text { Risk Ratio } \\
\text { M-H, Random, } 95 \% \mathrm{CI}\end{array}$ \\
\hline \multicolumn{6}{|c|}{ 16.4.1 Major infection } \\
\hline MyLupus 2011 & $5 / 42$ & $1 / 39$ & & $100 \%$ & $4.64[0.57,38]$ \\
\hline Subtotal $(95 \% \mathrm{Cl})$ & 42 & 39 & & $100 \%$ & $4.64[0.57,38]$ \\
\hline \multicolumn{6}{|c|}{ Total events: 5 (Standard dose), 1 (Reduced dose) } \\
\hline \multicolumn{6}{|c|}{ Heterogeneity: Not applicable } \\
\hline \multicolumn{6}{|c|}{ 16.4.2 Herpes zoster virus } \\
\hline MyLupus 2011 & $7 / 42$ & $0 / 39$ & & $100 \%$ & $13.95[0.82,236.48]$ \\
\hline Subtotal $(95 \% \mathrm{Cl})$ & 42 & 39 & & $100 \%$ & $13.95[0.82,236.48]$ \\
\hline \multicolumn{6}{|c|}{ Total events: 7 (Standard dose), 0 (Reduced dose) } \\
\hline \multicolumn{6}{|c|}{ Heterogeneity: Not applicable } \\
\hline
\end{tabular}

Analysis 16.5. Comparison 16 Standard versus reduced dose oral corticosteroids, Outcome 5 Gastrointestinal (GI) adverse events.

\begin{tabular}{|c|c|c|c|c|c|}
\hline Study or subgroup & $\begin{array}{c}\text { Standard dose } \\
\mathrm{n} / \mathrm{N} \\
\end{array}$ & $\begin{array}{c}\text { Reduced dose } \\
n / N \\
\end{array}$ & $\begin{array}{c}\text { Risk Ratio } \\
\text { M-H, Random, 95\% Cl }\end{array}$ & Weight & $\begin{array}{c}\text { Risk Ratio } \\
\text { M-H, Random, } 95 \% \mathrm{CI}\end{array}$ \\
\hline \multicolumn{6}{|l|}{ 16.5.1 Diarrhoea } \\
\hline MyLupus 2011 & $10 / 42$ & $8 / 39$ & & $100 \%$ & $1.16[0.51,2.64]$ \\
\hline Subtotal $(95 \% \mathrm{Cl})$ & 42 & 39 & & $100 \%$ & $1.16[0.51,2.64]$ \\
\hline \multicolumn{6}{|c|}{ Total events: 10 (Standard dose), 8 (Reduced dose) } \\
\hline \multicolumn{6}{|c|}{ Heterogeneity: Not applicable } \\
\hline \multicolumn{6}{|l|}{ 16.5.2 Vomiting } \\
\hline MyLupus 2011 & $4 / 42$ & $4 / 39$ & & $100 \%$ & $0.93[0.25,3.46]$ \\
\hline Subtotal $(95 \% \mathrm{Cl})$ & 42 & 39 & & $100 \%$ & $0.93[0.25,3.46]$ \\
\hline \multicolumn{6}{|c|}{ Total events: 4 (Standard dose), 4 (Reduced dose) } \\
\hline \multicolumn{6}{|c|}{ Heterogeneity: Not applicable } \\
\hline \multicolumn{6}{|l|}{ 16.5.3 Nausea } \\
\hline MyLupus 2011 & $3 / 42$ & $1 / 39$ & & $100 \%$ & $2.79[0.3,25.67]$ \\
\hline Subtotal $(95 \% \mathrm{Cl})$ & 42 & 39 & & $100 \%$ & $2.79[0.3,25.67]$ \\
\hline \multicolumn{6}{|c|}{ Total events: 3 (Standard dose), 1 (Reduced dose) } \\
\hline \multicolumn{6}{|c|}{ Heterogeneity: Not applicable } \\
\hline \multicolumn{6}{|c|}{ Test for overall effect: $Z=0.9(P=0.37)$} \\
\hline & Les & standard dose 0.01 & 0.1 & reduced & \\
\hline
\end{tabular}


Analysis 16.6. Comparison 16 Standard versus reduced dose oral corticosteroids, Outcome 6 Creatinine clearance.

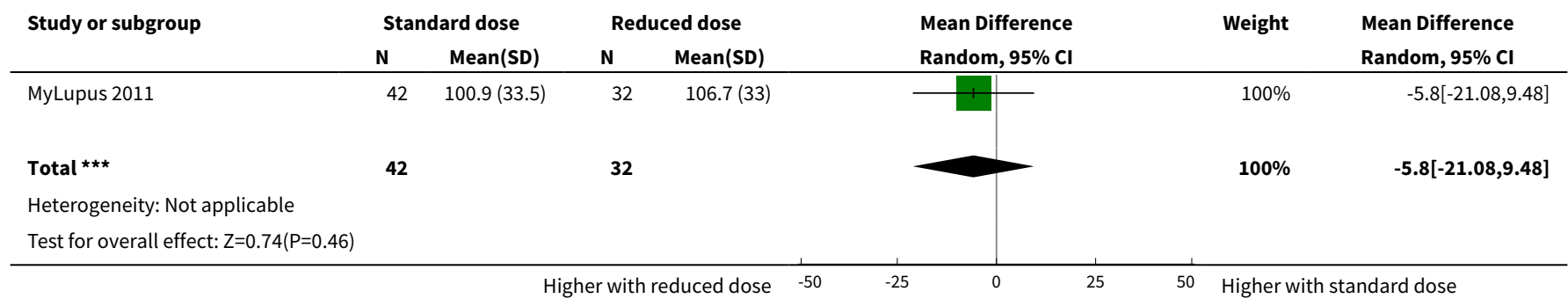

\section{Analysis 16.7. Comparison 16 Standard versus reduced dose oral corticosteroids, Outcome 7 Serum creatinine.}

\begin{tabular}{|c|c|c|c|c|c|c|c|}
\hline \multirow{3}{*}{$\begin{array}{l}\text { Study or subgroup } \\
\text { MyLupus } 2011\end{array}$} & \multicolumn{2}{|c|}{ Standard dose } & \multicolumn{2}{|c|}{ Reduced dose } & \multirow{2}{*}{$\begin{array}{l}\text { Mean Difference } \\
\text { Random, } 95 \% \mathrm{Cl}\end{array}$} & \multirow[t]{2}{*}{ Weight } & \multirow{2}{*}{$\begin{array}{l}\text { Mean Difference } \\
\text { Random, } 95 \% \mathrm{Cl}\end{array}$} \\
\hline & $\mathbf{N}$ & $\operatorname{Mean}(S D)$ & $\mathbf{N}$ & $\operatorname{Mean}(S D)$ & & & \\
\hline & 42 & $73.3(35)$ & 39 & $75.7(27.1)$ & & $100 \%$ & $-2.4[-15.98,11.18]$ \\
\hline 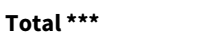 & 42 & & 39 & & & $100 \%$ & $-2.4[-15.98,11.18]$ \\
\hline \multicolumn{8}{|c|}{ Heterogeneity: Not applicable } \\
\hline Test for overall effect & & & & & & & \\
\hline
\end{tabular}

\section{Comparison 17. IV versus oral corticosteroids}

\begin{tabular}{lllll}
\hline $\begin{array}{l}\text { Outcome or subgroup } \\
\text { title }\end{array}$ & No. of studies & $\begin{array}{l}\text { No. of partici- } \\
\text { pants }\end{array}$ & Statistical method & Effect size \\
\hline 1 Death & 1 & 22 & Risk Ratio (M-H, Random, 95\% Cl) & $0.0[0.0,0.0]$ \\
\hline $\begin{array}{l}\text { 2 Adverse renal out- } \\
\text { comes }\end{array}$ & 1 & Risk Ratio (M-H, Random, 95\% Cl) & Subtotals only \\
\hline 2.1 Renal relapse & 1 & 22 & Risk Ratio $(\mathrm{M}-\mathrm{H}$, Random, 95\% Cl) & $0.95[0.44,2.04]$ \\
\hline
\end{tabular}

Analysis 17.1. Comparison 17 IV versus oral corticosteroids, Outcome 1 Death.

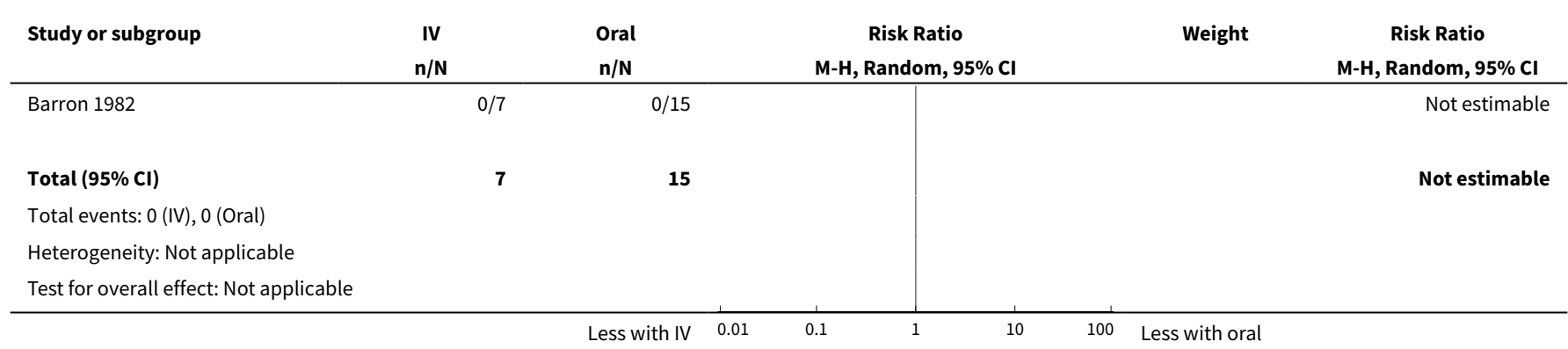


Analysis 17.2. Comparison 17 IV versus oral corticosteroids, Outcome 2 Adverse renal outcomes.

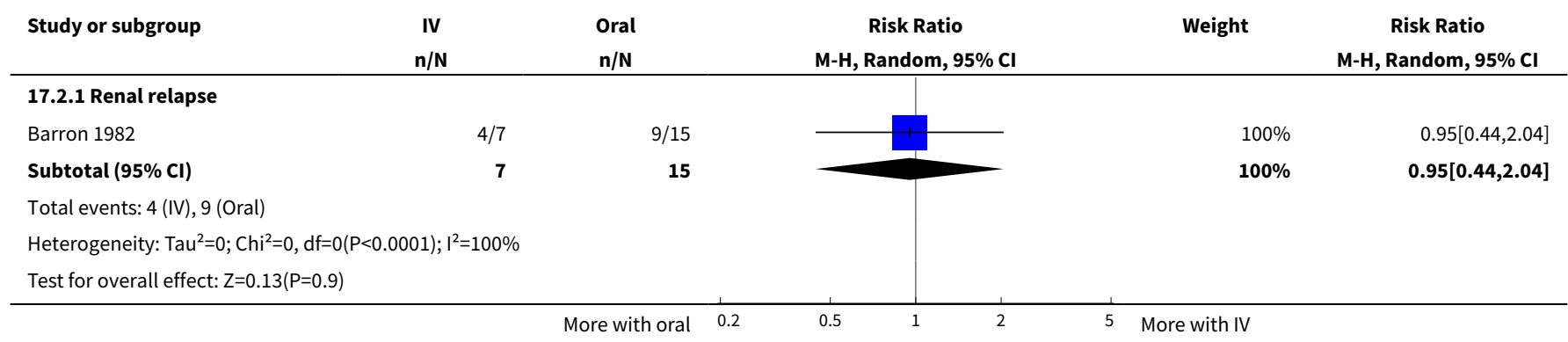

\section{Comparison 18. Cyclophosphamide (CPA) + corticosteroids versus corticosteroids}

\begin{tabular}{|c|c|c|c|c|}
\hline $\begin{array}{l}\text { Outcome or subgroup } \\
\text { title }\end{array}$ & No. of studies & $\begin{array}{l}\text { No. of partici- } \\
\text { pants }\end{array}$ & Statistical method & Effect size \\
\hline 1 Death & 5 & 226 & Risk Ratio (M-H, Random, 95\% Cl) & $0.98[0.53,1.82]$ \\
\hline $\begin{array}{l}2 \text { Complete remission of } \\
\text { proteinuria }\end{array}$ & 1 & 13 & Risk Ratio (M-H, Random, 95\% Cl) & $2.63[0.13,54.64]$ \\
\hline $\begin{array}{l}3 \text { Adverse renal out- } \\
\text { comes }\end{array}$ & 5 & & Risk Ratio (M-H, Random, 95\% Cl) & Subtotals only \\
\hline 3.1 ESKD & 5 & 278 & Risk Ratio (M-H, Random, 95\% Cl) & $0.63[0.39,1.03]$ \\
\hline 3.2 Renal relapse & 2 & 84 & Risk Ratio (M-H, Random, 95\% Cl) & $0.23[0.08,0.62]$ \\
\hline $\begin{array}{l}\text { 3.3 Doubling serum crea- } \\
\text { tinine }\end{array}$ & 4 & 228 & Risk Ratio (M-H, Random, 95\% Cl) & $0.59[0.40,0.88]$ \\
\hline $\begin{array}{l}4 \text { Deterioration of kidney } \\
\text { function }\end{array}$ & 5 & 179 & Risk Ratio (M-H, Random, 95\% Cl) & $0.78[0.52,1.18]$ \\
\hline 5 Stable kidney function & 5 & 278 & Risk Ratio (M-H, Random, 95\% Cl) & $1.20[1.00,1.45]$ \\
\hline 6 Ovarian failure & 3 & 147 & Risk Ratio (M-H, Random, 95\% Cl) & $2.18[1.10,4.34]$ \\
\hline 7 Infection & 6 & & Risk Ratio (M-H, Random, 95\% Cl) & Subtotals only \\
\hline 7.1 Major infection & 6 & 291 & Risk Ratio (M-H, Random, 95\% Cl) & $0.87[0.50,1.51]$ \\
\hline 7.2 Herpes zoster virus & 3 & 199 & Risk Ratio (M-H, Random, 95\% Cl) & $1.77[0.63,4.99]$ \\
\hline 8 Malignancy & 2 & 117 & Risk Ratio (M-H, Random, 95\% Cl) & $0.82[0.07,9.90]$ \\
\hline 9 Bone toxicity & 3 & 197 & Risk Ratio (M-H, Random, 95\% Cl) & $0.84[0.40,1.75]$ \\
\hline 10 Bladder toxicity & 2 & 65 & Risk Ratio (M-H, Random, 95\% Cl) & $2.66[0.33,21.68]$ \\
\hline 11 Daily proteinuria & 3 & 92 & Mean Difference (IV, Random, 95\% CI) & $0.15[-0.23,0.54]$ \\
\hline 12 Serum creatinine & 1 & 29 & Mean Difference (IV, Random, 95\% CI) & $-52.0[-111.39,7.39]$ \\
\hline
\end{tabular}




\begin{tabular}{lllll}
\hline $\begin{array}{l}\text { Outcome or subgroup } \\
\text { title }\end{array}$ & No. of studies & $\begin{array}{l}\text { No. of partici- } \\
\text { pants }\end{array}$ & Statistical method & Effect size \\
\hline 13 Creatinine clearance & 2 & 63 & Mean Difference (IV, Random, 95\% Cl) & $12.23[-0.13,24.58]$ \\
\hline
\end{tabular}

Analysis 18.1. Comparison 18 Cyclophosphamide (CPA) + corticosteroids versus corticosteroids, Outcome 1 Death.

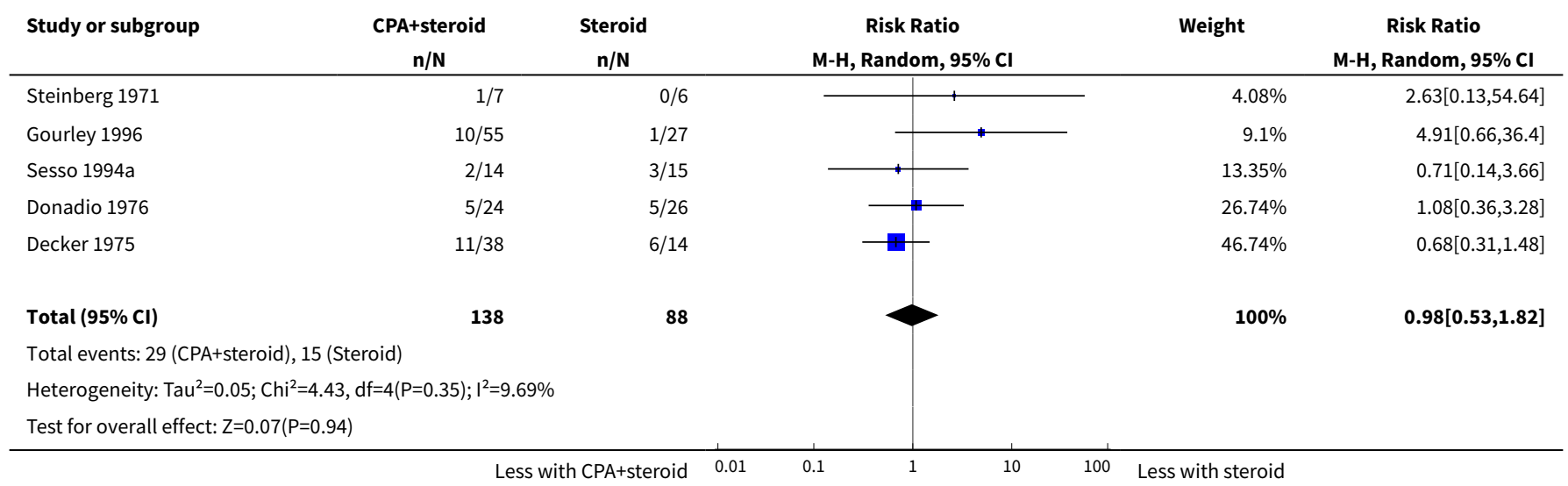

Analysis 18.2. Comparison 18 Cyclophosphamide (CPA) + corticosteroids versus corticosteroids, Outcome 2 Complete remission of proteinuria.

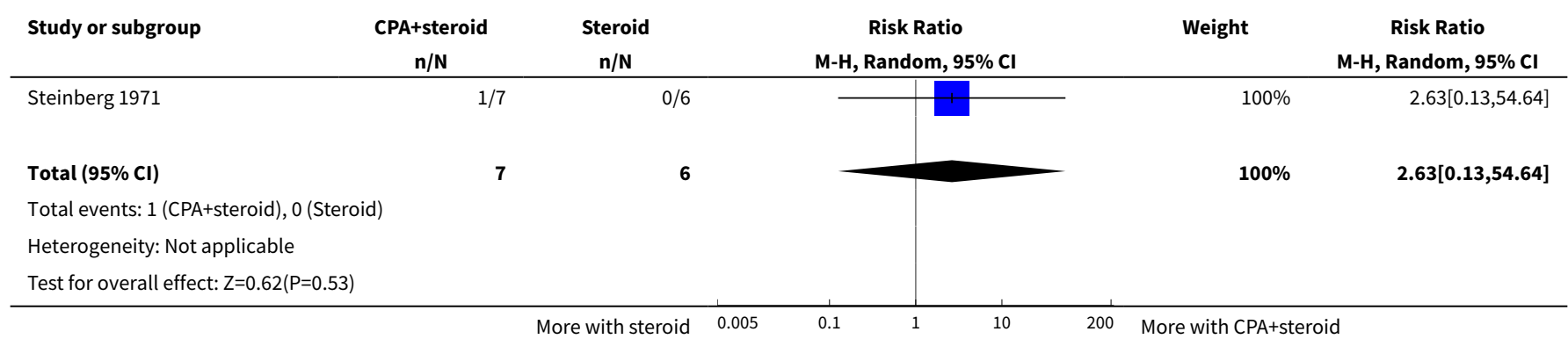

Analysis 18.3. Comparison 18 Cyclophosphamide (CPA) + corticosteroids versus corticosteroids, Outcome 3 Adverse renal outcomes.

\begin{tabular}{|c|c|c|c|c|c|}
\hline Study or subgroup & $\begin{array}{c}\text { CPA+steroid } \\
n / N\end{array}$ & $\begin{array}{c}\text { Steroid } \\
n / N\end{array}$ & $\begin{array}{c}\text { Risk Ratio } \\
\text { M-H, Random, } 95 \% \mathrm{Cl}\end{array}$ & Weight & $\begin{array}{c}\text { Risk Ratio } \\
\text { M-H, Random, } 95 \% \mathrm{Cl}\end{array}$ \\
\hline \multicolumn{6}{|l|}{ 18.3.1 ESKD } \\
\hline Sesso 1994a & $2 / 14$ & $3 / 15$ & $\rightarrow$ & $8.82 \%$ & $0.71[0.14,3.66]$ \\
\hline Donadio 1976 & $4 / 24$ & $6 / 26$ & $\longrightarrow$ & $18.23 \%$ & $0.72[0.23,2.25]$ \\
\hline Decker 1975 & $5 / 38$ & $5 / 14$ & - & $20.3 \%$ & $0.37[0.13,1.08]$ \\
\hline Boumpas 1992 & $7 / 40$ & $6 / 25$ & - & $25.09 \%$ & $0.73[0.28,1.92]$ \\
\hline Subtotal $(95 \% \mathrm{CI})$ & 171 & 107 & & $100 \%$ & $0.63[0.39,1.03]$ \\
\hline
\end{tabular}




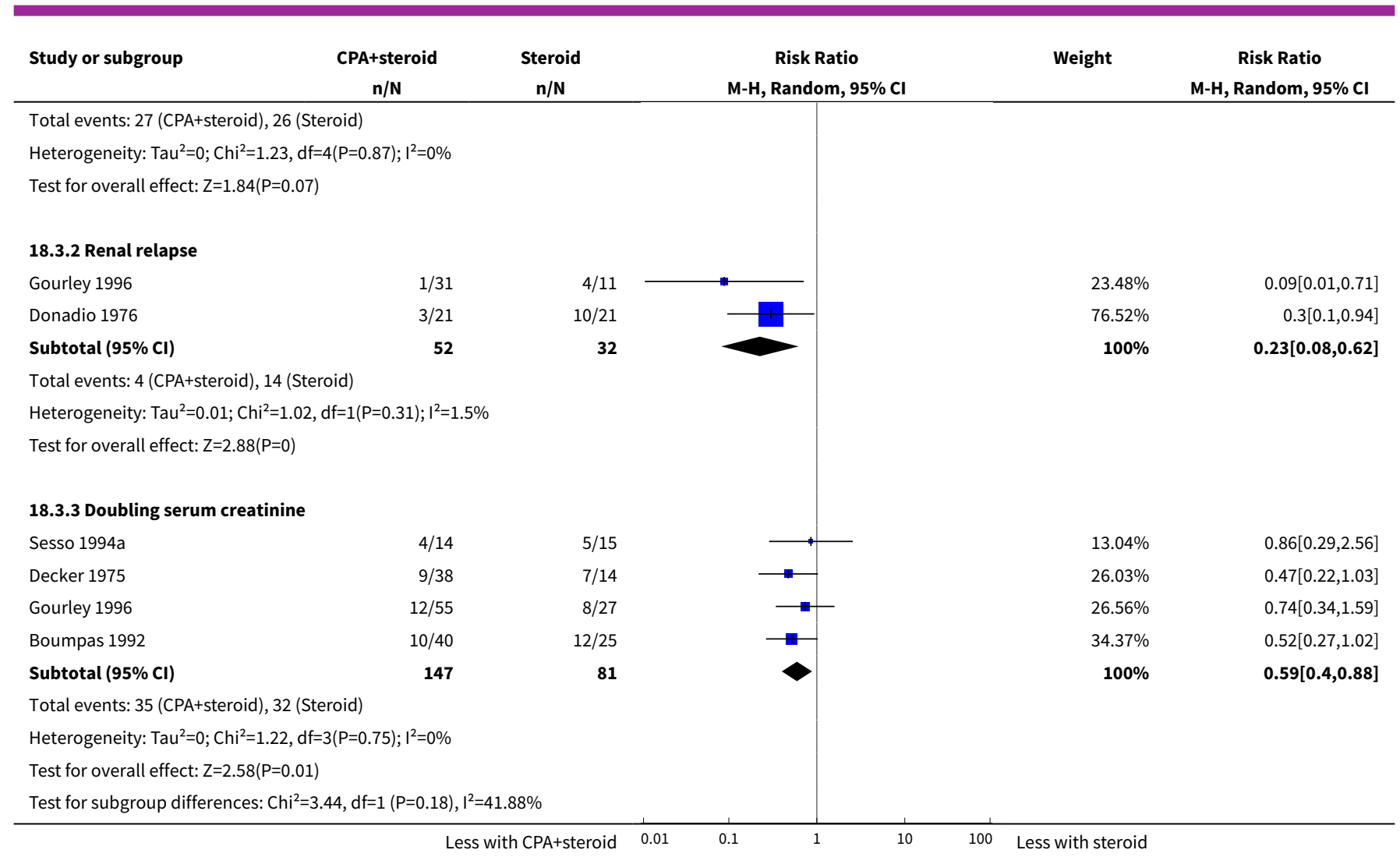

Analysis 18.4. Comparison 18 Cyclophosphamide (CPA) + corticosteroids versus corticosteroids, Outcome 4 Deterioration of kidney function.

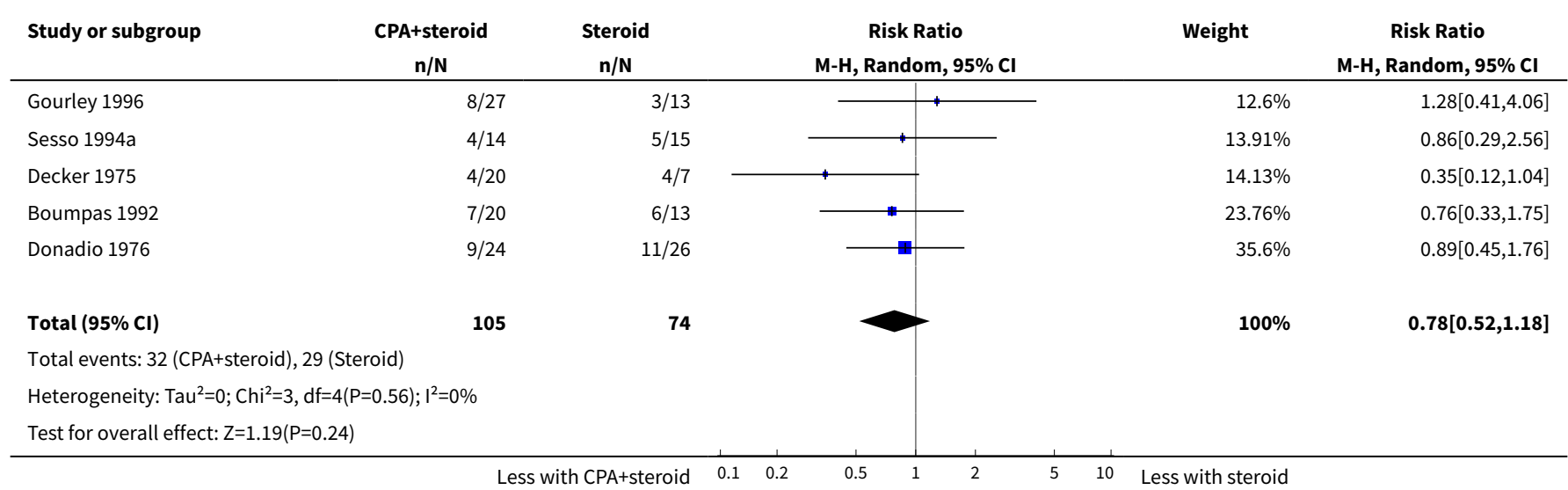

Analysis 18.5. Comparison 18 Cyclophosphamide (CPA) + corticosteroids versus corticosteroids, Outcome 5 Stable kidney function.

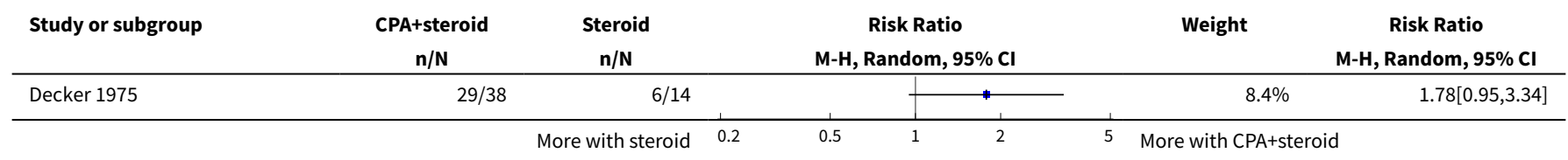




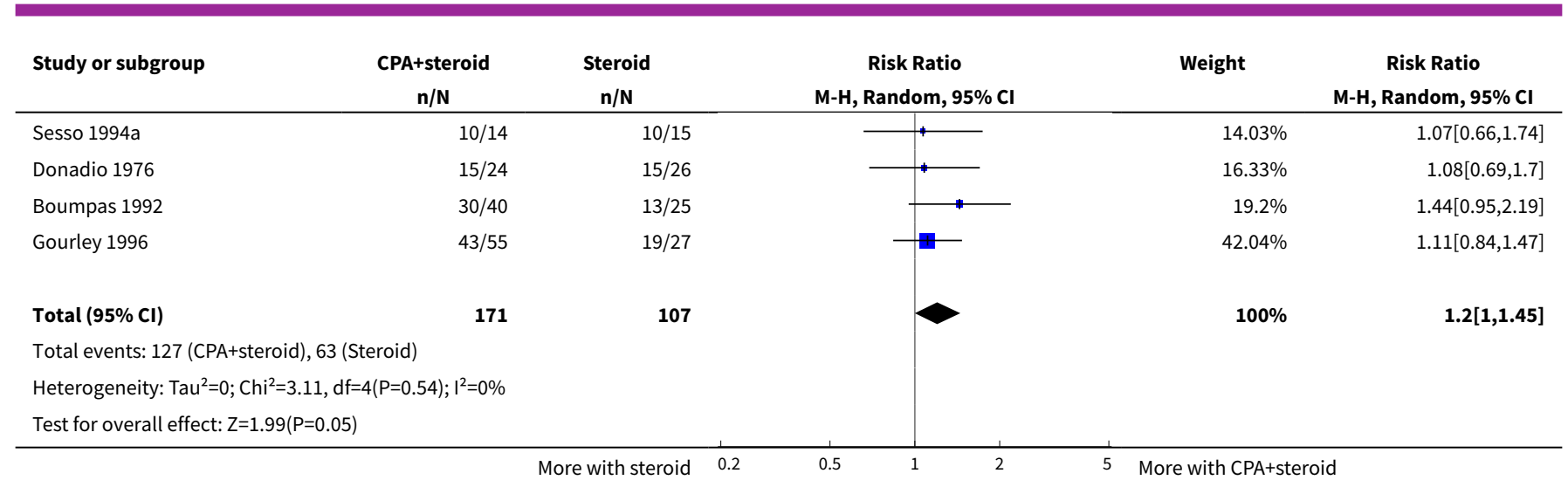

Analysis 18.6. Comparison 18 Cyclophosphamide (CPA) + corticosteroids versus corticosteroids, Outcome 6 Ovarian failure.

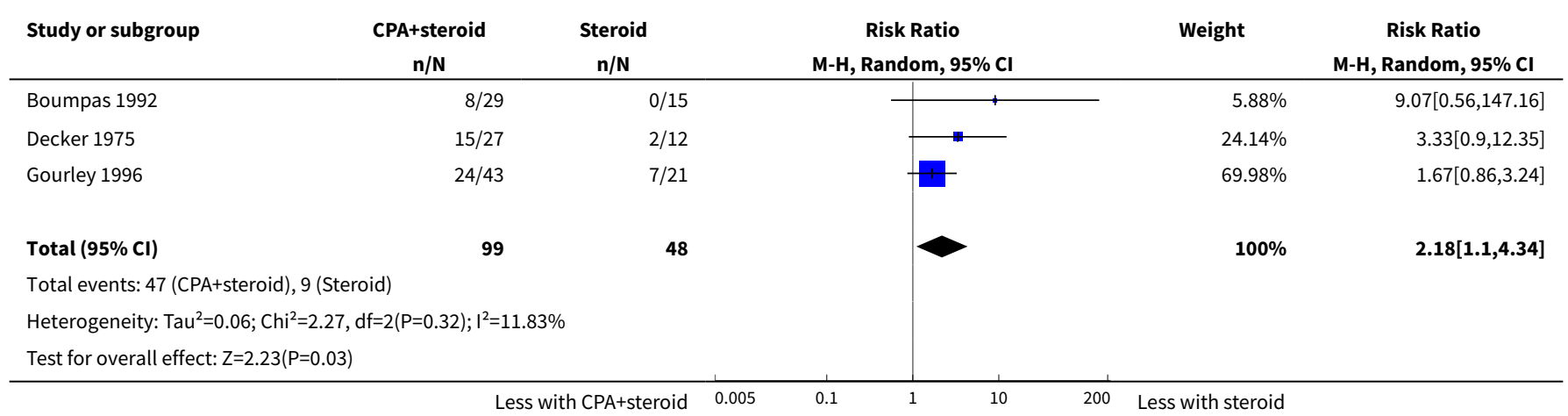

Analysis 18.7. Comparison 18 Cyclophosphamide (CPA) + corticosteroids versus corticosteroids, Outcome 7 Infection.

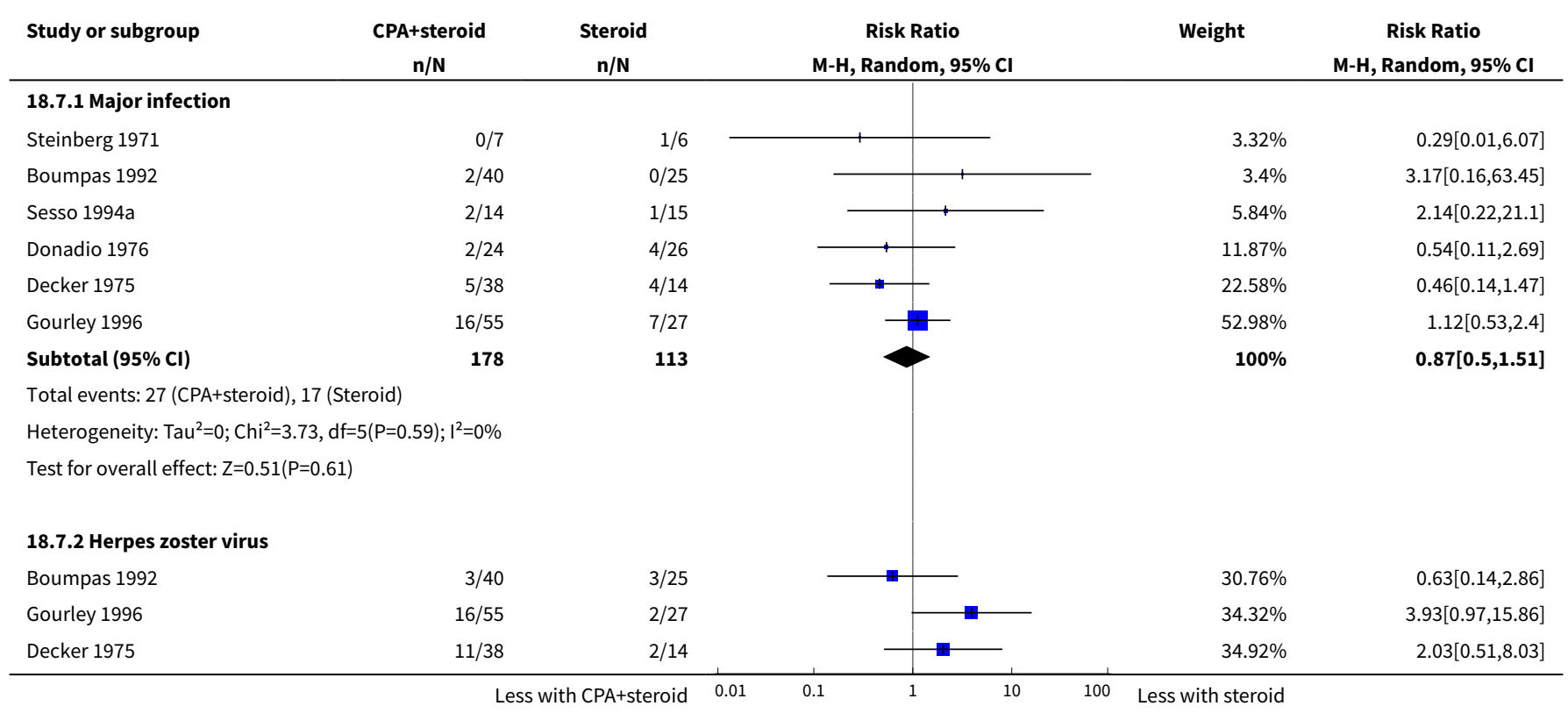




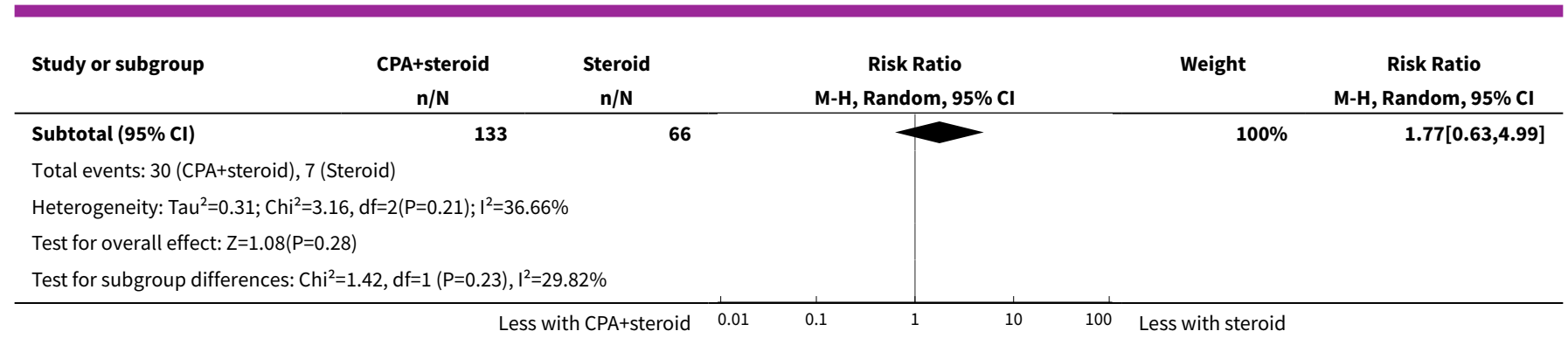

Analysis 18.8. Comparison 18 Cyclophosphamide (CPA) + corticosteroids versus corticosteroids, Outcome 8 Malignancy.

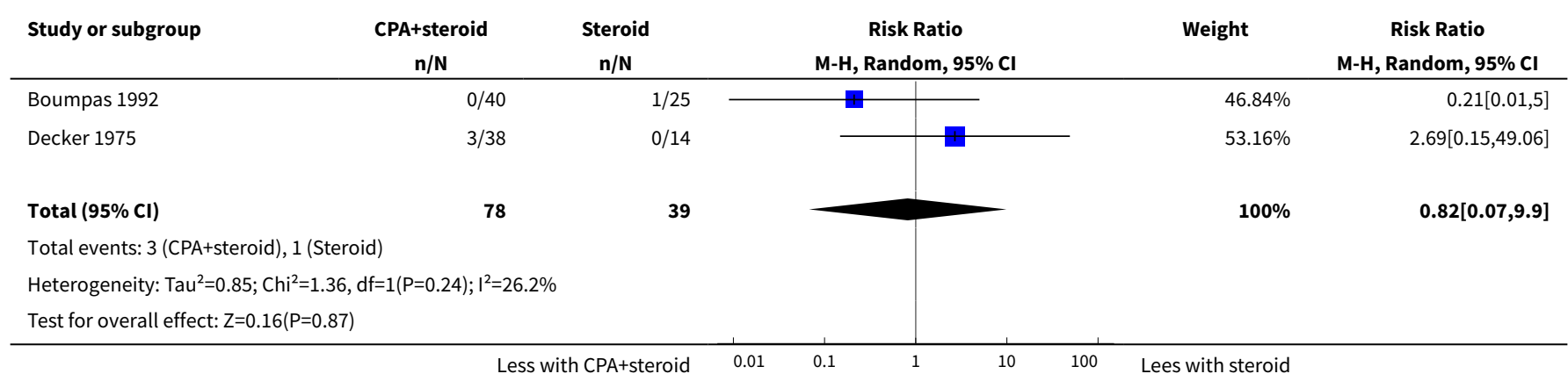

Analysis 18.9. Comparison 18 Cyclophosphamide (CPA) + corticosteroids versus corticosteroids, Outcome 9 Bone toxicity.

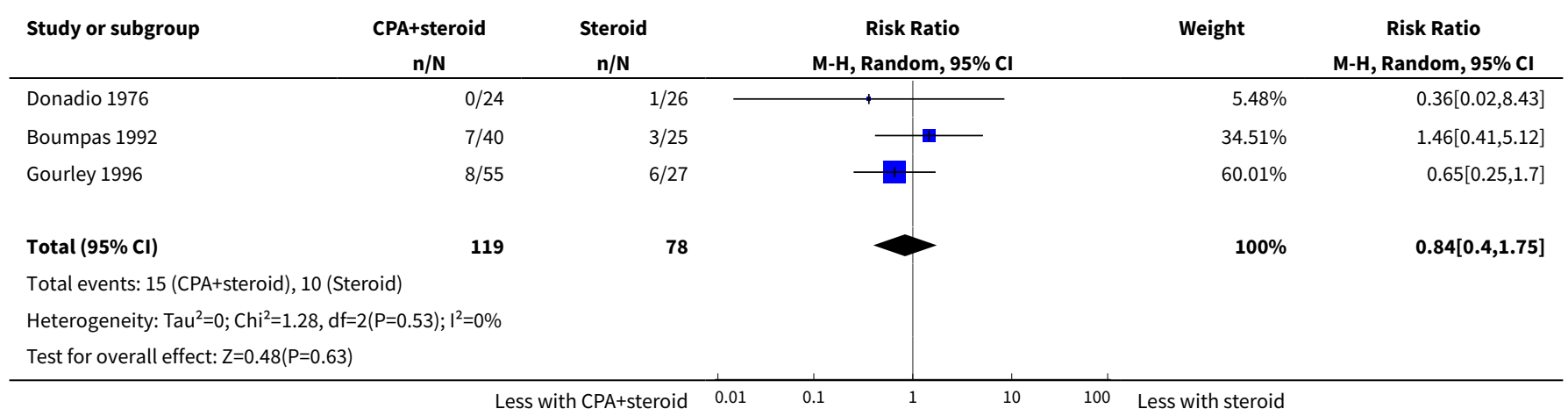

Analysis 18.10. Comparison 18 Cyclophosphamide (CPA) + corticosteroids versus corticosteroids, Outcome 10 Bladder toxicity.

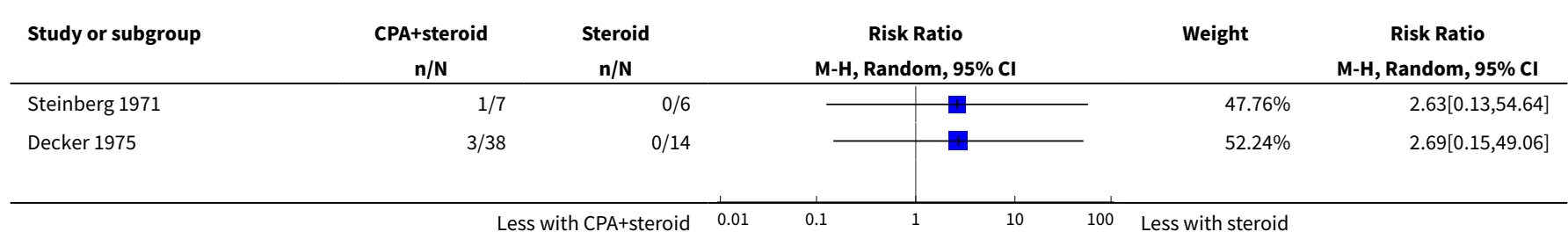




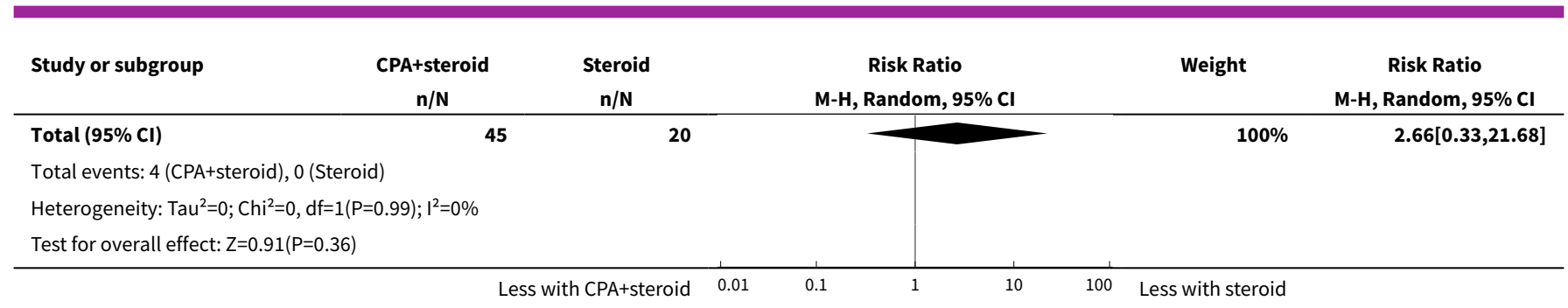

Analysis 18.11. Comparison 18 Cyclophosphamide (CPA) + corticosteroids versus corticosteroids, Outcome 11 Daily proteinuria.

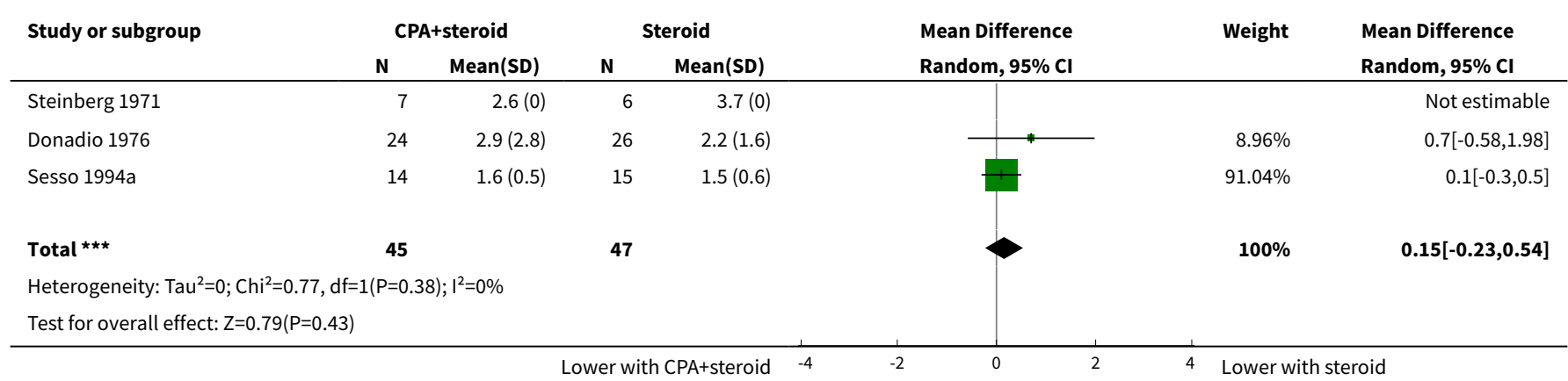

Analysis 18.12. Comparison 18 Cyclophosphamide (CPA) + corticosteroids versus corticosteroids, Outcome 12 Serum creatinine.

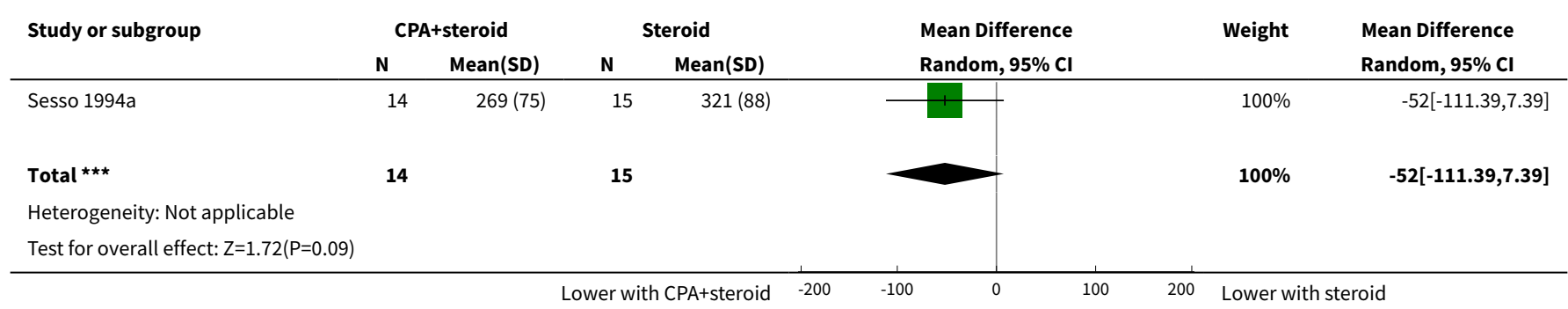

Analysis 18.13. Comparison 18 Cyclophosphamide (CPA) + corticosteroids versus corticosteroids, Outcome 13 Creatinine clearance.

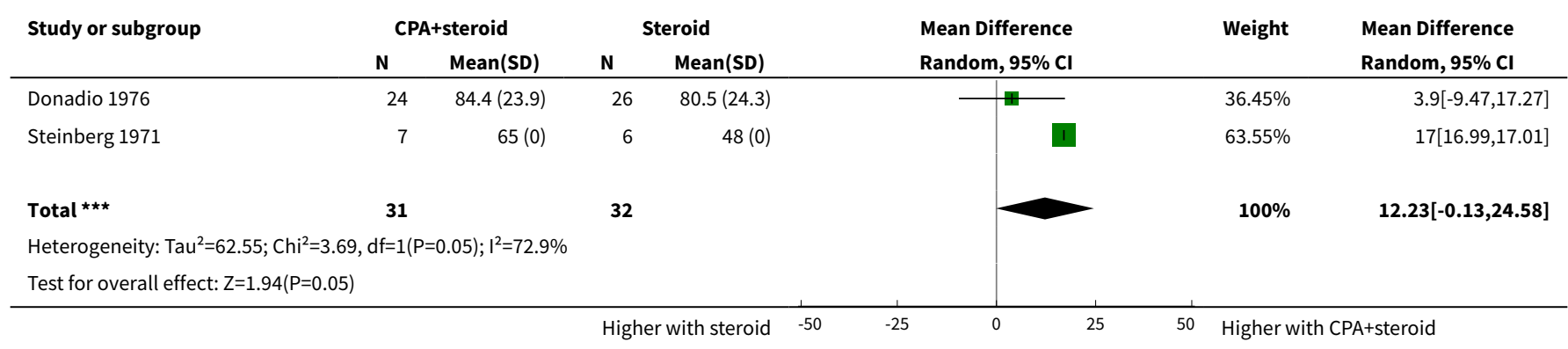


Comparison 19. Cyclophosphamide (CPA) + azathioprine (AZA) + corticosteroids versus corticosteroids alone

\begin{tabular}{|c|c|c|c|c|}
\hline $\begin{array}{l}\text { Outcome or subgroup } \\
\text { title }\end{array}$ & No. of studies & $\begin{array}{l}\text { No. of partici- } \\
\text { pants }\end{array}$ & Statistical method & Effect size \\
\hline 1 Death & 1 & 29 & Risk Ratio (M-H, Random, 95\% Cl) & $0.53[0.17,1.68]$ \\
\hline $\begin{array}{l}2 \text { Adverse renal out- } \\
\text { comes }\end{array}$ & 1 & & Risk Ratio (M-H, Random, 95\% Cl) & Subtotals only \\
\hline 2.1 ESKD & 1 & 29 & Risk Ratio (M-H, Random, 95\% Cl) & $0.21[0.04,1.02]$ \\
\hline $\begin{array}{l}\text { 2.2 Doubling of serum } \\
\text { creatinine }\end{array}$ & 1 & 29 & Risk Ratio (M-H, Random, 95\% Cl) & $0.16[0.04,0.69]$ \\
\hline 3 Stable kidney function & 1 & 29 & Risk Ratio (M-H, Random, 95\% Cl) & $1.59[0.83,3.06]$ \\
\hline 4 Ovarian failure & 1 & 27 & Risk Ratio (M-H, Random, 95\% Cl) & $7.32[0.49,108.96]$ \\
\hline 5 Infection & 1 & & Risk Ratio (M-H, Random, 95\% Cl) & Subtotals only \\
\hline 5.1 Major infection & 1 & 29 & Risk Ratio (M-H, Random, 95\% Cl) & $0.48[0.10,2.30]$ \\
\hline 5.2 Herpes zoster virus & 1 & 29 & Risk Ratio (M-H, Random, 95\% Cl) & $5.22[0.33,81.40]$ \\
\hline 6 Bladder toxicity & 1 & 29 & Risk Ratio (M-H, Random, 95\% Cl) & $2.43[0.14,42.17]$ \\
\hline
\end{tabular}

Analysis 19.1. Comparison 19 Cyclophosphamide (CPA) + azathioprine (AZA) + corticosteroids versus corticosteroids alone, Outcome 1 Death.

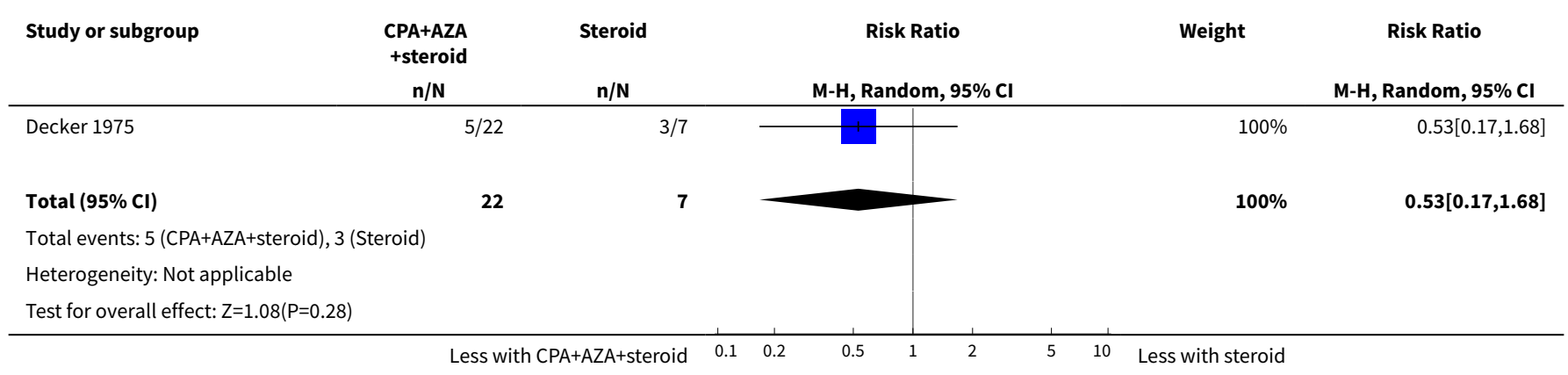

Analysis 19.2. Comparison 19 Cyclophosphamide (CPA) + azathioprine (AZA) + corticosteroids versus corticosteroids alone, Outcome 2 Adverse renal outcomes.

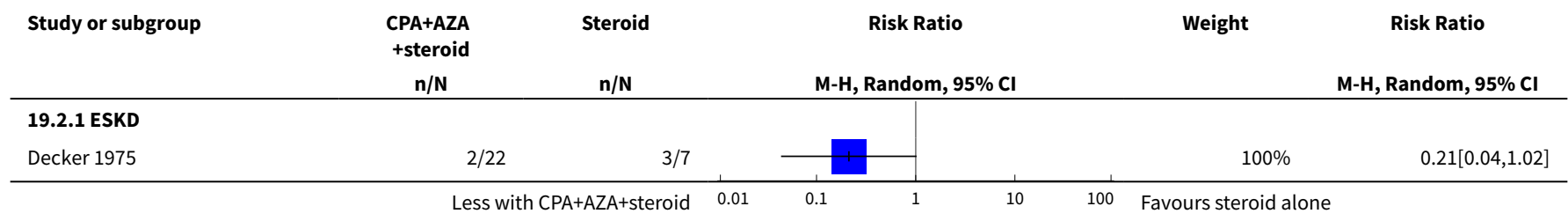




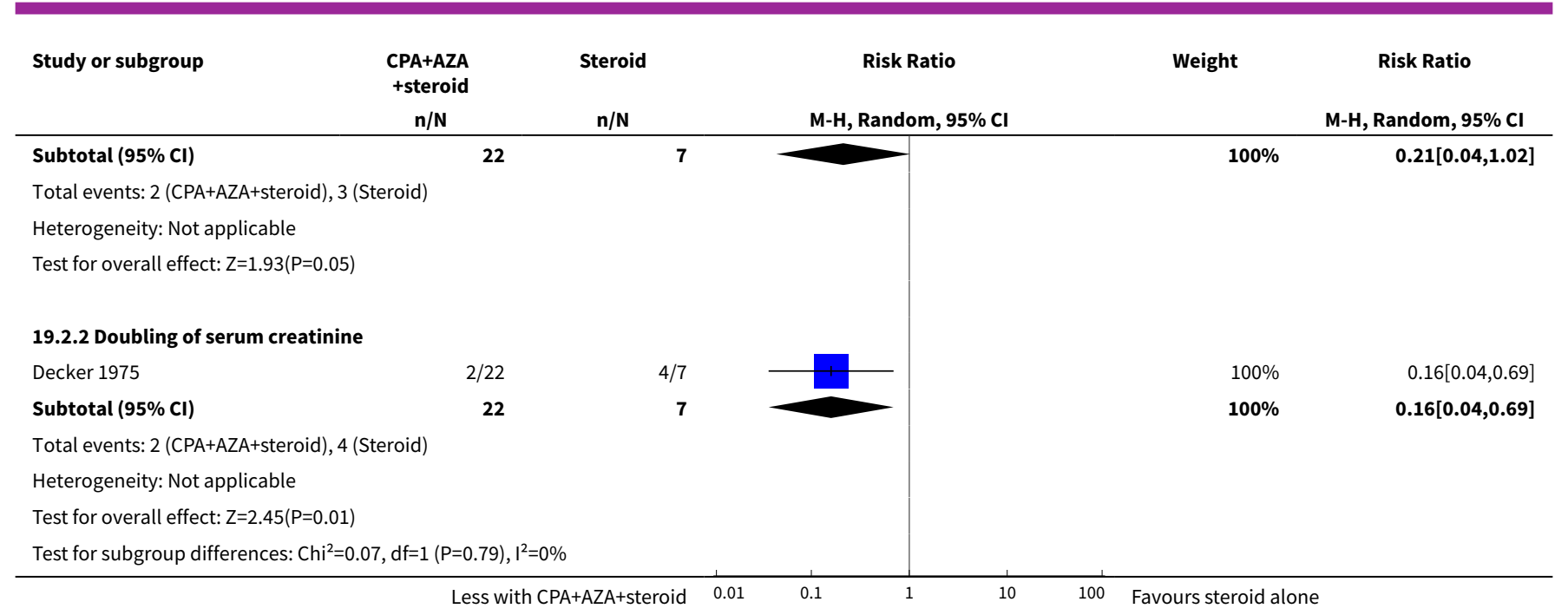

Analysis 19.3. Comparison 19 Cyclophosphamide (CPA) + azathioprine (AZA) + corticosteroids versus corticosteroids alone, Outcome 3 Stable kidney function.

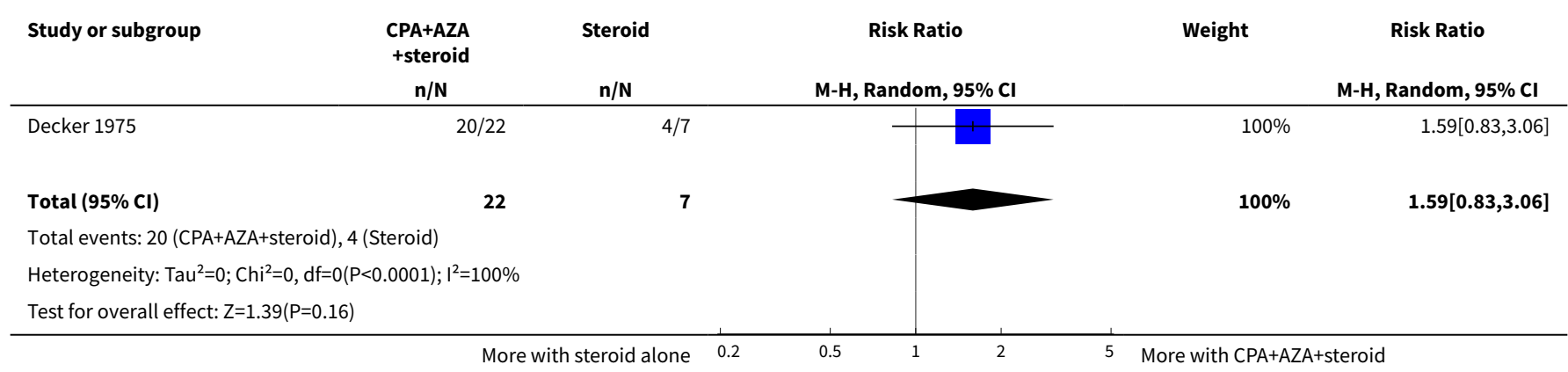

Analysis 19.4. Comparison 19 Cyclophosphamide (CPA) + azathioprine (AZA) + corticosteroids versus corticosteroids alone, Outcome 4 Ovarian failure.

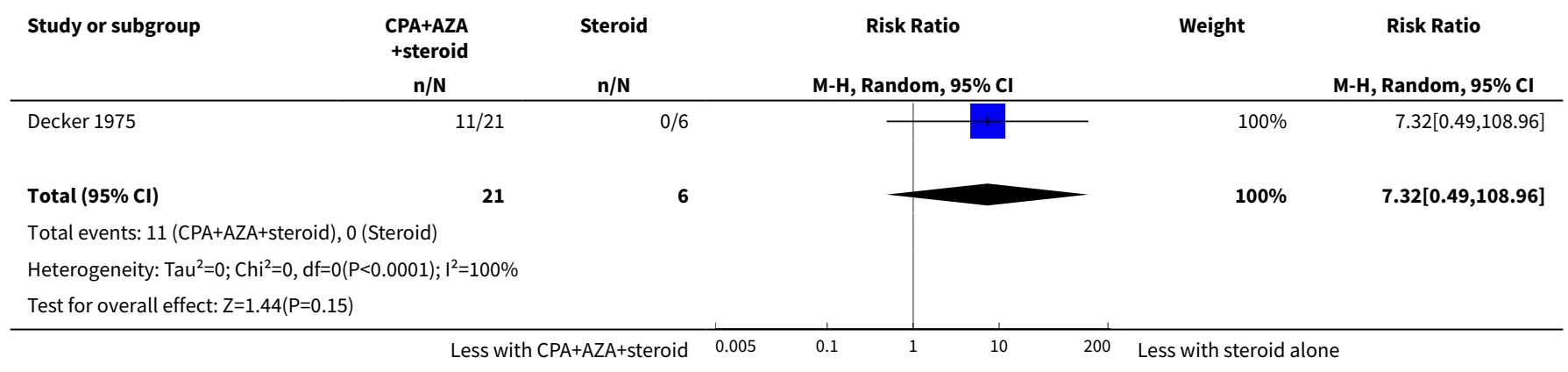


Analysis 19.5. Comparison 19 Cyclophosphamide (CPA) + azathioprine

$(A Z A)+$ corticosteroids versus corticosteroids alone, Outcome 5 Infection.

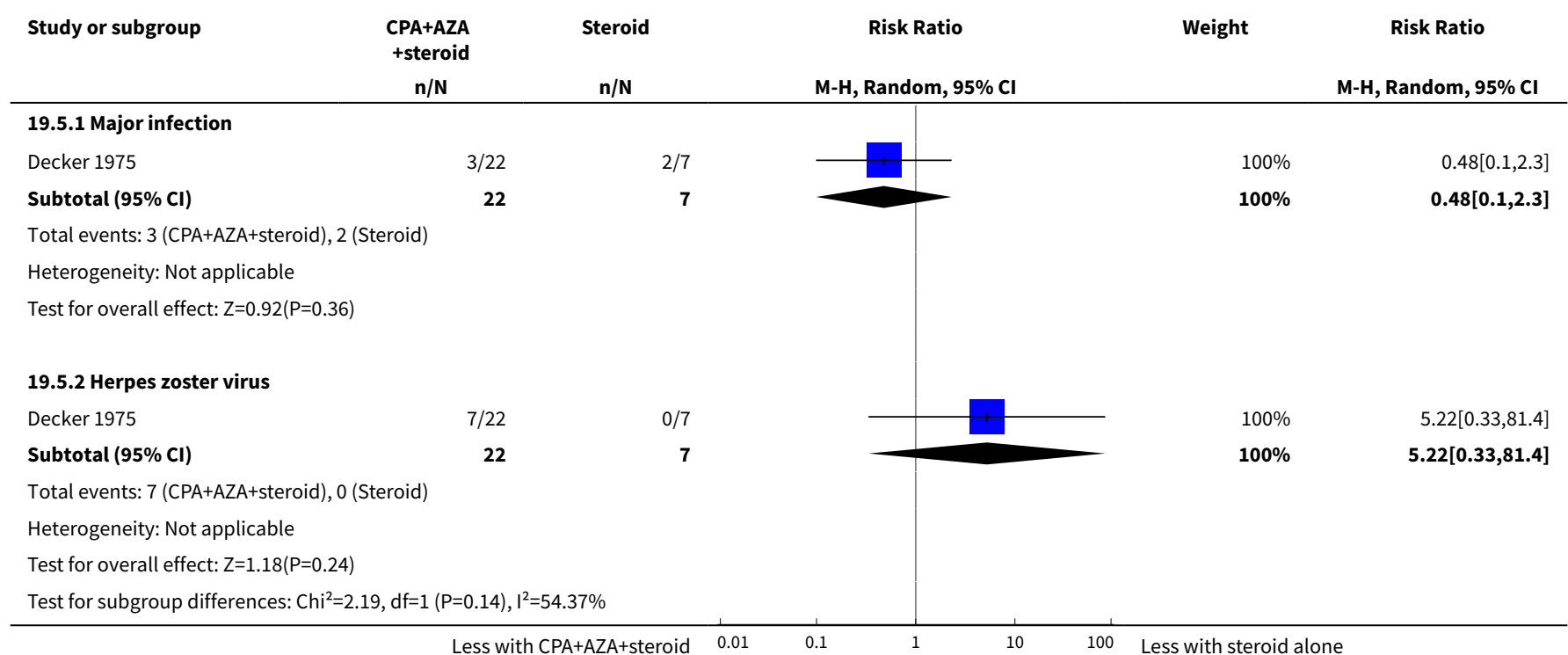

Analysis 19.6. Comparison 19 Cyclophosphamide (CPA) + azathioprine (AZA)

+ corticosteroids versus corticosteroids alone, Outcome 6 Bladder toxicity.

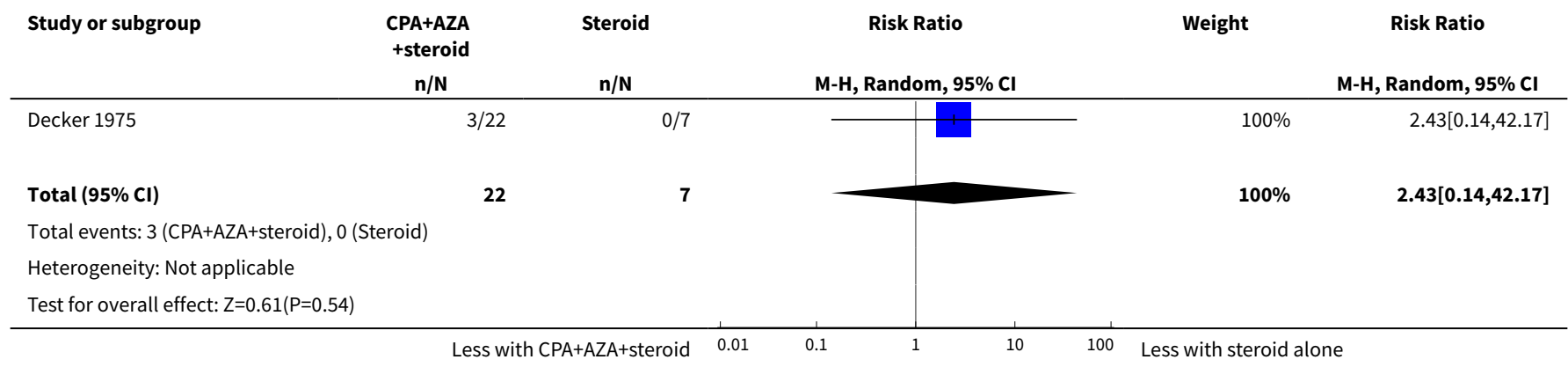

\section{Comparison 20. Azathioprine (AZA) + corticosteroids versus corticosteroids alone}

\begin{tabular}{lllll}
\hline $\begin{array}{l}\text { Outcome or subgroup } \\
\text { title }\end{array}$ & No. of studies & $\begin{array}{l}\text { No. of partici- } \\
\text { pants }\end{array}$ & Statistical method & Effect size \\
\hline 1 Death & 3 & 78 & Risk Ratio $(\mathrm{M}-\mathrm{H}$, Random, 95\% Cl) & $0.60[0.36,0.99]$ \\
\hline $\begin{array}{l}\text { 2 Complete remission of } \\
\text { proteinuria }\end{array}$ & 2 & 37 & Risk Ratio $(\mathrm{M}-\mathrm{H}$, Random, 95\% Cl) & $0.95[0.54,1.69]$ \\
\hline $\begin{array}{l}\text { 3 Adverse renal out- } \\
\text { comes }\end{array}$ & 3 & Risk Ratio $(\mathrm{M}-\mathrm{H}$, Random, $95 \% \mathrm{Cl})$ & Subtotals only \\
\hline 3.1 ESKD & 2 & 54 & Risk Ratio $(\mathrm{M}-\mathrm{H}$, Random, 95\% Cl) & $0.66[0.17,2.55]$ \\
\hline
\end{tabular}




\begin{tabular}{|c|c|c|c|c|}
\hline $\begin{array}{l}\text { Outcome or subgroup } \\
\text { title }\end{array}$ & No. of studies & $\begin{array}{l}\text { No. of partici- } \\
\text { pants }\end{array}$ & Statistical method & Effect size \\
\hline 3.2 Renal relapse & 1 & 16 & Risk Ratio (M-H, Random, 95\% Cl) & $0.78[0.22,2.74]$ \\
\hline $\begin{array}{l}\text { 3.3 Doubling of serum } \\
\text { creatinine }\end{array}$ & 1 & 26 & Risk Ratio (M-H, Random, 95\% Cl) & $0.98[0.36,2.68]$ \\
\hline 4 Stable kidney function & 1 & 26 & Risk Ratio (M-H, Random, 95\% Cl) & $1.01[0.48,2.14]$ \\
\hline 5 Ovarian failure & 1 & 24 & Risk Ratio (M-H, Random, 95\% Cl) & $2.58[0.15,43.86]$ \\
\hline 6 Infection & 2 & & Risk Ratio (M-H, Random, 95\% Cl) & Subtotals only \\
\hline 6.1 Herpes zoster virus & 2 & 42 & Risk Ratio (M-H, Random, 95\% Cl) & $3.56[0.46,27.79]$ \\
\hline 7 Malignancy & 1 & 26 & Risk Ratio (M-H, Random, 95\% Cl) & $2.0[0.11,37.22]$ \\
\hline 8 Bone toxicity & 1 & 24 & Risk Ratio (M-H, Random, 95\% Cl) & $3.55[0.43,29.42]$ \\
\hline 9 Creatinine clearance & 1 & 24 & Mean Difference (IV, Random, 95\% CI) & $5.0[-3.14,13.14]$ \\
\hline
\end{tabular}

Analysis 20.1. Comparison 20 Azathioprine (AZA) + corticosteroids versus corticosteroids alone, Outcome 1 Death.

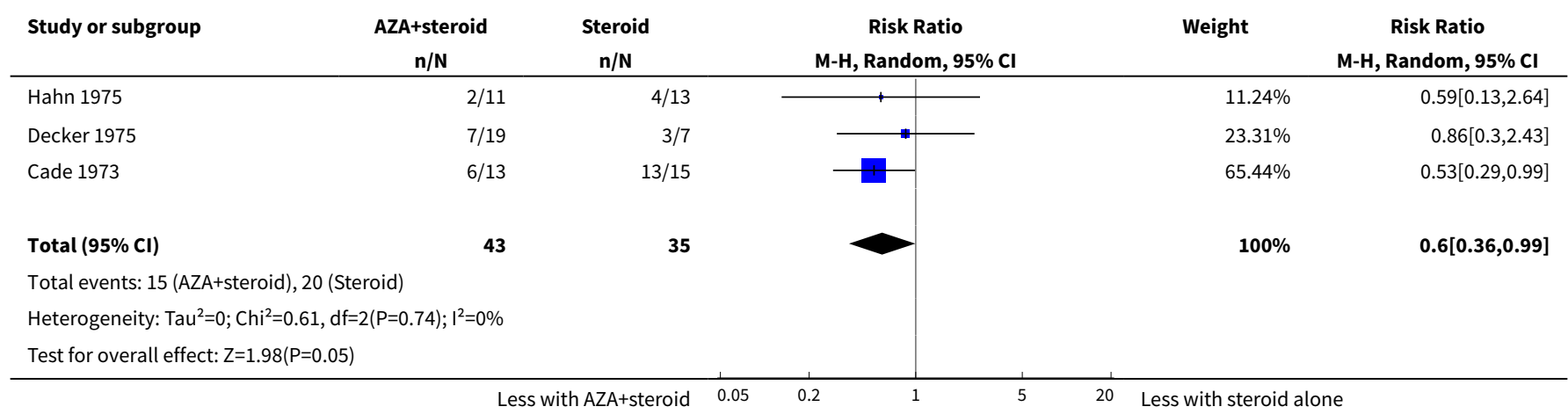

Analysis 20.2. Comparison 20 Azathioprine (AZA) + corticosteroids versus corticosteroids alone, Outcome 2 Complete remission of proteinuria.

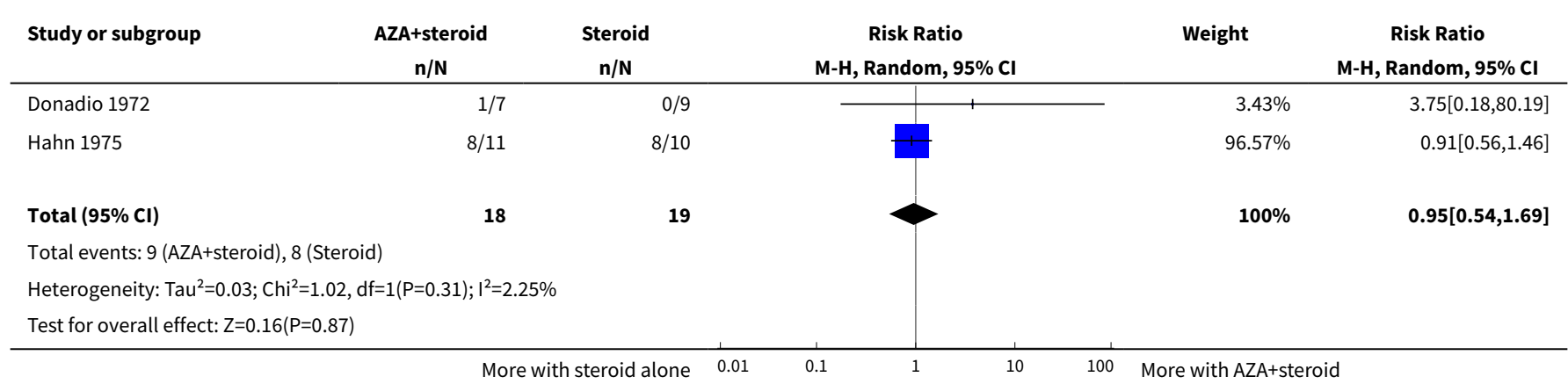


Analysis 20.3. Comparison 20 Azathioprine (AZA) + corticosteroids versus corticosteroids alone, Outcome 3 Adverse renal outcomes.

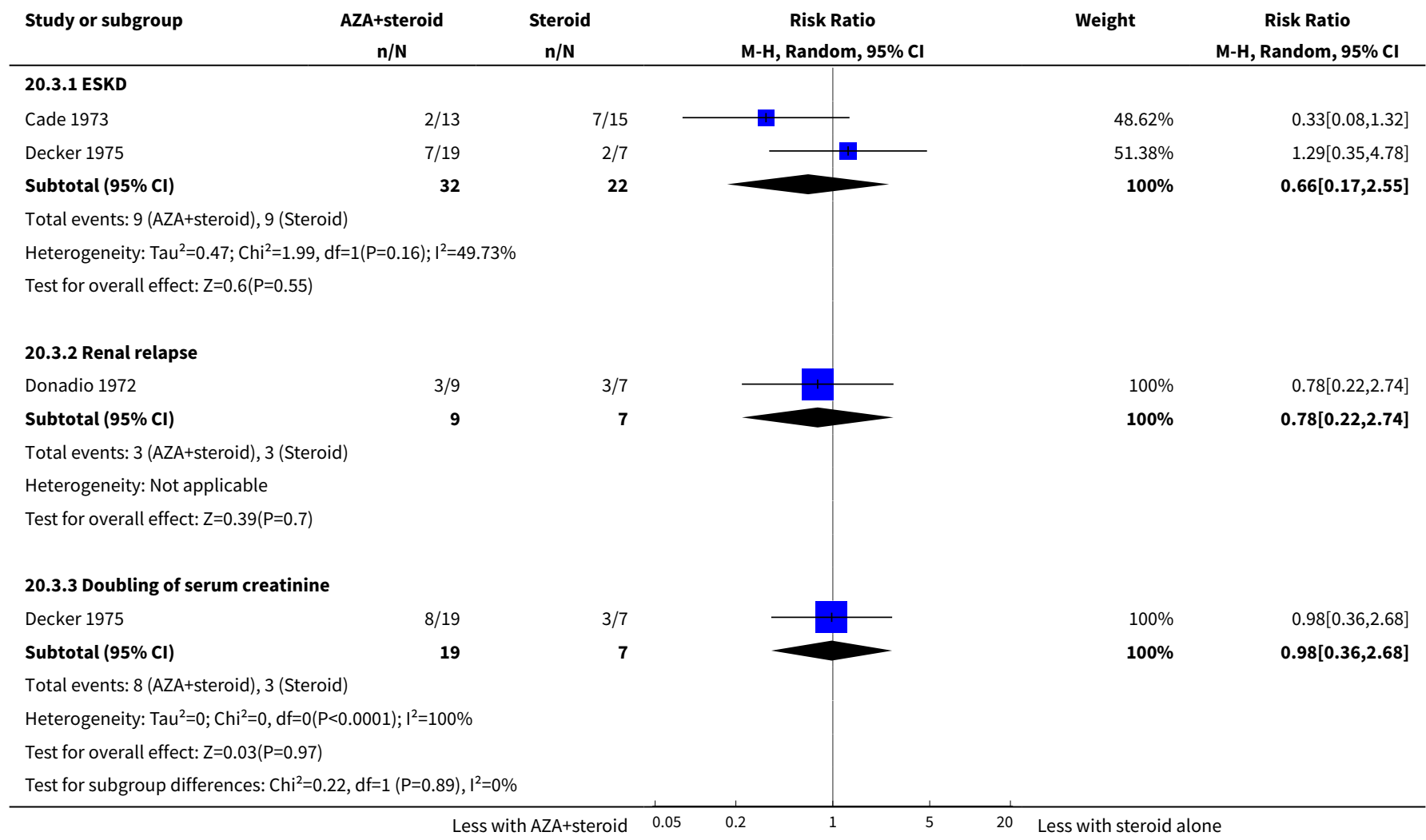

Analysis 20.4. Comparison 20 Azathioprine (AZA) + corticosteroids versus corticosteroids alone, Outcome 4 Stable kidney function.

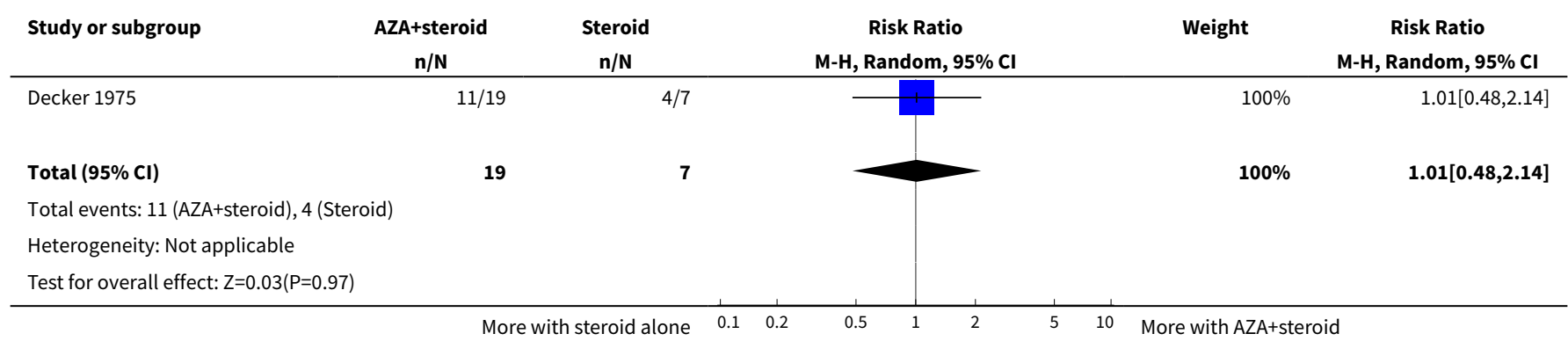


Analysis 20.5. Comparison 20 Azathioprine (AZA) + corticosteroids versus corticosteroids alone, Outcome 5 Ovarian failure.

\begin{tabular}{|c|c|c|c|c|c|}
\hline Study or subgroup & $\begin{array}{c}\text { AZA+steroid } \\
\mathrm{n} / \mathrm{N} \\
\end{array}$ & $\begin{array}{c}\text { Steroid } \\
\mathrm{n} / \mathrm{N}\end{array}$ & $\begin{array}{c}\text { Risk Ratio } \\
\text { M-H, Random, } 95 \% \text { Cl }\end{array}$ & Weight & $\begin{array}{c}\text { Risk Ratio } \\
\text { M-H, Random, } 95 \% \text { CI }\end{array}$ \\
\hline Decker 1975 & $3 / 18$ & $0 / 6$ & 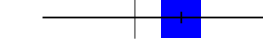 & $100 \%$ & $2.58[0.15,43.86]$ \\
\hline Total $(95 \% \mathrm{Cl})$ & 18 & 6 & & $100 \%$ & $2.58[0.15,43.86]$ \\
\hline \multicolumn{6}{|c|}{ Total events: 3 (AZA+steroid), 0 (Steroid) } \\
\hline \multicolumn{6}{|c|}{ Heterogeneity: Not applicable } \\
\hline Test for overall effec & & & & & \\
\hline
\end{tabular}

Analysis 20.6. Comparison 20 Azathioprine (AZA) + corticosteroids versus corticosteroids alone, Outcome 6 Infection.

\begin{tabular}{|c|c|c|c|c|c|}
\hline Study or subgroup & $\begin{array}{c}\text { AZA+steroid } \\
n / N\end{array}$ & $\begin{array}{c}\text { Steroid } \\
n / N\end{array}$ & $\begin{array}{c}\text { Risk Ratio } \\
\text { M-H, Random, } 95 \% \mathrm{CI}\end{array}$ & Weight & $\begin{array}{c}\text { Risk Ratio } \\
\text { M-H, Random, } 95 \% \mathrm{CI}\end{array}$ \\
\hline \multicolumn{6}{|c|}{ 20.6.1 Herpes zoster virus } \\
\hline Decker 1975 & $2 / 19$ & $0 / 7$ & 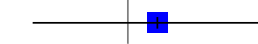 & $49.43 \%$ & $2[0.11,37.22]$ \\
\hline Donadio 1972 & $2 / 7$ & $0 / 9$ & & $50.57 \%$ & $6.25[0.35,112.52]$ \\
\hline Subtotal $(95 \% \mathrm{Cl})$ & 26 & 16 & & $100 \%$ & $3.56[0.46,27.79]$ \\
\hline \multicolumn{6}{|c|}{ Total events: 4 (AZA+steroid), 0 (Steroid) } \\
\hline \multicolumn{6}{|c|}{ Test for overall effect: $Z=1.21(P=0.23)$} \\
\hline
\end{tabular}

Analysis 20.7. Comparison 20 Azathioprine (AZA) + corticosteroids versus corticosteroids alone, Outcome 7 Malignancy.

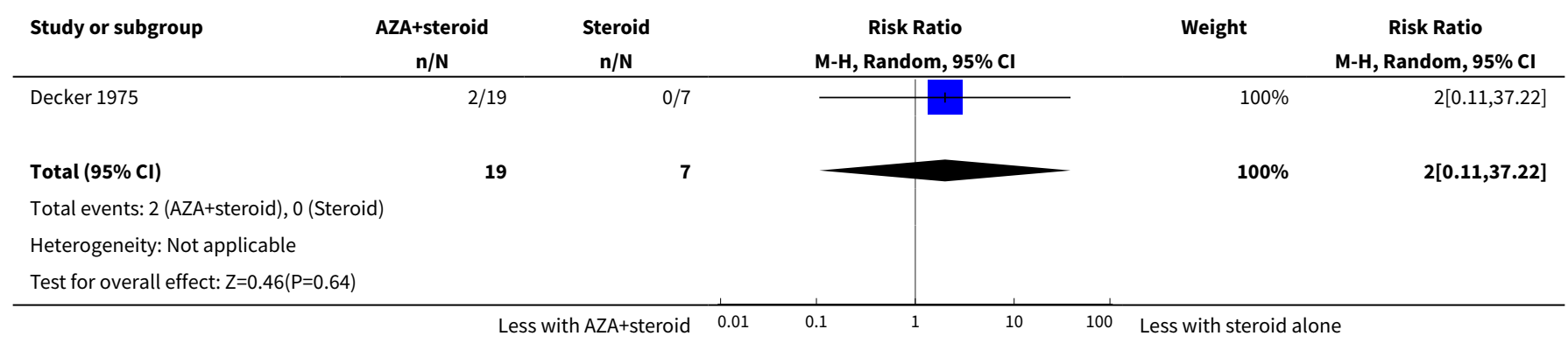

Analysis 20.8. Comparison 20 Azathioprine (AZA) + corticosteroids versus corticosteroids alone, Outcome 8 Bone toxicity.

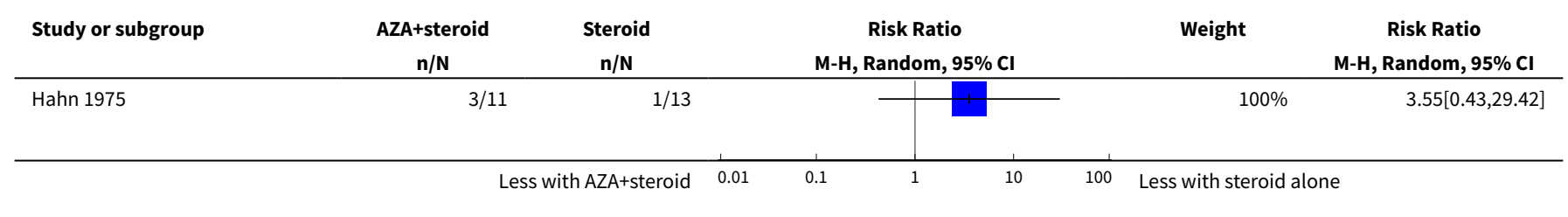




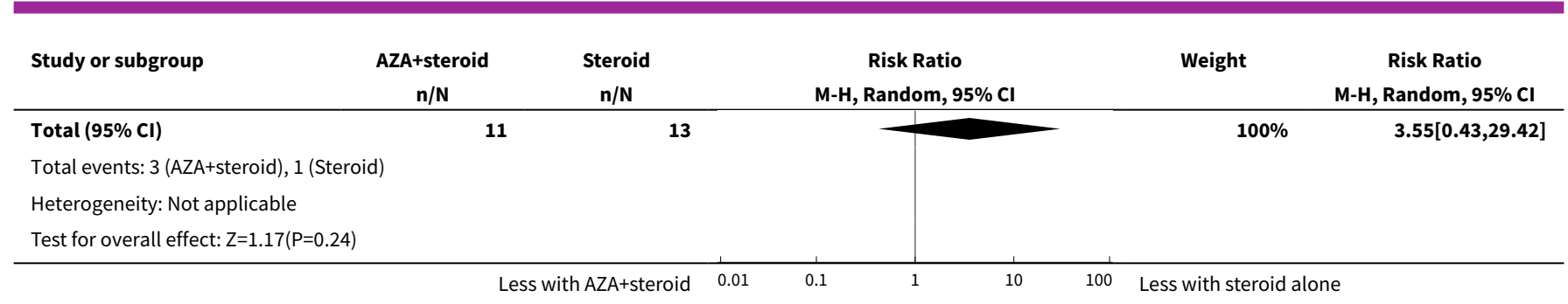

Analysis 20.9. Comparison 20 Azathioprine (AZA) + corticosteroids versus corticosteroids alone, Outcome 9 Creatinine clearance.

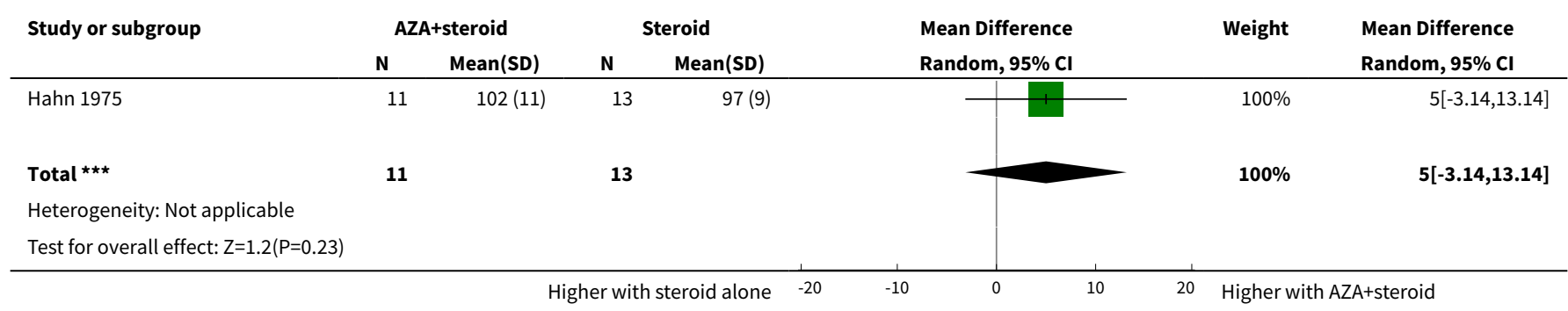

Comparison 21. Cyclosporin (CSA) + corticosteroids versus corticosteroids alone

\begin{tabular}{lllll}
\hline $\begin{array}{l}\text { Outcome or subgroup } \\
\text { title }\end{array}$ & No. of studies & $\begin{array}{l}\text { No. of partici- } \\
\text { pants }\end{array}$ & Statistical method & Effect size \\
\hline 1 Daily proteinuria & 1 & 10 & Mean Difference (IV, Random, 95\% Cl) & $-1.8[-2.59,-1.01]$ \\
\hline 2 Serum creatinine & 1 & 10 & Mean Difference (IV, Random, 95\% Cl) & $-31.90[-73.63,9.83]$ \\
\hline 3 Creatinine clearance & 1 & 10 & Mean Difference (IV, Random, 95\% Cl) & $-42.5[-85.02,0.02]$ \\
\hline
\end{tabular}

Analysis 21.1. Comparison 21 Cyclosporin (CSA) + corticosteroids versus corticosteroids alone, Outcome 1 Daily proteinuria.

\begin{tabular}{|c|c|c|c|c|c|c|c|}
\hline \multirow{3}{*}{$\begin{array}{l}\text { Study or subgroup } \\
\text { Balletta } 1992\end{array}$} & \multicolumn{2}{|c|}{ CSA+steroid } & \multicolumn{2}{|c|}{ Steroid } & \multirow{2}{*}{$\begin{array}{l}\text { Mean Difference } \\
\text { Random, } 95 \% \mathrm{Cl}\end{array}$} & \multirow[t]{2}{*}{ Weight } & \multirow{2}{*}{$\begin{array}{l}\text { Mean Difference } \\
\text { Random, } 95 \% \mathrm{Cl}\end{array}$} \\
\hline & $\mathbf{N}$ & Mean(SD) & $\mathbf{N}$ & Mean(SD) & & & \\
\hline & 5 & $0.3(0.1)$ & 5 & $2.1(0.9)$ & - & $100 \%$ & $-1.8[-2.59,-1.01]$ \\
\hline Total $\star \star \star ~$ & 5 & & 5 & & & $100 \%$ & $-1.8[-2.59,-1.01]$ \\
\hline \multicolumn{8}{|c|}{ Heterogeneity: Not applicable } \\
\hline \multicolumn{8}{|c|}{ Test for overall effect: $Z=4.44(P<0.0001)$} \\
\hline
\end{tabular}


Analysis 21.2. Comparison 21 Cyclosporin (CSA) + corticosteroids versus corticosteroids alone, Outcome 2 Serum creatinine.

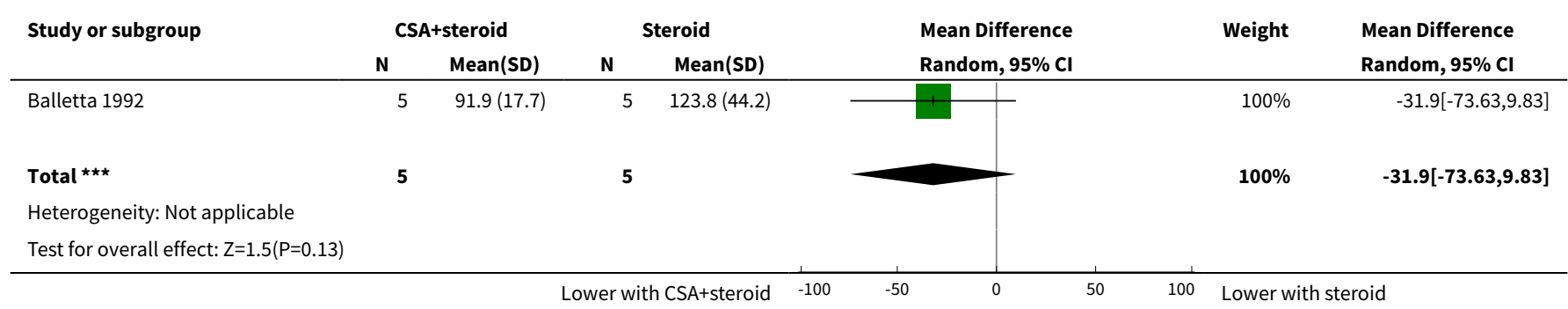

\section{Analysis 21.3. Comparison 21 Cyclosporin (CSA) + corticosteroids versus corticosteroids alone, Outcome 3 Creatinine clearance.}

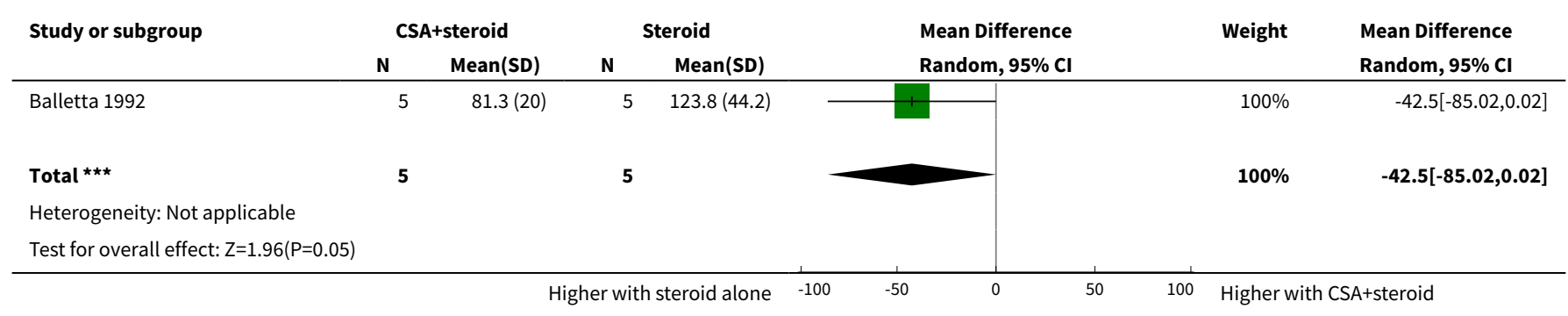

\section{Comparison 22. Misoprostol + corticosteroids versus corticosteroids alone}

\begin{tabular}{lllll}
\hline Outcome or subgroup title & No. of studies & $\begin{array}{l}\text { No. of partici- } \\
\text { pants }\end{array}$ & Statistical method & Effect size \\
\hline 1 Adverse renal outcomes & 1 & & Risk Ratio (M-H, Random, 95\% Cl) & Subtotals only \\
\hline 1.1 Doubling of serum creatinine & 1 & 14 & Risk Ratio (M-H, Random, 95\% Cl) & $0.0[0.0,0.0]$ \\
\hline
\end{tabular}

Analysis 22.1. Comparison 22 Misoprostol + corticosteroids versus corticosteroids alone, Outcome 1 Adverse renal outcomes.

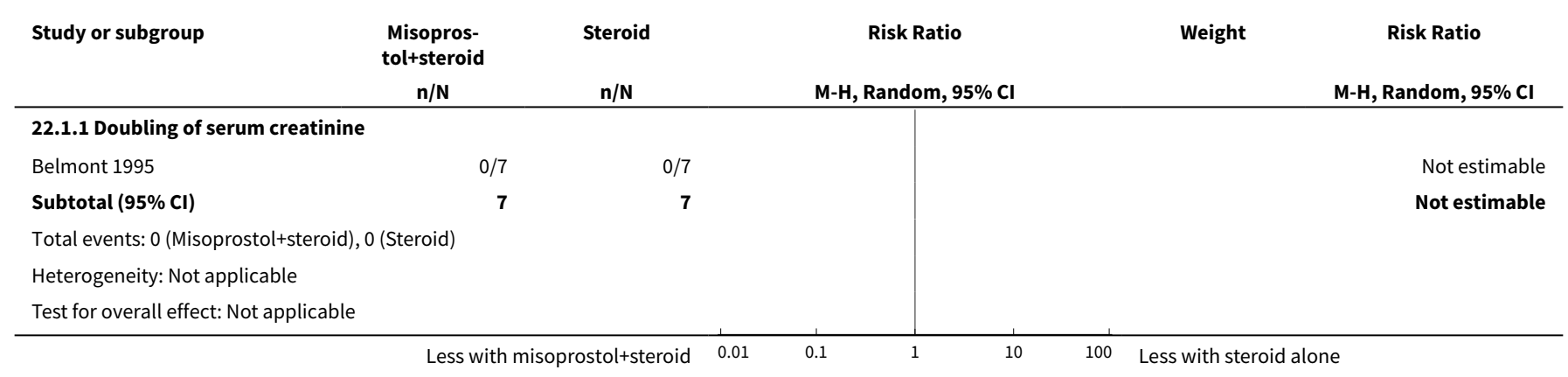


Comparison 23. Plasma exchange (PE) + immunosuppression (IS) versus IS alone

\begin{tabular}{|c|c|c|c|c|}
\hline $\begin{array}{l}\text { Outcome or subgroup } \\
\text { title }\end{array}$ & No. of studies & $\begin{array}{l}\text { No. of partici- } \\
\text { pants }\end{array}$ & Statistical method & Effect size \\
\hline 1 Death & 2 & 125 & Risk Ratio (M-H, Random, 95\% Cl) & $1.62[0.64,4.09]$ \\
\hline $\begin{array}{l}2 \text { Adverse renal out- } \\
\text { comes }\end{array}$ & 4 & 251 & Risk Ratio (M-H, Random, 95\% Cl) & $0.89[0.51,1.55]$ \\
\hline 2.1 ESKD & 3 & 143 & Risk Ratio (M-H, Random, 95\% Cl) & $1.24[0.60,2.57]$ \\
\hline $\begin{array}{l}\text { 2.2 Doubling of serum } \\
\text { creatinine }\end{array}$ & 2 & 51 & Risk Ratio (M-H, Random, 95\% Cl) & $0.17[0.02,1.26]$ \\
\hline $\begin{array}{l}2.3 \text { Deterioration of kid- } \\
\text { ney function }\end{array}$ & 2 & 57 & Risk Ratio (M-H, Random, 95\% Cl) & $0.53[0.06,4.83]$ \\
\hline 3 Stable kidney function & 3 & 75 & Risk Ratio (M-H, Random, 95\% Cl) & $1.10[0.94,1.30]$ \\
\hline 4 Infection & 3 & & Risk Ratio (M-H, Random, 95\% Cl) & Subtotals only \\
\hline 4.1 Major infection & 2 & 125 & Risk Ratio (M-H, Random, 95\% Cl) & $0.69[0.35,1.37]$ \\
\hline 4.2 Herpes zoster virus & 2 & 104 & Risk Ratio (M-H, Random, 95\% Cl) & $1.69[0.10,29.42]$ \\
\hline 5 Leucopenia & 1 & 18 & Risk Ratio (M-H, Random, 95\% Cl) & $2.60[0.20,34.07]$ \\
\hline 6 Daily proteinuria & 2 & 30 & Mean Difference (IV, Random, 95\% CI) & $-0.56[-5.23,4.11]$ \\
\hline 7 Serum creatinine & 3 & 69 & Mean Difference (IV, Random, 95\% CI) & $-17.90[-23.41,-12.39]$ \\
\hline 8 Creatinine clearance & 1 & 12 & Mean Difference (IV, Random, 95\% CI) & $26.0[-17.60,69.60]$ \\
\hline 9 Disease activity (SLAM) & 1 & 18 & Mean Difference (IV, Random, 95\% Cl) & $0.67[-3.47,4.81]$ \\
\hline
\end{tabular}

Analysis 23.1. Comparison 23 Plasma exchange (PE) + immunosuppression (IS) versus IS alone, Outcome 1 Death.

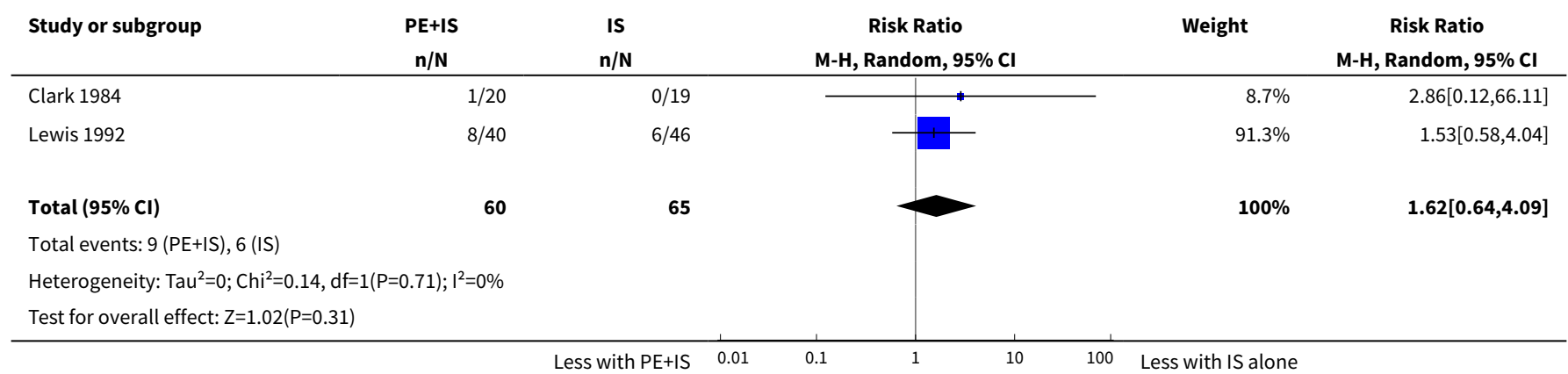


Analysis 23.2. Comparison 23 Plasma exchange (PE) + immunosuppression (IS) versus IS alone, Outcome 2 Adverse renal outcomes.

\begin{tabular}{|c|c|c|c|c|c|}
\hline Study or subgroup & $\begin{array}{c}\text { PE+IS } \\
n / N\end{array}$ & $\begin{array}{c}\text { IS } \\
\mathrm{n} / \mathrm{N}\end{array}$ & $\begin{array}{c}\text { Risk Ratio } \\
\text { M-H, Random, 95\% Cl }\end{array}$ & Weight & $\begin{array}{c}\text { Risk Ratio } \\
\text { M-H, Random, } 95 \% \text { Cl } \\
\end{array}$ \\
\hline \multicolumn{6}{|l|}{ 23.2.1 ESKD } \\
\hline Clark 1984 & $0 / 20$ & $1 / 19$ & r & $3.01 \%$ & $0.32[0.01,7.35]$ \\
\hline Wallace 1998 & $2 / 9$ & $2 / 9$ & & $9.4 \%$ & $1[0.18,5.63]$ \\
\hline Lewis 1992 & $10 / 40$ & $8 / 46$ & - & $32.36 \%$ & $1.44[0.63,3.29]$ \\
\hline Subtotal $(95 \% \mathrm{Cl})$ & 69 & 74 & & $44.77 \%$ & $1.24[0.6,2.57]$ \\
\hline \multicolumn{6}{|c|}{ Heterogeneity: $\mathrm{Tau}^{2}=0 ; \mathrm{Chi}^{2}=0.91, \mathrm{df}=2(\mathrm{P}=0.63) ; \mathrm{I}^{2}=0 \%$} \\
\hline \multicolumn{6}{|c|}{ Test for overall effect: $Z=0.59(P=0.56)$} \\
\hline \multicolumn{6}{|c|}{ 23.2.2 Doubling of serum creatinine } \\
\hline Clark 1984 & $0 / 20$ & $3 / 19$ & - & $3.52 \%$ & $0.14[0.01,2.47]$ \\
\hline Clark 1981 & $0 / 6$ & $2 / 6$ & & $3.64 \%$ & $0.2[0.01,3.46]$ \\
\hline \multicolumn{6}{|c|}{ Total events: 0 (PE+IS), 5 (IS) } \\
\hline \multicolumn{6}{|c|}{ Heterogeneity: $\mathrm{Tau}^{2}=0 ; \mathrm{Chi}^{2}=0.03, \mathrm{df}=1(\mathrm{P}=0.85) ; \mathrm{I}^{2}=0 \%$} \\
\hline \multicolumn{6}{|c|}{ Test for overall effect: $Z=1.73(P=0.08)$} \\
\hline \multicolumn{6}{|c|}{ 23.2.3 Deterioration of kidney function } \\
\hline Clark 1984 & $0 / 20$ & $3 / 19$ & 1 & $3.52 \%$ & $0.14[0.01,2.47]$ \\
\hline Wallace 1998 & $6 / 9$ & $6 / 9$ & & $44.54 \%$ & $1[0.52,1.92]$ \\
\hline Subtotal $(95 \% \mathrm{CI})$ & 29 & 28 & & $48.06 \%$ & $0.53[0.06,4.83]$ \\
\hline \multicolumn{6}{|c|}{ Total events: 6 (PE+IS), 9 (IS) } \\
\hline \multicolumn{6}{|c|}{ Heterogeneity: $\mathrm{Tau}^{2}=1.78 ; \mathrm{Chi}^{2}=2.55, \mathrm{df}=1(\mathrm{P}=0.11) ; \mathrm{I}^{2}=60.75 \%$} \\
\hline \multicolumn{6}{|c|}{ Test for overall effect: $Z=0.57(P=0.57)$} \\
\hline Total $(95 \% \mathrm{Cl})$ & 124 & 127 & & $100 \%$ & $0.89[0.51,1.55]$ \\
\hline \multicolumn{6}{|c|}{ Total events: 18 (PE+IS), 25 (IS) } \\
\hline \multicolumn{6}{|c|}{ Test for overall effect: $Z=0.41(P=0.68)$} \\
\hline Test for subgroup di & $\mathrm{f}=1(\mathrm{P}=0.16)$, & & & & \\
\hline
\end{tabular}

Analysis 23.3. Comparison 23 Plasma exchange (PE) + immunosuppression (IS) versus IS alone, Outcome 3 Stable kidney function.

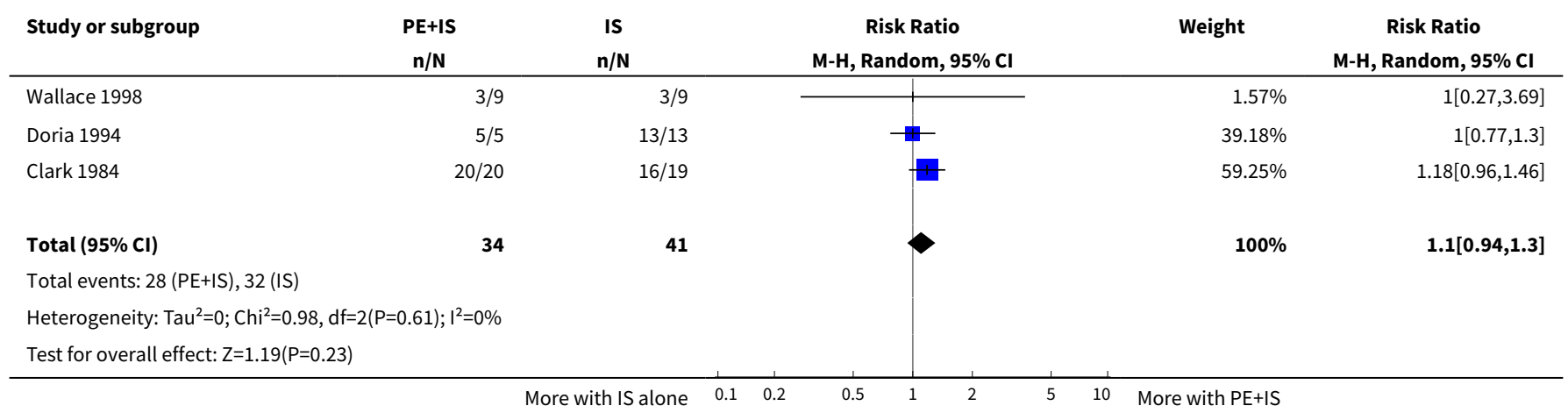


Analysis 23.4. Comparison 23 Plasma exchange (PE) + immunosuppression (IS) versus IS alone, Outcome 4 Infection.

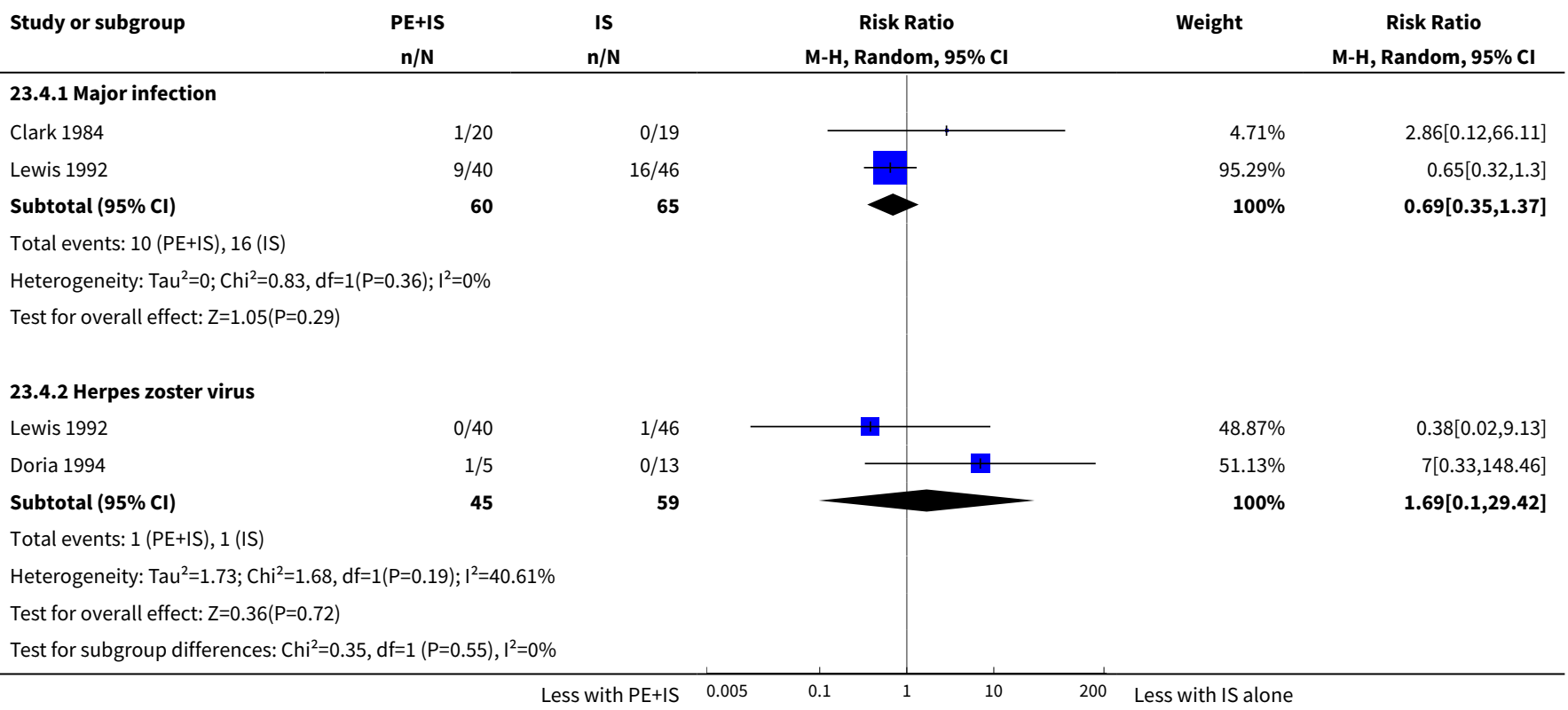

Analysis 23.5. Comparison 23 Plasma exchange (PE) + immunosuppression (IS) versus IS alone, Outcome 5 Leucopenia.

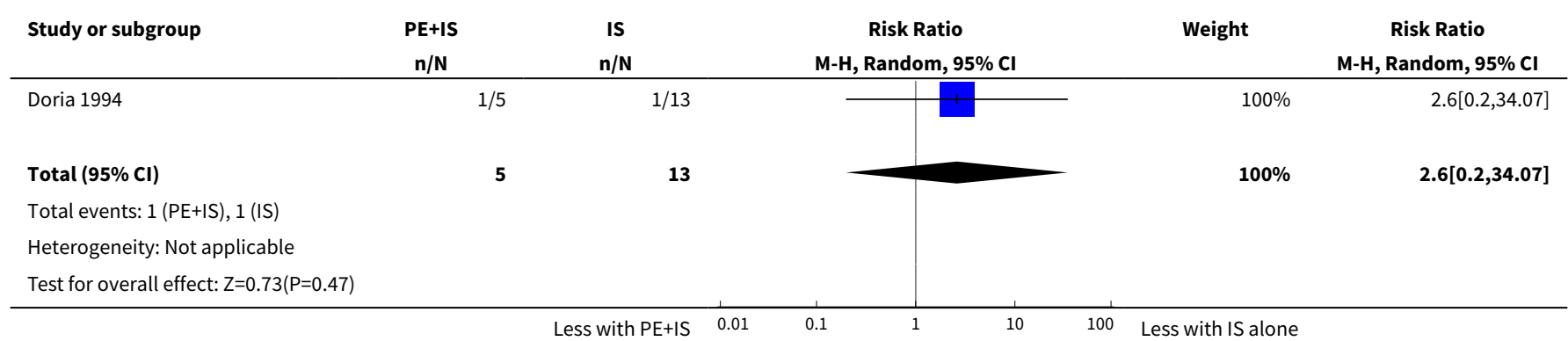

Analysis 23.6. Comparison 23 Plasma exchange (PE) + immunosuppression (IS) versus IS alone, Outcome 6 Daily proteinuria.

\begin{tabular}{|c|c|c|c|c|c|c|c|}
\hline \multirow[t]{2}{*}{ Study or subgroup } & \multicolumn{2}{|c|}{ PE+IS } & \multicolumn{2}{|r|}{ IS } & \multirow{2}{*}{$\begin{array}{l}\text { Mean Difference } \\
\text { Random, } 95 \% \mathrm{Cl}\end{array}$} & \multirow[t]{2}{*}{ Weight } & \multirow{2}{*}{$\begin{array}{l}\text { Mean Difference } \\
\text { Random, } 95 \% \mathrm{CI}\end{array}$} \\
\hline & $\mathbf{N}$ & Mean(SD) & $\mathbf{N}$ & Mean(SD) & & & \\
\hline Clark 1981 & 6 & $7.2(6.1)$ & 6 & $7.3(8.9)$ & & $29.24 \%$ & $-0.1[-8.73,8.53]$ \\
\hline Wallace 1998 & 9 & $4.4(6.5)$ & 9 & $5.2(5.5)$ & +1 & $70.76 \%$ & $-0.75[-6.3,4.8]$ \\
\hline Total $* \star \star$ & 15 & & 15 & & & $100 \%$ & $-0.56[-5.23,4.11]$ \\
\hline \multicolumn{8}{|c|}{ Heterogeneity: $\mathrm{Tau}^{2}=0 ; \mathrm{Chi}^{2}=0.02, \mathrm{df}=1(\mathrm{P}=0.9) ; \mathrm{I}^{2}=0 \%$} \\
\hline
\end{tabular}


Analysis 23.7. Comparison 23 Plasma exchange (PE) + immunosuppression (IS) versus IS alone, Outcome 7 Serum creatinine.

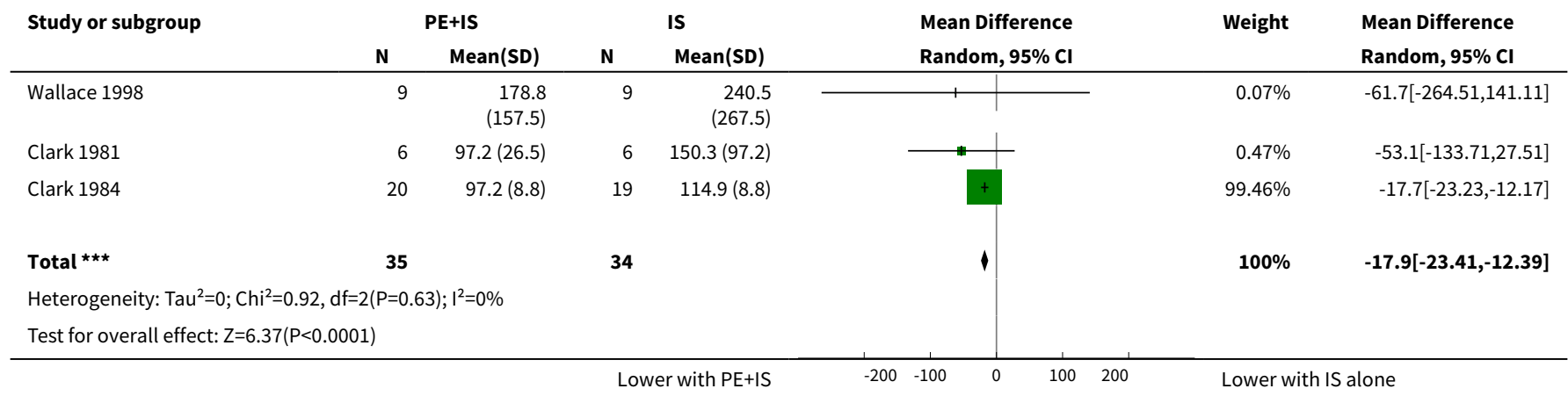

Analysis 23.8. Comparison 23 Plasma exchange (PE) + immunosuppression (IS) versus IS alone, Outcome 8 Creatinine clearance.

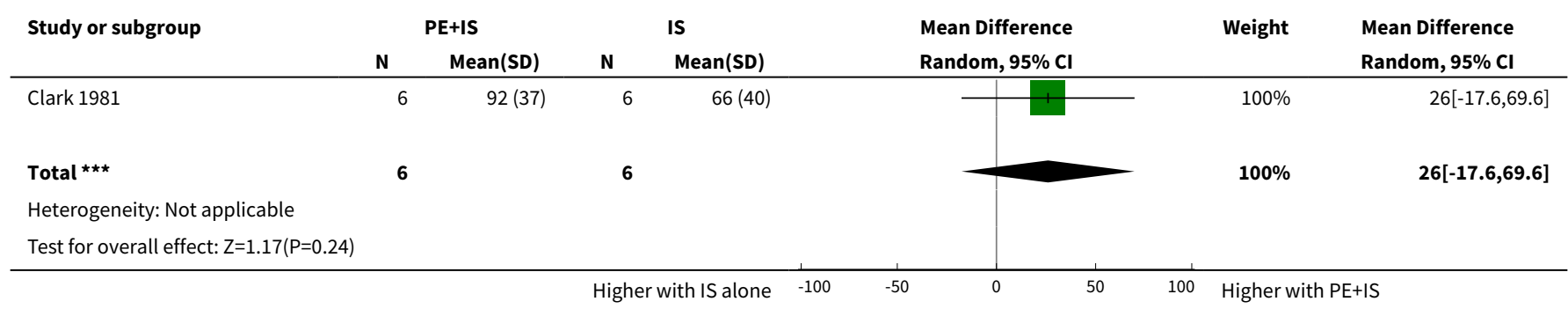

Analysis 23.9. Comparison 23 Plasma exchange (PE) + immunosuppression (IS) versus IS alone, Outcome 9 Disease activity (SLAM).

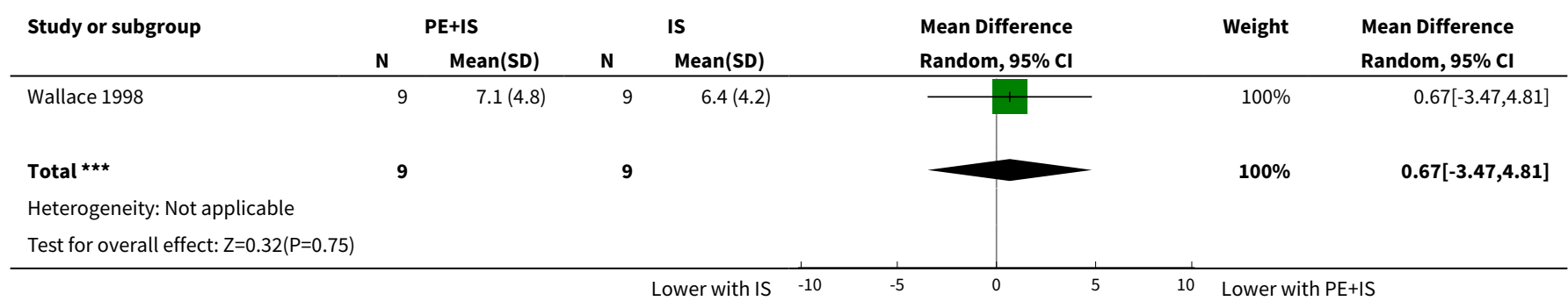

Comparison 24. Plasma exchange (PE) versus immunosuppression (IS)

\begin{tabular}{lllll}
\hline $\begin{array}{l}\text { Outcome or subgroup } \\
\text { title }\end{array}$ & No. of studies & $\begin{array}{l}\text { No. of partici- } \\
\text { pants }\end{array}$ & Statistical method & Effect size \\
\hline $\begin{array}{l}1 \text { Adverse renal out- } \\
\text { comes }\end{array}$ & 1 & Risk Ratio (M-H, Random, 95\% Cl) & Subtotals only \\
\hline
\end{tabular}




\begin{tabular}{lllll}
\hline $\begin{array}{l}\text { Outcome or subgroup } \\
\text { title }\end{array}$ & No. of studies & $\begin{array}{l}\text { No. of partici- } \\
\text { pants }\end{array}$ & Statistical method & Effect size \\
\hline 1.1 ESKD & 1 & 20 & Risk Ratio (M-H, Random, 95\% Cl) & $0.24[0.01,4.44]$ \\
\hline 2 Infection & 1 & Risk Ratio (M-H, Random, 95\% Cl) & Subtotals only \\
\hline 2.1 Major infection & 1 & 20 & Risk Ratio (M-H, Random, 95\% Cl) & $0.4[0.02,8.78]$ \\
\hline 2.2 Herpes zoster virus & 1 & 20 & Risk Ratio (M-H, Random, 95\% Cl) & $0.24[0.01,4.44]$ \\
\hline 3 Leucopenia & 1 & 20 & Risk Ratio (M-H, Random, 95\% Cl) & $0.0[0.0,0.0]$ \\
\hline 4 Alopecia & 1 & 20 & Mean Difference (IV, Random, 95\% Cl) & $-0.10[-0.45,0.25]$ \\
\hline 5 Daily proteinuria & 1 & 20 & Mean Difference (IV, Random, 95\% Cl) & $15.30[-5.40,36.00]$ \\
\hline 6 Creatinine clearance & 1 & 20 & $0.24[0.01,4.44]$ \\
\hline
\end{tabular}

Analysis 24.1. Comparison 24 Plasma exchange (PE) versus immunosuppression (IS), Outcome 1 Adverse renal outcomes.

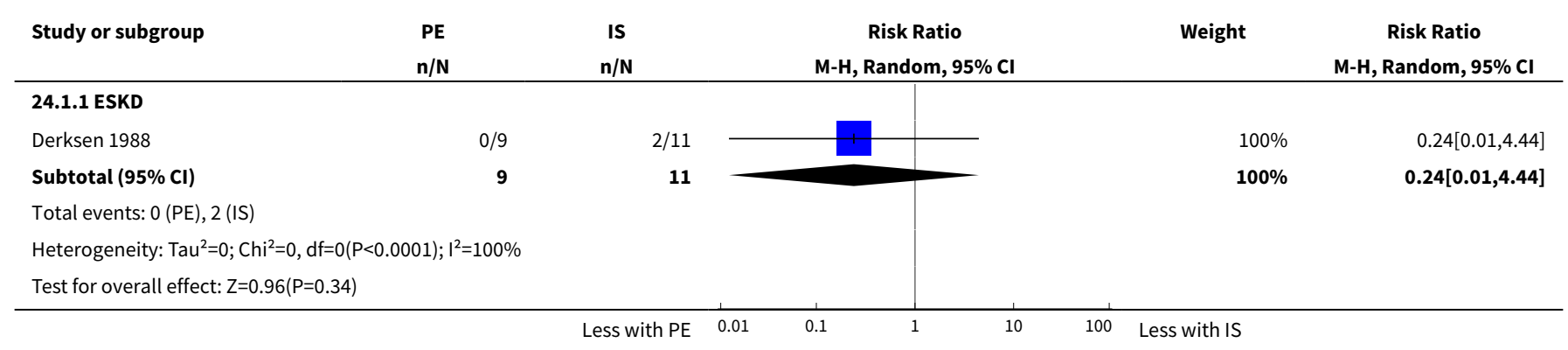

Analysis 24.2. Comparison 24 Plasma exchange (PE) versus immunosuppression (IS), Outcome 2 Infection.

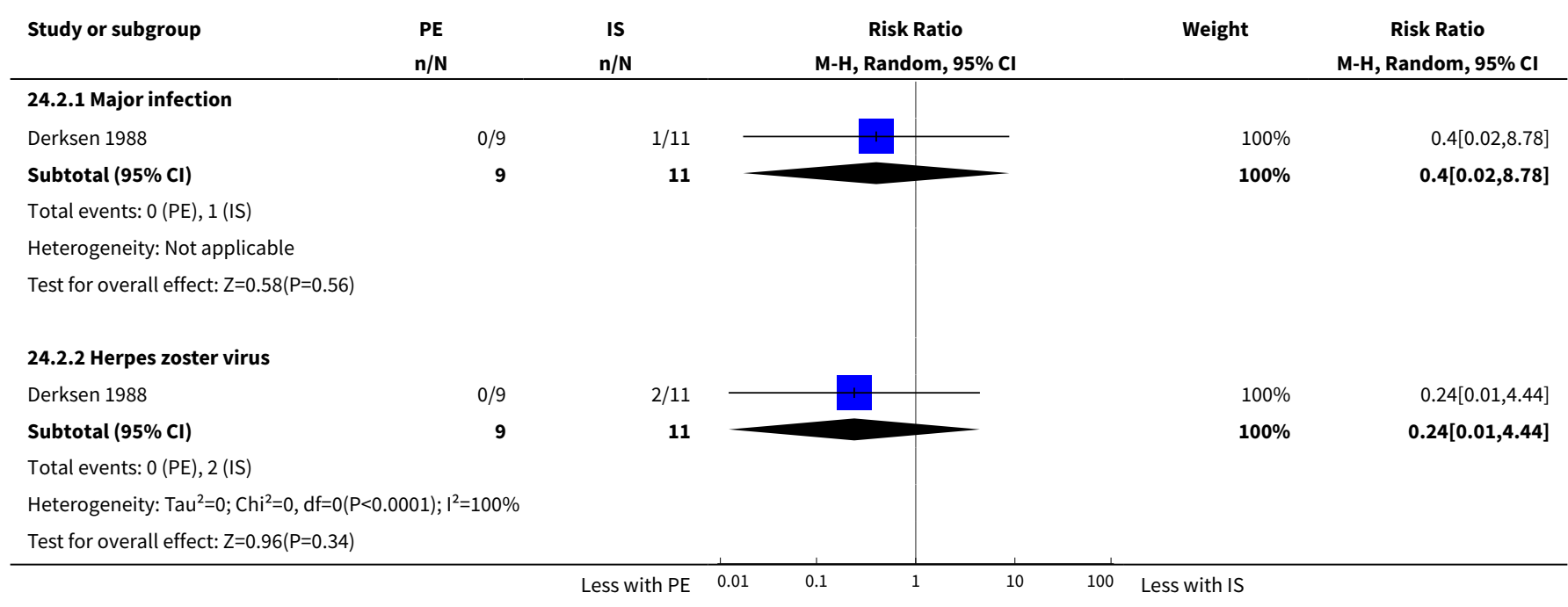


Analysis 24.3. Comparison 24 Plasma exchange (PE) versus immunosuppression (IS), Outcome 3 Leucopenia.

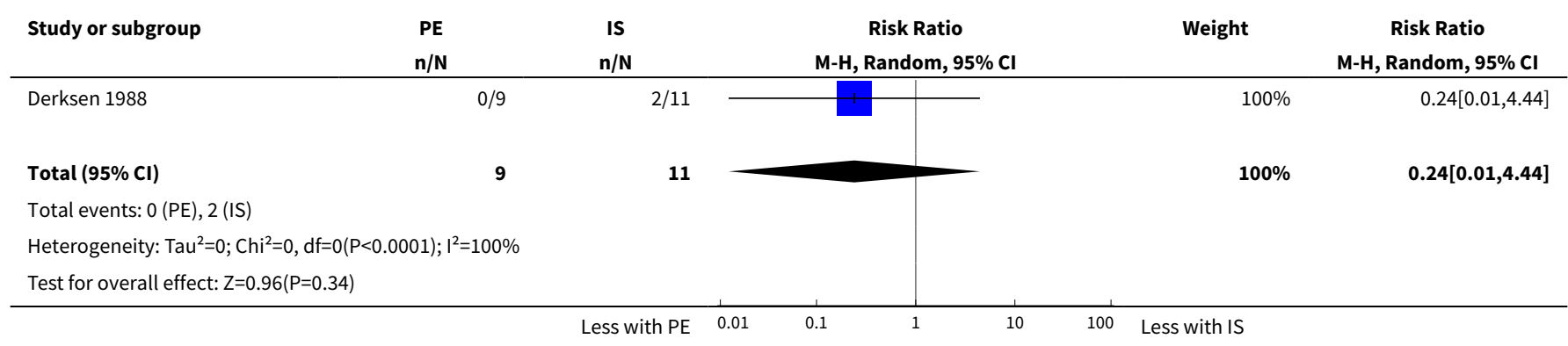

Analysis 24.4. Comparison 24 Plasma exchange (PE) versus immunosuppression (IS), Outcome 4 Alopecia.

\begin{tabular}{|c|c|c|c|c|c|}
\hline Study or subgroup & $\begin{array}{l}\mathrm{PE} \\
\mathrm{n} / \mathrm{N}\end{array}$ & $\begin{array}{l}\text { IS } \\
\mathrm{n} / \mathrm{N}\end{array}$ & $\begin{array}{c}\text { Risk Ratio } \\
\text { M-H, Random, } 95 \% \mathrm{Cl} \\
\end{array}$ & \multirow[t]{2}{*}{ Weight } & \multirow{2}{*}{\begin{tabular}{c}
\multicolumn{1}{c}{$\begin{array}{c}\text { Risk Ratio } \\
\text { M-H, Random, } 95 \% \mathrm{Cl}\end{array}$} \\
Not estimable
\end{tabular}} \\
\hline Derksen 1988 & $0 / 9$ & $0 / 11$ & & & \\
\hline Total $(95 \% \mathrm{Cl})$ & 9 & 11 & & & Not estimable \\
\hline \multicolumn{6}{|c|}{ Total events: 0 (PE), 0 (IS) } \\
\hline \multicolumn{6}{|c|}{ Heterogeneity: Not applicable } \\
\hline Test for overall effec & & & & & \\
\hline
\end{tabular}

Analysis 24.5. Comparison 24 Plasma exchange (PE) versus immunosuppression (IS), Outcome 5 Daily proteinuria.

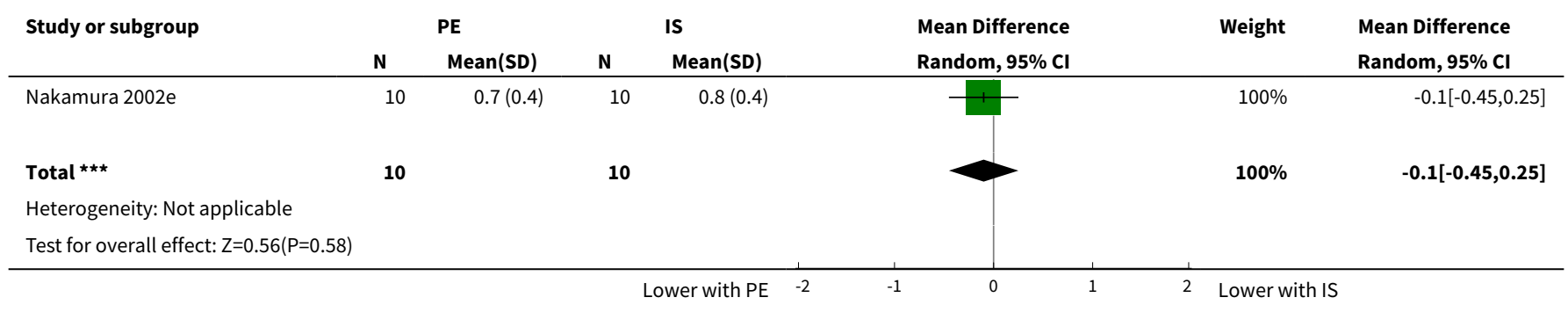

Analysis 24.6. Comparison 24 Plasma exchange (PE) versus immunosuppression (IS), Outcome 6 Creatinine clearance.

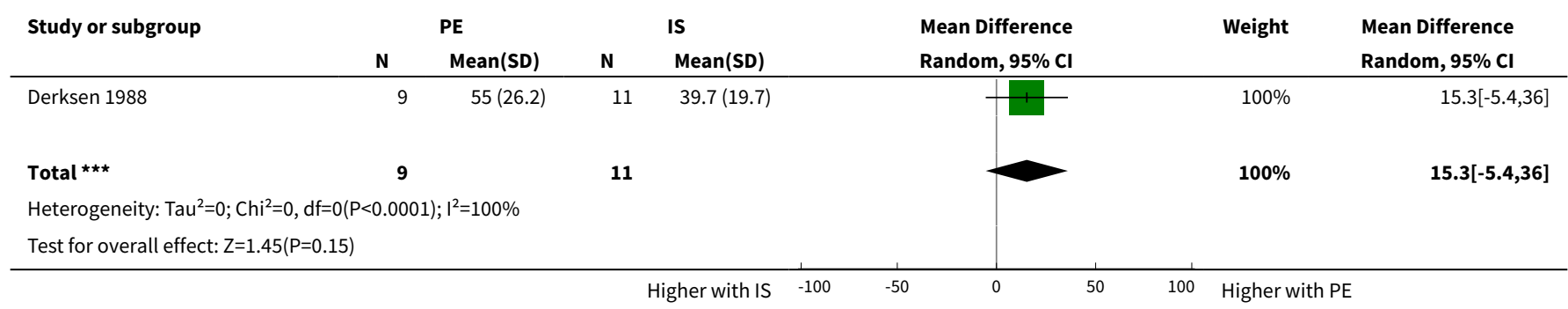


Comparison 25. Long versus short duration cyclophosphamide (CPA)

\begin{tabular}{|c|c|c|c|c|}
\hline $\begin{array}{l}\text { Outcome or subgroup } \\
\text { title }\end{array}$ & No. of studies & $\begin{array}{l}\text { No. of partici- } \\
\text { pants }\end{array}$ & Statistical method & Effect size \\
\hline $\begin{array}{l}1 \text { Adverse renal out- } \\
\text { comes }\end{array}$ & 1 & & Risk Ratio (M-H, Random, 95\% Cl) & Subtotals only \\
\hline $1.1 \mathrm{ESKD}$ & 1 & 40 & Risk Ratio (M-H, Random, 95\% Cl) & $0.4[0.09,1.83]$ \\
\hline $\begin{array}{l}1.2 \text { Doubling of serum } \\
\text { creatinine }\end{array}$ & 1 & 40 & Risk Ratio (M-H, Random, 95\% Cl) & $0.43[0.13,1.43]$ \\
\hline $\begin{array}{l}1.3 \text { Deterioration of kid- } \\
\text { ney function }\end{array}$ & 1 & 40 & Risk Ratio (M-H, Random, 95\% Cl) & $0.43[0.13,1.43]$ \\
\hline 2 Stable kidney function & 1 & 40 & Risk Ratio (M-H, Random, 95\% Cl) & $1.31[0.90,1.89]$ \\
\hline 3 Ovarian failure & 1 & 29 & Risk Ratio (M-H, Random, 95\% Cl) & $2.05[0.60,7.02]$ \\
\hline 4 Infection & 1 & & Risk Ratio (M-H, Random, 95\% Cl) & Subtotals only \\
\hline 4.1 Major infection & 1 & 40 & Risk Ratio (M-H, Random, 95\% Cl) & $1.0[0.07,14.90]$ \\
\hline 4.2 Herpes zoster virus & 1 & 40 & Risk Ratio (M-H, Random, 95\% Cl) & $0.5[0.05,5.08]$ \\
\hline 5 Malignancy & 1 & 40 & Risk Ratio (M-H, Random, 95\% Cl) & $3.0[0.13,69.52]$ \\
\hline 6 Bone toxicity & 1 & 40 & Risk Ratio (M-H, Random, 95\% Cl) & $1.33[0.34,5.21]$ \\
\hline 7 Bladder toxicity & 1 & 40 & Risk Ratio (M-H, Random, 95\% Cl) & $0.0[0.0,0.0]$ \\
\hline
\end{tabular}

Analysis 25.1. Comparison 25 Long versus short duration cyclophosphamide (CPA), Outcome 1 Adverse renal outcomes.

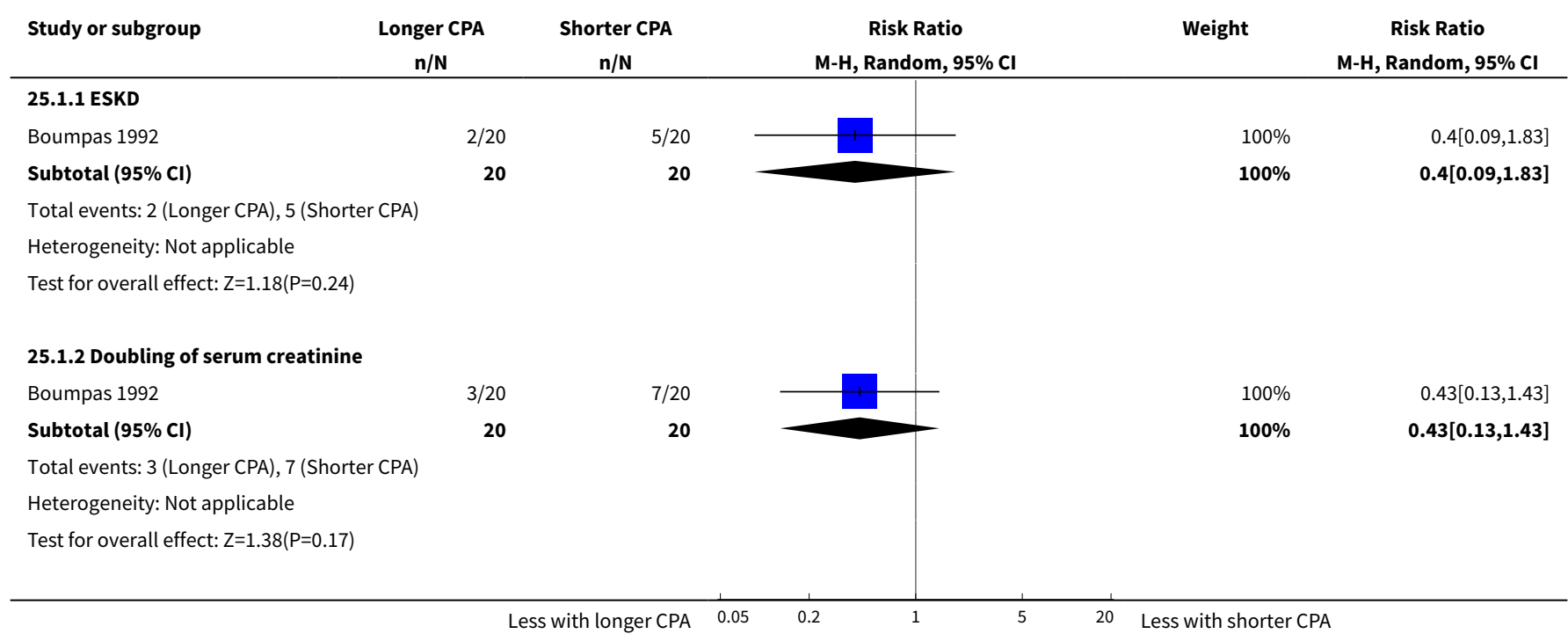




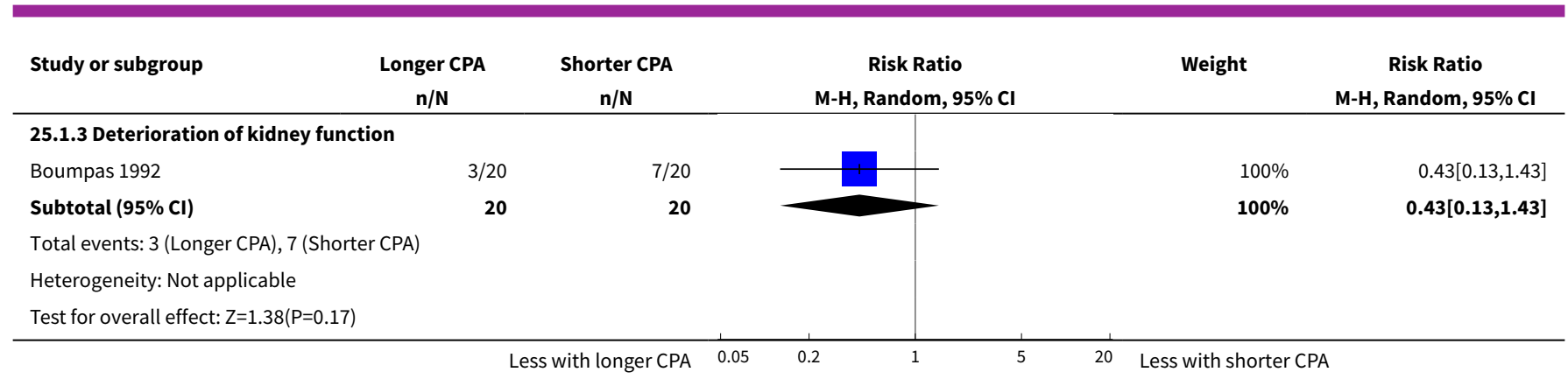

Analysis 25.2. Comparison 25 Long versus short duration cyclophosphamide (CPA), Outcome 2 Stable kidney function.

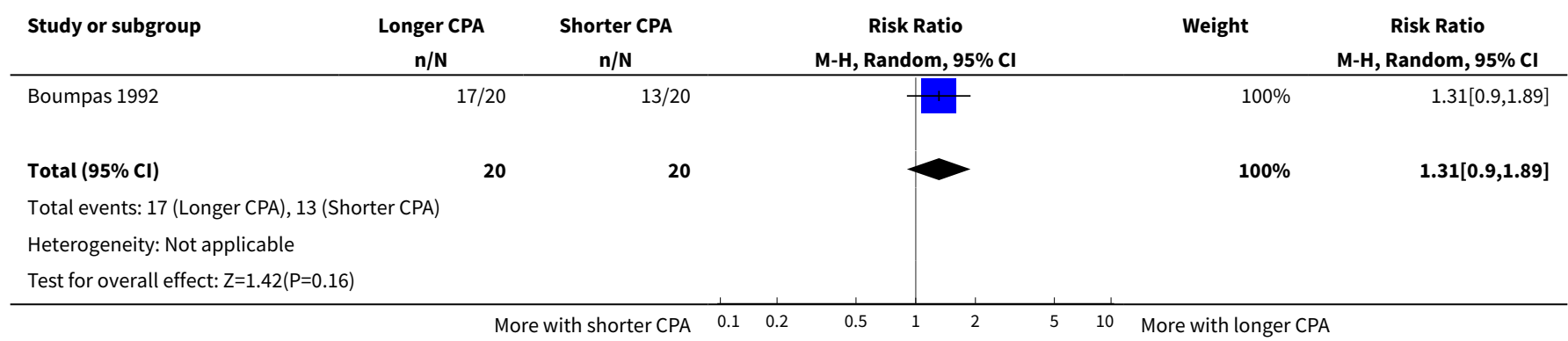

Analysis 25.3. Comparison 25 Long versus short duration cyclophosphamide (CPA), Outcome 3 Ovarian failure.

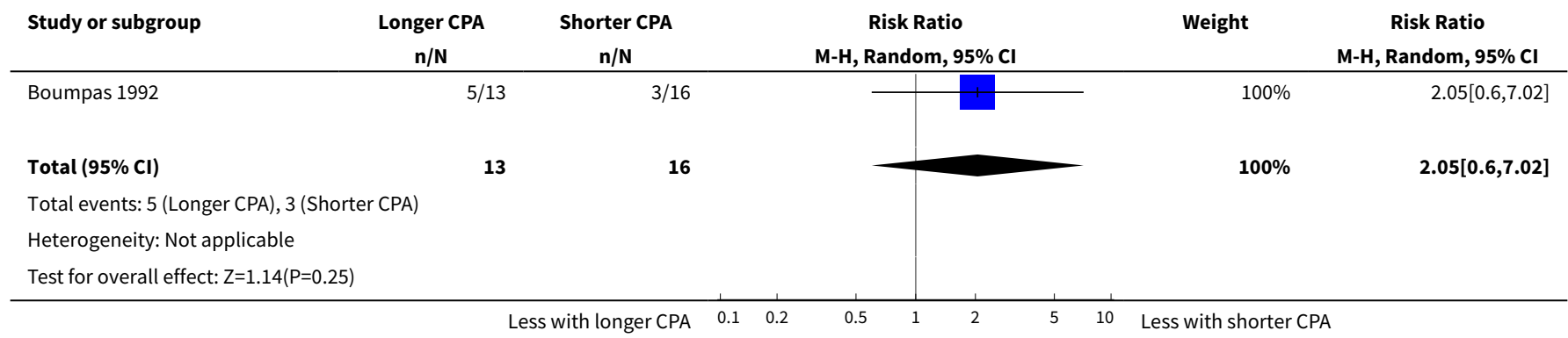

Analysis 25.4. Comparison 25 Long versus short duration cyclophosphamide (CPA), Outcome 4 Infection.

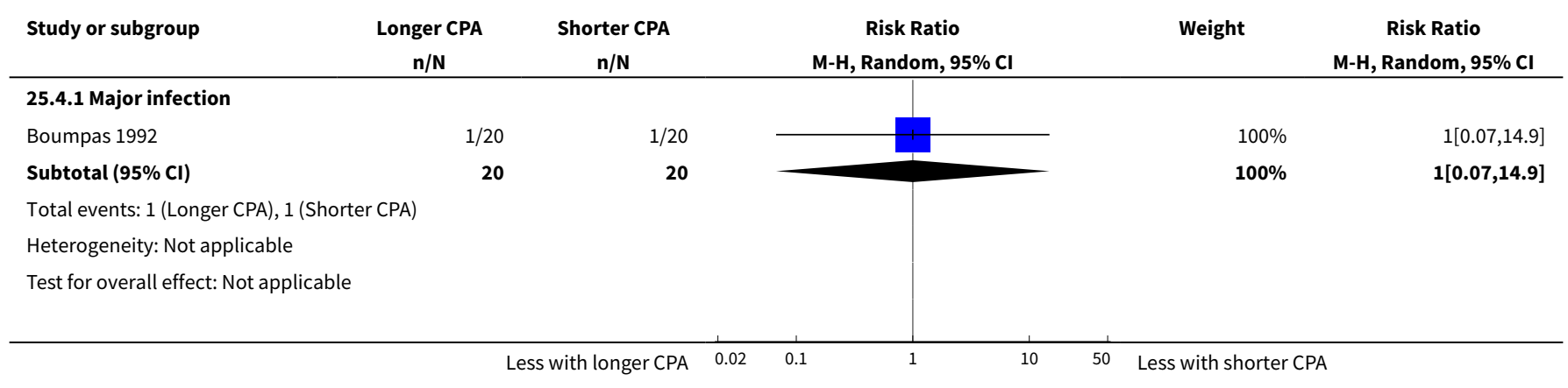




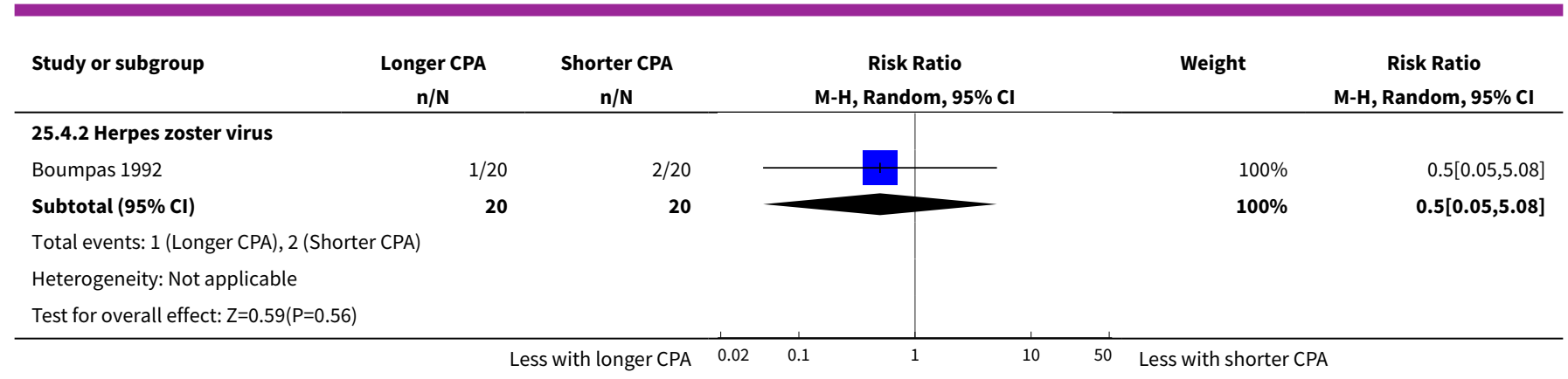

Analysis 25.5. Comparison 25 Long versus short duration cyclophosphamide (CPA), Outcome 5 Malignancy.

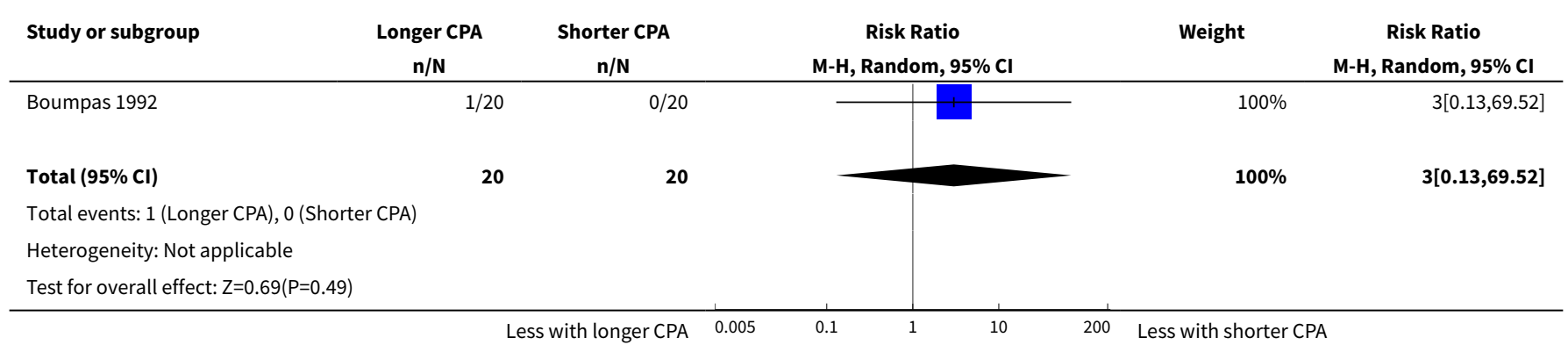

Analysis 25.6. Comparison 25 Long versus short duration cyclophosphamide (CPA), Outcome 6 Bone toxicity.

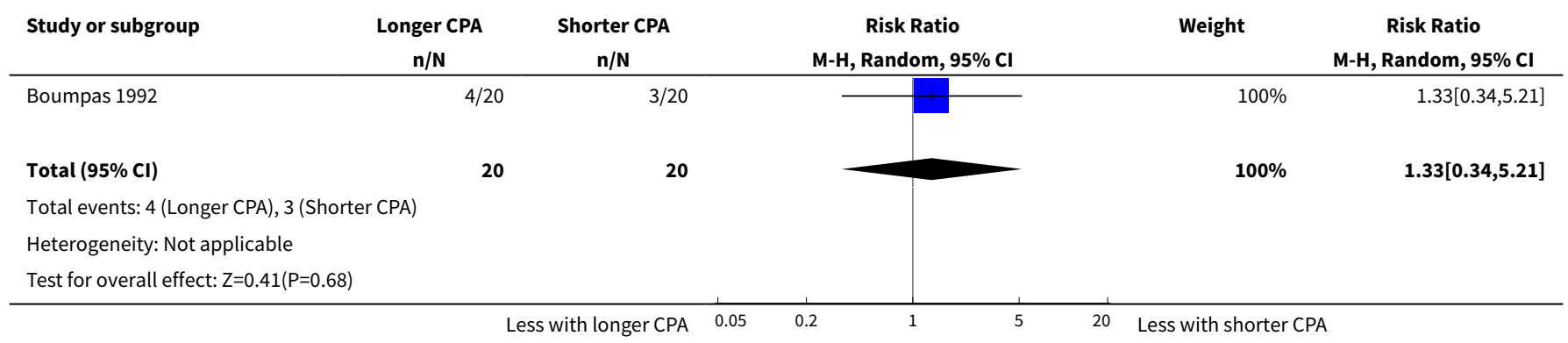

Analysis 25.7. Comparison 25 Long versus short duration cyclophosphamide (CPA), Outcome 7 Bladder toxicity.

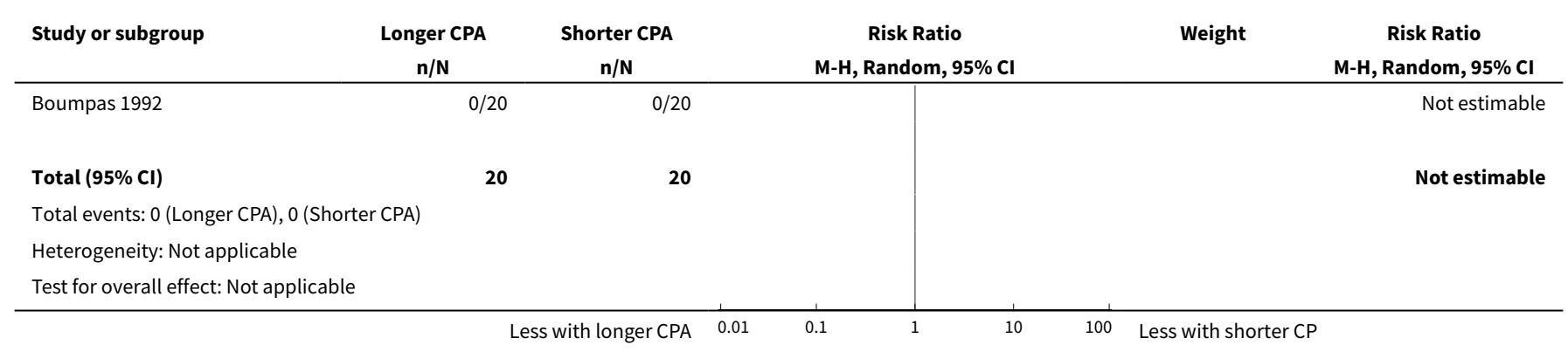


Comparison 26. Maintenance: azathioprine (AZA) versus mycophenolate mofetil (MMF)

\begin{tabular}{|c|c|c|c|c|}
\hline $\begin{array}{l}\text { Outcome or subgroup ti- } \\
\text { tle }\end{array}$ & No. of studies & $\begin{array}{l}\text { No. of partici- } \\
\text { pants }\end{array}$ & Statistical method & Effect size \\
\hline 1 Death & 4 & & Risk Ratio (M-H, Random, 95\% Cl) & Subtotals only \\
\hline $\begin{array}{l}\text { 1.1 At end of treatment du- } \\
\text { ration or follow-up }\end{array}$ & 4 & 451 & Risk Ratio (M-H, Random, 95\% Cl) & $1.15[0.34,3.87]$ \\
\hline 1.2 At 10 years & 1 & 87 & Risk Ratio (M-H, Random, 95\% Cl) & $0.62[0.11,3.54]$ \\
\hline 2 Renal relapse & 4 & & Risk Ratio (M-H, Random, 95\% Cl) & Subtotals only \\
\hline $\begin{array}{l}2.1 \text { At end of treatment du- } \\
\text { ration or follow-up }\end{array}$ & 4 & 452 & Risk Ratio (M-H, Random, 95\% Cl) & $1.75[1.20,2.55]$ \\
\hline 2.2 At 10 years & 1 & 87 & Risk Ratio (M-H, Random, 95\% Cl) & $1.08[0.69,1.69]$ \\
\hline 3 End-stage kidney disease & 4 & & Risk Ratio (M-H, Random, 95\% Cl) & Subtotals only \\
\hline $\begin{array}{l}3.1 \text { At end of treatment du- } \\
\text { ration or follow-up }\end{array}$ & 4 & 452 & Risk Ratio (M-H, Random, 95\% Cl) & $1.70[0.52,5.54]$ \\
\hline 3.2 At 10 years & 1 & 87 & Risk Ratio (M-H, Random, 95\% Cl) & $0.31[0.03,2.88]$ \\
\hline $\begin{array}{l}4 \text { Doubling of serum crea- } \\
\text { tinine }\end{array}$ & 4 & 452 & Risk Ratio (M-H, Random, 95\% Cl) & $2.19[1.03,4.66]$ \\
\hline 5 Ovarian failure & 2 & 177 & Risk Ratio (M-H, Random, 95\% Cl) & $0.77[0.17,3.42]$ \\
\hline 6 Infection & 3 & & Risk Ratio (M-H, Random, 95\% Cl) & Subtotals only \\
\hline 6.1 Major infection & 3 & 412 & Risk Ratio (M-H, Random, 95\% Cl) & $1.08[0.60,1.96]$ \\
\hline 6.2 Herpes zoster virus & 1 & 105 & Risk Ratio (M-H, Random, 95\% Cl) & $1.27[0.36,4.48]$ \\
\hline 7 Malignancy & 3 & & Risk Ratio (M-H, Random, 95\% Cl) & Subtotals only \\
\hline $\begin{array}{l}7.1 \text { At end of treatment du- } \\
\text { ration or follow-up }\end{array}$ & 3 & 370 & Risk Ratio (M-H, Random, 95\% Cl) & $4.04[0.45,36.07]$ \\
\hline 7.2 At 10 years & 1 & 87 & Risk Ratio (M-H, Random, 95\% Cl) & $1.87[0.18,19.84]$ \\
\hline 8 Leucopenia & 3 & 412 & Risk Ratio (M-H, Random, 95\% Cl) & $5.61[1.68,18.72]$ \\
\hline 9 Bone toxicity & 1 & 105 & Risk Ratio (M-H, Random, 95\% Cl) & $3.06[0.13,73.36]$ \\
\hline 10 Alopecia & 3 & 412 & Risk Ratio (M-H, Random, 95\% Cl) & $0.95[0.46,1.95]$ \\
\hline $\begin{array}{l}11 \text { Gastrointestinal (GI) ad- } \\
\text { verse events }\end{array}$ & 3 & & Risk Ratio (M-H, Random, 95\% Cl) & Subtotals only \\
\hline $11.1 \mathrm{GI}$ symptoms & 1 & 105 & Risk Ratio (M-H, Random, 95\% Cl) & $1.02[0.41,2.51]$ \\
\hline 11.2 Nausea & 2 & 307 & Risk Ratio (M-H, Random, 95\% Cl) & $1.08[0.65,1.80]$ \\
\hline
\end{tabular}




\begin{tabular}{lllll}
\hline $\begin{array}{l}\text { Outcome or subgroup ti- } \\
\text { tle }\end{array}$ & No. of studies & $\begin{array}{l}\text { No. of partici- } \\
\text { pants }\end{array}$ & Statistical method & Effect size \\
\hline 11.3 Diarrhoea & 2 & 307 & Risk Ratio (M-H, Random, 95\% Cl) & $0.74[0.31,1.73]$ \\
\hline 11.4 Vomiting & 2 & 307 & Risk Ratio (M-H, Random, 95\% Cl) & $0.81[0.18,3.62]$ \\
\hline 12 Daily proteinuria & 1 & 81 & Mean Difference (IV, Random, 95\% Cl) & $0.40[-0.53,1.33]$ \\
\hline
\end{tabular}

Analysis 26.1. Comparison 26 Maintenance: azathioprine (AZA) versus mycophenolate mofetil (MMF), Outcome 1 Death.

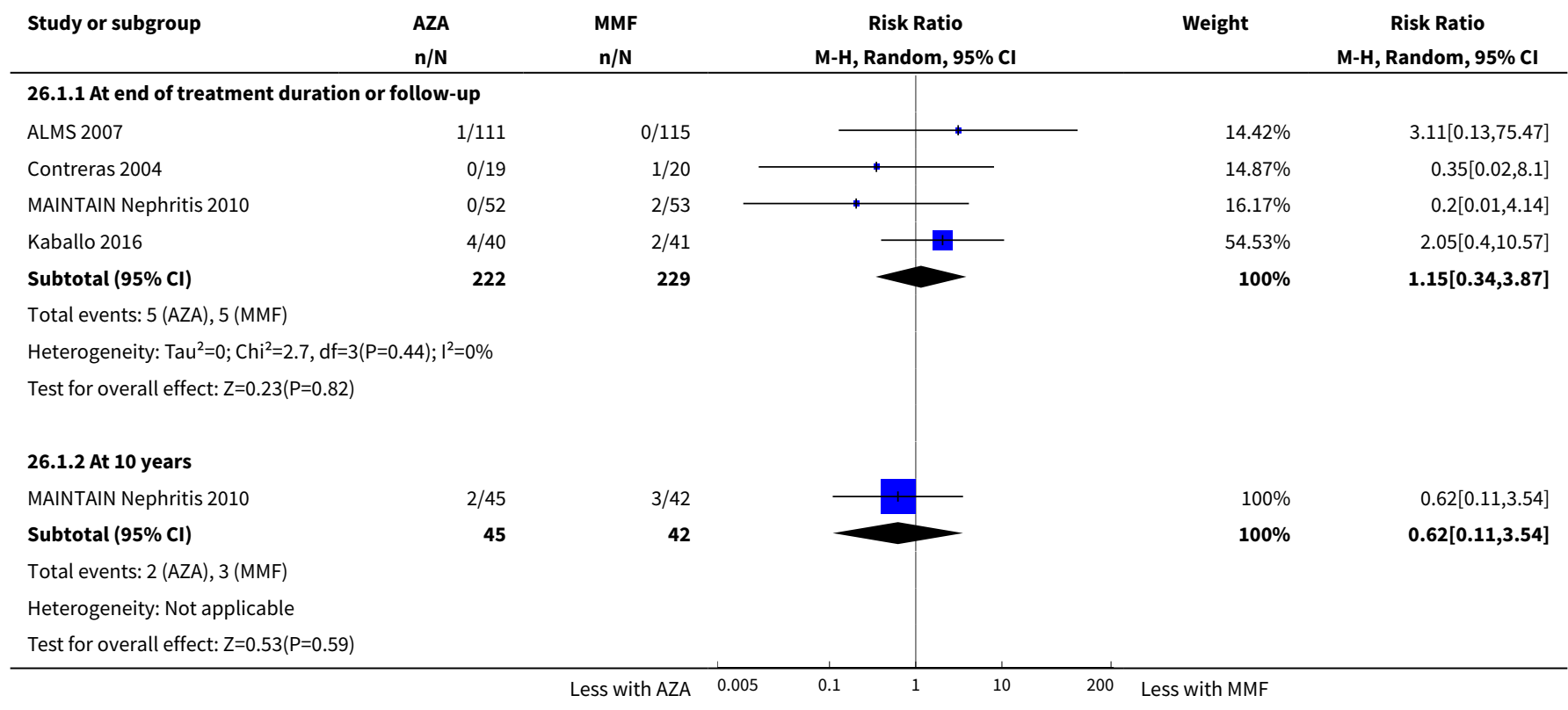

Analysis 26.2. Comparison 26 Maintenance: azathioprine (AZA) versus mycophenolate mofetil (MMF), Outcome 2 Renal relapse.

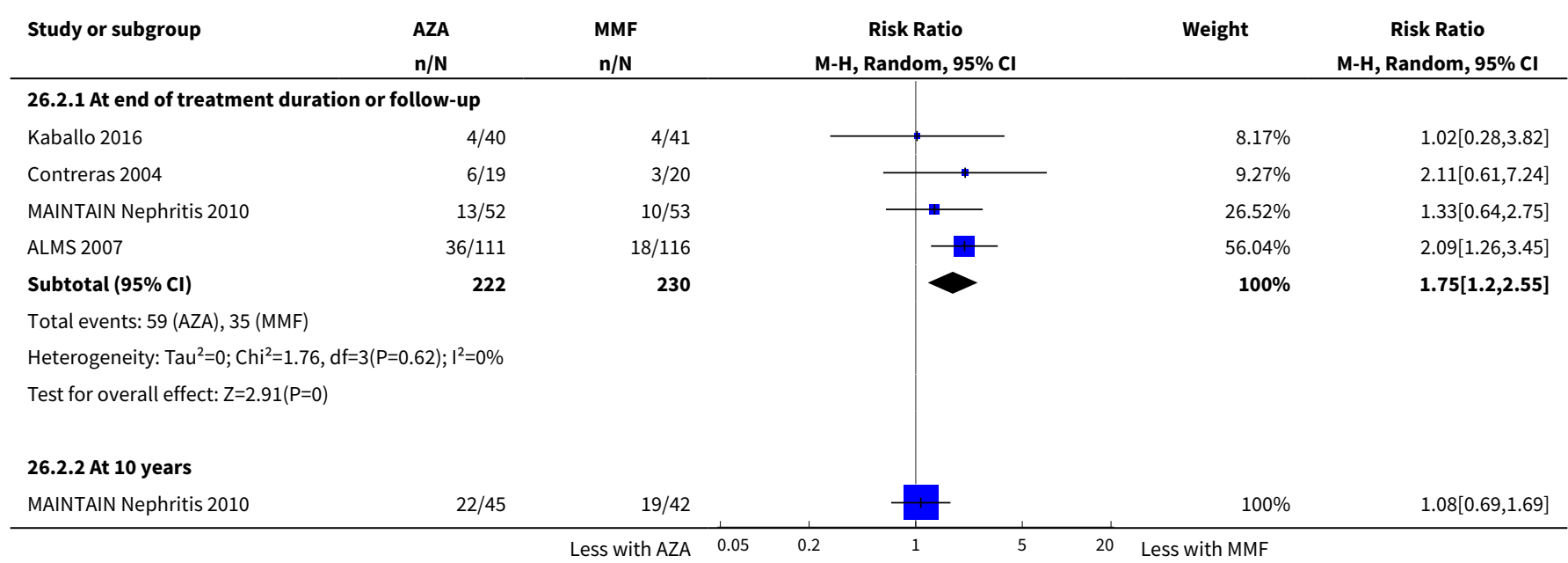




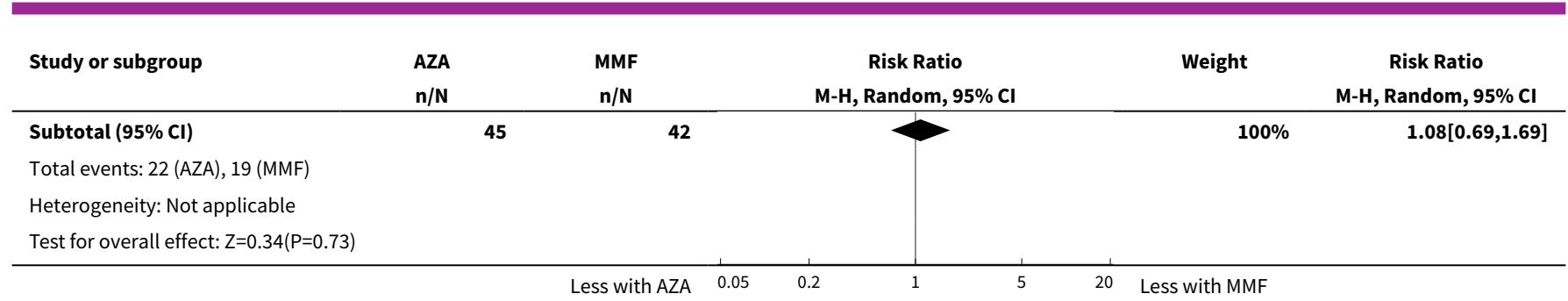

Analysis 26.3. Comparison 26 Maintenance: azathioprine (AZA) versus mycophenolate mofetil (MMF), Outcome 3 End-stage kidney disease.

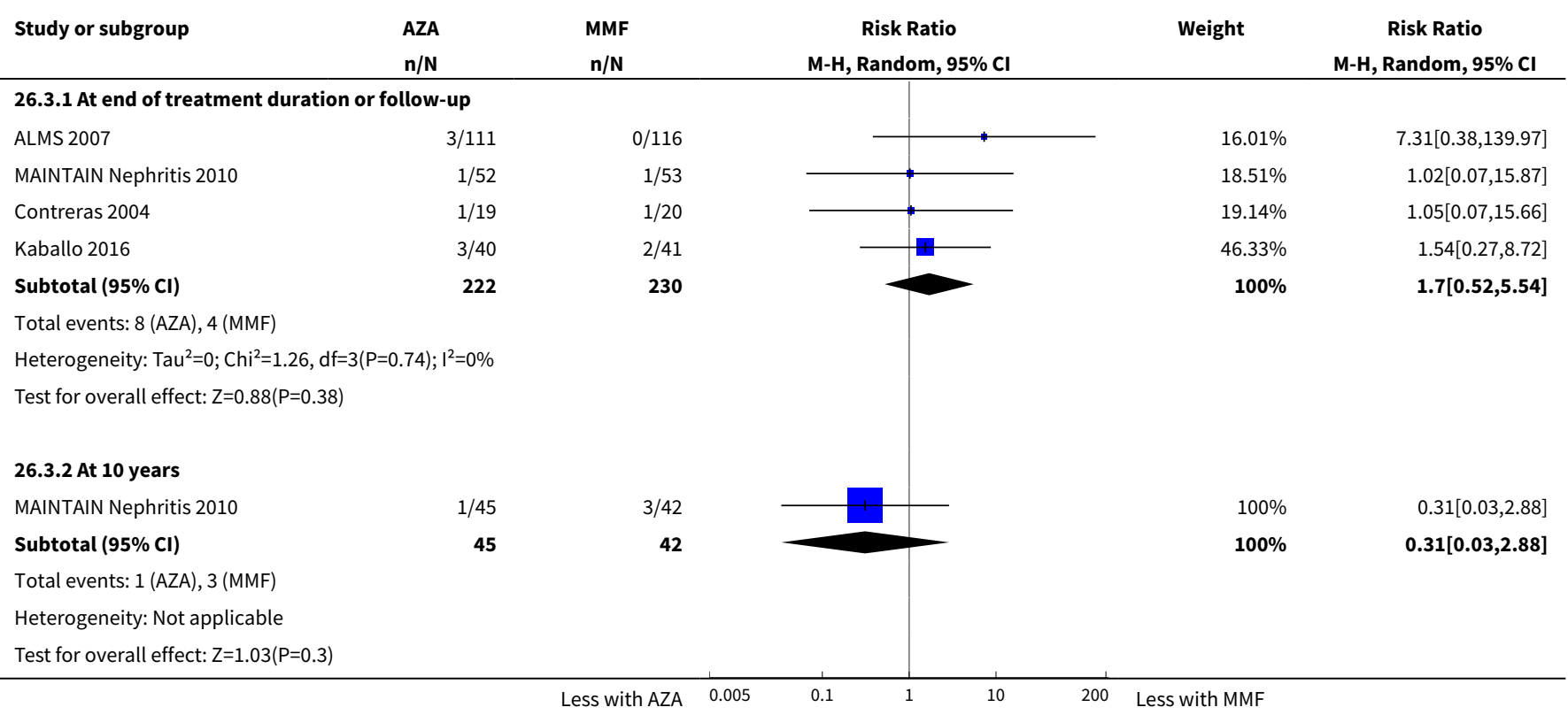

Analysis 26.4. Comparison 26 Maintenance: azathioprine (AZA) versus mycophenolate mofetil (MMF), Outcome 4 Doubling of serum creatinine.

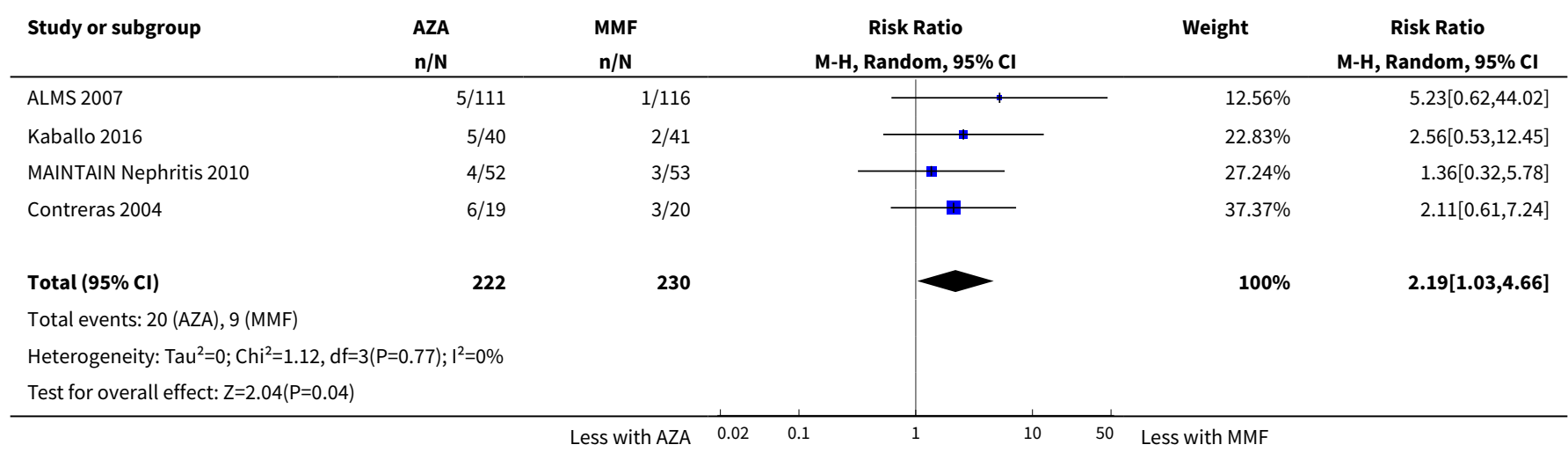


Analysis 26.5. Comparison 26 Maintenance: azathioprine (AZA) versus mycophenolate mofetil (MMF), Outcome 5 Ovarian failure.

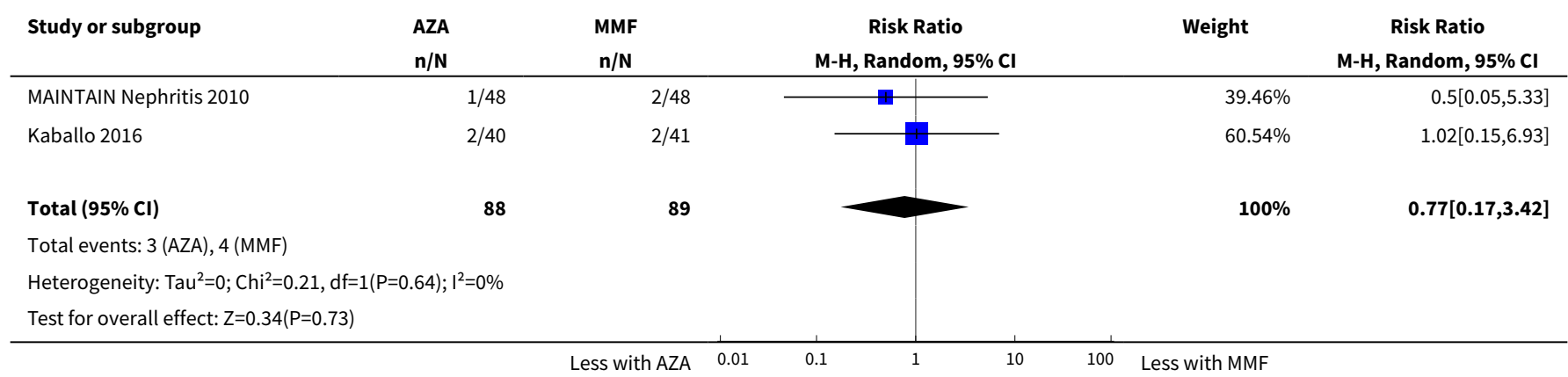

Analysis 26.6. Comparison 26 Maintenance: azathioprine (AZA) versus mycophenolate mofetil (MMF), Outcome 6 Infection.

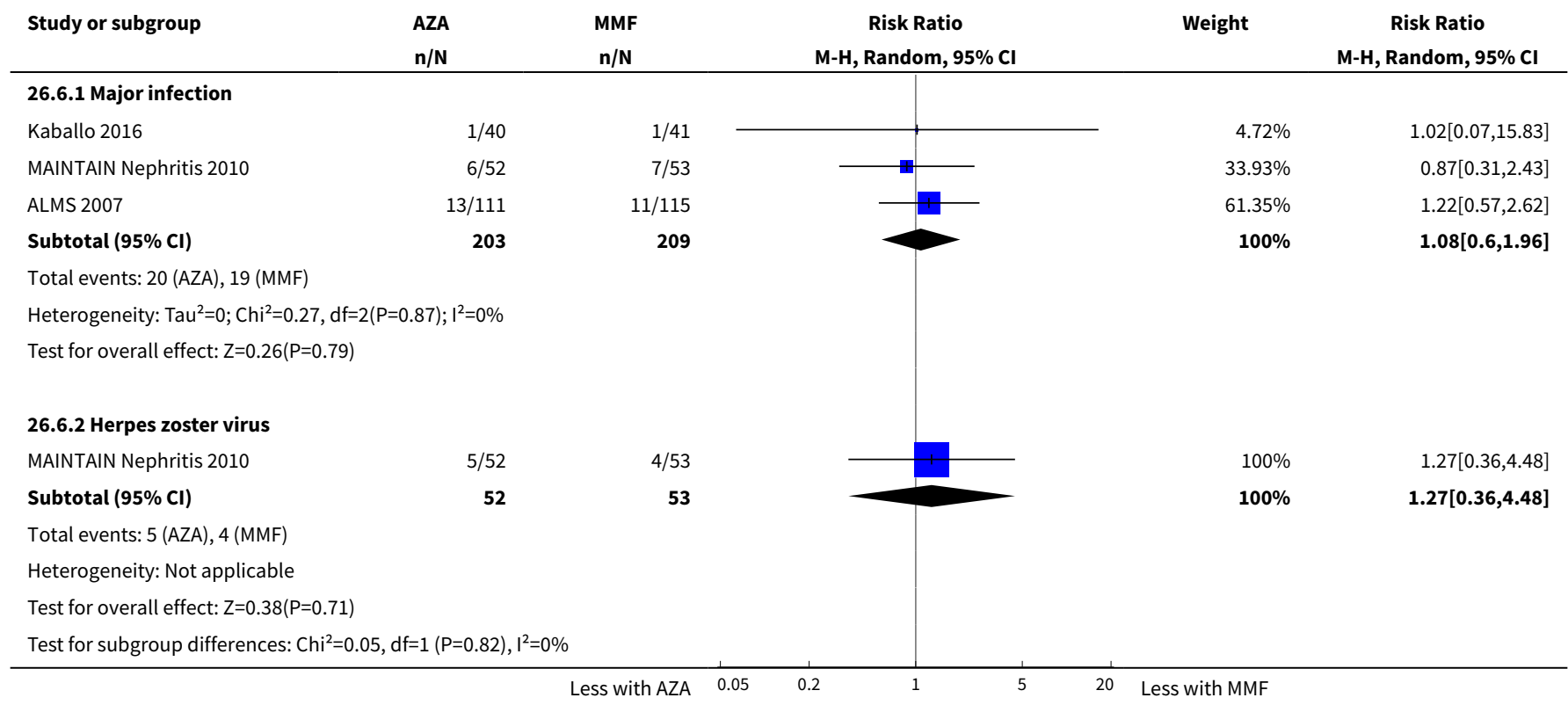

\section{Analysis 26.7. Comparison 26 Maintenance: azathioprine (AZA)} versus mycophenolate mofetil (MMF), Outcome 7 Malignancy.

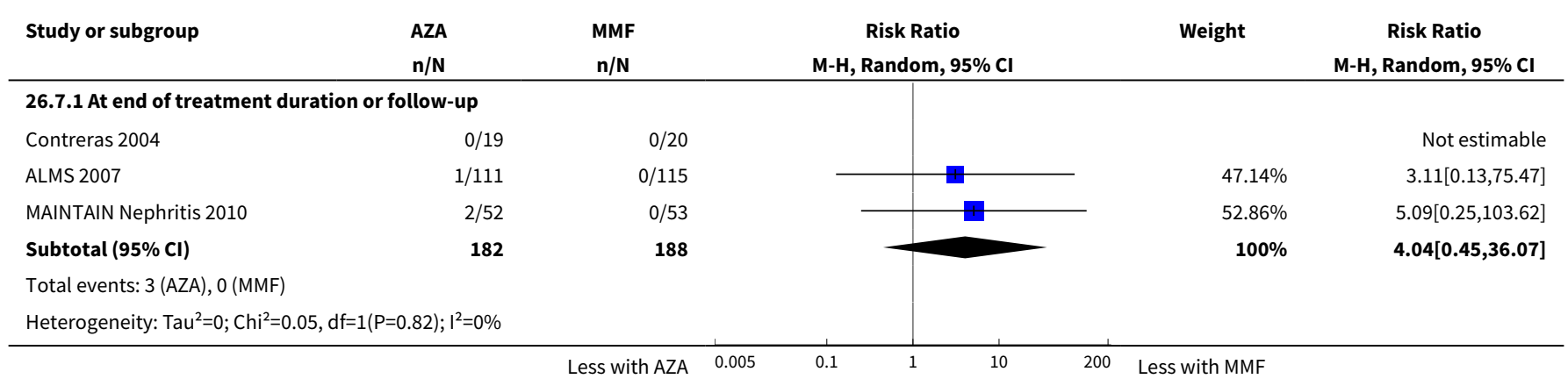




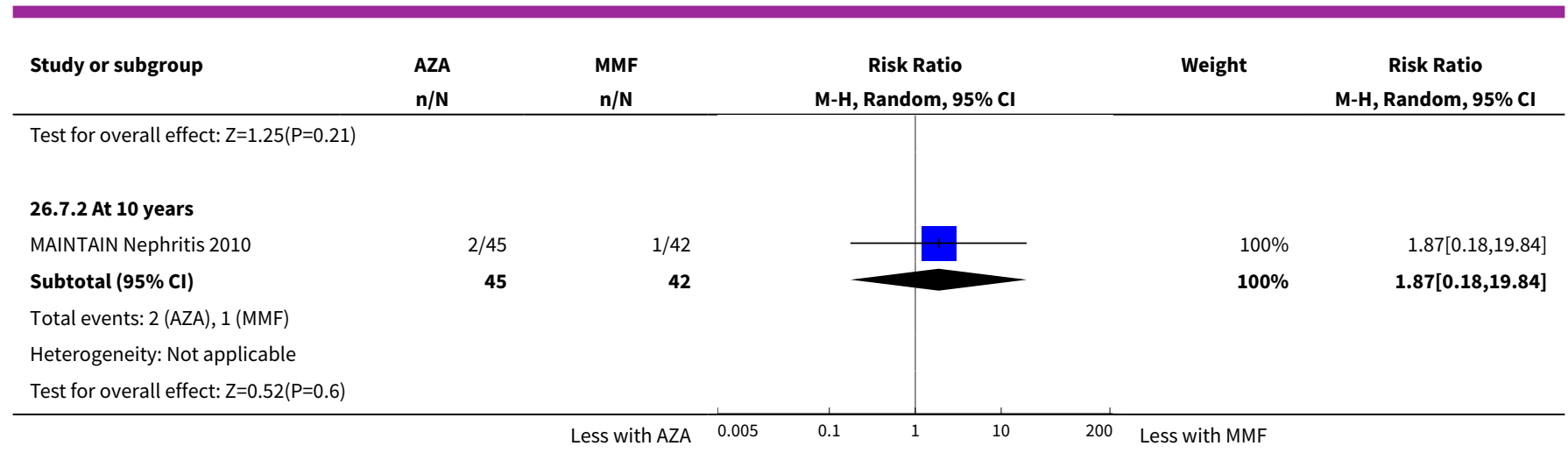

Analysis 26.8. Comparison 26 Maintenance: azathioprine (AZA) versus mycophenolate mofetil (MMF), Outcome 8 Leucopenia.

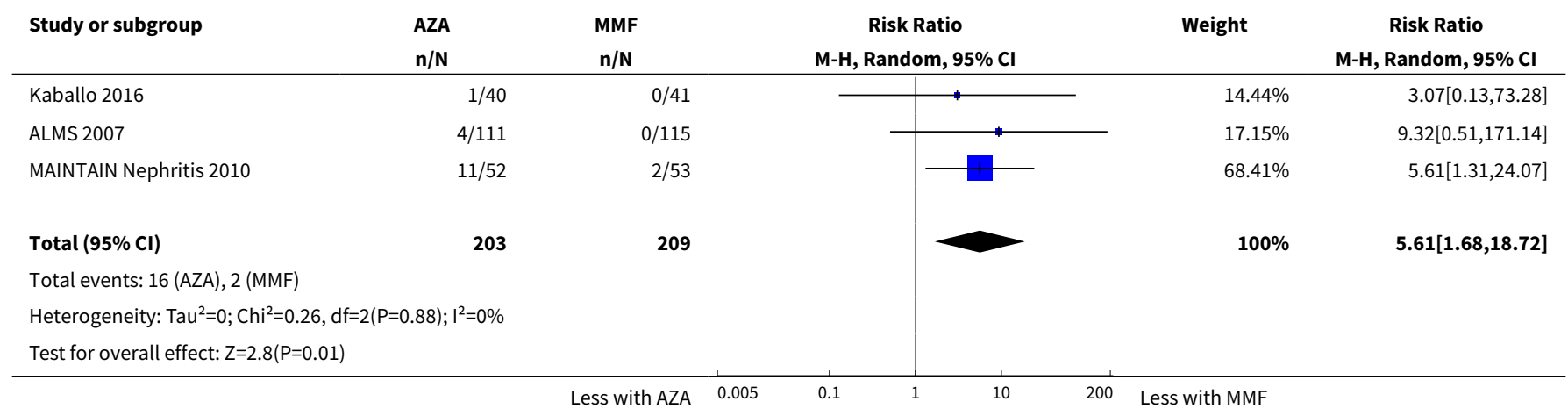

Analysis 26.9. Comparison 26 Maintenance: azathioprine (AZA) versus mycophenolate mofetil (MMF), Outcome 9 Bone toxicity.

\begin{tabular}{|c|c|c|c|c|c|}
\hline Study or subgroup & $\begin{array}{l}\text { AZA } \\
\mathrm{n} / \mathrm{N} \\
\end{array}$ & $\begin{array}{l}\text { MMF } \\
\mathbf{n} / \mathbf{N}\end{array}$ & $\begin{array}{c}\text { Risk Ratio } \\
\text { M-H, Random, 95\% Cl }\end{array}$ & Weight & $\begin{array}{c}\text { Risk Ratio } \\
\text { M-H, Random, } 95 \% \mathrm{CI}\end{array}$ \\
\hline MAINTAIN Nephritis 2010 & $1 / 52$ & $0 / 53$ & 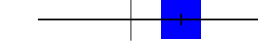 & $100 \%$ & $3.06[0.13,73.36]$ \\
\hline Total $(95 \% \mathrm{Cl})$ & 52 & 53 & & $100 \%$ & $3.06[0.13,73.36]$ \\
\hline \multicolumn{6}{|c|}{ Total events: 1 (AZA), 0 (MMF) } \\
\hline \multicolumn{6}{|c|}{ Heterogeneity: Not applicable } \\
\hline
\end{tabular}

Analysis 26.10. Comparison 26 Maintenance: azathioprine (AZA) versus mycophenolate mofetil (MMF), Outcome 10 Alopecia.

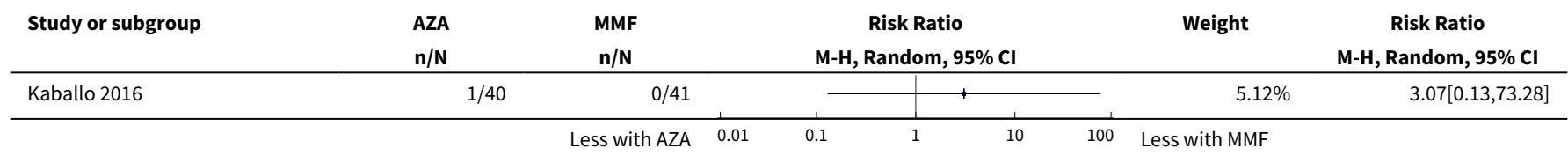




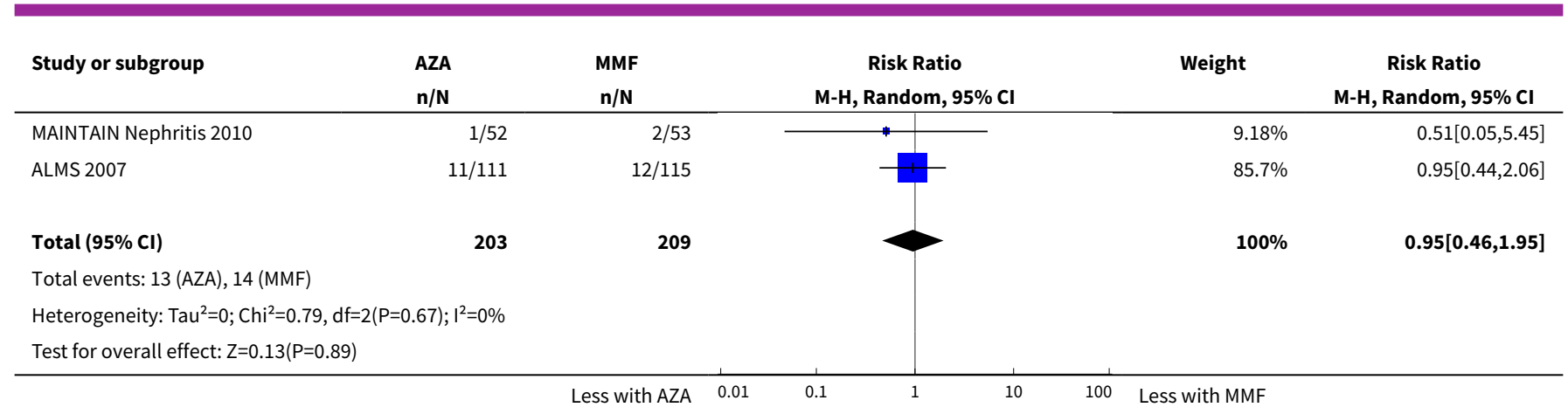

Analysis 26.11. Comparison 26 Maintenance: azathioprine (AZA) versus mycophenolate mofetil (MMF), Outcome 11 Gastrointestinal (GI) adverse events.

\begin{tabular}{|c|c|c|c|c|c|}
\hline Study or subgroup & $\begin{array}{l}\text { AZA } \\
n / N\end{array}$ & $\begin{array}{l}\text { MMF } \\
\text { n/N }\end{array}$ & $\begin{array}{c}\text { Risk Ratio } \\
\text { M-H, Random, } 95 \% \text { Cl }\end{array}$ & Weight & $\begin{array}{c}\text { Risk Ratio } \\
\text { M-H, Random, } 95 \% \text { CI }\end{array}$ \\
\hline \multicolumn{6}{|l|}{ 26.11.1 GI symptoms } \\
\hline MAINTAIN Nephritis 2010 & $8 / 52$ & $8 / 53$ & & $100 \%$ & $1.02[0.41,2.51]$ \\
\hline Subtotal $(95 \% \mathrm{Cl})$ & 52 & 53 & & $100 \%$ & $1.02[0.41,2.51]$ \\
\hline \multicolumn{6}{|c|}{ Total events: 8 (AZA), 8 (MMF) } \\
\hline \multicolumn{6}{|c|}{ Heterogeneity: Not applicable } \\
\hline \multicolumn{6}{|c|}{ Test for overall effect: $Z=0.04(P=0.97)$} \\
\hline \multicolumn{6}{|l|}{ 26.11.2 Nausea } \\
\hline Kaballo 2016 & $4 / 40$ & $4 / 41$ & & $15.06 \%$ & $1.02[0.28,3.82]$ \\
\hline ALMS 2007 & $21 / 111$ & $20 / 115$ & & $84.94 \%$ & $1.09[0.63,1.89]$ \\
\hline Subtotal $(95 \% \mathrm{Cl})$ & 151 & 156 & & $100 \%$ & $1.08[0.65,1.8]$ \\
\hline \multicolumn{6}{|c|}{ Total events: 25 (AZA), 24 (MMF) } \\
\hline \multicolumn{6}{|c|}{ Test for overall effect: $Z=0.29(P=0.77)$} \\
\hline \multicolumn{6}{|l|}{ 26.11.3 Diarrhoea } \\
\hline Kaballo 2016 & $2 / 40$ & $6 / 41$ & & $24.06 \%$ & $0.34[0.07,1.59]$ \\
\hline ALMS 2007 & $20 / 111$ & $22 / 115$ & & $75.94 \%$ & $0.94[0.55,1.63]$ \\
\hline Subtotal $(95 \% \mathrm{CI})$ & 151 & 156 & & $100 \%$ & $0.74[0.31,1.73]$ \\
\hline \multicolumn{6}{|c|}{ Total events: 22 (AZA), 28 (MMF) } \\
\hline \multicolumn{6}{|c|}{ Heterogeneity: $\mathrm{Tau}^{2}=0.17 ; \mathrm{Chi}^{2}=1.5, \mathrm{df}=1(\mathrm{P}=0.22) ; \mathrm{I}^{2}=33.16 \%$} \\
\hline \multicolumn{6}{|c|}{ Test for overall effect: $Z=0.7(P=0.49)$} \\
\hline \multicolumn{6}{|l|}{ 26.11.4 Vomiting } \\
\hline Kaballo 2016 & $1 / 40$ & $4 / 41$ & 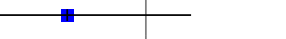 & $30.27 \%$ & $0.26[0.03,2.19]$ \\
\hline ALMS 2007 & $18 / 111$ & $14 / 115$ & & $69.73 \%$ & $1.33[0.7,2.55]$ \\
\hline \multicolumn{6}{|c|}{ Total events: 19 (AZA), 18 (MMF) } \\
\hline \multicolumn{6}{|c|}{ Heterogeneity: Tau $^{2}=0.73 ; \mathrm{Chi}^{2}=2.11, \mathrm{df}=1(\mathrm{P}=0.15) ; \mathrm{I}^{2}=52.64 \%$} \\
\hline \multicolumn{6}{|c|}{ Test for overall effect: $Z=0.28(P=0.78)$} \\
\hline Test for subgroup differen & $f=1(P=0.89)$, & & & & \\
\hline
\end{tabular}


Analysis 26.12. Comparison 26 Maintenance: azathioprine (AZA) versus mycophenolate mofetil (MMF), Outcome 12 Daily proteinuria.

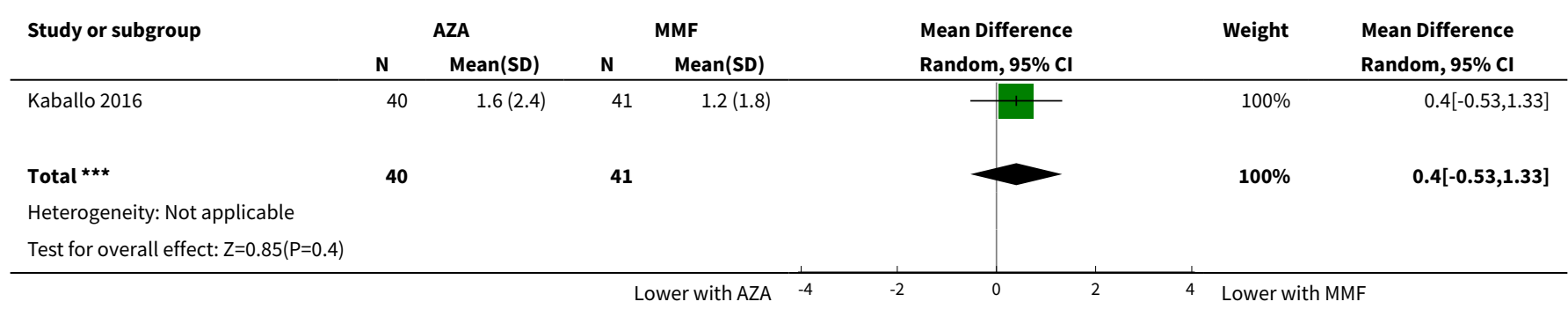

\section{Comparison 27. Maintenance: azathioprine (AZA) versus cyclosporin (CSA)}

\begin{tabular}{|c|c|c|c|c|}
\hline $\begin{array}{l}\text { Outcome or subgroup } \\
\text { title }\end{array}$ & No. of studies & $\begin{array}{l}\text { No. of partici- } \\
\text { pants }\end{array}$ & Statistical method & Effect size \\
\hline 1 Death & 1 & 69 & Risk Ratio (M-H, Random, 95\% Cl) & $0.0[0.0,0.0]$ \\
\hline $\begin{array}{l}2 \text { Adverse renal out- } \\
\text { comes }\end{array}$ & 1 & & Risk Ratio (M-H, Random, 95\% Cl) & Subtotals only \\
\hline 2.1 ESKD & 1 & 69 & Risk Ratio (M-H, Random, 95\% Cl) & $0.0[0.0,0.0]$ \\
\hline 2.2 Renal relapse & 1 & 69 & Risk Ratio (M-H, Random, 95\% Cl) & $1.25[0.51,3.06]$ \\
\hline 3 Infection & 1 & & Risk Ratio (M-H, Random, 95\% Cl) & Subtotals only \\
\hline 3.1 Major infection & 1 & 69 & Risk Ratio (M-H, Random, 95\% Cl) & $2.18[1.01,4.73]$ \\
\hline 4 Leucopenia & 1 & 69 & Risk Ratio (M-H, Random, 95\% Cl) & $2.73[0.95,7.86]$ \\
\hline $\begin{array}{l}5 \text { Gastrointestinal (GI) } \\
\text { adverse events }\end{array}$ & 1 & & Risk Ratio (M-H, Random, 95\% Cl) & Subtotals only \\
\hline $5.1 \mathrm{GI}$ disturbance & 1 & 69 & Risk Ratio (M-H, Random, 95\% Cl) & $0.30[0.09,0.97]$ \\
\hline 6 Daily proteinuria & 1 & 69 & Mean Difference (IV, Random, 95\% CI) & $0.15[-0.23,0.53]$ \\
\hline $\begin{array}{l}7 \text { Disease activity } \\
\text { (SLEDAI) }\end{array}$ & 1 & 69 & Mean Difference (IV, Random, 95\% CI) & $-3.20[-5.77,-0.63]$ \\
\hline
\end{tabular}

Analysis 27.1. Comparison 27 Maintenance: azathioprine (AZA) versus cyclosporin (CSA), Outcome 1 Death.

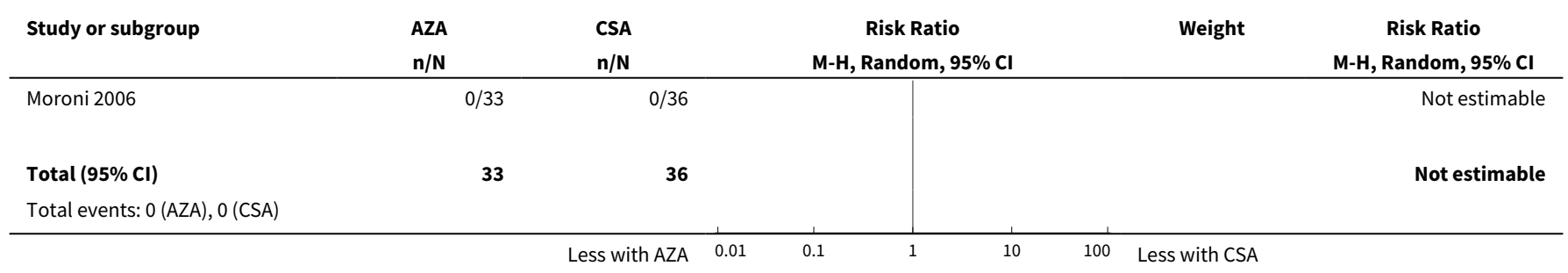




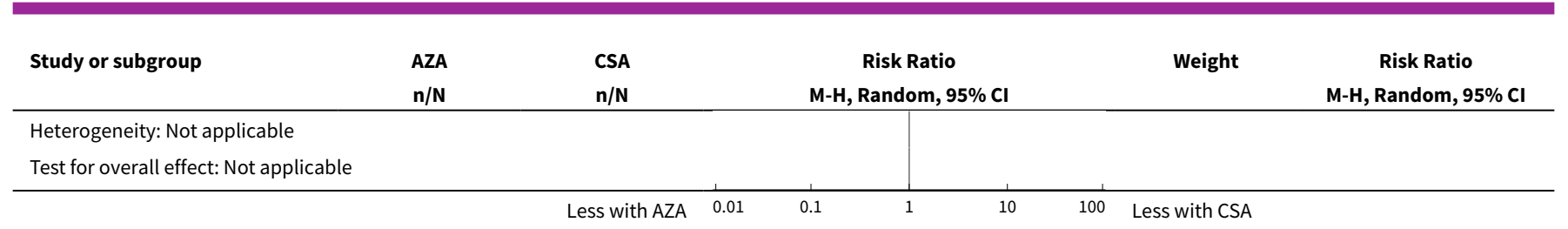

Analysis 27.2. Comparison 27 Maintenance: azathioprine (AZA)
versus cyclosporin (CSA), Outcome 2 Adverse renal outcomes.

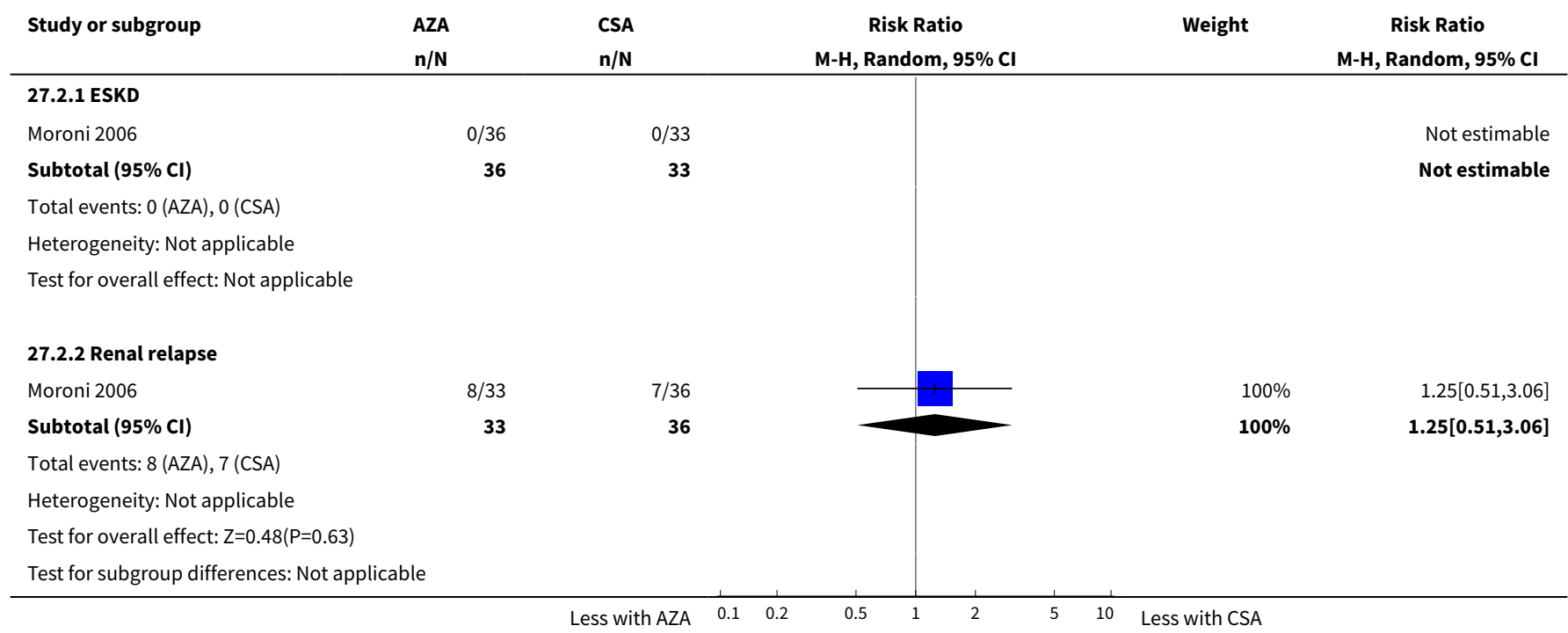

Analysis 27.3. Comparison 27 Maintenance: azathioprine (AZA) versus cyclosporin (CSA), Outcome 3 Infection.

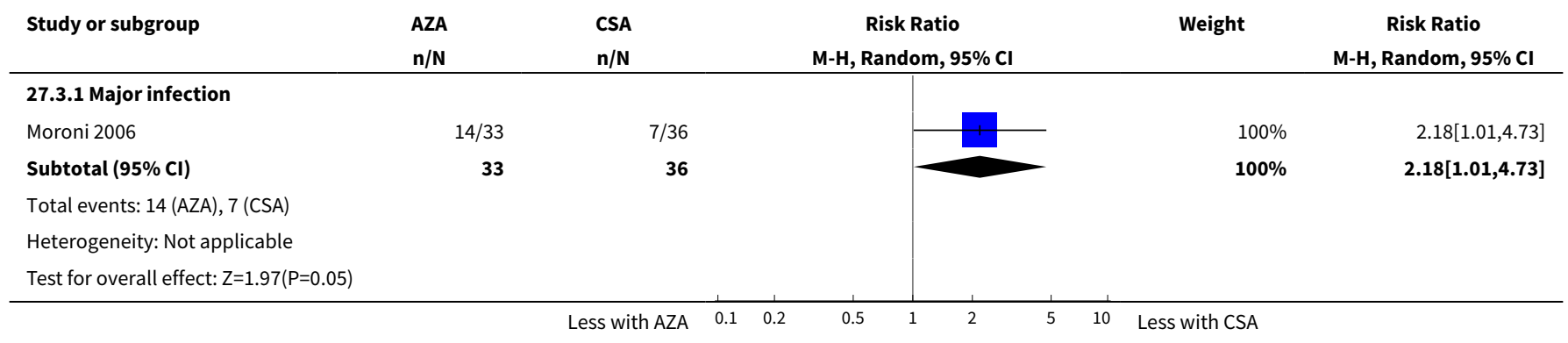

\section{Analysis 27.4. Comparison 27 Maintenance: azathioprine (AZA) versus cyclosporin (CSA), Outcome 4 Leucopenia.}

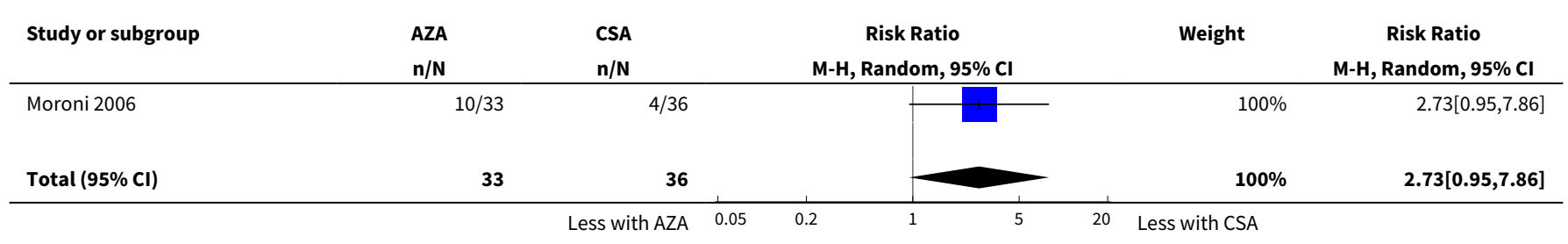




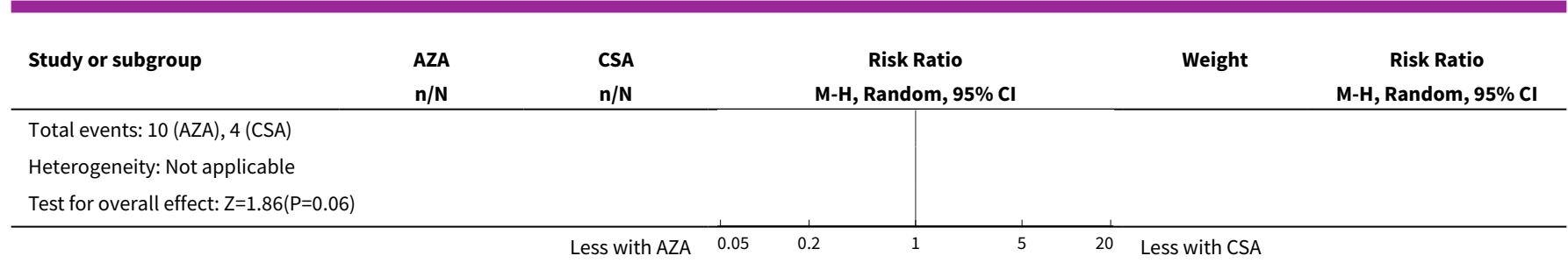

Analysis 27.5. Comparison 27 Maintenance: azathioprine (AZA) versus cyclosporin (CSA), Outcome 5 Gastrointestinal (GI) adverse events.

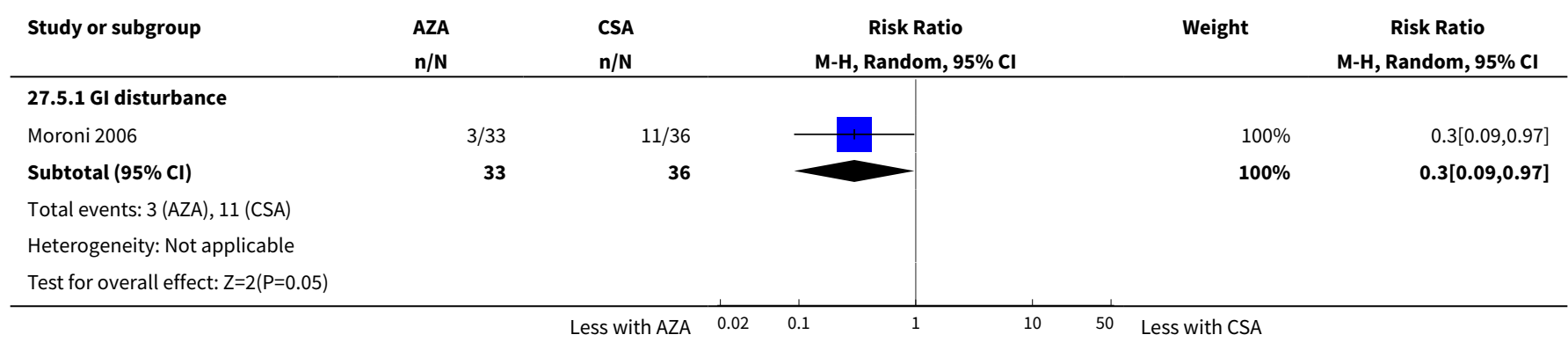

Analysis 27.6. Comparison 27 Maintenance: azathioprine (AZA) versus cyclosporin (CSA), Outcome 6 Daily proteinuria.

\begin{tabular}{|c|c|c|c|c|c|c|c|}
\hline \multirow[t]{2}{*}{ Study or subgroup } & \multicolumn{2}{|c|}{ AZA } & \multicolumn{2}{|c|}{ CSA } & \multirow{2}{*}{$\begin{array}{l}\text { Mean Difference } \\
\text { Random, } 95 \% \mathrm{Cl}\end{array}$} & \multirow[t]{2}{*}{ Weight } & \multirow{2}{*}{$\begin{array}{l}\text { Mean Difference } \\
\text { Random, } 95 \% \mathrm{Cl}\end{array}$} \\
\hline & $\mathbf{N}$ & Mean(SD) & $\mathbf{N}$ & Mean(SD) & & & \\
\hline Moroni 2006 & 33 & $0.5(0.8)$ & 36 & $0.4(0.9)$ & & $100 \%$ & $0.15[-0.23,0.53]$ \\
\hline 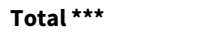 & 33 & & 36 & & & $100 \%$ & $0.15[-0.23,0.53]$ \\
\hline \multicolumn{8}{|c|}{ Heterogeneity: Not applicable } \\
\hline \multicolumn{8}{|c|}{ Test for overall effect: $Z=0.76(P=0.44)$} \\
\hline
\end{tabular}

Analysis 27.7. Comparison 27 Maintenance: azathioprine (AZA) versus cyclosporin (CSA), Outcome 7 Disease activity (SLEDAI).

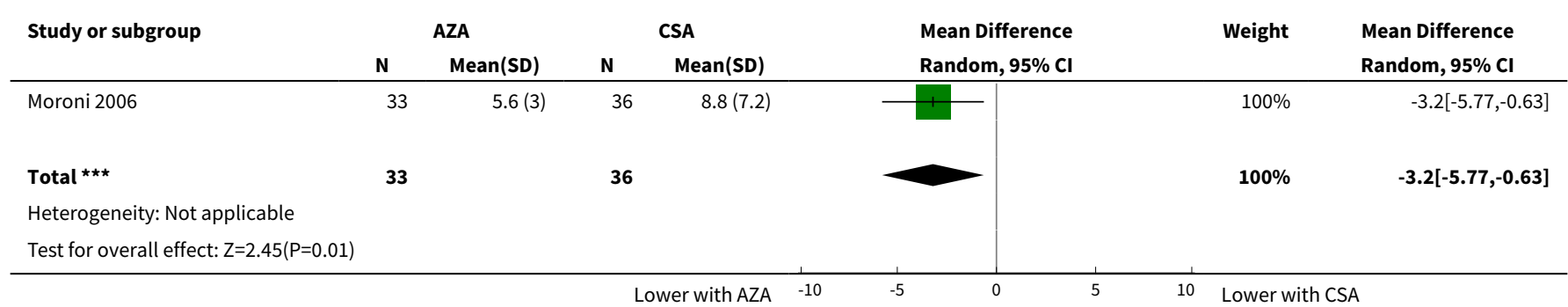


Comparison 28. Maintenance: azathioprine (AZA) versus cyclophosphamide (CPA)

\begin{tabular}{lllll}
\hline $\begin{array}{l}\text { Outcome or subgroup } \\
\text { title }\end{array}$ & No. of studies & $\begin{array}{l}\text { No. of partici- } \\
\text { pants }\end{array}$ & Statistical method & Effect size \\
\hline $\begin{array}{llll}\text { 1 Death } \\
\text { 2 Adverse renal out- } \\
\text { comes }\end{array}$ & 1 & 39 & Risk Ratio (M-H, Random, 95\% Cl) & $0.12[0.01,2.03]$ \\
\hline $\begin{array}{l}2.1 \text { ESKD } \\
\text { 2.2 Renal relapse }\end{array}$ & 1 & Risk Ratio (M-H, Random, 95\% Cl) & Subtotals only \\
\hline $\begin{array}{l}\text { 2.3 Doubling of serum } \\
\text { creatinine }\end{array}$ & 1 & 39 & Risk Ratio (M-H, Random, 95\% Cl) & $0.35[0.04,3.09]$ \\
\hline $\begin{array}{l}\text { 3 Bladder toxicity } \\
\text { 4 Creatinine clearance }\end{array}$ & 1 & 39 & Risk Ratio (M-H, Random, 95\% Cl) & $0.79[0.34,1.85]$ \\
\hline
\end{tabular}

Analysis 28.1. Comparison 28 Maintenance: azathioprine (AZA) versus cyclophosphamide (CPA), Outcome 1 Death.

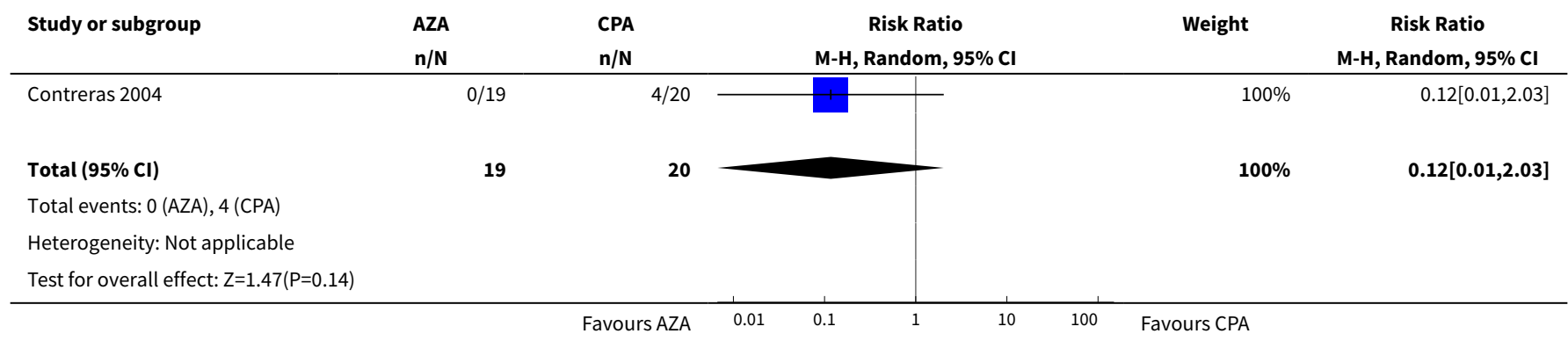

Analysis 28.2. Comparison 28 Maintenance: azathioprine (AZA) versus cyclophosphamide (CPA), Outcome 2 Adverse renal outcomes.

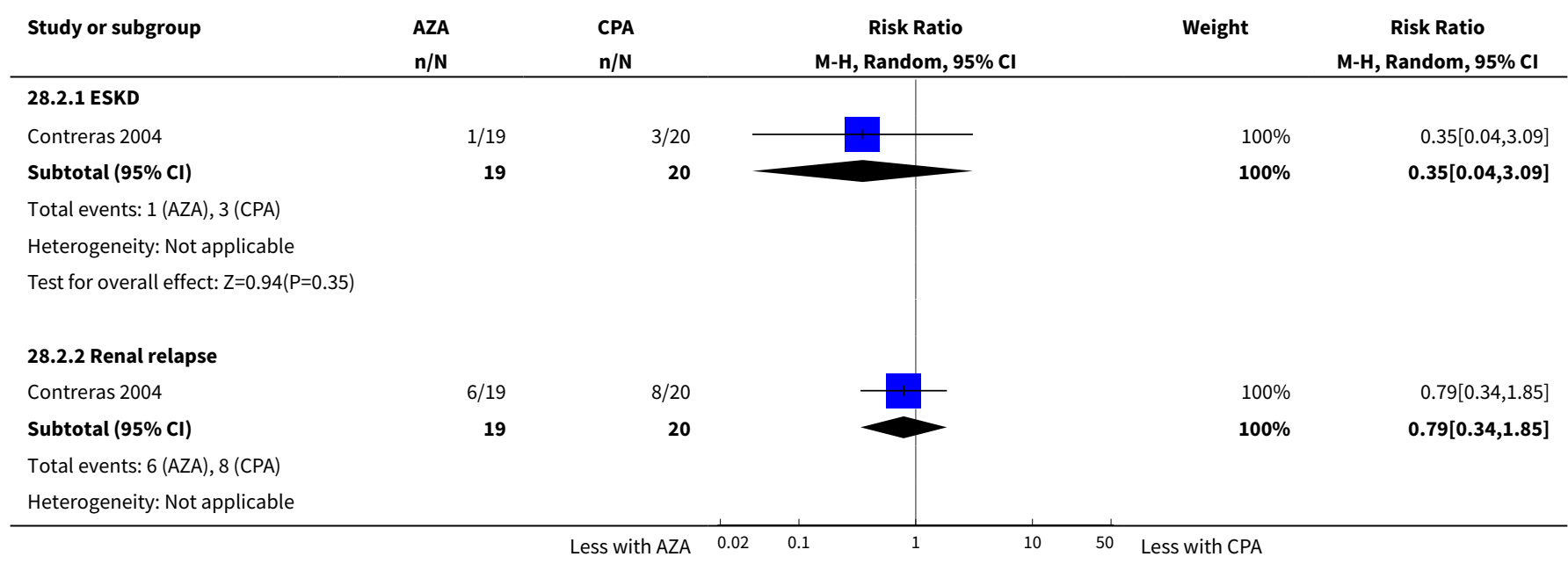




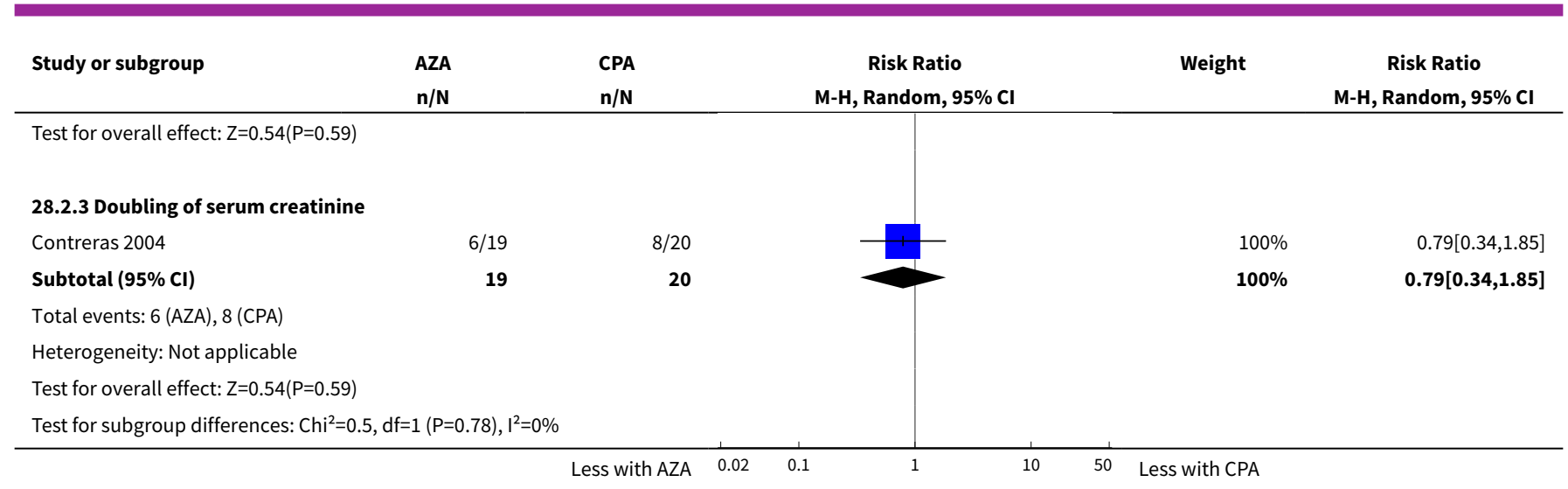

Analysis 28.3. Comparison 28 Maintenance: azathioprine (AZA) versus cyclophosphamide (CPA), Outcome 3 Bladder toxicity.

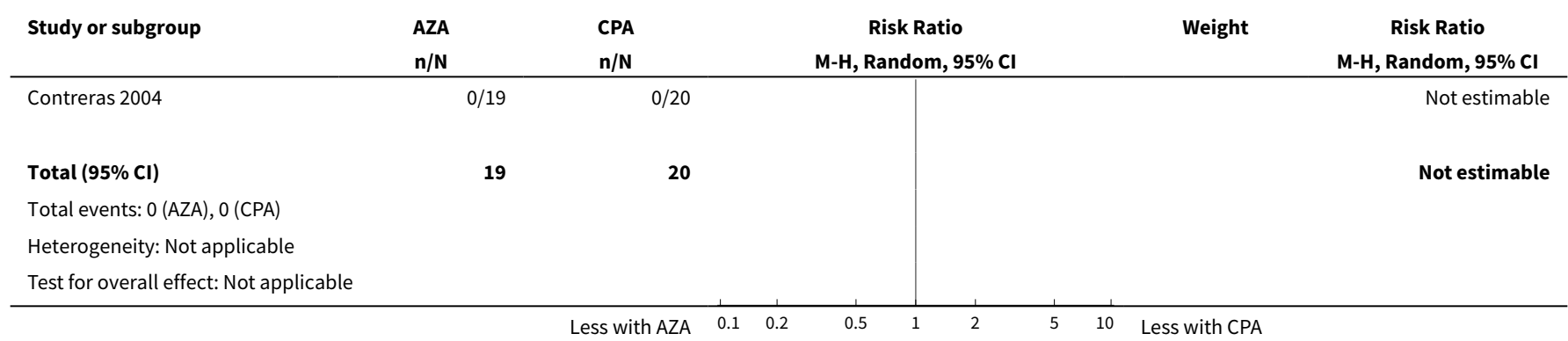

Analysis 28.4. Comparison 28 Maintenance: azathioprine (AZA) versus cyclophosphamide (CPA), Outcome 4 Creatinine clearance.

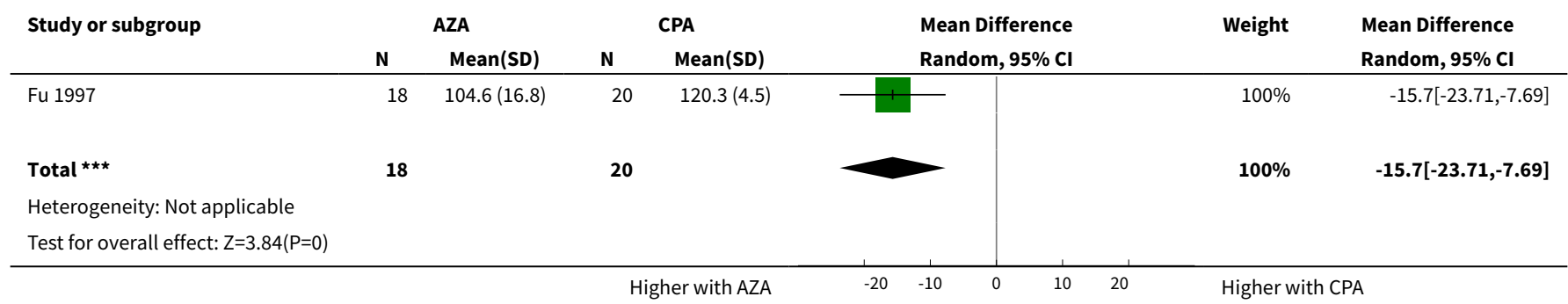

\section{Comparison 29. Maintenance: azathioprine (AZA) versus tacrolimus (TAC)}

\begin{tabular}{llllll}
\hline Outcome or subgroup title & No. of studies & $\begin{array}{l}\text { No. of partici- } \\
\text { pants }\end{array}$ & Statistical method & Effect size \\
\hline 1 Adverse renal outcomes & 1 & & Risk Ratio (M-H, Random, 95\% Cl) & Subtotals only \\
\hline 1.1 Renal relapse & 1 & 70 & Risk Ratio (M-H, Random, 95\% Cl) & $6.62[0.35,123.63]$ \\
\hline
\end{tabular}




\begin{tabular}{lllll}
\hline Outcome or subgroup title & No. of studies & $\begin{array}{l}\text { No. of partici- } \\
\text { pants }\end{array}$ & Statistical method & Effect size \\
\hline 2 Infection & 1 & & Risk Ratio (M-H, Random, 95\% Cl) & Subtotals only \\
\hline $\begin{array}{lllll}2.1 \text { Major infection } \\
\text { 3 Gastrointestinal (GI) ad- }\end{array}$ & 1 & 70 & Risk Ratio (M-H, Random, 95\% Cl) & $1.26[0.30,5.22]$ \\
\hline $\begin{array}{l}\text { verse events } \\
\text { 3.1 Gl disturbance }\end{array}$ & 1 & Risk Ratio (M-H, Random, 95\% Cl) & Subtotals only \\
\hline
\end{tabular}

Analysis 29.1. Comparison 29 Maintenance: azathioprine (AZA) versus tacrolimus (TAC), Outcome 1 Adverse renal outcomes.

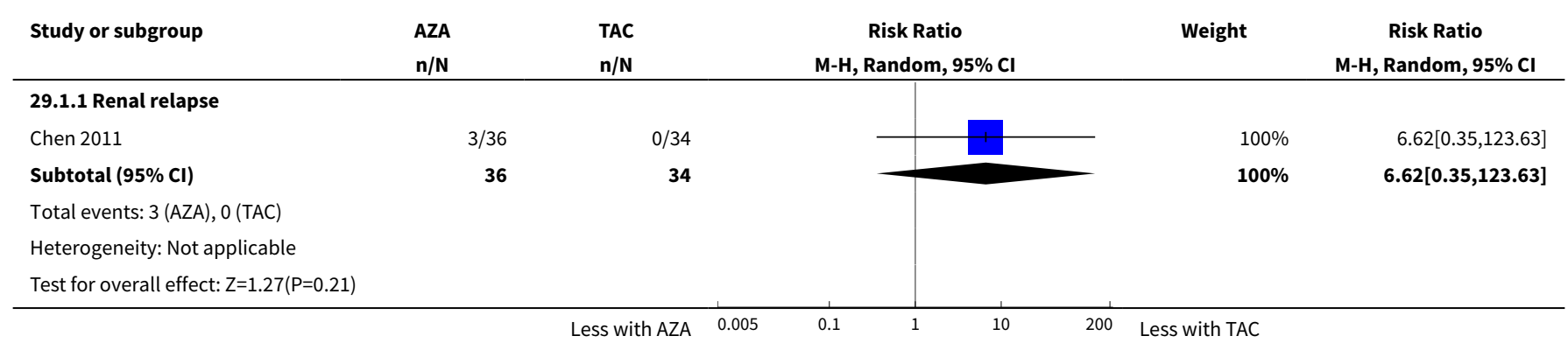

Analysis 29.2. Comparison 29 Maintenance: azathioprine (AZA) versus tacrolimus (TAC), Outcome 2 Infection.

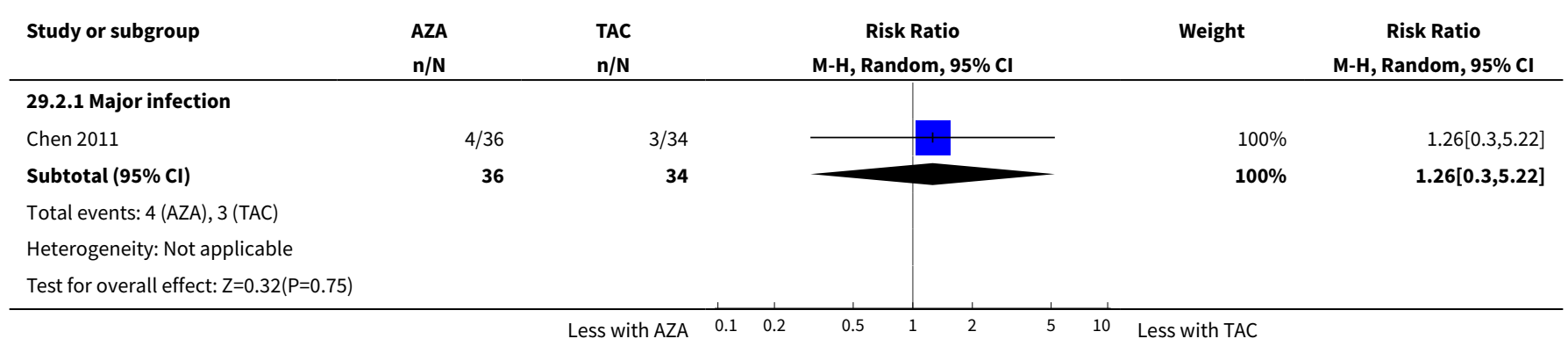

Analysis 29.3. Comparison 29 Maintenance: azathioprine (AZA) versus tacrolimus (TAC), Outcome 3 Gastrointestinal (GI) adverse events.

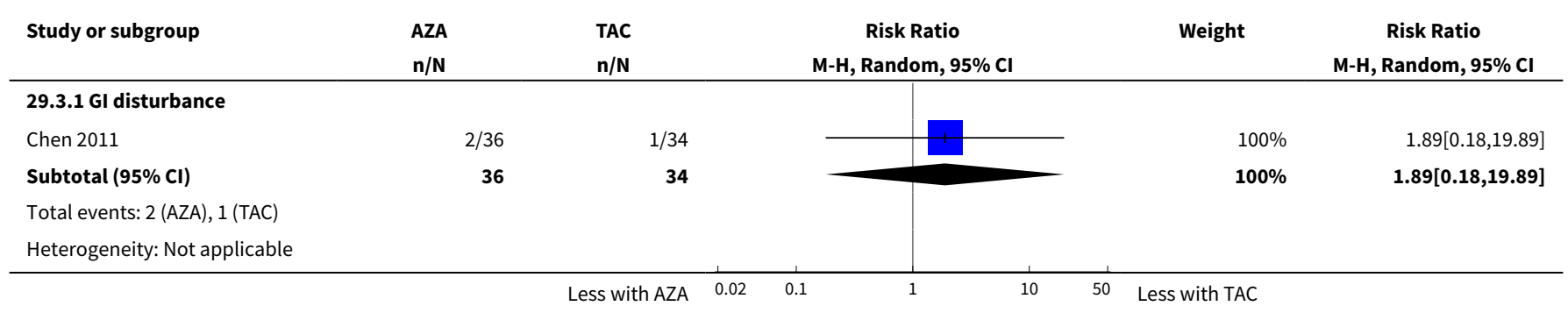




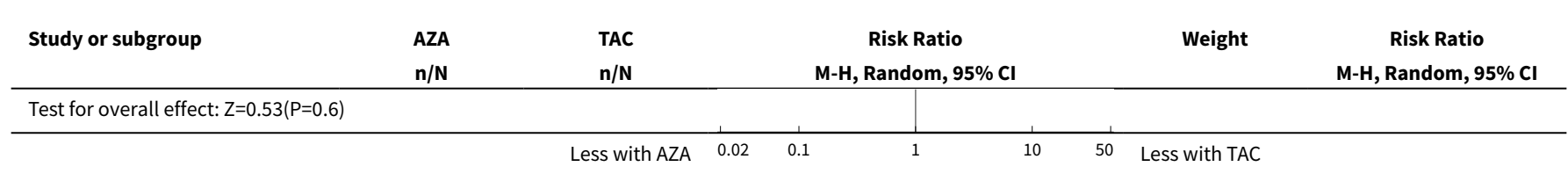

\section{Comparison 30. Maintenance: prednisone withdrawal versus prednisone continuation}

\begin{tabular}{lllll}
\hline $\begin{array}{l}\text { Outcome or subgroup } \\
\text { title }\end{array}$ & No. of studies & $\begin{array}{l}\text { No. of partici- } \\
\text { pants }\end{array}$ & Statistical method & Effect size \\
\hline 1 Relapse & 1 & & Risk Ratio (M-H, Random, 95\% Cl) & Subtotals only \\
\hline 1.1 Renal relapse & 1 & 15 & Risk Ratio (M-H, Random, 95\% Cl) & $0.38[0.05,2.88]$ \\
\hline 1.2 Non-renal relapse & 1 & 15 & Risk Ratio (M-H, Random, 95\% Cl) & $0.38[0.02,7.96]$ \\
\hline 2 Major infection & 1 & 15 & Risk Ratio (M-H, Random, 95\% Cl) & $0.57[0.06,5.03]$ \\
\hline
\end{tabular}

Analysis 30.1. Comparison 30 Maintenance: prednisone withdrawal versus prednisone continuation, Outcome 1 Relapse.

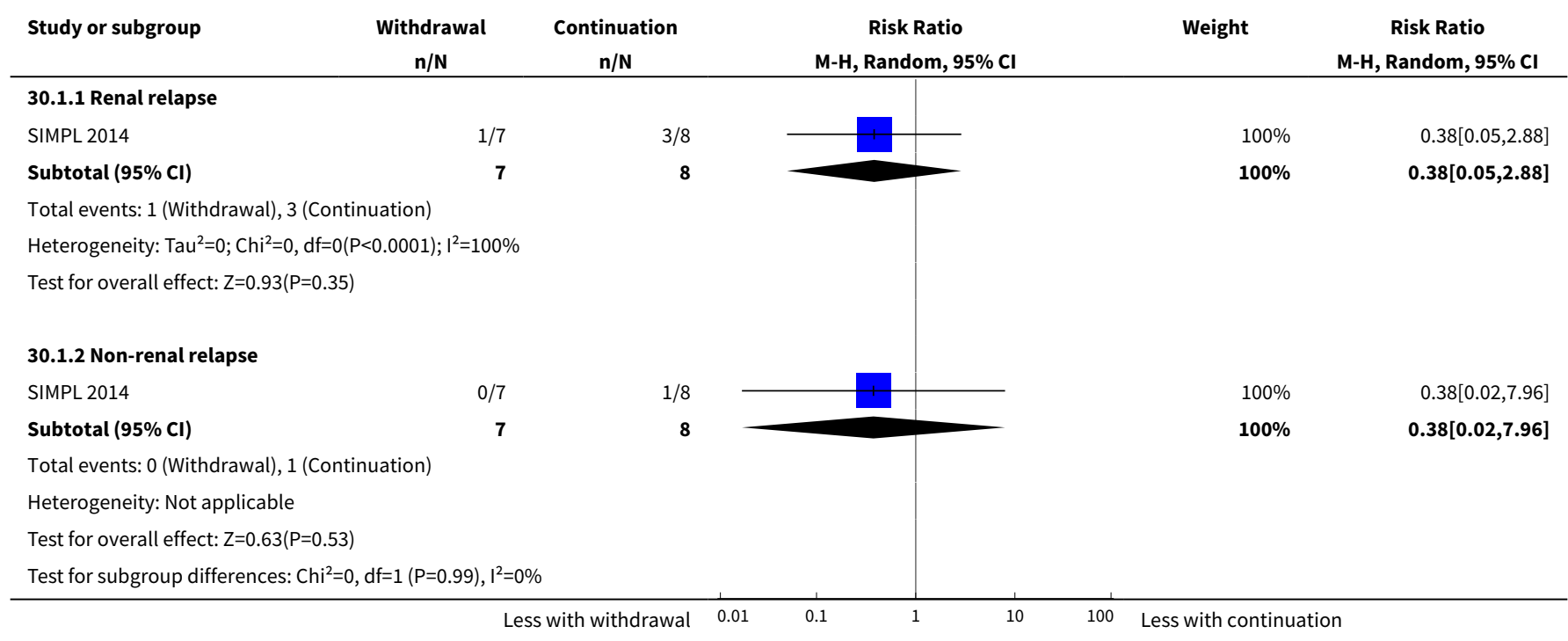

Analysis 30.2. Comparison 30 Maintenance: prednisone withdrawal versus prednisone continuation, Outcome 2 Major infection.

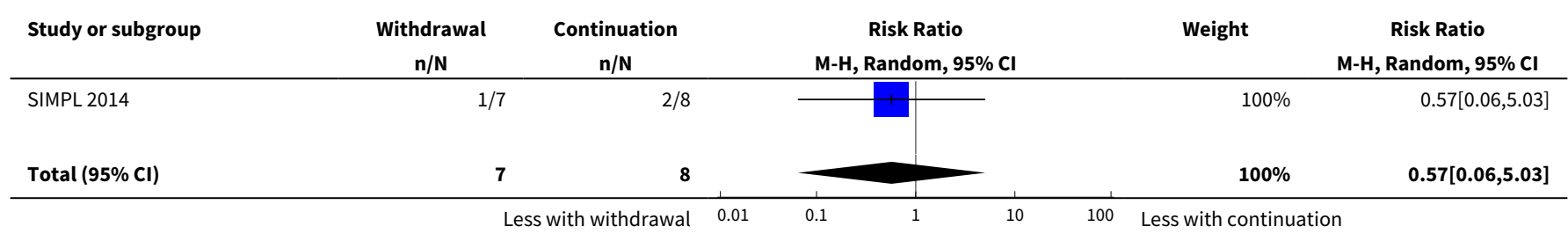




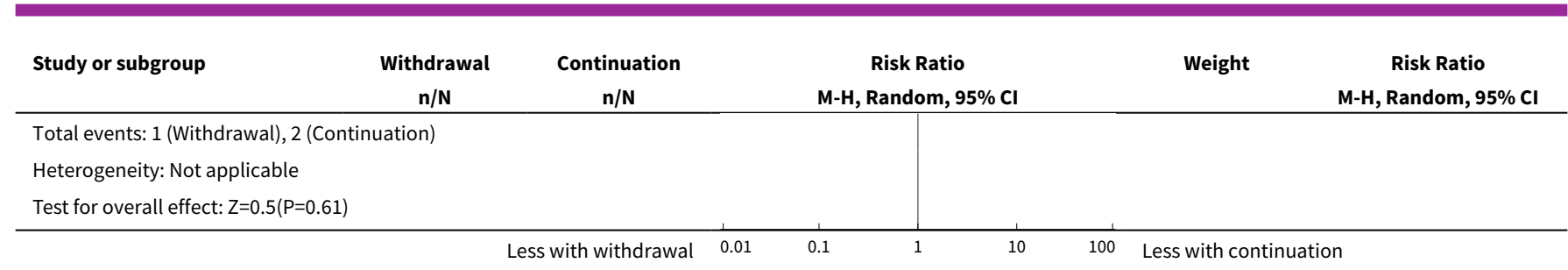

Comparison 31. Maintenance: intravenous immunoglobulin (IVIG) versus intravenous cyclophosphamide (IV CPA)

\begin{tabular}{lllll}
\hline $\begin{array}{l}\text { Outcome or subgroup } \\
\text { title }\end{array}$ & No. of studies & $\begin{array}{l}\text { No. of partici- } \\
\text { pants }\end{array}$ & Statistical method & Effect size \\
\hline 1 Creatinine clearance & 1 & 13 & Mean Difference (IV, Random, 95\% Cl) & $2.20[-37.85,42.25]$ \\
\hline 2 Daily proteinuria & 1 & 13 & Mean Difference (IV, Random, 95\% CI) & $-0.08[-0.95,0.79]$ \\
\hline 3 Serum creatinine & 1 & 14 & Mean Difference (IV, Random, 95\% Cl) & $-35.40[-128.90,58.10]$ \\
\hline
\end{tabular}

Analysis 31.1. Comparison 31 Maintenance: intravenous immunoglobulin (IVIG) versus intravenous cyclophosphamide (IV CPA), Outcome 1 Creatinine clearance.

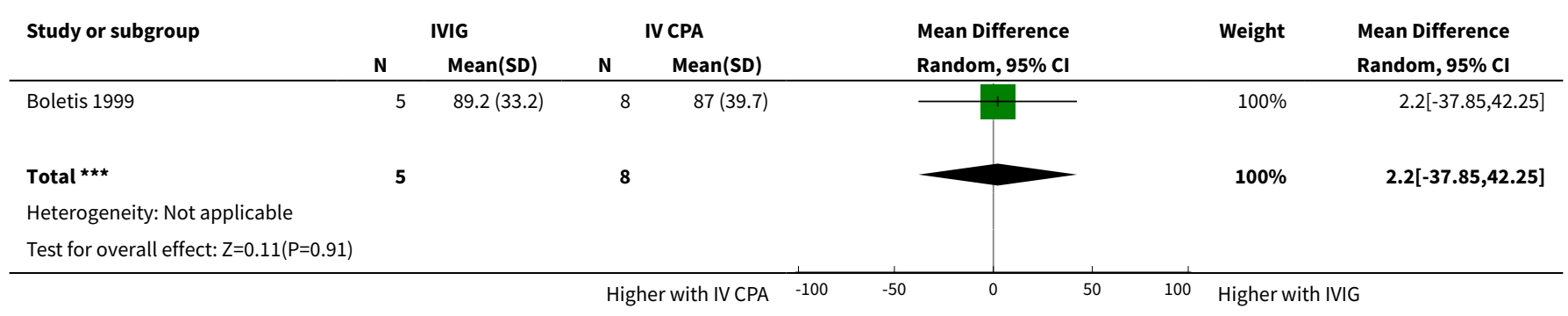

Analysis 31.2. Comparison 31 Maintenance: intravenous immunoglobulin (IVIG) versus intravenous cyclophosphamide (IV CPA), Outcome 2 Daily proteinuria.

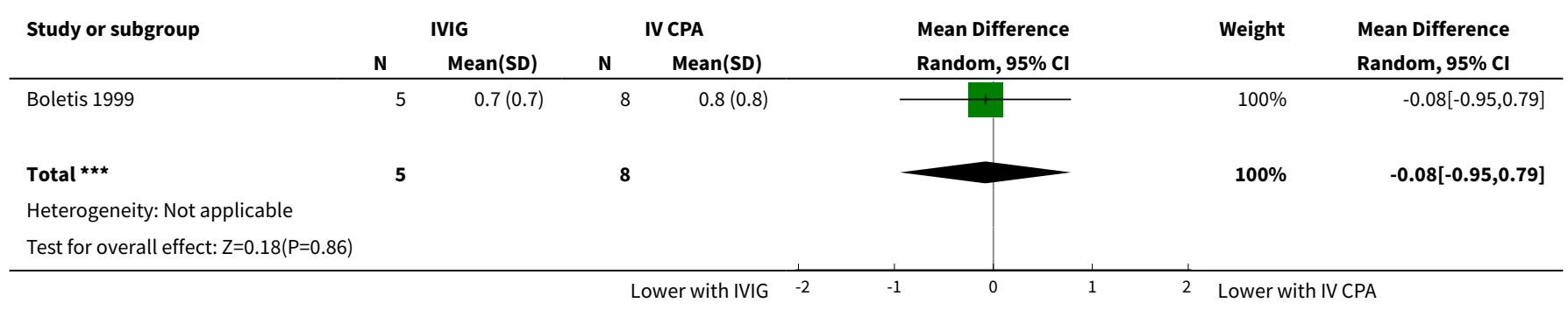


Analysis 31.3. Comparison 31 Maintenance: intravenous immunoglobulin (IVIG) versus intravenous cyclophosphamide (IV CPA), Outcome 3 Serum creatinine.

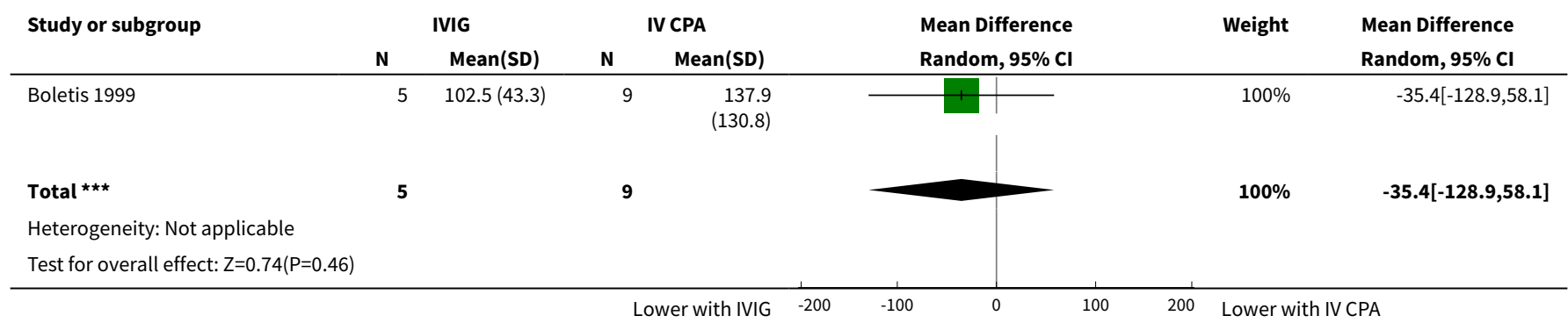

\section{ADDITIONAL TABLES}

Table 1. Description of health-related quality of life outcomes

\begin{tabular}{|c|c|c|c|c|c|}
\hline Study ID & Comparison & Therapy & Measure & Time point & Description of results \\
\hline ACCESS 2014 & $\begin{array}{l}\text { Abatacept ver- } \\
\text { sus placebo }\end{array}$ & Induction & $\begin{array}{l}\text { SF-36 physical } \\
\text { and mental } \\
\text { component } \\
\text { (mean } \pm \text { SD) }\end{array}$ & 6 months & $\begin{array}{l}\text { - In the abatacept group after } 6 \text { months of } \\
\text { therapy the physical component score in- } \\
\text { creased from } 39 \pm 11 \text { to } 45.3 \pm 11 \text {. In the } \\
\text { placebo + standard of care therapy group } \\
\text { after } 6 \text { months of therapy, the physical } \\
\text { component score increased from } 39 \pm 10 \\
\text { to } 46.5 \pm 11 \\
\text { - In the abatacept group after } 6 \text { months of } \\
\text { therapy the mental component score in- } \\
\text { creased from } 40 \pm 13 \text { to } 45.9 \pm 12 \text {. In the } \\
\text { placebo + standard of care group after } \\
6 \text { months of therapy, the mental compo- } \\
\text { nent score increased from } 40 \pm 13 \text { to } 46.5 \\
\pm 11\end{array}$ \\
\hline
\end{tabular}

\begin{tabular}{|c|c|c|c|}
\hline Furie 2014 & $\begin{array}{l}\text { Abatacept ver- } \\
\text { sus placebo }\end{array}$ & Induction & $\begin{array}{l}\text { SF-36 (ad- } \\
\text { justed mean }\end{array}$ \\
\hline
\end{tabular}

- In the high dose abatacept group after 12 months of therapy the adjusted mean \pm SE of SF-36 scores were: physical component $4.2 \pm 0.91$, mental component $2.5 \pm 1.0$, physical functioning $2.6 \pm 0.96$, role-physical $4.2 \pm 1.2$, bodily pain $4.5 \pm 1.1$, general health $4.7 \pm 0.9$, vitality $3.9 \pm 0.98$, social functioning $4.0 \pm 1.0$, role-emotional $1.6 \pm$ 1.3 , and mental health $3.1 \pm 1.1$

- In the low dose abatacept group after 12 months of therapy, the adjusted mean \pm SE of SF-36 scores were: physical component, $5.0 \pm 0.91$, mental component $4.7 \pm$ 1.0 , physical functioning $4.2 \pm 0.95$, rolephysical $6.9 \pm 1.2$, bodily pain $4.6 \pm 1.0$, general health $4.4 \pm 0.89$, vitality $4.6 \pm 0.97$, social functioning $6.1 \pm 1.0$, role-emotional $5.6 \pm 1.3$, and mental health $4.0 \pm 1.1$. In the placebo + standard of care group after 12 months of therapy, the adjusted mean \pm SE of SF-36 scores were: physical component $3.8 \pm 0.9$, mental component $4.4 \pm 1.0$, physical functioning $2.8 \pm 0.94$, role-phys- 
Table 1. Description of health-related quality of life outcomes (Continued)

ical $5.3 \pm 1.2$, bodily pain $4.3 \pm 1.0$, general health $4.0 \pm 0.88$, vitality $4.8 \pm 0.96$, social functioning $5.1 \pm 1.0$, role-emotional $4.7 \pm$ 1.3 , and mental health $3.2 \pm 1.1$

\begin{tabular}{|c|c|c|c|c|c|}
\hline LUNAR 2012 & $\begin{array}{l}\text { Rituximab ver- } \\
\text { sus placebo }\end{array}$ & Induction & $\begin{array}{l}\text { SF-36 - phys- } \\
\text { ical function- } \\
\text { ing (mean } \\
\text { change } \pm \text { SD) }\end{array}$ & 12 months & $\begin{array}{l}\text { - In the rituximab group after } 12 \text { months } \\
\text { of therapy the SF- } 36 \text { physical functioning } \\
\text { score increased by } 4.8 \pm 10.4 \\
\text { - In the placebo }+ \text { standard of care ther- } \\
\text { apy group, after } 12 \text { months of therapy } \\
\text { the SF- } 36 \text { physical functioning score in- } \\
\text { creased by } 5.7 \pm 9.4\end{array}$ \\
\hline
\end{tabular}

Table 2. Description of fatigue outcomes

\begin{tabular}{|c|c|c|c|c|c|}
\hline Study ID & Comparison & Therapy & Measure & Time point & Description of results \\
\hline \multirow[t]{6}{*}{ Furie 2014} & $\begin{array}{l}\text { Abatacept ver- } \\
\text { sus placebo }\end{array}$ & Induction & $\begin{array}{l}\text { Fatigue VAS } \\
\text { (adjusted } \\
\text { mean change }\end{array}$ & 6 months & $\begin{array}{l}\text { - In the high dose abatacept group after } \\
6 \text { months of therapy the fatigue VAS de- } \\
\text { creased by } 12.2 \pm 2.7\end{array}$ \\
\hline & & & $\pm \mathrm{SE})$ & & $\begin{array}{l}\text { - In the low dose abatacept group after } 6 \\
\text { months of therapy the fatigue VAS de- } \\
\text { creased by } 12.3 \pm 2.7\end{array}$ \\
\hline & & & & & $\begin{array}{l}\text { - In the placebo }+ \text { standard of care group af- } \\
\text { ter } 6 \text { months of therapy the fatigue VAS de- } \\
\text { creased by } 11.1 \pm 2.7\end{array}$ \\
\hline & & & $\begin{array}{l}\text { Fatigue sever- } \\
\text { ity score (ad- } \\
\text { justed mean }\end{array}$ & & $\begin{array}{l}\text { - In the high dose abatacept group after } \\
6 \text { months of therapy the fatigue VAS de- } \\
\text { creased by } 12.2 \pm 2.7\end{array}$ \\
\hline & & & change \pm SE) & & $\begin{array}{l}\text { - In the low dose abatacept group after } 6 \\
\text { months of therapy the fatigue VAS de- } \\
\text { creased by } 12.3 \pm 2.7\end{array}$ \\
\hline & & & & & $\begin{array}{l}\text { - In the placebo + standard of care group af- } \\
\text { ter } 6 \text { months of therapy the fatigue VAS de- } \\
\text { creased by } 11.1 \pm 2.7\end{array}$ \\
\hline
\end{tabular}

VAS - visual analogue scale

Table 3. Description of disease activity outcomes

\begin{tabular}{|c|c|c|c|c|}
\hline Study ID & Comparison & Measure & Time point & Description of results \\
\hline \multicolumn{5}{|c|}{ Induction therapy } \\
\hline ACCESS 2014 & $\begin{array}{l}\text { Abatacept versus } \\
\text { placebo }\end{array}$ & $\begin{array}{l}\text { BILAG (mean } \pm \\
\text { SD) }\end{array}$ & 6 months & $\begin{array}{l}\text { - In the placebo + standard of care therapy group } \\
\text { after } 6 \text { months of therapy the BILAG scores were } \\
3.4 \pm 1.8 \\
\text { - In the abatacept group after } 6 \text { months of therapy } \\
\text { the BILAG scores were } 3.8 \pm 3.0\end{array}$ \\
\hline ALMS 2007 & $\begin{array}{l}\text { MMF versus IV } \\
\text { CPA }\end{array}$ & $\begin{array}{l}\text { SLEDAI (mean } \\
\text { change } \pm \text { SD) }\end{array}$ & 6 months & $\begin{array}{l}\text { - In the IV CPA group after } 6 \text { months of therapy the } \\
\text { SLEDAI scores decreased by } 6.6 \pm 8.0\end{array}$ \\
\hline
\end{tabular}


Table 3. Description of disease activity outcomes (Continued)

- In the MMF group after 6 months of therapy the SLEDAl scores decreased by $6.2 \pm 10.1$

- The mean difference between the groups was 0.41 (95\% $\mathrm{Cl}-1.48$ to 2.30$)$

\begin{tabular}{llll}
\hline Deng 2016 & $\begin{array}{l}\text { Leflunomide ver- } \\
\text { sus CPA }\end{array}$ & SLEDAl & 6 months \\
\hline El-Shafey 2010 & $\begin{array}{l}\text { MMF versus IV } \\
\text { CPA }\end{array}$ & $\begin{array}{l}\text { SLAM (mean } \\
\text { change } \pm \text { SD) }\end{array}$ & 6 months
\end{tabular}

"SLEDAI scores were reduced"

- In the IV CPA group after 6 months of therapy SLAM scores decreased by $22.1 \pm 7.72$

- In the MMF group after 6 months of therapy SLAM scores decreased by $17.84 \pm 7.25$

\begin{tabular}{|c|c|c|c|c|}
\hline $\begin{array}{l}\text { Grootscholten } \\
2006\end{array}$ & $\begin{array}{l}\text { IV CPA versus } \\
\text { AZA }\end{array}$ & SLEDAI & 24 months & $\begin{array}{l}\text { "SLEDAI and VAS scores did not differ between } \\
\text { groups and decreased significantly and parallele } \\
\text { each other }(r=0.673, P<0.01) \text { " }\end{array}$ \\
\hline
\end{tabular}

\begin{tabular}{llll}
\hline Hong 2007 & TAC versus IC SLEDAl & 6 months & $\begin{array}{l}\text { "SLEDAl level of FK506 (TAC) group is better than } \\
\text { that of CPA group, }(\mathrm{P}<0.05) \text { " }\end{array}$
\end{tabular}

\begin{tabular}{lll}
\hline Houssiau 2002 & $\begin{array}{l}\text { High CPA versus } \\
\text { low CPA }\end{array}$ & 12 months
\end{tabular}

"ECLAM score significantly improved in both groups during the first year of follow-up. No significant difference was noted between patients in the low-dose and high-dose IV CYC groups for any of the parameters examined $(P>0.05)$ "

$\begin{array}{lll}\begin{array}{l}\text { Kamanamool MMF versus TAC } \\ 2017\end{array} & \begin{array}{l}\text { SLEDAI-2K (mean } 12 \text { months } \\ \pm \mathrm{SD})\end{array}\end{array}$
creased from $11.6 \pm 4.8$ to $6.3 \pm 3.9$ after 6 months
- In the MMF group, mean SLEDAI-2K was detherapy, and $5.4 \pm 4.4$ after 12 months

- In the TAC group, mean SLEDAI-2K was decreased from $9.0 \pm 3.7$ to $6.3 \pm 5.1$ after 6 months and to $7.1 \pm 5.4$ after 12 months

- The results showed a similar pattern with respect to renal SLEDAI and modified SLEDAI

\begin{tabular}{llll}
\hline Li 2009c & $\begin{array}{l}\text { Rituximab versus } \\
\text { rituximab + CPA }\end{array}$ & $\begin{array}{l}\text { SLEDAI (mean } \pm \\
\text { SD) }\end{array}$ & 12 months
\end{tabular}
rituximab + CPA SD)

- The overall SLEDAI of both groups at baseline was $9.2 \pm 3.4$, this decreased to $2.5 \pm 2.5$ after 12 months of therapy

- There was significant improvements in SLEDAI in both groups

\begin{tabular}{lll}
\hline Li 2012 & MMF versus TAC & SLEDAI (mean \pm \\
versus IV CPA & SD)
\end{tabular}

- In all three groups (IV CPA, MMF, TAC) after 6 months of therapy the SLEDAI across all three groups was $7.7 \pm 4.7$. In all three groups the SLEDAI scores decreased

\begin{tabular}{lll}
\hline Liu 2015 & $\begin{array}{l}\text { MMF + TAC ver- } \\
\text { Sus IV CPA }\end{array}$ & $\begin{array}{l}\text { SLEDAI (mean } \\
\text { change } \pm \text { SD) }\end{array}$
\end{tabular}

- In the IV CPA group after 6 months of therapy SLEDAI decreased by $11.01 \pm 6.07$

- In the MMF+TAC group after 6 months of therapy SLEDAI decreased by $8.55 \pm 5.05$

\begin{tabular}{lllll}
\hline Loo 2010 & PEX versus IA & SLEDAI & 6 months & $\begin{array}{l}\text { "The SLEDAl gap between the study groups re- } \\
\text { mained the same throughout the study. The im- } \\
\text { provements in SLEDAl score of both groups were } \\
\text { also significantly demonstrated." }\end{array}$ \\
\hline LUNAR 2012 & $\begin{array}{l}\text { Rituximab versus } \\
\text { placebo }\end{array}$ & $\begin{array}{l}\text { BILAG (Time ad- } \\
\text { justed area un- }\end{array}$ & 12 months & $\begin{array}{l}\text { In the rituximab group after } 12 \text { months of therapy } \\
\text { SLEDAl decreased to } 8.49 \pm 5.79\end{array}$ \\
\hline
\end{tabular}


Table 3. Description of disease activity outcomes (Continued)

der the curve

minus baseline

mean \pm SD)
- In the placebo + standard of care group after 12 months of therapy SLEDAI decreased to $8.58 \pm$ 5.14

\begin{tabular}{|c|c|c|c|c|}
\hline Mehra 2018 & $\begin{array}{l}\text { High-dose CPA } \\
\text { versus low-dose } \\
\text { CPA }\end{array}$ & Renal SLEDAI & 6 months & $\begin{array}{l}\text { At } 24 \text { weeks, renal SLEDAI were similar between } \\
\text { high-dose and low-dose cyclophosphamide }\end{array}$ \\
\hline \multirow[t]{2}{*}{ Mok 2016} & MMF versus TAC & $\begin{array}{l}\text { Renal SLEDAI } \\
\text { (mean } \pm \text { SD) }\end{array}$ & 6 months & $\begin{array}{l}\text { - In the MMF group after } 6 \text { months of therapy renal } \\
\text { SLEDAI scores were } 3.9 \pm 3.1 \\
\text { - In the tacrolimus group after } 6 \text { months of therapy } \\
\text { renal SLEDAI scores were } 3.3 \pm 3.1\end{array}$ \\
\hline & & $\begin{array}{l}\text { Extrarenal } \\
\text { SLEDAI (mean } \pm \\
\text { SD) }\end{array}$ & & $\begin{array}{l}\text { - In the MMF group after } 6 \text { months of therapy ex- } \\
\text { trarenal SLEDAI scores were } 1.7 \pm 1.9 \\
\text { - In the tacrolimus group after } 6 \text { months of therapy } \\
\text { extrarenal SLEDAI scores were } 1.9 \pm 1.7\end{array}$ \\
\hline \multirow[t]{2}{*}{ MyLupus 2011} & $\begin{array}{l}\text { Standard dose } \\
\text { PRED versus } \\
\text { reduced dose } \\
\text { PRED }\end{array}$ & $\begin{array}{l}\text { Global BILAG } \\
\text { (mean } \pm \text { SD) }\end{array}$ & 6 months & $\begin{array}{l}\text { For both groups (reduced dose and standard dose } \\
\text { corticosteroids) at the end of } 6 \text { months of treat- } \\
\text { ment global BILAG reduced from } 14 \pm 5.4 \text { to } 5.0 \pm \\
3.8(P<0.001)\end{array}$ \\
\hline & & $\begin{array}{l}\text { SLEDAI (mean } \pm \\
\text { SD) }\end{array}$ & & $\begin{array}{l}\text { For both groups (reduced dose and standard dose } \\
\text { corticosteroids) at the end of } 6 \text { months of treat- } \\
\text { ment SLEDAl reduced from } 16.2 \pm 6.9 \text { to } 6.2 \pm 5.1 \text { ( } P \\
<0.001 \text { ) }\end{array}$ \\
\hline Ong 2005 & $\begin{array}{l}\text { MMF versus IV } \\
\text { CPA }\end{array}$ & $\begin{array}{l}\text { SLEDAI (mean } \\
\text { change } \pm \text { SD) }\end{array}$ & 6 months & $\begin{array}{l}\text { - In the IV CPA group after } 6 \text { months of therapy } \\
\text { SLEDAI decreased by } 6.8 \pm 6.6 \\
\text { - In the MMF group after } 6 \text { months of therapy } \\
\text { SLEDAI decreased by }-7.2 \pm 7.7\end{array}$ \\
\hline
\end{tabular}

\begin{tabular}{|c|c|c|c|c|}
\hline Rathi 2016 & $\begin{array}{l}\text { MMF versus IV } \\
\text { CPA }\end{array}$ & SLEDAI & 6 months & $\begin{array}{l}\text { "SLEDAI improved significantly in both the groups } \\
\text { over the study period, and there were no differ- } \\
\text { ences between the treatment groups." }\end{array}$ \\
\hline \multirow[t]{2}{*}{ Rovin 2016} & $\begin{array}{l}\text { Sirukumab ver- } \\
\text { sus placebo }\end{array}$ & SLEDAI-2K & 6 months & $\begin{array}{l}\text { "Eighteen patients ( } 14 \text { in the sirukumab group and } \\
4 \text { in the placebo group) had a SLEDAI-2K RI-50 re- } \\
\text { sponse at any time through week } 24 . "\end{array}$ \\
\hline & & $\begin{array}{l}\text { Physician's and } \\
\text { patients global } \\
\text { assessment of } \\
\text { disease activity }\end{array}$ & & $\begin{array}{l}\text { "Neither the patient's nor the physician's global as- } \\
\text { sessment scores of disease activity showed notable } \\
\text { improvement over time in either treatment group } \\
\text { (data not shown)." }\end{array}$ \\
\hline Wallace 1998 & $\begin{array}{l}\text { PE versus stan- } \\
\text { dard of care }\end{array}$ & $\begin{array}{l}\text { SLAM (mean } \pm \\
\text { SD) }\end{array}$ & 12 months & $\begin{array}{l}\text { - In the standard of care group after } 12 \text { months of } \\
\text { therapy SLAM scores were } 6.44 \pm 4.16 \\
\text { - In the PEX group after } 12 \text { months of therapy SLAM } \\
\text { scores were } 7.11 \pm 4.78\end{array}$ \\
\hline
\end{tabular}

\section{Maintenance therapy}

\begin{tabular}{|c|c|c|c|c|}
\hline \multirow[t]{2}{*}{$\begin{array}{l}\text { MAINTAIN } \\
\text { Nephritis } 2010\end{array}$} & \multirow[t]{2}{*}{ AZA versus MMF } & SLEDAI & \multirow[t]{2}{*}{36 months } & \multirow[t]{2}{*}{$\begin{array}{l}\text { "SLEDAI and ECLAM scores decreased similarly in } \\
\text { both groups" }\end{array}$} \\
\hline & & ECLAM & & \\
\hline
\end{tabular}


Table 3. Description of disease activity outcomes (Continued)

$\begin{array}{lll}\text { Moroni } 2006 \quad \text { AZA versus CSA } & \text { SLEDAl (mean } \pm \\ & \text { SD) }\end{array} \quad 24$ months

- In the AZA group after 24 months of therapy SLEDAI scores were $5.6 \pm 3.0$

- In the CSA group after 24 months of therapy SLEDAI scores were $8.8 \pm 7.2$

AZA - azathioprine; BILAG - British Isles Lupus Assessment Group; CPA - cyclophosphamide; CSA - cyclosporin; ECLAM - European Consensus Lupus Activity Measurement; IA - immunoadsorption; MMF - mycophenolate mofetil; IV - intravenous; PE - plasma exchange; PEX plasmapheresis; PRED - corticosteroid; SLAM - Systemic Lupus Activity Measure; SLEDAI - Systemic Lupus Erythematosus Disease Activity Index; TAC - tacrolimus

\section{APPENDICES}

\section{Appendix 1. Electronic search strategies}

\begin{tabular}{ll}
\hline Database & Search terms \\
\hline MEDLINE & $\begin{array}{l}\text { 1. Lupus Nephritis/ } \\
\text { 2. lupus nephritis.tw } \\
\text { 3. or/1-2 }\end{array}$ \\
\hline CENTRAL & $\begin{array}{l}\text { 1. MeSH descriptor Lupus Nephritis, this term only } \\
\text { 2. (lupus):ti,ab,kw in Clinical Trials } \\
\text { 3. (\#1 OR \#2) }\end{array}$ \\
& $\begin{array}{l}\text { 1. exp Lupus Erythematosus Nephritis/ } \\
\text { 2. lupus nephritis.tw. } \\
\text { 3. or/1-2 }\end{array}$ \\
\hline
\end{tabular}

\section{Appendix 2. Risk of bias assessment tool}

\begin{tabular}{ll}
\hline Potential source of bias & Assessment criteria \\
\hline $\begin{array}{l}\text { Random sequence genera- } \\
\text { tion }\end{array}$ & $\begin{array}{l}\text { Low risk of bias: Random number table; computer random number generator; coin tossing; shuf- } \\
\text { fling cards or envelopes; throwing dice; drawing of lots; minimization (minimization may be imple- } \\
\text { mented without a random element, and this is considered to be equivalent to being random). }\end{array}$ \\
\cline { 2 - 2 } $\begin{array}{l}\text { Selection bias (biased alloca- } \\
\text { tion to interventions) due to } \\
\text { inadequate generation of a } \\
\text { randomised sequence }\end{array}$ & $\begin{array}{l}\text { High risk of bias: Sequence generated by odd or even date of birth; date (or day) of admission; se- } \\
\text { quence generated by hospital or clinic record number; allocation by judgement of the clinician; by } \\
\text { preference of the participant; based on the results of a laboratory test or a series of tests; by avail- } \\
\text { ability of the intervention. }\end{array}$ \\
\cline { 2 - 2 } & $\begin{array}{l}\text { Unclear: Insufficient information about the sequence generation process to permit judgement. } \\
\text { Allocation concealment }\end{array}$ \\
$\begin{array}{l}\text { Selection bias (biased alloca- } \\
\text { tion to interventions) due to } \\
\text { inadequate concealment of al- } \\
\text { locations prior to assignment }\end{array}$ & $\begin{array}{l}\text { Low risk of bias: Randomisation method described that would not allow investigator/participant to } \\
\text { allocation, including telephone, web-based, and pharmacy-controlled, randomisation; sequential- } \\
\text { ly numbered drug containers of identical appearance; sequentially numbered, opaque, sealed en- } \\
\text { velopes). }\end{array}$ \\
\cline { 2 - 2 }
\end{tabular}


High risk of bias: Using an open random allocation schedule (e.g. a list of random numbers); assignment envelopes were used without appropriate safeguards (e.g. if envelopes were unsealed or non-opaque or not sequentially numbered); alternation or rotation; date of birth; case record number; any other explicitly unconcealed procedure.

Unclear: Randomisation stated but no information on method used is available.

\section{Blinding of participants and personnel}

Performance bias due to knowledge of the allocated interventions by participants and personnel during the study
Low risk of bias: No blinding or incomplete blinding, but the review authors judge that the outcome is not likely to be influenced by lack of blinding; blinding of participants and key study personnel ensured, and unlikely that the blinding could have been broken.

High risk of bias: No blinding or incomplete blinding, and the outcome is likely to be influenced by lack of blinding; blinding of key study participants and personnel attempted, but likely that the blinding could have been broken, and the outcome is likely to be influenced by lack of blinding.

Unclear: Insufficient information to permit judgement

\section{Blinding of outcome assess- ment}

Detection bias due to knowledge of the allocated interventions by outcome assessors.
Low risk of bias: No blinding of outcome assessment, but the review authors judge that the outcome measurement is not likely to be influenced by lack of blinding; blinding of outcome assessment ensured, and unlikely that the blinding could have been broken.

High risk of bias: No blinding of outcome assessment, and the outcome measurement is likely to be influenced by lack of blinding; blinding of outcome assessment, but likely that the blinding could have been broken, and the outcome measurement is likely to be influenced by lack of blinding.

Unclear: Insufficient information to permit judgement

\section{Incomplete outcome data}

Attrition bias due to amount, nature or handling of incomplete outcome data.

Low risk of bias: No missing outcome data; reasons for missing outcome data unlikely to be related to true outcome (for survival data, censoring unlikely to be introducing bias); missing outcome data balanced in numbers across intervention groups, with similar reasons for missing data across groups; for dichotomous outcome data, the proportion of missing outcomes compared with observed event risk not enough to have a clinically relevant impact on the intervention effect estimate; for continuous outcome data, plausible effect size (difference in means or standardized difference in means) among missing outcomes not enough to have a clinically relevant impact on observed effect size; missing data have been imputed using appropriate methods.

High risk of bias: Reason for missing outcome data likely to be related to true outcome, with either imbalance in numbers or reasons for missing data across intervention groups; for dichotomous outcome data, the proportion of missing outcomes compared with observed event risk enough to induce clinically relevant bias in intervention effect estimate; for continuous outcome data, plausible effect size (difference in means or standardized difference in means) among missing outcomes enough to induce clinically relevant bias in observed effect size; 'as-treated' analysis done with substantial departure of the intervention received from that assigned at randomisation; potentially inappropriate application of simple imputation.

Unclear: Insufficient information to permit judgement

\section{Selective reporting}

Reporting bias due to selective outcome reporting
Low risk of bias: The study protocol is available and all of the study's pre-specified (primary and secondary) outcomes that are of interest in the review have been reported in the pre-specified way; the study protocol is not available but it is clear that the published reports include all expected outcomes, including those that were pre-specified (convincing text of this nature may be uncommon).

High risk of bias: Not all of the study's pre-specified primary outcomes have been reported; one or more primary outcomes is reported using measurements, analysis methods or subsets of the data (e.g. subscales) that were not pre-specified; one or more reported primary outcomes were not prespecified (unless clear justification for their reporting is provided, such as an unexpected adverse effect); one or more outcomes of interest in the review are reported incompletely so that they can- 
not be entered in a meta-analysis; the study report fails to include results for a key outcome that would be expected to have been reported for such a study.

Unclear: Insufficient information to permit judgement

\section{Other bias}

Bias due to problems not covered elsewhere in the table
Low risk of bias: The study appears to be free of other sources of bias.

High risk of bias: Had a potential source of bias related to the specific study design used; stopped early due to some data-dependent process (including a formal-stopping rule); had extreme baseline imbalance; has been claimed to have been fraudulent; had some other problem.

Unclear: Insufficient information to assess whether an important risk of bias exists; insufficient rationale or evidence that an identified problem will introduce bias.

WHAT'S NEW

\begin{tabular}{lll}
\hline Date & Event & Description \\
\hline 20 June 2018 & $\begin{array}{l}\text { New citation required and conclusions } \\
\text { have changed }\end{array}$ & New studies incorporated \\
\hline 20 June 2018 & New search has been performed & Review updated; 26 new studies added \\
\hline
\end{tabular}

\section{H ISTORY}

Protocol first published: Issue 1, 2001

Review first published: Issue 1, 2004

\begin{tabular}{lll}
\hline Date & Event & Description \\
\hline 7 November 2012 & $\begin{array}{l}\text { New citation required and conclusions } \\
\text { have changed }\end{array}$ & New studies, interventions and authors \\
\hline 7 November 2012 & New search has been performed & Review updated; 25 new studies added \\
\hline 15 October 2008 & Amended & Converted to new review format. \\
\hline
\end{tabular}

\section{CONTRIBUTIONS OF AUTHORS}

The work of this review update has been in the main conducted by David Tunnicliffe and Suetonia Palmer.

Each author individually contributed the following:

- David J Tunnicliffe: conduct data analysis, author

- Suetonia C Palmer: conduct data analysis, author

- Lorna Henderson: 2012 update design, analysis, reading drafts and co-author

- Philip Masson: 2012 update design, analysis, reading drafts and co-author

- Jonathan C Craig: reading drafts and co-author

- Allison Tong: reading drafts and co-author

- Davinder Singh-Grewal: reading drafts and co-author 
- Robert Flanc: original design and author

- Matthew Roberts: original design and author

- Angela Webster: 2012 update design, analysis, reading drafts and co-author

- Giovanni FM Strippoli: conduct data analysis, reading drafts, original design and author

\section{DECLARATIONS OF INTEREST}

None known.

\section{SOURCES OF SUPPORT}

\section{Internal sources}

- Cochrane Kidney and Transplant, Australia.

\section{External sources}

- Cochrane Review Support Programme 2017, UK.

\section{DIFFERENCES BETWEEN PROTOCOLANDREVIEW}

Risk of bias assessment tool has replaced quality assessment checklist.

\section{NOTES}

The numbering of comparisons for induction therapy in the data and analyses section is reflected throughout the main text.

\section{INDEX TERMS}

\section{Medical Subject Headings (MeSH)}

Azathioprine [adverse effects] [therapeutic use]; Calcineurin [therapeutic use]; Cyclophosphamide [adverse effects] [ ${ }^{\star}$ therapeutic use]; Glucocorticoids [adverse effects] [therapeutic use]; Immunosuppressive Agents [adverse effects] [*therapeutic use]; Induction Chemotherapy [methods]; Lupus Nephritis [*drug therapy]; Maintenance Chemotherapy [methods]; Mycophenolic Acid [ ${ }^{\star}$ analogs \& derivatives] [therapeutic use]; Randomized Controlled Trials as Topic; Recurrence; Tacrolimus [adverse effects] [therapeutic use]

\section{MeSH check words}

Adult; Child; Female; Humans; Male 